\title{
Towards Tetraradicaloid: The Effect of Fusion Mode on Radical Character and Chemical Reactivity
}

Pan Hu, ${ }^{\dagger}$ Sangsu Lee, ${ }^{\neq}$Tun Seng Herng ${ }^{\S}$ Naoki Aratani, $/$ Théo P. Gonçalves, ${ }^{\perp}$ Qingbiao Qi, ${ }^{\dagger}$ Xueliang Shi, ${ }^{+}$Hiroko Yamada,,$~ K u o-W e i$ Huang, ${ }^{\perp}$ Jun Ding, ${ }^{*}{ }^{\S}$ Dongho Kim, ${ }^{*, *}$ Jishan Wu ${ }^{*,+}, \#$

\section{Table of Contents}

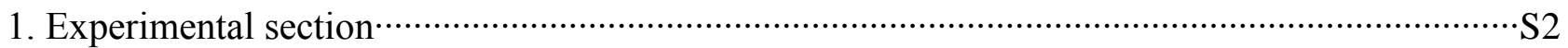

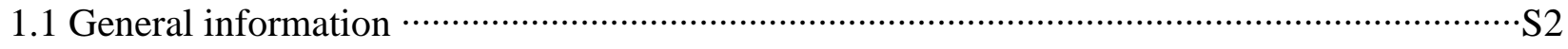

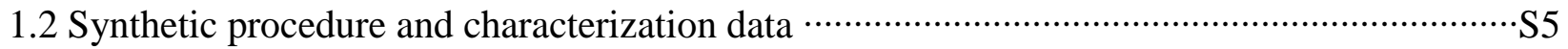

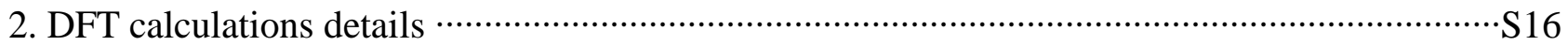

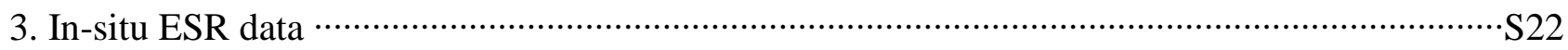

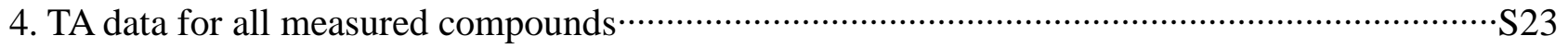

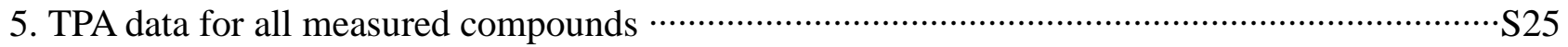

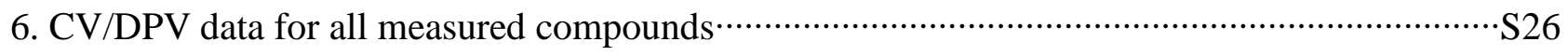

7. Additional optical data for those are not shown in the main manuscript ……........................S27

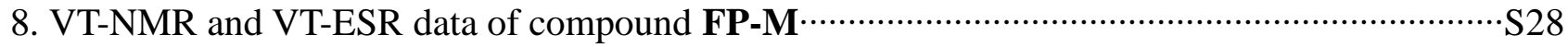

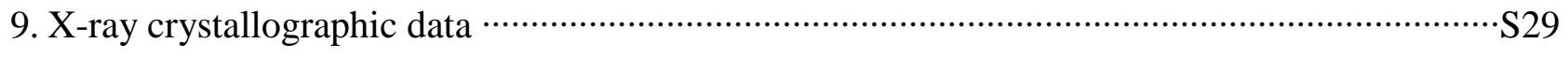

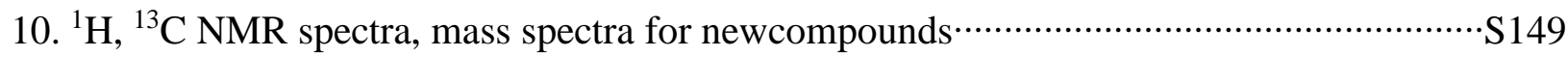

11. Appendix: energy, NICS data and Cartesian coordinates for all atoms of optimized geometries 


\section{Experimental Section}

\subsection{General information}

All reagents and starting materials were obtained from commercial suppliers and used without further purification. Anhydrous $N, N$-dimethylformamide (DMF) and dichloromathane (DCM) were distilled from $\mathrm{CaH}_{2}$. Anhydrous toluene and THF were distilled from sodium-benzophenone immediately prior to use. The ${ }^{1} \mathrm{H}$ NMR and ${ }^{13} \mathrm{C}$ NMR spectra were recorded in solution of $\mathrm{CDCl}_{3}, \mathrm{CD}_{2} \mathrm{Cl}_{2}$ and benzene-d ${ }^{6}$ on Bruker DPX 300/400/500 NMR spectrometers with tetramethylsilane (TMS) as the internal standard. The following abbreviations were used to explain the multiplicities: $\mathrm{s}=$ singlet, $\mathrm{d}=$ doublet, $\mathrm{t}=$ triplet, $\mathrm{m}=$ multiplet. HR-APCI mass spectra (MS) were recorded on a Bruker amazonX instrument. EI mass spectra were recorded on Agilent 5975C DIP/MS mass spectrometer. The solvents used for UV-vis and PL measurements are of HPLC grade (Merck). The electrochemical measurements were carried out in anhydrous methylene chloride with $0.1 \mathrm{M}$ tetrabutylammonium hexafluorophosphate $\left(n-\mathrm{Bu}_{4} \mathrm{NPF}_{6}\right)$ as the supporting electrolyte at room temperature under the protection of nitrogen. A gold disk was used as working electrode, platinum wire was used as counting electrode, and $\mathrm{Ag} / \mathrm{AgCl}(3 \mathrm{M} \mathrm{KCl}$ solution) as reference electrode. The potential was externally calibrated against the ferrocene/ferrocenium couple. Steady-state UV-vis absorption were recorded on a Shimadzu UV-1700 and UV-3600 spectrometer. Continuous wave X-band ESR spectra were obtained with a JEOL (FA200) spectrometer.

A superconducting quantum interference device magnetometer MPMS-XL was used for the magnetic characterization. Powder sample of FP-M with a weight of 5-10 mg was sealed in a plastic capsule. Magnetic moment was measured in the temperature range of 5 to $300 \mathrm{~K}$. The empty plastic capsule exhibited diamagnetic and its magnetic moment was measured for correction. After correction of diamagnetic signal of plastic capsule and sample holder, magnetic susceptibility was fitted with Bleaney-Bowers equation:

$$
\chi=\frac{N \beta^{2} g^{2}}{3 k T}\left[1+\frac{1}{3} \exp \left(\frac{J_{s-t}}{k T}\right)\right]^{-1}
$$

The femtosecond time-resolved transient absorption ( $f_{s}$-TA) spectrometer consists of an optical parametric amplifier (OPA; Palitra, Quantronix) pumped by a Ti:sapphire regenerative amplifier system (Integra-C, Quantronix) operating at $1 \mathrm{kHz}$ repetition rate and an optical detection system. The generated OPA pulses have a pulse width of $\sim 100 \mathrm{fs}$ and an average 
power of $1 \mathrm{~mW}$ in the range of $280-2700 \mathrm{~nm}$, which are used as pump pulses. White light continuum (WLC) probe pulses were generated using a sapphire window (3 $\mathrm{mm}$ thick) by focusing a small portion of the fundamental $800 \mathrm{~nm}$ pulses which was picked off by a quartz plate before entering the OPA. The time delay between pump and probe beams was carefully controlled by making the pump beam travel along a variable optical delay (ILS250, Newport). Intensities of the spectrally dispersed WLC probe pulses are monitored by a High Speed Spectrometer (Ultrafast Systems) for both visible and near-infrared measurements. To obtain the time-resolved transient absorption difference signal $(\Delta \mathrm{A})$ at a specific time, the pump pulses were chopped at $500 \mathrm{~Hz}$ and absorption spectra intensities were saved alternately with or without pump pulse. Typically, 4000 pulses excite the samples to obtain the fs-TA spectra at each delay time. The polarization angle between pump and probe beam was set at the magic angle $\left(54.7^{\circ}\right)$ using a Glan-laser polarizer with a half-wave retarder in order to prevent polarization-dependent signals. Cross-correlation fwhm in pump-probe experiments was less than $200 \mathrm{fs}$ and chirp of WLC probe pulses was measured to be $800 \mathrm{fs}$ in the $400-800 \mathrm{~nm}$ region. To minimize chirp, all reflection optics in the probe beam path and a quartz cell of $2 \mathrm{~mm}$ path length were used. After fs-TA experiments, the absorption spectra of all compounds were carefully examined to detect if there were artifacts due to degradation and photo-oxidation of samples. The three-dimensional data sets of $\Delta \mathrm{A}$ versus time and wavelength were subjected to singular value decomposition and global fitting to obtain the kinetic time constants and their associated spectra using Surface Xplorer software (Ultrafast Systems).

The two-photon absorption spectrum was measured in the NIR region using the open-aperture Z-scan method with 130 fs pulses from an optical parametric amplifier (Light Conversion, TOPAS) operating at a repetition rate of $1 \mathrm{kHz}$ generated from a Ti:sapphire regenerative amplifier system (Spectra-Physics, Hurricane). After passing through a $10 \mathrm{~cm}$ focal length lens, the laser beam was focused and passed through a $1 \mathrm{~mm}$ quartz cell. Since the position of the sample cell could be controlled along the laser beam direction ( $z$ axis) using the motor controlled delay stage, the local power density within the sample cell could be simply controlled under constant laser intensity. The transmitted laser beam from the sample cell was then detected by the same photodiode as used for reference monitoring. The on-axis peak intensity of the incident pulses at the focal point, $I_{0}$, ranged from 40 to $60 \mathrm{GW} \mathrm{cm}{ }^{-2}$. For a Gaussian beam profile, the nonlinear absorption coefficient can be obtained by curve fitting of 
the observed open-aperture traces $T(z)$ with the following equation:

$$
T(z)=1-\frac{\beta I_{0}\left(1-e^{-\alpha_{0} l}\right)}{2 \alpha_{0}\left[1+\left(z / z_{0}\right)^{2}\right]}
$$

where $\alpha_{0}$ is the linear absorption coefficient, $l$ is the sample length, and $z_{0}$ is the diffraction length of the incident beam. After the nonlinear absorption coefficient has been obtained, the TPA cross section $\sigma^{(2)}$ of one solute molecule (in units of GM, where $1 \mathrm{GM}=10^{-50} \mathrm{~cm}^{4} \mathrm{~s}$ photon molecule ${ }^{-1}$ ) can be determined by using the following relationship:

$$
\beta=\frac{10^{-3} \sigma^{(2)} N_{A} d}{h v}
$$

where $N_{\mathrm{A}}$ is the Avogadro constant, $d$ is the concentration of the compound in solution, $h$ is the Planck constant, and $v$ is the frequency of the incident laser beam. 


\subsection{Synthetic procedure and characterization data}

Compound 1<smiles>CCCCCOc1ccc2ccccc2c1-c1cc(C=O)c(Br)cc1C=O</smiles>

A mixture of compound 2,5-dibromobenzene-1,4-dicarboxaldehyde (4.38 g, $15.0 \mathrm{mmol})$, (2-(hexyloxy)naphthalen-1-yl)boronic acid (1.36 g, $5.0 \mathrm{mmol}), \mathrm{Pd}\left(\mathrm{PPh}_{3}\right)_{4}(0.87 \mathrm{mg}, 0.75 \mathrm{mmol})$, $\mathrm{P}(o \text {-tol })_{3}(91.20 \mathrm{mg}, 0.30 \mathrm{mmol})$ in toluene $(150 \mathrm{~mL})$, ethanol $(20 \mathrm{~mL})$ and $\mathrm{Na}_{2} \mathrm{CO}_{3}$ aqueous solution ( $2 \mathrm{M}, 20 \mathrm{~mL}$ ) was degassed by three freeze-pump-thaw cycles. The reaction mixture was heated at $105{ }^{\circ} \mathrm{C}$ for two days under nitrogen. After cooling to room temperature, the mixture was washed with water and extracted with DCM. The organic layer was dried over anhydrous $\mathrm{MgSO}_{4}$. The solvent was removed under vacuum and the residue was purified by silica gel column chromatography using DCM/hexane $(1 / 2, \mathrm{v} / \mathrm{v})$ as eluent to give compound $\mathbf{1}$ $(0.42 \mathrm{~g})$ as a yellow oil in $19 \%$ yield. ${ }^{1} \mathrm{H} \mathrm{NMR}\left(\mathrm{CD}_{2} \mathrm{Cl}_{2}, 300 \mathrm{MHz}\right) \delta \mathrm{ppm}: 10.47(\mathrm{~s}, 1 \mathrm{H}), 9.64(\mathrm{~s}$, $1 \mathrm{H}), 8.37(\mathrm{~s}, 1 \mathrm{H}), 8.00(\mathrm{~d}, J=9.0 \mathrm{~Hz}, 1 \mathrm{H}), 7.95(\mathrm{~s}, 1 \mathrm{H}), 7.89-7.86(\mathrm{~m}, 1 \mathrm{H}), 7.40-7.34(\mathrm{~m}, 4 \mathrm{H})$, 4.12-3.97 (m, 2H), 1.59-1.55 (m, 2H), 1.20-1.17 (m, 4H), 0.87-0.82 (t, 3H); ${ }^{13} \mathrm{C} \mathrm{NMR}\left(\mathrm{CD}_{2} \mathrm{Cl}_{2}\right.$, $75 \mathrm{MHz}) \delta$ ppm: $191.04(\mathrm{C}=\mathrm{O}), 190.57(\mathrm{C}=\mathrm{O}), 153.93,139.83,139.71,136.52,134.37,133.44$, 132.33, 131.15, 129.09, 128.39, 127.46, 125.56, 124.22, 124.06, 117.95, 113.97, 69.43, 31.38, 29.20, 25.54, 22.57, 13.82. HR-MS (ESI): caldc for $\mathrm{C}_{24} \mathrm{H}_{22} \mathrm{BrO}_{3}[\mathrm{M}-\mathrm{H}]^{+}$: 437.0758; found, 437.0754 (error: 0.8 ppm).

\section{Compound $\mathbf{3}$}

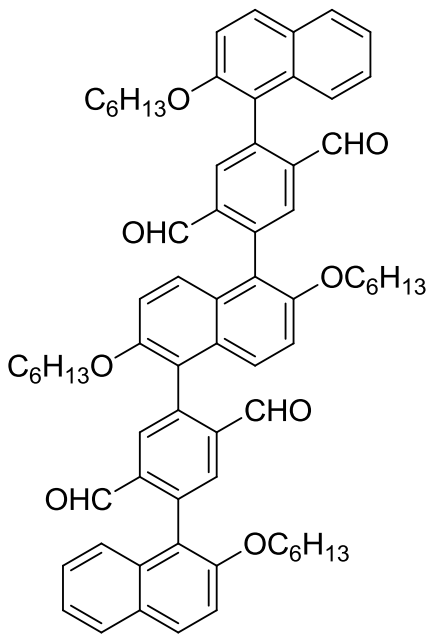


Compound 1 (1.31 g, 3.0 mmol), (2,6-bis(hexyloxy)naphthalene-1,5-diyl)diboronic acid (0.42 g, $1.0 \mathrm{mmol}), \mathrm{Pd}\left(\mathrm{PPh}_{3}\right)_{4}(57.78 \mathrm{mg}, 0.05 \mathrm{mmol})$ was added to the toluene $(25 \mathrm{~mL})$, ethanol $(3 \mathrm{~mL})$ and $\mathrm{K}_{2} \mathrm{CO}_{3}$ aqueous solution $(2 \mathrm{M}, 3 \mathrm{~mL}$ ). Following the similar procedure using DCM/hexane $(2 / 1, \mathrm{v} / \mathrm{v})$ as eluent gave compound $3(0.43 \mathrm{~g})$ as a yellow solid in $41 \%$ yield. ${ }^{1} \mathrm{H} \mathrm{NMR}\left(\mathrm{CDCl}_{3}\right.$, $300 \mathrm{MHz}): \delta$ 9.88-9.80 (m, 4H), 8.20-8.17 (m, 4H), 8.02 (d, $J=9.0 \mathrm{~Hz}, 2 \mathrm{H}), 7.93-7.90(\mathrm{~m}, 2 \mathrm{H})$, 7.69-7.59 (m, 2H), 7.53-7.29 (m, 10H), 4.20-3.98 (m, 8H), 1.70-1.58 (m, 8H), 1.29-1.24 (m,

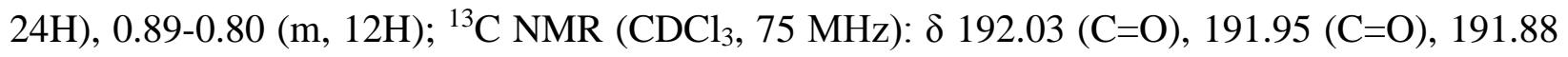
$(\mathrm{C}=\mathrm{O}), 153.82$, 152.44, 139.58, 139.52, 139.14, 138.45, 138.41, 138.33, 138.30, 133.60, 131.23, 131.13, 131.07, 130.70, 129.30, 129.01, 128.22, 127.21, 124.50, 123.91, 119.18, 115.41, 113.94, $113.85,69.33,69.25,69.14,50.74,31.45,31.30,29.22,29.15,29.06,25.56,25.46,25.33,22.50$, 22.40, 14.03, 13.90; HR-MS (APCI): caldc for $\mathrm{C}_{70} \mathrm{H}_{77} \mathrm{O}_{8}[\mathrm{M}+\mathrm{H}]^{+}$: 1045.5613; found, 1045.5615 (error: $-0.2 \mathrm{ppm})$.

\section{Compound HZD-M-ONE and HZD-F-ONE}

HZD-M-ONE: Mesitylmagnesium bromide $(1.0 \mathrm{M}, 2 \mathrm{ml})$ was added to the $15 \mathrm{ml}$ dry THF solution of compound $3(0.11 \mathrm{~g}, 0.10 \mathrm{mmol})$ under argon atmosphere, the mixture was stirred at room temperature overnight. The reaction mixture was quenched by water and extracted by chloroform. The organic layer was dried over $\mathrm{Na}_{2} \mathrm{SO}_{4}$ and the solvent was removed under reduced pressure. The crude product was then dissolved in $15 \mathrm{~mL}$ dry DCM under argon atmosphere and $0.1 \mathrm{~mL}$ of $\mathrm{BF}_{3} \cdot \mathrm{OEt}_{2}$ was added. The mixture was stirred for 5 minutes and quenched by methanol. The solvent was removed under reduced pressure. DDQ (45.40 g, 0.20 mmol) was added dropwise to the $20 \mathrm{~mL}$ dry toluene solution of compound $\mathbf{4 a}$. Upon addition of DDQ solution, the color of the reaction mixture changed quickly from yellow color to dark green, the mixture was allowed to stir for $1.5 \mathrm{~h}$. After evaporation of the solvent, the residue was purified by column chromatography (silica gel, hexane/ethyl acetate (4/1, v/v) as eluent) to give compound HZD-M-ONE (44.40 mg, 30\% in three steps) as a dark green solid. ${ }^{1} \mathrm{H}$ NMR $\left(\mathrm{CD}_{2} \mathrm{Cl}_{2}, 500 \mathrm{MHz}\right): \delta 10.45(\mathrm{~s}, 2 \mathrm{H}), 10.23(\mathrm{~s}, 2 \mathrm{H}), 8.54(\mathrm{~d}, J=15 \mathrm{~Hz}, 2 \mathrm{H}), 7.42-7.38(\mathrm{~m}, 4 \mathrm{H})$, $7.26(\mathrm{~s}, 4 \mathrm{H}), 7.17(\mathrm{~s}, 4 \mathrm{H}), 7.05(\mathrm{~s}, 2 \mathrm{H}), 6.50(\mathrm{~d}, J=15 \mathrm{~Hz}, 2 \mathrm{H}), 4.22-4.17(\mathrm{~m}, 4 \mathrm{H}), 3.88-3.83(\mathrm{~m}$, 4H), $2.56(\mathrm{~s}, 6 \mathrm{H}), 2.49(\mathrm{~s}, 6 \mathrm{H}), 1.95(\mathrm{~s}, 12 \mathrm{H}), 1.83(\mathrm{~s}, 12 \mathrm{H}), 1.52-1.50(\mathrm{~m}, 8 \mathrm{H}), 1.36-1.24(\mathrm{~m}$, 24H), 0.98-0.91 (m, 12H); ${ }^{13} \mathrm{C}$ NMR $\left(\mathrm{CD}_{2} \mathrm{Cl}_{2}, 125 \mathrm{MHz}\right): \delta 184.52(\mathrm{C}=\mathrm{O}), 161.92,156.09$, $139.57,137.80,137.37,137.30,136.06,134.65$, 134.56, 128.87, 128.41, 127.50, 123.50, 118.46, 
111.28, 104.64, 69.13, 68.04, 31.94, 31.72, 29.75, 28.28, 27.80, 25.57, 25.47, 22.94, 22.86, 21.24, 21.13, 20.02, 19.97, 13.98, 13.91, 0.83; HRMS (APCI, m/z): caldc for $\mathrm{C}_{106} \mathrm{H}_{111} \mathrm{O}_{6}[\mathrm{M}+1]^{+}$, 1479.8375; found, 1479.8385 (error $=-0.7 \mathrm{ppm}$ ).

HZD-F-ONE: Following the similar procedure to HZD-M-ONE, HZD-F-ONE was obtained as dark green solid in $28 \%$ yield. ${ }^{1} \mathrm{H}$ NMR $\left(\mathrm{CD}_{2} \mathrm{Cl}_{2}, 500 \mathrm{MHz}\right): \delta 10.25(\mathrm{~s}, 2 \mathrm{H}), 10.19(\mathrm{~s}, 2 \mathrm{H}), 8.63$ $(\mathrm{d}, J=8.7 \mathrm{~Hz}, 2 \mathrm{H}), 7.54(\mathrm{~d}, J=8.7 \mathrm{~Hz}, 2 \mathrm{H}), 7.40(\mathrm{~d}, J=10 \mathrm{~Hz}, 2 \mathrm{H}), 7.20(\mathrm{~s}, 2 \mathrm{H}), 6.68(\mathrm{~d}, J=$ $10 \mathrm{~Hz}, 2 \mathrm{H}), 4.37-4.34(\mathrm{~m}, 4 \mathrm{H}), 4.19-4.16(\mathrm{~m}, 4 \mathrm{H}), 1.73-1.66(\mathrm{~m}, 8 \mathrm{H}), 1.32-1.25(\mathrm{~m}, 24 \mathrm{H})$, 0.86-0.83 (m, 12H); ${ }^{13} \mathrm{C}$ NMR $\left(\mathrm{CD}_{2} \mathrm{Cl}_{2}, 125 \mathrm{MHz}\right): \delta 183.81(\mathrm{C}=\mathrm{O}), 161.94,157.01,137.45$, 131.20, 130.05, 129.45, 128.99, 128.38, 127.92, 127.01, 125.01, 123.51, 123.20, 118.59, 112.50, 105.00, 69.92, 69.62, 31.26, 29.57, 28.55, 28.31, 25.38, 25.28, 22.46, 13.51; HRMS (APCI, $m / z)$ : caldc for $\mathrm{C}_{94} \mathrm{H}_{67} \mathrm{~F}_{20} \mathrm{O}_{6}[\mathrm{M}+1]^{+}, 1671.4613$; found, 166.464 (error = - $0.1 \mathrm{ppm}$ ).

\section{Compound 5}

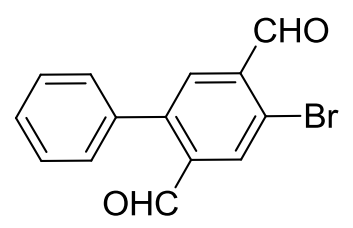

A mixture of 5-dibromobenzene-1,4-dicarboxaldehyde (11.68 g, $40.0 \mathrm{mmol}$ ), benzeneboronic acid $(1.94 \mathrm{~g}, 16.0 \mathrm{mmol}), \mathrm{Pd}\left(\mathrm{PPh}_{3}\right)_{4}(0.92 \mathrm{~g}, 0.80 \mathrm{mmol})$ in toluene $(200 \mathrm{~mL})$, ethanol $(30 \mathrm{~mL})$ and $\mathrm{Na}_{2} \mathrm{CO}_{3}$ aqueous solution $(2 \mathrm{M}, 30 \mathrm{~mL}$ ) was degassed by three freeze-pump-thaw cycles. The reaction mixture was heated at $105{ }^{\circ} \mathrm{C}$ overnight under nitrogen. After cooling to room temperature, the mixture was washed with water and extracted with DCM. The organic layer was dried over anhydrous $\mathrm{MgSO}_{4}$. The solvent was removed under vacuum and the residue was purified by silica gel column chromatography using DCM/hexane (1/1.5, v/v) as eluent to give the crude product as a yellow solid then recrystalliztion with ethanol for three times to get compound $5(0.69 \mathrm{~g})$ as a light yellow solid in $15 \%$ yield. ${ }^{1} \mathrm{H} \mathrm{NMR}\left(\mathrm{CDCl}_{3}, 300 \mathrm{MHz}\right) \delta \mathrm{ppm}$ : 10.45 (s, 1H), $9.97(\mathrm{~s}, 1 \mathrm{H}), 8.27(\mathrm{~s}, 1 \mathrm{H}), 8.00(\mathrm{~s}, 1 \mathrm{H}), 7.53-7.48(\mathrm{~m}, 3 \mathrm{H}), 7.41-7.35(\mathrm{~m}, 2 \mathrm{H}) .{ }^{13} \mathrm{C}$ NMR $\left(\mathrm{CDCl}_{3}, 75 \mathrm{MHz}\right) \delta$ ppm: $191.1(\mathrm{C}=\mathrm{O}), 190.3(\mathrm{C}=\mathrm{O}), 144.8,137.8,136.0,135.5,133.1$, 132.1, 129.8, 129.0, 128.8, 125.5. HR-MS (ESI): caldc for $\mathrm{C}_{14} \mathrm{H}_{8} \mathrm{BrO}_{2}[\mathrm{M}-\mathrm{H}]^{+}$: 286.9713; found, 286.9715 (error: $-0.7 \mathrm{ppm})$. 


\section{Compound 6}

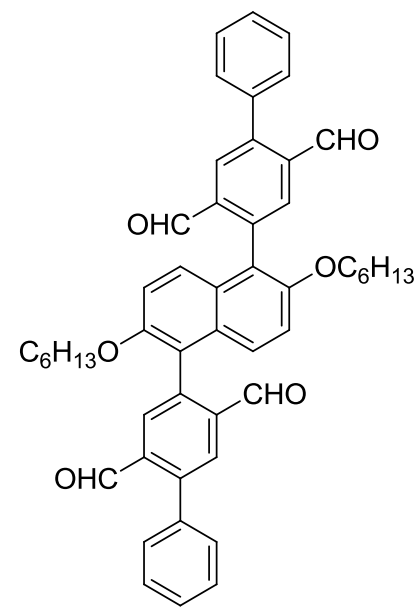

Compound 5 (0.87 g, $3.0 \mathrm{mmol})$, compound 2 (0.42 g, $1.0 \mathrm{mmol}), \mathrm{Pd}\left(\mathrm{PPh}_{3}\right)_{4}(58.0 \mathrm{mg}, 0.05$ mmol) was added to the toluene $(25 \mathrm{~mL})$, ethanol $(3 \mathrm{~mL}))$ and $\mathrm{K}_{2} \mathrm{CO}_{3}$ aqueous solution $(2 \mathrm{M}, 3$ $\mathrm{mL})$. Following a typical Suzuki coupling procedure as described before using DCM/hexane (1/2, v/v) as eluent gave compound $6(0.30 \mathrm{~g})$ as a yellow solid in $40 \%$ yield. ${ }^{1} \mathrm{H}$ NMR $\left(\mathrm{CD}_{2} \mathrm{Cl}_{2}, 300\right.$ MHz): $\delta 10.11(\mathrm{~s}, 1 \mathrm{H}), 9.79-9.76(\mathrm{~m}, 2 \mathrm{H}), 8.19(\mathrm{~s}, 2 \mathrm{H}), 8.02(\mathrm{~s}, 2 \mathrm{H}), 7.59-7.51(\mathrm{~m}, 12 \mathrm{H}), 7.31$ (d, $J=9 \mathrm{~Hz}, 2 \mathrm{H}), 4.06-3.91(\mathrm{~m}, 4 \mathrm{H}), 1.55-1.51(\mathrm{~m}, 4 \mathrm{H}), 1.20-1.13(\mathrm{~m}, 12 \mathrm{H}), 0.77(\mathrm{t}, J=6.4 \mathrm{~Hz}$, $6 \mathrm{H}) ;{ }^{13} \mathrm{C}$ NMR $\left(\mathrm{CD}_{2} \mathrm{Cl}_{2}, 75 \mathrm{MHz}\right): \delta 192.24(\mathrm{C}=\mathrm{O}), 192.20(\mathrm{C}=\mathrm{O}), 192.05(\mathrm{C}=\mathrm{O}), 152.99$, $145.25,139.60,138.74,137.34,137.25,132.43$, 130.59, 129.77, 129.10, 128.97, 127.77, 127.74, 119.97, 119.93, 115.92, 69.96, 69.91, 54.56, 54.20, 53.84, 53.48, 53.12, 31.69, 29.53, 25.82, 22.88, 14.09; HR-MS (EI): caldc for $\mathrm{C}_{50} \mathrm{H}_{48} \mathrm{O}_{6}[\mathrm{M}]^{+}$: 744.34509 ; found, 744.34697 (error: 2.53 ppm).

\section{Compound FP-M}

FP-M: Mesitylmagnesium bromide $(1.0 \mathrm{M}, 2 \mathrm{ml})$ was added to the $15 \mathrm{ml}$ dry THF solution of compound $6(74.40 \mathrm{mg}, 0.10 \mathrm{mmol})$ under argon atmosphere, and the mixture was stirred at room temperature overnight. The reaction mixture was quenched by water and extracted by ether. The organic layer was dried over $\mathrm{Na}_{2} \mathrm{SO}_{4}$ and the solvent was removed under reduced pressure. Then a $\mathrm{BF}_{3} \cdot \mathrm{OEt}_{2}$ catalyzed intramolecular Friedel-Crafts alkylation reaction was conducted to obtain the tetrahydro precursor 7a. DDQ $(45.40 \mathrm{~g}, 0.20 \mathrm{mmol})$ was added dropwise to the $20 \mathrm{ml}$ dry toluene solution of compound 7a. The mixture was heated to $80{ }^{\circ} \mathrm{C}$ and stirring for about 5 mins. The color of the reaction mixture changed from yellow color to dark green. The solvent 
was evaporated and the residue was purified by column chromatography (silica gel, hexane/DCM (4/1, v/v) as eluent) to give compound FP-M (45.1 mg, 39\% in three steps) as a dark green solid. HRMS (APCI, $m / z$ ): caldc for $\mathrm{C}_{86} \mathrm{H}_{85} \mathrm{O}_{2}[\mathrm{M}+1]^{+}, 1149.6544$; found, 1149.6519 (error $=+2.2 \mathrm{ppm})$. No NMR spectrum for aromatic protons can be recorded at room temperature due to the paramagnetic properties.

\section{Compound 9}

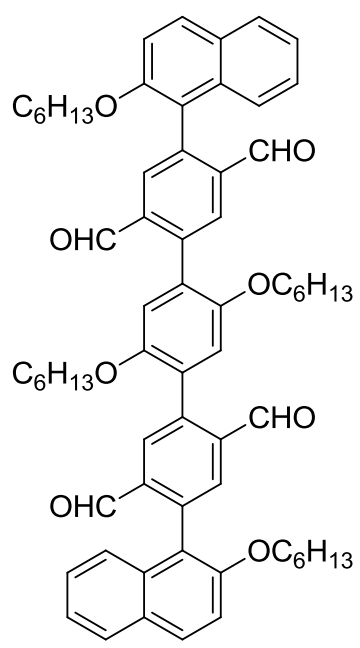

Compound 1 (1.31 g, $3.0 \mathrm{mmol})$, (2,5-bis(hexyloxy)-1,4-phenylene)diboronic acid (0.37 g, 1.0 $\mathrm{mmol}), \mathrm{Pd}\left(\mathrm{PPh}_{3}\right)_{4}(58.0 \mathrm{mg}, 0.05 \mathrm{mmol})$ was added to the toluene $(25 \mathrm{~mL})$, ethanol $(3 \mathrm{~mL})$ and $\mathrm{K}_{2} \mathrm{CO}_{3}$ aqueous solution ( $2 \mathrm{M}, 3 \mathrm{~mL}$ ) and mixture was degassed through three freeze-pump-thaw cycles. Following the similar procedure to compound 6 using DCM/hexane $(2 / 1, \mathrm{v} / \mathrm{v})$ as eluent gave compound $9(0.84 \mathrm{~g})$ as a yellow solid in $85 \%$ yield. ${ }^{1} \mathrm{H} \mathrm{NMR}\left(\mathrm{CDCl}_{3}, 300 \mathrm{MHz}\right): \delta 10.04$ (s, 2H), $9.76(\mathrm{~s}, 2 \mathrm{H}), 8.19(\mathrm{~s}, 2 \mathrm{H}), 8.06(\mathrm{~s}, 2 \mathrm{H}), 7.99$ (d, J = 9.0 Hz, 2H), 7.98-7.88 (m, 2H), 7.42-7.37 (m, 8H), 7.64-7.74 (m, 4H), $7.13(\mathrm{~s}, 2 \mathrm{H}), 4.10-3.00(\mathrm{~m}, 8 \mathrm{H}), 1.62-1.60(\mathrm{~m}, 8 \mathrm{H})$, 1.26-1.21 (m, 24H), 0.85-0.83 (m, 12H); ${ }^{13} \mathrm{C} \mathrm{NMR}\left(\mathrm{CDCl}_{3}, 75 \mathrm{MHz}\right): \delta 192.18,191.36,153.83$, 150.42, 139.98, 139.73, 138.37, 137.40, 133.69, 131.17, 130.77, 129.54, 128.27, 127.74, 127.27, 124.55, 123.96, 119.07, 115.29, 113.98, 69.47, 69.32, 31.38, 31.31, 29.11, 28.98, 25.52, 25.39, 22.46, 13.93; HRMS (ESI, $m / z$ ): caldc for $\mathrm{C}_{66} \mathrm{H}_{73} \mathrm{O}_{3}[\mathrm{M}-1]^{+}, 993.5311$; found, 993.5316 (error = $-0.5 \mathrm{ppm})$. 


\section{Compound PIF-M/F-ONE}

PIF-M-ONE: Mesitylmagnesium bromide $(1.0 \mathrm{M}, 2 \mathrm{ml})$ was added to the $15 \mathrm{ml}$ dry THF solution of compound 9 (99.30 mg, $0.10 \mathrm{mmol})$ under argon atmosphere, and the mixture was stirred at room temperature overnight. The reaction mixture was quenched by water and extracted by chloroform. The organic layer was dried over $\mathrm{Na}_{2} \mathrm{SO}_{4}$ and the solvent was removed under reduced pressure. The crude product was then dissolved in $15 \mathrm{~mL}$ dry DCM under argon atmosphere and $0.1 \mathrm{~mL}$ of $\mathrm{BF}_{3} \cdot \mathrm{OEt}_{2}$ was added. The mixture was stirred for 5 minutes and quenched by methanol. The solvent was removed under reduced pressure. DDQ (45.40 g, 0.20 $\mathrm{mmol}$ ) was added dropwise to the $20 \mathrm{ml}$ dry toluene solution of compound 10a. Upon addition of DDQ solution, the color of the reaction mixture changed quickly from yellow color to dark green, the mixture was allowed to stir for $2 \mathrm{~h}$. After evaporation of the solvent, the residue was purified by column chromatography (silica gel, Hexane/Ethyl acetate $(6 / 1, \mathrm{v} / \mathrm{v}$ ) as eluent) to give compound PIF-M-ONE (51.02 mg, 36\% in three steps) as a dark green solid. Due to strong aggregation, this compound showed a broad and unclear NMR spectrum in $\mathrm{CDCl}_{3}, \mathrm{CD}_{2} \mathrm{Cl}_{2}$ and $\mathrm{d}_{8}$-THF. Finally we found that it could exhibit clear and sharp NMR signal in $\mathrm{CD}_{2} \mathrm{Cl}_{4}$ at high temperature (at $\left.60{ }^{\circ} \mathrm{C}\right) .{ }^{1} \mathrm{H}$ NMR $\left(\mathrm{CD}_{2} \mathrm{Cl}_{4}, 500 \mathrm{MHz}\right): \delta 9.18(\mathrm{~s}, 2 \mathrm{H}), 7.92(\mathrm{~s}, 2 \mathrm{H}), 7.64-7.62(\mathrm{~m}$, 4H), $7.13(\mathrm{~d}, J=8.4 \mathrm{~Hz}, 2 \mathrm{H}), 7.03(\mathrm{~s}, 4 \mathrm{H}), 7.00(\mathrm{~s}, 4 \mathrm{H}), 6.34(\mathrm{~d}, J=8.4 \mathrm{~Hz}, 2 \mathrm{H}), 4.11-4.08$ (m, $4 \mathrm{H}), 3.29-3.26(\mathrm{~m}, 4 \mathrm{H}), 2.43(\mathrm{~s}, 6 \mathrm{H}), 2.41(\mathrm{~s}, 6 \mathrm{H}), 2.21(\mathrm{~s}, 12 \mathrm{H}), 1.77(\mathrm{~s}, 12 \mathrm{H}), 1.46-1.31(\mathrm{~m}$, $22 \mathrm{H}), 1.13-1.12(\mathrm{~m}, 6 \mathrm{H}), 1.00-0.95(\mathrm{~m}, 12 \mathrm{H})$. HRMS (APCI, $\mathrm{m} / z)$ : caldc for $\mathrm{C}_{102} \mathrm{H}_{109} \mathrm{O}_{6}[\mathrm{M}+1]^{+}$, 1429.8219; found, 1429.8176 (error $=3.0 \mathrm{ppm})$.

PIF-F-ONE: Following the similar procedure to PIF-M-ONE, PIF-F-ONE was obtained as dark green solid in $45 \%$ yield. ${ }^{1} \mathrm{H}$ NMR $\left(\mathrm{CD}_{2} \mathrm{Cl}_{4}, 500 \mathrm{MHz}\right): \delta 10.08(\mathrm{~s}, 2 \mathrm{H}), 9.30(\mathrm{~d}, J=15 \mathrm{~Hz}$, 2H), 8.42 (s, 2H), 8.12 (d, $J=15 \mathrm{~Hz}, 2 \mathrm{H}), 7.94$ (d, $J=15 \mathrm{~Hz}, 2 \mathrm{H}), 7.33$ (d, $J=15 \mathrm{HZ}, 2 \mathrm{H}), 5.00$ (br, 4H), 4.33 (br, 4H), 2.40-2.37 (m, 4H), 2.08-1.94 (m, 20H), 1.63-1.60 (m, 20H). HRMS (APCI, $m / z$ ): caldc for $\mathrm{C}_{90} \mathrm{H}_{65} \mathrm{~F}_{20} \mathrm{O}_{6}[\mathrm{M}+1]^{+}, 1621.4455$; found, 1621.4450 (error $=0.4 \mathrm{ppm}$ ).

\section{Compound 11}

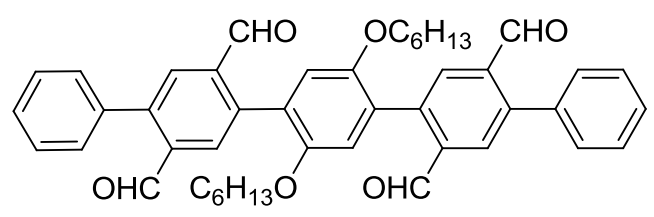


Compound 5 (0.87 g, $3.0 \mathrm{mmol})$, compound 8 (0.37 g, $1.0 \mathrm{mmol} \mathrm{1),} \mathrm{Pd}\left(\mathrm{PPh}_{3}\right)_{4}$ (57.78 mg, 0.05 mmol) was added to the toluene $(25 \mathrm{~mL})$, ethanol $(3 \mathrm{~mL})$ ) and $\mathrm{K}_{2} \mathrm{CO}_{3}$ aqueous solution $(2 \mathrm{M}, 3$ $\mathrm{mL}$ ), and the mixture was degassed through three freeze-pump-thaw cycles. Following the similar procedure to compound 9 using DCM/hexane (3/1, v/v) as eluent gave compound 11 (0.62 g) as a yellow solid in 90\% yield. ${ }^{1} \mathrm{H} \mathrm{NMR}\left(\mathrm{CDCl}_{3}, 500 \mathrm{MHz}\right): \delta 10.11(\mathrm{~s}, 2 \mathrm{H}), 9.99(\mathrm{~s}$, 2H), 8.13 (s, 2H), 8.09 (s, 2H), 7.54-7.47, $7.26(\mathrm{~m}, 10 \mathrm{H}), 7.04(\mathrm{~s}, 2 \mathrm{H}), 3.96(\mathrm{~m}, 4 \mathrm{H}), 1.61-1.58$ $(\mathrm{m}, 4 \mathrm{H}), 1.25-1.20(\mathrm{~m}, 12 \mathrm{H}), 0.81(\mathrm{t}, J=5.0 \mathrm{~Hz}, 6 \mathrm{H}) .{ }^{13} \mathrm{C} \mathrm{NMR}\left(125 \mathrm{MHz}, \mathrm{CDCl}_{3}\right) \delta 191.80$ $(\mathrm{C}=\mathrm{O}), 191.51(\mathrm{C}=\mathrm{O}), 150.28,146.36,144.97,139.80,139.18,137.43,137.14,136.73,136.55$, 136.51, 132.09, 130.45, 130.09, 129.97, 129.22, 128.75, 128.72, 128.67, 128.44, 128.22, 127.37, 115.17, 69.51, 31.26, 28.83, 25.43, 22.41, 13.87. HRMS (EI, $m / z)$ : caldc for $\mathrm{C}_{46} \mathrm{H}_{46} \mathrm{O}_{6}[\mathrm{M}]^{+}$, 694.32994; found, 694.32933 (error = -0.16 ppm).

\section{Compound IFD-CF $\mathbf{3}$}

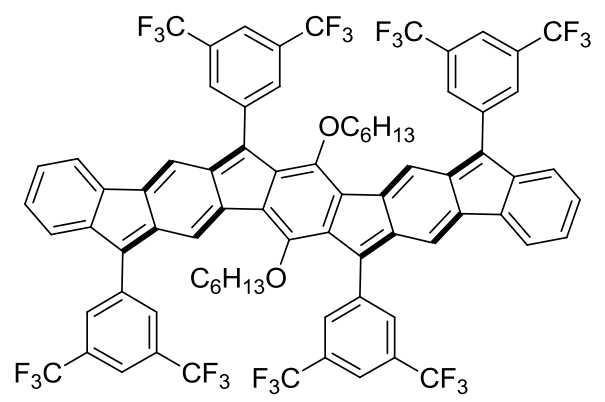

Compound IFD-CF3: 3,5-Bis(trifluoromethyl)phenyl magnesium bromide $(0.5 \mathrm{M}, 4 \mathrm{ml})$ was added to the $15 \mathrm{ml}$ dry THF solution of compound 11 (69.43 $\mathrm{mg}, 0.10 \mathrm{mmol})$ under argon atmosphere, and the mixture was stirred at room temperature overnight. The reaction mixture was quenched by water and extracted by chloroform. The organic layer was dried over $\mathrm{Na}_{2} \mathrm{SO}_{4}$ and the solvent was removed under reduced pressure. The crude product was then dissolved in $15 \mathrm{~mL}$ dry DCM under argon atmosphere and $0.1 \mathrm{~mL}$ of $\mathrm{BF}_{3} \cdot \mathrm{OEt}_{2}$ was added. The mixture was stirred for 5 minutes and quenched by methanol. The solvent was removed under reduced pressure. DDQ (45.40 g, $0.20 \mathrm{mmol}$ ) was added dropwise to the $20 \mathrm{ml}$ dry toluene solution of the precursor and the mixture was allowed to stir at $80{ }^{\circ} \mathrm{C}$ for further $12 \mathrm{~h}$ until the color change to dark green. After evaporation of the solvent, the residue was purified by column chromatography (silica gel, hexane/DCM (5/1, v/v) as eluent) to give compound IFD-CF3 (71.0 $\mathrm{mg}, 48 \%$ in three steps) as a black solid. ${ }^{1} \mathrm{H}$ NMR (d 8 -THF, $\left.500 \mathrm{MHz}\right): \delta 8.19(\mathrm{~s}, 8 \mathrm{H}), 8.14-8.13(\mathrm{~m}, 4 \mathrm{H}), 7.48(\mathrm{~s}$, 2H), $7.41(\mathrm{~d}, J=7.0 \mathrm{~Hz}, 2 \mathrm{H}), 7.14(\mathrm{~d}, J=7.0 \mathrm{~Hz}, 2 \mathrm{H}), 7.12(\mathrm{~s}, 2 \mathrm{H}), 7.10-7.04(\mathrm{~m}, 4 \mathrm{H}), 3.24(\mathrm{t}, J$ 
$=7.4 \mathrm{~Hz}, 4 \mathrm{H}), 0.95-0.89(\mathrm{~m}, 4 \mathrm{H}), 0.76-0.70(\mathrm{~m}, 14 \mathrm{H}), 0.66-0.61(\mathrm{~m}, 4 \mathrm{H}) ;{ }^{13} \mathrm{C}$ NMR $(125 \mathrm{MHz}$, $\mathrm{d}_{8}$-THF) $\delta 149.24,143.10,141.64,141.19,139.38,137.75,137.61,136.23,136.01,135.87$, $135.68,134.84,131.95,131.69,130.91,130.65,130.55,129.67,128.85,128.26,127.95,124.23$, 124.06, 122.86, 122.06, 121.89, 121.65, 120.62, 118.55, 73.81, 30.57, 29.22, 28.56, 21.44, 12.63. HRMS (APCI, m/z): caldc for $\mathrm{C}_{78} \mathrm{H}_{51} \mathrm{~F}_{24} \mathrm{O}_{2}[\mathrm{M}+1]^{+}$, 1475.3500 ; found, 1475.3494 (error = 0.4 ppm).

\section{Compound 13}

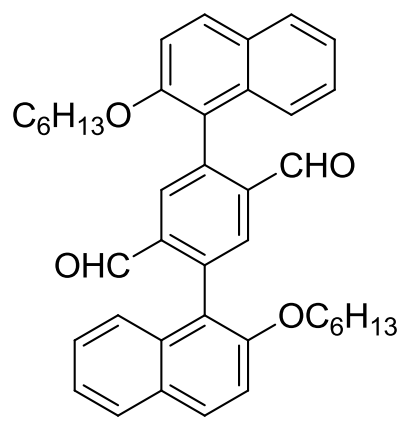

A mixture of 2,5-dibromobenzene-1,4-dicarboxaldehyde $(1.16 \quad \mathrm{~g}, \quad 4.0 \mathrm{mmol})$, (2-(hexyloxy)naphthalen-1-yl)boronic acid (2.69 g, $10.0 \mathrm{mmol}), \mathrm{Pd}\left(\mathrm{PPh}_{3}\right)_{4}(0.23 \mathrm{~g}, 0.20 \mathrm{mmol})$ in toluene $(40 \mathrm{~mL})$, ethanol $(5 \mathrm{~mL}))$ and $\mathrm{K}_{2} \mathrm{CO}_{3}$ aqueous solution $(2 \mathrm{M}, 5 \mathrm{~mL})$ was degassed by three freeze-pump-thaw cycles. The reaction mixture was heated at $105{ }^{\circ} \mathrm{C}$ overnight under nitrogen. After cooling to room temperature, the mixture was washed with water and extracted with DCM. The organic layer was dried over anhydrous $\mathrm{MgSO}_{4}$. The solvent was removed under vacuum and the residue was purified by silica gel column chromatography using DCM/hexane $(1 / 1, \mathrm{v} / \mathrm{v})$ as eluent to give compound $\mathbf{1 3}(1.99 \mathrm{~g})$ as a yellow solid in $85 \%$ yield. ${ }^{1} \mathrm{H}$ NMR $\left(\mathrm{CD}_{2} \mathrm{Cl}_{2}, 300 \mathrm{MHz}\right): \delta 9.73(\mathrm{~s}, 2 \mathrm{H}), 8.06(\mathrm{~s}, 2 \mathrm{H}), 8.02$ (d, $\left.J=8.04 \mathrm{~Hz}, 2 \mathrm{H}\right), 7.93-7.89(\mathrm{~m}, 2 \mathrm{H})$, 7.44-7.38 (m, 8H), 4.14-4.03 (m, 4H), 1.66-1.57 (m, 4H), 1.28-1.22 (m, 12H), $0.80(\mathrm{~m}, 3 \mathrm{H}) ;{ }^{13} \mathrm{C}$ NMR $\left(\mathrm{CD}_{2} \mathrm{Cl}_{2}, 75 \mathrm{MHz}\right): \delta 191.47(\mathrm{C}=\mathrm{O}), 153.58,139.02,138.03,133.26,130.77,130.68$, $130.14,128.63,127.77,126.66,124.07,123.40,118.89,113.64,68.80,30.97,28.82,28.70$, 25.15, 24.97, 22.08, 13.35. HR-MS (EI): caldc for $\mathrm{C}_{40} \mathrm{H}_{42} \mathrm{O}_{4}[\mathrm{M}]^{+}, 586.30831$; found, 586.30856 $($ error $=0.43 \mathrm{ppm})$.

\section{Compound HZ-M and HZ-F}


HZ-M: Following the similar procedure to compounds HZD-M-ONE, HZ-M was obtained as black solid in 56\% yield. ${ }^{1} \mathrm{H}$ NMR (d6-benzene, $\left.300 \mathrm{MHz}\right): \delta 9.02(\mathrm{~s}, 2 \mathrm{H}), 7.44(\mathrm{~d}, J=7.0 \mathrm{~Hz}$, 2H), $7.36(\mathrm{~d}, J=7.0 \mathrm{~Hz}, 2 \mathrm{H}), 7.04(\mathrm{~s}, 4 \mathrm{H}), 7.01-6.93(\mathrm{~m}, 6 \mathrm{H}), 3.71(\mathrm{t}, J=7.4 \mathrm{~Hz}, 4 \mathrm{H}), 2.38(\mathrm{~s}$, $6 \mathrm{H}), 2.17(\mathrm{~s}, 12 \mathrm{H}), 1.42-1.38(\mathrm{~m}, 4 \mathrm{H}), 1.27-1.16(\mathrm{~m}, 12 \mathrm{H}), 0.90(\mathrm{t}, J=6.8 \mathrm{~Hz}, 6 \mathrm{H}) ;{ }^{13} \mathrm{C} \mathrm{NMR}$ (d6-benzene, 75 MHz): $\delta$ 157.11, 138.67, 136.85, 136.80, 134.64, 134.56, 134.15, 131.27, 130.29, $129.53,129.20,127.70,127.16,125.73,123.73,119.55,115.48,69.26,32.45,29.41,26.27$, 23.57, 21.87, 20.71, 14.76. HRMS (ESI, m/z): caldc for $\mathrm{C}_{58} \mathrm{H}_{60} \mathrm{O}_{2}[\mathrm{M}]^{+}, 788.4588$; found, 788.4605 (error $=-2.1 \mathrm{ppm})$.

HZ-F: Black solid; 47\% yield. ${ }^{1} \mathrm{H}$ NMR $\left(\mathrm{C}_{6} \mathrm{D}_{6}, 300 \mathrm{MHz}\right): \delta 8.80$ (s, 2H), 7.41-7.36 (m, 4H), 7.12-7.07 (m, 2H), 6.89-6.81 (m, 4H), $3.64(\mathrm{t}, J=7.2 \mathrm{~Hz}, 4 \mathrm{H}), 1.39-1.36(\mathrm{~m}, 4 \mathrm{H}), 1.23-1.21(\mathrm{~m}$, $12 \mathrm{H}), 0.86(\mathrm{t}, J=6.7 \mathrm{~Hz}, 6 \mathrm{H})$. HRMS (ESI, $m / z)$ : caldc for $\mathrm{C}_{52} \mathrm{H}_{39} \mathrm{~F}_{10} \mathrm{O}_{2}[\mathrm{M}+1]^{+}, 885.2790$; found, 885.2815 (error $=-2.8 \mathrm{ppm})$.

\section{Compound 14}

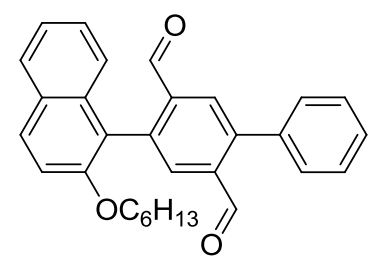

A mixture of compound 5 (0.29 g, $1.0 \mathrm{mmol})$, (2-(hexyloxy)naphthalen-1-yl)boronic acid (0.82 g, $3.0 \mathrm{mmol}), \mathrm{Pd}\left(\mathrm{PPh}_{3}\right)_{4}(57.78 \mathrm{mg}, 0.05 \mathrm{mmol})$ in toluene $(25 \mathrm{~mL})$, ethanol $\left.(3 \mathrm{~mL})\right)$ and $\mathrm{K}_{2} \mathrm{CO}_{3}$ aqueous solution ( $2 \mathrm{M}, 3 \mathrm{~mL}$ ) was degassed by three freeze-pump-thaw cycles. The reaction mixture was heated at $105{ }^{\circ} \mathrm{C}$ overnight under nitrogen. After cooling to the room temperature, the mixture was washed with water and extracted with DCM. The organic layer was dried over anhydrous $\mathrm{MgSO}_{4}$. The solvent was removed under vacuum and the residue was purified by silica gel column chromatography using DCM/hexane (1/1, v/v) as eluent to give compound 14 (0.35 g) as a yellow solid in $80 \%$ yield. ${ }^{1} \mathrm{H}$ NMR $\left(\mathrm{CD}_{2} \mathrm{Cl}_{2}, 300 \mathrm{MHz}\right) \delta \mathrm{ppm}: 10.12(\mathrm{~s}, 1 \mathrm{H}), 9.73$ (s, 1H), $8.19(\mathrm{~s}, 1 \mathrm{H}), 8.01-7.99(\mathrm{~m}, 2 \mathrm{H}), 7.91-7.88(\mathrm{~m}, 1 \mathrm{H})$, 7.57-7.51 (m, 5H), 7.43-7.37 (m, $4 \mathrm{H}) ;{ }^{13} \mathrm{C} \mathrm{NMR}\left(\mathrm{CD}_{2} \mathrm{Cl}_{2}, 75 \mathrm{MHz}\right) \delta \mathrm{ppm}: 191.7(\mathrm{C}=\mathrm{O}), 191.5(\mathrm{C}=\mathrm{O}), 153.86,144.58,139.21$, $138.15,136.88,136.74,133.61,131.96,130.59$, 130.06, 129.18, 128.97, 128.55, 128.40, 128.12, 127.06, 124.40, 123.79, 118.88, 113.98, 69.27, 31.20, 29.04, 25.33, 22.38, 13.60. HR-MS (ESI): 
calcd. for $\mathrm{C}_{30} \mathrm{H}_{29} \mathrm{O}_{3}[\mathrm{M}+\mathrm{H}]^{+}: \mathrm{m} / \mathrm{z}=437.2111$; found, 437.2107 (error: $1.0 \mathrm{ppm}$ ).

\section{Compound PF-M}

Mesitylmagnesium (1.0 M, $6 \mathrm{ml}$ ) was added to the $15 \mathrm{ml}$ dry THF solution of compound $\mathbf{1 4}$ $(0.44 \mathrm{~g}, 1.0 \mathrm{mmol})$ under argon atmosphere, and the mixture was stirred at room temperature for 8 hours. The reaction mixture was quenched by water and extracted by chloroform. The organic layer was dried over $\mathrm{Na}_{2} \mathrm{SO}_{4}$ and the solvent was removed under reduced pressure. The crude compound was then dissolved in $20 \mathrm{~mL}$ dry DCM under argon atmosphere and $2 \mathrm{~mL}$ of $\mathrm{BF}_{3} \cdot \mathrm{OEt}_{2}$ was added. The mixture was stirred for 5 minutes and quenched by methanol. The solvent was removed under reduced pressure. DDQ $(0.23 \mathrm{~g}, 1.0 \mathrm{mmol})$ was added dropwise to the $20 \mathrm{ml}$ dry toluene solution of the precursor. Upon addition of DDQ solution, the color of the reaction mixture changed slowly from light blue to dark green, and the reaction was monitored by TLC until completion. After evaporation of the solvent, the residue was purified by column chromatography (silica gel, DCM/hexane (1/3, v/v) as eluent) to give compound PF-M (0.22 g, $34 \%$ in three steps) as a green solid. ${ }^{1} \mathrm{H}$ NMR (d6-benzene, $\left.300 \mathrm{MHz}\right): \delta 9.28(\mathrm{~s}, 1 \mathrm{H}), 7.82(\mathrm{~s}$, 1H), $7.51(\mathrm{~s}, 1 \mathrm{H}), 7.48(\mathrm{~s}, 1 \mathrm{H}), 7.28(\mathrm{~d}, J=7.4 \mathrm{~Hz} 1 \mathrm{H}), 7.09-7.05(\mathrm{~m}, 3 \mathrm{H}), 7.02-6.96(\mathrm{~m}, 6 \mathrm{H})$, 6.91-6.85 (m, 1H), $3.61(\mathrm{t}, J=7.2 \mathrm{~Hz}, 2 \mathrm{H}), 2.35(\mathrm{~s}, 3 \mathrm{H}), 2.31(\mathrm{~s}, 3 \mathrm{H}), 2.24(\mathrm{~s}, 6 \mathrm{H}), 2.04(\mathrm{~s}, 6 \mathrm{H})$, $1.44(\mathrm{~m}, 2 \mathrm{H}), 1.29-1.08(\mathrm{~m}, 6 \mathrm{H}), 0.94(\mathrm{t}, J=6.9 \mathrm{~Hz}, 3 \mathrm{H}) ;{ }^{13} \mathrm{C}$ NMR (d 6 -benzene, $\left.300 \mathrm{MHz}\right): \delta$ $157.93,145.38,142.61,139.99,138.41,138.22,137.99,137.10,136.84,135.77,135.74,135.59$, $132.60,132.51,130.21,129.81,129.69,129.45,129.16,126.17,125.44,123.56,122.39,121.84$, $121.07,119.38,115.31,69.87,32.21,30.67,29.54,26.24,23.63,21.87,21.77,21.12,20.55$, 14.81. HRMS (ESI, $\mathrm{m} / z$ ): caldc for $\mathrm{C}_{48} \mathrm{H}_{47} \mathrm{O}$ [M+1], 639.3621; found, 639.3624 (error $=-0.4$ ppm).

\section{Compound PF-F}

Following the similar procedure to compound PF-M, PF-F was obtained as black solid in $30 \%$ yield. ${ }^{1} \mathrm{H}$ NMR $\left(\mathrm{CD}_{2} \mathrm{Cl}_{4}, 300 \mathrm{MHz}\right): \delta 9.07(\mathrm{~s}, 1 \mathrm{H}), 7.93-7.85(\mathrm{~m}, 2 \mathrm{H}), 7.78(\mathrm{~d}, J=9 \mathrm{~Hz}, 1 \mathrm{H})$, $7.52(\mathrm{~d}, J=9 \mathrm{~Hz}, 1 \mathrm{H}), 7.39-7.10(\mathrm{~m}, 6 \mathrm{H}), 4.32(\mathrm{t}, J=3.0 \mathrm{~Hz}, 2 \mathrm{H}), 1.83(\mathrm{br}, 2 \mathrm{H}), 1.28$ (br, 6H), 0.94-0.87 (m, 3H). HRMS (ESI, $m / z$ ): caldc for $\mathrm{C}_{42} \mathrm{H}_{25} \mathrm{~F}_{10} \mathrm{O}[\mathrm{M}+1]^{+}$, 735.1740; found, 735.1749 (error $=0.2 \mathrm{ppm})$. 
Compounds IF-M and IF-CF 3 were prepared according to the literature (Chase, D. T.; Fix, A. G.; Kang, S. J.; Rose, B. D.; Weber, C. D.; Zhong, Y.; Zakharov, L. N.; Lonergan, M. C.; Nuckolls, C.; Haley, M. M. J. Am. Chem. Soc. 2012, 134, 10349-10352.) 


\section{DFT calculations details}

Density functional theory calculations were employed with Gaussian 09 package, ${ }^{1}$ utilizing the UCAM-B3LYP ${ }^{2-4}$ level of theory with Pople basis set $6-31 \mathrm{G}^{* 5-7}$ for all molecules in the gas phase. NICS values were calculated using the standard GIAO procedure $\left(\right.$ NMR pop $=$ NCSall) ${ }^{8}$ The energies and Cartesian coordinates of the optimized structures, and the calculated NICS data are shown in the appendix.

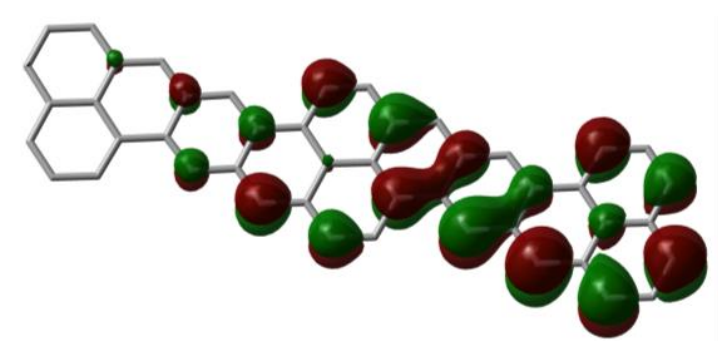

SOMO- $\alpha$

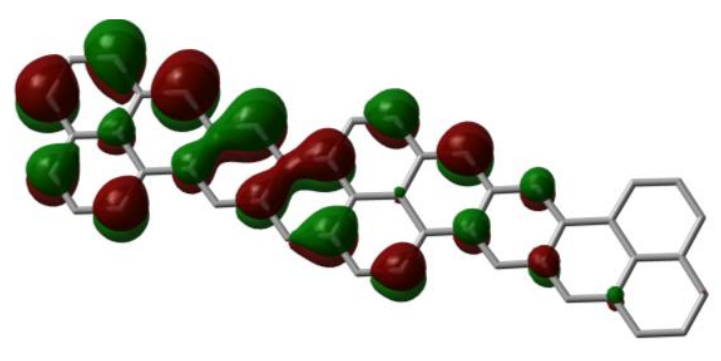

SOMO- $\beta$

Figure S1. Calculated (UCAM-B3LYP) SOMOs of $\alpha$ and $\beta$ electrons the parent HZD in the singlet diradical ground state.
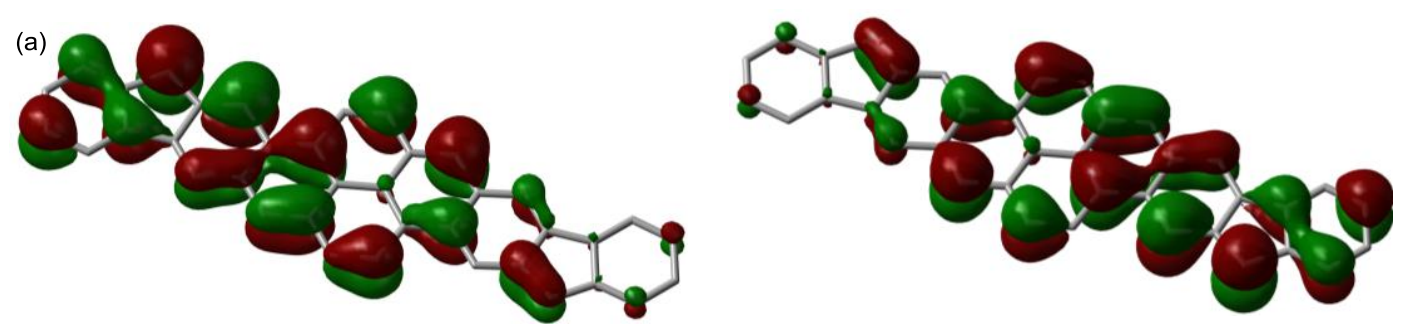

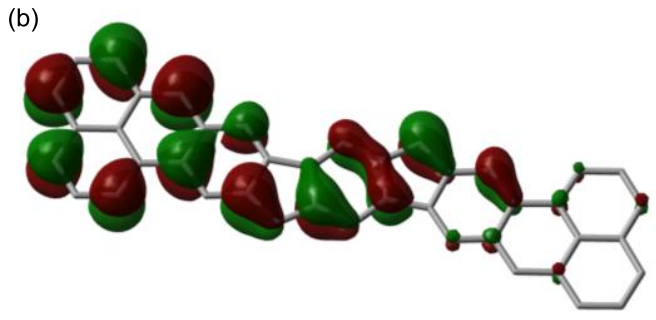

SOMO- $\alpha$

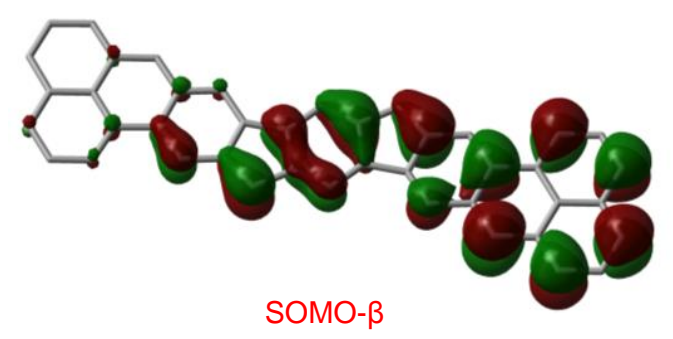

SOMO- $\beta$

Figure S2. Calculated (UCAM-B3LYP) SOMOs of $\alpha$ and $\beta$ electrons of parent FP (a) and PIF (b) in the singlet diradical ground state. 


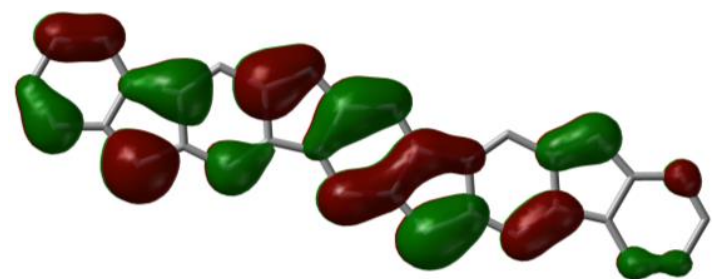

SOMO- $\alpha$

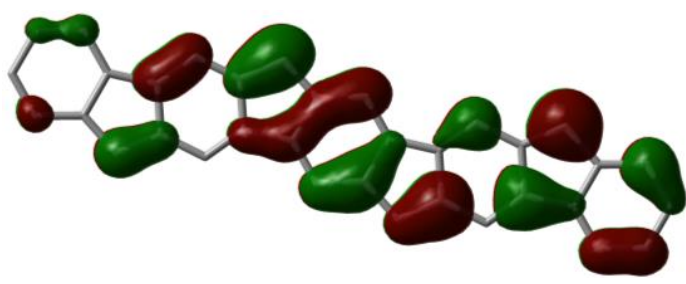

SOMO- $\beta$

Figure S3. Calculated (UCAM-B3LYP) SOMOs of $\alpha$ and $\beta$ electrons parent IFD molecule in the singlet diradical ground state.

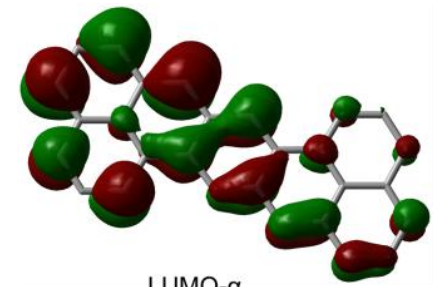

LUMO-a

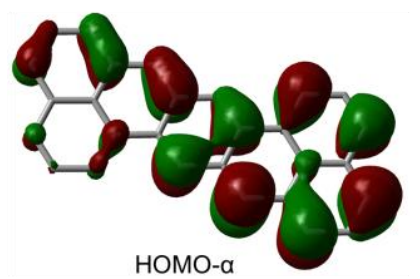

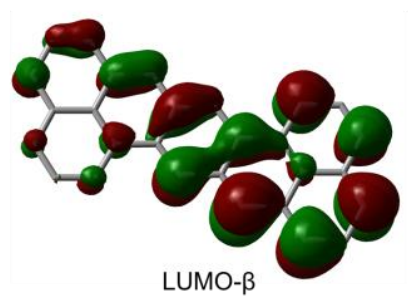

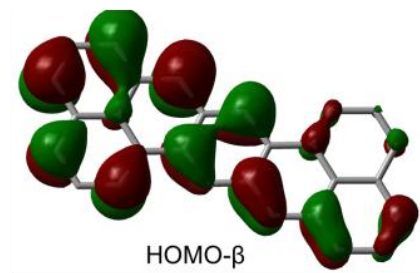

Figure S4. Calculated (UCAM-B3LYP) frontier molecular orbitals of parent $\mathbf{H Z}$ molecule in the singlet diradical ground state.
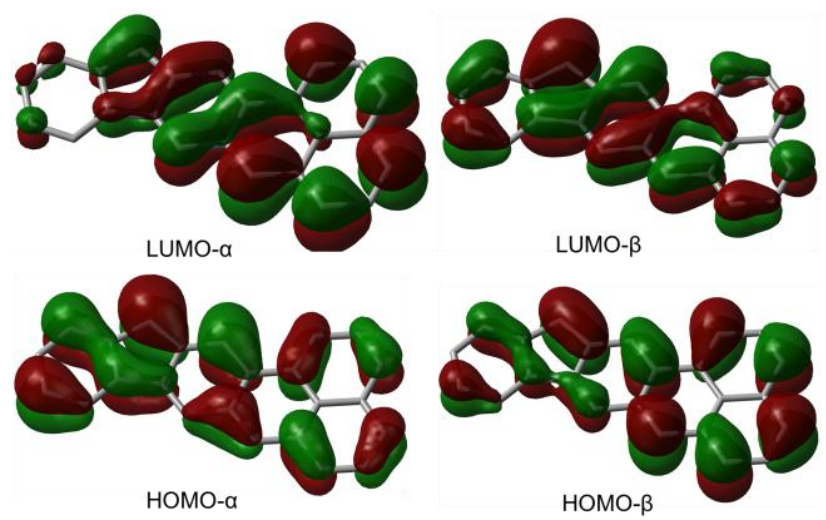

Figure S5. Calculated (UCAM-B3LYP) frontier molecular orbitals of parent PF molecule in the singlet diradical ground state. 


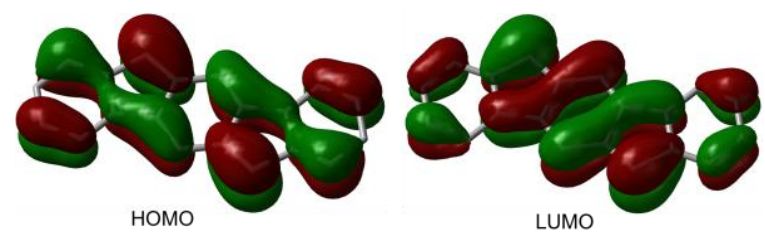

Figure S6. Calculated (CAM-B3LYP) frontier molecular orbitals of parent IF molecule in the closed-shell ground state.
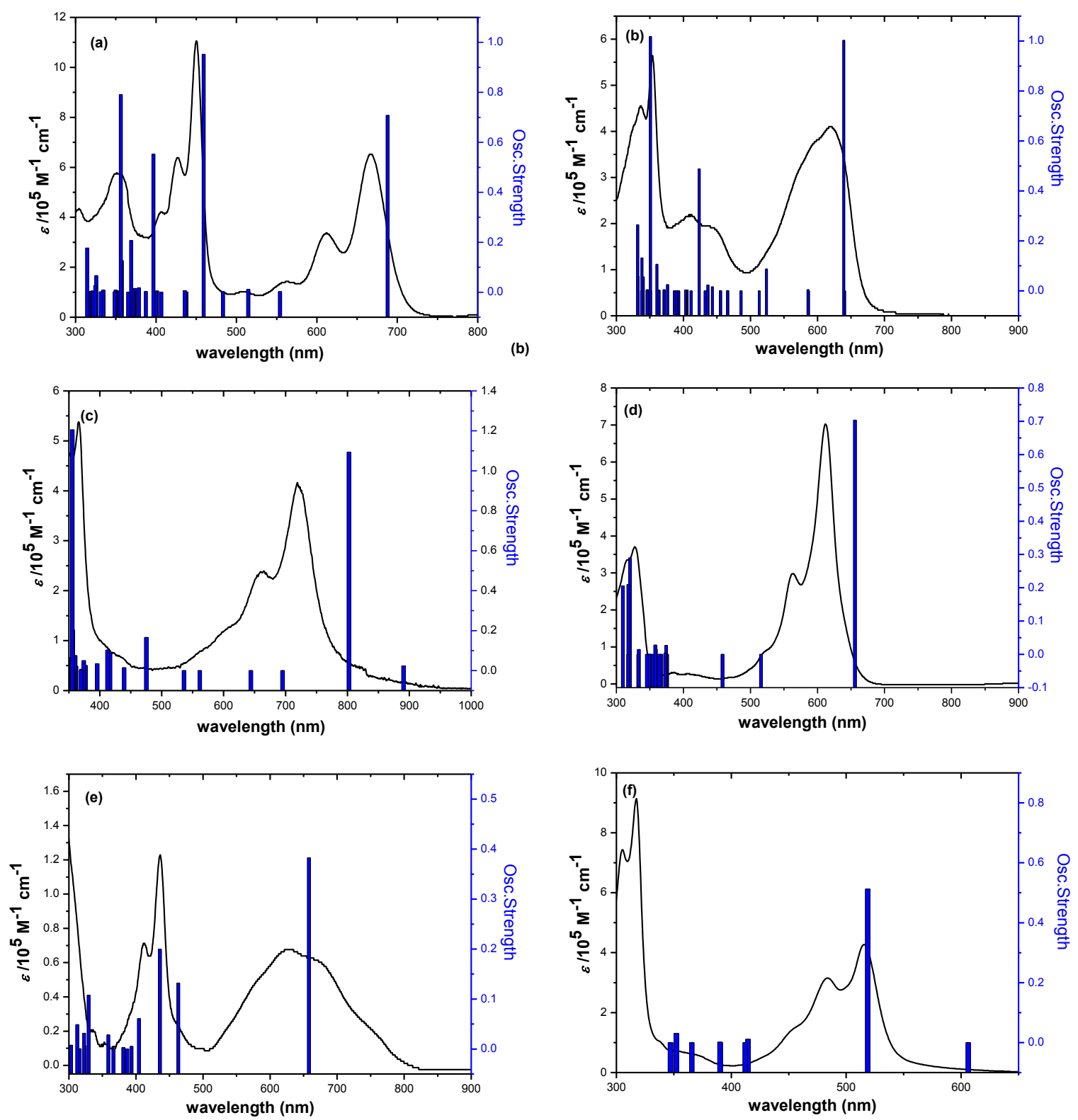

Figure S7. TD DFT simulated spectrum of HZD-M-ONE (a), PIF-M-ONE (b), IFD-CF3 (c), HZ-M (d), PF-M (e), IF-M (f) along with the experimental spectra. 
Table S1. Selected TD-DFT (B3LYP/6-31G*) calculated energies, oscillator strength and compositions of major electronic transitions of HZD-M-ONE. H=HOMO, L=LUMO, $\mathrm{L}+1=\mathrm{LUMO}+1$, etc.

\begin{tabular}{lll}
\hline Wavelength(nm) & Osc.Strength $(f)$ & Major contributions \\
688 & 0.7076 & $\mathrm{H}-0->\mathrm{L}+0(+99 \%)$ \\
514.7 & 0.0121 & $\mathrm{H}-0->\mathrm{L}+2(+60 \%), \mathrm{H}-2->\mathrm{L}+0(35 \%)$ \\
459.2 & 0.9521 & $\mathrm{H}-2->\mathrm{L}+0(+57 \%), \mathrm{H}-0->\mathrm{L}+2(+37 \%)$ \\
396.8 & 0.5531 & \\
\hline
\end{tabular}

Table S2. Selected TD-DFT (B3LYP/6-31G*) calculated energies, oscillator strength and compositions of major electronic transitions of PIF-M-ONE. H=HOMO, L=LUMO, $\mathrm{L}+1=\mathrm{LUMO}+1$, etc.

\begin{tabular}{lll}
\hline Wavelength $(\mathrm{nm})$ & Osc.Strength $(f)$ & Major contributions \\
639.2 & 1.0033 & $\mathrm{H}-0->\mathrm{L}+0(+96 \%)$ \\
523.9 & 0.0875 & $\mathrm{H}-4->\mathrm{L}+0(+92 \%)$ \\
423.5 & 0.488 & $\mathrm{H}-1->\mathrm{L}+1(+75 \%) \quad \mathrm{H}-9->\mathrm{L}+0(10 \%)$ \\
350.8 & $\mathrm{H}-1->\mathrm{L}+3(+71 \%) \mathrm{H}-16->\mathrm{L}+0(+12 \%), \mathrm{H}-4->\mathrm{L}+2(+9 \%)$ \\
\hline
\end{tabular}

Table S3. Selected TD-DFT (B3LYP/6-31G*) calculated energies, oscillator strength and compositions of major electronic transitions of IFD-CF3. H=HOMO, L=LUMO, L+1=LUMO+1, etc.

\section{Wavelength(nm) Osc.Strength $(f) \quad$ Major contributions}




$\begin{array}{llll}802.6 & 1.0936 & \mathrm{H}-0->\mathrm{L}+0(+97 \%) \\ 890.8 & 0.0238 & \mathrm{H}-1->\mathrm{L}+0(+94 \%) & \\ 475.2 & 0.1663 & \mathrm{H}-2->\mathrm{L}+1(+88 \%) & \mathrm{H}-3->\mathrm{L}+1(+8 \%) \\ 412.4 & 0.1031 & \mathrm{H}-0->\mathrm{L}+2(+63 \%) & \mathrm{H}-3->\mathrm{L}+1(+29 \%)\end{array}$

Table S4. Selected TD-DFT (B3LYP/6-31G*) calculated energies, oscillator strength and compositions of major electronic transitions of HZ-M. H=HOMO,L=LUMO,L+1=LUMO+1, etc

\begin{tabular}{|c|c|c|}
\hline Wavelength(nm) & Osc.Strength $(f)$ & Major contributions \\
\hline 655.9 & 0.7036 & $\mathrm{H}-0->\mathrm{L}+0(+101 \%)$ \\
\hline 374.2 & 0.0269 & $\mathrm{H}-0->\mathrm{L}+3(+29 \%), \mathrm{H}-3->\mathrm{L}+0(9 \%)$ \\
\hline 320.3 & 0.2896 & $\mathrm{H}-1->\mathrm{L}+1(+91 \%)$ \\
\hline
\end{tabular}

Table S5. Selected TD-DFT (B3LYP/6-31G*) calculated energies, oscillator strength and compositions of major electronic transitions of PF-M. H=HOMO, L=LUMO, L+1=LUMO+1, etc.

\begin{tabular}{llll}
\hline Wavelength $(\mathrm{nm})$ & Osc.Strength $(f)$ & Major contributions \\
658.0 & 0.3826 & $\mathrm{H}-0->\mathrm{L}+0(+92 \%)$ & $\mathrm{H}-1->\mathrm{L}+0(+6 \%$ \\
463.1 & 0.1319 & $\mathrm{H}-1->\mathrm{L}+0(+80 \%)$ & $\mathrm{H}-0->\mathrm{L}+1(10 \%)$ \\
435.6 & 0.1999 & $\mathrm{H}-0->\mathrm{L}+1(+76 \%)$ & $\mathrm{H}-1->\mathrm{L}+0(+9 \%) \mathrm{H}-2->\mathrm{L}+0(+8 \%)$ \\
\hline
\end{tabular}

Table S6. Selected TD-DFT (B3LYP/6-31G*) calculated energies, oscillator strength and 
compositions of major electronic transitions of IF-M. H=HOMO, L=LUMO, L+1=LUMO+1, etc.

\begin{tabular}{lll}
\hline Wavelength $(\mathrm{nm})$ & Osc.Strength $(f)$ & Major contributions \\
518.7 & 0.5126 & $\mathrm{H}-0->\mathrm{L}+0(+93 \%) \quad \mathrm{H}-1->\mathrm{L}+1(+7 \%)$ \\
606.2 & 0.0000 & $\mathrm{H}-1->\mathrm{L}+0(+100 \%)$ \\
414.5 & 0.0110 & $\mathrm{H}-2->\mathrm{L}+0(+99 \%$ \\
390.2 & 0.0017 & $\mathrm{H}-5->\mathrm{L}+0(+100 \%)$ \\
295.7 & $\mathrm{H}-1->\mathrm{L}+1(+55 \%) \mathrm{H}-8->\mathrm{L}+0(+31 \%), \mathrm{H}-0->\mathrm{L}+2(+6 \%)$
\end{tabular}

\section{References:}

1. Gaussian 09; Revision A.2; Frisch, M. J.; Trucks, G. W.; Schlegel, H. B.; Scuseria, G. E.; Robb, M. A.; Cheeseman, J. R.; Scalmani, G.; Barone, V.; Mennucci, B.; Petersson, G. A.; Nakatsuji, H.; Caricato, M.; Li, X.; Hratchian, H. P.; Izmaylov, A. F.; Bloino, J.; Zheng, G.; Sonnenberg, J. L.; Hada, M.; Ehara, M.; Toyota, K.; Fukuda, R.; Hasegawa, J.; Ishida, M.; Nakajima, T.; Honda, Y.; Kitao, O.; Nakai, H.; Vreven, T.; Montgomery, J., J. A.; Peralta, J. E.; Ogliaro, F.; Bearpark, M.; Heyd, J. J.; Brothers, E.; Kudin, K. N.; Staroverov, V. N.; Kobayashi, R.; Normand, J.; Raghavachari, K.; Rendell, A.; Burant, J. C.; Iyengar, S. S.; Tomasi, J.; Cossi, M.; Rega, N.; Millam, N. J.; Klene, M.; Knox, J. E.; Cross, J. B.; Bakken, V.; Adamo, C.; Jaramillo, J.; Gomperts, R.; Stratmann, R. E.; Yazyev, O.; Austin, A. J.; Cammi, R.; Pomelli, C.; Ochterski, J. W.; Martin, R. L.; Morokuma, K.; Zakrzewski, V. G.; Voth, G. A.; Salvador, P.; Dannenberg, J. J.; Dapprich, S.; Daniels, A. D.; Farkas, Ö.; Foresman, J. B.; Ortiz, J. V.; Cioslowski, J.; Fox, D. J.; Gaussian, Inc., Wallingford CT, 2009.

2. Becke, A. D. J. Chem. Phys. 1993, 98, 5648.

3. Lee, C.; Yang, W.; Parr, R. G. Phys. Rev. B: Condens. Matter 1988, 37, 785.

4. Yanai, T.; Tew, D.; and Handy, N. Chem. Phys. Lett. 2004, 393, 51.

5. Ditchfield, R.; Hehre, W. J.; Pople, J. A. J. Chem. Phys. 1971, 54, 724.

6. Hehre, W. J.; Ditchfield R.; Pople, J. A. J. Chem. Phys. 1972, 56, 2257.

7. Hariharan, P. C.; Pople, J. A. Theor. Chim. Acta 1973, 28, 213.

8. Fallah-Bagher-Shaidaei, H.; Wannere, S. S.; Corminboeuf, C.; Puchta, R.; Schleyer, P. V. R. Org. Lett. 2006, 8, 863. 


\section{In-situ ESR data}
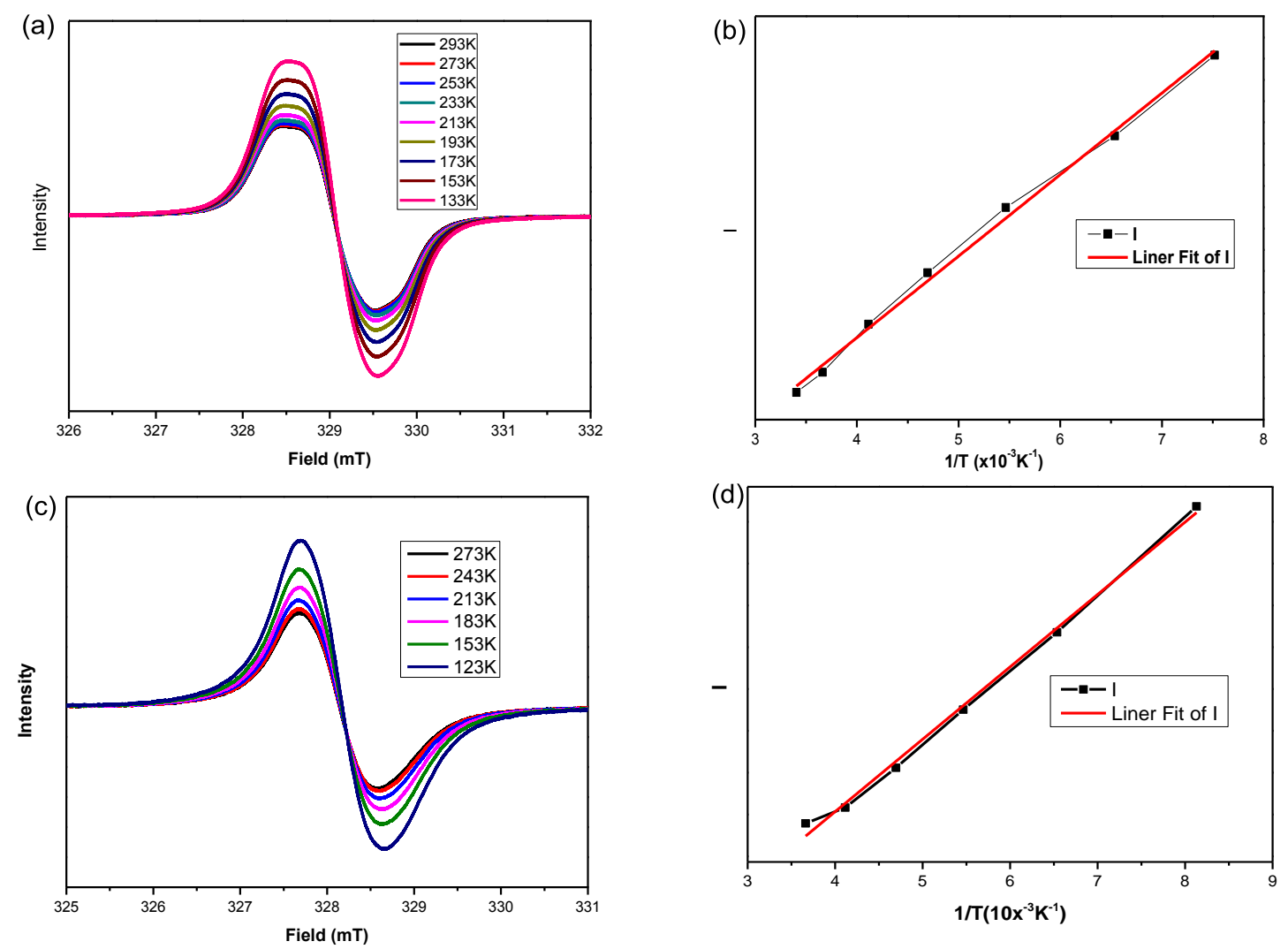

Figure S8. (a) VT ESR spectra of in-situ generated radical of HZD-M in solid form and the integrated ESR intensity $I-1 / T$ curve (b); (c) VT ESR spectra of in-situ generated radical of PIF-M in solid powder form and the integrated ESR intensity $I-1 / T$ curve (d). 


\section{TA data for all measured compounds}
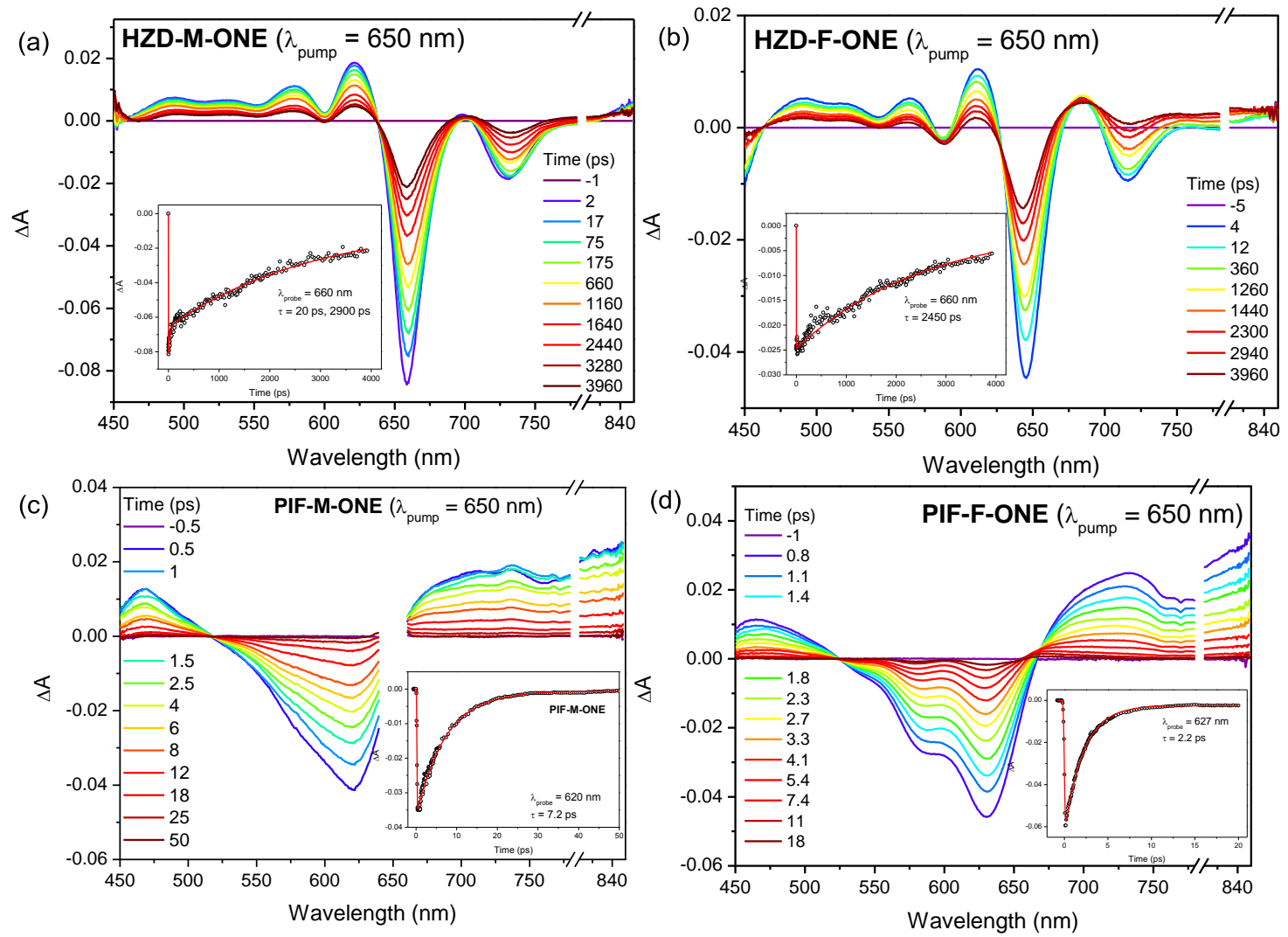

(e)

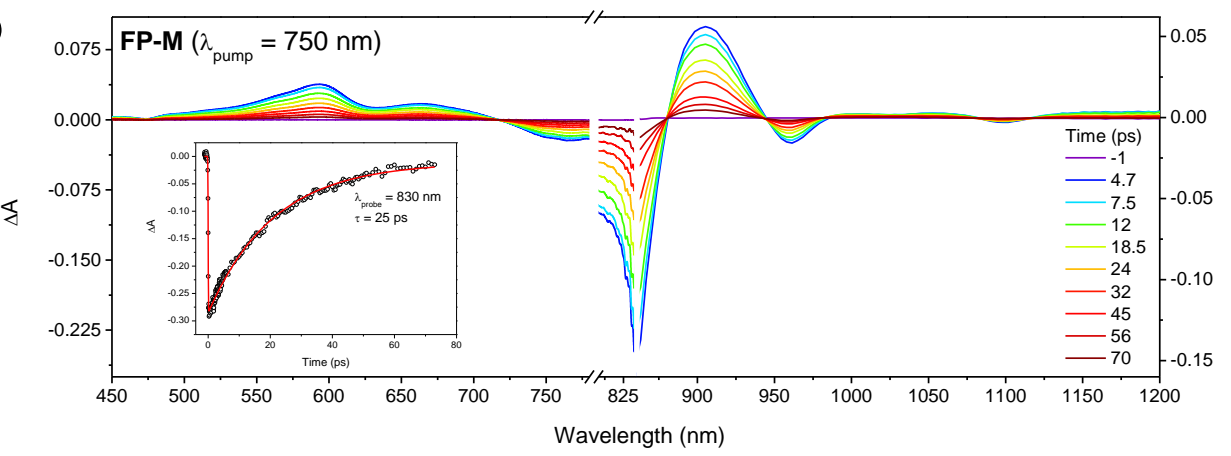



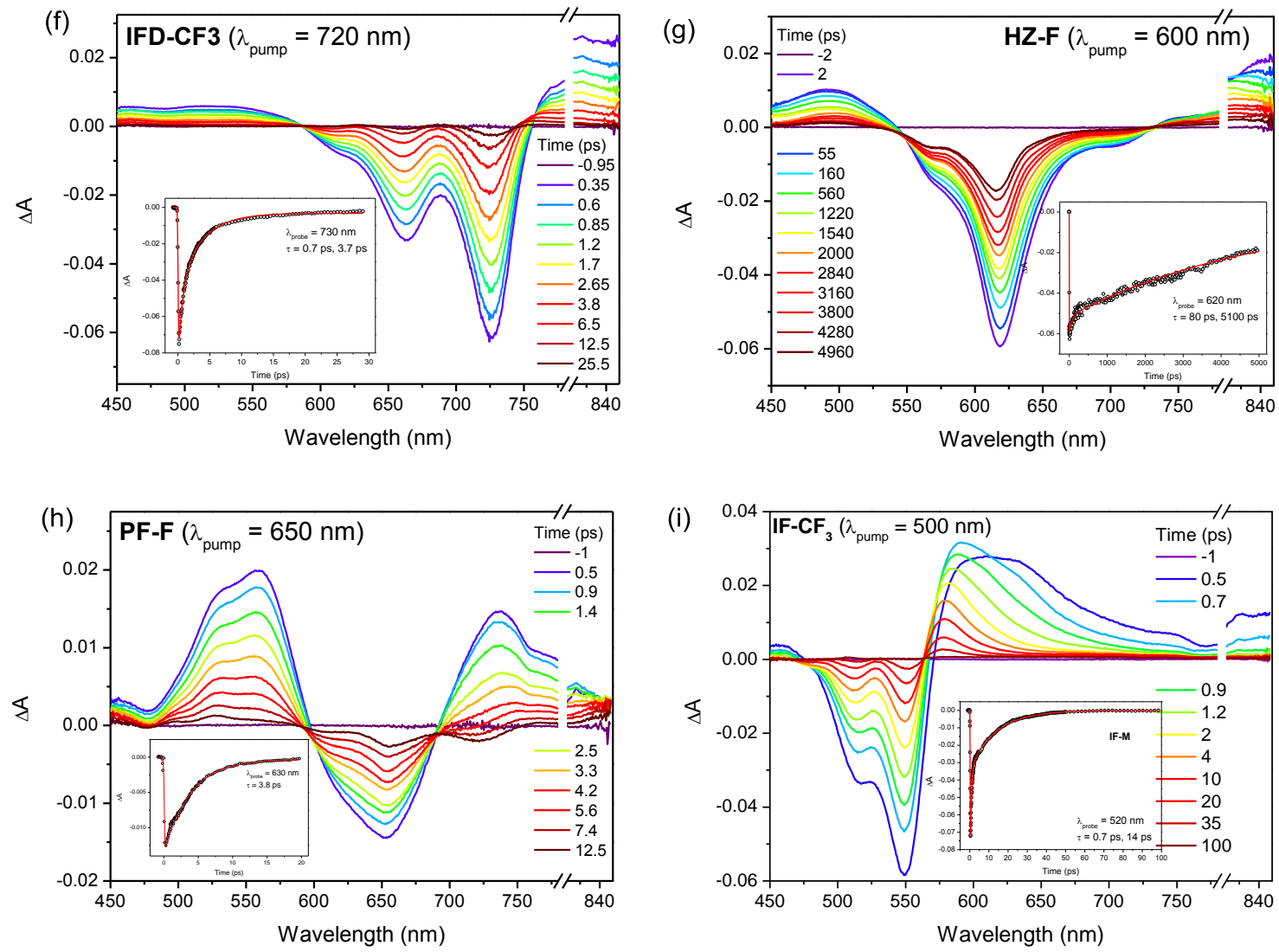

Figure S9. Transient absorption spectra of (a) HZD-M-ONE, (b) HZD-F-ONE, (c) PIF-M-ONE, (d) PIF-F-ONE, (e) FP-M, (f) IFD-CF3, (g) HZ-F, (h) PF-F and (i) IF-CF3 recorded in toluene. Inserts are the decay profiles. 


\section{TPA data for all measured compounds}

(a)

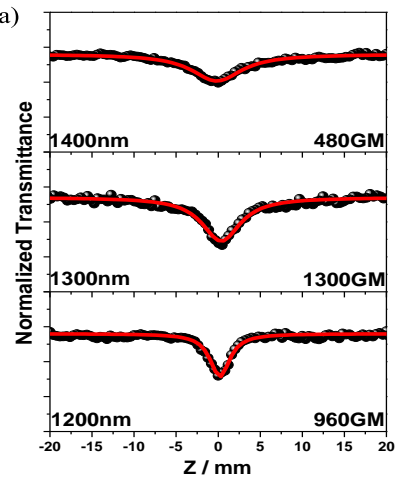

(d)

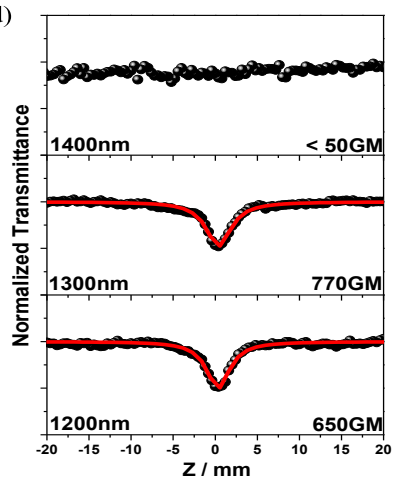

(g)

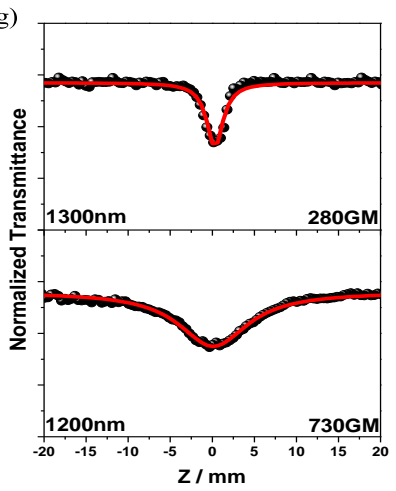

(j)

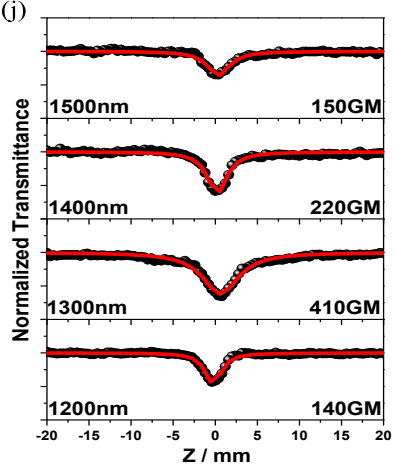

(b)

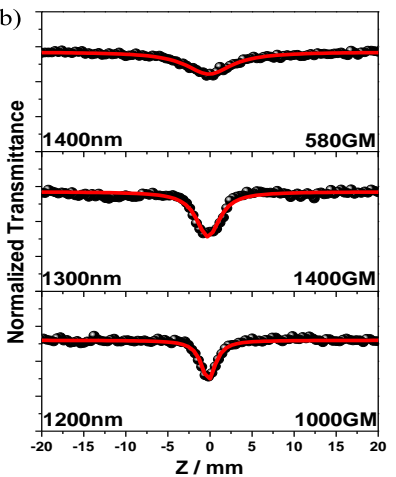

(e)

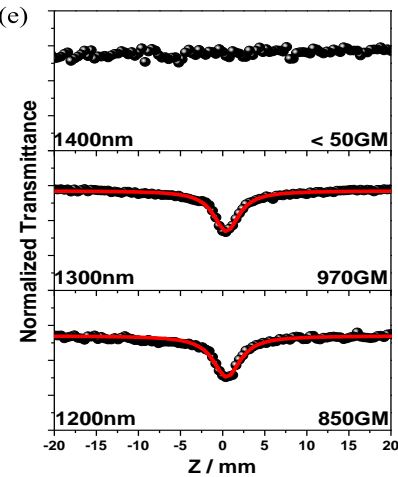

(h)

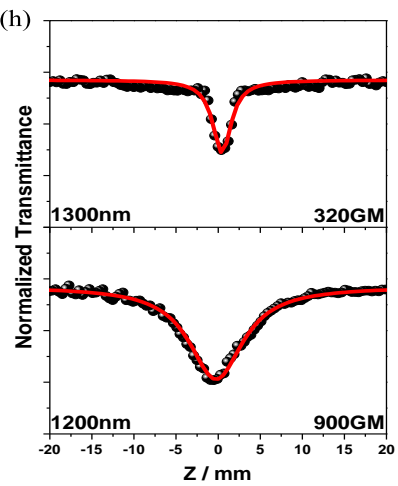

(c)

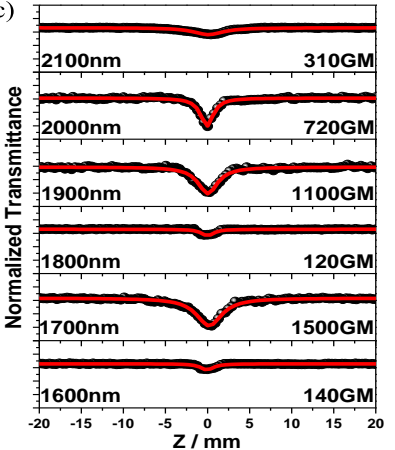

(f)

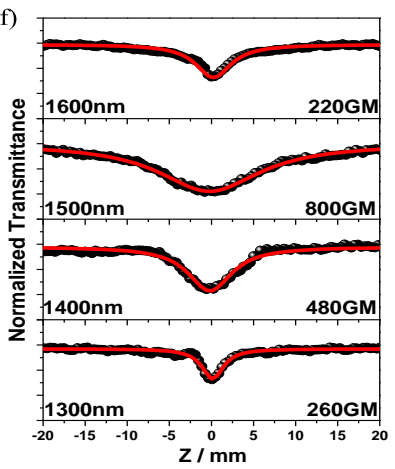

(i)

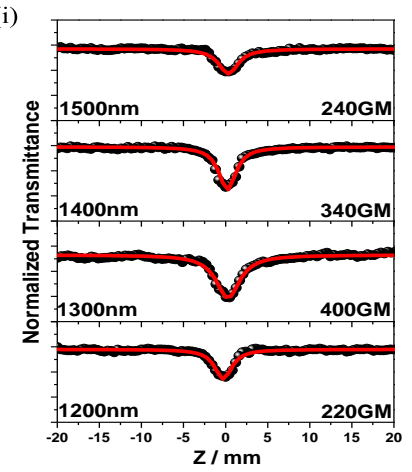

Figure S10. Z-scan curves of HZD-M-ONE (a), HZD-F-ONE (b), FP-M (c), PIF-M-ONE (d), PIF-F-ONE (e), IFD-CF 3 (f), HZ-M (g), HZ-F (h), PF-M (i) and PF-F (j) in toluene upon photoexcitation in the range from 1200 to $1800 \mathrm{~nm}$. 
6. CV/DPV data for all measured compounds

(a)

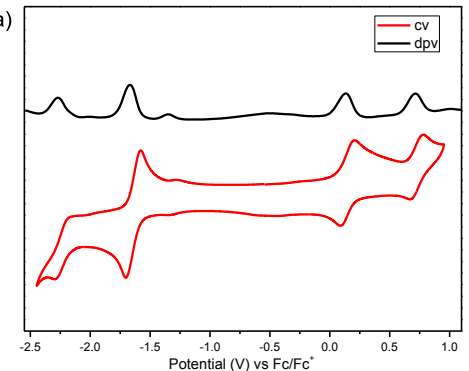

(d)

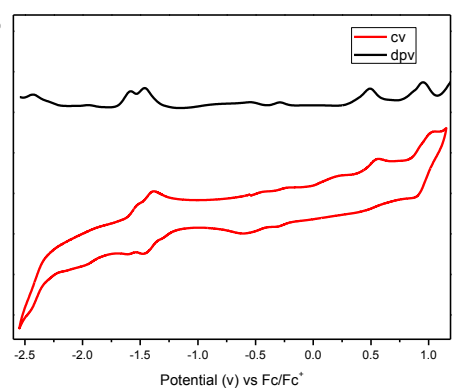

(g)

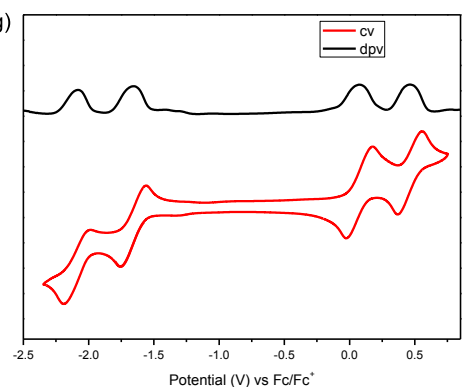

(j)

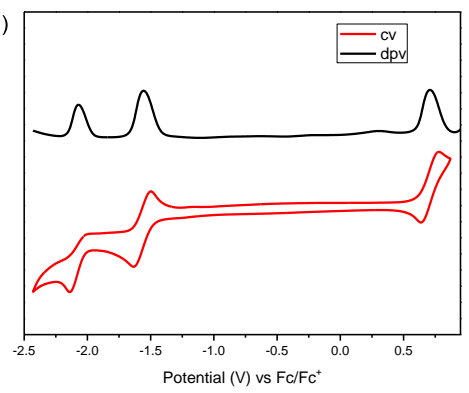

(b)

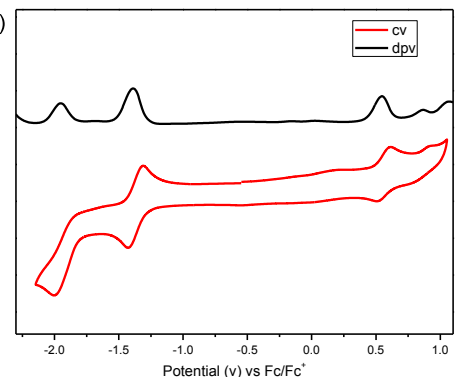

(e)

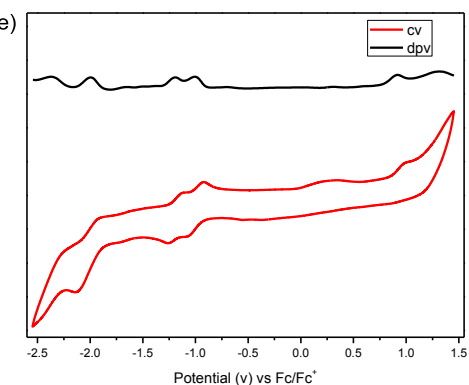

(h)

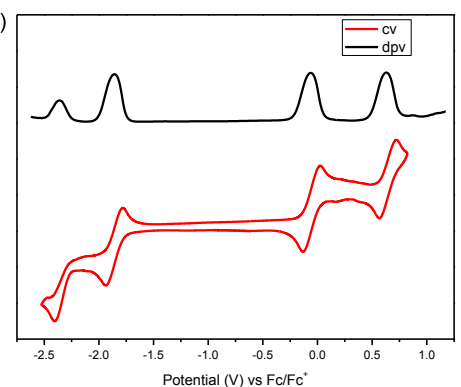

(k)

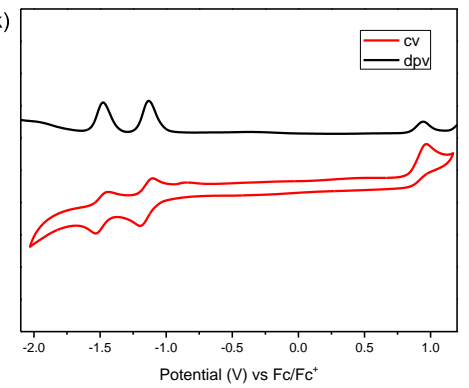

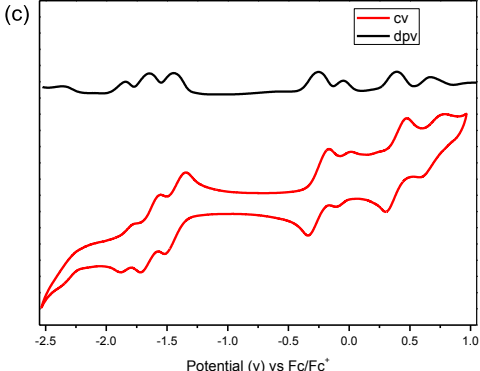
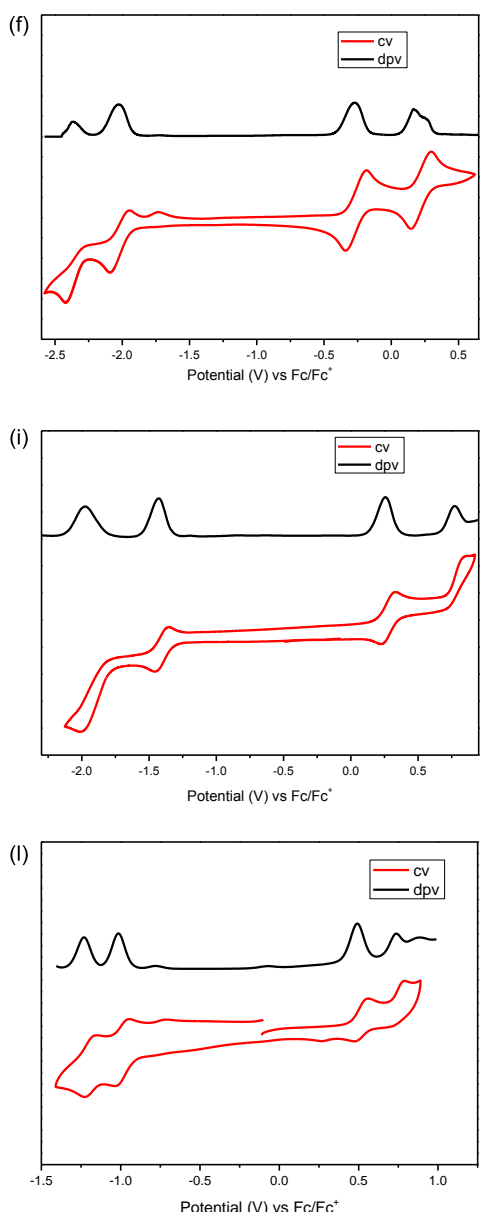

Figure S11. Cyclic voltammograms and differential pulse voltammograms of (a) HZD-M-ONE; (b) HZD-F-ONE; (c) FP-M; (d) PIF-M-ONE; (e) PIF-F-ONE; (f) HZ-M; (g) HZ-F; (h) PF-M; (i) PF-F; (j) IF-M; (k) IF-CF3; (1) IFD-CF 3 in dry DCM with $0.1 \mathrm{M} n$-Bu $4 \mathrm{NPF}_{6}$ as supporting electrolyte. 
7. Additional optical data for those are not shown in the main manuscript
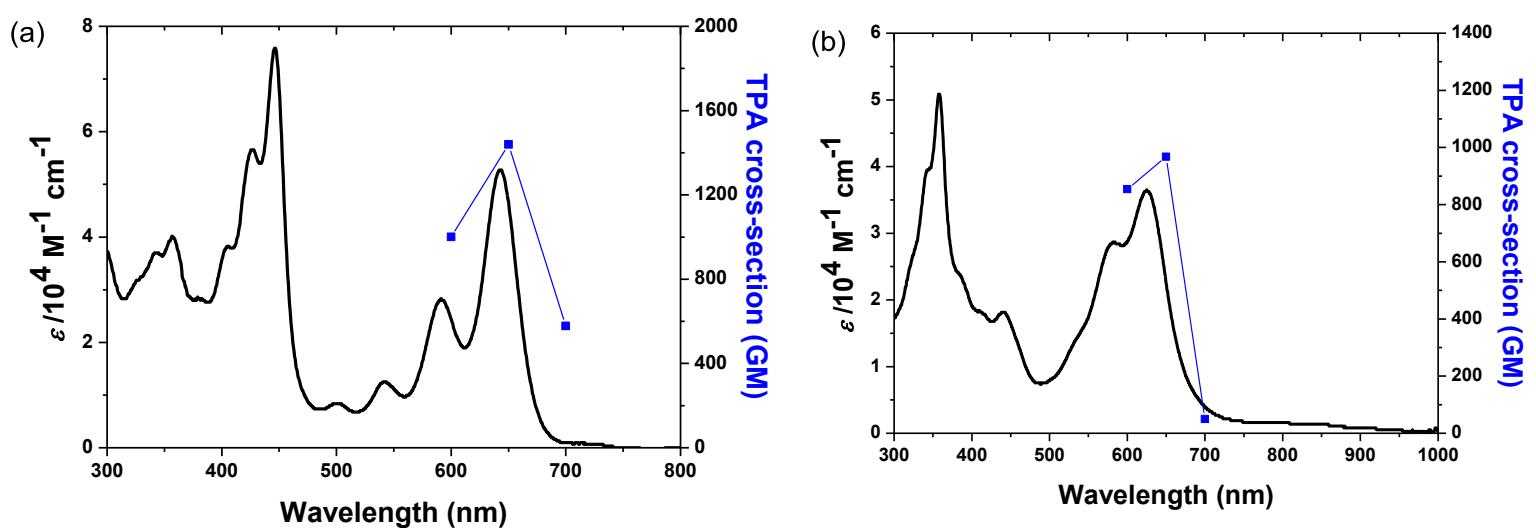

(c)

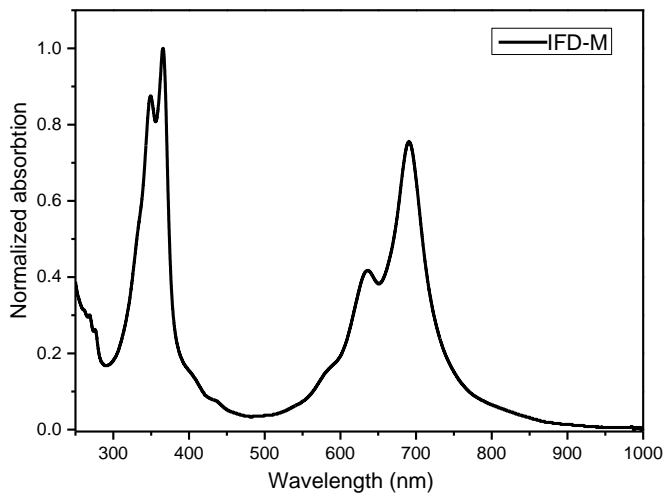

(e)

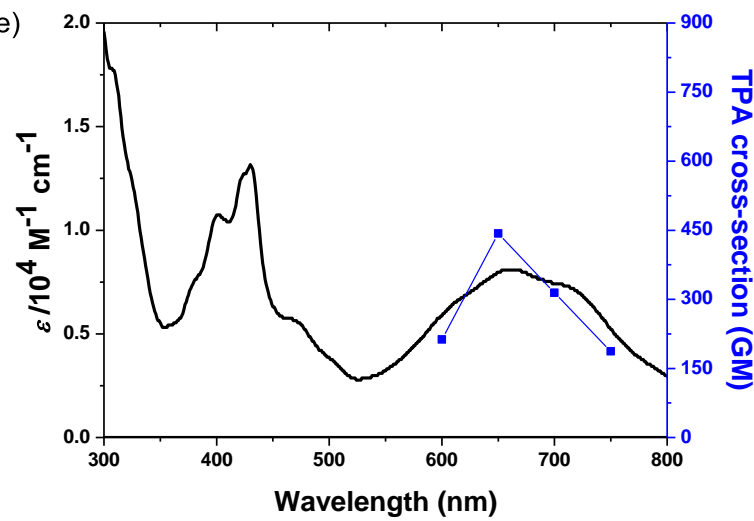

(d)

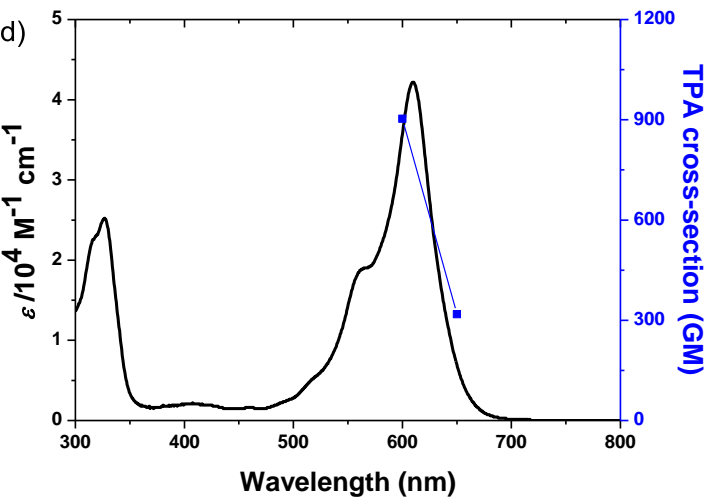

(f)

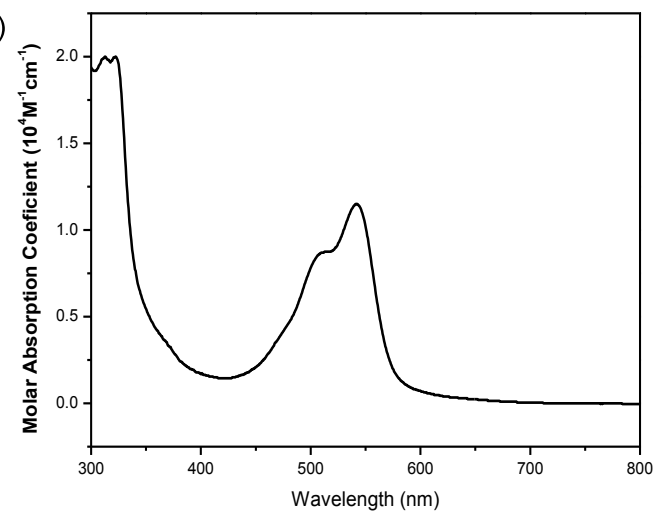

Figure S12. One-photon and two-photon absorption spectra of (a) HZD-F-ONE; (b) PIF-F-ONE; (c) IFD-M; (d) HZ-F; (e) PF-F; (f) IF-CF3 recorded in toluene. TPA spectra are plotted at $\lambda_{\mathrm{ex}} / 2$. 


\section{VT-NMR and VT-ESR data of compound FP-M}

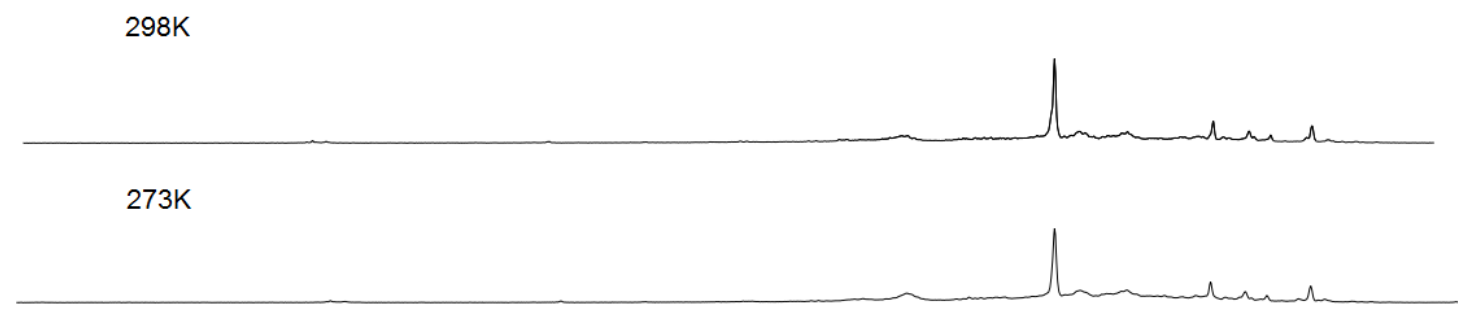

$243 K$

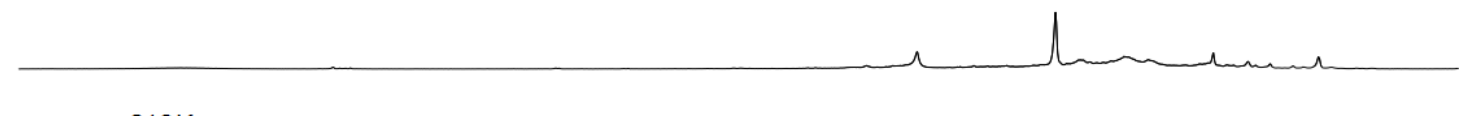

$213 \mathrm{~K}$

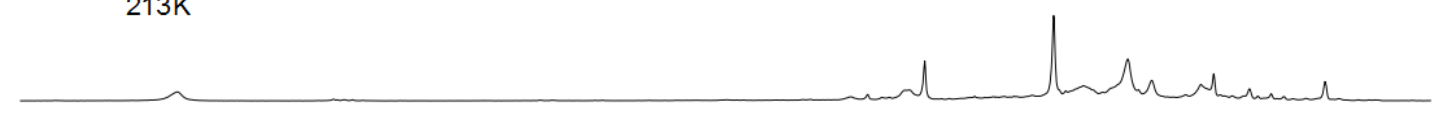

$183 \mathrm{~K}$

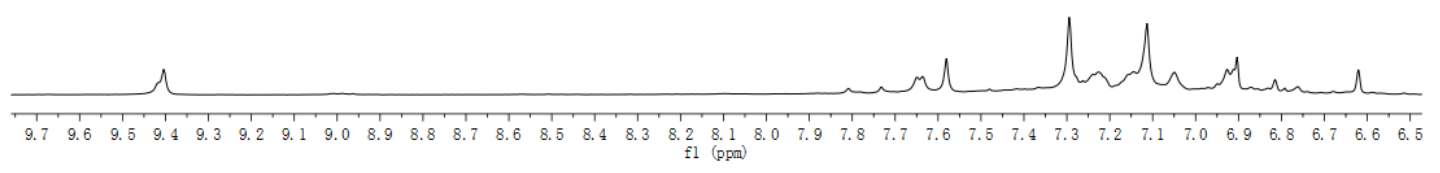

Figure S13. VT ${ }^{1} \mathrm{H}$ NMR spectrum (aromatic region) of compound FP-M $\left(500 \mathrm{MHz}, d_{8}\right.$-THF, from $298 \mathrm{~K}$ to $183 \mathrm{~K})$.
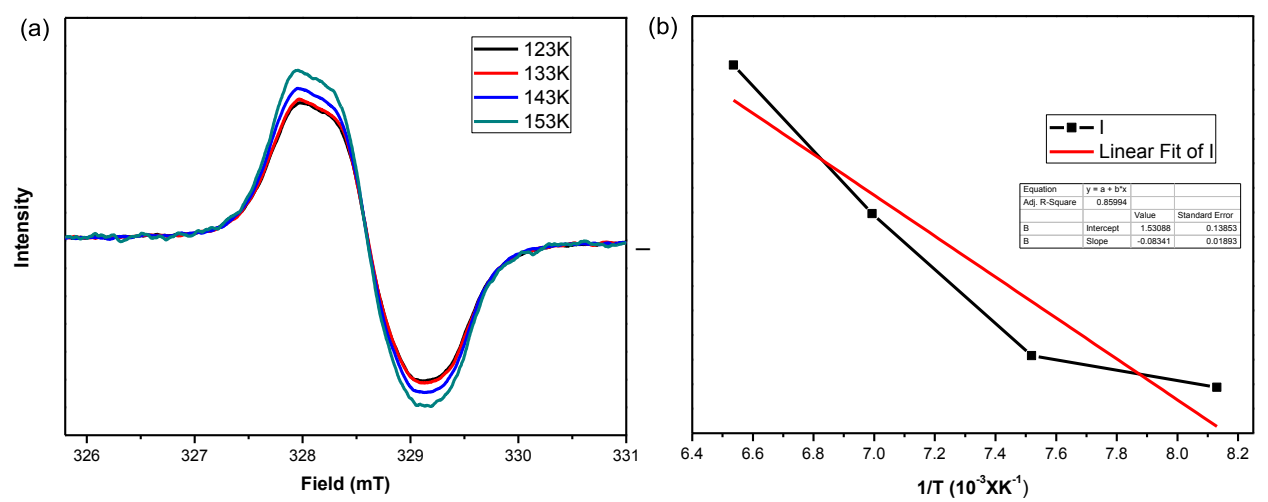

Figure S14. VT-ESR spectra (a) of the compound FP-M in the frozen $\mathrm{CH}_{2} \mathrm{Cl}_{2}$ solution and the integrated ESR intensity $I-1 / T$ curve (b). 


\section{X-ray crystallographic data}

\section{Data for HZD-M-ONE}

Table S7. Crystal data and structure refinement for HZD-M-ONE.

Identification code

Empirical formula

Formula weight

Temperature

Wavelength

Crystal system

Space group

Unit cell dimensions

Volume

Z,Density (calculated)

Absorption coefficient

$\mathrm{F}(000)$

Crystal size

Theta range for data collection

Index ranges

Reflections collected

Independent reflections

Completeness to theta $=25.242^{\circ}$

Absorption correction

Max. and min. transmission

Refinement method

Data / restraints / parameters

Goodness-of-fit on $\mathrm{F}^{2}$

Final $\mathrm{R}$ indices [I $>2 \operatorname{sigma}(\mathrm{I})]$

$\mathrm{R}$ indices (all data)

Extinction coefficient

Largest diff. peak and hole
HZD-M-ONE

C106 H110 O6

1480.04

$90 \mathrm{~K}$

$0.71073 \AA$

Triclinic

P-1

$$
\begin{array}{cr}
\mathrm{a}=15.7254(8) \AA & \alpha=88.0843(9)^{\circ} . \\
\mathrm{b}=16.3000(8) \AA & \beta=73.1877(9)^{\circ} . \\
\mathrm{c}=19.7644(10) & \gamma=70.5165(9)^{\circ} .
\end{array}
$$

4560.7(4) $\AA^{3}$

$2,1.252 \mathrm{Mg} / \mathrm{m}^{3}$

$0.244 \mathrm{~mm}^{-1}$

1820

$0.300 \times 0.200 \times 0.100 \mathrm{~mm}^{3}$

1.079 to $27.000^{\circ}$.

$-19<=\mathrm{h}<=20,-20<=\mathrm{k}<=17,-24<=1<=25$

28551

$19691[\mathrm{R}(\mathrm{int})=0.0206]$

$98.9 \%$

Semi-empirical from equivalents

0.976 and 0.893

Full-matrix least-squares on $\mathrm{F}^{2}$

19691 / 6 / 1153

1.024

$\mathrm{R} 1=0.0640, \mathrm{wR} 2=0.1703$

$\mathrm{R} 1=0.0871, \mathrm{wR} 2=0.1934$

$\mathrm{n} / \mathrm{a}$

1.371 and -1.073 e. $\AA^{-3}$ 
Table S8. Atomic coordinates $\left(\mathrm{x} \mathrm{10} 0^{4}\right)$ and equivalent isotropic displacement parameters $\left(\AA^{2} \times 10^{3}\right)$ for HZD-M-ONE. U(eq) is defined as one third of the trace of the orthogonalized $\mathrm{U}^{\mathrm{ij}}$ tensor.

\begin{tabular}{|c|c|c|c|c|}
\hline & & & $\mathrm{z}$ & \\
\hline $\mathrm{C}(1)$ & $3059(1)$ & 1399(1) & $5102(1)$ & $11(1)$ \\
\hline$C(2)$ & $3310(1)$ & $1696(1)$ & $4415(1)$ & $12(1)$ \\
\hline$C(3)$ & $2636(1)$ & $2377(1)$ & 4191(1) & $14(1)$ \\
\hline$C(4)$ & $2859(1)$ & $2760(1)$ & $3558(1)$ & $13(1)$ \\
\hline$C(5)$ & 2184(2) & $3468(1)$ & $3305(1)$ & $15(1)$ \\
\hline$C(6)$ & $1181(2)$ & $3767(2)$ & $3624(1)$ & $21(1)$ \\
\hline$C(7)$ & $592(2)$ & $4459(2)$ & $3362(1)$ & $24(1)$ \\
\hline $\mathrm{C}(8)$ & $965(2)$ & $4874(2)$ & 2794(1) & $23(1)$ \\
\hline $\mathrm{C}(9)$ & $1925(2)$ & $4600(1)$ & $2455(1)$ & $18(1)$ \\
\hline $\mathrm{C}(10)$ & $2276(2)$ & $5054(1)$ & $1829(1)$ & $21(1)$ \\
\hline $\mathrm{C}(11)$ & $3285(2)$ & $4753(2)$ & 1491(1) & $21(1)$ \\
\hline$C(12)$ & $3868(2)$ & $4068(1)$ & $1722(1)$ & $17(1)$ \\
\hline $\mathrm{C}(13)$ & $3542(2)$ & $3602(1)$ & $2324(1)$ & $14(1)$ \\
\hline$C(14)$ & $2541(2)$ & $3892(1)$ & $2704(1)$ & $15(1)$ \\
\hline$C(15)$ & $4166(1)$ & $2895(1)$ & $2522(1)$ & $13(1)$ \\
\hline$C(16)$ & $3838(1)$ & $2457(1)$ & $3138(1)$ & $13(1)$ \\
\hline$C(17)$ & $4492(1)$ & $1755(1)$ & $3336(1)$ & $12(1)$ \\
\hline $\mathrm{C}(18)$ & $4258(1)$ & $1327(1)$ & $3956(1)$ & $12(1)$ \\
\hline$C(19)$ & 4921(1) & $597(1)$ & $4169(1)$ & $11(1)$ \\
\hline $\mathrm{C}(20)$ & $4675(1)$ & $342(1)$ & $4866(1)$ & $10(1)$ \\
\hline $\mathrm{C}(21)$ & $3741(1)$ & $767(1)$ & $5338(1)$ & 11(1) \\
\hline $\mathrm{C}(22)$ & $6470(1)$ & $-541(1)$ & $3947(1)$ & $13(1)$ \\
\hline$C(23)$ & $5836(1)$ & $84(1)$ & $3697(1)$ & $12(1)$ \\
\hline $\mathrm{C}(24)$ & 2083(1) & $1770(1)$ & $5602(1)$ & $12(1)$ \\
\hline $\mathrm{C}(25)$ & $1829(2)$ & $2538(1)$ & $6026(1)$ & $15(1)$ \\
\hline$C(26)$ & $958(2)$ & $2814(2)$ & $6544(1)$ & $23(1)$ \\
\hline $\mathrm{C}(27)$ & $327(2)$ & $2356(2)$ & $6648(1)$ & $25(1)$ \\
\hline$C(28)$ & $590(2)$ & $1608(2)$ & $6212(1)$ & $21(1)$ \\
\hline C(29) & $1456(2)$ & $1310(1)$ & $5685(1)$ & $16(1)$ \\
\hline $\mathrm{C}(30)$ & 2494(2) & $3047(2)$ & $5938(1)$ & $26(1)$ \\
\hline $\mathrm{C}(31)$ & $-618(2)$ & $2670(2)$ & $7213(2)$ & $42(1)$ \\
\hline
\end{tabular}




\begin{tabular}{|c|c|c|c|c|}
\hline $\mathrm{C}(32)$ & $1715(2)$ & 493(2) & $5228(1)$ & $25(1)$ \\
\hline $\mathrm{C}(33)$ & $-170(5)$ & $3793(4)$ & $4523(4)$ & $17(1)$ \\
\hline $\mathrm{C}(34)$ & $-405(3)$ & $3352(3)$ & $5196(2)$ & $23(1)$ \\
\hline $\mathrm{C}(35)$ & $-1489(3)$ & $3626(3)$ & $5530(2)$ & $25(1)$ \\
\hline$C(36)$ & $-2016(4)$ & $4610(4)$ & $5663(3)$ & $33(1)$ \\
\hline C(37) & $-3030(3)$ & $4865(3)$ & $6125(2)$ & $28(1)$ \\
\hline $\mathrm{C}(38)$ & $-3648(6)$ & $4584(10)$ & $5763(7)$ & $26(2)$ \\
\hline$C(33 A)$ & $-194(7)$ & $3454(6)$ & $4537(6)$ & $27(2)$ \\
\hline$C(34 A)$ & $-463(4)$ & $4106(5)$ & 5161(4) & $36(2)$ \\
\hline$C(35 A)$ & $-1549(6)$ & $4348(6)$ & $5598(4)$ & $33(2)$ \\
\hline$C(36 \mathrm{~A})$ & $-2130(5)$ & $5048(5)$ & $5246(4)$ & $39(2)$ \\
\hline$C(37 A)$ & $-3189(4)$ & $5352(5)$ & $5681(4)$ & $35(2)$ \\
\hline $\mathrm{C}(38 \mathrm{~A})$ & $-3680(15)$ & $4692(17)$ & $5778(14)$ & $63(7)$ \\
\hline C(39) & $5192(1)$ & $2556(1)$ & 2104(1) & $13(1)$ \\
\hline $\mathrm{C}(40)$ & $5831(2)$ & 2914(1) & $2235(1)$ & $17(1)$ \\
\hline $\mathrm{C}(41)$ & $6773(2)$ & $2590(1)$ & $1830(1)$ & 19(1) \\
\hline $\mathrm{C}(42)$ & $7101(2)$ & $1918(1)$ & 1301(1) & $18(1)$ \\
\hline $\mathrm{C}(43)$ & $6460(2)$ & $1562(1)$ & 1194(1) & $17(1)$ \\
\hline $\mathrm{C}(44)$ & $5508(2)$ & $1865(1)$ & $1588(1)$ & $15(1)$ \\
\hline$C(45)$ & $5500(2)$ & $3641(2)$ & $2802(1)$ & $26(1)$ \\
\hline $\mathrm{C}(46)$ & $8123(2)$ & $1590(2)$ & $856(1)$ & $28(1)$ \\
\hline $\mathrm{C}(47)$ & $4850(2)$ & $1440(2)$ & $1461(1)$ & $27(1)$ \\
\hline $\mathrm{C}(48)$ & $6847(2)$ & $-289(2)$ & 2492(1) & $22(1)$ \\
\hline C(49) & $7678(2)$ & $-10(2)$ & $2479(1)$ & $25(1)$ \\
\hline$C(50)$ & $8588(2)$ & $-584(2)$ & 1949(1) & $30(1)$ \\
\hline $\mathrm{C}(51)$ & $9453(2)$ & $-378(2)$ & 1991(1) & $30(1)$ \\
\hline$C(52)$ & $10360(2)$ & $-874(2)$ & $1425(2)$ & $41(1)$ \\
\hline $\mathrm{C}(53)$ & 11211(2) & $-707(2)$ & $1537(2)$ & $50(1)$ \\
\hline$C(54)$ & $6907(1)$ & $8601(1)$ & $-24(1)$ & 11(1) \\
\hline$C(55)$ & $6605(1)$ & $8313(1)$ & $659(1)$ & $12(1)$ \\
\hline$C(56)$ & $7265(1)$ & $7665(1)$ & $918(1)$ & $13(1)$ \\
\hline $\mathrm{C}(57)$ & $7017(1)$ & $7295(1)$ & $1552(1)$ & $12(1)$ \\
\hline $\mathrm{C}(58)$ & $7684(1)$ & $6610(1)$ & $1826(1)$ & $12(1)$ \\
\hline $\mathrm{C}(59)$ & $8681(2)$ & $6386(1)$ & 1559(1) & $15(1)$ \\
\hline $\mathrm{C}(60)$ & $9283(2)$ & $5678(1)$ & $1803(1)$ & $16(1)$ \\
\hline $\mathrm{C}(61)$ & $8908(2)$ & $5203(1)$ & $2324(1)$ & $15(1)$ \\
\hline
\end{tabular}




\begin{tabular}{|c|c|c|c|c|}
\hline$C(62)$ & $7939(2)$ & $5428(1)$ & $2631(1)$ & $13(1)$ \\
\hline$C(63)$ & $7585(2)$ & 4933(1) & $3224(1)$ & $16(1)$ \\
\hline$C(64)$ & $6566(2)$ & $5227(1)$ & $3551(1)$ & $17(1)$ \\
\hline$C(65)$ & $5984(2)$ & $5918(1)$ & $3325(1)$ & $15(1)$ \\
\hline$C(66)$ & $6311(1)$ & $6404(1)$ & $2741(1)$ & $12(1)$ \\
\hline$C(67)$ & $7320(1)$ & $6140(1)$ & $2387(1)$ & $12(1)$ \\
\hline$C(68)$ & $5690(1)$ & 7112(1) & $2540(1)$ & $12(1)$ \\
\hline C(69) & $6027(1)$ & $7566(1)$ & 1934(1) & $11(1)$ \\
\hline $\mathrm{C}(70)$ & $5380(1)$ & $8244(1)$ & $1707(1)$ & $12(1)$ \\
\hline $\mathrm{C}(71)$ & $5640(1)$ & $8666(1)$ & $1083(1)$ & 11(1) \\
\hline $\mathrm{C}(72)$ & 4992(1) & $9377(1)$ & $833(1)$ & $10(1)$ \\
\hline$C(73)$ & $4031(1)$ & $9846(1)$ & $1253(1)$ & $13(1)$ \\
\hline $\mathrm{C}(74)$ & $3438(1)$ & $10486(1)$ & $982(1)$ & $13(1)$ \\
\hline$C(75)$ & $3731(1)$ & $10750(1)$ & $282(1)$ & 11(1) \\
\hline$C(76)$ & $5307(1)$ & $9657(1)$ & $147(1)$ & $10(1)$ \\
\hline $\mathrm{C}(77)$ & $7908(1)$ & 8191(1) & $-475(1)$ & $12(1)$ \\
\hline $\mathrm{C}(78)$ & $8184(2)$ & $7409(1)$ & $-888(1)$ & $16(1)$ \\
\hline $\mathrm{C}(79)$ & $9117(2)$ & $7058(2)$ & $-1314(1)$ & $20(1)$ \\
\hline $\mathrm{C}(80)$ & $9783(2)$ & $7453(2)$ & $-1340(1)$ & 21(1) \\
\hline $\mathrm{C}(81)$ & $9498(2)$ & $8221(2)$ & $-923(1)$ & $22(1)$ \\
\hline $\mathrm{C}(82)$ & $8572(2)$ & $8594(1)$ & $-487(1)$ & $17(1)$ \\
\hline$C(83)$ & $7491(2)$ & $6949(2)$ & $-875(1)$ & $25(1)$ \\
\hline $\mathrm{C}(84)$ & 10792(2) & $7056(2)$ & $-1805(2)$ & $33(1)$ \\
\hline$C(85)$ & $8287(2)$ & $9427(2)$ & $-39(1)$ & $28(1)$ \\
\hline$C(86)$ & $10012(2)$ & $6711(2)$ & $772(1)$ & $21(1)$ \\
\hline $\mathrm{C}(87)$ & $10455(2)$ & $5971(2)$ & $202(1)$ & $22(1)$ \\
\hline $\mathrm{C}(88)$ & $11521(2)$ & $5777(2)$ & $-75(1)$ & $23(1)$ \\
\hline C(89) & $12040(2)$ & 4973(2) & $-589(1)$ & $25(1)$ \\
\hline $\mathrm{C}(90)$ & $13112(2)$ & $4687(2)$ & $-748(1)$ & $29(1)$ \\
\hline $\mathrm{C}(91)$ & $13531(2)$ & $5344(2)$ & $-1126(2)$ & $44(1)$ \\
\hline $\mathrm{C}(92)$ & $4654(1)$ & $7442(1)$ & 2939(1) & $12(1)$ \\
\hline C(93) & $4025(2)$ & $7103(2)$ & $2763(1)$ & $19(1)$ \\
\hline $\mathrm{C}(94)$ & $3067(2)$ & $7457(2)$ & $3126(1)$ & $27(1)$ \\
\hline$C(95)$ & $2716(2)$ & $8125(2)$ & $3655(1)$ & $26(1)$ \\
\hline C(96) & $3351(2)$ & $8443(1)$ & $3821(1)$ & $18(1)$ \\
\hline C(97) & $4314(2)$ & $8115(1)$ & $3470(1)$ & $15(1)$ \\
\hline
\end{tabular}




\begin{tabular}{|c|c|c|c|c|}
\hline C(98) & $4374(2)$ & $6378(2)$ & 2194(1) & $28(1)$ \\
\hline C(99) & $1672(2)$ & $8520(2)$ & $4028(2)$ & $50(1)$ \\
\hline$C(100)$ & $4970(2)$ & $8503(2)$ & $3654(2)$ & $30(1)$ \\
\hline$C(101)$ & 2811(2) & $9982(2)$ & $2367(1)$ & $21(1)$ \\
\hline$C(102)$ & $2603(2)$ & $10842(2)$ & $2758(1)$ & $29(1)$ \\
\hline$C(103)$ & $1597(2)$ & $11141(2)$ & $3280(2)$ & $36(1)$ \\
\hline$C(104)$ & $834(2)$ & 11471(3) & 2922(2) & $69(1)$ \\
\hline$C(105)$ & $-171(2)$ & $11749(2)$ & $3422(2)$ & $60(1)$ \\
\hline$C(106)$ & $-455(3)$ & $11034(3)$ & $3763(3)$ & $86(2)$ \\
\hline$C(107)$ & $6379(2)$ & $2574(2)$ & $4638(2)$ & $34(1)$ \\
\hline$C(108)$ & $3784(2)$ & $7124(2)$ & $205(2)$ & $36(1)$ \\
\hline $\mathrm{Cl}(1)$ & $5216(1)$ & $3293(1)$ & $4820(1)$ & $56(1)$ \\
\hline $\mathrm{Cl}(2)$ & $7178(1)$ & $3051(1)$ & $4124(1)$ & $33(1)$ \\
\hline $\mathrm{Cl}(3)$ & 6493(1) & $1589(1)$ & $4238(1)$ & $57(1)$ \\
\hline $\mathrm{Cl}(4)$ & 2761(1) & $6967(1)$ & $751(1)$ & $45(1)$ \\
\hline $\mathrm{Cl}(5)$ & $3952(1)$ & $8030(1)$ & $528(1)$ & $61(1)$ \\
\hline $\mathrm{Cl}(6)$ & $4756(1)$ & $6170(1)$ & $145(1)$ & $67(1)$ \\
\hline $\mathrm{O}(1)$ & $831(1)$ & $3352(1)$ & $4181(1)$ & $34(1)$ \\
\hline $\mathrm{O}(2)$ & $1725(1)$ & $5652(1)$ & $1601(1)$ & $29(1)$ \\
\hline $\mathrm{O}(3)$ & $5986(1)$ & $241(1)$ & 2997(1) & $19(1)$ \\
\hline $\mathrm{O}(4)$ & $9010(1)$ & 6901(1) & $1073(1)$ & $21(1)$ \\
\hline $\mathrm{O}(5)$ & $8129(1)$ & $4322(1)$ & $3443(1)$ & $24(1)$ \\
\hline $\mathrm{O}(6)$ & $3778(1)$ & $9624(1)$ & 1933(1) & $20(1$ \\
\hline
\end{tabular}

Table S9. Bond lengths $[\AA ̊]$ and angles $\left[{ }^{\circ}\right]$ for HZD-M-ONE.

\begin{tabular}{lc}
\hline $\mathrm{C}(1)-\mathrm{C}(21)$ & $1.396(3)$ \\
$\mathrm{C}(1)-\mathrm{C}(2)$ & $1.417(3)$ \\
$\mathrm{C}(1)-\mathrm{C}(24)$ & $1.497(3)$ \\
$\mathrm{C}(2)-\mathrm{C}(3)$ & $1.417(3)$ \\
$\mathrm{C}(2)-\mathrm{C}(18)$ & $1.436(3)$ \\
$\mathrm{C}(3)-\mathrm{C}(4)$ & $1.383(3)$ \\
$\mathrm{C}(3)-\mathrm{H}(3)$ & 0.9500 \\
$\mathrm{C}(4)-\mathrm{C}(16)$ & $1.444(3)$ \\
$\mathrm{C}(4)-\mathrm{C}(5)$ & $1.470(3)$
\end{tabular}




\begin{tabular}{|c|c|}
\hline$C(5)-C(14)$ & $1.422(3)$ \\
\hline$C(5)-C(6)$ & $1.434(3)$ \\
\hline $\mathrm{C}(6)-\mathrm{O}(1)$ & $1.350(3)$ \\
\hline$C(6)-C(7)$ & $1.392(3)$ \\
\hline $\mathrm{C}(7)-\mathrm{C}(8)$ & $1.376(4)$ \\
\hline $\mathrm{C}(7)-\mathrm{H}(7)$ & 0.9500 \\
\hline $\mathrm{C}(8)-\mathrm{C}(9)$ & $1.383(3)$ \\
\hline $\mathrm{C}(8)-\mathrm{H}(8)$ & 0.9500 \\
\hline $\mathrm{C}(9)-\mathrm{C}(14)$ & $1.416(3)$ \\
\hline$C(9)-C(10)$ & $1.483(3)$ \\
\hline $\mathrm{C}(10)-\mathrm{O}(2)$ & $1.239(3)$ \\
\hline $\mathrm{C}(10)-\mathrm{C}(11)$ & $1.448(3)$ \\
\hline $\mathrm{C}(11)-\mathrm{C}(12)$ & $1.351(3)$ \\
\hline $\mathrm{C}(11)-\mathrm{H}(11)$ & 0.9500 \\
\hline $\mathrm{C}(12)-\mathrm{C}(13)$ & $1.449(3)$ \\
\hline $\mathrm{C}(12)-\mathrm{H}(12)$ & 0.9500 \\
\hline$C(13)-C(15)$ & $1.372(3)$ \\
\hline $\mathrm{C}(13)-\mathrm{C}(14)$ & $1.453(3)$ \\
\hline$C(15)-C(16)$ & $1.443(3)$ \\
\hline$C(15)-C(39)$ & $1.501(3)$ \\
\hline$C(16)-C(17)$ & $1.392(3)$ \\
\hline $\mathrm{C}(17)-\mathrm{C}(18)$ & $1.406(3)$ \\
\hline $\mathrm{C}(17)-\mathrm{H}(17)$ & 0.9500 \\
\hline $\mathrm{C}(18)-\mathrm{C}(19)$ & $1.441(3)$ \\
\hline $\mathrm{C}(19)-\mathrm{C}(20)$ & $1.407(3)$ \\
\hline $\mathrm{C}(19)-\mathrm{C}(23)$ & $1.454(3)$ \\
\hline$C(20)-C(21)$ & $1.441(3)$ \\
\hline$C(20)-C(20) \# 1$ & $1.444(4)$ \\
\hline $\mathrm{C}(21)-\mathrm{C}(22) \# 1$ & $1.420(3)$ \\
\hline$C(22)-C(23)$ & $1.360(3)$ \\
\hline $\mathrm{C}(22)-\mathrm{C}(21) \# 1$ & $1.420(3)$ \\
\hline $\mathrm{C}(22)-\mathrm{H}(22)$ & 0.9500 \\
\hline $\mathrm{C}(23)-\mathrm{O}(3)$ & $1.364(2)$ \\
\hline$C(24)-C(29)$ & $1.398(3)$ \\
\hline$C(24)-C(25)$ & $1.403(3)$ \\
\hline$C(25)-C(26)$ & $1.392(3)$ \\
\hline
\end{tabular}




\begin{tabular}{|c|c|}
\hline$C(25)-C(30)$ & $1.510(3)$ \\
\hline$C(26)-C(27)$ & $1.395(3)$ \\
\hline $\mathrm{C}(26)-\mathrm{H}(26)$ & 0.9500 \\
\hline $\mathrm{C}(27)-\mathrm{C}(28)$ & $1.388(3)$ \\
\hline $\mathrm{C}(27)-\mathrm{C}(31)$ & $1.513(3)$ \\
\hline $\mathrm{C}(28)-\mathrm{C}(29)$ & $1.396(3)$ \\
\hline $\mathrm{C}(28)-\mathrm{H}(28)$ & 0.9500 \\
\hline $\mathrm{C}(29)-\mathrm{C}(32)$ & $1.504(3)$ \\
\hline $\mathrm{C}(30)-\mathrm{H}(30 \mathrm{~A})$ & 0.9800 \\
\hline $\mathrm{C}(30)-\mathrm{H}(30 \mathrm{~B})$ & 0.9800 \\
\hline $\mathrm{C}(30)-\mathrm{H}(30 \mathrm{C})$ & 0.9800 \\
\hline $\mathrm{C}(31)-\mathrm{H}(31 \mathrm{~A})$ & 0.9800 \\
\hline $\mathrm{C}(31)-\mathrm{H}(31 \mathrm{~B})$ & 0.9800 \\
\hline $\mathrm{C}(31)-\mathrm{H}(31 \mathrm{C})$ & 0.9800 \\
\hline $\mathrm{C}(32)-\mathrm{H}(32 \mathrm{~A})$ & 0.9800 \\
\hline $\mathrm{C}(32)-\mathrm{H}(32 \mathrm{~B})$ & 0.9800 \\
\hline $\mathrm{C}(32)-\mathrm{H}(32 \mathrm{C})$ & 0.9800 \\
\hline $\mathrm{C}(33)-\mathrm{O}(1)$ & $1.455(7)$ \\
\hline $\mathrm{C}(33)-\mathrm{C}(34)$ & $1.501(8)$ \\
\hline $\mathrm{C}(33)-\mathrm{H}(33 \mathrm{~A})$ & 0.9900 \\
\hline $\mathrm{C}(33)-\mathrm{H}(33 \mathrm{~B})$ & 0.9900 \\
\hline$C(34)-C(35)$ & $1.550(5)$ \\
\hline $\mathrm{C}(34)-\mathrm{H}(34 \mathrm{~A})$ & 0.9900 \\
\hline $\mathrm{C}(34)-\mathrm{H}(34 \mathrm{~B})$ & 0.9900 \\
\hline $\mathrm{C}(35)-\mathrm{C}(36)$ & $1.531(7)$ \\
\hline $\mathrm{C}(35)-\mathrm{H}(35 \mathrm{~A})$ & 0.9900 \\
\hline $\mathrm{C}(35)-\mathrm{H}(35 \mathrm{~B})$ & 0.9900 \\
\hline$C(36)-C(37)$ & $1.514(6)$ \\
\hline $\mathrm{C}(36)-\mathrm{H}(36 \mathrm{~A})$ & 0.9900 \\
\hline $\mathrm{C}(36)-\mathrm{H}(36 \mathrm{~B})$ & 0.9900 \\
\hline $\mathrm{C}(37)-\mathrm{C}(38)$ & $1.544(11)$ \\
\hline $\mathrm{C}(37)-\mathrm{H}(37 \mathrm{~A})$ & 0.9900 \\
\hline $\mathrm{C}(37)-\mathrm{H}(37 \mathrm{~B})$ & 0.9900 \\
\hline $\mathrm{C}(38)-\mathrm{H}(38 \mathrm{~A})$ & 0.9800 \\
\hline $\mathrm{C}(38)-\mathrm{H}(38 \mathrm{~B})$ & 0.9800 \\
\hline $\mathrm{C}(38)-\mathrm{H}(38 \mathrm{C})$ & 0.9800 \\
\hline
\end{tabular}




\begin{tabular}{|c|c|}
\hline $\mathrm{C}(33 \mathrm{~A})-\mathrm{O}(1)$ & $1.515(10)$ \\
\hline $\mathrm{C}(33 \mathrm{~A})-\mathrm{C}(34 \mathrm{~A})$ & $1.520(13)$ \\
\hline $\mathrm{C}(33 \mathrm{~A})-\mathrm{H}(33 \mathrm{C})$ & 0.9900 \\
\hline $\mathrm{C}(33 \mathrm{~A})-\mathrm{H}(33 \mathrm{D})$ & 0.9900 \\
\hline $\mathrm{C}(34 \mathrm{~A})-\mathrm{C}(35 \mathrm{~A})$ & $1.594(10)$ \\
\hline $\mathrm{C}(34 \mathrm{~A})-\mathrm{H}(34 \mathrm{C})$ & 0.9900 \\
\hline $\mathrm{C}(34 \mathrm{~A})-\mathrm{H}(34 \mathrm{D})$ & 0.9900 \\
\hline$C(35 A)-C(36 A)$ & $1.497(10)$ \\
\hline $\mathrm{C}(35 \mathrm{~A})-\mathrm{H}(35 \mathrm{C})$ & 0.9900 \\
\hline $\mathrm{C}(35 \mathrm{~A})-\mathrm{H}(35 \mathrm{D})$ & 0.9900 \\
\hline $\mathrm{C}(36 \mathrm{~A})-\mathrm{C}(37 \mathrm{~A})$ & $1.551(9)$ \\
\hline $\mathrm{C}(36 \mathrm{~A})-\mathrm{H}(36 \mathrm{C})$ & 0.9900 \\
\hline $\mathrm{C}(36 \mathrm{~A})-\mathrm{H}(36 \mathrm{D})$ & 0.9900 \\
\hline $\mathrm{C}(37 \mathrm{~A})-\mathrm{C}(38 \mathrm{~A})$ & $1.50(2)$ \\
\hline $\mathrm{C}(37 \mathrm{~A})-\mathrm{H}(37 \mathrm{C})$ & 0.9900 \\
\hline $\mathrm{C}(37 \mathrm{~A})-\mathrm{H}(37 \mathrm{D})$ & 0.9900 \\
\hline $\mathrm{C}(38 \mathrm{~A})-\mathrm{H}(38 \mathrm{D})$ & 0.9800 \\
\hline $\mathrm{C}(38 \mathrm{~A})-\mathrm{H}(38 \mathrm{E})$ & 0.9800 \\
\hline $\mathrm{C}(38 \mathrm{~A})-\mathrm{H}(38 \mathrm{~F})$ & 0.9800 \\
\hline C(39)-C(44) & $1.401(3)$ \\
\hline $\mathrm{C}(39)-\mathrm{C}(40)$ & $1.404(3)$ \\
\hline$C(40)-C(41)$ & $1.391(3)$ \\
\hline$C(40)-C(45)$ & $1.507(3)$ \\
\hline$C(41)-C(42)$ & $1.395(3)$ \\
\hline $\mathrm{C}(41)-\mathrm{H}(41)$ & 0.9500 \\
\hline$C(42)-C(43)$ & $1.386(3)$ \\
\hline$C(42)-C(46)$ & $1.511(3)$ \\
\hline$C(43)-C(44)$ & $1.396(3)$ \\
\hline $\mathrm{C}(43)-\mathrm{H}(43)$ & 0.9500 \\
\hline $\mathrm{C}(44)-\mathrm{C}(47)$ & $1.501(3)$ \\
\hline $\mathrm{C}(45)-\mathrm{H}(45 \mathrm{~A})$ & 0.9800 \\
\hline $\mathrm{C}(45)-\mathrm{H}(45 \mathrm{~B})$ & 0.9800 \\
\hline $\mathrm{C}(45)-\mathrm{H}(45 \mathrm{C})$ & 0.9800 \\
\hline $\mathrm{C}(46)-\mathrm{H}(46 \mathrm{~A})$ & 0.9800 \\
\hline $\mathrm{C}(46)-\mathrm{H}(46 \mathrm{~B})$ & 0.9800 \\
\hline $\mathrm{C}(46)-\mathrm{H}(46 \mathrm{C})$ & 0.9800 \\
\hline
\end{tabular}




\begin{tabular}{|c|c|}
\hline $\mathrm{C}(47)-\mathrm{H}(47 \mathrm{~A})$ & 0.9800 \\
\hline $\mathrm{C}(47)-\mathrm{H}(47 \mathrm{~B})$ & 0.9800 \\
\hline $\mathrm{C}(47)-\mathrm{H}(47 \mathrm{C})$ & 0.9800 \\
\hline $\mathrm{C}(48)-\mathrm{O}(3)$ & $1.440(2)$ \\
\hline C(48)-C(49) & $1.514(3)$ \\
\hline $\mathrm{C}(48)-\mathrm{H}(48 \mathrm{~A})$ & 0.9900 \\
\hline $\mathrm{C}(48)-\mathrm{H}(48 \mathrm{~B})$ & 0.9900 \\
\hline $\mathrm{C}(49)-\mathrm{C}(50)$ & $1.525(3)$ \\
\hline $\mathrm{C}(49)-\mathrm{H}(49 \mathrm{~A})$ & 0.9900 \\
\hline $\mathrm{C}(49)-\mathrm{H}(49 \mathrm{~B})$ & 0.9900 \\
\hline $\mathrm{C}(50)-\mathrm{C}(51)$ & $1.530(4)$ \\
\hline $\mathrm{C}(50)-\mathrm{H}(50 \mathrm{~A})$ & 0.9900 \\
\hline $\mathrm{C}(50)-\mathrm{H}(50 \mathrm{~B})$ & 0.9900 \\
\hline $\mathrm{C}(51)-\mathrm{C}(52)$ & $1.519(4)$ \\
\hline $\mathrm{C}(51)-\mathrm{H}(51 \mathrm{~A})$ & 0.9900 \\
\hline $\mathrm{C}(51)-\mathrm{H}(51 \mathrm{~B})$ & 0.9900 \\
\hline$C(52)-C(53)$ & $1.526(4)$ \\
\hline $\mathrm{C}(52)-\mathrm{H}(52 \mathrm{~A})$ & 0.9900 \\
\hline $\mathrm{C}(52)-\mathrm{H}(52 \mathrm{~B})$ & 0.9900 \\
\hline $\mathrm{C}(53)-\mathrm{H}(53 \mathrm{~A})$ & 0.9800 \\
\hline $\mathrm{C}(53)-\mathrm{H}(53 \mathrm{~B})$ & 0.9800 \\
\hline $\mathrm{C}(53)-\mathrm{H}(53 \mathrm{C})$ & 0.9800 \\
\hline $\mathrm{C}(54)-\mathrm{C}(75) \# 2$ & $1.392(3)$ \\
\hline $\mathrm{C}(54)-\mathrm{C}(55)$ & $1.416(3)$ \\
\hline C(54)-C(77) & $1.499(3)$ \\
\hline$C(55)-C(56)$ & $1.411(3)$ \\
\hline $\mathrm{C}(55)-\mathrm{C}(71)$ & $1.432(3)$ \\
\hline$C(56)-C(57)$ & $1.383(3)$ \\
\hline $\mathrm{C}(56)-\mathrm{H}(56)$ & 0.9500 \\
\hline$C(57)-C(69)$ & $1.439(3)$ \\
\hline $\mathrm{C}(57)-\mathrm{C}(58)$ & $1.466(3)$ \\
\hline $\mathrm{C}(58)-\mathrm{C}(67)$ & $1.418(3)$ \\
\hline $\mathrm{C}(58)-\mathrm{C}(59)$ & $1.422(3)$ \\
\hline $\mathrm{C}(59)-\mathrm{O}(4)$ & $1.356(3)$ \\
\hline C(59)-C(60) & $1.399(3)$ \\
\hline $\mathrm{C}(60)-\mathrm{C}(61)$ & $1.380(3)$ \\
\hline
\end{tabular}




\begin{tabular}{|c|c|}
\hline $\mathrm{C}(60)-\mathrm{H}(60)$ & 0.9500 \\
\hline $\mathrm{C}(61)-\mathrm{C}(62)$ & $1.390(3)$ \\
\hline $\mathrm{C}(61)-\mathrm{H}(61)$ & 0.9500 \\
\hline $\mathrm{C}(62)-\mathrm{C}(67)$ & $1.416(3)$ \\
\hline $\mathrm{C}(62)-\mathrm{C}(63)$ & $1.482(3)$ \\
\hline $\mathrm{C}(63)-\mathrm{O}(5)$ & $1.235(3)$ \\
\hline $\mathrm{C}(63)-\mathrm{C}(64)$ & $1.457(3)$ \\
\hline $\mathrm{C}(64)-\mathrm{C}(65)$ & $1.349(3)$ \\
\hline $\mathrm{C}(64)-\mathrm{H}(64)$ & 0.9500 \\
\hline $\mathrm{C}(65)-\mathrm{C}(66)$ & $1.447(3)$ \\
\hline $\mathrm{C}(65)-\mathrm{H}(65)$ & 0.9500 \\
\hline $\mathrm{C}(66)-\mathrm{C}(68)$ & $1.372(3)$ \\
\hline $\mathrm{C}(66)-\mathrm{C}(67)$ & $1.454(3)$ \\
\hline $\mathrm{C}(68)-\mathrm{C}(69)$ & $1.450(3)$ \\
\hline $\mathrm{C}(68)-\mathrm{C}(92)$ & $1.504(3)$ \\
\hline C(69)-C(70) & $1.391(3)$ \\
\hline $\mathrm{C}(70)-\mathrm{C}(71)$ & $1.415(3)$ \\
\hline $\mathrm{C}(70)-\mathrm{H}(70)$ & 0.9500 \\
\hline $\mathrm{C}(71)-\mathrm{C}(72)$ & $1.446(3)$ \\
\hline$C(72)-C(76)$ & $1.419(3)$ \\
\hline $\mathrm{C}(72)-\mathrm{C}(73)$ & $1.449(3)$ \\
\hline $\mathrm{C}(73)-\mathrm{C}(74)$ & $1.358(3)$ \\
\hline $\mathrm{C}(73)-\mathrm{O}(6)$ & $1.361(2)$ \\
\hline $\mathrm{C}(74)-\mathrm{C}(75)$ & $1.426(3)$ \\
\hline $\mathrm{C}(74)-\mathrm{H}(74)$ & 0.9500 \\
\hline$C(75)-C(54) \# 2$ & $1.392(3)$ \\
\hline$C(75)-C(76) \# 2$ & $1.440(3)$ \\
\hline $\mathrm{C}(76)-\mathrm{C}(75) \# 2$ & $1.440(3)$ \\
\hline$C(76)-C(76) \# 2$ & $1.443(4)$ \\
\hline $\mathrm{C}(77)-\mathrm{C}(82)$ & $1.401(3)$ \\
\hline C(77)-C(78) & $1.404(3)$ \\
\hline $\mathrm{C}(78)-\mathrm{C}(79)$ & $1.395(3)$ \\
\hline $\mathrm{C}(78)-\mathrm{C}(83)$ & $1.509(3)$ \\
\hline $\mathrm{C}(79)-\mathrm{C}(80)$ & $1.390(3)$ \\
\hline $\mathrm{C}(79)-\mathrm{H}(79)$ & 0.9500 \\
\hline $\mathrm{C}(80)-\mathrm{C}(81)$ & $1.390(3)$ \\
\hline
\end{tabular}




\begin{tabular}{|c|c|}
\hline C(80)-C(84) & $1.513(3)$ \\
\hline $\mathrm{C}(81)-\mathrm{C}(82)$ & $1.397(3)$ \\
\hline $\mathrm{C}(81)-\mathrm{H}(81)$ & 0.9500 \\
\hline C(82)-C(85) & $1.508(3)$ \\
\hline $\mathrm{C}(83)-\mathrm{H}(83 \mathrm{~A})$ & 0.9800 \\
\hline $\mathrm{C}(83)-\mathrm{H}(83 \mathrm{~B})$ & 0.9800 \\
\hline $\mathrm{C}(83)-\mathrm{H}(83 \mathrm{C})$ & 0.9800 \\
\hline $\mathrm{C}(84)-\mathrm{H}(84 \mathrm{~A})$ & 0.9800 \\
\hline $\mathrm{C}(84)-\mathrm{H}(84 \mathrm{~B})$ & 0.9800 \\
\hline $\mathrm{C}(84)-\mathrm{H}(84 \mathrm{C})$ & 0.9800 \\
\hline $\mathrm{C}(85)-\mathrm{H}(85 \mathrm{~A})$ & 0.9800 \\
\hline $\mathrm{C}(85)-\mathrm{H}(85 \mathrm{~B})$ & 0.9800 \\
\hline $\mathrm{C}(85)-\mathrm{H}(85 \mathrm{C})$ & 0.9800 \\
\hline $\mathrm{C}(86)-\mathrm{O}(4)$ & $1.441(2)$ \\
\hline C(86)-C(87) & $1.512(3)$ \\
\hline $\mathrm{C}(86)-\mathrm{H}(86 \mathrm{~A})$ & 0.9900 \\
\hline $\mathrm{C}(86)-\mathrm{H}(86 \mathrm{~B})$ & 0.9900 \\
\hline C(87)-C(88) & $1.528(3)$ \\
\hline $\mathrm{C}(87)-\mathrm{H}(87 \mathrm{~A})$ & 0.9900 \\
\hline $\mathrm{C}(87)-\mathrm{H}(87 \mathrm{~B})$ & 0.9900 \\
\hline C(88)-C(89) & $1.521(3)$ \\
\hline $\mathrm{C}(88)-\mathrm{H}(88 \mathrm{~A})$ & 0.9900 \\
\hline $\mathrm{C}(88)-\mathrm{H}(88 \mathrm{~B})$ & 0.9900 \\
\hline C(89)-C(90) & $1.530(3)$ \\
\hline $\mathrm{C}(89)-\mathrm{H}(89 \mathrm{~A})$ & 0.9900 \\
\hline $\mathrm{C}(89)-\mathrm{H}(89 \mathrm{~B})$ & 0.9900 \\
\hline $\mathrm{C}(90)-\mathrm{C}(91)$ & $1.507(4)$ \\
\hline $\mathrm{C}(90)-\mathrm{H}(90 \mathrm{~A})$ & 0.9900 \\
\hline $\mathrm{C}(90)-\mathrm{H}(90 \mathrm{~B})$ & 0.9900 \\
\hline $\mathrm{C}(91)-\mathrm{H}(91 \mathrm{~A})$ & 0.9800 \\
\hline $\mathrm{C}(91)-\mathrm{H}(91 \mathrm{~B})$ & 0.9800 \\
\hline $\mathrm{C}(91)-\mathrm{H}(91 \mathrm{C})$ & 0.9800 \\
\hline C(92)-C(97) & $1.397(3)$ \\
\hline $\mathrm{C}(92)-\mathrm{C}(93)$ & $1.408(3)$ \\
\hline C(93)-C(94) & $1.392(3)$ \\
\hline C(93)-C(98) & $1.504(3)$ \\
\hline
\end{tabular}




\begin{tabular}{|c|c|}
\hline $\mathrm{C}(94)-\mathrm{C}(95)$ & $1.388(3)$ \\
\hline C(94)-H(94) & 0.9500 \\
\hline C(95)-C(96) & $1.387(3)$ \\
\hline $\mathrm{C}(95)-\mathrm{C}(99)$ & $1.510(3)$ \\
\hline C(96)-C(97) & $1.392(3)$ \\
\hline $\mathrm{C}(96)-\mathrm{H}(96)$ & 0.9500 \\
\hline$C(97)-C(100)$ & $1.504(3)$ \\
\hline $\mathrm{C}(98)-\mathrm{H}(98 \mathrm{~A})$ & 0.9800 \\
\hline $\mathrm{C}(98)-\mathrm{H}(98 \mathrm{~B})$ & 0.9800 \\
\hline C(98)-H(98C) & 0.9800 \\
\hline $\mathrm{C}(99)-\mathrm{H}(99 \mathrm{~A})$ & 0.9800 \\
\hline C(99)-H(99B) & 0.9800 \\
\hline C(99)-H(99C) & 0.9800 \\
\hline $\mathrm{C}(100)-\mathrm{H}(10 \mathrm{~A})$ & 0.9800 \\
\hline $\mathrm{C}(100)-\mathrm{H}(10 \mathrm{~B})$ & 0.9800 \\
\hline $\mathrm{C}(100)-\mathrm{H}(10 \mathrm{C})$ & 0.9800 \\
\hline $\mathrm{C}(101)-\mathrm{O}(6)$ & $1.440(2)$ \\
\hline$C(101)-C(102)$ & $1.513(4)$ \\
\hline $\mathrm{C}(101)-\mathrm{H}(10 \mathrm{D})$ & 0.9900 \\
\hline $\mathrm{C}(101)-\mathrm{H}(10 \mathrm{E})$ & 0.9900 \\
\hline$C(102)-C(103)$ & $1.543(3)$ \\
\hline $\mathrm{C}(102)-\mathrm{H}(10 \mathrm{~F})$ & 0.9900 \\
\hline $\mathrm{C}(102)-\mathrm{H}(10 \mathrm{G})$ & 0.9900 \\
\hline$C(103)-C(104)$ & $1.504(5)$ \\
\hline $\mathrm{C}(103)-\mathrm{H}(10 \mathrm{H})$ & 0.9900 \\
\hline $\mathrm{C}(103)-\mathrm{H}(10 \mathrm{I})$ & 0.9900 \\
\hline$C(104)-C(105)$ & $1.526(4)$ \\
\hline $\mathrm{C}(104)-\mathrm{H}(10 \mathrm{~J})$ & 0.9900 \\
\hline $\mathrm{C}(104)-\mathrm{H}(10 \mathrm{~K})$ & 0.9900 \\
\hline$C(105)-C(106)$ & $1.461(5)$ \\
\hline $\mathrm{C}(105)-\mathrm{H}(10 \mathrm{~L})$ & 0.9900 \\
\hline $\mathrm{C}(105)-\mathrm{H}(10 \mathrm{M})$ & 0.9900 \\
\hline $\mathrm{C}(106)-\mathrm{H}(10 \mathrm{~N})$ & 0.9800 \\
\hline $\mathrm{C}(106)-\mathrm{H}(10 \mathrm{O})$ & 0.9800 \\
\hline $\mathrm{C}(106)-\mathrm{H}(10 \mathrm{P})$ & 0.9800 \\
\hline $\mathrm{C}(107)-\mathrm{Cl}(3)$ & $1.745(3)$ \\
\hline
\end{tabular}




\begin{tabular}{|c|c|}
\hline $\mathrm{C}(107)-\mathrm{Cl}(1)$ & $1.751(3)$ \\
\hline $\mathrm{C}(107)-\mathrm{Cl}(2)$ & $1.754(3)$ \\
\hline $\mathrm{C}(107)-\mathrm{H}(107)$ & 1.0000 \\
\hline $\mathrm{C}(108)-\mathrm{Cl}(4)$ & $1.753(3)$ \\
\hline $\mathrm{C}(108)-\mathrm{Cl}(5)$ & $1.754(3)$ \\
\hline $\mathrm{C}(108)-\mathrm{Cl}(6)$ & $1.759(3)$ \\
\hline $\mathrm{C}(108)-\mathrm{H}(108)$ & 1.0000 \\
\hline $\mathrm{C}(21)-\mathrm{C}(1)-\mathrm{C}(2)$ & $119.82(18)$ \\
\hline $\mathrm{C}(21)-\mathrm{C}(1)-\mathrm{C}(24)$ & $118.32(17)$ \\
\hline $\mathrm{C}(2)-\mathrm{C}(1)-\mathrm{C}(24)$ & $121.82(17)$ \\
\hline $\mathrm{C}(3)-\mathrm{C}(2)-\mathrm{C}(1)$ & $120.01(18)$ \\
\hline $\mathrm{C}(3)-\mathrm{C}(2)-\mathrm{C}(18)$ & $120.02(18)$ \\
\hline $\mathrm{C}(1)-\mathrm{C}(2)-\mathrm{C}(18)$ & $119.94(18)$ \\
\hline $\mathrm{C}(4)-\mathrm{C}(3)-\mathrm{C}(2)$ & $122.98(18)$ \\
\hline $\mathrm{C}(4)-\mathrm{C}(3)-\mathrm{H}(3)$ & 118.5 \\
\hline $\mathrm{C}(2)-\mathrm{C}(3)-\mathrm{H}(3)$ & 118.5 \\
\hline $\mathrm{C}(3)-\mathrm{C}(4)-\mathrm{C}(16)$ & $116.64(18)$ \\
\hline$C(3)-C(4)-C(5)$ & $124.85(18)$ \\
\hline $\mathrm{C}(16)-\mathrm{C}(4)-\mathrm{C}(5)$ & $118.46(18)$ \\
\hline$C(14)-C(5)-C(6)$ & $117.15(19)$ \\
\hline $\mathrm{C}(14)-\mathrm{C}(5)-\mathrm{C}(4)$ & $118.39(18)$ \\
\hline$C(6)-C(5)-C(4)$ & $124.46(19)$ \\
\hline $\mathrm{O}(1)-\mathrm{C}(6)-\mathrm{C}(7)$ & $121.5(2)$ \\
\hline $\mathrm{O}(1)-\mathrm{C}(6)-\mathrm{C}(5)$ & $117.64(19)$ \\
\hline$C(7)-C(6)-C(5)$ & $120.9(2)$ \\
\hline$C(8)-C(7)-C(6)$ & $120.4(2)$ \\
\hline $\mathrm{C}(8)-\mathrm{C}(7)-\mathrm{H}(7)$ & 119.8 \\
\hline $\mathrm{C}(6)-\mathrm{C}(7)-\mathrm{H}(7)$ & 119.8 \\
\hline $\mathrm{C}(7)-\mathrm{C}(8)-\mathrm{C}(9)$ & $121.3(2)$ \\
\hline $\mathrm{C}(7)-\mathrm{C}(8)-\mathrm{H}(8)$ & 119.3 \\
\hline $\mathrm{C}(9)-\mathrm{C}(8)-\mathrm{H}(8)$ & 119.3 \\
\hline $\mathrm{C}(8)-\mathrm{C}(9)-\mathrm{C}(14)$ & $119.6(2)$ \\
\hline $\mathrm{C}(8)-\mathrm{C}(9)-\mathrm{C}(10)$ & $118.49(19)$ \\
\hline $\mathrm{C}(14)-\mathrm{C}(9)-\mathrm{C}(10)$ & $121.9(2)$ \\
\hline $\mathrm{O}(2)-\mathrm{C}(10)-\mathrm{C}(11)$ & $121.9(2)$ \\
\hline
\end{tabular}




\begin{tabular}{|c|c|}
\hline $\mathrm{O}(2)-\mathrm{C}(10)-\mathrm{C}(9)$ & $121.2(2)$ \\
\hline $\mathrm{C}(11)-\mathrm{C}(10)-\mathrm{C}(9)$ & $116.88(19)$ \\
\hline $\mathrm{C}(12)-\mathrm{C}(11)-\mathrm{C}(10)$ & $120.9(2)$ \\
\hline $\mathrm{C}(12)-\mathrm{C}(11)-\mathrm{H}(11)$ & 119.5 \\
\hline $\mathrm{C}(10)-\mathrm{C}(11)-\mathrm{H}(11)$ & 119.5 \\
\hline $\mathrm{C}(11)-\mathrm{C}(12)-\mathrm{C}(13)$ & $123.3(2)$ \\
\hline $\mathrm{C}(11)-\mathrm{C}(12)-\mathrm{H}(12)$ & 118.4 \\
\hline $\mathrm{C}(13)-\mathrm{C}(12)-\mathrm{H}(12)$ & 118.4 \\
\hline$C(15)-C(13)-C(12)$ & $120.54(19)$ \\
\hline$C(15)-C(13)-C(14)$ & $120.88(19)$ \\
\hline$C(12)-C(13)-C(14)$ & $118.57(18)$ \\
\hline $\mathrm{C}(9)-\mathrm{C}(14)-\mathrm{C}(5)$ & $120.68(19)$ \\
\hline$C(9)-C(14)-C(13)$ & $118.32(19)$ \\
\hline $\mathrm{C}(5)-\mathrm{C}(14)-\mathrm{C}(13)$ & $120.99(18)$ \\
\hline$C(13)-C(15)-C(16)$ & $119.95(18)$ \\
\hline$C(13)-C(15)-C(39)$ & $121.22(18)$ \\
\hline$C(16)-C(15)-C(39)$ & $118.83(17)$ \\
\hline$C(17)-C(16)-C(15)$ & $118.60(18)$ \\
\hline $\mathrm{C}(17)-\mathrm{C}(16)-\mathrm{C}(4)$ & $120.45(18)$ \\
\hline$C(15)-C(16)-C(4)$ & $120.91(18)$ \\
\hline $\mathrm{C}(16)-\mathrm{C}(17)-\mathrm{C}(18)$ & $123.00(18)$ \\
\hline $\mathrm{C}(16)-\mathrm{C}(17)-\mathrm{H}(17)$ & 118.5 \\
\hline $\mathrm{C}(18)-\mathrm{C}(17)-\mathrm{H}(17)$ & 118.5 \\
\hline $\mathrm{C}(17)-\mathrm{C}(18)-\mathrm{C}(2)$ & $116.27(18)$ \\
\hline $\mathrm{C}(17)-\mathrm{C}(18)-\mathrm{C}(19)$ & $123.96(18)$ \\
\hline $\mathrm{C}(2)-\mathrm{C}(18)-\mathrm{C}(19)$ & 119.61(18) \\
\hline$C(20)-C(19)-C(18)$ & $119.14(17)$ \\
\hline$C(20)-C(19)-C(23)$ & $117.22(17)$ \\
\hline $\mathrm{C}(18)-\mathrm{C}(19)-\mathrm{C}(23)$ & 123.61(18) \\
\hline$C(19)-C(20)-C(21)$ & $119.87(17)$ \\
\hline $\mathrm{C}(19)-\mathrm{C}(20)-\mathrm{C}(20) \# 1$ & $122.3(2)$ \\
\hline $\mathrm{C}(21)-\mathrm{C}(20)-\mathrm{C}(20) \# 1$ & $117.8(2)$ \\
\hline $\mathrm{C}(1)-\mathrm{C}(21)-\mathrm{C}(22) \# 1$ & $120.39(17)$ \\
\hline $\mathrm{C}(1)-\mathrm{C}(21)-\mathrm{C}(20)$ & $120.84(18)$ \\
\hline $\mathrm{C}(22) \# 1-\mathrm{C}(21)-\mathrm{C}(20)$ & $118.77(17)$ \\
\hline $\mathrm{C}(23)-\mathrm{C}(22)-\mathrm{C}(21) \# 1$ & $122.55(18)$ \\
\hline
\end{tabular}




\begin{tabular}{|c|c|}
\hline $\mathrm{C}(23)-\mathrm{C}(22)-\mathrm{H}(22)$ & 118.7 \\
\hline $\mathrm{C}(21) \# 1-\mathrm{C}(22)-\mathrm{H}(22)$ & 118.7 \\
\hline $\mathrm{C}(22)-\mathrm{C}(23)-\mathrm{O}(3)$ & $123.29(18)$ \\
\hline$C(22)-C(23)-C(19)$ & $120.76(18)$ \\
\hline $\mathrm{O}(3)-\mathrm{C}(23)-\mathrm{C}(19)$ & $115.87(17)$ \\
\hline$C(29)-C(24)-C(25)$ & $120.33(19)$ \\
\hline $\mathrm{C}(29)-\mathrm{C}(24)-\mathrm{C}(1)$ & $119.49(18)$ \\
\hline $\mathrm{C}(25)-\mathrm{C}(24)-\mathrm{C}(1)$ & 119.97(19) \\
\hline$C(26)-C(25)-C(24)$ & $118.8(2)$ \\
\hline$C(26)-C(25)-C(30)$ & $120.1(2)$ \\
\hline $\mathrm{C}(24)-\mathrm{C}(25)-\mathrm{C}(30)$ & $120.98(19)$ \\
\hline$C(25)-C(26)-C(27)$ & $121.9(2)$ \\
\hline $\mathrm{C}(25)-\mathrm{C}(26)-\mathrm{H}(26)$ & 119.0 \\
\hline $\mathrm{C}(27)-\mathrm{C}(26)-\mathrm{H}(26)$ & 119.0 \\
\hline$C(28)-C(27)-C(26)$ & $118.0(2)$ \\
\hline $\mathrm{C}(28)-\mathrm{C}(27)-\mathrm{C}(31)$ & $120.9(2)$ \\
\hline$C(26)-C(27)-C(31)$ & 121.1(2) \\
\hline $\mathrm{C}(27)-\mathrm{C}(28)-\mathrm{C}(29)$ & $121.9(2)$ \\
\hline $\mathrm{C}(27)-\mathrm{C}(28)-\mathrm{H}(28)$ & 119.1 \\
\hline $\mathrm{C}(29)-\mathrm{C}(28)-\mathrm{H}(28)$ & 119.1 \\
\hline $\mathrm{C}(28)-\mathrm{C}(29)-\mathrm{C}(24)$ & $119.0(2)$ \\
\hline $\mathrm{C}(28)-\mathrm{C}(29)-\mathrm{C}(32)$ & $120.2(2)$ \\
\hline$C(24)-C(29)-C(32)$ & $120.85(19)$ \\
\hline $\mathrm{C}(25)-\mathrm{C}(30)-\mathrm{H}(30 \mathrm{~A})$ & 109.5 \\
\hline $\mathrm{C}(25)-\mathrm{C}(30)-\mathrm{H}(30 \mathrm{~B})$ & 109.5 \\
\hline $\mathrm{H}(30 \mathrm{~A})-\mathrm{C}(30)-\mathrm{H}(30 \mathrm{~B})$ & 109.5 \\
\hline $\mathrm{C}(25)-\mathrm{C}(30)-\mathrm{H}(30 \mathrm{C})$ & 109.5 \\
\hline $\mathrm{H}(30 \mathrm{~A})-\mathrm{C}(30)-\mathrm{H}(30 \mathrm{C})$ & 109.5 \\
\hline $\mathrm{H}(30 \mathrm{~B})-\mathrm{C}(30)-\mathrm{H}(30 \mathrm{C})$ & 109.5 \\
\hline $\mathrm{C}(27)-\mathrm{C}(31)-\mathrm{H}(31 \mathrm{~A})$ & 109.5 \\
\hline $\mathrm{C}(27)-\mathrm{C}(31)-\mathrm{H}(31 \mathrm{~B})$ & 109.5 \\
\hline $\mathrm{H}(31 \mathrm{~A})-\mathrm{C}(31)-\mathrm{H}(31 \mathrm{~B})$ & 109.5 \\
\hline $\mathrm{C}(27)-\mathrm{C}(31)-\mathrm{H}(31 \mathrm{C})$ & 109.5 \\
\hline $\mathrm{H}(31 \mathrm{~A})-\mathrm{C}(31)-\mathrm{H}(31 \mathrm{C})$ & 109.5 \\
\hline $\mathrm{H}(31 \mathrm{~B})-\mathrm{C}(31)-\mathrm{H}(31 \mathrm{C})$ & 109.5 \\
\hline $\mathrm{C}(29)-\mathrm{C}(32)-\mathrm{H}(32 \mathrm{~A})$ & 109.5 \\
\hline
\end{tabular}




\begin{tabular}{|c|c|}
\hline $\mathrm{C}(29)-\mathrm{C}(32)-\mathrm{H}(32 \mathrm{~B})$ & 109.5 \\
\hline $\mathrm{H}(32 \mathrm{~A})-\mathrm{C}(32)-\mathrm{H}(32 \mathrm{~B})$ & 109.5 \\
\hline $\mathrm{C}(29)-\mathrm{C}(32)-\mathrm{H}(32 \mathrm{C})$ & 109.5 \\
\hline $\mathrm{H}(32 \mathrm{~A})-\mathrm{C}(32)-\mathrm{H}(32 \mathrm{C})$ & 109.5 \\
\hline $\mathrm{H}(32 \mathrm{~B})-\mathrm{C}(32)-\mathrm{H}(32 \mathrm{C})$ & 109.5 \\
\hline $\mathrm{O}(1)-\mathrm{C}(33)-\mathrm{C}(34)$ & $105.7(4)$ \\
\hline $\mathrm{O}(1)-\mathrm{C}(33)-\mathrm{H}(33 \mathrm{~A})$ & 110.6 \\
\hline $\mathrm{C}(34)-\mathrm{C}(33)-\mathrm{H}(33 \mathrm{~A})$ & 110.6 \\
\hline $\mathrm{O}(1)-\mathrm{C}(33)-\mathrm{H}(33 \mathrm{~B})$ & 110.6 \\
\hline $\mathrm{C}(34)-\mathrm{C}(33)-\mathrm{H}(33 \mathrm{~B})$ & 110.6 \\
\hline $\mathrm{H}(33 \mathrm{~A})-\mathrm{C}(33)-\mathrm{H}(33 \mathrm{~B})$ & 108.7 \\
\hline $\mathrm{C}(33)-\mathrm{C}(34)-\mathrm{C}(35)$ & $110.6(4)$ \\
\hline $\mathrm{C}(33)-\mathrm{C}(34)-\mathrm{H}(34 \mathrm{~A})$ & 109.5 \\
\hline $\mathrm{C}(35)-\mathrm{C}(34)-\mathrm{H}(34 \mathrm{~A})$ & 109.5 \\
\hline $\mathrm{C}(33)-\mathrm{C}(34)-\mathrm{H}(34 \mathrm{~B})$ & 109.5 \\
\hline $\mathrm{C}(35)-\mathrm{C}(34)-\mathrm{H}(34 \mathrm{~B})$ & 109.5 \\
\hline $\mathrm{H}(34 \mathrm{~A})-\mathrm{C}(34)-\mathrm{H}(34 \mathrm{~B})$ & 108.1 \\
\hline$C(36)-C(35)-C(34)$ & $115.4(4)$ \\
\hline $\mathrm{C}(36)-\mathrm{C}(35)-\mathrm{H}(35 \mathrm{~A})$ & 108.4 \\
\hline $\mathrm{C}(34)-\mathrm{C}(35)-\mathrm{H}(35 \mathrm{~A})$ & 108.4 \\
\hline $\mathrm{C}(36)-\mathrm{C}(35)-\mathrm{H}(35 \mathrm{~B})$ & 108.4 \\
\hline $\mathrm{C}(34)-\mathrm{C}(35)-\mathrm{H}(35 \mathrm{~B})$ & 108.4 \\
\hline $\mathrm{H}(35 \mathrm{~A})-\mathrm{C}(35)-\mathrm{H}(35 \mathrm{~B})$ & 107.5 \\
\hline $\mathrm{C}(37)-\mathrm{C}(36)-\mathrm{C}(35)$ & $114.8(4)$ \\
\hline $\mathrm{C}(37)-\mathrm{C}(36)-\mathrm{H}(36 \mathrm{~A})$ & 108.6 \\
\hline $\mathrm{C}(35)-\mathrm{C}(36)-\mathrm{H}(36 \mathrm{~A})$ & 108.6 \\
\hline $\mathrm{C}(37)-\mathrm{C}(36)-\mathrm{H}(36 \mathrm{~B})$ & 108.6 \\
\hline $\mathrm{C}(35)-\mathrm{C}(36)-\mathrm{H}(36 \mathrm{~B})$ & 108.6 \\
\hline$H(36 A)-C(36)-H(36 B)$ & 107.5 \\
\hline $\mathrm{C}(36)-\mathrm{C}(37)-\mathrm{C}(38)$ & $111.8(6)$ \\
\hline $\mathrm{C}(36)-\mathrm{C}(37)-\mathrm{H}(37 \mathrm{~A})$ & 109.3 \\
\hline $\mathrm{C}(38)-\mathrm{C}(37)-\mathrm{H}(37 \mathrm{~A})$ & 109.3 \\
\hline $\mathrm{C}(36)-\mathrm{C}(37)-\mathrm{H}(37 \mathrm{~B})$ & 109.3 \\
\hline $\mathrm{C}(38)-\mathrm{C}(37)-\mathrm{H}(37 \mathrm{~B})$ & 109.3 \\
\hline $\mathrm{H}(37 \mathrm{~A})-\mathrm{C}(37)-\mathrm{H}(37 \mathrm{~B})$ & 107.9 \\
\hline $\mathrm{C}(37)-\mathrm{C}(38)-\mathrm{H}(38 \mathrm{~A})$ & 109.5 \\
\hline
\end{tabular}




$\begin{array}{cc}\mathrm{C}(37)-\mathrm{C}(38)-\mathrm{H}(38 \mathrm{~B}) & 109.5 \\ \mathrm{H}(38 \mathrm{~A})-\mathrm{C}(38)-\mathrm{H}(38 \mathrm{~B}) & 109.5 \\ \mathrm{C}(37)-\mathrm{C}(38)-\mathrm{H}(38 \mathrm{C}) & 109.5 \\ \mathrm{H}(38 \mathrm{~A})-\mathrm{C}(38)-\mathrm{H}(38 \mathrm{C}) & 109.5 \\ \mathrm{H}(38 \mathrm{~B})-\mathrm{C}(38)-\mathrm{H}(38 \mathrm{C}) & 109.5 \\ \mathrm{O}(1)-\mathrm{C}(33 \mathrm{~A})-\mathrm{C}(34 \mathrm{~A}) & 102.6(6) \\ \mathrm{O}(1)-\mathrm{C}(33 \mathrm{~A})-\mathrm{H}(33 \mathrm{C}) & 111.2 \\ \mathrm{C}(34 \mathrm{~A})-\mathrm{C}(33 \mathrm{~A})-\mathrm{H}(33 \mathrm{C}) & 111.2 \\ \mathrm{O}(1)-\mathrm{C}(33 \mathrm{~A})-\mathrm{H}(33 \mathrm{D}) & 111.2 \\ \mathrm{C}(34 \mathrm{~A})-\mathrm{C}(33 \mathrm{~A})-\mathrm{H}(33 \mathrm{D}) & 111.2 \\ \mathrm{H}(33 \mathrm{C})-\mathrm{C}(33 \mathrm{~A})-\mathrm{H}(33 \mathrm{D}) & 109.2 \\ \mathrm{C}(33 \mathrm{~A})-\mathrm{C}(34 \mathrm{~A})-\mathrm{C}(35 \mathrm{~A}) & 111.3(6) \\ \mathrm{C}(33 \mathrm{~A})-\mathrm{C}(34 \mathrm{~A})-\mathrm{H}(34 \mathrm{C}) & 109.4 \\ \mathrm{C}(35 \mathrm{~A})-\mathrm{C}(34 \mathrm{~A})-\mathrm{H}(34 \mathrm{C}) & 109.4 \\ \mathrm{C}(33 \mathrm{~A})-\mathrm{C}(34 \mathrm{~A})-\mathrm{H}(34 \mathrm{D}) & 109.4 \\ \mathrm{C}(35 \mathrm{~A})-\mathrm{C}(34 \mathrm{~A})-\mathrm{H}(34 \mathrm{D}) & 109.4 \\ \mathrm{H}(34 \mathrm{C})-\mathrm{C}(34 \mathrm{~A})-\mathrm{H}(34 \mathrm{D}) & 108.0 \\ \mathrm{C}(36 \mathrm{~A})-\mathrm{C}(35 \mathrm{~A})-\mathrm{C}(34 \mathrm{~A}) & 109.0(6) \\ \mathrm{C}(36 \mathrm{~A})-\mathrm{C}(35 \mathrm{~A})-\mathrm{H}(35 \mathrm{C}) & 109.9 \\ \mathrm{C}(34 \mathrm{~A})-\mathrm{C}(35 \mathrm{~A})-\mathrm{H}(35 \mathrm{C}) & 109.9 \\ \mathrm{C}(36 \mathrm{~A})-\mathrm{C}(35 \mathrm{~A})-\mathrm{H}(35 \mathrm{D}) & 109.9 \\ \mathrm{C}(34 \mathrm{~A})-\mathrm{C}(35 \mathrm{~A})-\mathrm{H}(35 \mathrm{D}) & 109.9 \\ \mathrm{H}(35 \mathrm{C})-\mathrm{C}(35 \mathrm{~A})-\mathrm{H}(35 \mathrm{D}) & 108.3 \\ \mathrm{C}(35 \mathrm{~A})-\mathrm{C}(36 \mathrm{~A})-\mathrm{C}(37 \mathrm{~A}) & 111.0(6) \\ \mathrm{C}(35 \mathrm{~A})-\mathrm{C}(36 \mathrm{~A})-\mathrm{H}(36 \mathrm{C}) & 109.4 \\ \mathrm{C}(37 \mathrm{~A})-\mathrm{C}(36 \mathrm{~A})-\mathrm{H}(36 \mathrm{C}) & 109.4 \\ \mathrm{C}(35 \mathrm{~A})-\mathrm{C}(36 \mathrm{~A})-\mathrm{H}(36 \mathrm{D}) & 109.4 \\ \mathrm{C}(37 \mathrm{~A})-\mathrm{C}(36 \mathrm{~A})-\mathrm{H}(36 \mathrm{D}) & 109.4 \\ \mathrm{H}(36 \mathrm{C})-\mathrm{C}(36 \mathrm{~A})-\mathrm{H}(36 \mathrm{D}) & 108.0 \\ \mathrm{C}(38 \mathrm{~A}) \mathrm{C}) & 109.5\end{array}$




\begin{tabular}{|c|c|}
\hline $\mathrm{C}(37 \mathrm{~A})-\mathrm{C}(38 \mathrm{~A})-\mathrm{H}(38 \mathrm{E})$ & 109.5 \\
\hline $\mathrm{H}(38 \mathrm{D})-\mathrm{C}(38 \mathrm{~A})-\mathrm{H}(38 \mathrm{E})$ & 109.5 \\
\hline $\mathrm{C}(37 \mathrm{~A})-\mathrm{C}(38 \mathrm{~A})-\mathrm{H}(38 \mathrm{~F})$ & 109.5 \\
\hline $\mathrm{H}(38 \mathrm{D})-\mathrm{C}(38 \mathrm{~A})-\mathrm{H}(38 \mathrm{~F})$ & 109.5 \\
\hline $\mathrm{H}(38 \mathrm{E})-\mathrm{C}(38 \mathrm{~A})-\mathrm{H}(38 \mathrm{~F})$ & 109.5 \\
\hline C(44)-C(39)-C(40) & $120.23(19)$ \\
\hline $\mathrm{C}(44)-\mathrm{C}(39)-\mathrm{C}(15)$ & 119.24(19) \\
\hline C(40)-C(39)-C(15) & $120.51(18)$ \\
\hline $\mathrm{C}(41)-\mathrm{C}(40)-\mathrm{C}(39)$ & $118.8(2)$ \\
\hline$C(41)-C(40)-C(45)$ & $120.6(2)$ \\
\hline C(39)-C(40)-C(45) & $120.55(19)$ \\
\hline $\mathrm{C}(40)-\mathrm{C}(41)-\mathrm{C}(42)$ & $122.0(2)$ \\
\hline $\mathrm{C}(40)-\mathrm{C}(41)-\mathrm{H}(41)$ & 119.0 \\
\hline $\mathrm{C}(42)-\mathrm{C}(41)-\mathrm{H}(41)$ & 119.0 \\
\hline$C(43)-C(42)-C(41)$ & $118.0(2)$ \\
\hline$C(43)-C(42)-C(46)$ & $120.9(2)$ \\
\hline$C(41)-C(42)-C(46)$ & $121.1(2)$ \\
\hline$C(42)-C(43)-C(44)$ & $122.0(2)$ \\
\hline $\mathrm{C}(42)-\mathrm{C}(43)-\mathrm{H}(43)$ & 119.0 \\
\hline $\mathrm{C}(44)-\mathrm{C}(43)-\mathrm{H}(43)$ & 119.0 \\
\hline$C(43)-C(44)-C(39)$ & $118.9(2)$ \\
\hline C(43)-C(44)-C(47) & $119.70(19)$ \\
\hline C(39)-C(44)-C(47) & $121.39(19)$ \\
\hline $\mathrm{C}(40)-\mathrm{C}(45)-\mathrm{H}(45 \mathrm{~A})$ & 109.5 \\
\hline $\mathrm{C}(40)-\mathrm{C}(45)-\mathrm{H}(45 \mathrm{~B})$ & 109.5 \\
\hline $\mathrm{H}(45 \mathrm{~A})-\mathrm{C}(45)-\mathrm{H}(45 \mathrm{~B})$ & 109.5 \\
\hline $\mathrm{C}(40)-\mathrm{C}(45)-\mathrm{H}(45 \mathrm{C})$ & 109.5 \\
\hline $\mathrm{H}(45 \mathrm{~A})-\mathrm{C}(45)-\mathrm{H}(45 \mathrm{C})$ & 109.5 \\
\hline $\mathrm{H}(45 \mathrm{~B})-\mathrm{C}(45)-\mathrm{H}(45 \mathrm{C})$ & 109.5 \\
\hline $\mathrm{C}(42)-\mathrm{C}(46)-\mathrm{H}(46 \mathrm{~A})$ & 109.5 \\
\hline $\mathrm{C}(42)-\mathrm{C}(46)-\mathrm{H}(46 \mathrm{~B})$ & 109.5 \\
\hline$H(46 A)-C(46)-H(46 B)$ & 109.5 \\
\hline $\mathrm{C}(42)-\mathrm{C}(46)-\mathrm{H}(46 \mathrm{C})$ & 109.5 \\
\hline $\mathrm{H}(46 \mathrm{~A})-\mathrm{C}(46)-\mathrm{H}(46 \mathrm{C})$ & 109.5 \\
\hline $\mathrm{H}(46 \mathrm{~B})-\mathrm{C}(46)-\mathrm{H}(46 \mathrm{C})$ & 109.5 \\
\hline $\mathrm{C}(44)-\mathrm{C}(47)-\mathrm{H}(47 \mathrm{~A})$ & 109.5 \\
\hline
\end{tabular}




\begin{tabular}{|c|c|}
\hline $\mathrm{C}(44)-\mathrm{C}(47)-\mathrm{H}(47 \mathrm{~B})$ & 109.5 \\
\hline $\mathrm{H}(47 \mathrm{~A})-\mathrm{C}(47)-\mathrm{H}(47 \mathrm{~B})$ & 109.5 \\
\hline $\mathrm{C}(44)-\mathrm{C}(47)-\mathrm{H}(47 \mathrm{C})$ & 109.5 \\
\hline $\mathrm{H}(47 \mathrm{~A})-\mathrm{C}(47)-\mathrm{H}(47 \mathrm{C})$ & 109.5 \\
\hline $\mathrm{H}(47 \mathrm{~B})-\mathrm{C}(47)-\mathrm{H}(47 \mathrm{C})$ & 109.5 \\
\hline $\mathrm{O}(3)-\mathrm{C}(48)-\mathrm{C}(49)$ & $112.3(2)$ \\
\hline $\mathrm{O}(3)-\mathrm{C}(48)-\mathrm{H}(48 \mathrm{~A})$ & 109.1 \\
\hline $\mathrm{C}(49)-\mathrm{C}(48)-\mathrm{H}(48 \mathrm{~A})$ & 109.1 \\
\hline $\mathrm{O}(3)-\mathrm{C}(48)-\mathrm{H}(48 \mathrm{~B})$ & 109.1 \\
\hline $\mathrm{C}(49)-\mathrm{C}(48)-\mathrm{H}(48 \mathrm{~B})$ & 109.1 \\
\hline $\mathrm{H}(48 \mathrm{~A})-\mathrm{C}(48)-\mathrm{H}(48 \mathrm{~B})$ & 107.9 \\
\hline $\mathrm{C}(48)-\mathrm{C}(49)-\mathrm{C}(50)$ & $111.8(2)$ \\
\hline $\mathrm{C}(48)-\mathrm{C}(49)-\mathrm{H}(49 \mathrm{~A})$ & 109.3 \\
\hline $\mathrm{C}(50)-\mathrm{C}(49)-\mathrm{H}(49 \mathrm{~A})$ & 109.3 \\
\hline $\mathrm{C}(48)-\mathrm{C}(49)-\mathrm{H}(49 \mathrm{~B})$ & 109.3 \\
\hline $\mathrm{C}(50)-\mathrm{C}(49)-\mathrm{H}(49 \mathrm{~B})$ & 109.3 \\
\hline H(49A)-C(49)-H(49B) & 107.9 \\
\hline$C(49)-C(50)-C(51)$ & $112.4(2)$ \\
\hline $\mathrm{C}(49)-\mathrm{C}(50)-\mathrm{H}(50 \mathrm{~A})$ & 109.1 \\
\hline $\mathrm{C}(51)-\mathrm{C}(50)-\mathrm{H}(50 \mathrm{~A})$ & 109.1 \\
\hline $\mathrm{C}(49)-\mathrm{C}(50)-\mathrm{H}(50 \mathrm{~B})$ & 109.1 \\
\hline $\mathrm{C}(51)-\mathrm{C}(50)-\mathrm{H}(50 \mathrm{~B})$ & 109.1 \\
\hline $\mathrm{H}(50 \mathrm{~A})-\mathrm{C}(50)-\mathrm{H}(50 \mathrm{~B})$ & 107.9 \\
\hline $\mathrm{C}(52)-\mathrm{C}(51)-\mathrm{C}(50)$ & $114.1(2)$ \\
\hline $\mathrm{C}(52)-\mathrm{C}(51)-\mathrm{H}(51 \mathrm{~A})$ & 108.7 \\
\hline $\mathrm{C}(50)-\mathrm{C}(51)-\mathrm{H}(51 \mathrm{~A})$ & 108.7 \\
\hline $\mathrm{C}(52)-\mathrm{C}(51)-\mathrm{H}(51 \mathrm{~B})$ & 108.7 \\
\hline $\mathrm{C}(50)-\mathrm{C}(51)-\mathrm{H}(51 \mathrm{~B})$ & 108.7 \\
\hline $\mathrm{H}(51 \mathrm{~A})-\mathrm{C}(51)-\mathrm{H}(51 \mathrm{~B})$ & 107.6 \\
\hline $\mathrm{C}(51)-\mathrm{C}(52)-\mathrm{C}(53)$ & $111.6(3)$ \\
\hline $\mathrm{C}(51)-\mathrm{C}(52)-\mathrm{H}(52 \mathrm{~A})$ & 109.3 \\
\hline $\mathrm{C}(53)-\mathrm{C}(52)-\mathrm{H}(52 \mathrm{~A})$ & 109.3 \\
\hline $\mathrm{C}(51)-\mathrm{C}(52)-\mathrm{H}(52 \mathrm{~B})$ & 109.3 \\
\hline $\mathrm{C}(53)-\mathrm{C}(52)-\mathrm{H}(52 \mathrm{~B})$ & 109.3 \\
\hline $\mathrm{H}(52 \mathrm{~A})-\mathrm{C}(52)-\mathrm{H}(52 \mathrm{~B})$ & 108.0 \\
\hline $\mathrm{C}(52)-\mathrm{C}(53)-\mathrm{H}(53 \mathrm{~A})$ & 109.5 \\
\hline
\end{tabular}




\begin{tabular}{|c|c|}
\hline $\mathrm{C}(52)-\mathrm{C}(53)-\mathrm{H}(53 \mathrm{~B})$ & 109.5 \\
\hline $\mathrm{H}(53 \mathrm{~A})-\mathrm{C}(53)-\mathrm{H}(53 \mathrm{~B})$ & 109.5 \\
\hline $\mathrm{C}(52)-\mathrm{C}(53)-\mathrm{H}(53 \mathrm{C})$ & 109.5 \\
\hline $\mathrm{H}(53 \mathrm{~A})-\mathrm{C}(53)-\mathrm{H}(53 \mathrm{C})$ & 109.5 \\
\hline $\mathrm{H}(53 \mathrm{~B})-\mathrm{C}(53)-\mathrm{H}(53 \mathrm{C})$ & 109.5 \\
\hline $\mathrm{C}(75) \# 2-\mathrm{C}(54)-\mathrm{C}(55)$ & $119.99(18)$ \\
\hline $\mathrm{C}(75) \# 2-\mathrm{C}(54)-\mathrm{C}(77)$ & $120.12(17)$ \\
\hline $\mathrm{C}(55)-\mathrm{C}(54)-\mathrm{C}(77)$ & $119.88(17)$ \\
\hline $\mathrm{C}(56)-\mathrm{C}(55)-\mathrm{C}(54)$ & $119.28(18)$ \\
\hline $\mathrm{C}(56)-\mathrm{C}(55)-\mathrm{C}(71)$ & $120.12(18)$ \\
\hline $\mathrm{C}(54)-\mathrm{C}(55)-\mathrm{C}(71)$ & $120.60(18)$ \\
\hline$C(57)-C(56)-C(55)$ & $123.12(18)$ \\
\hline $\mathrm{C}(57)-\mathrm{C}(56)-\mathrm{H}(56)$ & 118.4 \\
\hline $\mathrm{C}(55)-\mathrm{C}(56)-\mathrm{H}(56)$ & 118.4 \\
\hline $\mathrm{C}(56)-\mathrm{C}(57)-\mathrm{C}(69)$ & $116.71(18)$ \\
\hline $\mathrm{C}(56)-\mathrm{C}(57)-\mathrm{C}(58)$ & $124.21(18)$ \\
\hline C(69)-C(57)-C(58) & $118.92(18)$ \\
\hline C(67)-C(58)-C(59) & 117.91(18) \\
\hline C(67)-C(58)-C(57) & $118.48(18)$ \\
\hline C(59)-C(58)-C(57) & $123.61(18)$ \\
\hline $\mathrm{O}(4)-\mathrm{C}(59)-\mathrm{C}(60)$ & $122.41(19)$ \\
\hline $\mathrm{O}(4)-\mathrm{C}(59)-\mathrm{C}(58)$ & $116.76(18)$ \\
\hline $\mathrm{C}(60)-\mathrm{C}(59)-\mathrm{C}(58)$ & $120.81(19)$ \\
\hline $\mathrm{C}(61)-\mathrm{C}(60)-\mathrm{C}(59)$ & 119.91(19) \\
\hline $\mathrm{C}(61)-\mathrm{C}(60)-\mathrm{H}(60)$ & 120.0 \\
\hline $\mathrm{C}(59)-\mathrm{C}(60)-\mathrm{H}(60)$ & 120.0 \\
\hline$C(60)-C(61)-C(62)$ & $121.38(19)$ \\
\hline $\mathrm{C}(60)-\mathrm{C}(61)-\mathrm{H}(61)$ & 119.3 \\
\hline $\mathrm{C}(62)-\mathrm{C}(61)-\mathrm{H}(61)$ & 119.3 \\
\hline$C(61)-C(62)-C(67)$ & $119.39(18)$ \\
\hline$C(61)-C(62)-C(63)$ & $118.69(18)$ \\
\hline$C(67)-C(62)-C(63)$ & $121.87(18)$ \\
\hline $\mathrm{O}(5)-\mathrm{C}(63)-\mathrm{C}(64)$ & $122.05(19)$ \\
\hline $\mathrm{O}(5)-\mathrm{C}(63)-\mathrm{C}(62)$ & $121.6(2)$ \\
\hline$C(64)-C(63)-C(62)$ & $116.31(18)$ \\
\hline$C(65)-C(64)-C(63)$ & $121.38(19)$ \\
\hline
\end{tabular}




\begin{tabular}{|c|c|}
\hline $\mathrm{C}(65)-\mathrm{C}(64)-\mathrm{H}(64)$ & 119.3 \\
\hline $\mathrm{C}(63)-\mathrm{C}(64)-\mathrm{H}(64)$ & 119.3 \\
\hline$C(64)-C(65)-C(66)$ & $123.31(19)$ \\
\hline $\mathrm{C}(64)-\mathrm{C}(65)-\mathrm{H}(65)$ & 118.3 \\
\hline $\mathrm{C}(66)-\mathrm{C}(65)-\mathrm{H}(65)$ & 118.3 \\
\hline$C(68)-C(66)-C(65)$ & $120.93(18)$ \\
\hline $\mathrm{C}(68)-\mathrm{C}(66)-\mathrm{C}(67)$ & $120.91(18)$ \\
\hline $\mathrm{C}(65)-\mathrm{C}(66)-\mathrm{C}(67)$ & $118.13(18)$ \\
\hline $\mathrm{C}(62)-\mathrm{C}(67)-\mathrm{C}(58)$ & $120.37(18)$ \\
\hline$C(62)-C(67)-C(66)$ & $118.97(18)$ \\
\hline$C(58)-C(67)-C(66)$ & $120.61(18)$ \\
\hline $\mathrm{C}(66)-\mathrm{C}(68)-\mathrm{C}(69)$ & $120.04(18)$ \\
\hline $\mathrm{C}(66)-\mathrm{C}(68)-\mathrm{C}(92)$ & $121.67(18)$ \\
\hline $\mathrm{C}(69)-\mathrm{C}(68)-\mathrm{C}(92)$ & $118.29(17)$ \\
\hline $\mathrm{C}(70)-\mathrm{C}(69)-\mathrm{C}(57)$ & $120.32(18)$ \\
\hline $\mathrm{C}(70)-\mathrm{C}(69)-\mathrm{C}(68)$ & $119.39(18)$ \\
\hline $\mathrm{C}(57)-\mathrm{C}(69)-\mathrm{C}(68)$ & $120.24(18)$ \\
\hline $\mathrm{C}(69)-\mathrm{C}(70)-\mathrm{C}(71)$ & $122.93(18)$ \\
\hline $\mathrm{C}(69)-\mathrm{C}(70)-\mathrm{H}(70)$ & 118.5 \\
\hline $\mathrm{C}(71)-\mathrm{C}(70)-\mathrm{H}(70)$ & 118.5 \\
\hline $\mathrm{C}(70)-\mathrm{C}(71)-\mathrm{C}(55)$ & $116.02(17)$ \\
\hline $\mathrm{C}(70)-\mathrm{C}(71)-\mathrm{C}(72)$ & $124.56(18)$ \\
\hline $\mathrm{C}(55)-\mathrm{C}(71)-\mathrm{C}(72)$ & $119.29(17)$ \\
\hline $\mathrm{C}(76)-\mathrm{C}(72)-\mathrm{C}(71)$ & $119.08(17)$ \\
\hline $\mathrm{C}(76)-\mathrm{C}(72)-\mathrm{C}(73)$ & $117.17(17)$ \\
\hline $\mathrm{C}(71)-\mathrm{C}(72)-\mathrm{C}(73)$ & $123.73(17)$ \\
\hline $\mathrm{C}(74)-\mathrm{C}(73)-\mathrm{O}(6)$ & $122.72(18)$ \\
\hline $\mathrm{C}(74)-\mathrm{C}(73)-\mathrm{C}(72)$ & $121.33(18)$ \\
\hline $\mathrm{O}(6)-\mathrm{C}(73)-\mathrm{C}(72)$ & $115.91(17)$ \\
\hline $\mathrm{C}(73)-\mathrm{C}(74)-\mathrm{C}(75)$ & $122.19(18)$ \\
\hline $\mathrm{C}(73)-\mathrm{C}(74)-\mathrm{H}(74)$ & 118.9 \\
\hline $\mathrm{C}(75)-\mathrm{C}(74)-\mathrm{H}(74)$ & 118.9 \\
\hline $\mathrm{C}(54) \# 2-\mathrm{C}(75)-\mathrm{C}(74)$ & $120.07(18)$ \\
\hline $\mathrm{C}(54) \# 2-\mathrm{C}(75)-\mathrm{C}(76) \# 2$ & $120.81(18)$ \\
\hline $\mathrm{C}(74)-\mathrm{C}(75)-\mathrm{C}(76) \# 2$ & 119.11(17) \\
\hline$C(72)-C(76)-C(75) \# 2$ & $119.98(17)$ \\
\hline
\end{tabular}




\begin{tabular}{|c|c|}
\hline $\mathrm{C}(72)-\mathrm{C}(76)-\mathrm{C}(76) \# 2$ & $122.3(2)$ \\
\hline $\mathrm{C}(75) \# 2-\mathrm{C}(76)-\mathrm{C}(76) \# 2$ & $117.8(2)$ \\
\hline $\mathrm{C}(82)-\mathrm{C}(77)-\mathrm{C}(78)$ & 119.92(19) \\
\hline $\mathrm{C}(82)-\mathrm{C}(77)-\mathrm{C}(54)$ & $119.59(18)$ \\
\hline $\mathrm{C}(78)-\mathrm{C}(77)-\mathrm{C}(54)$ & $120.49(19)$ \\
\hline $\mathrm{C}(79)-\mathrm{C}(78)-\mathrm{C}(77)$ & $118.9(2)$ \\
\hline $\mathrm{C}(79)-\mathrm{C}(78)-\mathrm{C}(83)$ & $120.0(2)$ \\
\hline $\mathrm{C}(77)-\mathrm{C}(78)-\mathrm{C}(83)$ & 121.17(19) \\
\hline $\mathrm{C}(80)-\mathrm{C}(79)-\mathrm{C}(78)$ & $122.2(2)$ \\
\hline $\mathrm{C}(80)-\mathrm{C}(79)-\mathrm{H}(79)$ & 118.9 \\
\hline $\mathrm{C}(78)-\mathrm{C}(79)-\mathrm{H}(79)$ & 118.9 \\
\hline $\mathrm{C}(81)-\mathrm{C}(80)-\mathrm{C}(79)$ & $118.0(2)$ \\
\hline $\mathrm{C}(81)-\mathrm{C}(80)-\mathrm{C}(84)$ & $120.9(2)$ \\
\hline $\mathrm{C}(79)-\mathrm{C}(80)-\mathrm{C}(84)$ & $121.1(2)$ \\
\hline $\mathrm{C}(80)-\mathrm{C}(81)-\mathrm{C}(82)$ & $121.7(2)$ \\
\hline $\mathrm{C}(80)-\mathrm{C}(81)-\mathrm{H}(81)$ & 119.1 \\
\hline $\mathrm{C}(82)-\mathrm{C}(81)-\mathrm{H}(81)$ & 119.1 \\
\hline $\mathrm{C}(81)-\mathrm{C}(82)-\mathrm{C}(77)$ & $119.3(2)$ \\
\hline $\mathrm{C}(81)-\mathrm{C}(82)-\mathrm{C}(85)$ & $120.3(2)$ \\
\hline $\mathrm{C}(77)-\mathrm{C}(82)-\mathrm{C}(85)$ & $120.39(19)$ \\
\hline $\mathrm{C}(78)-\mathrm{C}(83)-\mathrm{H}(83 \mathrm{~A})$ & 109.5 \\
\hline $\mathrm{C}(78)-\mathrm{C}(83)-\mathrm{H}(83 \mathrm{~B})$ & 109.5 \\
\hline $\mathrm{H}(83 \mathrm{~A})-\mathrm{C}(83)-\mathrm{H}(83 \mathrm{~B})$ & 109.5 \\
\hline $\mathrm{C}(78)-\mathrm{C}(83)-\mathrm{H}(83 \mathrm{C})$ & 109.5 \\
\hline $\mathrm{H}(83 \mathrm{~A})-\mathrm{C}(83)-\mathrm{H}(83 \mathrm{C})$ & 109.5 \\
\hline $\mathrm{H}(83 \mathrm{~B})-\mathrm{C}(83)-\mathrm{H}(83 \mathrm{C})$ & 109.5 \\
\hline $\mathrm{C}(80)-\mathrm{C}(84)-\mathrm{H}(84 \mathrm{~A})$ & 109.5 \\
\hline $\mathrm{C}(80)-\mathrm{C}(84)-\mathrm{H}(84 \mathrm{~B})$ & 109.5 \\
\hline $\mathrm{H}(84 \mathrm{~A})-\mathrm{C}(84)-\mathrm{H}(84 \mathrm{~B})$ & 109.5 \\
\hline $\mathrm{C}(80)-\mathrm{C}(84)-\mathrm{H}(84 \mathrm{C})$ & 109.5 \\
\hline $\mathrm{H}(84 \mathrm{~A})-\mathrm{C}(84)-\mathrm{H}(84 \mathrm{C})$ & 109.5 \\
\hline $\mathrm{H}(84 \mathrm{~B})-\mathrm{C}(84)-\mathrm{H}(84 \mathrm{C})$ & 109.5 \\
\hline $\mathrm{C}(82)-\mathrm{C}(85)-\mathrm{H}(85 \mathrm{~A})$ & 109.5 \\
\hline $\mathrm{C}(82)-\mathrm{C}(85)-\mathrm{H}(85 \mathrm{~B})$ & 109.5 \\
\hline $\mathrm{H}(85 \mathrm{~A})-\mathrm{C}(85)-\mathrm{H}(85 \mathrm{~B})$ & 109.5 \\
\hline $\mathrm{C}(82)-\mathrm{C}(85)-\mathrm{H}(85 \mathrm{C})$ & 109.5 \\
\hline
\end{tabular}




\begin{tabular}{|c|c|}
\hline $\mathrm{H}(85 \mathrm{~A})-\mathrm{C}(85)-\mathrm{H}(85 \mathrm{C})$ & 109.5 \\
\hline $\mathrm{H}(85 \mathrm{~B})-\mathrm{C}(85)-\mathrm{H}(85 \mathrm{C})$ & 109.5 \\
\hline $\mathrm{O}(4)-\mathrm{C}(86)-\mathrm{C}(87)$ & $112.67(19)$ \\
\hline $\mathrm{O}(4)-\mathrm{C}(86)-\mathrm{H}(86 \mathrm{~A})$ & 109.1 \\
\hline $\mathrm{C}(87)-\mathrm{C}(86)-\mathrm{H}(86 \mathrm{~A})$ & 109.1 \\
\hline $\mathrm{O}(4)-\mathrm{C}(86)-\mathrm{H}(86 \mathrm{~B})$ & 109.1 \\
\hline $\mathrm{C}(87)-\mathrm{C}(86)-\mathrm{H}(86 \mathrm{~B})$ & 109.1 \\
\hline $\mathrm{H}(86 \mathrm{~A})-\mathrm{C}(86)-\mathrm{H}(86 \mathrm{~B})$ & 107.8 \\
\hline C(86)-C(87)-C(88) & $110.15(19)$ \\
\hline $\mathrm{C}(86)-\mathrm{C}(87)-\mathrm{H}(87 \mathrm{~A})$ & 109.6 \\
\hline $\mathrm{C}(88)-\mathrm{C}(87)-\mathrm{H}(87 \mathrm{~A})$ & 109.6 \\
\hline $\mathrm{C}(86)-\mathrm{C}(87)-\mathrm{H}(87 \mathrm{~B})$ & 109.6 \\
\hline $\mathrm{C}(88)-\mathrm{C}(87)-\mathrm{H}(87 \mathrm{~B})$ & 109.6 \\
\hline $\mathrm{H}(87 \mathrm{~A})-\mathrm{C}(87)-\mathrm{H}(87 \mathrm{~B})$ & 108.1 \\
\hline $\mathrm{C}(89)-\mathrm{C}(88)-\mathrm{C}(87)$ & $113.6(2)$ \\
\hline $\mathrm{C}(89)-\mathrm{C}(88)-\mathrm{H}(88 \mathrm{~A})$ & 108.8 \\
\hline $\mathrm{C}(87)-\mathrm{C}(88)-\mathrm{H}(88 \mathrm{~A})$ & 108.8 \\
\hline $\mathrm{C}(89)-\mathrm{C}(88)-\mathrm{H}(88 \mathrm{~B})$ & 108.8 \\
\hline $\mathrm{C}(87)-\mathrm{C}(88)-\mathrm{H}(88 \mathrm{~B})$ & 108.8 \\
\hline $\mathrm{H}(88 \mathrm{~A})-\mathrm{C}(88)-\mathrm{H}(88 \mathrm{~B})$ & 107.7 \\
\hline C(88)-C(89)-C(90) & $112.8(2)$ \\
\hline $\mathrm{C}(88)-\mathrm{C}(89)-\mathrm{H}(89 \mathrm{~A})$ & 109.0 \\
\hline $\mathrm{C}(90)-\mathrm{C}(89)-\mathrm{H}(89 \mathrm{~A})$ & 109.0 \\
\hline $\mathrm{C}(88)-\mathrm{C}(89)-\mathrm{H}(89 \mathrm{~B})$ & 109.0 \\
\hline $\mathrm{C}(90)-\mathrm{C}(89)-\mathrm{H}(89 \mathrm{~B})$ & 109.0 \\
\hline $\mathrm{H}(89 \mathrm{~A})-\mathrm{C}(89)-\mathrm{H}(89 \mathrm{~B})$ & 107.8 \\
\hline C(91)-C(90)-C(89) & $113.6(2)$ \\
\hline $\mathrm{C}(91)-\mathrm{C}(90)-\mathrm{H}(90 \mathrm{~A})$ & 108.9 \\
\hline $\mathrm{C}(89)-\mathrm{C}(90)-\mathrm{H}(90 \mathrm{~A})$ & 108.9 \\
\hline $\mathrm{C}(91)-\mathrm{C}(90)-\mathrm{H}(90 \mathrm{~B})$ & 108.9 \\
\hline $\mathrm{C}(89)-\mathrm{C}(90)-\mathrm{H}(90 \mathrm{~B})$ & 108.9 \\
\hline $\mathrm{H}(90 \mathrm{~A})-\mathrm{C}(90)-\mathrm{H}(90 \mathrm{~B})$ & 107.7 \\
\hline $\mathrm{C}(90)-\mathrm{C}(91)-\mathrm{H}(91 \mathrm{~A})$ & 109.5 \\
\hline $\mathrm{C}(90)-\mathrm{C}(91)-\mathrm{H}(91 \mathrm{~B})$ & 109.5 \\
\hline $\mathrm{H}(91 \mathrm{~A})-\mathrm{C}(91)-\mathrm{H}(91 \mathrm{~B})$ & 109.5 \\
\hline $\mathrm{C}(90)-\mathrm{C}(91)-\mathrm{H}(91 \mathrm{C})$ & 109.5 \\
\hline
\end{tabular}




\begin{tabular}{|c|c|}
\hline $\mathrm{H}(91 \mathrm{~A})-\mathrm{C}(91)-\mathrm{H}(91 \mathrm{C})$ & 109.5 \\
\hline $\mathrm{H}(91 \mathrm{~B})-\mathrm{C}(91)-\mathrm{H}(91 \mathrm{C})$ & 109.5 \\
\hline $\mathrm{C}(97)-\mathrm{C}(92)-\mathrm{C}(93)$ & $119.99(19)$ \\
\hline $\mathrm{C}(97)-\mathrm{C}(92)-\mathrm{C}(68)$ & $119.33(18)$ \\
\hline $\mathrm{C}(93)-\mathrm{C}(92)-\mathrm{C}(68)$ & $120.63(18)$ \\
\hline $\mathrm{C}(94)-\mathrm{C}(93)-\mathrm{C}(92)$ & $118.5(2)$ \\
\hline $\mathrm{C}(94)-\mathrm{C}(93)-\mathrm{C}(98)$ & $120.5(2)$ \\
\hline $\mathrm{C}(92)-\mathrm{C}(93)-\mathrm{C}(98)$ & $121.0(2)$ \\
\hline $\mathrm{C}(95)-\mathrm{C}(94)-\mathrm{C}(93)$ & $122.3(2)$ \\
\hline $\mathrm{C}(95)-\mathrm{C}(94)-\mathrm{H}(94)$ & 118.9 \\
\hline $\mathrm{C}(93)-\mathrm{C}(94)-\mathrm{H}(94)$ & 118.9 \\
\hline $\mathrm{C}(96)-\mathrm{C}(95)-\mathrm{C}(94)$ & $118.0(2)$ \\
\hline $\mathrm{C}(96)-\mathrm{C}(95)-\mathrm{C}(99)$ & $120.3(2)$ \\
\hline $\mathrm{C}(94)-\mathrm{C}(95)-\mathrm{C}(99)$ & $121.6(2)$ \\
\hline $\mathrm{C}(95)-\mathrm{C}(96)-\mathrm{C}(97)$ & $121.7(2)$ \\
\hline $\mathrm{C}(95)-\mathrm{C}(96)-\mathrm{H}(96)$ & 119.1 \\
\hline $\mathrm{C}(97)-\mathrm{C}(96)-\mathrm{H}(96)$ & 119.1 \\
\hline C(96)-C(97)-C(92) & $119.4(2)$ \\
\hline $\mathrm{C}(96)-\mathrm{C}(97)-\mathrm{C}(100)$ & $119.6(2)$ \\
\hline $\mathrm{C}(92)-\mathrm{C}(97)-\mathrm{C}(100)$ & $120.94(19)$ \\
\hline $\mathrm{C}(93)-\mathrm{C}(98)-\mathrm{H}(98 \mathrm{~A})$ & 109.5 \\
\hline $\mathrm{C}(93)-\mathrm{C}(98)-\mathrm{H}(98 \mathrm{~B})$ & 109.5 \\
\hline $\mathrm{H}(98 \mathrm{~A})-\mathrm{C}(98)-\mathrm{H}(98 \mathrm{~B})$ & 109.5 \\
\hline $\mathrm{C}(93)-\mathrm{C}(98)-\mathrm{H}(98 \mathrm{C})$ & 109.5 \\
\hline $\mathrm{H}(98 \mathrm{~A})-\mathrm{C}(98)-\mathrm{H}(98 \mathrm{C})$ & 109.5 \\
\hline $\mathrm{H}(98 \mathrm{~B})-\mathrm{C}(98)-\mathrm{H}(98 \mathrm{C})$ & 109.5 \\
\hline $\mathrm{C}(95)-\mathrm{C}(99)-\mathrm{H}(99 \mathrm{~A})$ & 109.5 \\
\hline $\mathrm{C}(95)-\mathrm{C}(99)-\mathrm{H}(99 \mathrm{~B})$ & 109.5 \\
\hline $\mathrm{H}(99 \mathrm{~A})-\mathrm{C}(99)-\mathrm{H}(99 \mathrm{~B})$ & 109.5 \\
\hline $\mathrm{C}(95)-\mathrm{C}(99)-\mathrm{H}(99 \mathrm{C})$ & 109.5 \\
\hline H(99A)-C(99)-H(99C) & 109.5 \\
\hline H(99B)-C(99)-H(99C) & 109.5 \\
\hline $\mathrm{C}(97)-\mathrm{C}(100)-\mathrm{H}(10 \mathrm{~A})$ & 109.5 \\
\hline $\mathrm{C}(97)-\mathrm{C}(100)-\mathrm{H}(10 \mathrm{~B})$ & 109.5 \\
\hline $\mathrm{H}(10 \mathrm{~A})-\mathrm{C}(100)-\mathrm{H}(10 \mathrm{~B})$ & 109.5 \\
\hline $\mathrm{C}(97)-\mathrm{C}(100)-\mathrm{H}(10 \mathrm{C})$ & 109.5 \\
\hline
\end{tabular}




\begin{tabular}{|c|c|}
\hline $\mathrm{H}(10 \mathrm{~A})-\mathrm{C}(100)-\mathrm{H}(10 \mathrm{C})$ & 109.5 \\
\hline $\mathrm{H}(10 \mathrm{~B})-\mathrm{C}(100)-\mathrm{H}(10 \mathrm{C})$ & 109.5 \\
\hline $\mathrm{O}(6)-\mathrm{C}(101)-\mathrm{C}(102)$ & $112.6(2)$ \\
\hline $\mathrm{O}(6)-\mathrm{C}(101)-\mathrm{H}(10 \mathrm{D})$ & 109.1 \\
\hline $\mathrm{C}(102)-\mathrm{C}(101)-\mathrm{H}(10 \mathrm{D})$ & 109.1 \\
\hline $\mathrm{O}(6)-\mathrm{C}(101)-\mathrm{H}(10 \mathrm{E})$ & 109.1 \\
\hline $\mathrm{C}(102)-\mathrm{C}(101)-\mathrm{H}(10 \mathrm{E})$ & 109.1 \\
\hline $\mathrm{H}(10 \mathrm{D})-\mathrm{C}(101)-\mathrm{H}(10 \mathrm{E})$ & 107.8 \\
\hline$C(101)-C(102)-C(103)$ & $110.5(2)$ \\
\hline $\mathrm{C}(101)-\mathrm{C}(102)-\mathrm{H}(10 \mathrm{~F})$ & 109.6 \\
\hline $\mathrm{C}(103)-\mathrm{C}(102)-\mathrm{H}(10 \mathrm{~F})$ & 109.6 \\
\hline $\mathrm{C}(101)-\mathrm{C}(102)-\mathrm{H}(10 \mathrm{G})$ & 109.6 \\
\hline $\mathrm{C}(103)-\mathrm{C}(102)-\mathrm{H}(10 \mathrm{G})$ & 109.6 \\
\hline $\mathrm{H}(10 \mathrm{~F})-\mathrm{C}(102)-\mathrm{H}(10 \mathrm{G})$ & 108.1 \\
\hline$C(104)-C(103)-C(102)$ & $113.4(2)$ \\
\hline $\mathrm{C}(104)-\mathrm{C}(103)-\mathrm{H}(10 \mathrm{H})$ & 108.9 \\
\hline $\mathrm{C}(102)-\mathrm{C}(103)-\mathrm{H}(10 \mathrm{H})$ & 108.9 \\
\hline $\mathrm{C}(104)-\mathrm{C}(103)-\mathrm{H}(10 \mathrm{I})$ & 108.9 \\
\hline $\mathrm{C}(102)-\mathrm{C}(103)-\mathrm{H}(10 \mathrm{I})$ & 108.9 \\
\hline $\mathrm{H}(10 \mathrm{H})-\mathrm{C}(103)-\mathrm{H}(10 \mathrm{I})$ & 107.7 \\
\hline$C(103)-C(104)-C(105)$ & $114.9(3)$ \\
\hline $\mathrm{C}(103)-\mathrm{C}(104)-\mathrm{H}(10 \mathrm{~J})$ & 108.6 \\
\hline $\mathrm{C}(105)-\mathrm{C}(104)-\mathrm{H}(10 \mathrm{~J})$ & 108.6 \\
\hline $\mathrm{C}(103)-\mathrm{C}(104)-\mathrm{H}(10 \mathrm{~K})$ & 108.6 \\
\hline $\mathrm{C}(105)-\mathrm{C}(104)-\mathrm{H}(10 \mathrm{~K})$ & 108.6 \\
\hline $\mathrm{H}(10 \mathrm{~J})-\mathrm{C}(104)-\mathrm{H}(10 \mathrm{~K})$ & 107.5 \\
\hline$C(106)-C(105)-C(104)$ & $114.6(3)$ \\
\hline $\mathrm{C}(106)-\mathrm{C}(105)-\mathrm{H}(10 \mathrm{~L})$ & 108.6 \\
\hline $\mathrm{C}(104)-\mathrm{C}(105)-\mathrm{H}(10 \mathrm{~L})$ & 108.6 \\
\hline $\mathrm{C}(106)-\mathrm{C}(105)-\mathrm{H}(10 \mathrm{M})$ & 108.6 \\
\hline $\mathrm{C}(104)-\mathrm{C}(105)-\mathrm{H}(10 \mathrm{M})$ & 108.6 \\
\hline $\mathrm{H}(10 \mathrm{~L})-\mathrm{C}(105)-\mathrm{H}(10 \mathrm{M})$ & 107.6 \\
\hline $\mathrm{C}(105)-\mathrm{C}(106)-\mathrm{H}(10 \mathrm{~N})$ & 109.5 \\
\hline $\mathrm{C}(105)-\mathrm{C}(106)-\mathrm{H}(10 \mathrm{O})$ & 109.5 \\
\hline $\mathrm{H}(10 \mathrm{~N})-\mathrm{C}(106)-\mathrm{H}(10 \mathrm{O})$ & 109.5 \\
\hline $\mathrm{C}(105)-\mathrm{C}(106)-\mathrm{H}(10 \mathrm{P})$ & 109.5 \\
\hline
\end{tabular}




$\begin{array}{cc}\mathrm{H}(10 \mathrm{~N})-\mathrm{C}(106)-\mathrm{H}(10 \mathrm{P}) & 109.5 \\ \mathrm{H}(10 \mathrm{O})-\mathrm{C}(106)-\mathrm{H}(10 \mathrm{P}) & 109.5 \\ \mathrm{Cl}(3)-\mathrm{C}(107)-\mathrm{Cl}(1) & 110.27(16) \\ \mathrm{Cl}(3)-\mathrm{C}(107)-\mathrm{Cl}(2) & 112.12(16) \\ \mathrm{Cl}(1)-\mathrm{C}(107)-\mathrm{Cl}(2) & 110.96(15) \\ \mathrm{Cl}(3)-\mathrm{C}(107)-\mathrm{H}(107) & 107.8 \\ \mathrm{Cl}(1)-\mathrm{C}(107)-\mathrm{H}(107) & 107.8 \\ \mathrm{Cl}(2)-\mathrm{C}(107)-\mathrm{H}(107) & 107.8 \\ \mathrm{Cl}(4)-\mathrm{C}(108)-\mathrm{Cl}(5) & 110.86(17) \\ \mathrm{Cl}(4)-\mathrm{C}(108)-\mathrm{Cl}(6) & 109.07(15) \\ \mathrm{Cl}(5)-\mathrm{C}(108)-\mathrm{Cl}(6) & 111.29(16) \\ \mathrm{Cl}(4)-\mathrm{C}(108)-\mathrm{H}(108) & 108.5 \\ \mathrm{Cl}(5)-\mathrm{C}(108)-\mathrm{H}(108) & 108.5 \\ \mathrm{Cl}(6)-\mathrm{C}(108)-\mathrm{H}(108) & 108.5 \\ \mathrm{C}(6)-\mathrm{O}(1)-\mathrm{C}(33) & 112.4(3) \\ \mathrm{C}(6)-\mathrm{O}(1)-\mathrm{C}(33 \mathrm{~A}) & 127.5(4) \\ \mathrm{C}(23)-\mathrm{O}(3)-\mathrm{C}(48) & 118.88(16) \\ \mathrm{C}(59)-\mathrm{O}(4)-\mathrm{C}(86) & 120.09(16) \\ \mathrm{C}(73)-\mathrm{O}(6)-\mathrm{C}(101) & 119.97(16)\end{array}$

Symmetry transformations used to generate equivalent atoms:

$$
\# 1-\mathrm{x}+1,-\mathrm{y},-\mathrm{z}+1 \quad \# 2-\mathrm{x}+1,-\mathrm{y}+2,-\mathrm{z}
$$

Table S10. Anisotropic displacement parameters $\quad\left(\AA^{2} \times 10^{3}\right)$ for HZD-M-ONE. The anisotropic displacement factor exponent takes the form: $\quad-2 \mathrm{p}^{2}\left[\mathrm{~h}^{2} \mathrm{a}^{* 2} \mathrm{U}^{11}+\ldots+2 \mathrm{~h} \mathrm{k} \mathrm{a}^{*} \mathrm{~b}^{*} \mathrm{U}^{12}\right]$

\begin{tabular}{cccccccc}
\hline & \multicolumn{2}{c}{$\mathrm{U}^{11}$} & \multicolumn{2}{c}{$\mathrm{U}^{33}$} & \multicolumn{2}{c}{$\mathrm{U}^{23}$} & \multicolumn{2}{c}{$\mathrm{U}^{13}$} \\
\hline $\mathrm{C}(1)$ & $9(1)$ & $10(1)$ & $12(1)$ & $-1(1)$ & $-4(1)$ & $0(1)$ \\
$\mathrm{C}(2)$ & $9(1)$ & $12(1)$ & $12(1)$ & $0(1)$ & $-4(1)$ & $-1(1)$ \\
$\mathrm{C}(3)$ & $8(1)$ & $15(1)$ & $15(1)$ & $-1(1)$ & $-4(1)$ & $1(1)$ \\
$\mathrm{C}(4)$ & $10(1)$ & $13(1)$ & $14(1)$ & $0(1)$ & $-6(1)$ & $0(1)$ \\
$\mathrm{C}(5)$ & $12(1)$ & $16(1)$ & $13(1)$ & $-2(1)$ & $-6(1)$ & $3(1)$ \\
$\mathrm{C}(6)$ & $15(1)$ & $24(1)$ & $18(1)$ & $-2(1)$ & $-7(1)$ & $1(1)$ \\
$\mathrm{C}(7)$ & $13(1)$ & $25(1)$ & $31(1)$ & $-3(1)$ & $-12(1)$ & $3(1)$ \\
$\mathrm{C}(8)$ & $20(1)$ & $16(1)$ & $32(1)$ & $1(1)$ & $-18(1)$ & $3(1)$
\end{tabular}




\begin{tabular}{|c|c|c|c|c|c|}
\hline $\mathrm{C}(9)$ & $19(1)$ & $13(1)$ & $20(1)$ & $-1(1)$ & $-13(1)$ \\
\hline$C(10)$ & $28(1)$ & $11(1)$ & $25(1)$ & $1(1)$ & $-18(1)$ \\
\hline $\mathrm{C}(11)$ & $28(1)$ & $15(1)$ & $23(1)$ & $5(1)$ & $-13(1)$ \\
\hline$C(12)$ & $20(1)$ & $13(1)$ & $16(1)$ & $2(1)$ & $-8(1)$ \\
\hline$C(13)$ & $16(1)$ & 11(1) & $14(1)$ & $-2(1)$ & $-7(1)$ \\
\hline $\mathrm{C}(14)$ & $16(1)$ & $12(1)$ & $15(1)$ & $-2(1)$ & $-9(1)$ \\
\hline$C(15)$ & $14(1)$ & 11(1) & $13(1)$ & $0(1)$ & $-6(1)$ \\
\hline$C(16)$ & $12(1)$ & $13(1)$ & $13(1)$ & $0(1)$ & $-5(1)$ \\
\hline $\mathrm{C}(17)$ & $10(1)$ & $12(1)$ & $13(1)$ & $-1(1)$ & $-4(1)$ \\
\hline $\mathrm{C}(18)$ & $9(1)$ & 11(1) & $13(1)$ & $0(1)$ & $-4(1)$ \\
\hline$C(19)$ & $9(1)$ & $10(1)$ & $12(1)$ & $1(1)$ & $-4(1)$ \\
\hline$C(20)$ & $9(1)$ & $10(1)$ & $12(1)$ & $0(1)$ & $-4(1)$ \\
\hline $\mathrm{C}(21)$ & $8(1)$ & 11(1) & $12(1)$ & $0(1)$ & $-3(1)$ \\
\hline$C(22)$ & $8(1)$ & $12(1)$ & $13(1)$ & $0(1)$ & $0(1)$ \\
\hline$C(23)$ & $12(1)$ & $12(1)$ & 11(1) & $2(1)$ & $-2(1)$ \\
\hline$C(24)$ & $8(1)$ & $12(1)$ & $12(1)$ & $2(1)$ & $-4(1)$ \\
\hline$C(25)$ & $14(1)$ & $14(1)$ & $17(1)$ & $0(1)$ & $-5(1)$ \\
\hline$C(26)$ & $17(1)$ & 21(1) & $24(1)$ & $-8(1)$ & $-2(1)$ \\
\hline$C(27)$ & $15(1)$ & $28(1)$ & $23(1)$ & $-4(1)$ & $2(1)$ \\
\hline$C(28)$ & $14(1)$ & $23(1)$ & $24(1)$ & $3(1)$ & $-2(1)$ \\
\hline C(29) & $13(1)$ & $16(1)$ & $17(1)$ & $2(1)$ & $-5(1)$ \\
\hline$C(30)$ & $21(1)$ & $23(1)$ & $31(1)$ & $-10(1)$ & $-5(1)$ \\
\hline $\mathrm{C}(31)$ & $26(1)$ & $50(2)$ & $38(2)$ & $-16(1)$ & $14(1)$ \\
\hline$C(32)$ & 19(1) & $22(1)$ & $31(1)$ & $-5(1)$ & $-2(1)$ \\
\hline $\mathrm{C}(33)$ & $6(2)$ & $28(3)$ & $17(2)$ & $4(3)$ & $-3(2)$ \\
\hline$C(34)$ & $15(2)$ & $25(2)$ & $25(2)$ & $9(2)$ & $-5(2)$ \\
\hline$C(35)$ & $15(2)$ & $30(2)$ & $25(2)$ & $7(2)$ & $0(2)$ \\
\hline$C(36)$ & $14(3)$ & $33(3)$ & $41(3)$ & $-11(3)$ & $0(3)$ \\
\hline$C(37)$ & $18(2)$ & $34(3)$ & $27(2)$ & $-5(2)$ & $0(2)$ \\
\hline$C(38)$ & $8(3)$ & $34(4)$ & $37(6)$ & $-12(4)$ & $-2(3)$ \\
\hline$C(33 A)$ & $9(3)$ & $34(6)$ & $38(4)$ & $4(5)$ & $-5(3)$ \\
\hline$C(34 A)$ & $16(3)$ & $54(5)$ & $35(4)$ & $-3(3)$ & $-6(3)$ \\
\hline$C(35 A)$ & $41(5)$ & $39(5)$ & $25(4)$ & $6(3)$ & $-14(4)$ \\
\hline$C(36 \mathrm{~A})$ & $40(4)$ & $37(4)$ & $37(4)$ & $4(3)$ & $-10(3)$ \\
\hline $\mathrm{C}(37 \mathrm{~A})$ & $17(3)$ & $36(4)$ & $44(4)$ & $-6(3)$ & $-2(3)$ \\
\hline$C(38 \mathrm{~A})$ & $66(11)$ & $48(10)$ & $51(12)$ & $-26(8)$ & $0(8)$ \\
\hline
\end{tabular}




\begin{tabular}{|c|c|c|c|c|c|c|}
\hline C(39) & $12(1)$ & $12(1)$ & $12(1)$ & $4(1)$ & $-4(1)$ & $-1(1)$ \\
\hline$C(40)$ & $18(1)$ & $15(1)$ & $16(1)$ & $0(1)$ & $-5(1)$ & $-4(1)$ \\
\hline $\mathrm{C}(41)$ & $15(1)$ & $18(1)$ & $25(1)$ & $2(1)$ & $-6(1)$ & $-7(1)$ \\
\hline $\mathrm{C}(42)$ & $15(1)$ & $15(1)$ & $20(1)$ & $3(1)$ & $-1(1)$ & $-1(1)$ \\
\hline$C(43)$ & $17(1)$ & $12(1)$ & $16(1)$ & $-2(1)$ & $-3(1)$ & 1(1) \\
\hline $\mathrm{C}(44)$ & $15(1)$ & $13(1)$ & $17(1)$ & $1(1)$ & $-7(1)$ & $-2(1)$ \\
\hline$C(45)$ & $24(1)$ & $28(1)$ & $26(1)$ & $-8(1)$ & $-4(1)$ & $-10(1)$ \\
\hline$C(46)$ & $15(1)$ & $26(1)$ & $34(1)$ & $-4(1)$ & $2(1)$ & $-4(1)$ \\
\hline $\mathrm{C}(47)$ & $20(1)$ & $26(1)$ & $32(1)$ & $-13(1)$ & $-5(1)$ & $-6(1)$ \\
\hline $\mathrm{C}(48)$ & $16(1)$ & $24(1)$ & $13(1)$ & $0(1)$ & $1(1)$ & $4(1)$ \\
\hline C(49) & $23(1)$ & $24(1)$ & $21(1)$ & $6(1)$ & $-1(1)$ & $-3(1)$ \\
\hline $\mathrm{C}(50)$ & $19(1)$ & $41(2)$ & $24(1)$ & $-3(1)$ & $-2(1)$ & $-5(1)$ \\
\hline$C(51)$ & 21(1) & $36(2)$ & $28(1)$ & $1(1)$ & $-4(1)$ & $-5(1)$ \\
\hline$C(52)$ & $20(1)$ & $46(2)$ & $48(2)$ & $-7(1)$ & $-1(1)$ & $-5(1)$ \\
\hline$C(53)$ & $25(2)$ & $46(2)$ & $74(2)$ & $-2(2)$ & $-9(2)$ & $-10(1)$ \\
\hline $\mathrm{C}(54)$ & $10(1)$ & $12(1)$ & $11(1)$ & $0(1)$ & $-4(1)$ & $-2(1)$ \\
\hline$C(55)$ & $10(1)$ & 11(1) & $14(1)$ & $1(1)$ & $-4(1)$ & $-2(1)$ \\
\hline$C(56)$ & $10(1)$ & $13(1)$ & $14(1)$ & $2(1)$ & $-6(1)$ & $-1(1)$ \\
\hline$C(57)$ & $10(1)$ & 11(1) & $15(1)$ & $2(1)$ & $-5(1)$ & $-1(1)$ \\
\hline $\mathrm{C}(58)$ & $12(1)$ & $13(1)$ & $12(1)$ & $2(1)$ & $-6(1)$ & $-2(1)$ \\
\hline $\mathrm{C}(59)$ & $13(1)$ & $18(1)$ & $14(1)$ & $4(1)$ & $-6(1)$ & $-4(1)$ \\
\hline$C(60)$ & $12(1)$ & $18(1)$ & $18(1)$ & $2(1)$ & $-6(1)$ & $-1(1)$ \\
\hline $\mathrm{C}(61)$ & $15(1)$ & $14(1)$ & $16(1)$ & $2(1)$ & $-9(1)$ & $1(1)$ \\
\hline$C(62)$ & $15(1)$ & 11(1) & $12(1)$ & $2(1)$ & $-7(1)$ & $-1(1)$ \\
\hline$C(63)$ & $18(1)$ & $13(1)$ & $16(1)$ & $3(1)$ & $-6(1)$ & $-2(1)$ \\
\hline C(64) & $20(1)$ & $14(1)$ & $16(1)$ & $6(1)$ & $-4(1)$ & $-5(1)$ \\
\hline$C(65)$ & $14(1)$ & $14(1)$ & $16(1)$ & $2(1)$ & $-3(1)$ & $-3(1)$ \\
\hline$C(66)$ & $14(1)$ & $10(1)$ & $12(1)$ & $0(1)$ & $-5(1)$ & $-3(1)$ \\
\hline$C(67)$ & $12(1)$ & 11(1) & $12(1)$ & $-1(1)$ & $-6(1)$ & $-1(1)$ \\
\hline $\mathrm{C}(68)$ & 11(1) & $13(1)$ & 11(1) & $0(1)$ & $-4(1)$ & $-3(1)$ \\
\hline C(69) & 11(1) & 11(1) & $12(1)$ & $1(1)$ & $-5(1)$ & $-2(1)$ \\
\hline $\mathrm{C}(70)$ & $10(1)$ & $12(1)$ & $13(1)$ & $1(1)$ & $-4(1)$ & $-1(1)$ \\
\hline $\mathrm{C}(71)$ & 11(1) & $12(1)$ & $11(1)$ & $1(1)$ & $-6(1)$ & $-2(1)$ \\
\hline$C(72)$ & $10(1)$ & $10(1)$ & $11(1)$ & $0(1)$ & $-5(1)$ & $-2(1)$ \\
\hline $\mathrm{C}(73)$ & $13(1)$ & $13(1)$ & $10(1)$ & $2(1)$ & $-3(1)$ & $-3(1)$ \\
\hline $\mathrm{C}(74)$ & $9(1)$ & $13(1)$ & 12(1) & 1(1) & $-2(1)$ & \\
\hline
\end{tabular}




\begin{tabular}{|c|c|c|c|c|c|c|}
\hline$C(75)$ & $10(1)$ & $10(1)$ & $12(1)$ & $0(1)$ & $-4(1)$ & $-1(1)$ \\
\hline$C(76)$ & $10(1)$ & $9(1)$ & $12(1)$ & $0(1)$ & $-4(1)$ & $-2(1)$ \\
\hline$C(77)$ & $9(1)$ & $14(1)$ & $12(1)$ & $3(1)$ & $-4(1)$ & 1(1) \\
\hline $\mathrm{C}(78)$ & $15(1)$ & $14(1)$ & $18(1)$ & $2(1)$ & $-6(1)$ & $-3(1)$ \\
\hline $\mathrm{C}(79)$ & $17(1)$ & $15(1)$ & 21(1) & $-5(1)$ & $0(1)$ & $0(1)$ \\
\hline $\mathrm{C}(80)$ & $12(1)$ & $22(1)$ & $22(1)$ & $-1(1)$ & $-1(1)$ & $0(1)$ \\
\hline $\mathrm{C}(81)$ & $12(1)$ & $24(1)$ & $30(1)$ & $-2(1)$ & $-4(1)$ & $-5(1)$ \\
\hline $\mathrm{C}(82)$ & $12(1)$ & $17(1)$ & $19(1)$ & $-1(1)$ & $-4(1)$ & $-2(1)$ \\
\hline$C(83)$ & $19(1)$ & $22(1)$ & $32(1)$ & $-6(1)$ & $-4(1)$ & $-6(1)$ \\
\hline$C(84)$ & $16(1)$ & $32(1)$ & $37(2)$ & $-6(1)$ & $5(1)$ & $-2(1)$ \\
\hline$C(85)$ & $17(1)$ & $30(1)$ & $34(1)$ & $-14(1)$ & $-1(1)$ & $-9(1)$ \\
\hline$C(86)$ & 11(1) & $24(1)$ & $26(1)$ & $8(1)$ & $-6(1)$ & $-5(1)$ \\
\hline$C(87)$ & $19(1)$ & $28(1)$ & $24(1)$ & $5(1)$ & $-11(1)$ & $-8(1)$ \\
\hline $\mathrm{C}(88)$ & $18(1)$ & $26(1)$ & $23(1)$ & $-2(1)$ & $-4(1)$ & $-7(1)$ \\
\hline C(89) & $21(1)$ & $26(1)$ & $28(1)$ & $-3(1)$ & $-6(1)$ & $-8(1)$ \\
\hline $\mathrm{C}(90)$ & 21(1) & $25(1)$ & $34(1)$ & $-5(1)$ & $-4(1)$ & $-4(1)$ \\
\hline$C(91)$ & $31(2)$ & $33(2)$ & $54(2)$ & $-6(1)$ & 11(1) & $-10(1)$ \\
\hline$C(92)$ & 11(1) & $10(1)$ & $12(1)$ & $4(1)$ & $-3(1)$ & $-1(1)$ \\
\hline C(93) & $17(1)$ & $20(1)$ & 19(1) & $-1(1)$ & $-4(1)$ & $-7(1)$ \\
\hline $\mathrm{C}(94)$ & $16(1)$ & $36(1)$ & $30(1)$ & $-6(1)$ & $-5(1)$ & $-11(1)$ \\
\hline $\mathrm{C}(95)$ & $15(1)$ & $30(1)$ & $26(1)$ & $-3(1)$ & $0(1)$ & $-4(1)$ \\
\hline$C(96)$ & $18(1)$ & $16(1)$ & $15(1)$ & $-1(1)$ & $-2(1)$ & $-2(1)$ \\
\hline $\mathrm{C}(97)$ & $14(1)$ & $14(1)$ & $15(1)$ & $2(1)$ & $-4(1)$ & $-3(1)$ \\
\hline C(98) & $22(1)$ & $34(1)$ & $30(1)$ & $-12(1)$ & $-5(1)$ & $-12(1)$ \\
\hline C(99) & $17(1)$ & $65(2)$ & $56(2)$ & $-25(2)$ & $4(1)$ & $-10(1)$ \\
\hline$C(100)$ & $21(1)$ & $32(1)$ & $38(1)$ & $-17(1)$ & $-4(1)$ & $-9(1)$ \\
\hline$C(101)$ & $12(1)$ & $27(1)$ & $15(1)$ & $5(1)$ & $2(1)$ & $-1(1)$ \\
\hline$C(102)$ & $22(1)$ & $34(1)$ & $25(1)$ & $-5(1)$ & $-1(1)$ & $-7(1)$ \\
\hline$C(103)$ & $28(1)$ & $40(2)$ & $31(1)$ & $-6(1)$ & $2(1)$ & $-7(1)$ \\
\hline$C(104)$ & $27(2)$ & $98(3)$ & $48(2)$ & $22(2)$ & $2(2)$ & $9(2)$ \\
\hline$C(105)$ & $27(2)$ & $61(2)$ & $64(2)$ & $18(2)$ & $2(2)$ & $5(2)$ \\
\hline$C(106)$ & $41(2)$ & $58(3)$ & $141(5)$ & $21(3)$ & $-5(2)$ & $-13(2)$ \\
\hline$C(107)$ & $44(2)$ & $31(1)$ & $32(1)$ & $10(1)$ & $-16(1)$ & $-18(1)$ \\
\hline C(108) & $42(2)$ & $37(2)$ & $39(2)$ & 11(1) & $-24(1)$ & $-17(1)$ \\
\hline $\mathrm{Cl}(1)$ & $40(1)$ & $73(1)$ & $39(1)$ & $-6(1)$ & $3(1)$ & $-9(1)$ \\
\hline $\mathrm{Cl}(2)$ & $38(1)$ & $26(1)$ & $35(1)$ & $6(1)$ & $-8(1)$ & $-12(1)$ \\
\hline
\end{tabular}




$\begin{array}{lcccccc}\mathrm{Cl}(3) & 65(1) & 34(1) & 88(1) & -1(1) & -37(1) & -24(1) \\ \mathrm{Cl}(4) & 36(1) & 36(1) & 69(1) & 4(1) & -18(1) & -17(1) \\ \mathrm{Cl}(5) & 88(1) & 73(1) & 56(1) & 10(1) & -32(1) & -62(1) \\ \mathrm{Cl}(6) & 47(1) & 83(1) & 34(1) & 7(1) & -6(1) & 19(1) \\ \mathrm{O}(1) & 7(1) & 60(1) & 22(1) & 14(1) & -2(1) & 2(1) \\ \mathrm{O}(2) & 32(1) & 21(1) & 32(1) & 8(1) & -19(1) & 2(1) \\ \mathrm{O}(3) & 15(1) & 19(1) & 11(1) & 3(1) & 0(1) & 5(1) \\ \mathrm{O}(4) & 11(1) & 24(1) & 24(1) & 13(1) & -6(1) & -4(1) \\ \mathrm{O}(5) & 21(1) & 20(1) & 25(1) & 13(1) & -7(1) & 0(1) \\ \mathrm{O}(6) & 13(1) & 22(1) & 11(1) & 6(1) & 2(1) & 5(1)\end{array}$

Table S11. Hydrogen coordinates ( x 10 $0^{4}$ ) and isotropic displacement parameters $\left(\AA^{2} \times 10^{3}\right)$ for HZD-M-ONE.

\begin{tabular}{|c|c|c|c|c|}
\hline $\mathrm{x}$ & & & z & $\mathrm{U}(\mathrm{eq})$ \\
\hline $\mathrm{H}(3)$ & 2002 & 2581 & 4488 & 17 \\
\hline $\mathrm{H}(7)$ & -72 & 4645 & 3577 & 29 \\
\hline $\mathrm{H}(8)$ & 556 & 5357 & 2632 & 27 \\
\hline $\mathrm{H}(11)$ & 3541 & 5043 & 1100 & 26 \\
\hline $\mathrm{H}(12)$ & 4524 & 3881 & 1478 & 20 \\
\hline $\mathrm{H}(17)$ & 5126 & 1556 & 3039 & 15 \\
\hline $\mathrm{H}(22)$ & 7077 & -839 & 3631 & 15 \\
\hline $\mathrm{H}(26)$ & 788 & 3328 & 6837 & 28 \\
\hline $\mathrm{H}(28)$ & 167 & 1291 & 6274 & 25 \\
\hline $\mathrm{H}(30 \mathrm{~A})$ & 2137 & 3645 & 6145 & 38 \\
\hline $\mathrm{H}(30 \mathrm{~B})$ & 2808 & 3059 & 5433 & 38 \\
\hline $\mathrm{H}(30 \mathrm{C})$ & 2970 & 2768 & 6180 & 38 \\
\hline $\mathrm{H}(31 \mathrm{~A})$ & -1048 & 3171 & 7054 & 63 \\
\hline $\mathrm{H}(31 \mathrm{~B})$ & -534 & 2844 & 7652 & 63 \\
\hline $\mathrm{H}(31 \mathrm{C})$ & -885 & 2198 & 7298 & 63 \\
\hline $\mathrm{H}(32 \mathrm{~A})$ & 2172 & 12 & 5383 & 37 \\
\hline $\mathrm{H}(32 \mathrm{~B})$ & 1995 & 588 & 4733 & 37 \\
\hline $\mathrm{H}(32 \mathrm{C})$ & 1147 & 347 & 5269 & 37 \\
\hline
\end{tabular}




\begin{tabular}{|c|c|c|c|}
\hline $\mathrm{H}(33 \mathrm{~A})$ & -547 & 3735 & 4215 \\
\hline $\mathrm{H}(33 \mathrm{~B})$ & -302 & 4420 & 4627 \\
\hline $\mathrm{H}(34 \mathrm{~A})$ & -121 & 3513 & 5536 \\
\hline $\mathrm{H}(34 \mathrm{~B})$ & -134 & 2711 & 5095 \\
\hline $\mathrm{H}(35 \mathrm{~A})$ & -1749 & 3387 & 5214 \\
\hline $\mathrm{H}(35 \mathrm{~B})$ & -1617 & 3354 & 5987 \\
\hline $\mathrm{H}(36 \mathrm{~A})$ & -1670 & 4869 & 5889 \\
\hline $\mathrm{H}(36 \mathrm{~B})$ & -2007 & 4865 & 5201 \\
\hline $\mathrm{H}(37 \mathrm{~A})$ & -3291 & 5505 & 6229 \\
\hline $\mathrm{H}(37 \mathrm{~B})$ & -3050 & 4588 & 6580 \\
\hline $\mathrm{H}(38 \mathrm{~A})$ & -3538 & 4764 & 5277 \\
\hline $\mathrm{H}(38 \mathrm{~B})$ & -4315 & 4861 & 6028 \\
\hline $\mathrm{H}(38 \mathrm{C})$ & -3484 & 3948 & 5756 \\
\hline $\mathrm{H}(33 \mathrm{C})$ & -263 & 2892 & 4699 \\
\hline $\mathrm{H}(33 \mathrm{D})$ & -583 & 3685 & 4214 \\
\hline $\mathrm{H}(34 \mathrm{C})$ & -337 & 4642 & 4984 \\
\hline $\mathrm{H}(34 \mathrm{D})$ & -68 & 3854 & 5477 \\
\hline $\mathrm{H}(35 \mathrm{C})$ & -1741 & 3826 & 5616 \\
\hline $\mathrm{H}(35 \mathrm{D})$ & -1651 & 4556 & 6090 \\
\hline $\mathrm{H}(36 \mathrm{C})$ & -2055 & 4824 & 4765 \\
\hline $\mathrm{H}(36 \mathrm{D})$ & -1903 & 5552 & 5197 \\
\hline $\mathrm{H}(37 \mathrm{C})$ & -3246 & 5579 & 6157 \\
\hline $\mathrm{H}(37 \mathrm{D})$ & -3533 & 5847 & 5450 \\
\hline $\mathrm{H}(38 \mathrm{D})$ & -3639 & 4463 & 5314 \\
\hline $\mathrm{H}(38 \mathrm{E})$ & -4344 & 4968 & 6049 \\
\hline $\mathrm{H}(38 \mathrm{~F})$ & -3376 & 4213 & 6036 \\
\hline $\mathrm{H}(41)$ & 7206 & 2833 & 1916 \\
\hline $\mathrm{H}(43)$ & 6675 & 1097 & 841 \\
\hline $\mathrm{H}(45 \mathrm{~A})$ & 6048 & 3753 & 2867 \\
\hline $\mathrm{H}(45 \mathrm{~B})$ & 5150 & 3472 & 3248 \\
\hline $\mathrm{H}(45 \mathrm{C})$ & 5088 & 4171 & 2660 \\
\hline $\mathrm{H}(46 \mathrm{~A})$ & 8357 & 2082 & 758 \\
\hline $\mathrm{H}(46 \mathrm{~B})$ & 8177 & 1308 & 408 \\
\hline $\mathrm{H}(46 \mathrm{C})$ & 8499 & 1168 & 1113 \\
\hline $\mathrm{H}(47 \mathrm{~A})$ & 5141 & 1097 & 1004 \\
\hline $\mathrm{H}(47 \mathrm{~B})$ & 4256 & 1890 & 1455 \\
\hline
\end{tabular}




\begin{tabular}{|c|c|c|c|}
\hline $\mathrm{H}(47 \mathrm{C})$ & 4724 & 1056 & 1841 \\
\hline $\mathrm{H}(48 \mathrm{~A})$ & 6760 & -252 & 2014 \\
\hline $\mathrm{H}(48 \mathrm{~B})$ & 6986 & -905 & 2611 \\
\hline $\mathrm{H}(49 \mathrm{~A})$ & 7761 & -39 & 2958 \\
\hline $\mathrm{H}(49 \mathrm{~B})$ & 7545 & 604 & 2352 \\
\hline $\mathrm{H}(50 \mathrm{~A})$ & 8530 & -501 & 1464 \\
\hline $\mathrm{H}(50 \mathrm{~B})$ & 8679 & -1204 & 2043 \\
\hline $\mathrm{H}(51 \mathrm{~A})$ & 9329 & 255 & 1948 \\
\hline $\mathrm{H}(51 \mathrm{~B})$ & 9542 & -515 & 2462 \\
\hline $\mathrm{H}(52 \mathrm{~A})$ & 10302 & -694 & 953 \\
\hline $\mathrm{H}(52 \mathrm{~B})$ & 10461 & -1507 & 1436 \\
\hline $\mathrm{H}(53 \mathrm{~A})$ & 11111 & -81 & 1529 \\
\hline $\mathrm{H}(53 \mathrm{~B})$ & 11780 & -1024 & 1157 \\
\hline $\mathrm{H}(53 \mathrm{C})$ & 11287 & -909 & 1995 \\
\hline $\mathrm{H}(56)$ & 7909 & 7473 & 642 \\
\hline $\mathrm{H}(60)$ & 9948 & 5525 & 1611 \\
\hline $\mathrm{H}(61)$ & 9322 & 4712 & 2476 \\
\hline $\mathrm{H}(64)$ & 6307 & 4926 & 3930 \\
\hline $\mathrm{H}(65)$ & 5326 & 6095 & 3562 \\
\hline $\mathrm{H}(70)$ & 4736 & 8431 & 1983 \\
\hline $\mathrm{H}(74)$ & 2808 & 10767 & 1269 \\
\hline H(79) & 9302 & 6531 & -1595 \\
\hline $\mathrm{H}(81)$ & 9943 & 8501 & -935 \\
\hline $\mathrm{H}(83 \mathrm{~A})$ & 7072 & 7257 & -1154 \\
\hline $\mathrm{H}(83 \mathrm{~B})$ & 7115 & 6943 & -385 \\
\hline $\mathrm{H}(83 \mathrm{C})$ & 7836 & 6348 & -1078 \\
\hline $\mathrm{H}(84 \mathrm{~A})$ & 11153 & 6613 & -1552 \\
\hline $\mathrm{H}(84 \mathrm{~B})$ & 11076 & 7513 & -1922 \\
\hline $\mathrm{H}(84 \mathrm{C})$ & 10798 & 6784 & -2242 \\
\hline $\mathrm{H}(85 \mathrm{~A})$ & 8845 & 9591 & -82 \\
\hline $\mathrm{H}(85 \mathrm{~B})$ & 8007 & 9339 & 457 \\
\hline $\mathrm{H}(85 \mathrm{C})$ & 7824 & 9893 & -201 \\
\hline $\mathrm{H}(86 \mathrm{~A})$ & 10319 & 6558 & 1153 \\
\hline $\mathrm{H}(86 \mathrm{~B})$ & 10127 & 7241 & 568 \\
\hline $\mathrm{H}(87 \mathrm{~A})$ & 10175 & 6131 & -193 \\
\hline $\mathrm{H}(87 \mathrm{~B})$ & 10326 & 5443 & 397 \\
\hline
\end{tabular}




\begin{tabular}{|c|c|c|c|c|}
\hline $\mathrm{H}(88 \mathrm{~A})$ & 11641 & 6288 & -315 & 28 \\
\hline $\mathrm{H}(88 \mathrm{~B})$ & 11781 & 5695 & 333 & 28 \\
\hline H(89A) & 11876 & 5098 & -1038 & 30 \\
\hline $\mathrm{H}(89 \mathrm{~B})$ & 11823 & 4488 & -387 & 30 \\
\hline $\mathrm{H}(90 \mathrm{~A})$ & 13272 & 4581 & -296 & 35 \\
\hline $\mathrm{H}(90 \mathrm{~B})$ & 13404 & 4128 & -1042 & 35 \\
\hline $\mathrm{H}(91 \mathrm{~A})$ & 13393 & 5440 & -1580 & 67 \\
\hline $\mathrm{H}(91 \mathrm{~B})$ & 14216 & 5123 & -1209 & 67 \\
\hline $\mathrm{H}(91 \mathrm{C})$ & 13257 & 5896 & -834 & 67 \\
\hline $\mathrm{H}(94)$ & 2639 & 7233 & 3006 & 32 \\
\hline $\mathrm{H}(96)$ & 3123 & 8897 & 4185 & 22 \\
\hline $\mathrm{H}(98 \mathrm{~A})$ & 3835 & 6271 & 2110 & 43 \\
\hline $\mathrm{H}(98 \mathrm{~B})$ & 4771 & 5846 & 2346 & 43 \\
\hline $\mathrm{H}(98 \mathrm{C})$ & 4745 & 6544 & 1756 & 43 \\
\hline $\mathrm{H}(99 \mathrm{~A})$ & 1357 & 8886 & 3708 & 74 \\
\hline $\mathrm{H}(99 \mathrm{~B})$ & 1574 & 8876 & 4451 & 74 \\
\hline $\mathrm{H}(99 \mathrm{C})$ & 1406 & 8053 & 4166 & 74 \\
\hline $\mathrm{H}(10 \mathrm{~A})$ & 4616 & 8933 & 4059 & 46 \\
\hline $\mathrm{H}(10 \mathrm{~B})$ & 5244 & 8788 & 3246 & 46 \\
\hline $\mathrm{H}(10 \mathrm{C})$ & 5475 & 8040 & 3778 & 46 \\
\hline $\mathrm{H}(10 \mathrm{D})$ & 2672 & 9558 & 2715 & 26 \\
\hline $\mathrm{H}(10 \mathrm{E})$ & 2390 & 10068 & 2063 & 26 \\
\hline $\mathrm{H}(10 \mathrm{~F})$ & 2662 & 11289 & 2413 & 34 \\
\hline $\mathrm{H}(10 \mathrm{G})$ & 3068 & 10776 & 3021 & 34 \\
\hline $\mathrm{H}(10 \mathrm{H})$ & 1503 & 10645 & 3558 & 43 \\
\hline $\mathrm{H}(10 \mathrm{I})$ & 1536 & 11609 & 3616 & 43 \\
\hline $\mathrm{H}(10 \mathrm{~J})$ & 912 & 11008 & 2576 & 82 \\
\hline $\mathrm{H}(10 \mathrm{~K})$ & 922 & 11976 & 2655 & 82 \\
\hline $\mathrm{H}(10 \mathrm{~L})$ & -232 & 12175 & 3794 & 71 \\
\hline $\mathrm{H}(10 \mathrm{M})$ & -612 & 12050 & 3150 & 71 \\
\hline $\mathrm{H}(10 \mathrm{~N})$ & -296 & 10561 & 3406 & 129 \\
\hline $\mathrm{H}(10 \mathrm{O})$ & -1138 & 11245 & 3996 & 129 \\
\hline $\mathrm{H}(10 \mathrm{P})$ & -123 & 10817 & 4117 & 129 \\
\hline $\mathrm{H}(107)$ & 6521 & 2449 & 5099 & 40 \\
\hline $\mathrm{H}(108)$ & 3712 & 7234 & -280 & 43 \\
\hline
\end{tabular}


Table S12. Torsion angles $\left[{ }^{\circ}\right]$ for HZD-M-ONE.

\begin{tabular}{|c|c|}
\hline $\mathrm{C}(21)-\mathrm{C}(1)-\mathrm{C}(2)-\mathrm{C}(3)$ & $-175.95(19)$ \\
\hline $\mathrm{C}(24)-\mathrm{C}(1)-\mathrm{C}(2)-\mathrm{C}(3)$ & $1.5(3)$ \\
\hline $\mathrm{C}(21)-\mathrm{C}(1)-\mathrm{C}(2)-\mathrm{C}(18)$ & $1.9(3)$ \\
\hline $\mathrm{C}(24)-\mathrm{C}(1)-\mathrm{C}(2)-\mathrm{C}(18)$ & $179.42(18)$ \\
\hline $\mathrm{C}(1)-\mathrm{C}(2)-\mathrm{C}(3)-\mathrm{C}(4)$ & $173.1(2)$ \\
\hline $\mathrm{C}(18)-\mathrm{C}(2)-\mathrm{C}(3)-\mathrm{C}(4)$ & $-4.8(3)$ \\
\hline$C(2)-C(3)-C(4)-C(16)$ & $-2.7(3)$ \\
\hline $\mathrm{C}(2)-\mathrm{C}(3)-\mathrm{C}(4)-\mathrm{C}(5)$ & $-179.9(2)$ \\
\hline$C(3)-C(4)-C(5)-C(14)$ & $169.8(2)$ \\
\hline $\mathrm{C}(16)-\mathrm{C}(4)-\mathrm{C}(5)-\mathrm{C}(14)$ & $-7.3(3)$ \\
\hline$C(3)-C(4)-C(5)-C(6)$ & $-10.0(3)$ \\
\hline $\mathrm{C}(16)-\mathrm{C}(4)-\mathrm{C}(5)-\mathrm{C}(6)$ & $172.9(2)$ \\
\hline $\mathrm{C}(14)-\mathrm{C}(5)-\mathrm{C}(6)-\mathrm{O}(1)$ & $178.0(2)$ \\
\hline $\mathrm{C}(4)-\mathrm{C}(5)-\mathrm{C}(6)-\mathrm{O}(1)$ & $-2.2(3)$ \\
\hline $\mathrm{C}(14)-\mathrm{C}(5)-\mathrm{C}(6)-\mathrm{C}(7)$ & $-1.5(3)$ \\
\hline $\mathrm{C}(4)-\mathrm{C}(5)-\mathrm{C}(6)-\mathrm{C}(7)$ & $178.3(2)$ \\
\hline $\mathrm{O}(1)-\mathrm{C}(6)-\mathrm{C}(7)-\mathrm{C}(8)$ & $179.9(2)$ \\
\hline$C(5)-C(6)-C(7)-C(8)$ & $-0.6(4)$ \\
\hline $\mathrm{C}(6)-\mathrm{C}(7)-\mathrm{C}(8)-\mathrm{C}(9)$ & $2.2(4)$ \\
\hline $\mathrm{C}(7)-\mathrm{C}(8)-\mathrm{C}(9)-\mathrm{C}(14)$ & $-1.4(3)$ \\
\hline $\mathrm{C}(7)-\mathrm{C}(8)-\mathrm{C}(9)-\mathrm{C}(10)$ & $177.6(2)$ \\
\hline $\mathrm{C}(8)-\mathrm{C}(9)-\mathrm{C}(10)-\mathrm{O}(2)$ & $-1.6(3)$ \\
\hline $\mathrm{C}(14)-\mathrm{C}(9)-\mathrm{C}(10)-\mathrm{O}(2)$ & $177.4(2)$ \\
\hline $\mathrm{C}(8)-\mathrm{C}(9)-\mathrm{C}(10)-\mathrm{C}(11)$ & $179.6(2)$ \\
\hline $\mathrm{C}(14)-\mathrm{C}(9)-\mathrm{C}(10)-\mathrm{C}(11)$ & $-1.4(3)$ \\
\hline $\mathrm{O}(2)-\mathrm{C}(10)-\mathrm{C}(11)-\mathrm{C}(12)$ & $-176.2(2)$ \\
\hline $\mathrm{C}(9)-\mathrm{C}(10)-\mathrm{C}(11)-\mathrm{C}(12)$ & $2.7(3)$ \\
\hline $\mathrm{C}(10)-\mathrm{C}(11)-\mathrm{C}(12)-\mathrm{C}(13)$ & $-1.6(4)$ \\
\hline $\mathrm{C}(11)-\mathrm{C}(12)-\mathrm{C}(13)-\mathrm{C}(15)$ & $178.2(2)$ \\
\hline $\mathrm{C}(11)-\mathrm{C}(12)-\mathrm{C}(13)-\mathrm{C}(14)$ & $-0.8(3)$ \\
\hline $\mathrm{C}(8)-\mathrm{C}(9)-\mathrm{C}(14)-\mathrm{C}(5)$ & $-0.8(3)$ \\
\hline$C(10)-C(9)-C(14)-C(5)$ & $-179.8(2)$ \\
\hline $\mathrm{C}(8)-\mathrm{C}(9)-\mathrm{C}(14)-\mathrm{C}(13)$ & $178.1(2)$ \\
\hline $\mathrm{C}(10)-\mathrm{C}(9)-\mathrm{C}(14)-\mathrm{C}(13)$ & $-0.8(3)$ \\
\hline
\end{tabular}




\begin{tabular}{|c|c|}
\hline $\mathrm{C}(6)-\mathrm{C}(5)-\mathrm{C}(14)-\mathrm{C}(9)$ & $2.2(3)$ \\
\hline $\mathrm{C}(4)-\mathrm{C}(5)-\mathrm{C}(14)-\mathrm{C}(9)$ & $-177.58(19)$ \\
\hline $\mathrm{C}(6)-\mathrm{C}(5)-\mathrm{C}(14)-\mathrm{C}(13)$ & $-176.7(2)$ \\
\hline $\mathrm{C}(4)-\mathrm{C}(5)-\mathrm{C}(14)-\mathrm{C}(13)$ & $3.5(3)$ \\
\hline $\mathrm{C}(15)-\mathrm{C}(13)-\mathrm{C}(14)-\mathrm{C}(9)$ & $-177.1(2)$ \\
\hline$C(12)-C(13)-C(14)-C(9)$ & $2.0(3)$ \\
\hline $\mathrm{C}(15)-\mathrm{C}(13)-\mathrm{C}(14)-\mathrm{C}(5)$ & $1.9(3)$ \\
\hline $\mathrm{C}(12)-\mathrm{C}(13)-\mathrm{C}(14)-\mathrm{C}(5)$ & $-179.07(19)$ \\
\hline $\mathrm{C}(12)-\mathrm{C}(13)-\mathrm{C}(15)-\mathrm{C}(16)$ & $177.75(19)$ \\
\hline $\mathrm{C}(14)-\mathrm{C}(13)-\mathrm{C}(15)-\mathrm{C}(16)$ & $-3.2(3)$ \\
\hline $\mathrm{C}(12)-\mathrm{C}(13)-\mathrm{C}(15)-\mathrm{C}(39)$ & $-3.1(3)$ \\
\hline $\mathrm{C}(14)-\mathrm{C}(13)-\mathrm{C}(15)-\mathrm{C}(39)$ & $175.95(18)$ \\
\hline $\mathrm{C}(13)-\mathrm{C}(15)-\mathrm{C}(16)-\mathrm{C}(17)$ & $-178.44(19)$ \\
\hline $\mathrm{C}(39)-\mathrm{C}(15)-\mathrm{C}(16)-\mathrm{C}(17)$ & $2.4(3)$ \\
\hline $\mathrm{C}(13)-\mathrm{C}(15)-\mathrm{C}(16)-\mathrm{C}(4)$ & $-0.9(3)$ \\
\hline $\mathrm{C}(39)-\mathrm{C}(15)-\mathrm{C}(16)-\mathrm{C}(4)$ & $179.92(18)$ \\
\hline $\mathrm{C}(3)-\mathrm{C}(4)-\mathrm{C}(16)-\mathrm{C}(17)$ & $6.3(3)$ \\
\hline$C(5)-C(4)-C(16)-C(17)$ & $-176.31(19)$ \\
\hline$C(3)-C(4)-C(16)-C(15)$ & $-171.23(19)$ \\
\hline$C(5)-C(4)-C(16)-C(15)$ & $6.2(3)$ \\
\hline $\mathrm{C}(15)-\mathrm{C}(16)-\mathrm{C}(17)-\mathrm{C}(18)$ & $175.06(19)$ \\
\hline $\mathrm{C}(4)-\mathrm{C}(16)-\mathrm{C}(17)-\mathrm{C}(18)$ & $-2.5(3)$ \\
\hline $\mathrm{C}(16)-\mathrm{C}(17)-\mathrm{C}(18)-\mathrm{C}(2)$ & $-4.8(3)$ \\
\hline $\mathrm{C}(16)-\mathrm{C}(17)-\mathrm{C}(18)-\mathrm{C}(19)$ & $179.7(2)$ \\
\hline $\mathrm{C}(3)-\mathrm{C}(2)-\mathrm{C}(18)-\mathrm{C}(17)$ & $8.4(3)$ \\
\hline $\mathrm{C}(1)-\mathrm{C}(2)-\mathrm{C}(18)-\mathrm{C}(17)$ & $-169.52(19)$ \\
\hline $\mathrm{C}(3)-\mathrm{C}(2)-\mathrm{C}(18)-\mathrm{C}(19)$ & $-175.97(19)$ \\
\hline $\mathrm{C}(1)-\mathrm{C}(2)-\mathrm{C}(18)-\mathrm{C}(19)$ & $6.1(3)$ \\
\hline $\mathrm{C}(17)-\mathrm{C}(18)-\mathrm{C}(19)-\mathrm{C}(20)$ & $165.86(19)$ \\
\hline $\mathrm{C}(2)-\mathrm{C}(18)-\mathrm{C}(19)-\mathrm{C}(20)$ & $-9.4(3)$ \\
\hline $\mathrm{C}(17)-\mathrm{C}(18)-\mathrm{C}(19)-\mathrm{C}(23)$ & $-15.9(3)$ \\
\hline $\mathrm{C}(2)-\mathrm{C}(18)-\mathrm{C}(19)-\mathrm{C}(23)$ & $168.82(19)$ \\
\hline $\mathrm{C}(18)-\mathrm{C}(19)-\mathrm{C}(20)-\mathrm{C}(21)$ & $4.8(3)$ \\
\hline C(23)-C(19)-C(20)-C(21) & $-173.59(18)$ \\
\hline $\mathrm{C}(18)-\mathrm{C}(19)-\mathrm{C}(20)-\mathrm{C}(20) \# 1$ & $-175.6(2)$ \\
\hline$C(23)-C(19)-C(20)-C(20) \# 1$ & $6.0(3)$ \\
\hline
\end{tabular}




\begin{tabular}{|c|c|}
\hline $\mathrm{C}(2)-\mathrm{C}(1)-\mathrm{C}(21)-\mathrm{C}(22) \# 1$ & $172.18(19)$ \\
\hline $\mathrm{C}(24)-\mathrm{C}(1)-\mathrm{C}(21)-\mathrm{C}(22) \# 1$ & $-5.4(3)$ \\
\hline $\mathrm{C}(2)-\mathrm{C}(1)-\mathrm{C}(21)-\mathrm{C}(20)$ & $-6.7(3)$ \\
\hline $\mathrm{C}(24)-\mathrm{C}(1)-\mathrm{C}(21)-\mathrm{C}(20)$ & $175.71(18)$ \\
\hline $\mathrm{C}(19)-\mathrm{C}(20)-\mathrm{C}(21)-\mathrm{C}(1)$ & $3.3(3)$ \\
\hline $\mathrm{C}(20) \# 1-\mathrm{C}(20)-\mathrm{C}(21)-\mathrm{C}(1)$ & $-176.3(2)$ \\
\hline $\mathrm{C}(19)-\mathrm{C}(20)-\mathrm{C}(21)-\mathrm{C}(22) \# 1$ & $-175.59(19)$ \\
\hline $\mathrm{C}(20) \# 1-\mathrm{C}(20)-\mathrm{C}(21)-\mathrm{C}(22) \# 1$ & $4.8(3)$ \\
\hline $\mathrm{C}(21) \# 1-\mathrm{C}(22)-\mathrm{C}(23)-\mathrm{O}(3)$ & $-172.40(19)$ \\
\hline $\mathrm{C}(21) \# 1-\mathrm{C}(22)-\mathrm{C}(23)-\mathrm{C}(19)$ & $4.1(3)$ \\
\hline $\mathrm{C}(20)-\mathrm{C}(19)-\mathrm{C}(23)-\mathrm{C}(22)$ & $-8.3(3)$ \\
\hline $\mathrm{C}(18)-\mathrm{C}(19)-\mathrm{C}(23)-\mathrm{C}(22)$ & $173.4(2)$ \\
\hline $\mathrm{C}(20)-\mathrm{C}(19)-\mathrm{C}(23)-\mathrm{O}(3)$ & $168.47(18)$ \\
\hline $\mathrm{C}(18)-\mathrm{C}(19)-\mathrm{C}(23)-\mathrm{O}(3)$ & $-9.8(3)$ \\
\hline $\mathrm{C}(21)-\mathrm{C}(1)-\mathrm{C}(24)-\mathrm{C}(29)$ & $-81.6(2)$ \\
\hline $\mathrm{C}(2)-\mathrm{C}(1)-\mathrm{C}(24)-\mathrm{C}(29)$ & $100.9(2)$ \\
\hline $\mathrm{C}(21)-\mathrm{C}(1)-\mathrm{C}(24)-\mathrm{C}(25)$ & $93.2(2)$ \\
\hline $\mathrm{C}(2)-\mathrm{C}(1)-\mathrm{C}(24)-\mathrm{C}(25)$ & $-84.3(3)$ \\
\hline $\mathrm{C}(29)-\mathrm{C}(24)-\mathrm{C}(25)-\mathrm{C}(26)$ & $2.3(3)$ \\
\hline $\mathrm{C}(1)-\mathrm{C}(24)-\mathrm{C}(25)-\mathrm{C}(26)$ & $-172.46(19)$ \\
\hline $\mathrm{C}(29)-\mathrm{C}(24)-\mathrm{C}(25)-\mathrm{C}(30)$ & $-179.5(2)$ \\
\hline $\mathrm{C}(1)-\mathrm{C}(24)-\mathrm{C}(25)-\mathrm{C}(30)$ & $5.8(3)$ \\
\hline$C(24)-C(25)-C(26)-C(27)$ & $-0.9(4)$ \\
\hline$C(30)-C(25)-C(26)-C(27)$ & $-179.2(2)$ \\
\hline $\mathrm{C}(25)-\mathrm{C}(26)-\mathrm{C}(27)-\mathrm{C}(28)$ & $-0.3(4)$ \\
\hline $\mathrm{C}(25)-\mathrm{C}(26)-\mathrm{C}(27)-\mathrm{C}(31)$ & $-179.7(3)$ \\
\hline $\mathrm{C}(26)-\mathrm{C}(27)-\mathrm{C}(28)-\mathrm{C}(29)$ & $0.3(4)$ \\
\hline $\mathrm{C}(31)-\mathrm{C}(27)-\mathrm{C}(28)-\mathrm{C}(29)$ & $179.7(2)$ \\
\hline $\mathrm{C}(27)-\mathrm{C}(28)-\mathrm{C}(29)-\mathrm{C}(24)$ & $1.0(3)$ \\
\hline $\mathrm{C}(27)-\mathrm{C}(28)-\mathrm{C}(29)-\mathrm{C}(32)$ & $179.6(2)$ \\
\hline $\mathrm{C}(25)-\mathrm{C}(24)-\mathrm{C}(29)-\mathrm{C}(28)$ & $-2.3(3)$ \\
\hline $\mathrm{C}(1)-\mathrm{C}(24)-\mathrm{C}(29)-\mathrm{C}(28)$ & $172.45(19)$ \\
\hline$C(25)-C(24)-C(29)-C(32)$ & $179.1(2)$ \\
\hline $\mathrm{C}(1)-\mathrm{C}(24)-\mathrm{C}(29)-\mathrm{C}(32)$ & $-6.1(3)$ \\
\hline $\mathrm{O}(1)-\mathrm{C}(33)-\mathrm{C}(34)-\mathrm{C}(35)$ & $-167.8(4)$ \\
\hline $\mathrm{C}(33)-\mathrm{C}(34)-\mathrm{C}(35)-\mathrm{C}(36)$ & $-54.7(6)$ \\
\hline
\end{tabular}




\begin{tabular}{|c|c|}
\hline $\mathrm{C}(34)-\mathrm{C}(35)-\mathrm{C}(36)-\mathrm{C}(37)$ & $-167.7(4)$ \\
\hline $\mathrm{C}(35)-\mathrm{C}(36)-\mathrm{C}(37)-\mathrm{C}(38)$ & $-65.6(8)$ \\
\hline $\mathrm{O}(1)-\mathrm{C}(33 \mathrm{~A})-\mathrm{C}(34 \mathrm{~A})-\mathrm{C}(35 \mathrm{~A})$ & $177.3(6)$ \\
\hline$C(33 A)-C(34 A)-C(35 A)-C(36 A)$ & $-83.4(8)$ \\
\hline$C(34 A)-C(35 A)-C(36 A)-C(37 A)$ & $-176.1(6)$ \\
\hline $\mathrm{C}(35 \mathrm{~A})-\mathrm{C}(36 \mathrm{~A})-\mathrm{C}(37 \mathrm{~A})-\mathrm{C}(38 \mathrm{~A})$ & $-62.5(15)$ \\
\hline $\mathrm{C}(13)-\mathrm{C}(15)-\mathrm{C}(39)-\mathrm{C}(44)$ & $-95.3(2)$ \\
\hline $\mathrm{C}(16)-\mathrm{C}(15)-\mathrm{C}(39)-\mathrm{C}(44)$ & $83.9(2)$ \\
\hline $\mathrm{C}(13)-\mathrm{C}(15)-\mathrm{C}(39)-\mathrm{C}(40)$ & $85.8(3)$ \\
\hline $\mathrm{C}(16)-\mathrm{C}(15)-\mathrm{C}(39)-\mathrm{C}(40)$ & $-95.0(2)$ \\
\hline $\mathrm{C}(44)-\mathrm{C}(39)-\mathrm{C}(40)-\mathrm{C}(41)$ & $1.9(3)$ \\
\hline $\mathrm{C}(15)-\mathrm{C}(39)-\mathrm{C}(40)-\mathrm{C}(41)$ & $-179.19(19)$ \\
\hline $\mathrm{C}(44)-\mathrm{C}(39)-\mathrm{C}(40)-\mathrm{C}(45)$ & $-178.3(2)$ \\
\hline $\mathrm{C}(15)-\mathrm{C}(39)-\mathrm{C}(40)-\mathrm{C}(45)$ & $0.5(3)$ \\
\hline $\mathrm{C}(39)-\mathrm{C}(40)-\mathrm{C}(41)-\mathrm{C}(42)$ & $-0.2(3)$ \\
\hline $\mathrm{C}(45)-\mathrm{C}(40)-\mathrm{C}(41)-\mathrm{C}(42)$ & $-179.9(2)$ \\
\hline $\mathrm{C}(40)-\mathrm{C}(41)-\mathrm{C}(42)-\mathrm{C}(43)$ & $-1.3(3)$ \\
\hline $\mathrm{C}(40)-\mathrm{C}(41)-\mathrm{C}(42)-\mathrm{C}(46)$ & $178.6(2)$ \\
\hline $\mathrm{C}(41)-\mathrm{C}(42)-\mathrm{C}(43)-\mathrm{C}(44)$ & $1.1(3)$ \\
\hline $\mathrm{C}(46)-\mathrm{C}(42)-\mathrm{C}(43)-\mathrm{C}(44)$ & $-178.8(2)$ \\
\hline $\mathrm{C}(42)-\mathrm{C}(43)-\mathrm{C}(44)-\mathrm{C}(39)$ & $0.6(3)$ \\
\hline $\mathrm{C}(42)-\mathrm{C}(43)-\mathrm{C}(44)-\mathrm{C}(47)$ & $-178.6(2)$ \\
\hline $\mathrm{C}(40)-\mathrm{C}(39)-\mathrm{C}(44)-\mathrm{C}(43)$ & $-2.1(3)$ \\
\hline $\mathrm{C}(15)-\mathrm{C}(39)-\mathrm{C}(44)-\mathrm{C}(43)$ & $178.99(18)$ \\
\hline $\mathrm{C}(40)-\mathrm{C}(39)-\mathrm{C}(44)-\mathrm{C}(47)$ & $177.1(2)$ \\
\hline $\mathrm{C}(15)-\mathrm{C}(39)-\mathrm{C}(44)-\mathrm{C}(47)$ & $-1.8(3)$ \\
\hline $\mathrm{O}(3)-\mathrm{C}(48)-\mathrm{C}(49)-\mathrm{C}(50)$ & $179.17(19)$ \\
\hline $\mathrm{C}(48)-\mathrm{C}(49)-\mathrm{C}(50)-\mathrm{C}(51)$ & $-173.4(2)$ \\
\hline $\mathrm{C}(49)-\mathrm{C}(50)-\mathrm{C}(51)-\mathrm{C}(52)$ & $-174.4(2)$ \\
\hline $\mathrm{C}(50)-\mathrm{C}(51)-\mathrm{C}(52)-\mathrm{C}(53)$ & $-174.5(3)$ \\
\hline $\mathrm{C}(75) \# 2-\mathrm{C}(54)-\mathrm{C}(55)-\mathrm{C}(56)$ & $-178.92(19)$ \\
\hline $\mathrm{C}(77)-\mathrm{C}(54)-\mathrm{C}(55)-\mathrm{C}(56)$ & $2.5(3)$ \\
\hline $\mathrm{C}(75) \# 2-\mathrm{C}(54)-\mathrm{C}(55)-\mathrm{C}(71)$ & $1.8(3)$ \\
\hline $\mathrm{C}(77)-\mathrm{C}(54)-\mathrm{C}(55)-\mathrm{C}(71)$ & $-176.81(18)$ \\
\hline $\mathrm{C}(54)-\mathrm{C}(55)-\mathrm{C}(56)-\mathrm{C}(57)$ & $-174.54(19)$ \\
\hline $\mathrm{C}(71)-\mathrm{C}(55)-\mathrm{C}(56)-\mathrm{C}(57)$ & $4.8(3)$ \\
\hline
\end{tabular}




\begin{tabular}{|c|c|}
\hline $\mathrm{C}(55)-\mathrm{C}(56)-\mathrm{C}(57)-\mathrm{C}(69)$ & $3.4(3)$ \\
\hline $\mathrm{C}(55)-\mathrm{C}(56)-\mathrm{C}(57)-\mathrm{C}(58)$ & $178.9(2)$ \\
\hline$C(56)-C(57)-C(58)-C(67)$ & $-165.0(2)$ \\
\hline $\mathrm{C}(69)-\mathrm{C}(57)-\mathrm{C}(58)-\mathrm{C}(67)$ & $10.4(3)$ \\
\hline $\mathrm{C}(56)-\mathrm{C}(57)-\mathrm{C}(58)-\mathrm{C}(59)$ & $14.7(3)$ \\
\hline $\mathrm{C}(69)-\mathrm{C}(57)-\mathrm{C}(58)-\mathrm{C}(59)$ & $-169.94(19)$ \\
\hline $\mathrm{C}(67)-\mathrm{C}(58)-\mathrm{C}(59)-\mathrm{O}(4)$ & $-173.61(19)$ \\
\hline $\mathrm{C}(57)-\mathrm{C}(58)-\mathrm{C}(59)-\mathrm{O}(4)$ & $6.7(3)$ \\
\hline $\mathrm{C}(67)-\mathrm{C}(58)-\mathrm{C}(59)-\mathrm{C}(60)$ & $5.1(3)$ \\
\hline $\mathrm{C}(57)-\mathrm{C}(58)-\mathrm{C}(59)-\mathrm{C}(60)$ & $-174.6(2)$ \\
\hline $\mathrm{O}(4)-\mathrm{C}(59)-\mathrm{C}(60)-\mathrm{C}(61)$ & $176.8(2)$ \\
\hline $\mathrm{C}(58)-\mathrm{C}(59)-\mathrm{C}(60)-\mathrm{C}(61)$ & $-1.8(3)$ \\
\hline $\mathrm{C}(59)-\mathrm{C}(60)-\mathrm{C}(61)-\mathrm{C}(62)$ & $-2.0(3)$ \\
\hline $\mathrm{C}(60)-\mathrm{C}(61)-\mathrm{C}(62)-\mathrm{C}(67)$ & $2.3(3)$ \\
\hline $\mathrm{C}(60)-\mathrm{C}(61)-\mathrm{C}(62)-\mathrm{C}(63)$ & $-175.4(2)$ \\
\hline $\mathrm{C}(61)-\mathrm{C}(62)-\mathrm{C}(63)-\mathrm{O}(5)$ & $-1.1(3)$ \\
\hline $\mathrm{C}(67)-\mathrm{C}(62)-\mathrm{C}(63)-\mathrm{O}(5)$ & $-178.7(2)$ \\
\hline $\mathrm{C}(61)-\mathrm{C}(62)-\mathrm{C}(63)-\mathrm{C}(64)$ & $176.8(2)$ \\
\hline $\mathrm{C}(67)-\mathrm{C}(62)-\mathrm{C}(63)-\mathrm{C}(64)$ & $-0.8(3)$ \\
\hline $\mathrm{O}(5)-\mathrm{C}(63)-\mathrm{C}(64)-\mathrm{C}(65)$ & $177.2(2)$ \\
\hline $\mathrm{C}(62)-\mathrm{C}(63)-\mathrm{C}(64)-\mathrm{C}(65)$ & $-0.7(3)$ \\
\hline $\mathrm{C}(63)-\mathrm{C}(64)-\mathrm{C}(65)-\mathrm{C}(66)$ & $1.6(3)$ \\
\hline $\mathrm{C}(64)-\mathrm{C}(65)-\mathrm{C}(66)-\mathrm{C}(68)$ & $-178.9(2)$ \\
\hline $\mathrm{C}(64)-\mathrm{C}(65)-\mathrm{C}(66)-\mathrm{C}(67)$ & $-0.9(3)$ \\
\hline $\mathrm{C}(61)-\mathrm{C}(62)-\mathrm{C}(67)-\mathrm{C}(58)$ & $1.2(3)$ \\
\hline $\mathrm{C}(63)-\mathrm{C}(62)-\mathrm{C}(67)-\mathrm{C}(58)$ & $178.74(19)$ \\
\hline $\mathrm{C}(61)-\mathrm{C}(62)-\mathrm{C}(67)-\mathrm{C}(66)$ & $-176.17(19)$ \\
\hline $\mathrm{C}(63)-\mathrm{C}(62)-\mathrm{C}(67)-\mathrm{C}(66)$ & $1.4(3)$ \\
\hline $\mathrm{C}(59)-\mathrm{C}(58)-\mathrm{C}(67)-\mathrm{C}(62)$ & $-4.7(3)$ \\
\hline $\mathrm{C}(57)-\mathrm{C}(58)-\mathrm{C}(67)-\mathrm{C}(62)$ & $175.00(18)$ \\
\hline $\mathrm{C}(59)-\mathrm{C}(58)-\mathrm{C}(67)-\mathrm{C}(66)$ & $172.57(19)$ \\
\hline $\mathrm{C}(57)-\mathrm{C}(58)-\mathrm{C}(67)-\mathrm{C}(66)$ & $-7.7(3)$ \\
\hline $\mathrm{C}(68)-\mathrm{C}(66)-\mathrm{C}(67)-\mathrm{C}(62)$ & $177.45(19)$ \\
\hline $\mathrm{C}(65)-\mathrm{C}(66)-\mathrm{C}(67)-\mathrm{C}(62)$ & $-0.6(3)$ \\
\hline $\mathrm{C}(68)-\mathrm{C}(66)-\mathrm{C}(67)-\mathrm{C}(58)$ & $0.1(3)$ \\
\hline $\mathrm{C}(65)-\mathrm{C}(66)-\mathrm{C}(67)-\mathrm{C}(58)$ & $-177.89(19)$ \\
\hline
\end{tabular}




\begin{tabular}{|c|c|}
\hline $\mathrm{C}(65)-\mathrm{C}(66)-\mathrm{C}(68)-\mathrm{C}(69)$ & $-177.26(19)$ \\
\hline $\mathrm{C}(67)-\mathrm{C}(66)-\mathrm{C}(68)-\mathrm{C}(69)$ & $4.8(3)$ \\
\hline $\mathrm{C}(65)-\mathrm{C}(66)-\mathrm{C}(68)-\mathrm{C}(92)$ & $3.3(3)$ \\
\hline $\mathrm{C}(67)-\mathrm{C}(66)-\mathrm{C}(68)-\mathrm{C}(92)$ & $-174.68(18)$ \\
\hline $\mathrm{C}(56)-\mathrm{C}(57)-\mathrm{C}(69)-\mathrm{C}(70)$ & $-7.5(3)$ \\
\hline $\mathrm{C}(58)-\mathrm{C}(57)-\mathrm{C}(69)-\mathrm{C}(70)$ & $176.84(19)$ \\
\hline $\mathrm{C}(56)-\mathrm{C}(57)-\mathrm{C}(69)-\mathrm{C}(68)$ & $169.96(19)$ \\
\hline $\mathrm{C}(58)-\mathrm{C}(57)-\mathrm{C}(69)-\mathrm{C}(68)$ & $-5.7(3)$ \\
\hline $\mathrm{C}(66)-\mathrm{C}(68)-\mathrm{C}(69)-\mathrm{C}(70)$ & $175.58(19)$ \\
\hline $\mathrm{C}(92)-\mathrm{C}(68)-\mathrm{C}(69)-\mathrm{C}(70)$ & $-4.9(3)$ \\
\hline $\mathrm{C}(66)-\mathrm{C}(68)-\mathrm{C}(69)-\mathrm{C}(57)$ & $-1.9(3)$ \\
\hline $\mathrm{C}(92)-\mathrm{C}(68)-\mathrm{C}(69)-\mathrm{C}(57)$ & $177.57(18)$ \\
\hline $\mathrm{C}(57)-\mathrm{C}(69)-\mathrm{C}(70)-\mathrm{C}(71)$ & $3.4(3)$ \\
\hline $\mathrm{C}(68)-\mathrm{C}(69)-\mathrm{C}(70)-\mathrm{C}(71)$ & $-174.05(19)$ \\
\hline $\mathrm{C}(69)-\mathrm{C}(70)-\mathrm{C}(71)-\mathrm{C}(55)$ & $4.7(3)$ \\
\hline $\mathrm{C}(69)-\mathrm{C}(70)-\mathrm{C}(71)-\mathrm{C}(72)$ & $-179.40(19)$ \\
\hline $\mathrm{C}(56)-\mathrm{C}(55)-\mathrm{C}(71)-\mathrm{C}(70)$ & $-8.7(3)$ \\
\hline $\mathrm{C}(54)-\mathrm{C}(55)-\mathrm{C}(71)-\mathrm{C}(70)$ & $170.62(19)$ \\
\hline $\mathrm{C}(56)-\mathrm{C}(55)-\mathrm{C}(71)-\mathrm{C}(72)$ & $175.18(19)$ \\
\hline $\mathrm{C}(54)-\mathrm{C}(55)-\mathrm{C}(71)-\mathrm{C}(72)$ & $-5.5(3)$ \\
\hline $\mathrm{C}(70)-\mathrm{C}(71)-\mathrm{C}(72)-\mathrm{C}(76)$ & $-170.30(19)$ \\
\hline $\mathrm{C}(55)-\mathrm{C}(71)-\mathrm{C}(72)-\mathrm{C}(76)$ & $5.5(3)$ \\
\hline $\mathrm{C}(70)-\mathrm{C}(71)-\mathrm{C}(72)-\mathrm{C}(73)$ & $11.4(3)$ \\
\hline $\mathrm{C}(55)-\mathrm{C}(71)-\mathrm{C}(72)-\mathrm{C}(73)$ & $-172.84(19)$ \\
\hline $\mathrm{C}(76)-\mathrm{C}(72)-\mathrm{C}(73)-\mathrm{C}(74)$ & $4.4(3)$ \\
\hline $\mathrm{C}(71)-\mathrm{C}(72)-\mathrm{C}(73)-\mathrm{C}(74)$ & $-177.3(2)$ \\
\hline $\mathrm{C}(76)-\mathrm{C}(72)-\mathrm{C}(73)-\mathrm{O}(6)$ & $-173.55(18)$ \\
\hline $\mathrm{C}(71)-\mathrm{C}(72)-\mathrm{C}(73)-\mathrm{O}(6)$ & $4.8(3)$ \\
\hline $\mathrm{O}(6)-\mathrm{C}(73)-\mathrm{C}(74)-\mathrm{C}(75)$ & $175.98(19)$ \\
\hline $\mathrm{C}(72)-\mathrm{C}(73)-\mathrm{C}(74)-\mathrm{C}(75)$ & $-1.8(3)$ \\
\hline $\mathrm{C}(73)-\mathrm{C}(74)-\mathrm{C}(75)-\mathrm{C}(54) \# 2$ & $178.9(2)$ \\
\hline $\mathrm{C}(73)-\mathrm{C}(74)-\mathrm{C}(75)-\mathrm{C}(76) \# 2$ & $-1.8(3)$ \\
\hline $\mathrm{C}(71)-\mathrm{C}(72)-\mathrm{C}(76)-\mathrm{C}(75) \# 2$ & $-1.8(3)$ \\
\hline $\mathrm{C}(73)-\mathrm{C}(72)-\mathrm{C}(76)-\mathrm{C}(75) \# 2$ & $176.59(18)$ \\
\hline $\mathrm{C}(71)-\mathrm{C}(72)-\mathrm{C}(76)-\mathrm{C}(76) \# 2$ & $178.1(2)$ \\
\hline $\mathrm{C}(73)-\mathrm{C}(72)-\mathrm{C}(76)-\mathrm{C}(76) \# 2$ & $-3.5(3)$ \\
\hline
\end{tabular}




\begin{tabular}{|c|c|}
\hline $\mathrm{C}(75) \# 2-\mathrm{C}(54)-\mathrm{C}(77)-\mathrm{C}(82)$ & $84.7(3)$ \\
\hline $\mathrm{C}(55)-\mathrm{C}(54)-\mathrm{C}(77)-\mathrm{C}(82)$ & $-96.7(2)$ \\
\hline $\mathrm{C}(75) \# 2-\mathrm{C}(54)-\mathrm{C}(77)-\mathrm{C}(78)$ & $-94.9(2)$ \\
\hline $\mathrm{C}(55)-\mathrm{C}(54)-\mathrm{C}(77)-\mathrm{C}(78)$ & $83.7(3)$ \\
\hline $\mathrm{C}(82)-\mathrm{C}(77)-\mathrm{C}(78)-\mathrm{C}(79)$ & $-1.1(3)$ \\
\hline $\mathrm{C}(54)-\mathrm{C}(77)-\mathrm{C}(78)-\mathrm{C}(79)$ & $178.50(19)$ \\
\hline $\mathrm{C}(82)-\mathrm{C}(77)-\mathrm{C}(78)-\mathrm{C}(83)$ & $178.7(2)$ \\
\hline $\mathrm{C}(54)-\mathrm{C}(77)-\mathrm{C}(78)-\mathrm{C}(83)$ & $-1.6(3)$ \\
\hline $\mathrm{C}(77)-\mathrm{C}(78)-\mathrm{C}(79)-\mathrm{C}(80)$ & $0.4(3)$ \\
\hline $\mathrm{C}(83)-\mathrm{C}(78)-\mathrm{C}(79)-\mathrm{C}(80)$ & $-179.5(2)$ \\
\hline $\mathrm{C}(78)-\mathrm{C}(79)-\mathrm{C}(80)-\mathrm{C}(81)$ & $0.1(4)$ \\
\hline $\mathrm{C}(78)-\mathrm{C}(79)-\mathrm{C}(80)-\mathrm{C}(84)$ & $179.8(2)$ \\
\hline $\mathrm{C}(79)-\mathrm{C}(80)-\mathrm{C}(81)-\mathrm{C}(82)$ & $0.1(4)$ \\
\hline $\mathrm{C}(84)-\mathrm{C}(80)-\mathrm{C}(81)-\mathrm{C}(82)$ & $-179.6(2)$ \\
\hline $\mathrm{C}(80)-\mathrm{C}(81)-\mathrm{C}(82)-\mathrm{C}(77)$ & $-0.8(3)$ \\
\hline $\mathrm{C}(80)-\mathrm{C}(81)-\mathrm{C}(82)-\mathrm{C}(85)$ & $179.7(2)$ \\
\hline $\mathrm{C}(78)-\mathrm{C}(77)-\mathrm{C}(82)-\mathrm{C}(81)$ & $1.4(3)$ \\
\hline $\mathrm{C}(54)-\mathrm{C}(77)-\mathrm{C}(82)-\mathrm{C}(81)$ & $-178.28(19)$ \\
\hline $\mathrm{C}(78)-\mathrm{C}(77)-\mathrm{C}(82)-\mathrm{C}(85)$ & $-179.2(2)$ \\
\hline $\mathrm{C}(54)-\mathrm{C}(77)-\mathrm{C}(82)-\mathrm{C}(85)$ & $1.2(3)$ \\
\hline $\mathrm{O}(4)-\mathrm{C}(86)-\mathrm{C}(87)-\mathrm{C}(88)$ & $-177.70(18)$ \\
\hline $\mathrm{C}(86)-\mathrm{C}(87)-\mathrm{C}(88)-\mathrm{C}(89)$ & $173.3(2)$ \\
\hline $\mathrm{C}(87)-\mathrm{C}(88)-\mathrm{C}(89)-\mathrm{C}(90)$ & $-168.8(2)$ \\
\hline $\mathrm{C}(88)-\mathrm{C}(89)-\mathrm{C}(90)-\mathrm{C}(91)$ & $-64.6(3)$ \\
\hline $\mathrm{C}(66)-\mathrm{C}(68)-\mathrm{C}(92)-\mathrm{C}(97)$ & $94.3(2)$ \\
\hline $\mathrm{C}(69)-\mathrm{C}(68)-\mathrm{C}(92)-\mathrm{C}(97)$ & $-85.1(2)$ \\
\hline $\mathrm{C}(66)-\mathrm{C}(68)-\mathrm{C}(92)-\mathrm{C}(93)$ & $-88.3(3)$ \\
\hline $\mathrm{C}(69)-\mathrm{C}(68)-\mathrm{C}(92)-\mathrm{C}(93)$ & $92.2(2)$ \\
\hline $\mathrm{C}(97)-\mathrm{C}(92)-\mathrm{C}(93)-\mathrm{C}(94)$ & $0.3(3)$ \\
\hline $\mathrm{C}(68)-\mathrm{C}(92)-\mathrm{C}(93)-\mathrm{C}(94)$ & $-177.1(2)$ \\
\hline $\mathrm{C}(97)-\mathrm{C}(92)-\mathrm{C}(93)-\mathrm{C}(98)$ & $-180.0(2)$ \\
\hline $\mathrm{C}(68)-\mathrm{C}(92)-\mathrm{C}(93)-\mathrm{C}(98)$ & $2.7(3)$ \\
\hline $\mathrm{C}(92)-\mathrm{C}(93)-\mathrm{C}(94)-\mathrm{C}(95)$ & $-0.4(4)$ \\
\hline $\mathrm{C}(98)-\mathrm{C}(93)-\mathrm{C}(94)-\mathrm{C}(95)$ & $179.8(2)$ \\
\hline $\mathrm{C}(93)-\mathrm{C}(94)-\mathrm{C}(95)-\mathrm{C}(96)$ & $0.0(4)$ \\
\hline $\mathrm{C}(93)-\mathrm{C}(94)-\mathrm{C}(95)-\mathrm{C}(99)$ & 178.1(3) \\
\hline
\end{tabular}




$\begin{array}{cc}\mathrm{C}(94)-\mathrm{C}(95)-\mathrm{C}(96)-\mathrm{C}(97) & 0.5(4) \\ \mathrm{C}(99)-\mathrm{C}(95)-\mathrm{C}(96)-\mathrm{C}(97) & -177.7(3) \\ \mathrm{C}(95)-\mathrm{C}(96)-\mathrm{C}(97)-\mathrm{C}(92) & -0.6(3) \\ \mathrm{C}(95)-\mathrm{C}(96)-\mathrm{C}(97)-\mathrm{C}(100) & 178.0(2) \\ \mathrm{C}(93)-\mathrm{C}(92)-\mathrm{C}(97)-\mathrm{C}(96) & 0.2(3) \\ \mathrm{C}(68)-\mathrm{C}(92)-\mathrm{C}(97)-\mathrm{C}(96) & 177.60(18) \\ \mathrm{C}(93)-\mathrm{C}(92)-\mathrm{C}(97)-\mathrm{C}(100) & -178.4(2) \\ \mathrm{C}(68)-\mathrm{C}(92)-\mathrm{C}(97)-\mathrm{C}(100) & -1.0(3) \\ \mathrm{O}(6)-\mathrm{C}(101)-\mathrm{C}(102)-\mathrm{C}(103) & -173.3(2) \\ \mathrm{C}(101)-\mathrm{C}(102)-\mathrm{C}(103)-\mathrm{C}(104) & -73.5(3) \\ \mathrm{C}(102)-\mathrm{C}(103)-\mathrm{C}(104)-\mathrm{C}(105) & 178.4(3) \\ \mathrm{C}(103)-\mathrm{C}(104)-\mathrm{C}(105)-\mathrm{C}(106) & -68.3(6) \\ \mathrm{C}(7)-\mathrm{C}(6)-\mathrm{O}(1)-\mathrm{C}(33) & -9.1(4) \\ \mathrm{C}(5)-\mathrm{C}(6)-\mathrm{O}(1)-\mathrm{C}(33) & 171.4(3) \\ \mathrm{C}(7)-\mathrm{C}(6)-\mathrm{O}(1)-\mathrm{C}(33 \mathrm{~A}) & 9.1(6) \\ \mathrm{C}(5)-\mathrm{C}(6)-\mathrm{O}(1)-\mathrm{C}(33 \mathrm{~A}) & -170.4(5) \\ \mathrm{C}(34)-\mathrm{C}(33)-\mathrm{O}(1)-\mathrm{C}(6) & -170.0(3) \\ \mathrm{C}(34 \mathrm{~A})-\mathrm{C}(33 \mathrm{~A})-\mathrm{O}(1)-\mathrm{C}(6) & -96.4(7) \\ \mathrm{C}(22)-\mathrm{C}(23)-\mathrm{O}(3)-\mathrm{C}(48) & 1.4(3) \\ \mathrm{C}(19)-\mathrm{C}(23)-\mathrm{O}(3)-\mathrm{C}(48) & -175.35(19) \\ \mathrm{C}(49)-\mathrm{C}(48)-\mathrm{O}(3)-\mathrm{C}(23) & -79.1(2) \\ \mathrm{C}(60)-\mathrm{C}(59)-\mathrm{O}(4)-\mathrm{C}(86) & -179.37(19) \\ \mathrm{C}(58)-\mathrm{C}(59)-\mathrm{O}(4)-\mathrm{C}(86) & 78.3(2) \\ \mathrm{C}(87)-\mathrm{C}(86)-\mathrm{O}(4)-\mathrm{C}(59) & -1(3) \\ \mathrm{C}(74)-\mathrm{C}(73)-\mathrm{O}(6)-\mathrm{C}(101) & \\ \mathrm{C}(72)-\mathrm{C}(73)-\mathrm{O}(6)-\mathrm{C}(101) & \\ \mathrm{C}(102)-\mathrm{C}(101)-\mathrm{O}(6)-\mathrm{C}(73) & \\ & -173) \\ & \end{array}$

Symmetry transformations used to generate equivalent atoms:

$\# 1-x+1,-y,-z+1 \quad \# 2-x+1,-y+2,-z$ 


\section{Data for PIF-M-ONE}

Table S13. Crystal data and structure refinement for PIF-M-ONE.

Identification code

Empirical formula

Formula weight

Temperature

Wavelength

Crystal system

Space group

Unit cell dimensions

Volume

$\mathrm{Z}$

Density (calculated)

Absorption coefficient

$\mathrm{F}(000)$

Crystal size

Theta range for data collection

Index ranges

Reflections collected

Independent reflections

Completeness to theta $=66.500^{\circ}$

Absorption correction

Max. and min. transmission

Refinement method

Data / restraints / parameters

Goodness-of-fit on $\mathrm{F}^{2}$

Final $\mathrm{R}$ indices [I $>2 \operatorname{sigma}(\mathrm{I})]$

$\mathrm{R}$ indices (all data)

Extinction coefficient

Largest diff. peak and hole
PIF-M-ONE

$\mathrm{C}_{102} \mathrm{H}_{110} \mathrm{O}_{6}$

1430.83

100(2) K

$1.54178 \AA$

Triclinic

P-1

$a=10.9480(4) \AA$ $\mathrm{a}=69.553(2)^{\circ}$.

$\mathrm{b}=12.0802(4) \AA$

$\mathrm{b}=76.264(2)^{\circ}$.

$\mathrm{c}=16.2323(6) \AA$

$\mathrm{g}=79.814(2)^{\circ}$.

1943.58(12) $\AA^{3}$

1

$1.222 \mathrm{Mg} / \mathrm{m}^{3}$

$0.569 \mathrm{~mm}^{-1}$

768

$0.100 \times 0.060 \times 0.020 \mathrm{~mm}^{3}$

2.959 to $66.489^{\circ}$.

$-13<=\mathrm{h}<=13,-14<=\mathrm{k}<=14,-19<=1<=19$

26293

$6836[\mathrm{R}(\mathrm{int})=0.0765]$

$99.7 \%$

Semi-empirical from equivalents

0.7533 and 0.6338

Full-matrix least-squares on $\mathrm{F}^{2}$

$6836 / 36 / 549$

1.029

$\mathrm{R} 1=0.0570, \mathrm{wR} 2=0.1413$

$\mathrm{R} 1=0.0890, \mathrm{wR} 2=0.1578$

$\mathrm{n} / \mathrm{a}$

0.549 and -0.308 e. $\AA^{-3}$ 
Table S14. ( $\mathrm{x} 10^{4}$ ) and equivalent isotropic displacement parameters $\left(\AA^{2} \times 10^{3}\right)$ for PIF-M-ONE. U(eq) is defined as one third of the trace of the orthogonalized $\mathrm{U}^{\mathrm{ij}}$ tensor.

\begin{tabular}{|c|c|c|c|c|}
\hline $\mathrm{x}$ & & & $\mathrm{z}$ & $\mathrm{U}(\mathrm{eq})$ \\
\hline$C(27 X)$ & 1911(14) & $4464(15)$ & $5462(8)$ & $35(1)$ \\
\hline$C(28 X)$ & $1154(10)$ & $3755(10)$ & $5188(6)$ & $35(1)$ \\
\hline$C(29 X)$ & $315(9)$ & $3010(9)$ & $6022(6)$ & $35(1)$ \\
\hline$C(30 X)$ & $-452(9)$ & $2228(9)$ & $5818(7)$ & $35(1)$ \\
\hline$C(31 X)$ & $-1349(14)$ & $1498(15)$ & $6618(8)$ & $35(1)$ \\
\hline$C(32 X)$ & $-620(20)$ & $677(18)$ & $7329(13)$ & $35(1)$ \\
\hline $\mathrm{C}(32 \mathrm{Y})$ & $-570(20)$ & $447(15)$ & $7683(14)$ & $35(1)$ \\
\hline $\mathrm{C}(31 \mathrm{Y})$ & $-935(12)$ & $1606(13)$ & $6979(8)$ & $35(1)$ \\
\hline $\mathrm{C}(30 \mathrm{Y})$ & $186(10)$ & $2150(9)$ & $6281(7)$ & $35(1)$ \\
\hline C(29Y) & $-212(9)$ & $3344(8)$ & $5618(6)$ & $35(1)$ \\
\hline $\mathrm{C}(28 \mathrm{Y})$ & $897(9)$ & $3929(9)$ & $4933(7)$ & $35(1)$ \\
\hline$C(27 Y)$ & $1702(12)$ & $4399(16)$ & $5363(8)$ & $35(1)$ \\
\hline $\mathrm{C}(27)$ & $2075(6)$ & $3893(5)$ & $5405(3)$ & $36(1)$ \\
\hline$C(30)$ & $-374(5)$ & 2533(4) & $6236(3)$ & $40(1)$ \\
\hline $\mathrm{C}(29)$ & $863(4)$ & $2118(4)$ & $5722(3)$ & $38(1)$ \\
\hline $\mathrm{C}(28)$ & $1569(5)$ & $3123(5)$ & $5017(3)$ & $39(1)$ \\
\hline $\mathrm{C}(31)$ & $-1177(5)$ & $1529(6)$ & $6818(3)$ & $33(2)$ \\
\hline $\mathrm{C}(32)$ & $-529(13)$ & $580(9)$ & $7522(6)$ & $51(3)$ \\
\hline $\mathrm{O}(1)$ & $6730(2)$ & $9696(2)$ & $2836(1)$ & $51(1)$ \\
\hline $\mathrm{O}(2)$ & $2720(2)$ & 4899(2) & $4647(1)$ & $57(1)$ \\
\hline $\mathrm{O}(3)$ & $3405(1)$ & $3193(1)$ & $469(1)$ & $21(1)$ \\
\hline $\mathrm{C}(1)$ & $5474(3)$ & $8063(2)$ & $3305(2)$ & $35(1)$ \\
\hline$C(2)$ & $6101(3)$ & $8973(2)$ & $3463(2)$ & $50(1)$ \\
\hline$C(3)$ & $6338(7)$ & $8885(7)$ & 4293(3) & $55(2)$ \\
\hline $\mathrm{C}(4)$ & $5657(6)$ & $8055(5)$ & $5000(4)$ & $46(2)$ \\
\hline$C(5)$ & 4941(5) & $7284(6)$ & 4893(2) & $40(1)$ \\
\hline $\mathrm{C}(24)$ & $4235(5)$ & $6524(6)$ & $5639(3)$ & $40(1)$ \\
\hline$C(25)$ & $3418(12)$ & $5828(6)$ & $5553(3)$ & $40(1)$ \\
\hline $\mathrm{C}(3 \mathrm{~A})$ & $5692(7)$ & $9041(6)$ & 4331(3) & $55(2)$ \\
\hline$C(4 A)$ & $4946(7)$ & $8290(5)$ & $5058(4)$ & $54(2)$ \\
\hline$C(5 \mathrm{~A})$ & $4484(5)$ & $7364(5)$ & $4936(2)$ & $40(1)$ \\
\hline
\end{tabular}




\begin{tabular}{|c|c|c|c|c|}
\hline$C(24 \mathrm{~A})$ & $3791(5)$ & $6559(6)$ & $5703(4)$ & $40(1)$ \\
\hline$C(25 \mathrm{~A})$ & $3295(13)$ & $5623(6)$ & $5630(2)$ & $40(1)$ \\
\hline$C(6)$ & $4758(3)$ & $7252(2)$ & $4079(1)$ & $39(1)$ \\
\hline$C(7)$ & $4111(2)$ & $6398(2)$ & $3962(2)$ & $30(1)$ \\
\hline$C(8)$ & $4271(2)$ & $6298(2)$ & $3072(1)$ & $25(1)$ \\
\hline $\mathrm{C}(9)$ & $3701(2)$ & $5456(2)$ & 2889(1) & $25(1)$ \\
\hline$C(10)$ & $3987(2)$ & $5347(2)$ & $2047(1)$ & $21(1)$ \\
\hline $\mathrm{C}(11)$ & $4866(2)$ & $6047(2)$ & $1344(1)$ & $20(1)$ \\
\hline$C(12)$ & $5361(2)$ & $6915(2)$ & $1483(1)$ & $22(1)$ \\
\hline$C(13)$ & $5060(2)$ & $7067(2)$ & $2344(1)$ & $24(1)$ \\
\hline$C(14)$ & $5587(2)$ & $7998(2)$ & $2458(2)$ & $27(1)$ \\
\hline$C(15)$ & $6177(2)$ & $8918(2)$ & $1632(2)$ & $26(1)$ \\
\hline$C(16)$ & $5394(2)$ & $9905(2)$ & $1222(2)$ & $25(1)$ \\
\hline $\mathrm{C}(17)$ & $5937(2)$ & $10801(2)$ & $487(2)$ & $29(1)$ \\
\hline$C(18)$ & $7227(2)$ & $10731(2)$ & $137(2)$ & $32(1)$ \\
\hline$C(19)$ & $7970(2)$ & $9710(2)$ & $524(2)$ & $32(1)$ \\
\hline $\mathrm{C}(20)$ & $7469(2)$ & $8801(2)$ & $1268(2)$ & $31(1)$ \\
\hline $\mathrm{C}(21)$ & $3981(2)$ & $10014(2)$ & $1562(2)$ & $30(1)$ \\
\hline $\mathrm{C}(22)$ & $7786(3)$ & $11738(2)$ & $-632(2)$ & $40(1)$ \\
\hline $\mathrm{C}(23)$ & $8306(3)$ & $7723(2)$ & $1690(2)$ & $43(1)$ \\
\hline$C(26)$ & $3382(2)$ & $5663(2)$ & $4753(1)$ & $37(1)$ \\
\hline $\mathrm{C}(33)$ & $3544(2)$ & $4528(2)$ & $1726(1)$ & $21(1)$ \\
\hline$C(34)$ & $4195(2)$ & $4681(2)$ & $854(1)$ & $20(1)$ \\
\hline$C(35)$ & $4190(2)$ & $4080(2)$ & $230(1)$ & $19(1)$ \\
\hline$C(36)$ & $4971(2)$ & $4379(2)$ & $-581(1)$ & 19(1) \\
\hline $\mathrm{C}(37)$ & $2150(2)$ & $3668(2)$ & $299(2)$ & $24(1)$ \\
\hline$C(38)$ & $1281(2)$ & $2703(2)$ & $750(2)$ & $24(1)$ \\
\hline C(39) & $-87(2)$ & $3202(2)$ & $692(2)$ & $29(1)$ \\
\hline $\mathrm{C}(40)$ & $-1029(2)$ & $2286(2)$ & $1169(2)$ & $35(1)$ \\
\hline $\mathrm{C}(41)$ & $-2406(2)$ & $2827(2)$ & $1262(2)$ & $39(1)$ \\
\hline $\mathrm{C}(42)$ & $-2743(2)$ & $3470(3)$ & $1950(2)$ & $45(1)$ \\
\hline $\mathrm{C}(43)$ & $2569(2)$ & $3718(2)$ & $2284(1)$ & $22(1)$ \\
\hline $\mathrm{C}(44)$ & $1298(2)$ & $4187(2)$ & 2419(1) & $24(1)$ \\
\hline $\mathrm{C}(45)$ & $382(2)$ & $3430(2)$ & 2952(2) & $28(1)$ \\
\hline C(46) & $694(2)$ & $2228(2)$ & $3364(2)$ & $30(1)$ \\
\hline$C(47)$ & 1961(2) & $1787(2)$ & $3241(1)$ & \\
\hline
\end{tabular}




$\begin{array}{lrlll}\mathrm{C}(48) & 2907(2) & 2513(2) & 2708(1) & 25(1) \\ \mathrm{C}(49) & 898(2) & 5484(2) & 2000(2) & 33(1) \\ \mathrm{C}(50) & 4276(2) & 2011(2) & 2592(2) & 30(1) \\ \mathrm{C}(51) & -334(3) & 1444(2) & 3924(2) & 44(1)\end{array}$

Table S15. Bond lengths $[\AA]$ and angles $\left[^{\circ}\right]$ for PIF-M-ONE.

\begin{tabular}{|c|c|}
\hline $\mathrm{C}(27 \mathrm{X})-\mathrm{O}(2)$ & $1.391(12)$ \\
\hline$C(27 X)-C(28 X)$ & $1.520(9)$ \\
\hline $\mathrm{C}(27 \mathrm{X})-\mathrm{H}(27 \mathrm{X})$ & 0.9900 \\
\hline $\mathrm{C}(27 \mathrm{X})-\mathrm{H}(27 \mathrm{Y})$ & 0.9900 \\
\hline $\mathrm{C}(28 \mathrm{X})-\mathrm{C}(29 \mathrm{X})$ & $1.529(9)$ \\
\hline $\mathrm{C}(28 \mathrm{X})-\mathrm{H}(28 \mathrm{X})$ & 0.9900 \\
\hline $\mathrm{C}(28 \mathrm{X})-\mathrm{H}(28 \mathrm{Y})$ & 0.9900 \\
\hline$C(29 X)-C(30 X)$ & $1.528(9)$ \\
\hline $\mathrm{C}(29 \mathrm{X})-\mathrm{H}(29 \mathrm{X})$ & 0.9900 \\
\hline $\mathrm{C}(29 \mathrm{X})-\mathrm{H}(29 \mathrm{Y})$ & 0.9900 \\
\hline$C(30 X)-C(31 X)$ & $1.525(9)$ \\
\hline $\mathrm{C}(30 \mathrm{X})-\mathrm{H}(30 \mathrm{X})$ & 0.9900 \\
\hline $\mathrm{C}(30 \mathrm{X})-\mathrm{H}(30 \mathrm{Y})$ & 0.9900 \\
\hline$C(31 X)-C(32 X)$ & $1.523(9)$ \\
\hline $\mathrm{C}(31 \mathrm{X})-\mathrm{H}(31 \mathrm{X})$ & 0.9900 \\
\hline $\mathrm{C}(31 \mathrm{X})-\mathrm{H}(31 \mathrm{Y})$ & 0.9900 \\
\hline $\mathrm{C}(32 \mathrm{X})-\mathrm{H}(32 \mathrm{X})$ & 0.9800 \\
\hline $\mathrm{C}(32 \mathrm{X})-\mathrm{H}(32 \mathrm{Y})$ & 0.9800 \\
\hline $\mathrm{C}(32 \mathrm{X})-\mathrm{H}(32 \mathrm{Z})$ & 0.9800 \\
\hline $\mathrm{C}(32 \mathrm{Y})-\mathrm{C}(31 \mathrm{Y})$ & $1.523(10)$ \\
\hline $\mathrm{C}(32 \mathrm{Y})-\mathrm{H}(32 \mathrm{~A})$ & 0.9800 \\
\hline $\mathrm{C}(32 \mathrm{Y})-\mathrm{H}(32 \mathrm{~B})$ & 0.9800 \\
\hline $\mathrm{C}(32 \mathrm{Y})-\mathrm{H}(32 \mathrm{C})$ & 0.9800 \\
\hline $\mathrm{C}(31 \mathrm{Y})-\mathrm{C}(30 \mathrm{Y})$ & $1.526(9)$ \\
\hline $\mathrm{C}(31 \mathrm{Y})-\mathrm{H}(31 \mathrm{~A})$ & 0.9900 \\
\hline $\mathrm{C}(31 \mathrm{Y})-\mathrm{H}(31 \mathrm{~B})$ & 0.9900 \\
\hline $\mathrm{C}(30 \mathrm{Y})-\mathrm{C}(29 \mathrm{Y})$ & $1.531(9)$ \\
\hline $\mathrm{C}(30 \mathrm{Y})-\mathrm{H}(30 \mathrm{~A})$ & 0.9900 \\
\hline $\mathrm{C}(30 \mathrm{Y})-\mathrm{H}(30 \mathrm{~B})$ & 0.9900 \\
\hline
\end{tabular}




\begin{tabular}{|c|c|}
\hline $\mathrm{C}(29 \mathrm{Y})-\mathrm{C}(28 \mathrm{Y})$ & $1.524(8)$ \\
\hline $\mathrm{C}(29 \mathrm{Y})-\mathrm{H}(29 \mathrm{~A})$ & 0.9900 \\
\hline $\mathrm{C}(29 \mathrm{Y})-\mathrm{H}(29 \mathrm{~B})$ & 0.9900 \\
\hline $\mathrm{C}(28 \mathrm{Y})-\mathrm{C}(27 \mathrm{Y})$ & $1.527(9)$ \\
\hline $\mathrm{C}(28 \mathrm{Y})-\mathrm{H}(28 \mathrm{~A})$ & 0.9900 \\
\hline $\mathrm{C}(28 \mathrm{Y})-\mathrm{H}(28 \mathrm{~B})$ & 0.9900 \\
\hline $\mathrm{C}(27 \mathrm{Y})-\mathrm{O}(2)$ & $1.446(12)$ \\
\hline $\mathrm{C}(27 \mathrm{Y})-\mathrm{H}(27 \mathrm{~A})$ & 0.9900 \\
\hline $\mathrm{C}(27 \mathrm{Y})-\mathrm{H}(27 \mathrm{~B})$ & 0.9900 \\
\hline$C(27)-C(28)$ & $1.526(6)$ \\
\hline $\mathrm{C}(27)-\mathrm{O}(2)$ & $1.529(5)$ \\
\hline $\mathrm{C}(27)-\mathrm{H}(27 \mathrm{D})$ & 0.9900 \\
\hline $\mathrm{C}(27)-\mathrm{H}(27 \mathrm{E})$ & 0.9900 \\
\hline$C(30)-C(29)$ & $1.515(6)$ \\
\hline$C(30)-C(31)$ & $1.522(6)$ \\
\hline $\mathrm{C}(30)-\mathrm{H}(30 \mathrm{D})$ & 0.9900 \\
\hline $\mathrm{C}(30)-\mathrm{H}(30 \mathrm{E})$ & 0.9900 \\
\hline$C(29)-C(28)$ & $1.523(5)$ \\
\hline $\mathrm{C}(29)-\mathrm{H}(29 \mathrm{D})$ & 0.9900 \\
\hline C(29)-H(29E) & 0.9900 \\
\hline $\mathrm{C}(28)-\mathrm{H}(28 \mathrm{D})$ & 0.9900 \\
\hline $\mathrm{C}(28)-\mathrm{H}(28 \mathrm{E})$ & 0.9900 \\
\hline $\mathrm{C}(31)-\mathrm{C}(32)$ & $1.519(7)$ \\
\hline $\mathrm{C}(31)-\mathrm{H}(31 \mathrm{D})$ & 0.9900 \\
\hline $\mathrm{C}(31)-\mathrm{H}(31 \mathrm{E})$ & 0.9900 \\
\hline $\mathrm{C}(32)-\mathrm{H}(32 \mathrm{D})$ & 0.9800 \\
\hline $\mathrm{C}(32)-\mathrm{H}(32 \mathrm{E})$ & 0.9800 \\
\hline $\mathrm{C}(32)-\mathrm{H}(32 \mathrm{~F})$ & 0.9800 \\
\hline $\mathrm{O}(1)-\mathrm{C}(2)$ & $1.237(4)$ \\
\hline $\mathrm{O}(2)-\mathrm{C}(26)$ & $1.346(3)$ \\
\hline $\mathrm{O}(3)-\mathrm{C}(35)$ & $1.383(3)$ \\
\hline $\mathrm{O}(3)-\mathrm{C}(37)$ & $1.443(2)$ \\
\hline $\mathrm{C}(1)-\mathrm{C}(14)$ & $1.379(3)$ \\
\hline$C(1)-C(6)$ & $1.449(4)$ \\
\hline $\mathrm{C}(1)-\mathrm{C}(2)$ & $1.514(4)$ \\
\hline $\mathrm{C}(2)-\mathrm{C}(3)$ & $1.396(2)$ \\
\hline
\end{tabular}




\begin{tabular}{|c|c|}
\hline $\mathrm{C}(2)-\mathrm{C}(3 \mathrm{~A})$ & $1.401(2)$ \\
\hline$C(3)-C(4)$ & $1.390(2)$ \\
\hline $\mathrm{C}(3)-\mathrm{H}(3)$ & 0.9500 \\
\hline$C(4)-C(5)$ & $1.396(2)$ \\
\hline $\mathrm{C}(4)-\mathrm{H}(4)$ & 0.9500 \\
\hline$C(5)-C(24)$ & $1.392(2)$ \\
\hline$C(5)-C(6)$ & $1.398(2)$ \\
\hline$C(24)-C(25)$ & $1.390(2)$ \\
\hline $\mathrm{C}(24)-\mathrm{H}(24)$ & 0.9500 \\
\hline$C(25)-C(26)$ & $1.393(2)$ \\
\hline $\mathrm{C}(25)-\mathrm{H}(25)$ & 0.9500 \\
\hline$C(3 A)-C(4 A)$ & $1.392(2)$ \\
\hline $\mathrm{C}(3 \mathrm{~A})-\mathrm{H}(3 \mathrm{~A})$ & 0.9500 \\
\hline$C(4 A)-C(5 A)$ & $1.396(2)$ \\
\hline $\mathrm{C}(4 \mathrm{~A})-\mathrm{H}(4 \mathrm{~A})$ & 0.9500 \\
\hline$C(5 A)-C(6)$ & $1.401(2)$ \\
\hline$C(5 A)-C(24 A)$ & $1.427(7)$ \\
\hline$C(24 \mathrm{~A})-\mathrm{C}(25 \mathrm{~A})$ & $1.389(2)$ \\
\hline $\mathrm{C}(24 \mathrm{~A})-\mathrm{H}(24 \mathrm{~A})$ & 0.9500 \\
\hline$C(25 A)-C(26)$ & $1.388(2)$ \\
\hline $\mathrm{C}(25 \mathrm{~A})-\mathrm{H}(25 \mathrm{~A})$ & 0.9500 \\
\hline$C(6)-C(7)$ & $1.435(4)$ \\
\hline$C(7)-C(26)$ & $1.428(4)$ \\
\hline$C(7)-C(8)$ & $1.459(3)$ \\
\hline $\mathrm{C}(8)-\mathrm{C}(9)$ & $1.425(3)$ \\
\hline $\mathrm{C}(8)-\mathrm{C}(13)$ & $1.428(3)$ \\
\hline $\mathrm{C}(9)-\mathrm{C}(10)$ & $1.376(3)$ \\
\hline $\mathrm{C}(9)-\mathrm{H}(9)$ & 0.9500 \\
\hline $\mathrm{C}(10)-\mathrm{C}(11)$ & $1.427(3)$ \\
\hline $\mathrm{C}(10)-\mathrm{C}(33)$ & $1.461(3)$ \\
\hline$C(11)-C(12)$ & $1.367(3)$ \\
\hline $\mathrm{C}(11)-\mathrm{C}(36) \# 1$ & $1.461(3)$ \\
\hline $\mathrm{C}(12)-\mathrm{C}(13)$ & $1.426(3)$ \\
\hline $\mathrm{C}(12)-\mathrm{H}(12)$ & 0.9500 \\
\hline$C(13)-C(14)$ & $1.434(3)$ \\
\hline $\mathrm{C}(14)-\mathrm{C}(15)$ & $1.500(3)$ \\
\hline
\end{tabular}




\begin{tabular}{|c|c|}
\hline$C(15)-C(16)$ & $1.398(3)$ \\
\hline$C(15)-C(20)$ & $1.401(3)$ \\
\hline $\mathrm{C}(16)-\mathrm{C}(17)$ & $1.392(3)$ \\
\hline$C(16)-C(21)$ & $1.512(3)$ \\
\hline $\mathrm{C}(17)-\mathrm{C}(18)$ & $1.391(3)$ \\
\hline $\mathrm{C}(17)-\mathrm{H}(17)$ & 0.9500 \\
\hline$C(18)-C(19)$ & $1.388(3)$ \\
\hline C(18)-C(22) & $1.501(4)$ \\
\hline $\mathrm{C}(19)-\mathrm{C}(20)$ & $1.391(4)$ \\
\hline $\mathrm{C}(19)-\mathrm{H}(19)$ & 0.9500 \\
\hline$C(20)-C(23)$ & $1.502(3)$ \\
\hline $\mathrm{C}(21)-\mathrm{H}(21 \mathrm{~A})$ & 0.9800 \\
\hline $\mathrm{C}(21)-\mathrm{H}(21 \mathrm{~B})$ & 0.9800 \\
\hline $\mathrm{C}(21)-\mathrm{H}(21 \mathrm{C})$ & 0.9800 \\
\hline $\mathrm{C}(22)-\mathrm{H}(22 \mathrm{~A})$ & 0.9800 \\
\hline $\mathrm{C}(22)-\mathrm{H}(22 \mathrm{~B})$ & 0.9800 \\
\hline $\mathrm{C}(22)-\mathrm{H}(22 \mathrm{C})$ & 0.9800 \\
\hline $\mathrm{C}(23)-\mathrm{H}(23 \mathrm{~A})$ & 0.9800 \\
\hline $\mathrm{C}(23)-\mathrm{H}(23 \mathrm{~B})$ & 0.9800 \\
\hline $\mathrm{C}(23)-\mathrm{H}(23 \mathrm{C})$ & 0.9800 \\
\hline $\mathrm{C}(33)-\mathrm{C}(34)$ & $1.390(3)$ \\
\hline$C(33)-C(43)$ & $1.486(3)$ \\
\hline $\mathrm{C}(34)-\mathrm{C}(35)$ & $1.438(3)$ \\
\hline $\mathrm{C}(34)-\mathrm{C}(36) \# 1$ & $1.464(3)$ \\
\hline C(35)-C(36) & $1.356(3)$ \\
\hline $\mathrm{C}(36)-\mathrm{C}(11) \# 1$ & $1.461(3)$ \\
\hline $\mathrm{C}(36)-\mathrm{C}(34) \# 1$ & $1.464(3)$ \\
\hline $\mathrm{C}(37)-\mathrm{C}(38)$ & $1.506(3)$ \\
\hline $\mathrm{C}(37)-\mathrm{H}(37 \mathrm{~A})$ & 0.9900 \\
\hline $\mathrm{C}(37)-\mathrm{H}(37 \mathrm{~B})$ & 0.9900 \\
\hline C(38)-C(39) & $1.525(3)$ \\
\hline $\mathrm{C}(38)-\mathrm{H}(38 \mathrm{~A})$ & 0.9900 \\
\hline $\mathrm{C}(38)-\mathrm{H}(38 \mathrm{~B})$ & 0.9900 \\
\hline $\mathrm{C}(39)-\mathrm{C}(40)$ & $1.520(3)$ \\
\hline $\mathrm{C}(39)-\mathrm{H}(39 \mathrm{~A})$ & 0.9900 \\
\hline $\mathrm{C}(39)-\mathrm{H}(39 \mathrm{~B})$ & 0.9900 \\
\hline
\end{tabular}




\begin{tabular}{|c|c|}
\hline $\mathrm{C}(40)-\mathrm{C}(41)$ & $1.528(3)$ \\
\hline $\mathrm{C}(40)-\mathrm{H}(40 \mathrm{~A})$ & 0.9900 \\
\hline $\mathrm{C}(40)-\mathrm{H}(40 \mathrm{~B})$ & 0.9900 \\
\hline $\mathrm{C}(41)-\mathrm{C}(42)$ & $1.514(4)$ \\
\hline $\mathrm{C}(41)-\mathrm{H}(41 \mathrm{~A})$ & 0.9900 \\
\hline $\mathrm{C}(41)-\mathrm{H}(41 \mathrm{~B})$ & 0.9900 \\
\hline $\mathrm{C}(42)-\mathrm{H}(42 \mathrm{~A})$ & 0.9800 \\
\hline $\mathrm{C}(42)-\mathrm{H}(42 \mathrm{~B})$ & 0.9800 \\
\hline $\mathrm{C}(42)-\mathrm{H}(42 \mathrm{C})$ & 0.9800 \\
\hline C(43)-C(48) & $1.400(3)$ \\
\hline $\mathrm{C}(43)-\mathrm{C}(44)$ & $1.403(3)$ \\
\hline $\mathrm{C}(44)-\mathrm{C}(45)$ & $1.396(3)$ \\
\hline $\mathrm{C}(44)-\mathrm{C}(49)$ & $1.505(3)$ \\
\hline$C(45)-C(46)$ & $1.388(3)$ \\
\hline $\mathrm{C}(45)-\mathrm{H}(45)$ & 0.9500 \\
\hline $\mathrm{C}(46)-\mathrm{C}(47)$ & $1.389(3)$ \\
\hline$C(46)-C(51)$ & $1.510(4)$ \\
\hline $\mathrm{C}(47)-\mathrm{C}(48)$ & $1.399(3)$ \\
\hline $\mathrm{C}(47)-\mathrm{H}(47)$ & 0.9500 \\
\hline $\mathrm{C}(48)-\mathrm{C}(50)$ & $1.507(3)$ \\
\hline $\mathrm{C}(49)-\mathrm{H}(49 \mathrm{~A})$ & 0.9800 \\
\hline C(49)-H(49B) & 0.9800 \\
\hline $\mathrm{C}(49)-\mathrm{H}(49 \mathrm{C})$ & 0.9800 \\
\hline $\mathrm{C}(50)-\mathrm{H}(50 \mathrm{~A})$ & 0.9800 \\
\hline $\mathrm{C}(50)-\mathrm{H}(50 \mathrm{~B})$ & 0.9800 \\
\hline $\mathrm{C}(50)-\mathrm{H}(50 \mathrm{C})$ & 0.9800 \\
\hline $\mathrm{C}(51)-\mathrm{H}(51 \mathrm{~A})$ & 0.9800 \\
\hline $\mathrm{C}(51)-\mathrm{H}(51 \mathrm{~B})$ & 0.9800 \\
\hline $\mathrm{C}(51)-\mathrm{H}(51 \mathrm{C})$ & 0.9800 \\
\hline $\mathrm{O}(2)-\mathrm{C}(27 \mathrm{X})-\mathrm{C}(28 \mathrm{X})$ & $98.9(7)$ \\
\hline $\mathrm{O}(2)-\mathrm{C}(27 \mathrm{X})-\mathrm{H}(27 \mathrm{X})$ & 112.0 \\
\hline $\mathrm{C}(28 \mathrm{X})-\mathrm{C}(27 \mathrm{X})-\mathrm{H}(27 \mathrm{X})$ & 112.0 \\
\hline $\mathrm{O}(2)-\mathrm{C}(27 \mathrm{X})-\mathrm{H}(27 \mathrm{Y})$ & 112.0 \\
\hline $\mathrm{C}(28 \mathrm{X})-\mathrm{C}(27 \mathrm{X})-\mathrm{H}(27 \mathrm{Y})$ & 112.0 \\
\hline $\mathrm{H}(27 \mathrm{X})-\mathrm{C}(27 \mathrm{X})-\mathrm{H}(27 \mathrm{Y})$ & 109.7 \\
\hline
\end{tabular}




\begin{tabular}{|c|c|}
\hline $\mathrm{C}(27 \mathrm{X})-\mathrm{C}(28 \mathrm{X})-\mathrm{C}(29 \mathrm{X})$ & 109.3(8) \\
\hline $\mathrm{C}(27 \mathrm{X})-\mathrm{C}(28 \mathrm{X})-\mathrm{H}(28 \mathrm{X})$ & 109.8 \\
\hline $\mathrm{C}(29 \mathrm{X})-\mathrm{C}(28 \mathrm{X})-\mathrm{H}(28 \mathrm{X})$ & 109.8 \\
\hline $\mathrm{C}(27 \mathrm{X})-\mathrm{C}(28 \mathrm{X})-\mathrm{H}(28 \mathrm{Y})$ & 109.8 \\
\hline $\mathrm{C}(29 \mathrm{X})-\mathrm{C}(28 \mathrm{X})-\mathrm{H}(28 \mathrm{Y})$ & 109.8 \\
\hline $\mathrm{H}(28 \mathrm{X})-\mathrm{C}(28 \mathrm{X})-\mathrm{H}(28 \mathrm{Y})$ & 108.3 \\
\hline$C(30 X)-C(29 X)-C(28 X)$ & $113.2(8)$ \\
\hline $\mathrm{C}(30 \mathrm{X})-\mathrm{C}(29 \mathrm{X})-\mathrm{H}(29 \mathrm{X})$ & 108.9 \\
\hline $\mathrm{C}(28 \mathrm{X})-\mathrm{C}(29 \mathrm{X})-\mathrm{H}(29 \mathrm{X})$ & 108.9 \\
\hline $\mathrm{C}(30 \mathrm{X})-\mathrm{C}(29 \mathrm{X})-\mathrm{H}(29 \mathrm{Y})$ & 108.9 \\
\hline $\mathrm{C}(28 \mathrm{X})-\mathrm{C}(29 \mathrm{X})-\mathrm{H}(29 \mathrm{Y})$ & 108.9 \\
\hline $\mathrm{H}(29 \mathrm{X})-\mathrm{C}(29 \mathrm{X})-\mathrm{H}(29 \mathrm{Y})$ & 107.7 \\
\hline$C(31 X)-C(30 X)-C(29 X)$ & $115.8(10)$ \\
\hline $\mathrm{C}(31 \mathrm{X})-\mathrm{C}(30 \mathrm{X})-\mathrm{H}(30 \mathrm{X})$ & 108.3 \\
\hline $\mathrm{C}(29 \mathrm{X})-\mathrm{C}(30 \mathrm{X})-\mathrm{H}(30 \mathrm{X})$ & 108.3 \\
\hline $\mathrm{C}(31 \mathrm{X})-\mathrm{C}(30 \mathrm{X})-\mathrm{H}(30 \mathrm{Y})$ & 108.3 \\
\hline $\mathrm{C}(29 \mathrm{X})-\mathrm{C}(30 \mathrm{X})-\mathrm{H}(30 \mathrm{Y})$ & 108.3 \\
\hline $\mathrm{H}(30 \mathrm{X})-\mathrm{C}(30 \mathrm{X})-\mathrm{H}(30 \mathrm{Y})$ & 107.4 \\
\hline$C(32 X)-C(31 X)-C(30 X)$ & $110.3(13$ \\
\hline $\mathrm{C}(32 \mathrm{X})-\mathrm{C}(31 \mathrm{X})-\mathrm{H}(31 \mathrm{X})$ & 109.6 \\
\hline $\mathrm{C}(30 \mathrm{X})-\mathrm{C}(31 \mathrm{X})-\mathrm{H}(31 \mathrm{X})$ & 109.6 \\
\hline $\mathrm{C}(32 \mathrm{X})-\mathrm{C}(31 \mathrm{X})-\mathrm{H}(31 \mathrm{Y})$ & 109.6 \\
\hline $\mathrm{C}(30 \mathrm{X})-\mathrm{C}(31 \mathrm{X})-\mathrm{H}(31 \mathrm{Y})$ & 109.6 \\
\hline $\mathrm{H}(31 \mathrm{X})-\mathrm{C}(31 \mathrm{X})-\mathrm{H}(31 \mathrm{Y})$ & 108.1 \\
\hline $\mathrm{C}(31 \mathrm{X})-\mathrm{C}(32 \mathrm{X})-\mathrm{H}(32 \mathrm{X})$ & 109.5 \\
\hline $\mathrm{C}(31 \mathrm{X})-\mathrm{C}(32 \mathrm{X})-\mathrm{H}(32 \mathrm{Y})$ & 109.5 \\
\hline $\mathrm{H}(32 \mathrm{X})-\mathrm{C}(32 \mathrm{X})-\mathrm{H}(32 \mathrm{Y})$ & 109.5 \\
\hline $\mathrm{C}(31 \mathrm{X})-\mathrm{C}(32 \mathrm{X})-\mathrm{H}(32 \mathrm{Z})$ & 109.5 \\
\hline $\mathrm{H}(32 \mathrm{X})-\mathrm{C}(32 \mathrm{X})-\mathrm{H}(32 \mathrm{Z})$ & 109.5 \\
\hline $\mathrm{H}(32 \mathrm{Y})-\mathrm{C}(32 \mathrm{X})-\mathrm{H}(32 \mathrm{Z})$ & 109.5 \\
\hline $\mathrm{C}(31 \mathrm{Y})-\mathrm{C}(32 \mathrm{Y})-\mathrm{H}(32 \mathrm{~A})$ & 109.5 \\
\hline $\mathrm{C}(31 \mathrm{Y})-\mathrm{C}(32 \mathrm{Y})-\mathrm{H}(32 \mathrm{~B})$ & 109.5 \\
\hline $\mathrm{H}(32 \mathrm{~A})-\mathrm{C}(32 \mathrm{Y})-\mathrm{H}(32 \mathrm{~B})$ & 109.5 \\
\hline $\mathrm{C}(31 \mathrm{Y})-\mathrm{C}(32 \mathrm{Y})-\mathrm{H}(32 \mathrm{C})$ & 109.5 \\
\hline $\mathrm{H}(32 \mathrm{~A})-\mathrm{C}(32 \mathrm{Y})-\mathrm{H}(32 \mathrm{C})$ & 109.5 \\
\hline $\mathrm{H}(32 \mathrm{~B})-\mathrm{C}(32 \mathrm{Y})-\mathrm{H}(32 \mathrm{C})$ & 109.5 \\
\hline
\end{tabular}




\begin{tabular}{|c|c|}
\hline $\mathrm{C}(32 \mathrm{Y})-\mathrm{C}(31 \mathrm{Y})-\mathrm{C}(30 \mathrm{Y})$ & $114.0(14$ \\
\hline $\mathrm{C}(32 \mathrm{Y})-\mathrm{C}(31 \mathrm{Y})-\mathrm{H}(31 \mathrm{~A})$ & 108.7 \\
\hline $\mathrm{C}(30 \mathrm{Y})-\mathrm{C}(31 \mathrm{Y})-\mathrm{H}(31 \mathrm{~A})$ & 108.7 \\
\hline $\mathrm{C}(32 \mathrm{Y})-\mathrm{C}(31 \mathrm{Y})-\mathrm{H}(31 \mathrm{~B})$ & 108.7 \\
\hline $\mathrm{C}(30 \mathrm{Y})-\mathrm{C}(31 \mathrm{Y})-\mathrm{H}(31 \mathrm{~B})$ & 108.7 \\
\hline $\mathrm{H}(31 \mathrm{~A})-\mathrm{C}(31 \mathrm{Y})-\mathrm{H}(31 \mathrm{~B})$ & 107.6 \\
\hline $\mathrm{C}(31 \mathrm{Y})-\mathrm{C}(30 \mathrm{Y})-\mathrm{C}(29 \mathrm{Y})$ & 112.1(9) \\
\hline $\mathrm{C}(31 \mathrm{Y})-\mathrm{C}(30 \mathrm{Y})-\mathrm{H}(30 \mathrm{~A})$ & 109.2 \\
\hline $\mathrm{C}(29 \mathrm{Y})-\mathrm{C}(30 \mathrm{Y})-\mathrm{H}(30 \mathrm{~A})$ & 109.2 \\
\hline $\mathrm{C}(31 \mathrm{Y})-\mathrm{C}(30 \mathrm{Y})-\mathrm{H}(30 \mathrm{~B})$ & 109.2 \\
\hline $\mathrm{C}(29 \mathrm{Y})-\mathrm{C}(30 \mathrm{Y})-\mathrm{H}(30 \mathrm{~B})$ & 109.2 \\
\hline $\mathrm{H}(30 \mathrm{~A})-\mathrm{C}(30 \mathrm{Y})-\mathrm{H}(30 \mathrm{~B})$ & 107.9 \\
\hline $\mathrm{C}(28 \mathrm{Y})-\mathrm{C}(29 \mathrm{Y})-\mathrm{C}(30 \mathrm{Y})$ & $113.2(8)$ \\
\hline $\mathrm{C}(28 \mathrm{Y})-\mathrm{C}(29 \mathrm{Y})-\mathrm{H}(29 \mathrm{~A})$ & 108.9 \\
\hline $\mathrm{C}(30 \mathrm{Y})-\mathrm{C}(29 \mathrm{Y})-\mathrm{H}(29 \mathrm{~A})$ & 108.9 \\
\hline $\mathrm{C}(28 \mathrm{Y})-\mathrm{C}(29 \mathrm{Y})-\mathrm{H}(29 \mathrm{~B})$ & 108.9 \\
\hline $\mathrm{C}(30 \mathrm{Y})-\mathrm{C}(29 \mathrm{Y})-\mathrm{H}(29 \mathrm{~B})$ & 108.9 \\
\hline $\mathrm{H}(29 \mathrm{~A})-\mathrm{C}(29 \mathrm{Y})-\mathrm{H}(29 \mathrm{~B})$ & 107.8 \\
\hline $\mathrm{C}(29 \mathrm{Y})-\mathrm{C}(28 \mathrm{Y})-\mathrm{C}(27 \mathrm{Y})$ & $111.4(8$ \\
\hline $\mathrm{C}(29 \mathrm{Y})-\mathrm{C}(28 \mathrm{Y})-\mathrm{H}(28 \mathrm{~A})$ & 109.4 \\
\hline $\mathrm{C}(27 \mathrm{Y})-\mathrm{C}(28 \mathrm{Y})-\mathrm{H}(28 \mathrm{~A})$ & 109.4 \\
\hline $\mathrm{C}(29 \mathrm{Y})-\mathrm{C}(28 \mathrm{Y})-\mathrm{H}(28 \mathrm{~B})$ & 109.4 \\
\hline $\mathrm{C}(27 \mathrm{Y})-\mathrm{C}(28 \mathrm{Y})-\mathrm{H}(28 \mathrm{~B})$ & 109.4 \\
\hline $\mathrm{H}(28 \mathrm{~A})-\mathrm{C}(28 \mathrm{Y})-\mathrm{H}(28 \mathrm{~B})$ & 108.0 \\
\hline $\mathrm{O}(2)-\mathrm{C}(27 \mathrm{Y})-\mathrm{C}(28 \mathrm{Y})$ & $105.2(8$ \\
\hline $\mathrm{O}(2)-\mathrm{C}(27 \mathrm{Y})-\mathrm{H}(27 \mathrm{~A})$ & 110.7 \\
\hline $\mathrm{C}(28 \mathrm{Y})-\mathrm{C}(27 \mathrm{Y})-\mathrm{H}(27 \mathrm{~A})$ & 110.7 \\
\hline $\mathrm{O}(2)-\mathrm{C}(27 \mathrm{Y})-\mathrm{H}(27 \mathrm{~B})$ & 110.7 \\
\hline $\mathrm{C}(28 \mathrm{Y})-\mathrm{C}(27 \mathrm{Y})-\mathrm{H}(27 \mathrm{~B})$ & 110.7 \\
\hline $\mathrm{H}(27 \mathrm{~A})-\mathrm{C}(27 \mathrm{Y})-\mathrm{H}(27 \mathrm{~B})$ & 108.8 \\
\hline $\mathrm{C}(28)-\mathrm{C}(27)-\mathrm{O}(2)$ & $109.9(3$ \\
\hline $\mathrm{C}(28)-\mathrm{C}(27)-\mathrm{H}(27 \mathrm{D})$ & 109.7 \\
\hline $\mathrm{O}(2)-\mathrm{C}(27)-\mathrm{H}(27 \mathrm{D})$ & 109.7 \\
\hline $\mathrm{C}(28)-\mathrm{C}(27)-\mathrm{H}(27 \mathrm{E})$ & 109.7 \\
\hline $\mathrm{O}(2)-\mathrm{C}(27)-\mathrm{H}(27 \mathrm{E})$ & 109.7 \\
\hline $\mathrm{H}(27 \mathrm{D})-\mathrm{C}(27)-\mathrm{H}(27 \mathrm{E})$ & 108.2 \\
\hline
\end{tabular}




\begin{tabular}{|c|c|}
\hline $\mathrm{C}(29)-\mathrm{C}(30)-\mathrm{C}(31)$ & $113.4(4)$ \\
\hline $\mathrm{C}(29)-\mathrm{C}(30)-\mathrm{H}(30 \mathrm{D})$ & 108.9 \\
\hline $\mathrm{C}(31)-\mathrm{C}(30)-\mathrm{H}(30 \mathrm{D})$ & 108.9 \\
\hline $\mathrm{C}(29)-\mathrm{C}(30)-\mathrm{H}(30 \mathrm{E})$ & 108.9 \\
\hline $\mathrm{C}(31)-\mathrm{C}(30)-\mathrm{H}(30 \mathrm{E})$ & 108.9 \\
\hline $\mathrm{H}(30 \mathrm{D})-\mathrm{C}(30)-\mathrm{H}(30 \mathrm{E})$ & 107.7 \\
\hline $\mathrm{C}(30)-\mathrm{C}(29)-\mathrm{C}(28)$ & 114.1(4) \\
\hline $\mathrm{C}(30)-\mathrm{C}(29)-\mathrm{H}(29 \mathrm{D})$ & 108.7 \\
\hline $\mathrm{C}(28)-\mathrm{C}(29)-\mathrm{H}(29 \mathrm{D})$ & 108.7 \\
\hline $\mathrm{C}(30)-\mathrm{C}(29)-\mathrm{H}(29 \mathrm{E})$ & 108.7 \\
\hline $\mathrm{C}(28)-\mathrm{C}(29)-\mathrm{H}(29 \mathrm{E})$ & 108.7 \\
\hline $\mathrm{H}(29 \mathrm{D})-\mathrm{C}(29)-\mathrm{H}(29 \mathrm{E})$ & 107.6 \\
\hline $\mathrm{C}(29)-\mathrm{C}(28)-\mathrm{C}(27)$ & $113.9(4)$ \\
\hline $\mathrm{C}(29)-\mathrm{C}(28)-\mathrm{H}(28 \mathrm{D})$ & 108.8 \\
\hline $\mathrm{C}(27)-\mathrm{C}(28)-\mathrm{H}(28 \mathrm{D})$ & 108.8 \\
\hline $\mathrm{C}(29)-\mathrm{C}(28)-\mathrm{H}(28 \mathrm{E})$ & 108.8 \\
\hline $\mathrm{C}(27)-\mathrm{C}(28)-\mathrm{H}(28 \mathrm{E})$ & 108.8 \\
\hline $\mathrm{H}(28 \mathrm{D})-\mathrm{C}(28)-\mathrm{H}(28 \mathrm{E})$ & 107.7 \\
\hline $\mathrm{C}(32)-\mathrm{C}(31)-\mathrm{C}(30)$ & $113.7(6)$ \\
\hline $\mathrm{C}(32)-\mathrm{C}(31)-\mathrm{H}(31 \mathrm{D})$ & 108.8 \\
\hline $\mathrm{C}(30)-\mathrm{C}(31)-\mathrm{H}(31 \mathrm{D})$ & 108.8 \\
\hline $\mathrm{C}(32)-\mathrm{C}(31)-\mathrm{H}(31 \mathrm{E})$ & 108.8 \\
\hline $\mathrm{C}(30)-\mathrm{C}(31)-\mathrm{H}(31 \mathrm{E})$ & 108.8 \\
\hline $\mathrm{H}(31 \mathrm{D})-\mathrm{C}(31)-\mathrm{H}(31 \mathrm{E})$ & 107.7 \\
\hline $\mathrm{C}(31)-\mathrm{C}(32)-\mathrm{H}(32 \mathrm{D})$ & 109.5 \\
\hline $\mathrm{C}(31)-\mathrm{C}(32)-\mathrm{H}(32 \mathrm{E})$ & 109.5 \\
\hline $\mathrm{H}(32 \mathrm{D})-\mathrm{C}(32)-\mathrm{H}(32 \mathrm{E})$ & 109.5 \\
\hline $\mathrm{C}(31)-\mathrm{C}(32)-\mathrm{H}(32 \mathrm{~F})$ & 109.5 \\
\hline $\mathrm{H}(32 \mathrm{D})-\mathrm{C}(32)-\mathrm{H}(32 \mathrm{~F})$ & 109.5 \\
\hline $\mathrm{H}(32 \mathrm{E})-\mathrm{C}(32)-\mathrm{H}(32 \mathrm{~F})$ & 109.5 \\
\hline$C(26)-O(2)-C(27 X)$ & $106.3(4)$ \\
\hline $\mathrm{C}(26)-\mathrm{O}(2)-\mathrm{C}(27 \mathrm{Y})$ & $118.6(6)$ \\
\hline $\mathrm{C}(26)-\mathrm{O}(2)-\mathrm{C}(27)$ & $125.2(3)$ \\
\hline $\mathrm{C}(35)-\mathrm{O}(3)-\mathrm{C}(37)$ & $111.84(15)$ \\
\hline $\mathrm{C}(14)-\mathrm{C}(1)-\mathrm{C}(6)$ & $121.0(2)$ \\
\hline $\mathrm{C}(14)-\mathrm{C}(1)-\mathrm{C}(2)$ & $121.5(2)$ \\
\hline
\end{tabular}




\begin{tabular}{|c|c|}
\hline$C(6)-C(1)-C(2)$ & $117.5(2)$ \\
\hline $\mathrm{O}(1)-\mathrm{C}(2)-\mathrm{C}(3)$ & $112.2(4)$ \\
\hline $\mathrm{O}(1)-\mathrm{C}(2)-\mathrm{C}(3 \mathrm{~A})$ & $124.9(3)$ \\
\hline $\mathrm{O}(1)-\mathrm{C}(2)-\mathrm{C}(1)$ & $121.4(2)$ \\
\hline $\mathrm{C}(3)-\mathrm{C}(2)-\mathrm{C}(1)$ & $124.1(4)$ \\
\hline $\mathrm{C}(3 \mathrm{~A})-\mathrm{C}(2)-\mathrm{C}(1)$ & $112.7(4)$ \\
\hline $\mathrm{C}(4)-\mathrm{C}(3)-\mathrm{C}(2)$ & $112.6(5)$ \\
\hline $\mathrm{C}(4)-\mathrm{C}(3)-\mathrm{H}(3)$ & 123.7 \\
\hline $\mathrm{C}(2)-\mathrm{C}(3)-\mathrm{H}(3)$ & 123.7 \\
\hline $\mathrm{C}(3)-\mathrm{C}(4)-\mathrm{C}(5)$ & $124.0(6)$ \\
\hline $\mathrm{C}(3)-\mathrm{C}(4)-\mathrm{H}(4)$ & 118.0 \\
\hline $\mathrm{C}(5)-\mathrm{C}(4)-\mathrm{H}(4)$ & 118.0 \\
\hline $\mathrm{C}(24)-\mathrm{C}(5)-\mathrm{C}(4)$ & $120.1(4)$ \\
\hline$C(24)-C(5)-C(6)$ & $113.6(4)$ \\
\hline$C(4)-C(5)-C(6)$ & $126.0(5)$ \\
\hline$C(25)-C(24)-C(5)$ & $121.6(5)$ \\
\hline $\mathrm{C}(25)-\mathrm{C}(24)-\mathrm{H}(24)$ & 119.2 \\
\hline $\mathrm{C}(5)-\mathrm{C}(24)-\mathrm{H}(24)$ & 119.2 \\
\hline$C(24)-C(25)-C(26)$ & $124.2(6)$ \\
\hline $\mathrm{C}(24)-\mathrm{C}(25)-\mathrm{H}(25)$ & 117.9 \\
\hline $\mathrm{C}(26)-\mathrm{C}(25)-\mathrm{H}(25)$ & 117.9 \\
\hline$C(4 A)-C(3 A)-C(2)$ & $128.1(5)$ \\
\hline $\mathrm{C}(4 \mathrm{~A})-\mathrm{C}(3 \mathrm{~A})-\mathrm{H}(3 \mathrm{~A})$ & 116.0 \\
\hline $\mathrm{C}(2)-\mathrm{C}(3 \mathrm{~A})-\mathrm{H}(3 \mathrm{~A})$ & 116.0 \\
\hline$C(3 A)-C(4 A)-C(5 A)$ & $118.8(6)$ \\
\hline $\mathrm{C}(3 \mathrm{~A})-\mathrm{C}(4 \mathrm{~A})-\mathrm{H}(4 \mathrm{~A})$ & 120.6 \\
\hline $\mathrm{C}(5 \mathrm{~A})-\mathrm{C}(4 \mathrm{~A})-\mathrm{H}(4 \mathrm{~A})$ & 120.6 \\
\hline$C(4 A)-C(5 A)-C(6)$ & $118.5(5)$ \\
\hline$C(4 A)-C(5 A)-C(24 A)$ & $117.8(4)$ \\
\hline$C(6)-C(5 A)-C(24 A)$ & $123.6(4)$ \\
\hline$C(25 \mathrm{~A})-\mathrm{C}(24 \mathrm{~A})-\mathrm{C}(5 \mathrm{~A})$ & $120.9(5)$ \\
\hline $\mathrm{C}(25 \mathrm{~A})-\mathrm{C}(24 \mathrm{~A})-\mathrm{H}(24 \mathrm{~A})$ & 119.5 \\
\hline $\mathrm{C}(5 \mathrm{~A})-\mathrm{C}(24 \mathrm{~A})-\mathrm{H}(24 \mathrm{~A})$ & 119.5 \\
\hline$C(26)-C(25 A)-C(24 A)$ & $113.9(5)$ \\
\hline $\mathrm{C}(26)-\mathrm{C}(25 \mathrm{~A})-\mathrm{H}(25 \mathrm{~A})$ & 123.1 \\
\hline $\mathrm{C}(24 \mathrm{~A})-\mathrm{C}(25 \mathrm{~A})-\mathrm{H}(25 \mathrm{~A})$ & 123.1 \\
\hline
\end{tabular}




\begin{tabular}{|c|c|}
\hline$C(5)-C(6)-C(7)$ & $126.2(3)$ \\
\hline$C(5 A)-C(6)-C(7)$ & 116.1(3) \\
\hline$C(5)-C(6)-C(1)$ & $113.4(3)$ \\
\hline $\mathrm{C}(5 \mathrm{~A})-\mathrm{C}(6)-\mathrm{C}(1)$ & $123.4(3)$ \\
\hline$C(7)-C(6)-C(1)$ & $119.75(18)$ \\
\hline$C(26)-C(7)-C(6)$ & $116.4(2)$ \\
\hline$C(26)-C(7)-C(8)$ & $124.4(2)$ \\
\hline$C(6)-C(7)-C(8)$ & $119.2(2)$ \\
\hline $\mathrm{C}(9)-\mathrm{C}(8)-\mathrm{C}(13)$ & 117.92(19) \\
\hline $\mathrm{C}(9)-\mathrm{C}(8)-\mathrm{C}(7)$ & $123.6(2)$ \\
\hline $\mathrm{C}(13)-\mathrm{C}(8)-\mathrm{C}(7)$ & $118.4(2)$ \\
\hline $\mathrm{C}(10)-\mathrm{C}(9)-\mathrm{C}(8)$ & $120.1(2)$ \\
\hline $\mathrm{C}(10)-\mathrm{C}(9)-\mathrm{H}(9)$ & 120.0 \\
\hline $\mathrm{C}(8)-\mathrm{C}(9)-\mathrm{H}(9)$ & 120.0 \\
\hline $\mathrm{C}(9)-\mathrm{C}(10)-\mathrm{C}(11)$ & $121.5(2)$ \\
\hline $\mathrm{C}(9)-\mathrm{C}(10)-\mathrm{C}(33)$ & $128.9(2)$ \\
\hline$C(11)-C(10)-C(33)$ & $109.52(18)$ \\
\hline $\mathrm{C}(12)-\mathrm{C}(11)-\mathrm{C}(10)$ & $119.56(19)$ \\
\hline $\mathrm{C}(12)-\mathrm{C}(11)-\mathrm{C}(36) \# 1$ & $133.8(2)$ \\
\hline $\mathrm{C}(10)-\mathrm{C}(11)-\mathrm{C}(36) \# 1$ & $106.67(18)$ \\
\hline $\mathrm{C}(11)-\mathrm{C}(12)-\mathrm{C}(13)$ & $120.0(2)$ \\
\hline $\mathrm{C}(11)-\mathrm{C}(12)-\mathrm{H}(12)$ & 120.0 \\
\hline $\mathrm{C}(13)-\mathrm{C}(12)-\mathrm{H}(12)$ & 120.0 \\
\hline $\mathrm{C}(12)-\mathrm{C}(13)-\mathrm{C}(8)$ & $120.5(2)$ \\
\hline $\mathrm{C}(12)-\mathrm{C}(13)-\mathrm{C}(14)$ & $118.2(2)$ \\
\hline $\mathrm{C}(8)-\mathrm{C}(13)-\mathrm{C}(14)$ & $121.30(19)$ \\
\hline$C(1)-C(14)-C(13)$ & $119.8(2)$ \\
\hline $\mathrm{C}(1)-\mathrm{C}(14)-\mathrm{C}(15)$ & $122.2(2)$ \\
\hline $\mathrm{C}(13)-\mathrm{C}(14)-\mathrm{C}(15)$ & 117.91(19) \\
\hline$C(16)-C(15)-C(20)$ & $120.2(2)$ \\
\hline$C(16)-C(15)-C(14)$ & $118.0(2)$ \\
\hline$C(20)-C(15)-C(14)$ & 121.77(19) \\
\hline$C(17)-C(16)-C(15)$ & $118.7(2)$ \\
\hline$C(17)-C(16)-C(21)$ & $119.88(19)$ \\
\hline $\mathrm{C}(15)-\mathrm{C}(16)-\mathrm{C}(21)$ & $121.4(2)$ \\
\hline $\mathrm{C}(18)-\mathrm{C}(17)-\mathrm{C}(16)$ & $122.1(2)$ \\
\hline
\end{tabular}




\begin{tabular}{|c|c|}
\hline $\mathrm{C}(18)-\mathrm{C}(17)-\mathrm{H}(17)$ & 119.0 \\
\hline $\mathrm{C}(16)-\mathrm{C}(17)-\mathrm{H}(17)$ & 119.0 \\
\hline $\mathrm{C}(19)-\mathrm{C}(18)-\mathrm{C}(17)$ & $117.9(2)$ \\
\hline $\mathrm{C}(19)-\mathrm{C}(18)-\mathrm{C}(22)$ & $121.6(2)$ \\
\hline $\mathrm{C}(17)-\mathrm{C}(18)-\mathrm{C}(22)$ & $120.5(2)$ \\
\hline$C(18)-C(19)-C(20)$ & $121.8(2)$ \\
\hline $\mathrm{C}(18)-\mathrm{C}(19)-\mathrm{H}(19)$ & 119.1 \\
\hline $\mathrm{C}(20)-\mathrm{C}(19)-\mathrm{H}(19)$ & 119.1 \\
\hline $\mathrm{C}(19)-\mathrm{C}(20)-\mathrm{C}(15)$ & $119.1(2)$ \\
\hline $\mathrm{C}(19)-\mathrm{C}(20)-\mathrm{C}(23)$ & $120.6(2)$ \\
\hline$C(15)-C(20)-C(23)$ & $120.3(2)$ \\
\hline $\mathrm{C}(16)-\mathrm{C}(21)-\mathrm{H}(21 \mathrm{~A})$ & 109.5 \\
\hline $\mathrm{C}(16)-\mathrm{C}(21)-\mathrm{H}(21 \mathrm{~B})$ & 109.5 \\
\hline $\mathrm{H}(21 \mathrm{~A})-\mathrm{C}(21)-\mathrm{H}(21 \mathrm{~B})$ & 109.5 \\
\hline $\mathrm{C}(16)-\mathrm{C}(21)-\mathrm{H}(21 \mathrm{C})$ & 109.5 \\
\hline $\mathrm{H}(21 \mathrm{~A})-\mathrm{C}(21)-\mathrm{H}(21 \mathrm{C})$ & 109.5 \\
\hline $\mathrm{H}(21 \mathrm{~B})-\mathrm{C}(21)-\mathrm{H}(21 \mathrm{C})$ & 109.5 \\
\hline $\mathrm{C}(18)-\mathrm{C}(22)-\mathrm{H}(22 \mathrm{~A})$ & 109.5 \\
\hline $\mathrm{C}(18)-\mathrm{C}(22)-\mathrm{H}(22 \mathrm{~B})$ & 109.5 \\
\hline $\mathrm{H}(22 \mathrm{~A})-\mathrm{C}(22)-\mathrm{H}(22 \mathrm{~B})$ & 109.5 \\
\hline $\mathrm{C}(18)-\mathrm{C}(22)-\mathrm{H}(22 \mathrm{C})$ & 109.5 \\
\hline $\mathrm{H}(22 \mathrm{~A})-\mathrm{C}(22)-\mathrm{H}(22 \mathrm{C})$ & 109.5 \\
\hline $\mathrm{H}(22 \mathrm{~B})-\mathrm{C}(22)-\mathrm{H}(22 \mathrm{C})$ & 109.5 \\
\hline $\mathrm{C}(20)-\mathrm{C}(23)-\mathrm{H}(23 \mathrm{~A})$ & 109.5 \\
\hline $\mathrm{C}(20)-\mathrm{C}(23)-\mathrm{H}(23 \mathrm{~B})$ & 109.5 \\
\hline $\mathrm{H}(23 \mathrm{~A})-\mathrm{C}(23)-\mathrm{H}(23 \mathrm{~B})$ & 109.5 \\
\hline $\mathrm{C}(20)-\mathrm{C}(23)-\mathrm{H}(23 \mathrm{C})$ & 109.5 \\
\hline $\mathrm{H}(23 \mathrm{~A})-\mathrm{C}(23)-\mathrm{H}(23 \mathrm{C})$ & 109.5 \\
\hline $\mathrm{H}(23 \mathrm{~B})-\mathrm{C}(23)-\mathrm{H}(23 \mathrm{C})$ & 109.5 \\
\hline $\mathrm{O}(2)-\mathrm{C}(26)-\mathrm{C}(25 \mathrm{~A})$ & $115.6(4)$ \\
\hline $\mathrm{O}(2)-\mathrm{C}(26)-\mathrm{C}(25)$ & $126.8(4)$ \\
\hline $\mathrm{O}(2)-\mathrm{C}(26)-\mathrm{C}(7)$ & $116.99(17)$ \\
\hline$C(25 A)-C(26)-C(7)$ & $127.4(5)$ \\
\hline $\mathrm{C}(25)-\mathrm{C}(26)-\mathrm{C}(7)$ & $116.2(4)$ \\
\hline $\mathrm{C}(34)-\mathrm{C}(33)-\mathrm{C}(10)$ & $107.16(19)$ \\
\hline $\mathrm{C}(34)-\mathrm{C}(33)-\mathrm{C}(43)$ & $130.00(19$ \\
\hline
\end{tabular}




\begin{tabular}{|c|c|}
\hline $\mathrm{C}(10)-\mathrm{C}(33)-\mathrm{C}(43)$ & $122.84(18)$ \\
\hline $\mathrm{C}(33)-\mathrm{C}(34)-\mathrm{C}(35)$ & $131.6(2)$ \\
\hline $\mathrm{C}(33)-\mathrm{C}(34)-\mathrm{C}(36) \# 1$ & $109.75(18)$ \\
\hline $\mathrm{C}(35)-\mathrm{C}(34)-\mathrm{C}(36) \# 1$ & $118.62(18)$ \\
\hline $\mathrm{C}(36)-\mathrm{C}(35)-\mathrm{O}(3)$ & $120.77(18)$ \\
\hline$C(36)-C(35)-C(34)$ & $119.45(19)$ \\
\hline $\mathrm{O}(3)-\mathrm{C}(35)-\mathrm{C}(34)$ & $119.77(18)$ \\
\hline $\mathrm{C}(35)-\mathrm{C}(36)-\mathrm{C}(11) \# 1$ & $131.2(2)$ \\
\hline$C(35)-C(36)-C(34) \# 1$ & 121.91(19) \\
\hline $\mathrm{C}(11) \# 1-\mathrm{C}(36)-\mathrm{C}(34) \# 1$ & $106.81(18)$ \\
\hline $\mathrm{O}(3)-\mathrm{C}(37)-\mathrm{C}(38)$ & 108.93(16) \\
\hline $\mathrm{O}(3)-\mathrm{C}(37)-\mathrm{H}(37 \mathrm{~A})$ & 109.9 \\
\hline $\mathrm{C}(38)-\mathrm{C}(37)-\mathrm{H}(37 \mathrm{~A})$ & 109.9 \\
\hline $\mathrm{O}(3)-\mathrm{C}(37)-\mathrm{H}(37 \mathrm{~B})$ & 109.9 \\
\hline $\mathrm{C}(38)-\mathrm{C}(37)-\mathrm{H}(37 \mathrm{~B})$ & 109.9 \\
\hline $\mathrm{H}(37 \mathrm{~A})-\mathrm{C}(37)-\mathrm{H}(37 \mathrm{~B})$ & 108.3 \\
\hline C(37)-C(38)-C(39) & $110.78(18)$ \\
\hline $\mathrm{C}(37)-\mathrm{C}(38)-\mathrm{H}(38 \mathrm{~A})$ & 109.5 \\
\hline $\mathrm{C}(39)-\mathrm{C}(38)-\mathrm{H}(38 \mathrm{~A})$ & 109.5 \\
\hline $\mathrm{C}(37)-\mathrm{C}(38)-\mathrm{H}(38 \mathrm{~B})$ & 109.5 \\
\hline $\mathrm{C}(39)-\mathrm{C}(38)-\mathrm{H}(38 \mathrm{~B})$ & 109.5 \\
\hline $\mathrm{H}(38 \mathrm{~A})-\mathrm{C}(38)-\mathrm{H}(38 \mathrm{~B})$ & 108.1 \\
\hline $\mathrm{C}(40)-\mathrm{C}(39)-\mathrm{C}(38)$ & $113.78(19)$ \\
\hline $\mathrm{C}(40)-\mathrm{C}(39)-\mathrm{H}(39 \mathrm{~A})$ & 108.8 \\
\hline $\mathrm{C}(38)-\mathrm{C}(39)-\mathrm{H}(39 \mathrm{~A})$ & 108.8 \\
\hline $\mathrm{C}(40)-\mathrm{C}(39)-\mathrm{H}(39 \mathrm{~B})$ & 108.8 \\
\hline $\mathrm{C}(38)-\mathrm{C}(39)-\mathrm{H}(39 \mathrm{~B})$ & 108.8 \\
\hline $\mathrm{H}(39 \mathrm{~A})-\mathrm{C}(39)-\mathrm{H}(39 \mathrm{~B})$ & 107.7 \\
\hline$C(39)-C(40)-C(41)$ & $113.5(2)$ \\
\hline $\mathrm{C}(39)-\mathrm{C}(40)-\mathrm{H}(40 \mathrm{~A})$ & 108.9 \\
\hline $\mathrm{C}(41)-\mathrm{C}(40)-\mathrm{H}(40 \mathrm{~A})$ & 108.9 \\
\hline $\mathrm{C}(39)-\mathrm{C}(40)-\mathrm{H}(40 \mathrm{~B})$ & 108.9 \\
\hline $\mathrm{C}(41)-\mathrm{C}(40)-\mathrm{H}(40 \mathrm{~B})$ & 108.9 \\
\hline $\mathrm{H}(40 \mathrm{~A})-\mathrm{C}(40)-\mathrm{H}(40 \mathrm{~B})$ & 107.7 \\
\hline $\mathrm{C}(42)-\mathrm{C}(41)-\mathrm{C}(40)$ & $112.0(2)$ \\
\hline $\mathrm{C}(42)-\mathrm{C}(41)-\mathrm{H}(41 \mathrm{~A})$ & 109.2 \\
\hline
\end{tabular}




\begin{tabular}{|c|c|}
\hline $\mathrm{C}(40)-\mathrm{C}(41)-\mathrm{H}(41 \mathrm{~A})$ & 109.2 \\
\hline $\mathrm{C}(42)-\mathrm{C}(41)-\mathrm{H}(41 \mathrm{~B})$ & 109.2 \\
\hline $\mathrm{C}(40)-\mathrm{C}(41)-\mathrm{H}(41 \mathrm{~B})$ & 109.2 \\
\hline $\mathrm{H}(41 \mathrm{~A})-\mathrm{C}(41)-\mathrm{H}(41 \mathrm{~B})$ & 107.9 \\
\hline $\mathrm{C}(41)-\mathrm{C}(42)-\mathrm{H}(42 \mathrm{~A})$ & 109.5 \\
\hline $\mathrm{C}(41)-\mathrm{C}(42)-\mathrm{H}(42 \mathrm{~B})$ & 109.5 \\
\hline $\mathrm{H}(42 \mathrm{~A})-\mathrm{C}(42)-\mathrm{H}(42 \mathrm{~B})$ & 109.5 \\
\hline $\mathrm{C}(41)-\mathrm{C}(42)-\mathrm{H}(42 \mathrm{C})$ & 109.5 \\
\hline $\mathrm{H}(42 \mathrm{~A})-\mathrm{C}(42)-\mathrm{H}(42 \mathrm{C})$ & 109.5 \\
\hline $\mathrm{H}(42 \mathrm{~B})-\mathrm{C}(42)-\mathrm{H}(42 \mathrm{C})$ & 109.5 \\
\hline $\mathrm{C}(48)-\mathrm{C}(43)-\mathrm{C}(44)$ & $119.9(2)$ \\
\hline$C(48)-C(43)-C(33)$ & 121.03(19) \\
\hline$C(44)-C(43)-C(33)$ & $119.05(18)$ \\
\hline$C(45)-C(44)-C(43)$ & $119.15(19)$ \\
\hline$C(45)-C(44)-C(49)$ & $119.2(2)$ \\
\hline $\mathrm{C}(43)-\mathrm{C}(44)-\mathrm{C}(49)$ & $121.6(2)$ \\
\hline$C(46)-C(45)-C(44)$ & $121.9(2)$ \\
\hline $\mathrm{C}(46)-\mathrm{C}(45)-\mathrm{H}(45)$ & 119.0 \\
\hline $\mathrm{C}(44)-\mathrm{C}(45)-\mathrm{H}(45)$ & 119.0 \\
\hline$C(45)-C(46)-C(47)$ & $118.0(2)$ \\
\hline$C(45)-C(46)-C(51)$ & $119.9(2)$ \\
\hline $\mathrm{C}(47)-\mathrm{C}(46)-\mathrm{C}(51)$ & $122.1(2)$ \\
\hline $\mathrm{C}(46)-\mathrm{C}(47)-\mathrm{C}(48)$ & $121.9(2)$ \\
\hline $\mathrm{C}(46)-\mathrm{C}(47)-\mathrm{H}(47)$ & 119.1 \\
\hline $\mathrm{C}(48)-\mathrm{C}(47)-\mathrm{H}(47)$ & 119.1 \\
\hline$C(47)-C(48)-C(43)$ & $119.1(2)$ \\
\hline $\mathrm{C}(47)-\mathrm{C}(48)-\mathrm{C}(50)$ & 120.68(19) \\
\hline $\mathrm{C}(43)-\mathrm{C}(48)-\mathrm{C}(50)$ & $120.2(2)$ \\
\hline $\mathrm{C}(44)-\mathrm{C}(49)-\mathrm{H}(49 \mathrm{~A})$ & 109.5 \\
\hline $\mathrm{C}(44)-\mathrm{C}(49)-\mathrm{H}(49 \mathrm{~B})$ & 109.5 \\
\hline $\mathrm{H}(49 \mathrm{~A})-\mathrm{C}(49)-\mathrm{H}(49 \mathrm{~B})$ & 109.5 \\
\hline $\mathrm{C}(44)-\mathrm{C}(49)-\mathrm{H}(49 \mathrm{C})$ & 109.5 \\
\hline $\mathrm{H}(49 \mathrm{~A})-\mathrm{C}(49)-\mathrm{H}(49 \mathrm{C})$ & 109.5 \\
\hline H(49B)-C(49)-H(49C) & 109.5 \\
\hline $\mathrm{C}(48)-\mathrm{C}(50)-\mathrm{H}(50 \mathrm{~A})$ & 109.5 \\
\hline $\mathrm{C}(48)-\mathrm{C}(50)-\mathrm{H}(50 \mathrm{~B})$ & 109.5 \\
\hline
\end{tabular}




$\begin{array}{ll}\text { H(50A)-C(50)-H(50B) } & 109.5 \\ \text { C(48)-C(50)-H(50C) } & 109.5 \\ \text { H(50A)-C(50)-H(50C) } & 109.5 \\ \text { H(50B)-C(50)-H(50C) } & 109.5 \\ \text { C(46)-C(51)-H(51A) } & 109.5 \\ \text { C(46)-C(51)-H(51B) } & 109.5 \\ \text { H(51A)-C(51)-H(51B) } & 109.5 \\ \text { C(46)-C(51)-H(51C) } & 109.5 \\ \text { H(51A)-C(51)-H(51C) } & 109.5 \\ \text { H(51B)-C(51)-H(51C) } & 109.5\end{array}$

Symmetry transformations used to generate equivalent atoms:

$$
\# 1-\mathrm{x}+1,-\mathrm{y}+1,-\mathrm{z}
$$

Table S16. $\left(\AA^{2} \times 10^{3}\right)$ for PIF-M-ONE. The anisotropic displacement factor exponent takes the form: $-2 p^{2}\left[h^{2} a^{* 2} U^{11}+\ldots+2 h k a^{*} b^{*} U^{12}\right]$

\begin{tabular}{|c|c|c|c|c|c|c|}
\hline & J11 & $\mathrm{U}^{22}$ & $\mathrm{U}^{33}$ & $\mathrm{U}^{23}$ & $\mathrm{U}^{13}$ & $\mathrm{U}^{12}$ \\
\hline $\mathrm{C}(27)$ & $40(3)$ & $43(3)$ & $15(2)$ & $-3(2)$ & $7(2)$ & $-12(3)$ \\
\hline$C(30)$ & $39(3)$ & $25(3)$ & $47(3)$ & $1(2)$ & $-12(3)$ & $-4(3)$ \\
\hline $\mathrm{C}(29)$ & $46(3)$ & $43(3)$ & $26(2)$ & $-9(2)$ & $-5(2)$ & $-10(3)$ \\
\hline $\mathrm{C}(28)$ & $48(3)$ & $44(3)$ & $24(2)$ & $-5(2)$ & $-3(2)$ & $-16(3)$ \\
\hline $\mathrm{C}(31)$ & $32(3)$ & $35(3)$ & $33(4)$ & $-2(3)$ & $-14(3)$ & $-10(3)$ \\
\hline $\mathrm{C}(32)$ & $60(5)$ & $38(4)$ & $29(7)$ & $17(4)$ & $-6(5)$ & $-3(4)$ \\
\hline $\mathrm{O}(1)$ & $68(1)$ & $42(1)$ & $58(1)$ & $-25(1)$ & $-33(1)$ & $-1(1)$ \\
\hline $\mathrm{O}(2)$ & $49(1)$ & $98(2)$ & $21(1)$ & $-11(1)$ & $4(1)$ & $-34(1)$ \\
\hline $\mathrm{O}(3)$ & $19(1)$ & $17(1)$ & $26(1)$ & $-4(1)$ & $-3(1)$ & $-3(1)$ \\
\hline $\mathrm{C}(1)$ & $56(2)$ & $20(1)$ & $36(1)$ & $-11(1)$ & $-26(1)$ & $9(1)$ \\
\hline $\mathrm{C}(2)$ & $89(2)$ & $25(1)$ & $46(2)$ & $-12(1)$ & $-38(2)$ & $4(2)$ \\
\hline $\mathrm{C}(3)$ & $63(5)$ & $65(5)$ & $61(5)$ & $-28(4)$ & $-45(4)$ & $-5(4)$ \\
\hline $\mathrm{C}(4)$ & $64(4)$ & $49(4)$ & $35(3)$ & $-21(3)$ & $-25(3)$ & $5(4)$ \\
\hline$C(5)$ & $51(2)$ & $39(1)$ & $26(1)$ & $-12(1)$ & $-11(1)$ & $10(2)$ \\
\hline$C(24)$ & $51(2)$ & $39(1)$ & $26(1)$ & $-12(1)$ & $-11(1)$ & $10(2)$ \\
\hline $\mathrm{C}(25)$ & $51(2)$ & $39(1)$ & $26(1)$ & $-12(1)$ & $-11(1)$ & $10(2)$ \\
\hline $\mathrm{C}(3 \mathrm{~A})$ & $94(7)$ & $38(4)$ & $50(4)$ & $-7(3)$ & $-40(4)$ & $-27(4)$ \\
\hline
\end{tabular}




\begin{tabular}{|c|c|c|c|c|c|}
\hline $\mathrm{C}(4 \mathrm{~A})$ & $87(6)$ & $49(4)$ & $37(3)$ & $-27(3)$ & $-25(4)$ \\
\hline $\mathrm{C}(5 \mathrm{~A})$ & $51(2)$ & $39(1)$ & $26(1)$ & $-12(1)$ & $-11(1)$ \\
\hline $\mathrm{C}(24 \mathrm{~A})$ & $51(2)$ & $39(1)$ & $26(1)$ & $-12(1)$ & $-11(1)$ \\
\hline$C(25 \mathrm{~A})$ & $51(2)$ & $39(1)$ & $26(1)$ & $-12(1)$ & $-11(1)$ \\
\hline$C(6)$ & $65(2)$ & $25(1)$ & $32(1)$ & $-11(1)$ & $-24(1)$ \\
\hline$C(7)$ & $34(1)$ & $28(1)$ & $26(1)$ & $-9(1)$ & $-11(1)$ \\
\hline $\mathrm{C}(8)$ & $25(1)$ & $23(1)$ & $24(1)$ & $-7(1)$ & $-8(1)$ \\
\hline C(9) & $24(1)$ & $23(1)$ & $23(1)$ & $-5(1)$ & $-4(1)$ \\
\hline$C(10)$ & $20(1)$ & $18(1)$ & $22(1)$ & $-3(1)$ & $-5(1)$ \\
\hline $\mathrm{C}(11)$ & $18(1)$ & $17(1)$ & $22(1)$ & $-3(1)$ & $-6(1)$ \\
\hline$C(12)$ & $20(1)$ & $18(1)$ & $26(1)$ & $-5(1)$ & $-6(1)$ \\
\hline$C(13)$ & $24(1)$ & $22(1)$ & $26(1)$ & $-10(1)$ & $-10(1)$ \\
\hline$C(14)$ & $30(1)$ & $21(1)$ & $35(1)$ & $-12(1)$ & $-17(1)$ \\
\hline$C(15)$ & $30(1)$ & $19(1)$ & $35(1)$ & $-13(1)$ & $-15(1)$ \\
\hline$C(16)$ & $27(1)$ & $19(1)$ & $32(1)$ & $-9(1)$ & $-13(1)$ \\
\hline$C(17)$ & $31(1)$ & $18(1)$ & $38(1)$ & $-6(1)$ & $-14(1)$ \\
\hline$C(18)$ & $30(1)$ & $28(1)$ & $44(1)$ & $-14(1)$ & $-11(1)$ \\
\hline $\mathrm{C}(19)$ & $23(1)$ & $27(1)$ & $51(2)$ & $-17(1)$ & $-11(1)$ \\
\hline$C(20)$ & $28(1)$ & $22(1)$ & $50(1)$ & $-16(1)$ & $-18(1)$ \\
\hline$C(21)$ & $30(1)$ & $22(1)$ & $38(1)$ & $-6(1)$ & $-11(1)$ \\
\hline$C(22)$ & $34(1)$ & $34(1)$ & $50(2)$ & $-11(1)$ & $-7(1)$ \\
\hline $\mathrm{C}(23)$ & $32(1)$ & $30(1)$ & $70(2)$ & $-17(1)$ & $-22(1)$ \\
\hline$C(26)$ & $32(1)$ & $49(2)$ & $25(1)$ & $-11(1)$ & $-9(1)$ \\
\hline $\mathrm{C}(33)$ & $20(1)$ & $17(1)$ & $22(1)$ & $-1(1)$ & $-4(1)$ \\
\hline$C(34)$ & $18(1)$ & $17(1)$ & $22(1)$ & $-3(1)$ & $-5(1)$ \\
\hline$C(35)$ & $17(1)$ & $13(1)$ & $25(1)$ & $-2(1)$ & $-5(1)$ \\
\hline$C(36)$ & $18(1)$ & $16(1)$ & $22(1)$ & $-3(1)$ & $-5(1)$ \\
\hline $\mathrm{C}(37)$ & $21(1)$ & $21(1)$ & $29(1)$ & $-4(1)$ & $-7(1)$ \\
\hline$C(38)$ & $22(1)$ & $20(1)$ & $28(1)$ & $-5(1)$ & $-4(1)$ \\
\hline C(39) & $23(1)$ & $27(1)$ & $34(1)$ & $-5(1)$ & $-7(1)$ \\
\hline $\mathrm{C}(40)$ & $26(1)$ & $29(1)$ & $48(2)$ & $-7(1)$ & $-7(1)$ \\
\hline $\mathrm{C}(41)$ & $24(1)$ & $37(1)$ & $50(2)$ & $-6(1)$ & $-9(1)$ \\
\hline$C(42)$ & $28(1)$ & $55(2)$ & $38(1)$ & $-3(1)$ & $-5(1)$ \\
\hline$C(43)$ & $24(1)$ & 21(1) & $18(1)$ & $-3(1)$ & $-2(1)$ \\
\hline$C(44)$ & $24(1)$ & $22(1)$ & $23(1)$ & $-3(1)$ & $-3(1)$ \\
\hline$C(45)$ & $22(1)$ & $28(1)$ & $28(1)$ & $-7(1)$ & 1(1) \\
\hline
\end{tabular}




$\begin{array}{lllllll}\mathrm{C}(46) & 33(1) & 29(1) & 24(1) & -4(1) & -1(1) & -10(1) \\ \mathrm{C}(47) & 36(1) & 18(1) & 24(1) & 1(1) & -5(1) & -3(1) \\ \mathrm{C}(48) & 30(1) & 22(1) & 22(1) & -6(1) & -6(1) & -1(1) \\ \mathrm{C}(49) & 26(1) & 24(1) & 40(1) & -5(1) & -3(1) & 2(1) \\ \mathrm{C}(50) & 30(1) & 24(1) & 33(1) & -4(1) & -11(1) & 3(1) \\ \mathrm{C}(51) & 43(2) & 37(1) & 40(2) & -1(1) & 2(1) & -14(1)\end{array}$

Table S17. Displacement parameters $\left(\AA^{2} \mathrm{x} 10^{3}\right)$ for PIF-M-ONE.

\begin{tabular}{|c|c|c|c|c|}
\hline $\mathrm{x}$ & & & $\mathrm{z}$ & $\mathrm{U}(\mathrm{eq})$ \\
\hline $\mathrm{H}(27 \mathrm{X})$ & 1373 & 5110 & 5658 & 42 \\
\hline $\mathrm{H}(27 \mathrm{Y})$ & 2376 & 3952 & 5938 & 42 \\
\hline $\mathrm{H}(28 \mathrm{X})$ & 1735 & 3229 & 4883 & 42 \\
\hline $\mathrm{H}(28 \mathrm{Y})$ & 624 & 4302 & 4765 & 42 \\
\hline $\mathrm{H}(29 \mathrm{X})$ & 853 & 2498 & 6451 & 42 \\
\hline $\mathrm{H}(29 \mathrm{Y})$ & -274 & 3547 & 6312 & 42 \\
\hline $\mathrm{H}(30 \mathrm{X})$ & -953 & 2743 & 5365 & 42 \\
\hline $\mathrm{H}(30 \mathrm{Y})$ & 145 & 1676 & 5546 & 42 \\
\hline $\mathrm{H}(31 \mathrm{X})$ & -1990 & 2037 & 6875 & 42 \\
\hline $\mathrm{H}(31 \mathrm{Y})$ & -1794 & 1021 & 6418 & 42 \\
\hline $\mathrm{H}(32 \mathrm{X})$ & 104 & 236 & 7051 & 52 \\
\hline $\mathrm{H}(32 \mathrm{Y})$ & -1173 & 118 & 7788 & 52 \\
\hline $\mathrm{H}(32 \mathrm{Z})$ & -310 & 1149 & 7607 & 52 \\
\hline $\mathrm{H}(32 \mathrm{~A})$ & -144 & -137 & 7388 & 52 \\
\hline $\mathrm{H}(32 \mathrm{~B})$ & -1340 & 143 & 8100 & 52 \\
\hline $\mathrm{H}(32 \mathrm{C})$ & -9 & 590 & 8013 & 52 \\
\hline $\mathrm{H}(31 \mathrm{~A})$ & -1375 & 2187 & 7285 & 42 \\
\hline $\mathrm{H}(31 \mathrm{~B})$ & -1537 & 1458 & 6670 & 42 \\
\hline $\mathrm{H}(30 \mathrm{~A})$ & 600 & 1593 & 5948 & 42 \\
\hline $\mathrm{H}(30 \mathrm{~B})$ & 811 & 2265 & 6588 & 42 \\
\hline $\mathrm{H}(29 \mathrm{~A})$ & -815 & 3220 & 5297 & 42 \\
\hline $\mathrm{H}(29 \mathrm{~B})$ & -658 & 3888 & 5955 & 42 \\
\hline $\mathrm{H}(28 \mathrm{~A})$ & 574 & 4593 & 4448 & 42 \\
\hline
\end{tabular}




\begin{tabular}{|c|c|c|c|}
\hline $\mathrm{H}(28 \mathrm{~B})$ & 1429 & 3343 & 4664 \\
\hline $\mathrm{H}(27 \mathrm{~A})$ & 1198 & 5014 & 5613 \\
\hline $\mathrm{H}(27 \mathrm{~B})$ & 2038 & 3748 & 5850 \\
\hline $\mathrm{H}(27 \mathrm{D})$ & 2688 & 3402 & 5784 \\
\hline $\mathrm{H}(27 \mathrm{E})$ & 1370 & 4230 & 5785 \\
\hline $\mathrm{H}(30 \mathrm{D})$ & -865 & 3121 & 5805 \\
\hline $\mathrm{H}(30 \mathrm{E})$ & -190 & 2938 & 6622 \\
\hline $\mathrm{H}(29 \mathrm{D})$ & 689 & 1589 & 5425 \\
\hline $\mathrm{H}(29 \mathrm{E})$ & 1416 & 1645 & 6153 \\
\hline $\mathrm{H}(28 \mathrm{D})$ & 993 & 3633 & 4615 \\
\hline $\mathrm{H}(28 \mathrm{E})$ & 2286 & 2777 & 4651 \\
\hline $\mathrm{H}(31 \mathrm{D})$ & -1973 & 1868 & 7120 \\
\hline $\mathrm{H}(31 \mathrm{E})$ & -1400 & 1153 & 6427 \\
\hline $\mathrm{H}(32 \mathrm{D})$ & 233 & 202 & 7227 \\
\hline $\mathrm{H}(32 \mathrm{E})$ & -1107 & -20 & 7886 \\
\hline $\mathrm{H}(32 \mathrm{~F})$ & -293 & 944 & 7907 \\
\hline $\mathrm{H}(3)$ & 6903 & 9341 & 4366 \\
\hline $\mathrm{H}(4)$ & 5680 & 8011 & 5592 \\
\hline $\mathrm{H}(24)$ & 4314 & 6479 & 6221 \\
\hline $\mathrm{H}(25)$ & 2846 & 5441 & 6075 \\
\hline $\mathrm{H}(3 \mathrm{~A})$ & 5956 & 9679 & 4437 \\
\hline $\mathrm{H}(4 \mathrm{~A})$ & 4754 & 8406 & 5626 \\
\hline $\mathrm{H}(24 \mathrm{~A})$ & 3668 & 6665 & 6271 \\
\hline $\mathrm{H}(25 \mathrm{~A})$ & 2930 & 5011 & 6134 \\
\hline $\mathrm{H}(9)$ & 3124 & 4970 & 3350 \\
\hline $\mathrm{H}(12)$ & 5905 & 7416 & 1007 \\
\hline $\mathrm{H}(17)$ & 5411 & 11482 & 216 \\
\hline $\mathrm{H}(19)$ & 8846 & 9628 & 274 \\
\hline $\mathrm{H}(21 \mathrm{~A})$ & 3612 & 10806 & 1247 \\
\hline $\mathrm{H}(21 \mathrm{~B})$ & 3811 & 9895 & 2206 \\
\hline $\mathrm{H}(21 \mathrm{C})$ & 3606 & 9411 & 1453 \\
\hline $\mathrm{H}(22 \mathrm{~A})$ & 8710 & 11596 & -729 \\
\hline $\mathrm{H}(22 \mathrm{~B})$ & 7512 & 12483 & -496 \\
\hline $\mathrm{H}(22 \mathrm{C})$ & 7500 & 11793 & -1175 \\
\hline $\mathrm{H}(23 \mathrm{~A})$ & 9138 & 7720 & 1297 \\
\hline $\mathrm{H}(23 \mathrm{~B})$ & 7924 & 7005 & 1777 \\
\hline
\end{tabular}




\begin{tabular}{|c|c|c|c|c|}
\hline $\mathrm{H}(23 \mathrm{C})$ & 8402 & 7741 & 2270 & 64 \\
\hline $\mathrm{H}(37 \mathrm{~A})$ & 2167 & 3963 & -354 & 29 \\
\hline $\mathrm{H}(37 \mathrm{~B})$ & 1843 & 4341 & 535 & 29 \\
\hline $\mathrm{H}(38 \mathrm{~A})$ & 1359 & 2335 & 1387 & 29 \\
\hline $\mathrm{H}(38 \mathrm{~B})$ & 1531 & 2078 & 460 & 29 \\
\hline $\mathrm{H}(39 \mathrm{~A})$ & -312 & 3854 & 955 & 35 \\
\hline $\mathrm{H}(39 \mathrm{~B})$ & -160 & 3543 & 53 & 35 \\
\hline $\mathrm{H}(40 \mathrm{~A})$ & -846 & 1834 & 1773 & 42 \\
\hline $\mathrm{H}(40 \mathrm{~B})$ & -911 & 1718 & 833 & 42 \\
\hline $\mathrm{H}(41 \mathrm{~A})$ & -2553 & 3392 & 675 & 47 \\
\hline $\mathrm{H}(41 \mathrm{~B})$ & -2965 & 2190 & 1440 & 47 \\
\hline $\mathrm{H}(42 \mathrm{~A})$ & -2654 & 2902 & 2541 & 68 \\
\hline $\mathrm{H}(42 \mathrm{~B})$ & -3618 & 3836 & 1969 & 68 \\
\hline $\mathrm{H}(42 \mathrm{C})$ & -2174 & 4087 & 1786 & 68 \\
\hline $\mathrm{H}(45)$ & -479 & 3747 & 3035 & 33 \\
\hline $\mathrm{H}(47)$ & 2192 & 969 & 3528 & 34 \\
\hline $\mathrm{H}(49 \mathrm{~A})$ & -27 & 5624 & 2122 & 49 \\
\hline $\mathrm{H}(49 \mathrm{~B})$ & 1209 & 5716 & 1351 & 49 \\
\hline $\mathrm{H}(49 \mathrm{C})$ & 1251 & 5956 & 2253 & 49 \\
\hline $\mathrm{H}(50 \mathrm{~A})$ & 4584 & 2023 & 1972 & 45 \\
\hline $\mathrm{H}(50 \mathrm{~B})$ & 4356 & 1192 & 2996 & 45 \\
\hline $\mathrm{H}(50 \mathrm{C})$ & 4777 & 2493 & 2735 & 45 \\
\hline $\mathrm{H}(51 \mathrm{~A})$ & -803 & 1748 & 4409 & 65 \\
\hline $\mathrm{H}(51 \mathrm{~B})$ & 47 & 632 & 4180 & 65 \\
\hline $\mathrm{H}(51 \mathrm{C})$ & -912 & 1442 & 3547 & 65 \\
\hline
\end{tabular}

\section{Data for FP-M}

Table S18. Crystal data and structure refinement for FP-M

Empirical formula

Formula weight

Temperature

Wavelength

Crystal system

Space group
$\mathrm{C}_{50} \mathrm{H}_{50} \mathrm{O}$

666.90

100(2) K

$1.54178 \AA$

Monoclinic

$\mathrm{P} 2(1) / \mathrm{c}$ 
Unit cell dimensions

$\mathrm{b}=27.6365(10) \AA$

$\mathrm{c}=14.8907(5) \AA$

Volume

Z

Density (calculated)

Absorption coefficient

$\mathrm{F}(000)$

Crystal size

Theta range for data collection

Index ranges

Reflections collected

Independent reflections

Completeness to theta $=67.679^{\circ}$

Refinement method

Data / restraints / parameters

Goodness-of-fit on $\mathrm{F}^{2}$

Final $\mathrm{R}$ indices [I>2sigma(I)]

$\mathrm{R}$ indices (all data)

Extinction coefficient

Largest diff. peak and hole $\mathrm{a}=9.2447(4) \AA \quad \alpha=90^{\circ}$

$\beta=98.658(3)^{\circ}$

$\gamma=90^{\circ}$

3761.1(2) $\AA^{3}$

4

$1.178 \mathrm{Mg} / \mathrm{m}^{3}$

$0.514 \mathrm{~mm}^{-1}$

1432

$0.311 \times 0.101 \times 0.055 \mathrm{~mm}^{3}$

4.839 to $74.566^{\circ}$

$-11<=\mathrm{h}<=9,-34<=\mathrm{k}<=30,-16<=1<=17$

19304

$7238[\mathrm{R}($ int $)=0.0800]$

$97.1 \%$

Full-matrix least-squares on $\mathrm{F}^{2}$

7238 / 0 / 468

1.015

$\mathrm{R} 1=0.0530, \mathrm{wR} 2=0.1185$

$\mathrm{R} 1=0.0962, \mathrm{wR} 2=0.1368$

$\mathrm{n} / \mathrm{a}$

0.262 and -0.254 e. $\AA^{-3}$

Table S19. Atomic coordinates ( x 10 $)$ and equivalent isotropic displacement parameters $\left(\AA^{2} \times 10^{3}\right.$ ) for FP-M. $\mathrm{U}(\mathrm{eq})$ is defined as one third of the trace of the orthogonalized $\mathrm{Uij}^{\mathrm{ij}}$ tensor.

\begin{tabular}{lrrrr}
\hline \multicolumn{2}{c}{$\mathrm{x}$} & \multicolumn{2}{c}{$\mathrm{z}(\mathrm{eq})$} \\
\hline & & & & \\
$\mathrm{O}(1)$ & $2676(2)$ & $5605(1)$ & $1650(1)$ & $22(1)$ \\
$\mathrm{C}(1)$ & $3171(2)$ & $4831(1)$ & $1051(1)$ & $21(1)$ \\
$\mathrm{C}(2)$ & $3346(2)$ & $5324(1)$ & $1079(1)$ & $20(1)$ \\
$\mathrm{C}(3)$ & $4241(2)$ & $5564(1)$ & $513(1)$ & $18(1)$ \\
$\mathrm{C}(4)$ & $4369(2)$ & $6086(1)$ & $441(1)$ & $18(1)$ \\
$\mathrm{C}(5)$ & $3444(2)$ & $6415(1)$ & $785(1)$ & $19(1)$ \\
$\mathrm{C}(6)$ & $3583(2)$ & $6916(1)$ & $665(1)$ & $19(1)$ \\
$\mathrm{C}(7)$ & $2715(2)$ & $7301(1)$ & $886(1)$ & $19(1)$
\end{tabular}




$\begin{array}{lrrrr}\mathrm{C}(8) & 1380(2) & 7254(1) & 1320(1) & 20(1) \\ \mathrm{C}(9) & 1485(2) & 7089(1) & 2224(1) & 22(1) \\ \mathrm{C}(10) & 212(3) & 7032(1) & 2605(1) & 25(1) \\ \mathrm{C}(11) & -1157(3) & 7144(1) & 2131(1) & 25(1) \\ \mathrm{C}(12) & -1229(2) & 7318(1) & 1251(1) & 25(1) \\ \mathrm{C}(13) & 7(2) & 7371(1) & 833(1) & 21(1) \\ \mathrm{C}(14) & 3345(2) & 7746(1) & 595(1) & 20(1) \\ \mathrm{C}(15) & 2907(2) & 8227(1) & 640(1) & 23(1) \\ \mathrm{C}(16) & 3723(2) & 8584(1) & 295(1) & 25(1) \\ \mathrm{C}(17) & 4971(2) & 8471(1) & -78(1) & 25(1) \\ \mathrm{C}(18) & 5423(2) & 7991(1) & -128(1) & 23(1) \\ \mathrm{C}(19) & 4611(2) & 7631(1) & 201(1) & 20(1) \\ \mathrm{C}(20) & 4754(2) & 7104(1) & 224(1) & 19(1) \\ \mathrm{C}(21) & 5676(2) & 6797(1) & -114(1) & 20(1) \\ \mathrm{C}(22) & 5485(2) & 6282(1) & -51(1) & 19(1) \\ \mathrm{C}(23) & 6338(2) & 5977(1) & -495(1) & 20(1) \\ \mathrm{C}(24) & 6078(2) & 5470(1) & -517(1) & 20(1) \\ \mathrm{C}(25) & 5067(2) & 5258(1) & 2(1) & 19(1) \\ \mathrm{C}(26) & 1914(2) & 5365(1) & 2296(1) & 24(1) \\ \mathrm{C}(27) & 1395(3) & 5748(1) & 2898(1) & 26(1) \\ \mathrm{C}(28) & 714(3) & 5520(1) & 3667(1) & 27(1) \\ \mathrm{C}(29) & 30(3) & 5888(1) & 4238(1) & 27(1) \\ & & & & \end{array}$

\begin{tabular}{lllll}
\hline $\mathrm{x}$ & $\mathrm{y}$ & $\mathrm{z}$ & $\mathrm{U}(\mathrm{eq})$ \\
\hline
\end{tabular}

C(30)

$\mathrm{C}(31)$

$\mathrm{C}(32)$

$\mathrm{C}(33)$

C(34)

$\mathrm{C}(35)$

$\mathrm{C}(36)$

$\mathrm{C}(37)$

$\mathrm{C}(39)$

$\mathrm{C}(40)$

$\begin{array}{rrrr}-543(3) & 5664(1) & 5043(2) & 36(1) \\ -1129(3) & 6027(1) & 5660(2) & 45(1) \\ 7574(2) & 6174(1) & -933(1) & 19(1) \\ 7346(2) & 6380(1) & -1801(1) & 24(1) \\ 8548(3) & 6550(1) & -2173(1) & 26(1) \\ 9958(3) & 6512(1) & -1714(1) & 26(1) \\ 10160(2) & 6307(1) & -854(1) & 25(1) \\ 8993(2) & 6140(1) & -450(1) & 23(1) \\ 2952(2) & 6999(1) & 2794(1) & 27(1) \\ -2536(3) & 7084(1) & 2558(2) & 36(1)\end{array}$




\begin{tabular}{lrrrr}
$\mathrm{C}(41)$ & $-175(3)$ & $7546(1)$ & $-138(1)$ & $26(1)$ \\
$\mathrm{C}(42)$ & $5826(3)$ & $6427(1)$ & $-2332(2)$ & $36(1)$ \\
$\mathrm{C}(43)$ & $11261(3)$ & $6686(1)$ & $-2124(2)$ & $37(1)$ \\
$\mathrm{C}(44)$ & $9269(3)$ & $5935(1)$ & $496(2)$ & $34(1)$ \\
& & & & \\
\hline & $\mathrm{x}$ & $\mathrm{y}$ & $\mathrm{z}$ & $\mathrm{U}(\mathrm{eq})$ \\
& & & & \\
\hline $\mathrm{C}(1 \mathrm{~T})$ & & & & \\
$\mathrm{C}(2 \mathrm{~T})$ & $5372(3)$ & $5364(1)$ & $3662(2)$ & $44(1)$ \\
$\mathrm{C}(3 \mathrm{~T})$ & $5900(3)$ & $5636(1)$ & $3005(2)$ & $44(1)$ \\
$\mathrm{C}(4 \mathrm{~T})$ & $6746(3)$ & $5423(1)$ & $2424(2)$ & $40(1)$ \\
$\mathrm{C}(5 \mathrm{~T})$ & $7071(3)$ & $4932(1)$ & $2486(2)$ & $36(1)$ \\
$\mathrm{C}(6 \mathrm{~T})$ & $6548(3)$ & $4668(1)$ & $3153(2)$ & $41(1)$ \\
$\mathrm{C}(7 \mathrm{~T})$ & $5695(3)$ & $4879(1)$ & $3734(2)$ & $47(1)$ \\
& $7966(3)$ & $4691(1)$ & $1846(2)$ & $45(1)$
\end{tabular}

Table S20. Bond lengths $[\AA]$ and angles $\left[{ }^{\circ}\right]$ for FP-M.

\begin{tabular}{lclc}
\hline & & & \\
$\mathrm{O}(1)-\mathrm{C}(2)$ & $1.367(2)$ & $\mathrm{O}(1)-\mathrm{C}(26)$ & $1.437(2)$ \\
$\mathrm{C}(1)-\mathrm{C}(2)$ & $1.372(3)$ & $\mathrm{C}(1)-\mathrm{C}(24) \# 1$ & $1.405(3)$ \\
$\mathrm{C}(1)-\mathrm{H}(1)$ & 0.9500 & $\mathrm{C}(2)-\mathrm{C}(3)$ & $1.429(3)$ \\
$\mathrm{C}(3)-\mathrm{C}(25)$ & $1.433(3)$ & $\mathrm{C}(3)-\mathrm{C}(4)$ & $1.452(3)$ \\
$\mathrm{C}(4)-\mathrm{C}(5)$ & $1.398(3)$ & $\mathrm{C}(4)-\mathrm{C}(22)$ & $1.456(3)$ \\
$\mathrm{C}(5)-\mathrm{C}(6)$ & $1.405(3)$ & $\mathrm{C}(5)-\mathrm{H}(5)$ & 0.9500 \\
$\mathrm{C}(6)-\mathrm{C}(7)$ & $1.400(3)$ & $\mathrm{C}(6)-\mathrm{C}(20)$ & $1.444(3)$ \\
$\mathrm{C}(7)-\mathrm{C}(14)$ & $1.455(3)$ & $\mathrm{C}(7)-\mathrm{C}(8)$ & $1.482(3)$ \\
$\mathrm{C}(8)-\mathrm{C}(13)$ & $1.403(3)$ & $\mathrm{C}(8)-\mathrm{C}(9)$ & $1.410(3)$ \\
$\mathrm{C}(9)-\mathrm{C}(10)$ & $1.390(3)$ & $\mathrm{C}(9)-\mathrm{C}(39)$ & $1.509(3)$ \\
$\mathrm{C}(10)-\mathrm{C}(11)$ & $1.387(3)$ & $\mathrm{C}(10)-\mathrm{H}(10)$ & 0.9500 \\
$\mathrm{C}(11)-\mathrm{C}(12)$ & $1.389(3)$ & $\mathrm{C}(11)-\mathrm{C}(40)$ & $1.517(3)$ \\
$\mathrm{C}(12)-\mathrm{C}(13)$ & $1.388(3)$ & $\mathrm{C}(12)-\mathrm{H}(12)$ & 0.9500 \\
$\mathrm{C}(13)-\mathrm{C}(41)$ & $1.509(3)$ & $\mathrm{C}(14)-\mathrm{C}(15)$ & $1.394(3)$ \\
$\mathrm{C}(14)-\mathrm{C}(19)$ & $1.421(3)$ & $\mathrm{C}(15)-\mathrm{C}(16)$ & $1.388(3)$ \\
$\mathrm{C}(15)-\mathrm{H}(15)$ & 0.9500 & $\mathrm{C}(16)-\mathrm{C}(17)$ & $1.389(3)$ \\
$\mathrm{C}(16)-\mathrm{H}(16)$ & 0.9500 & $\mathrm{C}(17)-\mathrm{C}(18)$ & $1.394(3)$
\end{tabular}




\begin{tabular}{|c|c|c|c|}
\hline $\mathrm{C}(17)-\mathrm{H}(17)$ & 0.9500 & C(18)-C(19) & $1.381(3)$ \\
\hline $\mathrm{C}(18)-\mathrm{H}(18)$ & 0.9500 & $\mathrm{C}(19)-\mathrm{C}(20)$ & $1.461(3)$ \\
\hline $\mathrm{C}(20)-\mathrm{C}(21)$ & $1.353(3)$ & $\mathrm{C}(21)-\mathrm{C}(22)$ & $1.440(3)$ \\
\hline $\mathrm{C}(21)-\mathrm{H}(21)$ & 0.9500 & $\mathrm{C}(22)-\mathrm{C}(23)$ & $1.387(3)$ \\
\hline $\mathrm{C}(23)-\mathrm{C}(24)$ & $1.422(3)$ & $\mathrm{C}(23)-\mathrm{C}(32)$ & $1.500(3)$ \\
\hline $\mathrm{C}(24)-\mathrm{C}(1) \# 1$ & $1.405(3)$ & $\mathrm{C}(24)-\mathrm{C}(25)$ & $1.425(3)$ \\
\hline $\mathrm{C}(25)-\mathrm{C}(25) \# 1$ & $1.432(4)$ & $C(26)-C(27)$ & $1.511(3)$ \\
\hline $\mathrm{C}(26)-\mathrm{H}(26 \mathrm{~A})$ & 0.9900 & $\mathrm{C}(26)-\mathrm{H}(26 \mathrm{~B})$ & 0.9900 \\
\hline $\mathrm{C}(27)-\mathrm{C}(28)$ & $1.525(3)$ & $\mathrm{C}(27)-\mathrm{H}(27 \mathrm{~A})$ & 0.9900 \\
\hline $\mathrm{C}(27)-\mathrm{H}(27 \mathrm{~B})$ & 0.9900 & $\mathrm{C}(28)-\mathrm{C}(29)$ & $1.523(3)$ \\
\hline $\mathrm{C}(28)-\mathrm{H}(28 \mathrm{~A})$ & 0.9900 & $\mathrm{C}(28)-\mathrm{H}(28 \mathrm{~B})$ & 0.9900 \\
\hline C(29)-C(30) & $1.515(3)$ & $\mathrm{C}(29)-\mathrm{H}(29 \mathrm{~A})$ & 0.9900 \\
\hline $\mathrm{C}(29)-\mathrm{H}(29 \mathrm{~B})$ & 0.9900 & $\mathrm{C}(30)-\mathrm{C}(31)$ & $1.515(3)$ \\
\hline $\mathrm{C}(30)-\mathrm{H}(30 \mathrm{~A})$ & 0.9900 & $\mathrm{C}(30)-\mathrm{H}(30 \mathrm{~B})$ & 0.9900 \\
\hline $\mathrm{C}(31)-\mathrm{H}(31 \mathrm{~A})$ & 0.9800 & $\mathrm{C}(31)-\mathrm{H}(31 \mathrm{~B})$ & 0.9800 \\
\hline $\mathrm{C}(31)-\mathrm{H}(31 \mathrm{C})$ & 0.9800 & $\mathrm{C}(32)-\mathrm{C}(33)$ & $1.400(3)$ \\
\hline C(32)-C(37) & $1.400(3)$ & $\mathrm{C}(33)-\mathrm{C}(34)$ & $1.396(3)$ \\
\hline $\mathrm{C}(33)-\mathrm{C}(42)$ & $1.511(3)$ & $\mathrm{C}(34)-\mathrm{C}(35)$ & $1.382(3)$ \\
\hline $\mathrm{C}(34)-\mathrm{H}(34)$ & 0.9500 & $\mathrm{C}(35)-\mathrm{C}(36)$ & $1.388(3)$ \\
\hline$C(35)-C(43)$ & $1.508(3)$ & $\mathrm{C}(36)-\mathrm{C}(37)$ & $1.390(3)$ \\
\hline $\mathrm{C}(36)-\mathrm{H}(36)$ & 0.9500 & $\mathrm{C}(37)-\mathrm{C}(44)$ & $1.504(3)$ \\
\hline $\mathrm{C}(39)-\mathrm{H}(39 \mathrm{~A})$ & 0.9800 & $\mathrm{C}(39)-\mathrm{H}(39 \mathrm{~B})$ & 0.9800 \\
\hline $\mathrm{C}(39)-\mathrm{H}(39 \mathrm{C})$ & 0.9800 & $\mathrm{C}(40)-\mathrm{H}(40 \mathrm{~A})$ & 0.9800 \\
\hline $\mathrm{C}(40)-\mathrm{H}(40 \mathrm{~B})$ & 0.9800 & $\mathrm{C}(40)-\mathrm{H}(40 \mathrm{C})$ & 0.9800 \\
\hline $\mathrm{C}(41)-\mathrm{H}(41 \mathrm{~A})$ & 0.9800 & $\mathrm{C}(41)-\mathrm{H}(41 \mathrm{~B})$ & 0.9800 \\
\hline $\mathrm{C}(41)-\mathrm{H}(41 \mathrm{C})$ & 0.9800 & $\mathrm{C}(42)-\mathrm{H}(42 \mathrm{~A})$ & 0.9800 \\
\hline $\mathrm{C}(42)-\mathrm{H}(42 \mathrm{~B})$ & 0.9800 & $\mathrm{C}(42)-\mathrm{H}(42 \mathrm{C})$ & 0.9800 \\
\hline $\mathrm{C}(43)-\mathrm{H}(43 \mathrm{~A})$ & 0.9800 & $\mathrm{C}(43)-\mathrm{H}(43 \mathrm{~B})$ & 0.9800 \\
\hline $\mathrm{C}(43)-\mathrm{H}(43 \mathrm{C})$ & 0.9800 & $\mathrm{C}(44)-\mathrm{H}(44 \mathrm{~A})$ & 0.9800 \\
\hline $\mathrm{C}(44)-\mathrm{H}(44 \mathrm{~B})$ & 0.9800 & $\mathrm{C}(44)-\mathrm{H}(44 \mathrm{C})$ & 0.9800 \\
\hline $\mathrm{C}(1 \mathrm{~T})-\mathrm{C}(6 \mathrm{~T})$ & $1.374(4)$ & $\mathrm{C}(1 \mathrm{~T})-\mathrm{C}(2 \mathrm{~T})$ & $1.380(4)$ \\
\hline $\mathrm{C}(1 \mathrm{~T})-\mathrm{H}(1 \mathrm{~T})$ & 0.9500 & $\mathrm{C}(2 \mathrm{~T})-\mathrm{C}(3 \mathrm{~T})$ & $1.383(4)$ \\
\hline $\mathrm{C}(2 \mathrm{~T})-\mathrm{H}(2 \mathrm{~T})$ & 0.9500 & $\mathrm{C}(3 \mathrm{~T})-\mathrm{C}(4 \mathrm{~T})$ & $1.388(3)$ \\
\hline $\mathrm{C}(3 \mathrm{~T})-\mathrm{H}(3 \mathrm{~T})$ & 0.9500 & $\mathrm{C}(4 \mathrm{~T})-\mathrm{C}(5 \mathrm{~T})$ & $1.377(3)$ \\
\hline $\mathrm{C}(4 \mathrm{~T})-\mathrm{C}(7 \mathrm{~T})$ & $1.509(3)$ & $\mathrm{C}(5 \mathrm{~T})-\mathrm{C}(6 \mathrm{~T})$ & $1.384(4)$ \\
\hline
\end{tabular}




\begin{tabular}{|c|c|c|c|}
\hline $\mathrm{C}(5 \mathrm{~T})-\mathrm{H}(5 \mathrm{~T})$ & 0.9500 & $\mathrm{C}(6 \mathrm{~T})-\mathrm{H}(6 \mathrm{~T})$ & 0.9500 \\
\hline $\mathrm{C}(7 \mathrm{~T})-\mathrm{H}(7 \mathrm{~T} 1)$ & 0.9800 & $\mathrm{C}(7 \mathrm{~T})-\mathrm{H}(7 \mathrm{~T} 2)$ & 0.9800 \\
\hline $\mathrm{C}(7 \mathrm{~T})-\mathrm{H}(7 \mathrm{~T} 3)$ & 0.9800 & & \\
\hline $\mathrm{C}(2)-\mathrm{O}(1)-\mathrm{C}(26)$ & $117.96(15)$ & $\mathrm{C}(2)-\mathrm{C}(1)-\mathrm{C}(24) \# 1$ & $122.58(19)$ \\
\hline $\mathrm{C}(2)-\mathrm{C}(1)-\mathrm{H}(1)$ & 118.7 & $\mathrm{C}(24) \# 1-\mathrm{C}(1)-\mathrm{H}(1)$ & 118.7 \\
\hline $\mathrm{O}(1)-\mathrm{C}(2)-\mathrm{C}(1)$ & $121.26(18)$ & $\mathrm{O}(1)-\mathrm{C}(2)-\mathrm{C}(3)$ & $117.40(17)$ \\
\hline $\mathrm{C}(1)-\mathrm{C}(2)-\mathrm{C}(3)$ & $121.34(18)$ & $C(2)-C(3)-C(25)$ & $116.12(18)$ \\
\hline $\mathrm{C}(2)-\mathrm{C}(3)-\mathrm{C}(4)$ & $124.48(18)$ & $C(25)-C(3)-C(4)$ & $119.40(17)$ \\
\hline $\mathrm{C}(5)-\mathrm{C}(4)-\mathrm{C}(3)$ & $123.85(18)$ & $C(5)-C(4)-C(22)$ & $117.54(18)$ \\
\hline$C(3)-C(4)-C(22)$ & $118.56(17)$ & $C(4)-C(5)-C(6)$ & $121.43(18)$ \\
\hline $\mathrm{C}(4)-\mathrm{C}(5)-\mathrm{H}(5)$ & 119.3 & $\mathrm{C}(6)-\mathrm{C}(5)-\mathrm{H}(5)$ & 119.3 \\
\hline$C(7)-C(6)-C(5)$ & $130.60(19)$ & $C(7)-C(6)-C(20)$ & $109.25(18)$ \\
\hline$C(5)-C(6)-C(20)$ & $120.12(19)$ & $C(6)-C(7)-C(14)$ & $107.54(18)$ \\
\hline$C(6)-C(7)-C(8)$ & $125.48(18)$ & $\mathrm{C}(14)-\mathrm{C}(7)-\mathrm{C}(8)$ & $126.96(18)$ \\
\hline $\mathrm{C}(13)-\mathrm{C}(8)-\mathrm{C}(9)$ & $119.65(19)$ & $\mathrm{C}(13)-\mathrm{C}(8)-\mathrm{C}(7)$ & $120.16(17)$ \\
\hline $\mathrm{C}(9)-\mathrm{C}(8)-\mathrm{C}(7)$ & $120.18(19)$ & $\mathrm{C}(10)-\mathrm{C}(9)-\mathrm{C}(8)$ & 119.1(2) \\
\hline $\mathrm{C}(10)-\mathrm{C}(9)-\mathrm{C}(39)$ & $119.77(19)$ & $\mathrm{C}(8)-\mathrm{C}(9)-\mathrm{C}(39)$ & 121.11(19) \\
\hline $\mathrm{C}(11)-\mathrm{C}(10)-\mathrm{C}(9)$ & $122.1(2)$ & $\mathrm{C}(11)-\mathrm{C}(10)-\mathrm{H}(10)$ & 119.0 \\
\hline $\mathrm{C}(9)-\mathrm{C}(10)-\mathrm{H}(10)$ & 119.0 & $\mathrm{C}(10)-\mathrm{C}(11)-\mathrm{C}(12)$ & $117.9(2)$ \\
\hline$C(10)-C(11)-C(40)$ & $121.5(2)$ & $\mathrm{C}(12)-\mathrm{C}(11)-\mathrm{C}(40)$ & $120.6(2)$ \\
\hline $\mathrm{C}(13)-\mathrm{C}(12)-\mathrm{C}(11)$ & $122.3(2)$ & $\mathrm{C}(13)-\mathrm{C}(12)-\mathrm{H}(12)$ & 118.9 \\
\hline $\mathrm{C}(11)-\mathrm{C}(12)-\mathrm{H}(12)$ & 118.9 & $\mathrm{C}(12)-\mathrm{C}(13)-\mathrm{C}(8)$ & 119.07(19) \\
\hline $\mathrm{C}(12)-\mathrm{C}(13)-\mathrm{C}(41)$ & $118.8(2)$ & $\mathrm{C}(8)-\mathrm{C}(13)-\mathrm{C}(41)$ & $122.09(18)$ \\
\hline $\mathrm{C}(15)-\mathrm{C}(14)-\mathrm{C}(19)$ & 119.73(19) & $\mathrm{C}(15)-\mathrm{C}(14)-\mathrm{C}(7)$ & $131.24(19)$ \\
\hline $\mathrm{C}(19)-\mathrm{C}(14)-\mathrm{C}(7)$ & 109.03(17) & $\mathrm{C}(16)-\mathrm{C}(15)-\mathrm{C}(14)$ & $118.9(2)$ \\
\hline $\mathrm{C}(16)-\mathrm{C}(15)-\mathrm{H}(15)$ & 120.6 & $\mathrm{C}(14)-\mathrm{C}(15)-\mathrm{H}(15)$ & 120.6 \\
\hline $\mathrm{C}(15)-\mathrm{C}(16)-\mathrm{C}(17)$ & $121.2(2)$ & $\mathrm{C}(15)-\mathrm{C}(16)-\mathrm{H}(16)$ & 119.4 \\
\hline $\mathrm{C}(17)-\mathrm{C}(16)-\mathrm{H}(16)$ & 119.4 & $\mathrm{C}(16)-\mathrm{C}(17)-\mathrm{C}(18)$ & $120.6(2)$ \\
\hline $\mathrm{C}(16)-\mathrm{C}(17)-\mathrm{H}(17)$ & 119.7 & $\mathrm{C}(18)-\mathrm{C}(17)-\mathrm{H}(17)$ & 119.7 \\
\hline $\mathrm{C}(19)-\mathrm{C}(18)-\mathrm{C}(17)$ & $118.9(2)$ & $\mathrm{C}(19)-\mathrm{C}(18)-\mathrm{H}(18)$ & 120.6 \\
\hline $\mathrm{C}(17)-\mathrm{C}(18)-\mathrm{H}(18)$ & 120.6 & $C(18)-C(19)-C(14)$ & $120.73(19)$ \\
\hline $\mathrm{C}(18)-\mathrm{C}(19)-\mathrm{C}(20)$ & $132.42(19)$ & $C(14)-C(19)-C(20)$ & $106.84(17)$ \\
\hline$C(21)-C(20)-C(6)$ & $120.05(18)$ & $C(21)-C(20)-C(19)$ & $132.55(19)$ \\
\hline$C(6)-C(20)-C(19)$ & $107.30(17)$ & $C(20)-C(21)-C(22)$ & $120.44(18)$ \\
\hline $\mathrm{C}(20)-\mathrm{C}(21)-\mathrm{H}(21)$ & 119.8 & $\mathrm{C}(22)-\mathrm{C}(21)-\mathrm{H}(21)$ & 119.8 \\
\hline
\end{tabular}




\begin{tabular}{|c|c|c|c|}
\hline $\mathrm{C}(23)-\mathrm{C}(22)-\mathrm{C}(21)$ & $119.03(18)$ & $\mathrm{C}(23)-\mathrm{C}(22)-\mathrm{C}(4)$ & $120.76(18)$ \\
\hline $\mathrm{C}(21)-\mathrm{C}(22)-\mathrm{C}(4)$ & $120.16(18)$ & $\mathrm{C}(22)-\mathrm{C}(23)-\mathrm{C}(24)$ & $120.14(18)$ \\
\hline $\mathrm{C}(22)-\mathrm{C}(23)-\mathrm{C}(32)$ & $120.84(18)$ & $\mathrm{C}(24)-\mathrm{C}(23)-\mathrm{C}(32)$ & $118.99(17)$ \\
\hline $\mathrm{C}(1) \# 1-\mathrm{C}(24)-\mathrm{C}(23)$ & $120.00(18)$ & $\mathrm{C}(1) \# 1-\mathrm{C}(24)-\mathrm{C}(25)$ & $118.98(18)$ \\
\hline $\mathrm{C}(23)-\mathrm{C}(24)-\mathrm{C}(25)$ & $121.02(18)$ & $C(24)-C(25)-C(25) \# 1$ & $118.0(2)$ \\
\hline $\mathrm{C}(24)-\mathrm{C}(25)-\mathrm{C}(3)$ & $119.40(18)$ & $\mathrm{C}(25) \# 1-\mathrm{C}(25)-\mathrm{C}(3)$ & $122.6(2)$ \\
\hline $\mathrm{O}(1)-\mathrm{C}(26)-\mathrm{C}(27)$ & $107.72(16)$ & $\mathrm{O}(1)-\mathrm{C}(26)-\mathrm{H}(26 \mathrm{~A})$ & 110.2 \\
\hline $\mathrm{C}(27)-\mathrm{C}(26)-\mathrm{H}(26 \mathrm{~A})$ & 110.2 & $\mathrm{O}(1)-\mathrm{C}(26)-\mathrm{H}(26 \mathrm{~B})$ & 110.2 \\
\hline $\mathrm{C}(27)-\mathrm{C}(26)-\mathrm{H}(26 \mathrm{~B})$ & 110.2 & $\mathrm{H}(26 \mathrm{~A})-\mathrm{C}(26)-\mathrm{H}(26 \mathrm{~B})$ & 108.5 \\
\hline $\mathrm{C}(26)-\mathrm{C}(27)-\mathrm{C}(28)$ & $111.02(17)$ & $\mathrm{C}(26)-\mathrm{C}(27)-\mathrm{H}(27 \mathrm{~A})$ & 109.4 \\
\hline $\mathrm{C}(28)-\mathrm{C}(27)-\mathrm{H}(27 \mathrm{~A})$ & 109.4 & $\mathrm{C}(26)-\mathrm{C}(27)-\mathrm{H}(27 \mathrm{~B})$ & 109.4 \\
\hline $\mathrm{C}(28)-\mathrm{C}(27)-\mathrm{H}(27 \mathrm{~B})$ & 109.4 & $\mathrm{H}(27 \mathrm{~A})-\mathrm{C}(27)-\mathrm{H}(27 \mathrm{~B})$ & 108.0 \\
\hline$C(29)-C(28)-C(27)$ & $113.27(18)$ & $\mathrm{C}(29)-\mathrm{C}(28)-\mathrm{H}(28 \mathrm{~A})$ & 108.9 \\
\hline $\mathrm{C}(27)-\mathrm{C}(28)-\mathrm{H}(28 \mathrm{~A})$ & 108.9 & $\mathrm{C}(29)-\mathrm{C}(28)-\mathrm{H}(28 \mathrm{~B})$ & 108.9 \\
\hline $\mathrm{C}(27)-\mathrm{C}(28)-\mathrm{H}(28 \mathrm{~B})$ & 108.9 & $\mathrm{H}(28 \mathrm{~A})-\mathrm{C}(28)-\mathrm{H}(28 \mathrm{~B})$ & 107.7 \\
\hline $\mathrm{C}(30)-\mathrm{C}(29)-\mathrm{C}(28)$ & 113.01(19) & $\mathrm{C}(30)-\mathrm{C}(29)-\mathrm{H}(29 \mathrm{~A})$ & 109.0 \\
\hline $\mathrm{C}(28)-\mathrm{C}(29)-\mathrm{H}(29 \mathrm{~A})$ & 109.0 & $\mathrm{C}(30)-\mathrm{C}(29)-\mathrm{H}(29 \mathrm{~B})$ & 109.0 \\
\hline $\mathrm{C}(28)-\mathrm{C}(29)-\mathrm{H}(29 \mathrm{~B})$ & 109.0 & $\mathrm{H}(29 \mathrm{~A})-\mathrm{C}(29)-\mathrm{H}(29 \mathrm{~B})$ & 107.8 \\
\hline $\mathrm{C}(29)-\mathrm{C}(30)-\mathrm{C}(31)$ & $114.1(2)$ & $\mathrm{C}(29)-\mathrm{C}(30)-\mathrm{H}(30 \mathrm{~A})$ & 108.7 \\
\hline $\mathrm{C}(31)-\mathrm{C}(30)-\mathrm{H}(30 \mathrm{~A})$ & 108.7 & $\mathrm{C}(29)-\mathrm{C}(30)-\mathrm{H}(30 \mathrm{~B})$ & 108.7 \\
\hline $\mathrm{C}(31)-\mathrm{C}(30)-\mathrm{H}(30 \mathrm{~B})$ & 108.7 & $\mathrm{H}(30 \mathrm{~A})-\mathrm{C}(30)-\mathrm{H}(30 \mathrm{~B})$ & 107.6 \\
\hline $\mathrm{C}(30)-\mathrm{C}(31)-\mathrm{H}(31 \mathrm{~A})$ & 109.5 & $\mathrm{C}(30)-\mathrm{C}(31)-\mathrm{H}(31 \mathrm{~B})$ & 109.5 \\
\hline $\mathrm{H}(31 \mathrm{~A})-\mathrm{C}(31)-\mathrm{H}(31 \mathrm{~B})$ & 109.5 & $\mathrm{C}(30)-\mathrm{C}(31)-\mathrm{H}(31 \mathrm{C})$ & 109.5 \\
\hline $\mathrm{H}(31 \mathrm{~A})-\mathrm{C}(31)-\mathrm{H}(31 \mathrm{C})$ & 109.5 & $\mathrm{H}(31 \mathrm{~B})-\mathrm{C}(31)-\mathrm{H}(31 \mathrm{C})$ & 109.5 \\
\hline C(33)-C(32)-C(37) & $120.02(19)$ & $\mathrm{C}(33)-\mathrm{C}(32)-\mathrm{C}(23)$ & $121.98(19)$ \\
\hline $\mathrm{C}(37)-\mathrm{C}(32)-\mathrm{C}(23)$ & $118.00(18)$ & $\mathrm{C}(34)-\mathrm{C}(33)-\mathrm{C}(32)$ & $119.0(2)$ \\
\hline $\mathrm{C}(34)-\mathrm{C}(33)-\mathrm{C}(42)$ & $119.80(19)$ & $\mathrm{C}(32)-\mathrm{C}(33)-\mathrm{C}(42)$ & $121.19(19)$ \\
\hline $\mathrm{C}(35)-\mathrm{C}(34)-\mathrm{C}(33)$ & $121.8(2)$ & $\mathrm{C}(35)-\mathrm{C}(34)-\mathrm{H}(34)$ & 119.1 \\
\hline $\mathrm{C}(33)-\mathrm{C}(34)-\mathrm{H}(34)$ & 119.1 & $\mathrm{C}(34)-\mathrm{C}(35)-\mathrm{C}(36)$ & $118.24(19)$ \\
\hline $\mathrm{C}(34)-\mathrm{C}(35)-\mathrm{C}(43)$ & $121.8(2)$ & $\mathrm{C}(36)-\mathrm{C}(35)-\mathrm{C}(43)$ & $119.9(2)$ \\
\hline $\mathrm{C}(35)-\mathrm{C}(36)-\mathrm{C}(37)$ & $121.9(2)$ & $\mathrm{C}(35)-\mathrm{C}(36)-\mathrm{H}(36)$ & 119.0 \\
\hline $\mathrm{C}(37)-\mathrm{C}(36)-\mathrm{H}(36)$ & 119.0 & $\mathrm{C}(36)-\mathrm{C}(37)-\mathrm{C}(32)$ & $118.98(19)$ \\
\hline $\mathrm{C}(36)-\mathrm{C}(37)-\mathrm{C}(44)$ & $119.8(2)$ & $\mathrm{C}(32)-\mathrm{C}(37)-\mathrm{C}(44)$ & $121.26(19)$ \\
\hline $\mathrm{C}(9)-\mathrm{C}(39)-\mathrm{H}(39 \mathrm{~A})$ & 109.5 & $\mathrm{C}(9)-\mathrm{C}(39)-\mathrm{H}(39 \mathrm{~B})$ & 109.5 \\
\hline $\mathrm{H}(39 \mathrm{~A})-\mathrm{C}(39)-\mathrm{H}(39 \mathrm{~B})$ & 109.5 & C(9)-C(39)-H(39C) & 109.5 \\
\hline $\mathrm{H}(39 \mathrm{~A})-\mathrm{C}(39)-\mathrm{H}(39 \mathrm{C})$ & 109.5 & $\mathrm{H}(39 \mathrm{~B})-\mathrm{C}(39)-\mathrm{H}(39 \mathrm{C})$ & 109.5 \\
\hline
\end{tabular}




\begin{tabular}{|c|c|c|c|}
\hline $\mathrm{C}(11)-\mathrm{C}(40)-\mathrm{H}(40 \mathrm{~A})$ & 109.5 & $\mathrm{C}(11)-\mathrm{C}(40)-\mathrm{H}(40 \mathrm{~B})$ & 109.5 \\
\hline $\mathrm{H}(40 \mathrm{~A})-\mathrm{C}(40)-\mathrm{H}(40 \mathrm{~B})$ & 109.5 & $\mathrm{C}(11)-\mathrm{C}(40)-\mathrm{H}(40 \mathrm{C})$ & 109.5 \\
\hline $\mathrm{H}(40 \mathrm{~A})-\mathrm{C}(40)-\mathrm{H}(40 \mathrm{C})$ & 109.5 & $\mathrm{H}(40 \mathrm{~B})-\mathrm{C}(40)-\mathrm{H}(40 \mathrm{C})$ & 109.5 \\
\hline $\mathrm{C}(13)-\mathrm{C}(41)-\mathrm{H}(41 \mathrm{~A})$ & 109.5 & $\mathrm{C}(13)-\mathrm{C}(41)-\mathrm{H}(41 \mathrm{~B})$ & 109.5 \\
\hline $\mathrm{H}(41 \mathrm{~A})-\mathrm{C}(41)-\mathrm{H}(41 \mathrm{~B})$ & 109.5 & $\mathrm{C}(13)-\mathrm{C}(41)-\mathrm{H}(41 \mathrm{C})$ & 109.5 \\
\hline $\mathrm{H}(41 \mathrm{~A})-\mathrm{C}(41)-\mathrm{H}(41 \mathrm{C})$ & 109.5 & $\mathrm{H}(41 \mathrm{~B})-\mathrm{C}(41)-\mathrm{H}(41 \mathrm{C})$ & 109.5 \\
\hline $\mathrm{C}(33)-\mathrm{C}(42)-\mathrm{H}(42 \mathrm{~A})$ & 109.5 & $\mathrm{C}(33)-\mathrm{C}(42)-\mathrm{H}(42 \mathrm{~B})$ & 109.5 \\
\hline $\mathrm{H}(42 \mathrm{~A})-\mathrm{C}(42)-\mathrm{H}(42 \mathrm{~B})$ & 109.5 & $\mathrm{C}(33)-\mathrm{C}(42)-\mathrm{H}(42 \mathrm{C})$ & 109.5 \\
\hline $\mathrm{H}(42 \mathrm{~A})-\mathrm{C}(42)-\mathrm{H}(42 \mathrm{C})$ & 109.5 & $\mathrm{H}(42 \mathrm{~B})-\mathrm{C}(42)-\mathrm{H}(42 \mathrm{C})$ & 109.5 \\
\hline $\mathrm{C}(35)-\mathrm{C}(43)-\mathrm{H}(43 \mathrm{~A})$ & 109.5 & $\mathrm{C}(35)-\mathrm{C}(43)-\mathrm{H}(43 \mathrm{~B})$ & 109.5 \\
\hline $\mathrm{H}(43 \mathrm{~A})-\mathrm{C}(43)-\mathrm{H}(43 \mathrm{~B})$ & 109.5 & $\mathrm{C}(35)-\mathrm{C}(43)-\mathrm{H}(43 \mathrm{C})$ & 109.5 \\
\hline $\mathrm{H}(43 \mathrm{~A})-\mathrm{C}(43)-\mathrm{H}(43 \mathrm{C})$ & 109.5 & $\mathrm{H}(43 \mathrm{~B})-\mathrm{C}(43)-\mathrm{H}(43 \mathrm{C})$ & 109.5 \\
\hline $\mathrm{C}(37)-\mathrm{C}(44)-\mathrm{H}(44 \mathrm{~A})$ & 109.5 & $\mathrm{C}(37)-\mathrm{C}(44)-\mathrm{H}(44 \mathrm{~B})$ & 109.5 \\
\hline $\mathrm{H}(44 \mathrm{~A})-\mathrm{C}(44)-\mathrm{H}(44 \mathrm{~B})$ & 109.5 & $\mathrm{C}(37)-\mathrm{C}(44)-\mathrm{H}(44 \mathrm{C})$ & 109.5 \\
\hline $\mathrm{H}(44 \mathrm{~A})-\mathrm{C}(44)-\mathrm{H}(44 \mathrm{C})$ & 109.5 & $\mathrm{H}(44 \mathrm{~B})-\mathrm{C}(44)-\mathrm{H}(44 \mathrm{C})$ & 109.5 \\
\hline $\mathrm{C}(6 \mathrm{~T})-\mathrm{C}(1 \mathrm{~T})-\mathrm{C}(2 \mathrm{~T})$ & $119.5(3)$ & $\mathrm{C}(6 \mathrm{~T})-\mathrm{C}(1 \mathrm{~T})-\mathrm{H}(1 \mathrm{~T})$ & 120.3 \\
\hline $\mathrm{C}(2 \mathrm{~T})-\mathrm{C}(1 \mathrm{~T})-\mathrm{H}(1 \mathrm{~T})$ & 120.3 & $\mathrm{C}(1 \mathrm{~T})-\mathrm{C}(2 \mathrm{~T})-\mathrm{C}(3 \mathrm{~T})$ & $120.3(3)$ \\
\hline $\mathrm{C}(1 \mathrm{~T})-\mathrm{C}(2 \mathrm{~T})-\mathrm{H}(2 \mathrm{~T})$ & 119.9 & $\mathrm{C}(3 \mathrm{~T})-\mathrm{C}(2 \mathrm{~T})-\mathrm{H}(2 \mathrm{~T})$ & 119.9 \\
\hline $\mathrm{C}(2 \mathrm{~T})-\mathrm{C}(3 \mathrm{~T})-\mathrm{C}(4 \mathrm{~T})$ & $120.8(2)$ & $\mathrm{C}(2 \mathrm{~T})-\mathrm{C}(3 \mathrm{~T})-\mathrm{H}(3 \mathrm{~T})$ & 119.6 \\
\hline $\mathrm{C}(4 \mathrm{~T})-\mathrm{C}(3 \mathrm{~T})-\mathrm{H}(3 \mathrm{~T})$ & 119.6 & $\mathrm{C}(5 \mathrm{~T})-\mathrm{C}(4 \mathrm{~T})-\mathrm{C}(3 \mathrm{~T})$ & $118.0(2)$ \\
\hline $\mathrm{C}(5 \mathrm{~T})-\mathrm{C}(4 \mathrm{~T})-\mathrm{C}(7 \mathrm{~T})$ & $120.5(2)$ & $\mathrm{C}(3 \mathrm{~T})-\mathrm{C}(4 \mathrm{~T})-\mathrm{C}(7 \mathrm{~T})$ & $121.6(2)$ \\
\hline $\mathrm{C}(4 \mathrm{~T})-\mathrm{C}(5 \mathrm{~T})-\mathrm{C}(6 \mathrm{~T})$ & $121.6(2)$ & $\mathrm{C}(4 \mathrm{~T})-\mathrm{C}(5 \mathrm{~T})-\mathrm{H}(5 \mathrm{~T})$ & 119.2 \\
\hline $\mathrm{C}(6 \mathrm{~T})-\mathrm{C}(5 \mathrm{~T})-\mathrm{H}(5 \mathrm{~T})$ & 119.2 & $\mathrm{C}(1 \mathrm{~T})-\mathrm{C}(6 \mathrm{~T})-\mathrm{C}(5 \mathrm{~T})$ & 119.9(3) \\
\hline $\mathrm{C}(1 \mathrm{~T})-\mathrm{C}(6 \mathrm{~T})-\mathrm{H}(6 \mathrm{~T})$ & 120.1 & $\mathrm{C}(5 \mathrm{~T})-\mathrm{C}(6 \mathrm{~T})-\mathrm{H}(6 \mathrm{~T})$ & 120.1 \\
\hline $\mathrm{C}(4 \mathrm{~T})-\mathrm{C}(7 \mathrm{~T})-\mathrm{H}(7 \mathrm{~T} 1)$ & 109.5 & $\mathrm{C}(4 \mathrm{~T})-\mathrm{C}(7 \mathrm{~T})-\mathrm{H}(7 \mathrm{~T} 2)$ & 109.5 \\
\hline $\mathrm{H}(7 \mathrm{~T} 1)-\mathrm{C}(7 \mathrm{~T})-\mathrm{H}(7 \mathrm{~T} 2)$ & 109.5 & $\mathrm{C}(4 \mathrm{~T})-\mathrm{C}(7 \mathrm{~T})-\mathrm{H}(7 \mathrm{~T} 3)$ & 109.5 \\
\hline H(7T1)-C(7T)-H(7T3) & 109.5 & $\mathrm{H}(7 \mathrm{~T} 2)-\mathrm{C}(7 \mathrm{~T})-\mathrm{H}(7 \mathrm{~T} 3)$ & 109.5 \\
\hline
\end{tabular}

Symmetry transformations used to generate equivalent atoms: $\quad \# 1-\mathrm{x}+1,-\mathrm{y}+1,-\mathrm{z}$ 
Table S21. Anisotropic displacement parameters $\left(\AA^{2} \times 10^{3}\right)$ for FP-M. The anisotropic

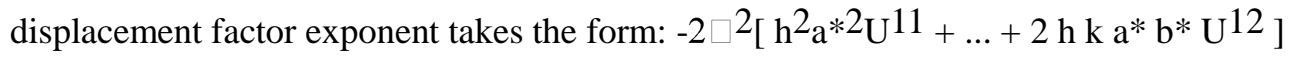

$\begin{array}{llllll}\mathrm{U}^{11} & \mathrm{U}^{22} & \mathrm{U}^{33} & \mathrm{U}^{23} & \mathrm{U}^{13} & \mathrm{U}^{12}\end{array}$

\begin{tabular}{|c|c|c|c|c|c|c|}
\hline $\mathrm{O}(1)$ & $28(1)$ & $16(1)$ & $27(1)$ & $0(1)$ & $17(1)$ & $-1(1)$ \\
\hline $\mathrm{C}(1)$ & $22(1)$ & $20(1)$ & $24(1)$ & $-1(1)$ & $9(1)$ & $-2(1)$ \\
\hline$C(2)$ & $18(1)$ & $20(1)$ & $24(1)$ & $-1(1)$ & $9(1)$ & $2(1)$ \\
\hline$C(3)$ & $17(1)$ & $16(1)$ & 21(1) & $-2(1)$ & $6(1)$ & $-1(1)$ \\
\hline $\mathrm{C}(4)$ & $18(1)$ & $17(1)$ & $21(1)$ & $-1(1)$ & $6(1)$ & $0(1)$ \\
\hline$C(5)$ & $20(1)$ & $17(1)$ & 21(1) & $-1(1)$ & $6(1)$ & $-3(1)$ \\
\hline$C(6)$ & 21(1) & $20(1)$ & $18(1)$ & $-2(1)$ & $4(1)$ & $-2(1)$ \\
\hline$C(7)$ & $22(1)$ & $15(1)$ & 21(1) & $-1(1)$ & $6(1)$ & $-1(1)$ \\
\hline$C(8)$ & $24(1)$ & $14(1)$ & $23(1)$ & $-2(1)$ & $10(1)$ & $-1(1)$ \\
\hline $\mathrm{C}(9)$ & $28(1)$ & $16(1)$ & $24(1)$ & $-3(1)$ & $7(1)$ & $2(1)$ \\
\hline$C(10)$ & $34(1)$ & $22(1)$ & $22(1)$ & $1(1)$ & 11(1) & $-1(1)$ \\
\hline$C(11)$ & $28(1)$ & $22(1)$ & $27(1)$ & $-4(1)$ & $12(1)$ & $-2(1)$ \\
\hline $\mathrm{C}(12)$ & $23(1)$ & $23(1)$ & $28(1)$ & $-3(1)$ & $6(1)$ & 2(1) \\
\hline $\mathrm{C}(13)$ & $25(1)$ & $15(1)$ & $23(1)$ & $-3(1)$ & $7(1)$ & $0(1)$ \\
\hline$C(14)$ & $22(1)$ & 19(1) & $20(1)$ & $0(1)$ & $5(1)$ & $-1(1)$ \\
\hline $\mathrm{C}(15)$ & $27(1)$ & $20(1)$ & $25(1)$ & $-2(1)$ & $9(1)$ & 1(1) \\
\hline$C(16)$ & $33(1)$ & $15(1)$ & $28(1)$ & $0(1)$ & $4(1)$ & $3(1)$ \\
\hline$C(17)$ & $27(1)$ & $19(1)$ & $29(1)$ & $3(1)$ & $5(1)$ & $-6(1)$ \\
\hline $\mathrm{C}(18)$ & $23(1)$ & $20(1)$ & $26(1)$ & $0(1)$ & $6(1)$ & $-1(1)$ \\
\hline$C(19)$ & $21(1)$ & $19(1)$ & $20(1)$ & $0(1)$ & $4(1)$ & $0(1)$ \\
\hline$C(20)$ & $19(1)$ & $17(1)$ & 21(1) & $0(1)$ & $5(1)$ & $-4(1)$ \\
\hline$C(21)$ & $19(1)$ & $20(1)$ & $25(1)$ & $0(1)$ & $8(1)$ & $-2(1)$ \\
\hline $\mathrm{C}(22)$ & $17(1)$ & $17(1)$ & $23(1)$ & $0(1)$ & $6(1)$ & $0(1)$ \\
\hline$C(23)$ & 21(1) & $19(1)$ & $22(1)$ & $1(1)$ & $6(1)$ & $0(1)$ \\
\hline $\mathrm{C}(24)$ & $20(1)$ & $17(1)$ & $22(1)$ & $0(1)$ & $7(1)$ & 1(1) \\
\hline$C(25)$ & $17(1)$ & $18(1)$ & $23(1)$ & $-1(1)$ & $7(1)$ & $-1(1)$ \\
\hline$C(26)$ & $29(1)$ & 19(1) & $25(1)$ & $1(1)$ & $14(1)$ & $-1(1)$ \\
\hline $\mathrm{C}(27)$ & $30(1)$ & $20(1)$ & $29(1)$ & $-1(1)$ & $14(1)$ & $0(1)$ \\
\hline$C(28)$ & $32(1)$ & $25(1)$ & $28(1)$ & $1(1)$ & $14(1)$ & $0(1)$ \\
\hline $\mathrm{C}(29)$ & $29(1)$ & $27(1)$ & $28(1)$ & $-4(1)$ & $10(1)$ & 1(1) \\
\hline
\end{tabular}




\begin{tabular}{|c|c|c|c|c|c|c|}
\hline \multicolumn{2}{|c|}{$\mathrm{U}^{11}$} & $\mathrm{U}^{22}$ & $\mathrm{U}^{33}$ & $\mathrm{U}^{23}$ & $\mathrm{U}^{13}$ & $\mathrm{U}^{12}$ \\
\hline $\mathrm{C}(30)$ & $44(2)$ & $36(1)$ & $33(1)$ & $-3(1)$ & $20(1)$ & $-1(1)$ \\
\hline $\mathrm{C}(31)$ & $46(2)$ & $53(2)$ & $42(2)$ & $-12(1)$ & $25(1)$ & $-3(1)$ \\
\hline $\mathrm{C}(32)$ & $24(1)$ & $14(1)$ & $23(1)$ & $-2(1)$ & $12(1)$ & $-1(1)$ \\
\hline $\mathrm{C}(33)$ & $30(1)$ & $17(1)$ & $26(1)$ & $-3(1)$ & $10(1)$ & $-1(1)$ \\
\hline $\mathrm{C}(34)$ & $37(1)$ & $20(1)$ & $22(1)$ & $2(1)$ & $13(1)$ & $0(1)$ \\
\hline $\mathrm{C}(35)$ & $33(1)$ & $18(1)$ & $29(1)$ & $-2(1)$ & $17(1)$ & $-4(1)$ \\
\hline $\mathrm{C}(36)$ & $21(1)$ & $23(1)$ & $33(1)$ & $1(1)$ & $9(1)$ & $-1(1)$ \\
\hline $\mathrm{C}(37)$ & $24(1)$ & 19(1) & $27(1)$ & $0(1)$ & $10(1)$ & $0(1)$ \\
\hline $\mathrm{C}(39)$ & $29(1)$ & $28(1)$ & $24(1)$ & $1(1)$ & $5(1)$ & $2(1)$ \\
\hline$C(40)$ & $31(1)$ & $45(2)$ & $35(1)$ & $2(1)$ & $15(1)$ & $-3(1)$ \\
\hline $\mathrm{C}(41)$ & $28(1)$ & $27(1)$ & $26(1)$ & $1(1)$ & $7(1)$ & $2(1)$ \\
\hline$C(42)$ & $36(2)$ & $40(2)$ & $32(1)$ & $3(1)$ & $3(1)$ & $-5(1)$ \\
\hline $\mathrm{C}(43)$ & $42(2)$ & $32(1)$ & $44(1)$ & $4(1)$ & $26(1)$ & $-3(1)$ \\
\hline$C(44)$ & $27(1)$ & $42(2)$ & $32(1)$ & $12(1)$ & $5(1)$ & $-3(1)$ \\
\hline & & $\mathrm{U}^{22}$ & $\mathrm{U}^{33}$ & $\mathrm{U}^{23}$ & $\mathrm{U}^{13}$ & $\mathrm{U}^{12}$ \\
\hline
\end{tabular}

\begin{tabular}{lllllll}
\hline $\mathrm{C}(1 \mathrm{~T})$ & $49(2)$ & $35(2)$ & $48(2)$ & $-7(1)$ & $7(1)$ & $-1(1)$ \\
$\mathrm{C}(2 \mathrm{~T})$ & $49(2)$ & $33(1)$ & $48(2)$ & $-1(1)$ & $0(1)$ & $-2(1)$ \\
$\mathrm{C}(3 \mathrm{~T})$ & $40(2)$ & $34(1)$ & $45(2)$ & $7(1)$ & $1(1)$ & $-6(1)$ \\
$\mathrm{C}(4 \mathrm{~T})$ & $36(2)$ & $31(1)$ & $39(1)$ & $1(1)$ & $-1(1)$ & $-4(1)$ \\
$\mathrm{C}(5 \mathrm{~T})$ & $50(2)$ & $31(1)$ & $43(2)$ & $2(1)$ & $8(1)$ & $-2(1)$ \\
$\mathrm{C}(6 \mathrm{~T})$ & $60(2)$ & $36(2)$ & $47(2)$ & $5(1)$ & $12(1)$ & $-1(1)$ \\
$\mathrm{C}(7 \mathrm{~T})$ & $43(2)$ & $46(2)$ & $47(2)$ & $2(1)$ & $9(1)$ & $0(1)$
\end{tabular}


Table S22. Hydrogen coordinates ( $\left.\mathrm{x} 10^{4}\right)$ and isotropic displacement parameters $\left(\AA^{2} \times 10^{3}\right)$ for FP-M

\begin{tabular}{|c|c|c|c|c|}
\hline $\mathrm{x}$ & & & $\mathrm{z}$ & $\mathrm{U}(\mathrm{eq})$ \\
\hline $\mathrm{H}(1)$ & 2515 & 4688 & 1407 & 25 \\
\hline $\mathrm{H}(5)$ & 2704 & 6298 & 1108 & 23 \\
\hline $\mathrm{H}(10)$ & 281 & 6912 & 3208 & 30 \\
\hline $\mathrm{H}(12)$ & -2155 & 7405 & 924 & 30 \\
\hline $\mathrm{H}(15)$ & 2065 & 8308 & 903 & 28 \\
\hline $\mathrm{H}(16)$ & 3423 & 8912 & 313 & 30 \\
\hline $\mathrm{H}(17)$ & 5522 & 8722 & -300 & 30 \\
\hline $\mathrm{H}(18)$ & 6275 & 7914 & -385 & 27 \\
\hline $\mathrm{H}(21)$ & 6454 & 6922 & -395 & 25 \\
\hline $\mathrm{H}(26 \mathrm{~A})$ & 2575 & 5135 & 2666 & 28 \\
\hline $\mathrm{H}(26 \mathrm{~B})$ & 1069 & 5182 & 1977 & 28 \\
\hline $\mathrm{H}(27 \mathrm{~A})$ & 664 & 5958 & 2530 & 31 \\
\hline $\mathrm{H}(27 \mathrm{~B})$ & 2233 & 5954 & 3156 & 31 \\
\hline $\mathrm{H}(28 \mathrm{~A})$ & -48 & 5286 & 3406 & 32 \\
\hline $\mathrm{H}(28 \mathrm{~B})$ & 1477 & 5337 & 4067 & 32 \\
\hline $\mathrm{H}(29 \mathrm{~A})$ & 771 & 6136 & 4461 & 33 \\
\hline $\mathrm{H}(29 \mathrm{~B})$ & -786 & 6053 & 3849 & 33 \\
\hline $\mathrm{H}(30 \mathrm{~A})$ & 256 & 5477 & 5403 & 43 \\
\hline $\mathrm{H}(30 \mathrm{~B})$ & -1333 & 5433 & 4815 & 43 \\
\hline $\mathrm{H}(31 \mathrm{~A})$ & -332 & 6238 & 5935 & 68 \\
\hline $\mathrm{H}(31 \mathrm{~B})$ & -1538 & 5855 & 6139 & 68 \\
\hline $\mathrm{H}(31 \mathrm{C})$ & -1895 & 6222 & 5305 & 68 \\
\hline $\mathrm{H}(34)$ & 8392 & 6696 & -2757 & 31 \\
\hline $\mathrm{H}(36)$ & 11124 & 6280 & -531 & 30 \\
\hline $\mathrm{H}(39 \mathrm{~A})$ & 2815 & 6933 & 3423 & 40 \\
\hline $\mathrm{H}(39 \mathrm{~B})$ & 3574 & 7285 & 2778 & 40 \\
\hline $\mathrm{H}(39 \mathrm{C})$ & 3421 & 6719 & 2552 & 40 \\
\hline $\mathrm{H}(40 \mathrm{~A})$ & -2777 & 7393 & 2825 & 54 \\
\hline $\mathrm{H}(40 \mathrm{~B})$ & -2377 & 6836 & 3033 & 54 \\
\hline $\mathrm{H}(40 \mathrm{C})$ & -3346 & 6986 & 2091 & 54 \\
\hline
\end{tabular}




\begin{tabular}{|c|c|c|c|c|}
\hline $\mathrm{x}$ & & & $\mathrm{z}$ & $\mathrm{U}(\mathrm{eq})$ \\
\hline $\mathrm{H}(41 \mathrm{~A})$ & -1066 & 7405 & -478 & 40 \\
\hline $\mathrm{H}(41 \mathrm{~B})$ & 673 & 7445 & -416 & 40 \\
\hline $\mathrm{H}(41 \mathrm{C})$ & -251 & 7899 & -150 & 40 \\
\hline $\mathrm{H}(42 \mathrm{~A})$ & 5265 & 6663 & -2033 & 54 \\
\hline $\mathrm{H}(42 \mathrm{~B})$ & 5333 & 6112 & -2357 & 54 \\
\hline $\mathrm{H}(42 \mathrm{C})$ & 5892 & 6537 & -2950 & 54 \\
\hline $\mathrm{H}(43 \mathrm{~A})$ & 11711 & 6412 & -2393 & 56 \\
\hline $\mathrm{H}(43 \mathrm{~B})$ & 11976 & 6833 & -1649 & 56 \\
\hline $\mathrm{H}(43 \mathrm{C})$ & 10941 & 6927 & -2595 & 56 \\
\hline $\mathrm{H}(44 \mathrm{~A})$ & 10326 & 5910 & 697 & 50 \\
\hline $\mathrm{H}(44 \mathrm{~B})$ & 8827 & 5613 & 497 & 50 \\
\hline $\mathrm{H}(44 \mathrm{C})$ & 8835 & 6148 & 909 & 50 \\
\hline $\mathrm{x}$ & & & $\mathrm{z}$ & $\mathrm{U}(\mathrm{eq})$ \\
\hline $\mathrm{H}(1 \mathrm{~T})$ & 4789 & 5510 & 4060 & 53 \\
\hline $\mathrm{H}(2 \mathrm{~T})$ & 5682 & 5972 & 2952 & 53 \\
\hline $\mathrm{H}(3 \mathrm{~T})$ & 7109 & 5614 & 1977 & 48 \\
\hline $\mathrm{H}(5 \mathrm{~T})$ & 6779 & 4334 & 3216 & 50 \\
\hline $\mathrm{H}(6 \mathrm{~T})$ & 5333 & 4688 & 4183 & 56 \\
\hline $\mathrm{H}(7 \mathrm{~T} 1)$ & 8862 & 4557 & 2193 & 68 \\
\hline $\mathrm{H}(7 \mathrm{~T} 2)$ & 8223 & 4929 & 1408 & 68 \\
\hline $\mathrm{H}(7 \mathrm{~T} 3)$ & 7395 & 4429 & 1520 & 68 \\
\hline
\end{tabular}

Table S23. Torsion angles $\left[{ }^{\circ}\right]$ for FP-M.

$\begin{array}{ll}\mathrm{C}(26)-\mathrm{O}(1)-\mathrm{C}(2)-\mathrm{C}(1) & \mathrm{C}(26)-\mathrm{O}(1)-\mathrm{C}(2)-\mathrm{C}(3) \\ \mathrm{C}(24) \# 1-\mathrm{C}(1)-\mathrm{C}(2)-\mathrm{O}(1) & \mathrm{C}(24) \# 1-\mathrm{C}(1)-\mathrm{C}(2)-\mathrm{C}(3)\end{array}$




\begin{tabular}{|c|c|}
\hline $\mathrm{O}(1)-\mathrm{C}(2)-\mathrm{C}(3)-\mathrm{C}(25)$ & $\mathrm{C}(1)-\mathrm{C}(2)-\mathrm{C}(3)-\mathrm{C}(25)$ \\
\hline $\mathrm{O}(1)-\mathrm{C}(2)-\mathrm{C}(3)-\mathrm{C}(4)$ & $\mathrm{C}(1)-\mathrm{C}(2)-\mathrm{C}(3)-\mathrm{C}(4)$ \\
\hline $\mathrm{C}(2)-\mathrm{C}(3)-\mathrm{C}(4)-\mathrm{C}(5)$ & $\mathrm{C}(25)-\mathrm{C}(3)-\mathrm{C}(4)-\mathrm{C}(5)$ \\
\hline $\mathrm{C}(2)-\mathrm{C}(3)-\mathrm{C}(4)-\mathrm{C}(22)$ & $\mathrm{C}(25)-\mathrm{C}(3)-\mathrm{C}(4)-\mathrm{C}(22)$ \\
\hline $\mathrm{C}(3)-\mathrm{C}(4)-\mathrm{C}(5)-\mathrm{C}(6)$ & $\mathrm{C}(22)-\mathrm{C}(4)-\mathrm{C}(5)-\mathrm{C}(6)$ \\
\hline $\mathrm{C}(4)-\mathrm{C}(5)-\mathrm{C}(6)-\mathrm{C}(7)$ & $\mathrm{C}(4)-\mathrm{C}(5)-\mathrm{C}(6)-\mathrm{C}(20)$ \\
\hline$C(5)-C(6)-C(7)-C(14)$ & $C(20)-C(6)-C(7)-C(14)$ \\
\hline $\mathrm{C}(5)-\mathrm{C}(6)-\mathrm{C}(7)-\mathrm{C}(8)$ & $C(20)-C(6)-C(7)-C(8)$ \\
\hline $\mathrm{C}(6)-\mathrm{C}(7)-\mathrm{C}(8)-\mathrm{C}(13)$ & $\mathrm{C}(14)-\mathrm{C}(7)-\mathrm{C}(8)-\mathrm{C}(13)$ \\
\hline $\mathrm{C}(6)-\mathrm{C}(7)-\mathrm{C}(8)-\mathrm{C}(9)$ & $\mathrm{C}(14)-\mathrm{C}(7)-\mathrm{C}(8)-\mathrm{C}(9)$ \\
\hline $\mathrm{C}(13)-\mathrm{C}(8)-\mathrm{C}(9)-\mathrm{C}(10)$ & $\mathrm{C}(7)-\mathrm{C}(8)-\mathrm{C}(9)-\mathrm{C}(10)$ \\
\hline $\mathrm{C}(13)-\mathrm{C}(8)-\mathrm{C}(9)-\mathrm{C}(39)$ & $\mathrm{C}(7)-\mathrm{C}(8)-\mathrm{C}(9)-\mathrm{C}(39)$ \\
\hline $\mathrm{C}(8)-\mathrm{C}(9)-\mathrm{C}(10)-\mathrm{C}(11)$ & $\mathrm{C}(39)-\mathrm{C}(9)-\mathrm{C}(10)-\mathrm{C}(11)$ \\
\hline $\mathrm{C}(9)-\mathrm{C}(10)-\mathrm{C}(11)-\mathrm{C}(12)$ & $\mathrm{C}(9)-\mathrm{C}(10)-\mathrm{C}(11)-\mathrm{C}(40)$ \\
\hline $\mathrm{C}(10)-\mathrm{C}(11)-\mathrm{C}(12)-\mathrm{C}(13)$ & $\mathrm{C}(40)-\mathrm{C}(11)-\mathrm{C}(12)-\mathrm{C}(13)$ \\
\hline $\mathrm{C}(11)-\mathrm{C}(12)-\mathrm{C}(13)-\mathrm{C}(8)$ & $\mathrm{C}(11)-\mathrm{C}(12)-\mathrm{C}(13)-\mathrm{C}(41)$ \\
\hline $\mathrm{C}(9)-\mathrm{C}(8)-\mathrm{C}(13)-\mathrm{C}(12)$ & $\mathrm{C}(7)-\mathrm{C}(8)-\mathrm{C}(13)-\mathrm{C}(12)$ \\
\hline $\mathrm{C}(9)-\mathrm{C}(8)-\mathrm{C}(13)-\mathrm{C}(41)$ & $\mathrm{C}(7)-\mathrm{C}(8)-\mathrm{C}(13)-\mathrm{C}(41)$ \\
\hline $\mathrm{C}(6)-\mathrm{C}(7)-\mathrm{C}(14)-\mathrm{C}(15)$ & $\mathrm{C}(8)-\mathrm{C}(7)-\mathrm{C}(14)-\mathrm{C}(15)$ \\
\hline $\mathrm{C}(6)-\mathrm{C}(7)-\mathrm{C}(14)-\mathrm{C}(19)$ & $\mathrm{C}(8)-\mathrm{C}(7)-\mathrm{C}(14)-\mathrm{C}(19)$ \\
\hline $\mathrm{C}(19)-\mathrm{C}(14)-\mathrm{C}(15)-\mathrm{C}(16)$ & $\mathrm{C}(7)-\mathrm{C}(14)-\mathrm{C}(15)-\mathrm{C}(16)$ \\
\hline $\mathrm{C}(14)-\mathrm{C}(15)-\mathrm{C}(16)-\mathrm{C}(17)$ & $\mathrm{C}(15)-\mathrm{C}(16)-\mathrm{C}(17)-\mathrm{C}(18)$ \\
\hline $\mathrm{C}(16)-\mathrm{C}(17)-\mathrm{C}(18)-\mathrm{C}(19)$ & $\mathrm{C}(17)-\mathrm{C}(18)-\mathrm{C}(19)-\mathrm{C}(14)$ \\
\hline $\mathrm{C}(17)-\mathrm{C}(18)-\mathrm{C}(19)-\mathrm{C}(20)$ & $\mathrm{C}(15)-\mathrm{C}(14)-\mathrm{C}(19)-\mathrm{C}(18)$ \\
\hline $\mathrm{C}(7)-\mathrm{C}(14)-\mathrm{C}(19)-\mathrm{C}(18)$ & $\mathrm{C}(15)-\mathrm{C}(14)-\mathrm{C}(19)-\mathrm{C}(20)$ \\
\hline $\mathrm{C}(7)-\mathrm{C}(14)-\mathrm{C}(19)-\mathrm{C}(20)$ & $C(7)-C(6)-C(20)-C(21)$ \\
\hline$C(5)-C(6)-C(20)-C(21)$ & $C(7)-C(6)-C(20)-C(19)$ \\
\hline $\mathrm{C}(5)-\mathrm{C}(6)-\mathrm{C}(20)-\mathrm{C}(19)$ & $\mathrm{C}(18)-\mathrm{C}(19)-\mathrm{C}(20)-\mathrm{C}(21)$ \\
\hline $\mathrm{C}(14)-\mathrm{C}(19)-\mathrm{C}(20)-\mathrm{C}(21)$ & $\mathrm{C}(18)-\mathrm{C}(19)-\mathrm{C}(20)-\mathrm{C}(6)$ \\
\hline $\mathrm{C}(14)-\mathrm{C}(19)-\mathrm{C}(20)-\mathrm{C}(6)$ & $C(6)-C(20)-C(21)-C(22)$ \\
\hline $\mathrm{C}(19)-\mathrm{C}(20)-\mathrm{C}(21)-\mathrm{C}(22)$ & $\mathrm{C}(20)-\mathrm{C}(21)-\mathrm{C}(22)-\mathrm{C}(23)$ \\
\hline$C(20)-C(21)-C(22)-C(4)$ & $\mathrm{C}(5)-\mathrm{C}(4)-\mathrm{C}(22)-\mathrm{C}(23)$ \\
\hline $\mathrm{C}(3)-\mathrm{C}(4)-\mathrm{C}(22)-\mathrm{C}(23)$ & $C(5)-C(4)-C(22)-C(21)$ \\
\hline$C(3)-C(4)-C(22)-C(21)$ & $\mathrm{C}(21)-\mathrm{C}(22)-\mathrm{C}(23)-\mathrm{C}(24)$ \\
\hline $\mathrm{C}(4)-\mathrm{C}(22)-\mathrm{C}(23)-\mathrm{C}(24)$ & $\mathrm{C}(21)-\mathrm{C}(22)-\mathrm{C}(23)-\mathrm{C}(32)$ \\
\hline $\mathrm{C}(4)-\mathrm{C}(22)-\mathrm{C}(23)-\mathrm{C}(32)$ & $\mathrm{C}(22)-\mathrm{C}(23)-\mathrm{C}(24)-\mathrm{C}(1) \# 1$ \\
\hline
\end{tabular}




$\begin{array}{ll}\mathrm{C}(32)-\mathrm{C}(23)-\mathrm{C}(24)-\mathrm{C}(1) \# 1 & \mathrm{C}(22)-\mathrm{C}(23)-\mathrm{C}(24)-\mathrm{C}(25) \\ \mathrm{C}(32)-\mathrm{C}(23)-\mathrm{C}(24)-\mathrm{C}(25) & \mathrm{C}(1) \# 1-\mathrm{C}(24)-\mathrm{C}(25)-\mathrm{C}(25) \# 1 \\ \mathrm{C}(23)-\mathrm{C}(24)-\mathrm{C}(25)-\mathrm{C}(25) \# 1 & \mathrm{C}(1) \# 1-\mathrm{C}(24)-\mathrm{C}(25)-\mathrm{C}(3) \\ \mathrm{C}(23)-\mathrm{C}(24)-\mathrm{C}(25)-\mathrm{C}(3) & \mathrm{C}(2)-\mathrm{C}(3)-\mathrm{C}(25)-\mathrm{C}(24) \\ \mathrm{C}(4)-\mathrm{C}(3)-\mathrm{C}(25)-\mathrm{C}(24) & \mathrm{C}(2)-\mathrm{C}(3)-\mathrm{C}(25)-\mathrm{C}(25) \# 1 \\ \mathrm{C}(4)-\mathrm{C}(3)-\mathrm{C}(25)-\mathrm{C}(25) \# 1 & \mathrm{C}(2)-\mathrm{O}(1)-\mathrm{C}(26)-\mathrm{C}(27) \\ \mathrm{O}(1)-\mathrm{C}(26)-\mathrm{C}(27)-\mathrm{C}(28) & \mathrm{C}(26)-\mathrm{C}(27)-\mathrm{C}(28)-\mathrm{C}(29) \\ \mathrm{C}(27)-\mathrm{C}(28)-\mathrm{C}(29)-\mathrm{C}(30) & \mathrm{C}(28)-\mathrm{C}(29)-\mathrm{C}(30)-\mathrm{C}(31) \\ \mathrm{C}(22)-\mathrm{C}(23)-\mathrm{C}(32)-\mathrm{C}(33) & \mathrm{C}(24)-\mathrm{C}(23)-\mathrm{C}(32)-\mathrm{C}(33) \\ \mathrm{C}(22)-\mathrm{C}(23)-\mathrm{C}(32)-\mathrm{C}(37) & \mathrm{C}(24)-\mathrm{C}(23)-\mathrm{C}(32)-\mathrm{C}(37) \\ \mathrm{C}(37)-\mathrm{C}(32)-\mathrm{C}(33)-\mathrm{C}(34) & \mathrm{C}(23)-\mathrm{C}(32)-\mathrm{C}(33)-\mathrm{C}(34) \\ \mathrm{C}(37)-\mathrm{C}(32)-\mathrm{C}(33)-\mathrm{C}(42) & \mathrm{C}(23)-\mathrm{C}(32)-\mathrm{C}(33)-\mathrm{C}(42) \\ \mathrm{C}(32)-\mathrm{C}(33)-\mathrm{C}(34)-\mathrm{C}(35) & \mathrm{C}(42)-\mathrm{C}(33)-\mathrm{C}(34)-\mathrm{C}(35) \\ \mathrm{C}(33)-\mathrm{C}(34)-\mathrm{C}(35)-\mathrm{C}(36) & \mathrm{C}(33)-\mathrm{C}(34)-\mathrm{C}(35)-\mathrm{C}(43) \\ \mathrm{C}(34)-\mathrm{C}(35)-\mathrm{C}(36)-\mathrm{C}(37) & \mathrm{C}(43)-\mathrm{C}(35)-\mathrm{C}(36)-\mathrm{C}(37) \\ \mathrm{C}(35)-\mathrm{C}(36)-\mathrm{C}(37)-\mathrm{C}(32) & \mathrm{C}(35)-\mathrm{C}(36)-\mathrm{C}(37)-\mathrm{C}(44) \\ \mathrm{C}(33)-\mathrm{C}(32)-\mathrm{C}(37)-\mathrm{C}(36) & \mathrm{C}(23)-\mathrm{C}(32)-\mathrm{C}(37)-\mathrm{C}(36) \\ \mathrm{C}(33)-\mathrm{C}(32)-\mathrm{C}(37)-\mathrm{C}(44) & \mathrm{C}(23)-\mathrm{C}(32)-\mathrm{C}(37)-\mathrm{C}(44) \\ \mathrm{C}(6 \mathrm{~T})-\mathrm{C}(1 \mathrm{~T})-\mathrm{C}(2 \mathrm{~T})-\mathrm{C}(3 \mathrm{~T}) & \mathrm{C}(1 \mathrm{~T})-\mathrm{C}(2 \mathrm{~T})-\mathrm{C}(3 \mathrm{~T})-\mathrm{C}(4 \mathrm{~T}) \\ \mathrm{C}(2 \mathrm{~T})-\mathrm{C}(3 \mathrm{~T})-\mathrm{C}(4 \mathrm{~T})-\mathrm{C}(5 \mathrm{~T}) & \mathrm{C}(2 \mathrm{~T})-\mathrm{C}(3 \mathrm{~T})-\mathrm{C}(4 \mathrm{~T})-\mathrm{C}(7 \mathrm{~T}) \\ \mathrm{C}(3 \mathrm{~T})-\mathrm{C}(4 \mathrm{~T})-\mathrm{C}(5 \mathrm{~T})-\mathrm{C}(6 \mathrm{~T}) & \mathrm{C}(7 \mathrm{~T})-\mathrm{C}(4 \mathrm{~T})-\mathrm{C}(5 \mathrm{~T})-\mathrm{C}(6 \mathrm{~T}) \\ \mathrm{C}(2 \mathrm{~T})-\mathrm{C}(1 \mathrm{~T})-\mathrm{C}(6 \mathrm{~T})-\mathrm{C}(5 \mathrm{~T}) & \mathrm{C}(4 \mathrm{~T})-\mathrm{C}(5 \mathrm{~T})-\mathrm{C}(6 \mathrm{~T})-\mathrm{C}(1 \mathrm{~T})\end{array}$

Symmetry transformations used to generate equivalent atoms: $\# 1-x+1,-y+1,-z$

\section{Data for IFD-CF 3}

Table S24. Crystal data and structure refinement for $\mathbf{I F D}_{\mathbf{C}} \mathbf{C F}_{\mathbf{3}}$.

$\begin{array}{ll}\text { Empirical formula } & \mathrm{C}_{78} \mathrm{H}_{50} \mathrm{~F}_{24} \mathrm{O}_{2} \\ \text { Formula weight } & 1475.22 \\ \text { Temperature } & 160(2) \mathrm{K} \\ \text { Wavelength } & 1.54178 \AA \\ \text { Crystal system } & \text { Monoclinic } \\ \text { Space group } & \mathrm{C} 2 / \mathrm{c}\end{array}$


Unit cell dimensions

Volume

Z

Density (calculated)

Absorption coefficient

$\mathrm{F}(000)$

Crystal size

Theta range for data collection

Index ranges

Reflections collected

Independent reflections

Completeness to theta $=67.679^{\circ}$

Refinement method

Data / restraints / parameters

Goodness-of-fit on $\mathrm{F}^{2}$

Final $\mathrm{R}$ indices [I>2sigma(I)]

$\mathrm{R}$ indices (all data)

Extinction coefficient

Largest diff. peak and hole

$$
\begin{array}{cc}
a=32.7089(12) \AA & \alpha=90^{\circ} \\
b=14.2715(5) \AA & \beta=104.233(2)^{\circ} \\
c=15.6260(6) \AA & \gamma=90^{\circ}
\end{array}
$$

7070.4(5) $\AA^{3}$

8

$1.386 \mathrm{Mg} / \mathrm{m}^{3}$

$1.109 \mathrm{~mm}^{-1}$

3000

$0.178 \times 0.102 \times 0.075 \mathrm{~mm}^{3}$

2.787 to $74.614^{\circ}$

$-39<=\mathrm{h}<=39,-17<=\mathrm{k}<=5,-19<=\mathrm{l}<=18$

23484

$7000[\mathrm{R}($ int $)=0.0495]$

$98.5 \%$

Full-matrix least-squares on $\mathrm{F}^{2}$

$7000 / 120 / 506$

1.778

$\mathrm{R} 1=0.1243, \mathrm{wR} 2=0.3729$

$\mathrm{R} 1=0.1386, \mathrm{wR} 2=0.3981$

$\mathrm{n} / \mathrm{a}$

3.368 and -0.465 e. $\AA^{-3}$ 
Table S25. Atomic coordinates ( x 10 $)$ and equivalent isotropic displacement parameters $\left(\AA^{2} \times 10^{3}\right)$ for IFD-CF 3 . U(eq) is defined as one third of the trace of the orthogonalized $\mathrm{U}^{\mathrm{ij}}$ tensor.

\begin{tabular}{|c|c|c|c|c|}
\hline $\mathrm{x}$ & & & $\mathrm{z}$ & $\mathrm{U}(\mathrm{eq})$ \\
\hline $\mathrm{O}(1)$ & $4268(1)$ & $836(2)$ & 293(2) & $28(1)$ \\
\hline $\mathrm{C}(1)$ & $4846(1)$ & $4328(2)$ & $1151(2)$ & $29(1)$ \\
\hline $\mathrm{C}(2)$ & $5169(1)$ & $5063(2)$ & $1244(2)$ & $30(1)$ \\
\hline$C(3)$ & $5173(1)$ & $6004(2)$ & $1503(3)$ & $37(1)$ \\
\hline$C(4)$ & $5535(1)$ & $6534(2)$ & $1521(3)$ & $42(1)$ \\
\hline$C(5)$ & $5888(1)$ & $6152(3)$ & $1323(3)$ & $42(1)$ \\
\hline$C(6)$ & 5891(1) & $5199(2)$ & 1092(3) & $35(1)$ \\
\hline$C(7)$ & $5534(1)$ & $4677(2)$ & $1053(2)$ & $31(1)$ \\
\hline $\mathrm{C}(8)$ & $5443(1)$ & $3687(2)$ & $832(2)$ & $29(1)$ \\
\hline $\mathrm{C}(9)$ & $5689(1)$ & $3000(2)$ & $614(2)$ & $28(1)$ \\
\hline $\mathrm{C}(10)$ & $5512(1)$ & $2084(2)$ & $428(2)$ & $29(1)$ \\
\hline $\mathrm{C}(11)$ & $5072(1)$ & $1925(2)$ & $434(2)$ & $27(1)$ \\
\hline$C(12)$ & $4826(1)$ & $2611(2)$ & $656(2)$ & $27(1)$ \\
\hline$C(13)$ & $5015(1)$ & $3516(2)$ & $894(2)$ & $29(1)$ \\
\hline$C(14)$ & $4429(1)$ & $4454(2)$ & $1309(2)$ & $29(1)$ \\
\hline$C(15)$ & $4224(1)$ & $3741(2)$ & $1657(2)$ & $30(1)$ \\
\hline$C(16)$ & $3825(1)$ & $3880(2)$ & 1791(2) & $34(1)$ \\
\hline$C(17)$ & $3624(1)$ & $4744(3)$ & $1624(3)$ & $40(1)$ \\
\hline $\mathrm{C}(18)$ & $3828(1)$ & $5457(3)$ & 1301(3) & $40(1)$ \\
\hline C(19) & $4220(1)$ & $5316(2)$ & $1121(2)$ & $34(1)$ \\
\hline $\mathrm{C}(20)$ & $5686(1)$ & $1264(2)$ & $199(2)$ & $26(1)$ \\
\hline $\mathrm{C}(21)$ & $6120(1)$ & $1163(2)$ & $98(2)$ & $28(1)$ \\
\hline $\mathrm{C}(22)$ & $6468(1)$ & $1386(2)$ & $789(3)$ & $34(1)$ \\
\hline $\mathrm{C}(23)$ & 6871(1) & $1312(3)$ & $677(3)$ & $42(1)$ \\
\hline $\mathrm{C}(24)$ & $6937(1)$ & 1039(3) & $-138(3)$ & $42(1)$ \\
\hline$C(25)$ & $6599(1)$ & $815(3)$ & $-810(3)$ & $39(1)$ \\
\hline$C(26)$ & $6190(1)$ & $854(2)$ & $-698(2)$ & $35(1)$ \\
\hline $\mathrm{C}(27)$ & 4984(1) & $946(2)$ & 200(2) & $26(1)$ \\
\hline$C(28)$ & $4624(1)$ & $415(2)$ & $138(2)$ & $26(1)$ \\
\hline $\mathrm{C}(29)$ & $3966(1)$ & $1145(2)$ & $-498(2)$ & $30(1)$ \\
\hline
\end{tabular}




\begin{tabular}{|c|c|c|c|c|}
\hline $\mathrm{x}$ & & & $\mathrm{z}$ & \\
\hline$C(30)$ & $3627(1)$ & $1699(2)$ & $-217(2)$ & $34(1)$ \\
\hline$C(31)$ & $3246(1)$ & $1890(3)$ & $-997(3)$ & $39(1)$ \\
\hline$C(32)$ & 2930(1) & $2560(3)$ & $-755(3)$ & $46(1)$ \\
\hline$C(33)$ & $3067(2)$ & $3579(3)$ & $-689(4)$ & $52(1)$ \\
\hline$C(34)$ & $2747(2)$ & $4212(4)$ & $-396(5)$ & $77(2)$ \\
\hline$C(35)$ & $4637(1)$ & $-543(2)$ & $-59(2)$ & $27(1)$ \\
\hline$C(36)$ & $3594(1)$ & $3102(3)$ & $2128(3)$ & $40(1)$ \\
\hline $\mathrm{F}(1 \mathrm{~A})$ & $3832(1)$ & $2347(2)$ & $2368(2)$ & $57(1)$ \\
\hline $\mathrm{F}(2 \mathrm{~A})$ & $3256(1)$ & $2828(2)$ & $1516(2)$ & $67(1)$ \\
\hline $\mathrm{F}(3 \mathrm{~A})$ & $3464(1)$ & $3368(2)$ & $2833(2)$ & $50(1)$ \\
\hline$C(37 \mathrm{~A})$ & $3641(2)$ & $6429(5)$ & $1185(4)$ & $40(2)$ \\
\hline $\mathrm{F}(4 \mathrm{~A})$ & $3402(2)$ & $6526(4)$ & $373(4)$ & $70(2)$ \\
\hline $\mathrm{F}(5 \mathrm{~A})$ & $3401(2)$ & $6631(3)$ & 1732(3) & $53(1)$ \\
\hline $\mathrm{F}(6 \mathrm{~A})$ & $3933(2)$ & 7093(6) & $1305(5)$ & $58(2)$ \\
\hline$C(37 B)$ & $3582(4)$ & $6325(11)$ & $894(9)$ & $46(5)$ \\
\hline $\mathrm{F}(4 \mathrm{~B})$ & $3402(5)$ & $6344(13)$ & $37(9)$ & $77(5)$ \\
\hline $\mathrm{F}(5 \mathrm{~B})$ & $3279(5)$ & $6481(11)$ & $1304(10)$ & $84(4)$ \\
\hline $\mathrm{F}(6 \mathrm{~B})$ & $3836(5)$ & $7062(16)$ & $1050(11)$ & $66(5)$ \\
\hline $\mathrm{C}(38)$ & $7244(2)$ & $1528(4)$ & $1424(4)$ & $62(1)$ \\
\hline $\mathrm{F}(7)$ & $7143(1)$ & $1675(3)$ & 2192(3) & $96(1)$ \\
\hline $\mathrm{F}(8)$ & $7450(2)$ & $2272(4)$ & $1267(4)$ & $117(2)$ \\
\hline $\mathrm{F}(9)$ & $7520(1)$ & $849(3)$ & 1584(3) & $99(1)$ \\
\hline C(39) & $6660(2)$ & $515(4)$ & $-1693(3)$ & $56(1)$ \\
\hline $\mathrm{F}(10)$ & $7040(1)$ & $725(3)$ & $-1798(3)$ & $87(1)$ \\
\hline $\mathrm{F}(11)$ & $6623(2)$ & $-405(3)$ & $-1801(3)$ & $87(1)$ \\
\hline $\mathrm{F}(12)$ & $6389(1)$ & $929(3)$ & $-2347(2)$ & $89(1)$ \\
\hline
\end{tabular}


Table S26. Bond lengths $[\AA]$ and angles $\left[^{\circ}\right]$ for IFD-CF3.

\begin{tabular}{|c|c|c|c|}
\hline $\mathrm{O}(1)-\mathrm{C}(28)$ & $1.385(4)$ & $\mathrm{O}(1)-\mathrm{C}(29)$ & $1.447(4)$ \\
\hline $\mathrm{C}(1)-\mathrm{C}(13)$ & $1.385(5)$ & $\mathrm{C}(1)-\mathrm{C}(14)$ & $1.457(5)$ \\
\hline $\mathrm{C}(1)-\mathrm{C}(2)$ & $1.470(5)$ & $\mathrm{C}(2)-\mathrm{C}(3)$ & $1.401(5)$ \\
\hline $\mathrm{C}(2)-\mathrm{C}(7)$ & $1.413(5)$ & $\mathrm{C}(3)-\mathrm{C}(4)$ & $1.397(6)$ \\
\hline $\mathrm{C}(3)-\mathrm{H}(3)$ & 0.9500 & $C(4)-C(5)$ & $1.380(6)$ \\
\hline $\mathrm{C}(4)-\mathrm{H}(4)$ & 0.9500 & $C(5)-C(6)$ & $1.407(5)$ \\
\hline $\mathrm{C}(5)-\mathrm{H}(5)$ & 0.9500 & $C(6)-C(7)$ & $1.373(5)$ \\
\hline $\mathrm{C}(6)-\mathrm{H}(6)$ & 0.9500 & $\mathrm{C}(7)-\mathrm{C}(8)$ & $1.467(4)$ \\
\hline $\mathrm{C}(8)-\mathrm{C}(9)$ & $1.366(5)$ & $\mathrm{C}(8)-\mathrm{C}(13)$ & $1.448(5)$ \\
\hline $\mathrm{C}(9)-\mathrm{C}(10)$ & $1.431(4)$ & $\mathrm{C}(9)-\mathrm{H}(9)$ & 0.9500 \\
\hline$C(10)-C(20)$ & $1.387(5)$ & $\mathrm{C}(10)-\mathrm{C}(11)$ & $1.458(5)$ \\
\hline $\mathrm{C}(11)-\mathrm{C}(12)$ & $1.365(5)$ & $\mathrm{C}(11)-\mathrm{C}(27)$ & $1.456(4)$ \\
\hline $\mathrm{C}(12)-\mathrm{C}(13)$ & $1.440(4)$ & $\mathrm{C}(12)-\mathrm{H}(12)$ & 0.9500 \\
\hline $\mathrm{C}(14)-\mathrm{C}(15)$ & $1.399(5)$ & $\mathrm{C}(14)-\mathrm{C}(19)$ & $1.403(4)$ \\
\hline$C(15)-C(16)$ & $1.386(5)$ & $\mathrm{C}(15)-\mathrm{H}(15)$ & 0.9500 \\
\hline $\mathrm{C}(16)-\mathrm{C}(17)$ & $1.392(5)$ & $C(16)-C(36)$ & $1.509(5)$ \\
\hline $\mathrm{C}(17)-\mathrm{C}(18)$ & $1.380(6)$ & $\mathrm{C}(17)-\mathrm{H}(17)$ & 0.9500 \\
\hline $\mathrm{C}(18)-\mathrm{C}(19)$ & $1.391(6)$ & $\mathrm{C}(18)-\mathrm{C}(37 \mathrm{~A})$ & $1.509(8)$ \\
\hline $\mathrm{C}(18)-\mathrm{C}(37 \mathrm{~B})$ & $1.529(15)$ & C(19)-H(19) & 0.9500 \\
\hline $\mathrm{C}(20)-\mathrm{C}(35) \# 1$ & $1.451(4)$ & $C(20)-C(21)$ & $1.474(5)$ \\
\hline$C(21)-C(26)$ & $1.390(5)$ & $\mathrm{C}(21)-\mathrm{C}(22)$ & $1.400(5)$ \\
\hline$C(22)-C(23)$ & $1.376(6)$ & $\mathrm{C}(22)-\mathrm{H}(22)$ & 0.9500 \\
\hline $\mathrm{C}(23)-\mathrm{C}(24)$ & $1.399(6)$ & $\mathrm{C}(23)-\mathrm{C}(38)$ & $1.499(6)$ \\
\hline$C(24)-C(25)$ & $1.363(6)$ & $\mathrm{C}(24)-\mathrm{H}(24)$ & 0.9500 \\
\hline$C(25)-C(26)$ & $1.394(6)$ & $\mathrm{C}(25)-\mathrm{C}(39)$ & $1.505(6)$ \\
\hline $\mathrm{C}(26)-\mathrm{H}(26)$ & 0.9500 & $\mathrm{C}(27)-\mathrm{C}(28)$ & $1.383(5)$ \\
\hline $\mathrm{C}(27)-\mathrm{C}(35) \# 1$ & $1.433(5)$ & $\mathrm{C}(28)-\mathrm{C}(35)$ & $1.404(4)$ \\
\hline $\mathrm{C}(29)-\mathrm{C}(30)$ & $1.513(5)$ & $\mathrm{C}(29)-\mathrm{H}(29 \mathrm{~A})$ & 0.9900 \\
\hline $\mathrm{C}(29)-\mathrm{H}(29 \mathrm{~B})$ & 0.9900 & $\mathrm{C}(30)-\mathrm{C}(31)$ & $1.537(5)$ \\
\hline $\mathrm{C}(30)-\mathrm{H}(30 \mathrm{~A})$ & 0.9900 & $\mathrm{C}(30)-\mathrm{H}(30 \mathrm{~B})$ & 0.9900 \\
\hline $\mathrm{C}(31)-\mathrm{C}(32)$ & $1.525(6)$ & $\mathrm{C}(31)-\mathrm{H}(31 \mathrm{~A})$ & 0.9900 \\
\hline $\mathrm{C}(31)-\mathrm{H}(31 \mathrm{~B})$ & 0.9900 & $\mathrm{C}(32)-\mathrm{C}(33)$ & $1.518(7)$ \\
\hline $\mathrm{C}(32)-\mathrm{H}(32 \mathrm{~A})$ & 0.9900 & $\mathrm{C}(32)-\mathrm{H}(32 \mathrm{~B})$ & 0.9900 \\
\hline
\end{tabular}




\begin{tabular}{|c|c|c|c|}
\hline $\mathrm{C}(33)-\mathrm{C}(34)$ & $1.534(7)$ & $\mathrm{C}(33)-\mathrm{H}(33 \mathrm{~A})$ & 0.9900 \\
\hline $\mathrm{C}(33)-\mathrm{H}(33 \mathrm{~B})$ & 0.9900 & $\mathrm{C}(34)-\mathrm{H}(34 \mathrm{~A})$ & 0.9800 \\
\hline $\mathrm{C}(34)-\mathrm{H}(34 \mathrm{~B})$ & 0.9800 & $\mathrm{C}(34)-\mathrm{H}(34 \mathrm{C})$ & 0.9800 \\
\hline $\mathrm{C}(35)-\mathrm{C}(27) \# 1$ & $1.433(5)$ & C(35)-C(20)\#1 & $1.451(4)$ \\
\hline $\mathrm{C}(36)-\mathrm{F}(1 \mathrm{~A})$ & $1.329(5)$ & $\mathrm{C}(36)-\mathrm{F}(3 \mathrm{~A})$ & $1.331(5)$ \\
\hline $\mathrm{C}(36)-\mathrm{F}(2 \mathrm{~A})$ & $1.332(5)$ & $\mathrm{C}(37 \mathrm{~A})-\mathrm{F}(4 \mathrm{~A})$ & $1.324(4)$ \\
\hline $\mathrm{C}(37 \mathrm{~A})-\mathrm{F}(5 \mathrm{~A})$ & $1.324(4)$ & $\mathrm{C}(37 \mathrm{~A})-\mathrm{F}(6 \mathrm{~A})$ & $1.326(4)$ \\
\hline $\mathrm{C}(37 \mathrm{~B})-\mathrm{F}(5 \mathrm{~B})$ & $1.323(5)$ & $\mathrm{C}(37 \mathrm{~B})-\mathrm{F}(4 \mathrm{~B})$ & $1.324(5)$ \\
\hline $\mathrm{C}(37 \mathrm{~B})-\mathrm{F}(6 \mathrm{~B})$ & $1.325(5)$ & $\mathrm{C}(38)-\mathrm{F}(9)$ & $1.309(6)$ \\
\hline $\mathrm{C}(38)-\mathrm{F}(8)$ & $1.314(7)$ & $\mathrm{C}(38)-\mathrm{F}(7)$ & $1.337(7)$ \\
\hline $\mathrm{C}(39)-\mathrm{F}(12)$ & $1.317(7)$ & $\mathrm{C}(39)-\mathrm{F}(11)$ & $1.325(6)$ \\
\hline $\mathrm{C}(39)-\mathrm{F}(10)$ & $1.328(6)$ & & \\
\hline $\mathrm{C}(28)-\mathrm{O}(1)-\mathrm{C}(29)$ & $114.2(3)$ & $\mathrm{C}(13)-\mathrm{C}(1)-\mathrm{C}(14)$ & $127.5(3)$ \\
\hline $\mathrm{C}(13)-\mathrm{C}(1)-\mathrm{C}(2)$ & $107.4(3)$ & $\mathrm{C}(14)-\mathrm{C}(1)-\mathrm{C}(2)$ & $125.1(3)$ \\
\hline $\mathrm{C}(3)-\mathrm{C}(2)-\mathrm{C}(7)$ & $119.0(3)$ & $\mathrm{C}(3)-\mathrm{C}(2)-\mathrm{C}(1)$ & $131.9(3)$ \\
\hline$C(7)-C(2)-C(1)$ & 109.0(3) & $\mathrm{C}(4)-\mathrm{C}(3)-\mathrm{C}(2)$ & 118.3(4) \\
\hline $\mathrm{C}(4)-\mathrm{C}(3)-\mathrm{H}(3)$ & 120.9 & $\mathrm{C}(2)-\mathrm{C}(3)-\mathrm{H}(3)$ & 120.9 \\
\hline$C(5)-C(4)-C(3)$ & $122.1(3)$ & $\mathrm{C}(5)-\mathrm{C}(4)-\mathrm{H}(4)$ & 118.9 \\
\hline $\mathrm{C}(3)-\mathrm{C}(4)-\mathrm{H}(4)$ & 118.9 & $\mathrm{C}(4)-\mathrm{C}(5)-\mathrm{C}(6)$ & $119.9(4)$ \\
\hline $\mathrm{C}(4)-\mathrm{C}(5)-\mathrm{H}(5)$ & 120.1 & $\mathrm{C}(6)-\mathrm{C}(5)-\mathrm{H}(5)$ & 120.1 \\
\hline$C(7)-C(6)-C(5)$ & $118.5(4)$ & $\mathrm{C}(7)-\mathrm{C}(6)-\mathrm{H}(6)$ & 120.8 \\
\hline $\mathrm{C}(5)-\mathrm{C}(6)-\mathrm{H}(6)$ & 120.8 & $\mathrm{C}(6)-\mathrm{C}(7)-\mathrm{C}(2)$ & $122.2(3)$ \\
\hline $\mathrm{C}(6)-\mathrm{C}(7)-\mathrm{C}(8)$ & $131.0(3)$ & $C(2)-C(7)-C(8)$ & $106.9(3)$ \\
\hline $\mathrm{C}(9)-\mathrm{C}(8)-\mathrm{C}(13)$ & $122.4(3)$ & $\mathrm{C}(9)-\mathrm{C}(8)-\mathrm{C}(7)$ & $130.6(3)$ \\
\hline $\mathrm{C}(13)-\mathrm{C}(8)-\mathrm{C}(7)$ & 107.0(3) & $\mathrm{C}(8)-\mathrm{C}(9)-\mathrm{C}(10)$ & 118.1(3) \\
\hline $\mathrm{C}(8)-\mathrm{C}(9)-\mathrm{H}(9)$ & 120.9 & $\mathrm{C}(10)-\mathrm{C}(9)-\mathrm{H}(9)$ & 120.9 \\
\hline $\mathrm{C}(20)-\mathrm{C}(10)-\mathrm{C}(9)$ & $130.7(3)$ & $\mathrm{C}(20)-\mathrm{C}(10)-\mathrm{C}(11)$ & 109.7(3) \\
\hline $\mathrm{C}(9)-\mathrm{C}(10)-\mathrm{C}(11)$ & $119.5(3)$ & $\mathrm{C}(12)-\mathrm{C}(11)-\mathrm{C}(27)$ & 131.2(3) \\
\hline $\mathrm{C}(12)-\mathrm{C}(11)-\mathrm{C}(10)$ & $122.3(3)$ & $\mathrm{C}(27)-\mathrm{C}(11)-\mathrm{C}(10)$ & $106.5(3)$ \\
\hline $\mathrm{C}(11)-\mathrm{C}(12)-\mathrm{C}(13)$ & $117.9(3)$ & $\mathrm{C}(11)-\mathrm{C}(12)-\mathrm{H}(12)$ & 121.1 \\
\hline $\mathrm{C}(13)-\mathrm{C}(12)-\mathrm{H}(12)$ & 121.1 & $\mathrm{C}(1)-\mathrm{C}(13)-\mathrm{C}(12)$ & $130.6(3)$ \\
\hline $\mathrm{C}(1)-\mathrm{C}(13)-\mathrm{C}(8)$ & $109.8(3)$ & $\mathrm{C}(12)-\mathrm{C}(13)-\mathrm{C}(8)$ & $119.5(3)$ \\
\hline $\mathrm{C}(15)-\mathrm{C}(14)-\mathrm{C}(19)$ & $117.6(3)$ & $\mathrm{C}(15)-\mathrm{C}(14)-\mathrm{C}(1)$ & $122.3(3)$ \\
\hline $\mathrm{C}(19)-\mathrm{C}(14)-\mathrm{C}(1)$ & 120.1(3) & $\mathrm{C}(16)-\mathrm{C}(15)-\mathrm{C}(14)$ & $120.9(3)$ \\
\hline $\mathrm{C}(16)-\mathrm{C}(15)-\mathrm{H}(15)$ & 119.6 & $\mathrm{C}(14)-\mathrm{C}(15)-\mathrm{H}(15)$ & 119.6 \\
\hline
\end{tabular}




\begin{tabular}{|c|c|c|c|}
\hline$C(15)-C(16)-C(17)$ & $121.2(3)$ & $C(15)-C(16)-C(36)$ & $121.3(3)$ \\
\hline$C(17)-C(16)-C(36)$ & $117.5(4)$ & $\mathrm{C}(18)-\mathrm{C}(17)-\mathrm{C}(16)$ & $118.2(4)$ \\
\hline $\mathrm{C}(18)-\mathrm{C}(17)-\mathrm{H}(17)$ & 120.9 & $\mathrm{C}(16)-\mathrm{C}(17)-\mathrm{H}(17)$ & 120.9 \\
\hline $\mathrm{C}(17)-\mathrm{C}(18)-\mathrm{C}(19)$ & $121.3(3)$ & $\mathrm{C}(17)-\mathrm{C}(18)-\mathrm{C}(37 \mathrm{~A})$ & $120.2(4)$ \\
\hline $\mathrm{C}(19)-\mathrm{C}(18)-\mathrm{C}(37 \mathrm{~A})$ & $118.4(4)$ & $\mathrm{C}(17)-\mathrm{C}(18)-\mathrm{C}(37 \mathrm{~B})$ & $119.9(6)$ \\
\hline $\mathrm{C}(19)-\mathrm{C}(18)-\mathrm{C}(37 \mathrm{~B})$ & $116.8(6)$ & $\mathrm{C}(18)-\mathrm{C}(19)-\mathrm{C}(14)$ & $120.7(3)$ \\
\hline $\mathrm{C}(18)-\mathrm{C}(19)-\mathrm{H}(19)$ & 119.7 & $\mathrm{C}(14)-\mathrm{C}(19)-\mathrm{H}(19)$ & 119.7 \\
\hline $\mathrm{C}(10)-\mathrm{C}(20)-\mathrm{C}(35) \# 1$ & $108.0(3)$ & $\mathrm{C}(10)-\mathrm{C}(20)-\mathrm{C}(21)$ & $125.3(3)$ \\
\hline $\mathrm{C}(35) \# 1-\mathrm{C}(20)-\mathrm{C}(21)$ & $126.8(3)$ & $\mathrm{C}(26)-\mathrm{C}(21)-\mathrm{C}(22)$ & $118.8(3)$ \\
\hline$C(26)-C(21)-C(20)$ & $120.1(3)$ & $\mathrm{C}(22)-\mathrm{C}(21)-\mathrm{C}(20)$ & $121.1(3)$ \\
\hline $\mathrm{C}(23)-\mathrm{C}(22)-\mathrm{C}(21)$ & $120.4(4)$ & $\mathrm{C}(23)-\mathrm{C}(22)-\mathrm{H}(22)$ & 119.8 \\
\hline $\mathrm{C}(21)-\mathrm{C}(22)-\mathrm{H}(22)$ & 119.8 & $\mathrm{C}(22)-\mathrm{C}(23)-\mathrm{C}(24)$ & $120.5(4)$ \\
\hline $\mathrm{C}(22)-\mathrm{C}(23)-\mathrm{C}(38)$ & $120.4(4)$ & $\mathrm{C}(24)-\mathrm{C}(23)-\mathrm{C}(38)$ & $119.2(4)$ \\
\hline$C(25)-C(24)-C(23)$ & $119.1(4)$ & $\mathrm{C}(25)-\mathrm{C}(24)-\mathrm{H}(24)$ & 120.4 \\
\hline $\mathrm{C}(23)-\mathrm{C}(24)-\mathrm{H}(24)$ & 120.4 & $\mathrm{C}(24)-\mathrm{C}(25)-\mathrm{C}(26)$ & $121.2(4)$ \\
\hline $\mathrm{C}(24)-\mathrm{C}(25)-\mathrm{C}(39)$ & $120.5(4)$ & $\mathrm{C}(26)-\mathrm{C}(25)-\mathrm{C}(39)$ & $118.3(4)$ \\
\hline$C(21)-C(26)-C(25)$ & $119.9(3)$ & $\mathrm{C}(21)-\mathrm{C}(26)-\mathrm{H}(26)$ & 120.0 \\
\hline $\mathrm{C}(25)-\mathrm{C}(26)-\mathrm{H}(26)$ & 120.0 & $\mathrm{C}(28)-\mathrm{C}(27)-\mathrm{C}(35) \# 1$ & $121.6(3)$ \\
\hline $\mathrm{C}(28)-\mathrm{C}(27)-\mathrm{C}(11)$ & 131.1(3) & $\mathrm{C}(35) \# 1-\mathrm{C}(27)-\mathrm{C}(11)$ & $107.3(3)$ \\
\hline $\mathrm{C}(27)-\mathrm{C}(28)-\mathrm{O}(1)$ & 119.2(3) & $\mathrm{C}(27)-\mathrm{C}(28)-\mathrm{C}(35)$ & $118.5(3)$ \\
\hline $\mathrm{O}(1)-\mathrm{C}(28)-\mathrm{C}(35)$ & $122.2(3)$ & $\mathrm{O}(1)-\mathrm{C}(29)-\mathrm{C}(30)$ & $107.8(3)$ \\
\hline $\mathrm{O}(1)-\mathrm{C}(29)-\mathrm{H}(29 \mathrm{~A})$ & 110.1 & $\mathrm{C}(30)-\mathrm{C}(29)-\mathrm{H}(29 \mathrm{~A})$ & 110.1 \\
\hline $\mathrm{O}(1)-\mathrm{C}(29)-\mathrm{H}(29 \mathrm{~B})$ & 110.1 & $\mathrm{C}(30)-\mathrm{C}(29)-\mathrm{H}(29 \mathrm{~B})$ & 110.1 \\
\hline $\mathrm{H}(29 \mathrm{~A})-\mathrm{C}(29)-\mathrm{H}(29 \mathrm{~B})$ & 108.5 & $\mathrm{C}(29)-\mathrm{C}(30)-\mathrm{C}(31)$ & $111.8(3)$ \\
\hline $\mathrm{C}(29)-\mathrm{C}(30)-\mathrm{H}(30 \mathrm{~A})$ & 109.2 & $\mathrm{C}(31)-\mathrm{C}(30)-\mathrm{H}(30 \mathrm{~A})$ & 109.2 \\
\hline $\mathrm{C}(29)-\mathrm{C}(30)-\mathrm{H}(30 \mathrm{~B})$ & 109.2 & $\mathrm{C}(31)-\mathrm{C}(30)-\mathrm{H}(30 \mathrm{~B})$ & 109.2 \\
\hline $\mathrm{H}(30 \mathrm{~A})-\mathrm{C}(30)-\mathrm{H}(30 \mathrm{~B})$ & 107.9 & $\mathrm{C}(32)-\mathrm{C}(31)-\mathrm{C}(30)$ & $112.5(3)$ \\
\hline $\mathrm{C}(32)-\mathrm{C}(31)-\mathrm{H}(31 \mathrm{~A})$ & 109.1 & $\mathrm{C}(30)-\mathrm{C}(31)-\mathrm{H}(31 \mathrm{~A})$ & 109.1 \\
\hline $\mathrm{C}(32)-\mathrm{C}(31)-\mathrm{H}(31 \mathrm{~B})$ & 109.1 & $\mathrm{C}(30)-\mathrm{C}(31)-\mathrm{H}(31 \mathrm{~B})$ & 109.1 \\
\hline $\mathrm{H}(31 \mathrm{~A})-\mathrm{C}(31)-\mathrm{H}(31 \mathrm{~B})$ & 107.8 & $\mathrm{C}(33)-\mathrm{C}(32)-\mathrm{C}(31)$ & $114.2(4)$ \\
\hline $\mathrm{C}(33)-\mathrm{C}(32)-\mathrm{H}(32 \mathrm{~A})$ & 108.7 & $\mathrm{C}(31)-\mathrm{C}(32)-\mathrm{H}(32 \mathrm{~A})$ & 108.7 \\
\hline $\mathrm{C}(33)-\mathrm{C}(32)-\mathrm{H}(32 \mathrm{~B})$ & 108.7 & $\mathrm{C}(31)-\mathrm{C}(32)-\mathrm{H}(32 \mathrm{~B})$ & 108.7 \\
\hline $\mathrm{H}(32 \mathrm{~A})-\mathrm{C}(32)-\mathrm{H}(32 \mathrm{~B})$ & 107.6 & $\mathrm{C}(32)-\mathrm{C}(33)-\mathrm{C}(34)$ & $111.9(5)$ \\
\hline $\mathrm{C}(32)-\mathrm{C}(33)-\mathrm{H}(33 \mathrm{~A})$ & 109.2 & $\mathrm{C}(34)-\mathrm{C}(33)-\mathrm{H}(33 \mathrm{~A})$ & 109.2 \\
\hline $\mathrm{C}(32)-\mathrm{C}(33)-\mathrm{H}(33 \mathrm{~B})$ & 109.2 & $\mathrm{C}(34)-\mathrm{C}(33)-\mathrm{H}(33 \mathrm{~B})$ & 109.2 \\
\hline $\mathrm{H}(33 \mathrm{~A})-\mathrm{C}(33)-\mathrm{H}(33 \mathrm{~B})$ & 107.9 & $\mathrm{C}(33)-\mathrm{C}(34)-\mathrm{H}(34 \mathrm{~A})$ & 109.5 \\
\hline
\end{tabular}




$\begin{array}{lclc}\mathrm{C}(33)-\mathrm{C}(34)-\mathrm{H}(34 \mathrm{~B}) & 109.5 & \mathrm{H}(34 \mathrm{~A})-\mathrm{C}(34)-\mathrm{H}(34 \mathrm{~B}) & 109.5 \\ \mathrm{C}(33)-\mathrm{C}(34)-\mathrm{H}(34 \mathrm{C}) & 109.5 & \mathrm{H}(34 \mathrm{~A})-\mathrm{C}(34)-\mathrm{H}(34 \mathrm{C}) & 109.5 \\ \mathrm{H}(34 \mathrm{~B})-\mathrm{C}(34)-\mathrm{H}(34 \mathrm{C}) & 109.5 & \mathrm{C}(28)-\mathrm{C}(35)-\mathrm{C}(27) \# 1 & 119.8(3) \\ \mathrm{C}(28)-\mathrm{C}(35)-\mathrm{C}(20) \# 1 & 131.5(3) & \mathrm{C}(27) \# 1-\mathrm{C}(35)-\mathrm{C}(20) \# 1 & 108.6(3) \\ \mathrm{F}(1 \mathrm{~A})-\mathrm{C}(36)-\mathrm{F}(3 \mathrm{~A}) & 106.4(3) & \mathrm{F}(1 \mathrm{~A})-\mathrm{C}(36)-\mathrm{F}(2 \mathrm{~A}) & 106.9(3) \\ \mathrm{F}(3 \mathrm{~A})-\mathrm{C}(36)-\mathrm{F}(2 \mathrm{~A}) & 107.4(4) & \mathrm{F}(1 \mathrm{~A})-\mathrm{C}(36)-\mathrm{C}(16) & 112.7(3) \\ \mathrm{F}(3 \mathrm{~A})-\mathrm{C}(36)-\mathrm{C}(16) & 111.7(3) & \mathrm{F}(2 \mathrm{~A})-\mathrm{C}(36)-\mathrm{C}(16) & 111.4(3) \\ \mathrm{F}(4 \mathrm{~A})-\mathrm{C}(37 \mathrm{~A})-\mathrm{F}(5 \mathrm{~A}) & 107.1(5) & \mathrm{F}(4 \mathrm{~A})-\mathrm{C}(37 \mathrm{~A})-\mathrm{F}(6 \mathrm{~A}) & 107.1(5) \\ \mathrm{F}(5 \mathrm{~A})-\mathrm{C}(37 \mathrm{~A})-\mathrm{F}(6 \mathrm{~A}) & 105.8(4) & \mathrm{F}(4 \mathrm{~A})-\mathrm{C}(37 \mathrm{~A})-\mathrm{C}(18) & 109.7(5) \\ \mathrm{F}(5 \mathrm{~A})-\mathrm{C}(37 \mathrm{~A})-\mathrm{C}(18) & 114.2(5) & \mathrm{F}(6 \mathrm{~A})-\mathrm{C}(37 \mathrm{~A})-\mathrm{C}(18) & 112.5(5) \\ \mathrm{F}(5 \mathrm{~B})-\mathrm{C}(37 \mathrm{~B})-\mathrm{F}(4 \mathrm{~B}) & 106.7(7) & \mathrm{F}(5 \mathrm{~B})-\mathrm{C}(37 \mathrm{~B})-\mathrm{F}(6 \mathrm{~B}) & 106.7(7) \\ \mathrm{F}(4 \mathrm{~B})-\mathrm{C}(37 \mathrm{~B})-\mathrm{F}(6 \mathrm{~B}) & 105.9(7) & \mathrm{F}(5 \mathrm{~B})-\mathrm{C}(37 \mathrm{~B})-\mathrm{C}(18) & 108.3(11) \\ \mathrm{F}(4 \mathrm{~B})-\mathrm{C}(37 \mathrm{~B})-\mathrm{C}(18) & 119.6(12) & \mathrm{F}(6 \mathrm{~B})-\mathrm{C}(37 \mathrm{~B})-\mathrm{C}(18) & 109.0(13) \\ \mathrm{F}(9)-\mathrm{C}(38)-\mathrm{F}(8) & 105.7(5) & \mathrm{F}(9)-\mathrm{C}(38)-\mathrm{F}(7) & 104.8(5) \\ \mathrm{F}(8)-\mathrm{C}(38)-\mathrm{F}(7) & 107.0(5) & \mathrm{F}(9)-\mathrm{C}(38)-\mathrm{C}(23) & 112.8(4) \\ \mathrm{F}(8)-\mathrm{C}(38)-\mathrm{C}(23) & 112.3(5) & \mathrm{F}(7)-\mathrm{C}(38)-\mathrm{C}(23) & 113.6(4) \\ \mathrm{F}(12)-\mathrm{C}(39)-\mathrm{F}(11) & 108.8(5) & \mathrm{F}(12)-\mathrm{C}(39)-\mathrm{F}(10) & 106.0(4) \\ \mathrm{F}(11)-\mathrm{C}(39)-\mathrm{F}(10) & 105.4(4) & \mathrm{F}(12)-\mathrm{C}(39)-\mathrm{C}(25) & 111.6(4) \\ \mathrm{F}(11)-\mathrm{C}(39)-\mathrm{C}(25) & 111.8(4) & \mathrm{F}(10)-\mathrm{C}(39)-\mathrm{C}(25) & 112.8(4) \\ & & & \\ & & & \\ & & & \end{array}$

Symmetry transformations used to generate equivalent atoms: $\quad \# 1-x+1,-y,-z$

Table S27. Anisotropic displacement parameters $\left(\AA^{2} \times 10^{3}\right)$ for IFD-CF3. The anisotropic displacement factor exponent takes the form: $-2 \square^{2}\left[h^{2} a * 2 U^{11}+\ldots+2 h k a^{*} b^{*} U^{12}\right]$

\begin{tabular}{ccccccc}
\hline & \multirow{2}{*}{$\mathrm{U}^{11}$} & \multicolumn{2}{c}{$\mathrm{U}^{33}$} & $\mathrm{U}^{23}$ & $\mathrm{U}^{13}$ & \multicolumn{2}{c}{$\mathrm{U}^{12}$} \\
& & & & & & \\
$\mathrm{O}(1)$ & $28(1)$ & $26(1)$ & $29(1)$ & $-1(1)$ & $4(1)$ & $4(1)$ \\
$\mathrm{C}(1)$ & $35(2)$ & $22(2)$ & $28(2)$ & $-1(1)$ & $4(1)$ & $-1(1)$ \\
$\mathrm{C}(2)$ & $36(2)$ & $23(2)$ & $30(2)$ & $1(1)$ & $9(1)$ & $-1(1)$ \\
$\mathrm{C}(3)$ & $50(2)$ & $21(2)$ & $42(2)$ & $-2(1)$ & $16(2)$ & $0(1)$ \\
$\mathrm{C}(4)$ & $53(2)$ & $22(2)$ & $53(2)$ & $-7(2)$ & $17(2)$ & $-8(1)$ \\
$\mathrm{C}(5)$ & $49(2)$ & $26(2)$ & $53(2)$ & $-3(2)$ & $15(2)$ & $-11(1)$ \\
$\mathrm{C}(6)$ & $37(2)$ & $28(2)$ & $40(2)$ & $-2(1)$ & $7(2)$ & $-4(1)$ \\
$\mathrm{C}(7)$ & $35(2)$ & $23(2)$ & $34(2)$ & $-1(1)$ & $6(1)$ & $-1(1)$
\end{tabular}




$\begin{array}{lllllll}\mathrm{C}(8) & 32(2) & 21(2) & 30(2) & -1(1) & 3(1) & -1(1) \\ \mathrm{C}(9) & 27(2) & 22(2) & 33(2) & 0(1) & 3(1) & -1(1) \\ \mathrm{C}(10) & 29(2) & 23(2) & 32(2) & -2(1) & 5(1) & 0(1) \\ \mathrm{C}(11) & 25(2) & 21(2) & 30(2) & -2(1) & 0(1) & 0(1) \\ \mathrm{C}(12) & 28(2) & 24(2) & 29(2) & -3(1) & 4(1) & 0(1) \\ \mathrm{C}(13) & 32(2) & 24(2) & 30(2) & 2(1) & 4(1) & 1(1) \\ \mathrm{C}(14) & 35(2) & 20(2) & 30(2) & -1(1) & 7(1) & 2(1) \\ \mathrm{C}(15) & 40(2) & 21(2) & 26(2) & -2(1) & 6(1) & -2(1) \\ \mathrm{C}(16) & 40(2) & 31(2) & 31(2) & 2(1) & 8(2) & -4(1) \\ \mathrm{C}(17) & 37(2) & 37(2) & 49(2) & 5(2) & 16(2) & 2(2) \\ \mathrm{C}(18) & 39(2) & 34(2) & 50(2) & 8(2) & 18(2) & 7(2) \\ \mathrm{C}(19) & 41(2) & 22(2) & 40(2) & 6(1) & 14(2) & 8(1) \\ \mathrm{C}(20) & 27(2) & 24(2) & 26(1) & -4(1) & 2(1) & -1(1) \\ \mathrm{C}(21) & 31(2) & 18(1) & 36(2) & 1(1) & 10(1) & 1(1) \\ \mathrm{C}(22) & 31(2) & 27(2) & 44(2) & -4(1) & 10(2) & 0(1) \\ \mathrm{C}(23) & 33(2) & 37(2) & 55(2) & -6(2) & 8(2) & 2(1) \\ \mathrm{C}(24) & 39(2) & 34(2) & 59(2) & -2(2) & 23(2) & 4(1) \\ \mathrm{C}(25) & 51(2) & 29(2) & 46(2) & 4(1) & 26(2) & 5(1) \\ \mathrm{C}(26) & 43(2) & 25(2) & 37(2) & 2(1) & 11(2) & -1(1) \\ \mathrm{C}(27) & 26(2) & 21(2) & 29(2) & -3(1) & 3(1) & 0(1) \\ \mathrm{C}(28) & 24(2) & 22(2) & 28(2) & -1(1) & 2(1) & 1(1) \\ \mathrm{C}(29) & 32(2) & 25(2) & 31(2) & 1(1) & 2(1) & 6(1) \\ & & & & & & \\ \end{array}$

$\begin{array}{llllll}\mathrm{U}^{11} & \mathrm{U}^{22} & \mathrm{U}^{33} & \mathrm{U}^{23} & \mathrm{U}^{13} & \mathrm{U}^{12}\end{array}$

$\begin{array}{lllrrrr}\mathrm{C}(30) & 31(2) & 31(2) & 37(2) & -1(1) & 3(1) & 5(1) \\ \mathrm{C}(31) & 31(2) & 39(2) & 42(2) & -1(2) & 1(2) & 5(1) \\ \mathrm{C}(32) & 29(2) & 53(2) & 51(2) & -2(2) & 2(2) & 8(2) \\ \mathrm{C}(33) & 44(2) & 46(2) & 65(3) & 3(2) & 10(2) & 14(2) \\ \mathrm{C}(34) & 60(3) & 66(4) & 103(5) & -6(3) & 14(3) & 24(3) \\ \mathrm{C}(35) & 28(2) & 22(2) & 28(2) & -1(1) & 2(1) & 1(1) \\ \mathrm{C}(36) & 43(2) & 38(2) & 38(2) & 3(2) & 10(2) & -5(2) \\ \mathrm{F}(1 \mathrm{~A}) & 73(2) & 34(1) & 72(2) & 13(1) & 33(2) & -4(1) \\ \mathrm{F}(2 \mathrm{~A}) & 68(2) & 75(2) & 49(2) & 13(1) & -2(1) & -39(2) \\ \mathrm{F}(3 \mathrm{~A}) & 55(2) & 55(2) & 47(1) & 6(1) & 23(1) & -8(1) \\ & & & & & & \mathrm{S} 111\end{array}$




$\begin{array}{lcccccc}\mathrm{C}(37 \mathrm{~A}) & 40(4) & 36(4) & 49(5) & 4(3) & 21(3) & 10(3) \\ \mathrm{F}(4 \mathrm{~A}) & 87(4) & 56(3) & 56(3) & 7(3) & -5(3) & 34(2) \\ \mathrm{F}(5 \mathrm{~A}) & 56(3) & 44(2) & 67(3) & 5(2) & 31(2) & 18(2) \\ \mathrm{F}(6 \mathrm{~A}) & 56(3) & 28(2) & 91(4) & 10(3) & 22(3) & 8(2) \\ \mathrm{C}(37 \mathrm{~B}) & 40(10) & 48(11) & 51(14) & 14(10) & 13(9) & 9(7) \\ \mathrm{F}(4 \mathrm{~B}) & 71(7) & 73(8) & 79(8) & 13(6) & 3(6) & 9(6) \\ \mathrm{F}(5 \mathrm{~B}) & 76(8) & 87(8) & 102(8) & 13(7) & 46(7) & 32(6) \\ \mathrm{F}(6 \mathrm{~B}) & 74(9) & 45(7) & 84(9) & 14(6) & 28(7) & 21(6) \\ \mathrm{C}(38) & 30(2) & 77(4) & 76(4) & -27(3) & 8(2) & -2(2) \\ \mathrm{F}(7) & 45(2) & 156(4) & 76(2) & -38(2) & -6(2) & -4(2) \\ \mathrm{F}(8) & 84(3) & 107(3) & 141(4) & -10(3) & -7(3) & -50(2) \\ \mathrm{F}(9) & 54(2) & 111(3) & 114(3) & -19(2) & -13(2) & 28(2) \\ \mathrm{C}(39) & 64(3) & 60(3) & 52(3) & 4(2) & 31(2) & 3(2) \\ \mathrm{F}(10) & 82(2) & 113(3) & 84(2) & 2(2) & 58(2) & 4(2) \\ \mathrm{F}(11) & 137(3) & 65(2) & 74(2) & -27(2) & 55(2) & -10(2) \\ \mathrm{F}(12) & 93(3) & 132(3) & 49(2) & 17(2) & 30(2) & 20(2)\end{array}$

Table S28. Torsion angles [ $\left.{ }^{\circ}\right]$ for IFD-CF3.

$\begin{array}{lr}\mathrm{C}(13)-\mathrm{C}(1)-\mathrm{C}(2)-\mathrm{C}(3) & -178.5(4) \\ \mathrm{C}(14)-\mathrm{C}(1)-\mathrm{C}(2)-\mathrm{C}(3) & 1.1(6) \\ \mathrm{C}(13)-\mathrm{C}(1)-\mathrm{C}(2)-\mathrm{C}(7) & -0.8(4) \\ \mathrm{C}(14)-\mathrm{C}(1)-\mathrm{C}(2)-\mathrm{C}(7) & 178.8(3) \\ \mathrm{C}(7)-\mathrm{C}(2)-\mathrm{C}(3)-\mathrm{C}(4) & 2.9(5) \\ \mathrm{C}(1)-\mathrm{C}(2)-\mathrm{C}(3)-\mathrm{C}(4) & -179.6(4) \\ \mathrm{C}(2)-\mathrm{C}(3)-\mathrm{C}(4)-\mathrm{C}(5) & -2.2(6) \\ \mathrm{C}(3)-\mathrm{C}(4)-\mathrm{C}(5)-\mathrm{C}(6) & 0.0(7) \\ \mathrm{C}(4)-\mathrm{C}(5)-\mathrm{C}(6)-\mathrm{C}(7) & 1.3(6) \\ \mathrm{C}(5)-\mathrm{C}(6)-\mathrm{C}(7)-\mathrm{C}(2) & -0.6(6) \\ \mathrm{C}(5)-\mathrm{C}(6)-\mathrm{C}(7)-\mathrm{C}(8) & 179.7(4) \\ \mathrm{C}(3)-\mathrm{C}(2)-\mathrm{C}(7)-\mathrm{C}(6) & -1.6(5) \\ \mathrm{C}(1)-\mathrm{C}(2)-\mathrm{C}(7)-\mathrm{C}(6) & -179.6(3) \\ \mathrm{C}(3)-\mathrm{C}(2)-\mathrm{C}(7)-\mathrm{C}(8) & 178.3(3) \\ \mathrm{C}(1)-\mathrm{C}(2)-\mathrm{C}(7)-\mathrm{C}(8) & 0.2(4)\end{array}$




\begin{tabular}{|c|c|}
\hline $\mathrm{C}(6)-\mathrm{C}(7)-\mathrm{C}(8)-\mathrm{C}(9)$ & $1.0(6)$ \\
\hline $\mathrm{C}(2)-\mathrm{C}(7)-\mathrm{C}(8)-\mathrm{C}(9)$ & $-178.8(3)$ \\
\hline$C(6)-C(7)-C(8)-C(13)$ & $-179.8(4)$ \\
\hline $\mathrm{C}(2)-\mathrm{C}(7)-\mathrm{C}(8)-\mathrm{C}(13)$ & $0.4(4)$ \\
\hline $\mathrm{C}(13)-\mathrm{C}(8)-\mathrm{C}(9)-\mathrm{C}(10)$ & $0.9(5)$ \\
\hline $\mathrm{C}(7)-\mathrm{C}(8)-\mathrm{C}(9)-\mathrm{C}(10)$ & $-180.0(3)$ \\
\hline $\mathrm{C}(8)-\mathrm{C}(9)-\mathrm{C}(10)-\mathrm{C}(20)$ & $-179.5(3)$ \\
\hline $\mathrm{C}(8)-\mathrm{C}(9)-\mathrm{C}(10)-\mathrm{C}(11)$ & $3.1(5)$ \\
\hline $\mathrm{C}(20)-\mathrm{C}(10)-\mathrm{C}(11)-\mathrm{C}(12)$ & $178.6(3)$ \\
\hline $\mathrm{C}(9)-\mathrm{C}(10)-\mathrm{C}(11)-\mathrm{C}(12)$ & $-3.5(5)$ \\
\hline $\mathrm{C}(20)-\mathrm{C}(10)-\mathrm{C}(11)-\mathrm{C}(27)$ & $0.5(4)$ \\
\hline $\mathrm{C}(9)-\mathrm{C}(10)-\mathrm{C}(11)-\mathrm{C}(27)$ & $178.4(3)$ \\
\hline $\mathrm{C}(27)-\mathrm{C}(11)-\mathrm{C}(12)-\mathrm{C}(13)$ & $177.3(3)$ \\
\hline $\mathrm{C}(10)-\mathrm{C}(11)-\mathrm{C}(12)-\mathrm{C}(13)$ & $-0.3(5)$ \\
\hline $\mathrm{C}(14)-\mathrm{C}(1)-\mathrm{C}(13)-\mathrm{C}(12)$ & $4.9(6)$ \\
\hline $\mathrm{C}(2)-\mathrm{C}(1)-\mathrm{C}(13)-\mathrm{C}(12)$ & $-175.5(3)$ \\
\hline $\mathrm{C}(14)-\mathrm{C}(1)-\mathrm{C}(13)-\mathrm{C}(8)$ & $-178.5(3)$ \\
\hline $\mathrm{C}(2)-\mathrm{C}(1)-\mathrm{C}(13)-\mathrm{C}(8)$ & $1.1(4)$ \\
\hline $\mathrm{C}(11)-\mathrm{C}(12)-\mathrm{C}(13)-\mathrm{C}(1)$ & $-179.5(3)$ \\
\hline $\mathrm{C}(11)-\mathrm{C}(12)-\mathrm{C}(13)-\mathrm{C}(8)$ & $4.2(5)$ \\
\hline $\mathrm{C}(9)-\mathrm{C}(8)-\mathrm{C}(13)-\mathrm{C}(1)$ & $178.3(3)$ \\
\hline $\mathrm{C}(7)-\mathrm{C}(8)-\mathrm{C}(13)-\mathrm{C}(1)$ & $-1.0(4)$ \\
\hline $\mathrm{C}(9)-\mathrm{C}(8)-\mathrm{C}(13)-\mathrm{C}(12)$ & $-4.7(5)$ \\
\hline $\mathrm{C}(7)-\mathrm{C}(8)-\mathrm{C}(13)-\mathrm{C}(12)$ & $176.0(3)$ \\
\hline $\mathrm{C}(13)-\mathrm{C}(1)-\mathrm{C}(14)-\mathrm{C}(15)$ & $33.5(5)$ \\
\hline $\mathrm{C}(2)-\mathrm{C}(1)-\mathrm{C}(14)-\mathrm{C}(15)$ & $-146.1(3)$ \\
\hline $\mathrm{C}(13)-\mathrm{C}(1)-\mathrm{C}(14)-\mathrm{C}(19)$ & $-147.1(4)$ \\
\hline $\mathrm{C}(2)-\mathrm{C}(1)-\mathrm{C}(14)-\mathrm{C}(19)$ & $33.3(5)$ \\
\hline $\mathrm{C}(19)-\mathrm{C}(14)-\mathrm{C}(15)-\mathrm{C}(16)$ & $1.4(5)$ \\
\hline $\mathrm{C}(1)-\mathrm{C}(14)-\mathrm{C}(15)-\mathrm{C}(16)$ & $-179.3(3)$ \\
\hline $\mathrm{C}(14)-\mathrm{C}(15)-\mathrm{C}(16)-\mathrm{C}(17)$ & $-3.1(5)$ \\
\hline $\mathrm{C}(14)-\mathrm{C}(15)-\mathrm{C}(16)-\mathrm{C}(36)$ & $177.3(3)$ \\
\hline $\mathrm{C}(15)-\mathrm{C}(16)-\mathrm{C}(17)-\mathrm{C}(18)$ & $1.5(6)$ \\
\hline$C(36)-C(16)-C(17)-C(18)$ & $-178.9(4)$ \\
\hline $\mathrm{C}(16)-\mathrm{C}(17)-\mathrm{C}(18)-\mathrm{C}(19)$ & $1.7(6)$ \\
\hline $\mathrm{C}(16)-\mathrm{C}(17)-\mathrm{C}(18)-\mathrm{C}(37 \mathrm{~A})$ & $-174.6(4)$ \\
\hline
\end{tabular}




\begin{tabular}{|c|c|}
\hline $\mathrm{C}(16)-\mathrm{C}(17)-\mathrm{C}(18)-\mathrm{C}(37 \mathrm{~B})$ & $164.7(7)$ \\
\hline $\mathrm{C}(17)-\mathrm{C}(18)-\mathrm{C}(19)-\mathrm{C}(14)$ & $-3.4(6)$ \\
\hline $\mathrm{C}(37 \mathrm{~A})-\mathrm{C}(18)-\mathrm{C}(19)-\mathrm{C}(14)$ & $173.0(4)$ \\
\hline $\mathrm{C}(37 \mathrm{~B})-\mathrm{C}(18)-\mathrm{C}(19)-\mathrm{C}(14)$ & $-166.9(7)$ \\
\hline $\mathrm{C}(15)-\mathrm{C}(14)-\mathrm{C}(19)-\mathrm{C}(18)$ & $1.8(5)$ \\
\hline $\mathrm{C}(1)-\mathrm{C}(14)-\mathrm{C}(19)-\mathrm{C}(18)$ & $-177.6(3)$ \\
\hline $\mathrm{C}(9)-\mathrm{C}(10)-\mathrm{C}(20)-\mathrm{C}(35) \# 1$ & $-178.3(3)$ \\
\hline$C(11)-C(10)-C(20)-C(35) \# 1$ & $-0.6(4)$ \\
\hline $\mathrm{C}(9)-\mathrm{C}(10)-\mathrm{C}(20)-\mathrm{C}(21)$ & $0.3(6)$ \\
\hline $\mathrm{C}(11)-\mathrm{C}(10)-\mathrm{C}(20)-\mathrm{C}(21)$ & $178.0(3)$ \\
\hline $\mathrm{C}(10)-\mathrm{C}(20)-\mathrm{C}(21)-\mathrm{C}(26)$ & $-122.3(4)$ \\
\hline $\mathrm{C}(35) \# 1-\mathrm{C}(20)-\mathrm{C}(21)-\mathrm{C}(26)$ & $56.1(5)$ \\
\hline $\mathrm{C}(10)-\mathrm{C}(20)-\mathrm{C}(21)-\mathrm{C}(22)$ & $56.8(5)$ \\
\hline $\mathrm{C}(35) \# 1-\mathrm{C}(20)-\mathrm{C}(21)-\mathrm{C}(22)$ & $-124.8(4)$ \\
\hline $\mathrm{C}(26)-\mathrm{C}(21)-\mathrm{C}(22)-\mathrm{C}(23)$ & $0.8(5)$ \\
\hline$C(20)-C(21)-C(22)-C(23)$ & $-178.3(3)$ \\
\hline $\mathrm{C}(21)-\mathrm{C}(22)-\mathrm{C}(23)-\mathrm{C}(24)$ & $1.9(6)$ \\
\hline $\mathrm{C}(21)-\mathrm{C}(22)-\mathrm{C}(23)-\mathrm{C}(38)$ & $-178.5(4)$ \\
\hline $\mathrm{C}(22)-\mathrm{C}(23)-\mathrm{C}(24)-\mathrm{C}(25)$ & $-2.3(6)$ \\
\hline $\mathrm{C}(38)-\mathrm{C}(23)-\mathrm{C}(24)-\mathrm{C}(25)$ & $178.1(4)$ \\
\hline $\mathrm{C}(23)-\mathrm{C}(24)-\mathrm{C}(25)-\mathrm{C}(26)$ & $0.0(6)$ \\
\hline $\mathrm{C}(23)-\mathrm{C}(24)-\mathrm{C}(25)-\mathrm{C}(39)$ & $-179.7(4)$ \\
\hline$C(22)-C(21)-C(26)-C(25)$ & $-3.1(5)$ \\
\hline$C(20)-C(21)-C(26)-C(25)$ & $176.0(3)$ \\
\hline $\mathrm{C}(24)-\mathrm{C}(25)-\mathrm{C}(26)-\mathrm{C}(21)$ & $2.7(5)$ \\
\hline $\mathrm{C}(39)-\mathrm{C}(25)-\mathrm{C}(26)-\mathrm{C}(21)$ & $-177.5(3)$ \\
\hline $\mathrm{C}(12)-\mathrm{C}(11)-\mathrm{C}(27)-\mathrm{C}(28)$ & $-0.4(6)$ \\
\hline $\mathrm{C}(10)-\mathrm{C}(11)-\mathrm{C}(27)-\mathrm{C}(28)$ & $177.4(3)$ \\
\hline $\mathrm{C}(12)-\mathrm{C}(11)-\mathrm{C}(27)-\mathrm{C}(35) \# 1$ & $-178.0(3)$ \\
\hline $\mathrm{C}(10)-\mathrm{C}(11)-\mathrm{C}(27)-\mathrm{C}(35) \# 1$ & $-0.1(4)$ \\
\hline $\mathrm{C}(35) \# 1-\mathrm{C}(27)-\mathrm{C}(28)-\mathrm{O}(1)$ & 179.1(3) \\
\hline $\mathrm{C}(11)-\mathrm{C}(27)-\mathrm{C}(28)-\mathrm{O}(1)$ & $1.9(5)$ \\
\hline $\mathrm{C}(35) \# 1-\mathrm{C}(27)-\mathrm{C}(28)-\mathrm{C}(35)$ & $0.6(5)$ \\
\hline $\mathrm{C}(11)-\mathrm{C}(27)-\mathrm{C}(28)-\mathrm{C}(35)$ & $-176.6(3)$ \\
\hline $\mathrm{C}(29)-\mathrm{O}(1)-\mathrm{C}(28)-\mathrm{C}(27)$ & $95.9(3)$ \\
\hline $\mathrm{C}(29)-\mathrm{O}(1)-\mathrm{C}(28)-\mathrm{C}(35)$ & $-85.7(4)$ \\
\hline
\end{tabular}




\begin{tabular}{|c|c|}
\hline $\mathrm{C}(28)-\mathrm{O}(1)-\mathrm{C}(29)-\mathrm{C}(30)$ & $-172.6(3)$ \\
\hline $\mathrm{O}(1)-\mathrm{C}(29)-\mathrm{C}(30)-\mathrm{C}(31)$ & $-168.5(3)$ \\
\hline $\mathrm{C}(29)-\mathrm{C}(30)-\mathrm{C}(31)-\mathrm{C}(32)$ & $-172.0(3)$ \\
\hline $\mathrm{C}(30)-\mathrm{C}(31)-\mathrm{C}(32)-\mathrm{C}(33)$ & $75.7(5)$ \\
\hline $\mathrm{C}(31)-\mathrm{C}(32)-\mathrm{C}(33)-\mathrm{C}(34)$ & $-177.0(4)$ \\
\hline $\mathrm{C}(27)-\mathrm{C}(28)-\mathrm{C}(35)-\mathrm{C}(27) \# 1$ & $-0.6(5)$ \\
\hline $\mathrm{O}(1)-\mathrm{C}(28)-\mathrm{C}(35)-\mathrm{C}(27) \# 1$ & $-179.1(3)$ \\
\hline $\mathrm{C}(27)-\mathrm{C}(28)-\mathrm{C}(35)-\mathrm{C}(20) \# 1$ & $-177.4(3)$ \\
\hline $\mathrm{O}(1)-\mathrm{C}(28)-\mathrm{C}(35)-\mathrm{C}(20) \# 1$ & $4.2(5)$ \\
\hline $\mathrm{C}(15)-\mathrm{C}(16)-\mathrm{C}(36)-\mathrm{F}(1 \mathrm{~A})$ & $7.4(5)$ \\
\hline $\mathrm{C}(17)-\mathrm{C}(16)-\mathrm{C}(36)-\mathrm{F}(1 \mathrm{~A})$ & $-172.2(4)$ \\
\hline $\mathrm{C}(15)-\mathrm{C}(16)-\mathrm{C}(36)-\mathrm{F}(3 \mathrm{~A})$ & 127.1(4) \\
\hline $\mathrm{C}(17)-\mathrm{C}(16)-\mathrm{C}(36)-\mathrm{F}(3 \mathrm{~A})$ & $-52.5(5)$ \\
\hline $\mathrm{C}(15)-\mathrm{C}(16)-\mathrm{C}(36)-\mathrm{F}(2 \mathrm{~A})$ & $-112.7(4)$ \\
\hline $\mathrm{C}(17)-\mathrm{C}(16)-\mathrm{C}(36)-\mathrm{F}(2 \mathrm{~A})$ & $67.7(5)$ \\
\hline $\mathrm{C}(17)-\mathrm{C}(18)-\mathrm{C}(37 \mathrm{~A})-\mathrm{F}(4 \mathrm{~A})$ & $-92.5(6)$ \\
\hline $\mathrm{C}(19)-\mathrm{C}(18)-\mathrm{C}(37 \mathrm{~A})-\mathrm{F}(4 \mathrm{~A})$ & $91.1(5)$ \\
\hline $\mathrm{C}(17)-\mathrm{C}(18)-\mathrm{C}(37 \mathrm{~A})-\mathrm{F}(5 \mathrm{~A})$ & $27.7(7)$ \\
\hline $\mathrm{C}(19)-\mathrm{C}(18)-\mathrm{C}(37 \mathrm{~A})-\mathrm{F}(5 \mathrm{~A})$ & $-148.7(4)$ \\
\hline $\mathrm{C}(17)-\mathrm{C}(18)-\mathrm{C}(37 \mathrm{~A})-\mathrm{F}(6 \mathrm{~A})$ & $148.4(5)$ \\
\hline $\mathrm{C}(19)-\mathrm{C}(18)-\mathrm{C}(37 \mathrm{~A})-\mathrm{F}(6 \mathrm{~A})$ & $-28.1(6)$ \\
\hline $\mathrm{C}(17)-\mathrm{C}(18)-\mathrm{C}(37 \mathrm{~B})-\mathrm{F}(5 \mathrm{~B})$ & $32.1(11)$ \\
\hline $\mathrm{C}(19)-\mathrm{C}(18)-\mathrm{C}(37 \mathrm{~B})-\mathrm{F}(5 \mathrm{~B})$ & $-164.1(8)$ \\
\hline $\mathrm{C}(17)-\mathrm{C}(18)-\mathrm{C}(37 \mathrm{~B})-\mathrm{F}(4 \mathrm{~B})$ & $-90.3(10)$ \\
\hline $\mathrm{C}(19)-\mathrm{C}(18)-\mathrm{C}(37 \mathrm{~B})-\mathrm{F}(4 \mathrm{~B})$ & $73.4(11)$ \\
\hline $\mathrm{C}(17)-\mathrm{C}(18)-\mathrm{C}(37 \mathrm{~B})-\mathrm{F}(6 \mathrm{~B})$ & $147.8(8)$ \\
\hline $\mathrm{C}(19)-\mathrm{C}(18)-\mathrm{C}(37 \mathrm{~B})-\mathrm{F}(6 \mathrm{~B})$ & $-48.4(9)$ \\
\hline $\mathrm{C}(22)-\mathrm{C}(23)-\mathrm{C}(38)-\mathrm{F}(9)$ & $126.8(5)$ \\
\hline $\mathrm{C}(24)-\mathrm{C}(23)-\mathrm{C}(38)-\mathrm{F}(9)$ & $-53.6(7)$ \\
\hline $\mathrm{C}(22)-\mathrm{C}(23)-\mathrm{C}(38)-\mathrm{F}(8)$ & $-113.9(5)$ \\
\hline $\mathrm{C}(24)-\mathrm{C}(23)-\mathrm{C}(38)-\mathrm{F}(8)$ & $65.7(6)$ \\
\hline $\mathrm{C}(22)-\mathrm{C}(23)-\mathrm{C}(38)-\mathrm{F}(7)$ & $7.7(7)$ \\
\hline $\mathrm{C}(24)-\mathrm{C}(23)-\mathrm{C}(38)-\mathrm{F}(7)$ & $-172.7(5)$ \\
\hline $\mathrm{C}(24)-\mathrm{C}(25)-\mathrm{C}(39)-\mathrm{F}(12)$ & $-135.4(5)$ \\
\hline $\mathrm{C}(26)-\mathrm{C}(25)-\mathrm{C}(39)-\mathrm{F}(12)$ & $44.8(6)$ \\
\hline $\mathrm{C}(24)-\mathrm{C}(25)-\mathrm{C}(39)-\mathrm{F}(11)$ & $102.5(5)$ \\
\hline
\end{tabular}



$\mathrm{C}(26)-\mathrm{C}(25)-\mathrm{C}(39)-\mathrm{F}(11)$
$-77.3(5)$
$\mathrm{C}(24)-\mathrm{C}(25)-\mathrm{C}(39)-\mathrm{F}(10)$
$-16.1(6)$
$\mathrm{C}(26)-\mathrm{C}(25)-\mathrm{C}(39)-\mathrm{F}(10)$
$164.1(4)$

Symmetry transformations used to generate equivalent atoms: $\quad \# 1-x+1,-y,-z$

\section{Data for HZ-M}

Table S29. Crystal data and structure refinement for HZ-M.

Identification code

Empirical formula

Formula weight

Temperature

Wavelength

Crystal system

Space group

Unit cell dimensions

Volume

Z

Density (calculated)

Absorption coefficient

$\mathrm{F}(000)$

Crystal size

Theta range for data collection

Index ranges

Reflections collected

Independent reflections

Completeness to theta $=25.242^{\circ}$

Absorption correction

Max. and min. transmission

Refinement method

Data / restraints / parameters

Goodness-of-fit on $\mathrm{F}^{2}$
HZ-M

$\mathrm{C}_{58} \mathrm{H}_{60} \mathrm{O}_{2}$

789.06

100(2) K

$0.71073 \AA$

Monoclinic

$\mathrm{C} 2 / \mathrm{c}$

$$
\begin{array}{ll}
\mathrm{a}=26.3642(16) \AA & \mathrm{a}=90^{\circ} . \\
\mathrm{b}=16.1386(9) \AA & \mathrm{b}=119.4610(18)^{\circ} . \\
\mathrm{c}=23.420(2) \AA & \mathrm{g}=90^{\circ} .
\end{array}
$$

8676.1(11) $\AA^{3}$

8

$1.208 \mathrm{Mg} / \mathrm{m}^{3}$

$0.071 \mathrm{~mm}^{-1}$

3392

$0.260 \times 0.240 \times 0.200 \mathrm{~mm}^{3}$

1.911 to $28.282^{\circ}$.

$-34<=\mathrm{h}<=35,-21<=\mathrm{k}<=21,-31<=1<=31$

163291

$10778[\mathrm{R}$ (int) $=0.0332]$

$99.9 \%$

Semi-empirical from equivalents

0.7457 and 0.7251

Full-matrix least-squares on $\mathrm{F}^{2}$

10778 / 0 / 549

1.021 
Final $\mathrm{R}$ indices [I>2sigma(I)]

$\mathrm{R} 1=0.0513, \mathrm{wR} 2=0.1386$

$\mathrm{R}$ indices (all data)

$\mathrm{R} 1=0.0613, \mathrm{wR} 2=0.1481$

Extinction coefficient

$\mathrm{n} / \mathrm{a}$

Largest diff. peak and hole

0.548 and -0.248 e. $\AA^{-3}$

Table S30. ( x 10 $)$ and equivalent isotropic displacement parameters $\left(\AA^{2} \times 10^{3}\right)$ for HZ-M. U(eq) is defined as one third of the trace of the orthogonalized $U^{i j}$ tensor.

\begin{tabular}{|c|c|c|c|c|}
\hline & & & $\mathrm{z}$ & $\mathrm{U}(\mathrm{eq})$ \\
\hline $\mathrm{O}(1)$ & $5878(1)$ & $6729(1)$ & $4194(1)$ & $23(1)$ \\
\hline $\mathrm{O}(2)$ & $3443(1)$ & $5982(1)$ & $3953(1)$ & $28(1)$ \\
\hline $\mathrm{C}(1)$ & $7877(1)$ & $8226(1)$ & $5219(1)$ & $17(1)$ \\
\hline $\mathrm{C}(2)$ & 8101(1) & $7442(1)$ & $5300(1)$ & 19(1) \\
\hline$C(3)$ & $7753(1)$ & $6702(1)$ & $5092(1)$ & $18(1)$ \\
\hline$C(4)$ & $8027(1)$ & $5943(1)$ & $5192(1)$ & $20(1)$ \\
\hline $\mathrm{C}(5)$ & $7690(1)$ & $5192(1)$ & 4999(1) & $20(1)$ \\
\hline$C(6)$ & 7961(1) & $4419(1)$ & $5111(1)$ & $24(1)$ \\
\hline $\mathrm{C}(7)$ & $7638(1)$ & $3682(1)$ & $4918(1)$ & $26(1)$ \\
\hline $\mathrm{C}(8)$ & $7045(1)$ & $3707(1)$ & $4623(1)$ & $25(1)$ \\
\hline $\mathrm{C}(9)$ & $6748(1)$ & $4474(1)$ & $4512(1)$ & $21(1)$ \\
\hline$C(10)$ & $6136(1)$ & $4503(1)$ & $4230(1)$ & $24(1)$ \\
\hline $\mathrm{C}(11)$ & $5852(1)$ & $5239(1)$ & $4130(1)$ & $23(1)$ \\
\hline$C(12)$ & $6158(1)$ & 5994(1) & $4292(1)$ & $20(1)$ \\
\hline$C(13)$ & $6772(1)$ & $6011(1)$ & $4577(1)$ & $18(1)$ \\
\hline$C(14)$ & $7066(1)$ & $5234(1)$ & $4692(1)$ & $18(1)$ \\
\hline$C(15)$ & $5258(1)$ & $6751(1)$ & $3968(1)$ & $24(1)$ \\
\hline$C(16)$ & 4901(1) & $6669(1)$ & $3226(1)$ & $25(1)$ \\
\hline$C(17)$ & $4247(1)$ & $6610(1)$ & 2986(1) & $27(1)$ \\
\hline $\mathrm{C}(18)$ & $3890(1)$ & $6453(1)$ & $2248(1)$ & $26(1)$ \\
\hline$C(19)$ & $3232(1)$ & $6389(1)$ & 1992(1) & $28(1)$ \\
\hline$C(20)$ & $2946(1)$ & $7208(1)$ & 1970(1) & $38(1)$ \\
\hline$C(21)$ & $8677(1)$ & $5902(1)$ & $5484(1)$ & $21(1)$ \\
\hline $\mathrm{C}(22)$ & $9039(1)$ & $5831(1)$ & $6163(1)$ & $29(1)$ \\
\hline$C(23)$ & $9645(1)$ & $5817(1)$ & $6419(1)$ & $34(1)$ \\
\hline $\mathrm{C}(24)$ & $9896(1)$ & $5884(1)$ & $6023(1)$ & $31(1)$ \\
\hline
\end{tabular}




\begin{tabular}{|c|c|c|c|c|}
\hline$C(25)$ & $9530(1)$ & $5960(1)$ & $5352(1)$ & $28(1)$ \\
\hline$C(26)$ & $8925(1)$ & $5968(1)$ & $5074(1)$ & $24(1)$ \\
\hline $\mathrm{C}(27)$ & $8786(1)$ & $5803(1)$ & $6615(1)$ & $44(1)$ \\
\hline $\mathrm{C}(28)$ & 10551(1) & $5889(1)$ & $6306(1)$ & $42(1)$ \\
\hline $\mathrm{C}(29)$ & $8547(1)$ & $6079(1)$ & $4347(1)$ & $35(1)$ \\
\hline $\mathrm{C}(30)$ & $5339(1)$ & $4251(1)$ & $5115(1)$ & $16(1)$ \\
\hline $\mathrm{C}(31)$ & $5534(1)$ & $4888(1)$ & $5563(1)$ & $16(1)$ \\
\hline $\mathrm{C}(32)$ & $5227(1)$ & $5651(1)$ & $5470(1)$ & $16(1)$ \\
\hline $\mathrm{C}(33)$ & $5481(1)$ & $6289(1)$ & $5918(1)$ & $17(1)$ \\
\hline$C(34)$ & $5205(1)$ & 7090(1) & $5789(1)$ & 18(1) \\
\hline $\mathrm{C}(35)$ & $5481(1)$ & $7763(1)$ & $6201(1)$ & $22(1)$ \\
\hline$C(36)$ & $5213(1)$ & $8542(1)$ & $6089(1)$ & $24(1)$ \\
\hline $\mathrm{C}(37)$ & $4662(1)$ & $8651(1)$ & $5573(1)$ & $23(1)$ \\
\hline $\mathrm{C}(38)$ & $4360(1)$ & 7984(1) & $5146(1)$ & $21(1)$ \\
\hline C(39) & $3783(1)$ & $8076(1)$ & $4634(1)$ & $26(1)$ \\
\hline $\mathrm{C}(40)$ & $3481(1)$ & $7417(1)$ & $4250(1)$ & $28(1)$ \\
\hline $\mathrm{C}(41)$ & $3749(1)$ & $6639(1)$ & 4334(1) & $22(1)$ \\
\hline $\mathrm{C}(42)$ & $4338(1)$ & $6522(1)$ & $4802(1)$ & $17(1)$ \\
\hline $\mathrm{C}(43)$ & $4634(1)$ & $7198(1)$ & $5236(1)$ & $18(1)$ \\
\hline$C(44)$ & $2826(1)$ & $5928(1)$ & $3744(1)$ & $26(1)$ \\
\hline$C(45)$ & $2452(1)$ & 6191(1) & $3035(1)$ & $26(1)$ \\
\hline$C(46)$ & $1802(1)$ & $6120(1)$ & $2815(1)$ & $27(1)$ \\
\hline $\mathrm{C}(47)$ & $1599(1)$ & $6731(1)$ & $3160(1)$ & $29(1)$ \\
\hline $\mathrm{C}(48)$ & $962(1)$ & $6650(1)$ & 2974(1) & $35(1)$ \\
\hline $\mathrm{C}(49)$ & $784(1)$ & $7262(1)$ & $3333(1)$ & $34(1)$ \\
\hline$C(50)$ & $6053(1)$ & $6165(1)$ & $6530(1)$ & $17(1)$ \\
\hline $\mathrm{C}(51)$ & $6575(1)$ & $6217(1)$ & $6510(1)$ & $21(1)$ \\
\hline $\mathrm{C}(52)$ & 7101(1) & 6081(1) & $7082(1)$ & $24(1)$ \\
\hline$C(53)$ & $7123(1)$ & $5895(1)$ & $7672(1)$ & $24(1)$ \\
\hline $\mathrm{C}(54)$ & $6604(1)$ & $5855(1)$ & $7688(1)$ & $23(1)$ \\
\hline$C(55)$ & $6067(1)$ & $5986(1)$ & $7125(1)$ & $20(1)$ \\
\hline$C(56)$ & $6570(1)$ & $6386(1)$ & $5875(1)$ & $30(1)$ \\
\hline $\mathrm{C}(57)$ & $7698(1)$ & $5716(1)$ & $8275(1)$ & $36(1)$ \\
\hline$C(58)$ & $5513(1)$ & $5919(1)$ & $7161(1)$ & \\
\hline
\end{tabular}


Table S31. Bond lengths $[\AA]$ and angles $\left[{ }^{\circ}\right]$ for HZ-M.

\begin{tabular}{|c|c|c|c|}
\hline $\mathrm{O}(1)-\mathrm{C}(12)$ & $1.3552(15)$ & $\mathrm{C}(16)-\mathrm{H}(16 \mathrm{~A})$ & 0.9900 \\
\hline $\mathrm{O}(1)-\mathrm{C}(15)$ & $1.4488(14)$ & $\mathrm{C}(16)-\mathrm{H}(16 \mathrm{~B})$ & 0.9900 \\
\hline $\mathrm{O}(2)-\mathrm{C}(41)$ & $1.3648(15)$ & $\mathrm{C}(17)-\mathrm{C}(18)$ & $1.5276(19)$ \\
\hline $\mathrm{O}(2)-\mathrm{C}(44)$ & $1.4515(15)$ & $\mathrm{C}(17)-\mathrm{H}(17 \mathrm{~A})$ & 0.9900 \\
\hline C(1)-C(2) & $1.3699(17)$ & $\mathrm{C}(17)-\mathrm{H}(17 \mathrm{~B})$ & 0.9900 \\
\hline $\mathrm{C}(1)-\mathrm{C}(3) \# 1$ & $1.4543(16)$ & $\mathrm{C}(18)-\mathrm{C}(19)$ & $1.5356(18)$ \\
\hline$C(1)-C(13) \# 1$ & $1.4712(16)$ & $\mathrm{C}(18)-\mathrm{H}(18 \mathrm{~A})$ & 0.9900 \\
\hline$C(2)-C(3)$ & $1.4350(16)$ & $\mathrm{C}(18)-\mathrm{H}(18 \mathrm{~B})$ & 0.9900 \\
\hline $\mathrm{C}(2)-\mathrm{H}(2)$ & 0.9500 & $\mathrm{C}(19)-\mathrm{C}(20)$ & $1.510(2)$ \\
\hline$C(3)-C(4)$ & $1.3828(17)$ & $\mathrm{C}(19)-\mathrm{H}(19 \mathrm{~A})$ & 0.9900 \\
\hline $\mathrm{C}(3)-\mathrm{C}(1) \# 1$ & $1.4542(16)$ & $\mathrm{C}(19)-\mathrm{H}(19 \mathrm{~B})$ & 0.9900 \\
\hline C(4)-C(5) & $1.4371(16)$ & $\mathrm{C}(20)-\mathrm{H}(20 \mathrm{~A})$ & 0.9800 \\
\hline$C(4)-C(21)$ & $1.5024(17)$ & $\mathrm{C}(20)-\mathrm{H}(20 \mathrm{~B})$ & 0.9800 \\
\hline$C(5)-C(6)$ & $1.3962(17)$ & $\mathrm{C}(20)-\mathrm{H}(20 \mathrm{C})$ & 0.9800 \\
\hline$C(5)-C(14)$ & $1.4392(17)$ & $\mathrm{C}(21)-\mathrm{C}(22)$ & $1.3990(19)$ \\
\hline $\mathrm{C}(6)-\mathrm{C}(7)$ & $1.4029(18)$ & $\mathrm{C}(21)-\mathrm{C}(26)$ & $1.4053(19)$ \\
\hline $\mathrm{C}(6)-\mathrm{H}(6)$ & 0.9500 & $C(22)-C(23)$ & $1.402(2)$ \\
\hline $\mathrm{C}(7)-\mathrm{C}(8)$ & $1.363(2)$ & $\mathrm{C}(22)-\mathrm{C}(27)$ & $1.506(2)$ \\
\hline $\mathrm{C}(7)-\mathrm{H}(7)$ & 0.9500 & $\mathrm{C}(23)-\mathrm{C}(24)$ & $1.384(2)$ \\
\hline $\mathrm{C}(8)-\mathrm{C}(9)$ & $1.4206(18)$ & $\mathrm{C}(23)-\mathrm{H}(23)$ & 0.9500 \\
\hline $\mathrm{C}(8)-\mathrm{H}(8)$ & 0.9500 & $C(24)-C(25)$ & $1.388(2)$ \\
\hline$C(9)-C(10)$ & $1.4118(18)$ & $\mathrm{C}(24)-\mathrm{C}(28)$ & $1.516(2)$ \\
\hline $\mathrm{C}(9)-\mathrm{C}(14)$ & $1.4263(16)$ & $C(25)-C(26)$ & $1.3954(18)$ \\
\hline$C(10)-C(11)$ & $1.3617(19)$ & $\mathrm{C}(25)-\mathrm{H}(25)$ & 0.9500 \\
\hline $\mathrm{C}(10)-\mathrm{H}(10)$ & 0.9500 & $\mathrm{C}(26)-\mathrm{C}(29)$ & $1.502(2)$ \\
\hline $\mathrm{C}(11)-\mathrm{C}(12)$ & $1.4068(17)$ & $\mathrm{C}(27)-\mathrm{H}(27 \mathrm{~A})$ & 0.9800 \\
\hline $\mathrm{C}(11)-\mathrm{H}(11)$ & 0.9500 & $\mathrm{C}(27)-\mathrm{H}(27 \mathrm{~B})$ & 0.9800 \\
\hline $\mathrm{C}(12)-\mathrm{C}(13)$ & $1.4156(17)$ & $\mathrm{C}(27)-\mathrm{H}(27 \mathrm{C})$ & 0.9800 \\
\hline $\mathrm{C}(13)-\mathrm{C}(14)$ & $1.4289(17)$ & $\mathrm{C}(28)-\mathrm{H}(28 \mathrm{~A})$ & 0.9800 \\
\hline $\mathrm{C}(13)-\mathrm{C}(1) \# 1$ & $1.4712(16)$ & $\mathrm{C}(28)-\mathrm{H}(28 \mathrm{~B})$ & 0.9800 \\
\hline$C(15)-C(16)$ & $1.5222(19)$ & $\mathrm{C}(28)-\mathrm{H}(28 \mathrm{C})$ & 0.9800 \\
\hline $\mathrm{C}(15)-\mathrm{H}(15 \mathrm{~A})$ & 0.9900 & C(29)-H(29A) & 0.9800 \\
\hline $\mathrm{C}(15)-\mathrm{H}(15 \mathrm{~B})$ & 0.9900 & $\mathrm{C}(29)-\mathrm{H}(29 \mathrm{~B})$ & 0.9800 \\
\hline $\mathrm{C}(16)-\mathrm{C}(17)$ & $1.5314(17)$ & C(29)-H(29C) & 0.9800 \\
\hline
\end{tabular}




\begin{tabular}{|c|c|c|c|}
\hline $\mathrm{C}(30)-\mathrm{C}(31)$ & $1.3752(16)$ & $\mathrm{C}(30)-\mathrm{C}(32) \# 2$ & $1.4556(15)$ \\
\hline $\mathrm{C}(30)-\mathrm{C}(42) \# 2$ & $1.4673(16)$ & & \\
\hline $\mathrm{C}(31)-\mathrm{C}(32)$ & $1.4305(16)$ & $\mathrm{C}(47)-\mathrm{H}(47 \mathrm{~B})$ & 0.9900 \\
\hline $\mathrm{C}(31)-\mathrm{H}(31)$ & 0.9500 & $\mathrm{C}(48)-\mathrm{C}(49)$ & $1.513(2)$ \\
\hline $\mathrm{C}(32)-\mathrm{C}(33)$ & $1.3854(16)$ & $\mathrm{C}(48)-\mathrm{H}(48 \mathrm{~A})$ & 0.9900 \\
\hline $\mathrm{C}(32)-\mathrm{C}(30) \# 2$ & $1.4555(15)$ & $\mathrm{C}(48)-\mathrm{H}(48 \mathrm{~B})$ & 0.9900 \\
\hline C(33)-C(34) & $1.4398(17)$ & $\mathrm{C}(49)-\mathrm{H}(49 \mathrm{~A})$ & 0.9800 \\
\hline $\mathrm{C}(33)-\mathrm{C}(50)$ & $1.4977(16)$ & $\mathrm{C}(49)-\mathrm{H}(49 \mathrm{~B})$ & 0.9800 \\
\hline$C(34)-C(35)$ & $1.3963(17)$ & $\mathrm{C}(49)-\mathrm{H}(49 \mathrm{C})$ & 0.9800 \\
\hline C(34)-C(43) & $1.4355(16)$ & $\mathrm{C}(50)-\mathrm{C}(51)$ & $1.4015(17)$ \\
\hline$C(35)-C(36)$ & $1.4014(18)$ & $\mathrm{C}(50)-\mathrm{C}(55)$ & $1.4072(17)$ \\
\hline $\mathrm{C}(35)-\mathrm{H}(35)$ & 0.9500 & $\mathrm{C}(51)-\mathrm{C}(52)$ & $1.3925(17)$ \\
\hline $\mathrm{C}(36)-\mathrm{C}(37)$ & $1.3687(18)$ & $\mathrm{C}(51)-\mathrm{C}(56)$ & $1.5049(18)$ \\
\hline $\mathrm{C}(36)-\mathrm{H}(36)$ & 0.9500 & $\mathrm{C}(52)-\mathrm{C}(53)$ & $1.3893(19)$ \\
\hline C(37)-C(38) & $1.4188(18)$ & $\mathrm{C}(52)-\mathrm{H}(52)$ & 0.9500 \\
\hline $\mathrm{C}(37)-\mathrm{H}(37)$ & 0.9500 & $\mathrm{C}(53)-\mathrm{C}(54)$ & $1.3871(19)$ \\
\hline C(38)-C(39) & $1.4075(17)$ & $\mathrm{C}(53)-\mathrm{C}(57)$ & $1.5054(18)$ \\
\hline $\mathrm{C}(38)-\mathrm{C}(43)$ & $1.4225(17)$ & $\mathrm{C}(54)-\mathrm{C}(55)$ & $1.3968(17)$ \\
\hline C(39)-C(40) & $1.3666(19)$ & $\mathrm{C}(54)-\mathrm{H}(54)$ & 0.9500 \\
\hline $\mathrm{C}(39)-\mathrm{H}(39)$ & 0.9500 & $\mathrm{C}(55)-\mathrm{C}(58)$ & $1.5061(18)$ \\
\hline $\mathrm{C}(40)-\mathrm{C}(41)$ & $1.4068(18)$ & $\mathrm{C}(56)-\mathrm{H}(56 \mathrm{~A})$ & 0.9800 \\
\hline $\mathrm{C}(40)-\mathrm{H}(40)$ & 0.9500 & $\mathrm{C}(56)-\mathrm{H}(56 \mathrm{~B})$ & 0.9800 \\
\hline $\mathrm{C}(41)-\mathrm{C}(42)$ & $1.4050(16)$ & $\mathrm{C}(56)-\mathrm{H}(56 \mathrm{C})$ & 0.9800 \\
\hline $\mathrm{C}(42)-\mathrm{C}(43)$ & $1.4326(16)$ & $\mathrm{C}(57)-\mathrm{H}(57 \mathrm{~A})$ & 0.9800 \\
\hline $\mathrm{C}(42)-\mathrm{C}(30) \# 2$ & $1.4674(16)$ & $\mathrm{C}(57)-\mathrm{H}(57 \mathrm{~B})$ & 0.9800 \\
\hline $\mathrm{C}(44)-\mathrm{C}(45)$ & $1.5164(19)$ & $\mathrm{C}(57)-\mathrm{H}(57 \mathrm{C})$ & 0.9800 \\
\hline $\mathrm{C}(44)-\mathrm{H}(44 \mathrm{~A})$ & 0.9900 & $\mathrm{C}(58)-\mathrm{H}(58 \mathrm{~A})$ & 0.9800 \\
\hline $\mathrm{C}(44)-\mathrm{H}(44 \mathrm{~B})$ & 0.9900 & $\mathrm{C}(58)-\mathrm{H}(58 \mathrm{~B})$ & 0.9800 \\
\hline$C(45)-C(46)$ & $1.5328(17)$ & $\mathrm{C}(58)-\mathrm{H}(58 \mathrm{C})$ & 0.9800 \\
\hline $\mathrm{C}(45)-\mathrm{H}(45 \mathrm{~A})$ & 0.9900 & & \\
\hline $\mathrm{C}(45)-\mathrm{H}(45 \mathrm{~B})$ & 0.9900 & $\mathrm{C}(12)-\mathrm{O}(1)-\mathrm{C}(15)$ & $120.43(10)$ \\
\hline $\mathrm{C}(46)-\mathrm{C}(47)$ & $1.529(2)$ & $\mathrm{C}(41)-\mathrm{O}(2)-\mathrm{C}(44)$ & $118.13(10)$ \\
\hline $\mathrm{C}(46)-\mathrm{H}(46 \mathrm{~A})$ & 0.9900 & $\mathrm{C}(2)-\mathrm{C}(1)-\mathrm{C}(3) \# 1$ & $116.75(10)$ \\
\hline $\mathrm{C}(46)-\mathrm{H}(46 \mathrm{~B})$ & 0.9900 & $\mathrm{C}(2)-\mathrm{C}(1)-\mathrm{C}(13) \# 1$ & $124.69(11)$ \\
\hline $\mathrm{C}(47)-\mathrm{C}(48)$ & $1.5179(19)$ & $\mathrm{C}(3) \# 1-\mathrm{C}(1)-\mathrm{C}(13) \# 1$ & $118.57(10)$ \\
\hline $\mathrm{C}(47)-\mathrm{H}(47 \mathrm{~A})$ & 0.9900 & $\mathrm{C}(1)-\mathrm{C}(2)-\mathrm{C}(3)$ & $124.21(11)$ \\
\hline
\end{tabular}




\begin{tabular}{|c|c|c|c|}
\hline $\mathrm{C}(1)-\mathrm{C}(2)-\mathrm{H}(2)$ & 117.9 & $\mathrm{C}(9)-\mathrm{C}(14)-\mathrm{C}(5)$ & $118.01(11)$ \\
\hline $\mathrm{C}(3)-\mathrm{C}(2)-\mathrm{H}(2)$ & 117.9 & $C(13)-C(14)-C(5)$ & $121.08(10)$ \\
\hline $\mathrm{C}(4)-\mathrm{C}(3)-\mathrm{C}(2)$ & 119.11(11) & $\mathrm{O}(1)-\mathrm{C}(15)-\mathrm{C}(16)$ & $111.54(11)$ \\
\hline $\mathrm{C}(4)-\mathrm{C}(3)-\mathrm{C}(1) \# 1$ & $121.84(11)$ & $\mathrm{O}(1)-\mathrm{C}(15)-\mathrm{H}(15 \mathrm{~A})$ & 109.3 \\
\hline $\mathrm{C}(2)-\mathrm{C}(3)-\mathrm{C}(1) \# 1$ & $119.04(10)$ & $C(16)-C(15)-H(15 A)$ & 109.3 \\
\hline$C(3)-C(4)-C(5)$ & $120.27(11)$ & $\mathrm{O}(1)-\mathrm{C}(15)-\mathrm{H}(15 \mathrm{~B})$ & 109.3 \\
\hline $\mathrm{C}(3)-\mathrm{C}(4)-\mathrm{C}(21)$ & $119.97(11)$ & $\mathrm{C}(16)-\mathrm{C}(15)-\mathrm{H}(15 \mathrm{~B})$ & 109.3 \\
\hline $\mathrm{C}(5)-\mathrm{C}(4)-\mathrm{C}(21)$ & $119.73(11)$ & $\mathrm{H}(15 \mathrm{~A})-\mathrm{C}(15)-\mathrm{H}(15 \mathrm{~B})$ & 108.0 \\
\hline$C(6)-C(5)-C(4)$ & $121.01(11)$ & $\mathrm{C}(15)-\mathrm{C}(16)-\mathrm{C}(17)$ & $112.11(11)$ \\
\hline$C(6)-C(5)-C(14)$ & $119.26(11)$ & $\mathrm{C}(15)-\mathrm{C}(16)-\mathrm{H}(16 \mathrm{~A})$ & 109.2 \\
\hline$C(4)-C(5)-C(14)$ & $119.73(11)$ & $\mathrm{C}(17)-\mathrm{C}(16)-\mathrm{H}(16 \mathrm{~A})$ & 109.2 \\
\hline$C(5)-C(6)-C(7)$ & $121.65(12)$ & $\mathrm{C}(15)-\mathrm{C}(16)-\mathrm{H}(16 \mathrm{~B})$ & 109.2 \\
\hline $\mathrm{C}(5)-\mathrm{C}(6)-\mathrm{H}(6)$ & 119.2 & $\mathrm{C}(17)-\mathrm{C}(16)-\mathrm{H}(16 \mathrm{~B})$ & 109.2 \\
\hline $\mathrm{C}(7)-\mathrm{C}(6)-\mathrm{H}(6)$ & 119.2 & $\mathrm{H}(16 \mathrm{~A})-\mathrm{C}(16)-\mathrm{H}(16 \mathrm{~B})$ & 107.9 \\
\hline$C(8)-C(7)-C(6)$ & $120.05(12)$ & $\mathrm{C}(18)-\mathrm{C}(17)-\mathrm{C}(16)$ & $112.49(11)$ \\
\hline $\mathrm{C}(8)-\mathrm{C}(7)-\mathrm{H}(7)$ & 120.0 & $\mathrm{C}(18)-\mathrm{C}(17)-\mathrm{H}(17 \mathrm{~A})$ & 109.1 \\
\hline $\mathrm{C}(6)-\mathrm{C}(7)-\mathrm{H}(7)$ & 120.0 & $\mathrm{C}(16)-\mathrm{C}(17)-\mathrm{H}(17 \mathrm{~A})$ & 109.1 \\
\hline $\mathrm{C}(7)-\mathrm{C}(8)-\mathrm{C}(9)$ & $120.75(12)$ & $\mathrm{C}(18)-\mathrm{C}(17)-\mathrm{H}(17 \mathrm{~B})$ & 109.1 \\
\hline $\mathrm{C}(7)-\mathrm{C}(8)-\mathrm{H}(8)$ & 119.6 & $\mathrm{C}(16)-\mathrm{C}(17)-\mathrm{H}(17 \mathrm{~B})$ & 109.1 \\
\hline $\mathrm{C}(9)-\mathrm{C}(8)-\mathrm{H}(8)$ & 119.6 & $\mathrm{H}(17 \mathrm{~A})-\mathrm{C}(17)-\mathrm{H}(17 \mathrm{~B})$ & 107.8 \\
\hline $\mathrm{C}(10)-\mathrm{C}(9)-\mathrm{C}(8)$ & $121.02(11)$ & $\mathrm{C}(17)-\mathrm{C}(18)-\mathrm{C}(19)$ & $113.76(11)$ \\
\hline$C(10)-C(9)-C(14)$ & $118.73(11)$ & $\mathrm{C}(17)-\mathrm{C}(18)-\mathrm{H}(18 \mathrm{~A})$ & 108.8 \\
\hline $\mathrm{C}(8)-\mathrm{C}(9)-\mathrm{C}(14)$ & $120.24(12)$ & $\mathrm{C}(19)-\mathrm{C}(18)-\mathrm{H}(18 \mathrm{~A})$ & 108.8 \\
\hline $\mathrm{C}(11)-\mathrm{C}(10)-\mathrm{C}(9)$ & $120.89(11)$ & $\mathrm{C}(17)-\mathrm{C}(18)-\mathrm{H}(18 \mathrm{~B})$ & 108.8 \\
\hline $\mathrm{C}(11)-\mathrm{C}(10)-\mathrm{H}(10)$ & 119.6 & $\mathrm{C}(19)-\mathrm{C}(18)-\mathrm{H}(18 \mathrm{~B})$ & 108.8 \\
\hline $\mathrm{C}(9)-\mathrm{C}(10)-\mathrm{H}(10)$ & 119.6 & $\mathrm{H}(18 \mathrm{~A})-\mathrm{C}(18)-\mathrm{H}(18 \mathrm{~B})$ & 107.7 \\
\hline $\mathrm{C}(10)-\mathrm{C}(11)-\mathrm{C}(12)$ & $121.09(12)$ & $\mathrm{C}(20)-\mathrm{C}(19)-\mathrm{C}(18)$ & $113.74(12)$ \\
\hline $\mathrm{C}(10)-\mathrm{C}(11)-\mathrm{H}(11)$ & 119.5 & $\mathrm{C}(20)-\mathrm{C}(19)-\mathrm{H}(19 \mathrm{~A})$ & 108.8 \\
\hline $\mathrm{C}(12)-\mathrm{C}(11)-\mathrm{H}(11)$ & 119.5 & $\mathrm{C}(18)-\mathrm{C}(19)-\mathrm{H}(19 \mathrm{~A})$ & 108.8 \\
\hline $\mathrm{O}(1)-\mathrm{C}(12)-\mathrm{C}(11)$ & $121.30(11)$ & $\mathrm{C}(20)-\mathrm{C}(19)-\mathrm{H}(19 \mathrm{~B})$ & 108.8 \\
\hline $\mathrm{O}(1)-\mathrm{C}(12)-\mathrm{C}(13)$ & $117.71(10)$ & $\mathrm{C}(18)-\mathrm{C}(19)-\mathrm{H}(19 \mathrm{~B})$ & 108.8 \\
\hline $\mathrm{C}(11)-\mathrm{C}(12)-\mathrm{C}(13)$ & $120.98(11)$ & $\mathrm{H}(19 \mathrm{~A})-\mathrm{C}(19)-\mathrm{H}(19 \mathrm{~B})$ & 107.7 \\
\hline$C(12)-C(13)-C(14)$ & $117.39(10)$ & $\mathrm{C}(19)-\mathrm{C}(20)-\mathrm{H}(20 \mathrm{~A})$ & 109.5 \\
\hline $\mathrm{C}(12)-\mathrm{C}(13)-\mathrm{C}(1) \# 1$ & $124.07(11)$ & $\mathrm{C}(19)-\mathrm{C}(20)-\mathrm{H}(20 \mathrm{~B})$ & 109.5 \\
\hline $\mathrm{C}(14)-\mathrm{C}(13)-\mathrm{C}(1) \# 1$ & $118.50(10)$ & $\mathrm{H}(20 \mathrm{~A})-\mathrm{C}(20)-\mathrm{H}(20 \mathrm{~B})$ & 109.5 \\
\hline $\mathrm{C}(9)-\mathrm{C}(14)-\mathrm{C}(13)$ & $120.90(11)$ & $\mathrm{C}(19)-\mathrm{C}(20)-\mathrm{H}(20 \mathrm{C})$ & 109.5 \\
\hline
\end{tabular}




\begin{tabular}{|c|c|c|c|}
\hline $\mathrm{H}(20 \mathrm{~A})-\mathrm{C}(20)-\mathrm{H}(20 \mathrm{C})$ & 109.5 & $\mathrm{H}(29 \mathrm{~A})-\mathrm{C}(29)-\mathrm{H}(29 \mathrm{C})$ & 109.5 \\
\hline $\mathrm{H}(20 \mathrm{~B})-\mathrm{C}(20)-\mathrm{H}(20 \mathrm{C})$ & 109.5 & $\mathrm{H}(29 \mathrm{~B})-\mathrm{C}(29)-\mathrm{H}(29 \mathrm{C})$ & 109.5 \\
\hline$C(22)-C(21)-C(26)$ & $119.66(12)$ & $\mathrm{C}(31)-\mathrm{C}(30)-\mathrm{C}(32) \# 2$ & $117.52(10)$ \\
\hline $\mathrm{C}(22)-\mathrm{C}(21)-\mathrm{C}(4)$ & $120.62(12)$ & $\mathrm{C}(31)-\mathrm{C}(30)-\mathrm{C}(42) \# 2$ & $123.95(10)$ \\
\hline$C(26)-C(21)-C(4)$ & $119.68(12)$ & $\mathrm{C}(32) \# 2-\mathrm{C}(30)-\mathrm{C}(42) \# 2$ & $118.52(10)$ \\
\hline $\mathrm{C}(21)-\mathrm{C}(22)-\mathrm{C}(23)$ & $119.05(13)$ & $\mathrm{C}(30)-\mathrm{C}(31)-\mathrm{C}(32)$ & $123.80(10)$ \\
\hline $\mathrm{C}(21)-\mathrm{C}(22)-\mathrm{C}(27)$ & $120.67(13)$ & $\mathrm{C}(30)-\mathrm{C}(31)-\mathrm{H}(31)$ & 118.1 \\
\hline $\mathrm{C}(23)-\mathrm{C}(22)-\mathrm{C}(27)$ & $120.23(14)$ & $\mathrm{C}(32)-\mathrm{C}(31)-\mathrm{H}(31)$ & 118.1 \\
\hline $\mathrm{C}(24)-\mathrm{C}(23)-\mathrm{C}(22)$ & $122.00(14)$ & $\mathrm{C}(33)-\mathrm{C}(32)-\mathrm{C}(31)$ & $119.93(10)$ \\
\hline $\mathrm{C}(24)-\mathrm{C}(23)-\mathrm{H}(23)$ & 119.0 & $\mathrm{C}(33)-\mathrm{C}(32)-\mathrm{C}(30) \# 2$ & $121.43(11)$ \\
\hline $\mathrm{C}(22)-\mathrm{C}(23)-\mathrm{H}(23)$ & 119.0 & $\mathrm{C}(31)-\mathrm{C}(32)-\mathrm{C}(30) \# 2$ & $118.59(10)$ \\
\hline $\mathrm{C}(23)-\mathrm{C}(24)-\mathrm{C}(25)$ & $118.16(13)$ & $C(32)-C(33)-C(34)$ & $120.18(10)$ \\
\hline $\mathrm{C}(23)-\mathrm{C}(24)-\mathrm{C}(28)$ & $121.65(15)$ & $C(32)-C(33)-C(50)$ & $120.59(10)$ \\
\hline $\mathrm{C}(25)-\mathrm{C}(24)-\mathrm{C}(28)$ & $120.18(14)$ & $\mathrm{C}(34)-\mathrm{C}(33)-\mathrm{C}(50)$ & $119.20(10)$ \\
\hline$C(24)-C(25)-C(26)$ & $121.74(13)$ & $\mathrm{C}(35)-\mathrm{C}(34)-\mathrm{C}(43)$ & $119.21(11)$ \\
\hline $\mathrm{C}(24)-\mathrm{C}(25)-\mathrm{H}(25)$ & 119.1 & $\mathrm{C}(35)-\mathrm{C}(34)-\mathrm{C}(33)$ & $121.08(11)$ \\
\hline $\mathrm{C}(26)-\mathrm{C}(25)-\mathrm{H}(25)$ & 119.1 & $\mathrm{C}(43)-\mathrm{C}(34)-\mathrm{C}(33)$ & $119.70(10)$ \\
\hline$C(25)-C(26)-C(21)$ & $119.37(13)$ & $\mathrm{C}(34)-\mathrm{C}(35)-\mathrm{C}(36)$ & $121.64(11)$ \\
\hline $\mathrm{C}(25)-\mathrm{C}(26)-\mathrm{C}(29)$ & 119.81(13) & $\mathrm{C}(34)-\mathrm{C}(35)-\mathrm{H}(35)$ & 119.2 \\
\hline$C(21)-C(26)-C(29)$ & $120.79(12)$ & $\mathrm{C}(36)-\mathrm{C}(35)-\mathrm{H}(35)$ & 119.2 \\
\hline $\mathrm{C}(22)-\mathrm{C}(27)-\mathrm{H}(27 \mathrm{~A})$ & 109.5 & $\mathrm{C}(37)-\mathrm{C}(36)-\mathrm{C}(35)$ & $120.01(12)$ \\
\hline $\mathrm{C}(22)-\mathrm{C}(27)-\mathrm{H}(27 \mathrm{~B})$ & 109.5 & $\mathrm{C}(37)-\mathrm{C}(36)-\mathrm{H}(36)$ & 120.0 \\
\hline $\mathrm{H}(27 \mathrm{~A})-\mathrm{C}(27)-\mathrm{H}(27 \mathrm{~B})$ & 109.5 & $\mathrm{C}(35)-\mathrm{C}(36)-\mathrm{H}(36)$ & 120.0 \\
\hline $\mathrm{C}(22)-\mathrm{C}(27)-\mathrm{H}(27 \mathrm{C})$ & 109.5 & $\mathrm{C}(36)-\mathrm{C}(37)-\mathrm{C}(38)$ & $120.51(11)$ \\
\hline $\mathrm{H}(27 \mathrm{~A})-\mathrm{C}(27)-\mathrm{H}(27 \mathrm{C})$ & 109.5 & $\mathrm{C}(36)-\mathrm{C}(37)-\mathrm{H}(37)$ & 119.7 \\
\hline $\mathrm{H}(27 \mathrm{~B})-\mathrm{C}(27)-\mathrm{H}(27 \mathrm{C})$ & 109.5 & $\mathrm{C}(38)-\mathrm{C}(37)-\mathrm{H}(37)$ & 119.7 \\
\hline $\mathrm{C}(24)-\mathrm{C}(28)-\mathrm{H}(28 \mathrm{~A})$ & 109.5 & $\mathrm{C}(39)-\mathrm{C}(38)-\mathrm{C}(37)$ & $121.12(11)$ \\
\hline $\mathrm{C}(24)-\mathrm{C}(28)-\mathrm{H}(28 \mathrm{~B})$ & 109.5 & $\mathrm{C}(39)-\mathrm{C}(38)-\mathrm{C}(43)$ & $118.53(11)$ \\
\hline $\mathrm{H}(28 \mathrm{~A})-\mathrm{C}(28)-\mathrm{H}(28 \mathrm{~B})$ & 109.5 & $\mathrm{C}(37)-\mathrm{C}(38)-\mathrm{C}(43)$ & $120.33(11)$ \\
\hline $\mathrm{C}(24)-\mathrm{C}(28)-\mathrm{H}(28 \mathrm{C})$ & 109.5 & $\mathrm{C}(40)-\mathrm{C}(39)-\mathrm{C}(38)$ & $120.93(12)$ \\
\hline $\mathrm{H}(28 \mathrm{~A})-\mathrm{C}(28)-\mathrm{H}(28 \mathrm{C})$ & 109.5 & $\mathrm{C}(40)-\mathrm{C}(39)-\mathrm{H}(39)$ & 119.5 \\
\hline $\mathrm{H}(28 \mathrm{~B})-\mathrm{C}(28)-\mathrm{H}(28 \mathrm{C})$ & 109.5 & $\mathrm{C}(38)-\mathrm{C}(39)-\mathrm{H}(39)$ & 119.5 \\
\hline $\mathrm{C}(26)-\mathrm{C}(29)-\mathrm{H}(29 \mathrm{~A})$ & 109.5 & $C(39)-C(40)-C(41)$ & $120.81(12)$ \\
\hline $\mathrm{C}(26)-\mathrm{C}(29)-\mathrm{H}(29 \mathrm{~B})$ & 109.5 & $\mathrm{C}(39)-\mathrm{C}(40)-\mathrm{H}(40)$ & 119.6 \\
\hline $\mathrm{H}(29 \mathrm{~A})-\mathrm{C}(29)-\mathrm{H}(29 \mathrm{~B})$ & 109.5 & $\mathrm{C}(41)-\mathrm{C}(40)-\mathrm{H}(40)$ & 119.6 \\
\hline $\mathrm{C}(26)-\mathrm{C}(29)-\mathrm{H}(29 \mathrm{C})$ & 109.5 & $\mathrm{O}(2)-\mathrm{C}(41)-\mathrm{C}(42)$ & $118.46(11)$ \\
\hline
\end{tabular}




\begin{tabular}{|c|c|c|c|}
\hline $\mathrm{O}(2)-\mathrm{C}(41)-\mathrm{C}(40)$ & $120.45(11)$ & $\mathrm{C}(47)-\mathrm{C}(48)-\mathrm{H}(48 \mathrm{~B})$ & 109.0 \\
\hline$C(42)-C(41)-C(40)$ & $121.08(11)$ & $\mathrm{H}(48 \mathrm{~A})-\mathrm{C}(48)-\mathrm{H}(48 \mathrm{~B})$ & 107.8 \\
\hline$C(41)-C(42)-C(43)$ & $117.22(11)$ & $\mathrm{C}(48)-\mathrm{C}(49)-\mathrm{H}(49 \mathrm{~A})$ & 109.5 \\
\hline $\mathrm{C}(41)-\mathrm{C}(42)-\mathrm{C}(30) \# 2$ & $124.40(11)$ & $\mathrm{C}(48)-\mathrm{C}(49)-\mathrm{H}(49 \mathrm{~B})$ & 109.5 \\
\hline $\mathrm{C}(43)-\mathrm{C}(42)-\mathrm{C}(30) \# 2$ & $118.35(10)$ & $\mathrm{H}(49 \mathrm{~A})-\mathrm{C}(49)-\mathrm{H}(49 \mathrm{~B})$ & 109.5 \\
\hline $\mathrm{C}(38)-\mathrm{C}(43)-\mathrm{C}(42)$ & $120.97(11)$ & $\mathrm{C}(48)-\mathrm{C}(49)-\mathrm{H}(49 \mathrm{C})$ & 109.5 \\
\hline $\mathrm{C}(38)-\mathrm{C}(43)-\mathrm{C}(34)$ & $118.20(11)$ & $\mathrm{H}(49 \mathrm{~A})-\mathrm{C}(49)-\mathrm{H}(49 \mathrm{C})$ & 109.5 \\
\hline $\mathrm{C}(42)-\mathrm{C}(43)-\mathrm{C}(34)$ & $120.78(11)$ & $\mathrm{H}(49 \mathrm{~B})-\mathrm{C}(49)-\mathrm{H}(49 \mathrm{C})$ & 109.5 \\
\hline $\mathrm{O}(2)-\mathrm{C}(44)-\mathrm{C}(45)$ & $111.68(11)$ & $\mathrm{C}(51)-\mathrm{C}(50)-\mathrm{C}(55)$ & $119.93(11)$ \\
\hline $\mathrm{O}(2)-\mathrm{C}(44)-\mathrm{H}(44 \mathrm{~A})$ & 109.3 & $\mathrm{C}(51)-\mathrm{C}(50)-\mathrm{C}(33)$ & $120.19(11)$ \\
\hline $\mathrm{C}(45)-\mathrm{C}(44)-\mathrm{H}(44 \mathrm{~A})$ & 109.3 & $C(55)-C(50)-C(33)$ & $119.87(11)$ \\
\hline $\mathrm{O}(2)-\mathrm{C}(44)-\mathrm{H}(44 \mathrm{~B})$ & 109.3 & $\mathrm{C}(52)-\mathrm{C}(51)-\mathrm{C}(50)$ & $119.18(12)$ \\
\hline $\mathrm{C}(45)-\mathrm{C}(44)-\mathrm{H}(44 \mathrm{~B})$ & 109.3 & $\mathrm{C}(52)-\mathrm{C}(51)-\mathrm{C}(56)$ & $119.94(12)$ \\
\hline $\mathrm{H}(44 \mathrm{~A})-\mathrm{C}(44)-\mathrm{H}(44 \mathrm{~B})$ & 107.9 & $\mathrm{C}(50)-\mathrm{C}(51)-\mathrm{C}(56)$ & $120.83(11)$ \\
\hline $\mathrm{C}(44)-\mathrm{C}(45)-\mathrm{C}(46)$ & $111.40(11)$ & $\mathrm{C}(53)-\mathrm{C}(52)-\mathrm{C}(51)$ & $121.75(12)$ \\
\hline $\mathrm{C}(44)-\mathrm{C}(45)-\mathrm{H}(45 \mathrm{~A})$ & 109.3 & $\mathrm{C}(53)-\mathrm{C}(52)-\mathrm{H}(52)$ & 119.1 \\
\hline $\mathrm{C}(46)-\mathrm{C}(45)-\mathrm{H}(45 \mathrm{~A})$ & 109.3 & $\mathrm{C}(51)-\mathrm{C}(52)-\mathrm{H}(52)$ & 119.1 \\
\hline $\mathrm{C}(44)-\mathrm{C}(45)-\mathrm{H}(45 \mathrm{~B})$ & 109.3 & $\mathrm{C}(54)-\mathrm{C}(53)-\mathrm{C}(52)$ & $118.49(11)$ \\
\hline $\mathrm{C}(46)-\mathrm{C}(45)-\mathrm{H}(45 \mathrm{~B})$ & 109.3 & $\mathrm{C}(54)-\mathrm{C}(53)-\mathrm{C}(57)$ & $121.17(13)$ \\
\hline $\mathrm{H}(45 \mathrm{~A})-\mathrm{C}(45)-\mathrm{H}(45 \mathrm{~B})$ & 108.0 & $\mathrm{C}(52)-\mathrm{C}(53)-\mathrm{C}(57)$ & $120.31(13)$ \\
\hline$C(47)-C(46)-C(45)$ & $113.44(11)$ & $\mathrm{C}(53)-\mathrm{C}(54)-\mathrm{C}(55)$ & $121.64(12)$ \\
\hline $\mathrm{C}(47)-\mathrm{C}(46)-\mathrm{H}(46 \mathrm{~A})$ & 108.9 & $\mathrm{C}(53)-\mathrm{C}(54)-\mathrm{H}(54)$ & 119.2 \\
\hline $\mathrm{C}(45)-\mathrm{C}(46)-\mathrm{H}(46 \mathrm{~A})$ & 108.9 & $\mathrm{C}(55)-\mathrm{C}(54)-\mathrm{H}(54)$ & 119.2 \\
\hline $\mathrm{C}(47)-\mathrm{C}(46)-\mathrm{H}(46 \mathrm{~B})$ & 108.9 & $\mathrm{C}(54)-\mathrm{C}(55)-\mathrm{C}(50)$ & $119.00(12)$ \\
\hline $\mathrm{C}(45)-\mathrm{C}(46)-\mathrm{H}(46 \mathrm{~B})$ & 108.9 & $\mathrm{C}(54)-\mathrm{C}(55)-\mathrm{C}(58)$ & $119.83(12)$ \\
\hline $\mathrm{H}(46 \mathrm{~A})-\mathrm{C}(46)-\mathrm{H}(46 \mathrm{~B})$ & 107.7 & $\mathrm{C}(50)-\mathrm{C}(55)-\mathrm{C}(58)$ & $121.16(11)$ \\
\hline $\mathrm{C}(48)-\mathrm{C}(47)-\mathrm{C}(46)$ & $114.69(12)$ & $\mathrm{C}(51)-\mathrm{C}(56)-\mathrm{H}(56 \mathrm{~A})$ & 109.5 \\
\hline $\mathrm{C}(48)-\mathrm{C}(47)-\mathrm{H}(47 \mathrm{~A})$ & 108.6 & $\mathrm{C}(51)-\mathrm{C}(56)-\mathrm{H}(56 \mathrm{~B})$ & 109.5 \\
\hline $\mathrm{C}(46)-\mathrm{C}(47)-\mathrm{H}(47 \mathrm{~A})$ & 108.6 & $\mathrm{H}(56 \mathrm{~A})-\mathrm{C}(56)-\mathrm{H}(56 \mathrm{~B})$ & 109.5 \\
\hline $\mathrm{C}(48)-\mathrm{C}(47)-\mathrm{H}(47 \mathrm{~B})$ & 108.6 & $\mathrm{C}(51)-\mathrm{C}(56)-\mathrm{H}(56 \mathrm{C})$ & 109.5 \\
\hline $\mathrm{C}(46)-\mathrm{C}(47)-\mathrm{H}(47 \mathrm{~B})$ & 108.6 & $\mathrm{H}(56 \mathrm{~A})-\mathrm{C}(56)-\mathrm{H}(56 \mathrm{C})$ & 109.5 \\
\hline $\mathrm{H}(47 \mathrm{~A})-\mathrm{C}(47)-\mathrm{H}(47 \mathrm{~B})$ & 107.6 & $\mathrm{H}(56 \mathrm{~B})-\mathrm{C}(56)-\mathrm{H}(56 \mathrm{C})$ & 109.5 \\
\hline $\mathrm{C}(49)-\mathrm{C}(48)-\mathrm{C}(47)$ & $112.74(13)$ & $\mathrm{C}(53)-\mathrm{C}(57)-\mathrm{H}(57 \mathrm{~A})$ & 109.5 \\
\hline $\mathrm{C}(49)-\mathrm{C}(48)-\mathrm{H}(48 \mathrm{~A})$ & 109.0 & $\mathrm{C}(53)-\mathrm{C}(57)-\mathrm{H}(57 \mathrm{~B})$ & 109.5 \\
\hline $\mathrm{C}(47)-\mathrm{C}(48)-\mathrm{H}(48 \mathrm{~A})$ & 109.0 & $\mathrm{H}(57 \mathrm{~A})-\mathrm{C}(57)-\mathrm{H}(57 \mathrm{~B})$ & 109.5 \\
\hline $\mathrm{C}(49)-\mathrm{C}(48)-\mathrm{H}(48 \mathrm{~B})$ & 109.0 & $\mathrm{C}(53)-\mathrm{C}(57)-\mathrm{H}(57 \mathrm{C})$ & 109.5 \\
\hline
\end{tabular}




$\begin{array}{llll}\mathrm{H}(57 \mathrm{~A})-\mathrm{C}(57)-\mathrm{H}(57 \mathrm{C}) & 109.5 & \mathrm{C}(55)-\mathrm{C}(58)-\mathrm{H}(58 \mathrm{C}) & 109.5 \\ \mathrm{H}(57 \mathrm{~B})-\mathrm{C}(57)-\mathrm{H}(57 \mathrm{C}) & 109.5 & \mathrm{H}(58 \mathrm{~A})-\mathrm{C}(58)-\mathrm{H}(58 \mathrm{C}) & 109.5 \\ \mathrm{C}(55)-\mathrm{C}(58)-\mathrm{H}(58 \mathrm{~A}) & 109.5 & \mathrm{H}(58 \mathrm{~B})-\mathrm{C}(58)-\mathrm{H}(58 \mathrm{C}) & 109.5 \\ \mathrm{C}(55)-\mathrm{C}(58)-\mathrm{H}(58 \mathrm{~B}) & 109.5 & & \\ \mathrm{H}(58 \mathrm{~A})-\mathrm{C}(58)-\mathrm{H}(58 \mathrm{~B}) & 109.5 & & \end{array}$

Symmetry transformations used to generate equivalent atoms:

$$
\# 1-x+3 / 2,-y+3 / 2,-z+1 \quad \# 2-x+1,-y+1,-z+1
$$


Table S32. $\left(\AA^{2} \mathrm{x} 10^{3}\right)$ for HZ-M. The anisotropic displacement factor exponent takes the form: $-2 p^{2}\left[h^{2} a^{* 2} U^{11}+\ldots+2 h k a^{*} b^{*} U^{12}\right]$

\begin{tabular}{|c|c|c|c|c|c|c|}
\hline & $\mathrm{U}^{11}$ & $\mathrm{U}^{22}$ & $\mathrm{U}^{33}$ & $\mathrm{U}^{23}$ & $\mathrm{U}^{13}$ & $\mathrm{U}^{12}$ \\
\hline $\mathrm{O}(1)$ & $16(1)$ & 21(1) & $28(1)$ & $0(1)$ & $9(1)$ & $-3(1)$ \\
\hline $\mathrm{O}(2)$ & $12(1)$ & $30(1)$ & $33(1)$ & $-12(1)$ & $3(1)$ & $2(1)$ \\
\hline $\mathrm{C}(1)$ & $18(1)$ & $17(1)$ & $17(1)$ & $-2(1)$ & $10(1)$ & $-3(1)$ \\
\hline $\mathrm{C}(2)$ & $17(1)$ & $18(1)$ & $22(1)$ & $-1(1)$ & $10(1)$ & $-3(1)$ \\
\hline $\mathrm{C}(3)$ & 19(1) & $16(1)$ & $20(1)$ & $-1(1)$ & 11(1) & $-2(1)$ \\
\hline $\mathrm{C}(4)$ & $19(1)$ & $18(1)$ & $22(1)$ & $0(1)$ & 11(1) & $-2(1)$ \\
\hline$C(5)$ & $24(1)$ & $16(1)$ & 21(1) & $0(1)$ & $13(1)$ & $-1(1)$ \\
\hline$C(6)$ & $27(1)$ & 19(1) & $28(1)$ & $1(1)$ & $15(1)$ & 1(1) \\
\hline$C(7)$ & $37(1)$ & $16(1)$ & $30(1)$ & $1(1)$ & 19(1) & 1(1) \\
\hline $\mathrm{C}(8)$ & $34(1)$ & $15(1)$ & $25(1)$ & $-1(1)$ & $14(1)$ & $-6(1)$ \\
\hline $\mathrm{C}(9)$ & $28(1)$ & $18(1)$ & 19(1) & $-1(1)$ & $12(1)$ & $-5(1)$ \\
\hline$C(10)$ & $27(1)$ & $20(1)$ & 22(1) & $-1(1)$ & 10(1) & $-10(1)$ \\
\hline $\mathrm{C}(11)$ & $21(1)$ & $25(1)$ & 21(1) & $-1(1)$ & $8(1)$ & $-8(1)$ \\
\hline $\mathrm{C}(12)$ & 21(1) & $20(1)$ & $17(1)$ & $0(1)$ & $9(1)$ & $-4(1)$ \\
\hline$C(13)$ & $20(1)$ & $17(1)$ & $16(1)$ & $-1(1)$ & $10(1)$ & $-4(1)$ \\
\hline $\mathrm{C}(14)$ & $24(1)$ & $17(1)$ & $16(1)$ & $-1(1)$ & $12(1)$ & $-4(1)$ \\
\hline $\mathrm{C}(15)$ & $17(1)$ & $29(1)$ & $28(1)$ & $-1(1)$ & $12(1)$ & $-3(1)$ \\
\hline$C(16)$ & $19(1)$ & $31(1)$ & $25(1)$ & $2(1)$ & 11(1) & $-2(1)$ \\
\hline $\mathrm{C}(17)$ & $20(1)$ & $34(1)$ & $26(1)$ & $0(1)$ & $12(1)$ & $-1(1)$ \\
\hline $\mathrm{C}(18)$ & $22(1)$ & $32(1)$ & $26(1)$ & $1(1)$ & $13(1)$ & $1(1)$ \\
\hline$C(19)$ & $22(1)$ & $36(1)$ & $25(1)$ & $-2(1)$ & $10(1)$ & $-2(1)$ \\
\hline$C(20)$ & $25(1)$ & $42(1)$ & $45(1)$ & $10(1)$ & $16(1)$ & $8(1)$ \\
\hline $\mathrm{C}(21)$ & $20(1)$ & $16(1)$ & $28(1)$ & $1(1)$ & $12(1)$ & $1(1)$ \\
\hline $\mathrm{C}(22)$ & $24(1)$ & $32(1)$ & $31(1)$ & $7(1)$ & $14(1)$ & $3(1)$ \\
\hline $\mathrm{C}(23)$ & $24(1)$ & $40(1)$ & $31(1)$ & $9(1)$ & $9(1)$ & $4(1)$ \\
\hline $\mathrm{C}(24)$ & $22(1)$ & $27(1)$ & $43(1)$ & $5(1)$ & $15(1)$ & $3(1)$ \\
\hline$C(25)$ & $25(1)$ & $24(1)$ & $39(1)$ & $0(1)$ & $20(1)$ & $0(1)$ \\
\hline$C(26)$ & $24(1)$ & 19(1) & $31(1)$ & $-2(1)$ & $15(1)$ & $-1(1)$ \\
\hline $\mathrm{C}(27)$ & $32(1)$ & 71(1) & $32(1)$ & 13(1) & $17(1)$ & $5(1)$ \\
\hline $\mathrm{C}(28)$ & $22(1)$ & $47(1)$ & $53(1)$ & $10(1)$ & $16(1)$ & $4(1)$ \\
\hline $\mathrm{C}(29)$ & $30(1)$ & $48(1)$ & $29(1)$ & $-7(1)$ & $17(1)$ & $-9(1)$ \\
\hline $\mathrm{C}(30)$ & $13(1)$ & $18(1)$ & $16(1)$ & $-1(1)$ & $6(1)$ & $-1(1)$ \\
\hline $\mathrm{C}(31)$ & $13(1)$ & $19(1)$ & $15(1)$ & $-1(1)$ & $5(1)$ & $\begin{array}{l}-1(1) \\
\text { S125 }\end{array}$ \\
\hline
\end{tabular}




\begin{tabular}{|c|c|c|c|c|c|c|}
\hline $\mathrm{C}(32)$ & $13(1)$ & 19(1) & 14(1) & $-1(1)$ & $5(1)$ & $-1(1)$ \\
\hline $\mathrm{C}(33)$ & $15(1)$ & 19(1) & $16(1)$ & $-1(1)$ & $6(1)$ & $-1(1)$ \\
\hline $\mathrm{C}(34)$ & $18(1)$ & $19(1)$ & $17(1)$ & $-1(1)$ & $8(1)$ & $-1(1)$ \\
\hline$C(35)$ & 21(1) & 21(1) & 20(1) & $-2(1)$ & $6(1)$ & $-1(1)$ \\
\hline$C(36)$ & $27(1)$ & $19(1)$ & $23(1)$ & $-4(1)$ & $10(1)$ & $-2(1)$ \\
\hline$C(37)$ & $27(1)$ & $18(1)$ & $25(1)$ & $0(1)$ & $13(1)$ & $3(1)$ \\
\hline $\mathrm{C}(38)$ & $22(1)$ & $21(1)$ & $20(1)$ & $-1(1)$ & $10(1)$ & $2(1)$ \\
\hline C(39) & $23(1)$ & $25(1)$ & $26(1)$ & $-1(1)$ & $9(1)$ & $8(1)$ \\
\hline $\mathrm{C}(40)$ & $19(1)$ & $31(1)$ & $27(1)$ & $-4(1)$ & $4(1)$ & $7(1)$ \\
\hline $\mathrm{C}(41)$ & $17(1)$ & $24(1)$ & $22(1)$ & $-5(1)$ & $7(1)$ & $2(1)$ \\
\hline $\mathrm{C}(42)$ & $16(1)$ & $20(1)$ & $16(1)$ & $-1(1)$ & $7(1)$ & $0(1)$ \\
\hline $\mathrm{C}(43)$ & $17(1)$ & $19(1)$ & $17(1)$ & $-1(1)$ & $9(1)$ & $0(1)$ \\
\hline $\mathrm{C}(44)$ & $15(1)$ & $33(1)$ & $25(1)$ & $-2(1)$ & $6(1)$ & $2(1)$ \\
\hline $\mathrm{C}(45)$ & $18(1)$ & $35(1)$ & $24(1)$ & $-1(1)$ & $9(1)$ & 1(1) \\
\hline $\mathrm{C}(46)$ & $15(1)$ & $36(1)$ & $22(1)$ & $0(1)$ & $4(1)$ & $2(1)$ \\
\hline$C(47)$ & $22(1)$ & $34(1)$ & $28(1)$ & $0(1)$ & $10(1)$ & 1(1) \\
\hline$C(48)$ & $21(1)$ & $46(1)$ & $34(1)$ & $-4(1)$ & $10(1)$ & $2(1)$ \\
\hline $\mathrm{C}(49)$ & $27(1)$ & $42(1)$ & $34(1)$ & $2(1)$ & $16(1)$ & $5(1)$ \\
\hline $\mathrm{C}(50)$ & $16(1)$ & $15(1)$ & $15(1)$ & $-4(1)$ & $4(1)$ & $-1(1)$ \\
\hline $\mathrm{C}(51)$ & $19(1)$ & $23(1)$ & $18(1)$ & $-2(1)$ & $7(1)$ & $-4(1)$ \\
\hline $\mathrm{C}(52)$ & $16(1)$ & $27(1)$ & $23(1)$ & $-4(1)$ & $6(1)$ & $-4(1)$ \\
\hline $\mathrm{C}(53)$ & $22(1)$ & 21(1) & $18(1)$ & $-3(1)$ & $2(1)$ & $-1(1)$ \\
\hline $\mathrm{C}(54)$ & $28(1)$ & $22(1)$ & $15(1)$ & $-2(1)$ & $7(1)$ & $-2(1)$ \\
\hline $\mathrm{C}(55)$ & $22(1)$ & $17(1)$ & 19(1) & $-4(1)$ & $9(1)$ & $-2(1)$ \\
\hline $\mathrm{C}(56)$ & $22(1)$ & $46(1)$ & $21(1)$ & $0(1)$ & $10(1)$ & $-7(1)$ \\
\hline$C(57)$ & $28(1)$ & $40(1)$ & $21(1)$ & $0(1)$ & $-2(1)$ & 1(1) \\
\hline $\mathrm{C}(58)$ & $27(1)$ & $35(1)$ & $24(1)$ & $-4(1)$ & $15(1)$ & $-3(1)$ \\
\hline
\end{tabular}


Table S33. displacement parameters $\left(\AA^{2} \times 10^{3}\right)$ for HZ-M.

\begin{tabular}{|c|c|c|c|c|}
\hline \multicolumn{5}{|c|}{-} \\
\hline & & & & \\
\hline $\mathrm{H}(2)$ & 8512 & 7383 & 5507 & 22 \\
\hline $\mathrm{H}(6)$ & 8374 & 4392 & 5324 & 28 \\
\hline $\mathrm{H}(7)$ & 7832 & 3164 & 4992 & 32 \\
\hline $\mathrm{H}(8)$ & 6829 & 3205 & 4490 & 30 \\
\hline $\mathrm{H}(10)$ & 5919 & 4001 & 4110 & 29 \\
\hline $\mathrm{H}(11)$ & 5441 & 5243 & 3946 & 28 \\
\hline $\mathrm{H}(15 \mathrm{~A})$ & 5160 & 7280 & 4105 & 29 \\
\hline $\mathrm{H}(15 \mathrm{~B})$ & 5156 & 6293 & 4175 & 29 \\
\hline $\mathrm{H}(16 \mathrm{~A})$ & 5028 & 6168 & 3086 & 30 \\
\hline $\mathrm{H}(16 \mathrm{~B})$ & 4976 & 7156 & 3020 & 30 \\
\hline $\mathrm{H}(17 \mathrm{~A})$ & 4177 & 6154 & 3222 & 32 \\
\hline $\mathrm{H}(17 \mathrm{~B})$ & 4114 & 7132 & 3092 & 32 \\
\hline $\mathrm{H}(18 \mathrm{~A})$ & 4026 & 5932 & 2144 & 32 \\
\hline $\mathrm{H}(18 \mathrm{~B})$ & 3962 & 6909 & 2015 & 32 \\
\hline $\mathrm{H}(19 \mathrm{~A})$ & 3043 & 6149 & 1545 & 34 \\
\hline $\mathrm{H}(19 \mathrm{~B})$ & 3164 & 6003 & 2277 & 34 \\
\hline $\mathrm{H}(20 \mathrm{~A})$ & 3138 & 7459 & 2407 & 56 \\
\hline $\mathrm{H}(20 \mathrm{~B})$ & 2533 & 7118 & 1827 & 56 \\
\hline $\mathrm{H}(20 \mathrm{C})$ & 2982 & 7579 & 1660 & 56 \\
\hline $\mathrm{H}(23)$ & 9890 & 5760 & 6880 & 41 \\
\hline $\mathrm{H}(25)$ & 9696 & 6008 & 5073 & 33 \\
\hline $\mathrm{H}(27 \mathrm{~A})$ & 8690 & 6367 & 6686 & 67 \\
\hline $\mathrm{H}(27 \mathrm{~B})$ & 9071 & 5561 & 7036 & 67 \\
\hline $\mathrm{H}(27 \mathrm{C})$ & 8432 & 5464 & 6417 & 67 \\
\hline $\mathrm{H}(28 \mathrm{~A})$ & 10740 & 5726 & 6769 & 63 \\
\hline $\mathrm{H}(28 \mathrm{~B})$ & 10679 & 6446 & 6270 & 63 \\
\hline $\mathrm{H}(28 \mathrm{C})$ & 10658 & 5496 & 6064 & 63 \\
\hline $\mathrm{H}(29 \mathrm{~A})$ & 8403 & 6650 & 4254 & 52 \\
\hline $\mathrm{H}(29 \mathrm{~B})$ & 8217 & 5695 & 4184 & 52 \\
\hline $\mathrm{H}(29 \mathrm{C})$ & 8776 & 5963 & 4129 & 52 \\
\hline $\mathrm{H}(31)$ & 5893 & 4816 & 5957 & $\begin{array}{l}19 \\
\text { S127 }\end{array}$ \\
\hline
\end{tabular}




\begin{tabular}{|c|c|c|c|}
\hline $\mathrm{H}(35)$ & 5860 & 7692 & 6566 \\
\hline $\mathrm{H}(36)$ & 5413 & 8993 & 6371 \\
\hline $\mathrm{H}(37)$ & 4480 & 9178 & 5501 \\
\hline H(39) & 3601 & 8604 & 4553 \\
\hline $\mathrm{H}(40)$ & 3084 & 7485 & 3924 \\
\hline $\mathrm{H}(44 \mathrm{~A})$ & 2739 & 6288 & 4028 \\
\hline $\mathrm{H}(44 \mathrm{~B})$ & 2729 & 5351 & 3797 \\
\hline $\mathrm{H}(45 \mathrm{~A})$ & 2543 & 5837 & 2752 \\
\hline $\mathrm{H}(45 \mathrm{~B})$ & 2545 & 6771 & 2984 \\
\hline $\mathrm{H}(46 \mathrm{~A})$ & 1574 & 6216 & 2336 \\
\hline $\mathrm{H}(46 \mathrm{~B})$ & 1719 & 5549 & 2901 \\
\hline $\mathrm{H}(47 \mathrm{~A})$ & 1841 & 6652 & 3640 \\
\hline $\mathrm{H}(47 \mathrm{~B})$ & 1668 & 7301 & 3058 \\
\hline $\mathrm{H}(48 \mathrm{~A})$ & 717 & 6738 & 2496 \\
\hline $\mathrm{H}(48 \mathrm{~B})$ & 890 & 6081 & 3074 \\
\hline $\mathrm{H}(49 \mathrm{~A})$ & 998 & 7144 & 3804 \\
\hline $\mathrm{H}(49 \mathrm{~B})$ & 365 & 7213 & 3172 \\
\hline $\mathrm{H}(49 \mathrm{C})$ & 873 & 7826 & 3254 \\
\hline $\mathrm{H}(52)$ & 7454 & 6116 & 7067 \\
\hline $\mathrm{H}(54)$ & 6614 & 5735 & 8090 \\
\hline $\mathrm{H}(56 \mathrm{~A})$ & 6443 & 5887 & 5601 \\
\hline $\mathrm{H}(56 \mathrm{~B})$ & 6963 & 6538 & 5967 \\
\hline $\mathrm{H}(56 \mathrm{C})$ & 6301 & 6841 & 5644 \\
\hline $\mathrm{H}(57 \mathrm{~A})$ & 7820 & 5150 & 8250 \\
\hline $\mathrm{H}(57 \mathrm{~B})$ & 7655 & 5772 & 8666 \\
\hline $\mathrm{H}(57 \mathrm{C})$ & 7991 & 6109 & 8300 \\
\hline $\mathrm{H}(58 \mathrm{~A})$ & 5609 & 5798 & 7614 \\
\hline $\mathrm{H}(58 \mathrm{~B})$ & 5271 & 5472 & 6872 \\
\hline $\mathrm{H}(58 \mathrm{C})$ & 5300 & 6444 & 7022 \\
\hline
\end{tabular}




\section{Data for HZ-F}

Table S34. Crystal data and structure refinement for HZ-F.

\begin{tabular}{|c|c|c|}
\hline Empirical formula & \multicolumn{2}{|l|}{$\mathrm{C}_{52} \mathrm{H}_{38} \mathrm{~F}_{10} \mathrm{O}_{2}$} \\
\hline Formula weight & \multicolumn{2}{|l|}{884.86} \\
\hline Temperature & \multicolumn{2}{|l|}{$100(2) \mathrm{K}$} \\
\hline Wavelength & \multicolumn{2}{|l|}{$0.71073 \AA$} \\
\hline Crystal system & \multicolumn{2}{|l|}{ Triclinic } \\
\hline Space group & \multicolumn{2}{|l|}{$\mathrm{P}-1$} \\
\hline \multirow[t]{3}{*}{ Unit cell dimensions } & $a=7.7536(3) \AA$ & $\alpha=83.656(2)^{\circ}$ \\
\hline & $\mathrm{b}=8.4647(4) \AA$ & $\beta=80.330(2)^{\circ}$ \\
\hline & $c=16.2224(8) \AA$ & $\gamma=65.6020(10)^{\circ}$ \\
\hline Volume & \multicolumn{2}{|l|}{$954.85(8) \AA^{3}$} \\
\hline $\mathrm{Z}$ & \multicolumn{2}{|l|}{2} \\
\hline Density (calculated) & \multicolumn{2}{|l|}{$1.539 \mathrm{Mg} / \mathrm{m}^{3}$} \\
\hline Absorption coefficient & \multicolumn{2}{|l|}{$0.126 \mathrm{~mm}^{-1}$} \\
\hline $\mathrm{F}(000)$ & \multicolumn{2}{|l|}{456} \\
\hline Crystal size & \multicolumn{2}{|c|}{$0.294 \times 0.229 \times 0.150 \mathrm{~mm}^{3}$} \\
\hline Theta range for data collection & \multicolumn{2}{|l|}{2.645 to $30.508^{\circ}$} \\
\hline Index ranges & \multicolumn{2}{|c|}{$-11<=\mathrm{h}<=8,-12<=\mathrm{k}<=11,-23<=1<=23$} \\
\hline Reflections collected & \multicolumn{2}{|l|}{24081} \\
\hline Independent reflections & \multicolumn{2}{|c|}{$5820[\mathrm{R}(\mathrm{int})=0.0237]$} \\
\hline Completeness to theta $=25.242^{\circ}$ & \multicolumn{2}{|l|}{$99.9 \%$} \\
\hline Refinement method & \multicolumn{2}{|c|}{ Full-matrix least-squares on $\mathrm{F}^{2}$} \\
\hline Data / restraints / parameters & \multicolumn{2}{|l|}{$5820 / 0 / 290$} \\
\hline Goodness-of-fit on $\mathrm{F}^{2}$ & \multicolumn{2}{|l|}{1.035} \\
\hline Final $R$ indices [I>2sigma $(\mathrm{I})]$ & \multicolumn{2}{|c|}{$\mathrm{R} 1=0.0457, \mathrm{wR} 2=0.1188$} \\
\hline $\mathrm{R}$ indices (all data) & \multicolumn{2}{|c|}{$\mathrm{R} 1=0.0610, \mathrm{wR} 2=0.1282$} \\
\hline Extinction coefficient & \multicolumn{2}{|c|}{$\mathrm{n} / \mathrm{a}$} \\
\hline Largest diff. peak and hole & \multicolumn{2}{|c|}{0.609 and -0.269 e. $\AA^{-3}$} \\
\hline
\end{tabular}

Table S35. Atomic coordinates ( x 104) and equivalent isotropic displacement parameters $\left(\AA^{2} \mathrm{x}\right.$ $10^{3}$ ) for HZ-F. U(eq) is defined as one third of the trace of the orthogonalized Uij tensor. 


$\begin{array}{lrrrr}\mathrm{O}(1) & 1223(1) & 682(1) & -1151(1) & 14(1) \\ \mathrm{F}(1) & 4247(1) & 4301(1) & 2013(1) & 17(1) \\ \mathrm{F}(2) & 3744(1) & 5652(1) & 3507(1) & 29(1) \\ \mathrm{F}(3) & 390(2) & 6401(1) & 4539(1) & 32(1) \\ \mathrm{F}(4) & -2516(1) & 5862(1) & 4054(1) & 30(1) \\ \mathrm{F}(5) & -2049(1) & 4449(1) & 2583(1) & 17(1) \\ \mathrm{C}(1) & 3469(2) & 713(2) & 2266(1) & 14(1)\end{array}$

\begin{tabular}{|c|c|c|c|c|}
\hline & $\mathrm{x}$ & $\mathrm{y}$ & $\mathrm{z}$ & $\mathrm{U}(\mathrm{eq})$ \\
\hline$C(2)$ & $4604(2)$ & $-1072(2)$ & $2329(1)$ & $15(1)$ \\
\hline $\mathrm{C}(3)$ & $4845(2)$ & $-2102(2)$ & $1686(1)$ & $15(1)$ \\
\hline $\mathrm{C}(4)$ & $3942(2)$ & $-1376(2)$ & $963(1)$ & $13(1)$ \\
\hline$C(5)$ & 4092(2) & $-2458(2)$ & $333(1)$ & $15(1)$ \\
\hline$C(6)$ & $3180(2)$ & $-1778(2)$ & $-355(1)$ & $15(1)$ \\
\hline$C(7)$ & $2126(2)$ & $24(2)$ & $-462(1)$ & $12(1)$ \\
\hline$C(8)$ & $1958(2)$ & $1195(1)$ & 132(1) & $11(1)$ \\
\hline $\mathrm{C}(9)$ & $968(2)$ & $3106(1)$ & $40(1)$ & 11(1) \\
\hline$C(10)$ & $740(2)$ & $4136(1)$ & $746(1)$ & $11(1)$ \\
\hline $\mathrm{C}(11)$ & $1486(2)$ & $3342(2)$ & $1476(1)$ & $12(1)$ \\
\hline$C(12)$ & $2617(2)$ & $1500(1)$ & $1547(1)$ & $11(1)$ \\
\hline$C(13)$ & $2845(2)$ & $447(1)$ & $869(1)$ & $11(1)$ \\
\hline$C(14)$ & $1130(2)$ & $4333(2)$ & $2238(1)$ & $14(1)$ \\
\hline$C(15)$ & $-571(2)$ & $4751(2)$ & 2782(1) & $16(1)$ \\
\hline$C(16)$ & $-832(2)$ & $5436(2)$ & $3555(1)$ & $21(1)$ \\
\hline$C(17)$ & $633(2)$ & $5719(2)$ & $3799(1)$ & $23(1)$ \\
\hline $\mathrm{C}(18)$ & $2334(2)$ & $5350(2)$ & $3273(1)$ & $20(1)$ \\
\hline C(19) & 2551(2) & $4675(2)$ & 2503(1) & $16(1)$ \\
\hline$C(20)$ & $1585(2)$ & $-469(2)$ & $-1811(1)$ & $13(1)$ \\
\hline$C(21)$ & $605(2)$ & $669(2)$ & $-2521(1)$ & $14(1)$ \\
\hline $\mathrm{C}(22)$ & $973(2)$ & $-302(2)$ & $-3317(1)$ & $16(1)$ \\
\hline $\mathrm{C}(23)$ & $3076(2)$ & $-1110(2)$ & $-3691(1)$ & $22(1)$ \\
\hline$C(24)$ & $3418(2)$ & $-1838(2)$ & $-4553(1)$ & $28(1)$ \\
\hline$C(25)$ & $2783(2)$ & $-454(2)$ & $-5248(1)$ & $30(1)$ \\
\hline$C(26)$ & $-238(2)$ & 5997(1) & $669(1)$ & $12(1)$ \\
\hline
\end{tabular}

Table S36. Bond lengths $[\AA]$ and angles $\left[^{\circ}\right]$ for HZ-F. 


\begin{tabular}{|c|c|c|c|}
\hline $\mathrm{O}(1)-\mathrm{C}(7)$ & $1.3605(14)$ & $\mathrm{O}(1)-\mathrm{C}(20)$ & $1.4408(13)$ \\
\hline $\mathrm{F}(1)-\mathrm{C}(19)$ & $1.3495(15)$ & $\mathrm{F}(2)-\mathrm{C}(18)$ & $1.3407(16)$ \\
\hline $\mathrm{F}(3)-\mathrm{C}(17)$ & $1.3400(14)$ & $\mathrm{F}(4)-\mathrm{C}(16)$ & $1.3455(16)$ \\
\hline$F(5)-C(15)$ & $1.3665(15)$ & $C(1)-C(12)$ & $1.4006(16)$ \\
\hline$C(1)-C(2)$ & $1.4023(16)$ & $\mathrm{C}(1)-\mathrm{H}(1)$ & 0.9500 \\
\hline $\mathrm{C}(2)-\mathrm{C}(3)$ & $1.3729(17)$ & $\mathrm{C}(2)-\mathrm{H}(2)$ & 0.9500 \\
\hline$C(3)-C(4)$ & $1.4156(16)$ & $\mathrm{C}(3)-\mathrm{H}(3)$ & 0.9500 \\
\hline$C(4)-C(5)$ & $1.4053(16)$ & $\mathrm{C}(4)-\mathrm{C}(13)$ & $1.4267(15)$ \\
\hline$C(5)-C(6)$ & $1.3660(17)$ & $\mathrm{C}(5)-\mathrm{H}(5)$ & 0.9500 \\
\hline$C(6)-C(7)$ & $1.4087(16)$ & $\mathrm{C}(6)-\mathrm{H}(6)$ & 0.9500 \\
\hline$C(7)-C(8)$ & $1.4111(15)$ & $\mathrm{C}(8)-\mathrm{C}(13)$ & $1.4331(15)$ \\
\hline$C(8)-C(9)$ & $1.4787(15)$ & $\mathrm{C}(9)-\mathrm{C}(26) \# 1$ & $1.3756(16)$ \\
\hline $\mathrm{C}(9)-\mathrm{C}(10)$ & $1.4587(15)$ & $\mathrm{C}(10)-\mathrm{C}(11)$ & $1.3869(15)$ \\
\hline$C(10)-C(26)$ & $1.4398(15)$ & $\mathrm{C}(11)-\mathrm{C}(12)$ & $1.4414(15)$ \\
\hline $\mathrm{C}(11)-\mathrm{C}(14)$ & $1.4925(16)$ & $\mathrm{C}(12)-\mathrm{C}(13)$ & $1.4338(15)$ \\
\hline $\mathrm{C}(14)-\mathrm{C}(19)$ & $1.3906(17)$ & $\mathrm{C}(14)-\mathrm{C}(15)$ & $1.3920(17)$ \\
\hline$C(15)-C(16)$ & $1.3881(17)$ & $\mathrm{C}(16)-\mathrm{C}(17)$ & $1.379(2)$ \\
\hline $\mathrm{C}(17)-\mathrm{C}(18)$ & $1.381(2)$ & $\mathrm{C}(18)-\mathrm{C}(19)$ & $1.3865(17)$ \\
\hline$C(20)-C(21)$ & $1.5094(16)$ & $\mathrm{C}(20)-\mathrm{H}(20 \mathrm{~A})$ & 0.9900 \\
\hline $\mathrm{C}(20)-\mathrm{H}(20 \mathrm{~B})$ & 0.9900 & $\mathrm{C}(21)-\mathrm{C}(22)$ & $1.5299(16)$ \\
\hline $\mathrm{C}(21)-\mathrm{H}(21 \mathrm{~A})$ & 0.9900 & $\mathrm{C}(21)-\mathrm{H}(21 \mathrm{~B})$ & 0.9900 \\
\hline $\mathrm{C}(22)-\mathrm{C}(23)$ & $1.5280(18)$ & $\mathrm{C}(22)-\mathrm{H}(22 \mathrm{~A})$ & 0.9900 \\
\hline $\mathrm{C}(22)-\mathrm{H}(22 \mathrm{~B})$ & 0.9900 & $C(23)-C(24)$ & $1.5285(18)$ \\
\hline $\mathrm{C}(23)-\mathrm{H}(23 \mathrm{~A})$ & 0.9900 & $\mathrm{C}(23)-\mathrm{H}(23 \mathrm{~B})$ & 0.9900 \\
\hline$C(24)-C(25)$ & $1.518(2)$ & $\mathrm{C}(24)-\mathrm{H}(24 \mathrm{~A})$ & 0.9900 \\
\hline $\mathrm{C}(24)-\mathrm{H}(24 \mathrm{~B})$ & 0.9900 & $\mathrm{C}(25)-\mathrm{H}(25 \mathrm{~A})$ & 0.9800 \\
\hline $\mathrm{C}(25)-\mathrm{H}(25 \mathrm{~B})$ & 0.9800 & $\mathrm{C}(25)-\mathrm{H}(25 \mathrm{C})$ & 0.9800 \\
\hline $\mathrm{C}(26)-\mathrm{C}(9) \# 1$ & $1.3755(16)$ & $\mathrm{C}(26)-\mathrm{H}(26)$ & 0.9500 \\
\hline $\mathrm{C}(7)-\mathrm{O}(1)-\mathrm{C}(20)$ & $118.33(9)$ & $\mathrm{C}(12)-\mathrm{C}(1)-\mathrm{C}(2)$ & $121.80(11)$ \\
\hline $\mathrm{C}(12)-\mathrm{C}(1)-\mathrm{H}(1)$ & 119.1 & $\mathrm{C}(2)-\mathrm{C}(1)-\mathrm{H}(1)$ & 119.1 \\
\hline $\mathrm{C}(3)-\mathrm{C}(2)-\mathrm{C}(1)$ & $119.70(11)$ & $\mathrm{C}(3)-\mathrm{C}(2)-\mathrm{H}(2)$ & 120.2 \\
\hline $\mathrm{C}(1)-\mathrm{C}(2)-\mathrm{H}(2)$ & 120.2 & $\mathrm{C}(2)-\mathrm{C}(3)-\mathrm{C}(4)$ & $120.47(11)$ \\
\hline $\mathrm{C}(2)-\mathrm{C}(3)-\mathrm{H}(3)$ & 119.8 & $\mathrm{C}(4)-\mathrm{C}(3)-\mathrm{H}(3)$ & 119.8 \\
\hline$C(5)-C(4)-C(3)$ & $120.02(10)$ & $C(5)-C(4)-C(13)$ & $119.17(10)$ \\
\hline $\mathrm{C}(3)-\mathrm{C}(4)-\mathrm{C}(13)$ & $120.81(10)$ & $\mathrm{C}(6)-\mathrm{C}(5)-\mathrm{C}(4)$ & $120.51(11)$ \\
\hline $\mathrm{C}(6)-\mathrm{C}(5)-\mathrm{H}(5)$ & 119.7 & $\mathrm{C}(4)-\mathrm{C}(5)-\mathrm{H}(5)$ & 119.7 \\
\hline
\end{tabular}




\begin{tabular}{|c|c|c|c|}
\hline$C(5)-C(6)-C(7)$ & $120.77(11)$ & $\mathrm{C}(5)-\mathrm{C}(6)-\mathrm{H}(6)$ & 119.6 \\
\hline $\mathrm{C}(7)-\mathrm{C}(6)-\mathrm{H}(6)$ & 119.6 & $\mathrm{O}(1)-\mathrm{C}(7)-\mathrm{C}(6)$ & $120.09(10)$ \\
\hline $\mathrm{O}(1)-\mathrm{C}(7)-\mathrm{C}(8)$ & $118.05(10)$ & $\mathrm{C}(6)-\mathrm{C}(7)-\mathrm{C}(8)$ & $121.85(10)$ \\
\hline $\mathrm{C}(7)-\mathrm{C}(8)-\mathrm{C}(13)$ & $116.47(10)$ & $\mathrm{C}(7)-\mathrm{C}(8)-\mathrm{C}(9)$ & $124.86(10)$ \\
\hline $\mathrm{C}(13)-\mathrm{C}(8)-\mathrm{C}(9)$ & $118.67(10)$ & $\mathrm{C}(26) \# 1-\mathrm{C}(9)-\mathrm{C}(10)$ & $116.75(10)$ \\
\hline $\mathrm{C}(26) \# 1-\mathrm{C}(9)-\mathrm{C}(8)$ & $124.79(10)$ & $\mathrm{C}(10)-\mathrm{C}(9)-\mathrm{C}(8)$ & $118.46(10)$ \\
\hline $\mathrm{C}(11)-\mathrm{C}(10)-\mathrm{C}(26)$ & $120.25(10)$ & $\mathrm{C}(11)-\mathrm{C}(10)-\mathrm{C}(9)$ & $120.63(10)$ \\
\hline $\mathrm{C}(26)-\mathrm{C}(10)-\mathrm{C}(9)$ & 119.11(10) & $\mathrm{C}(10)-\mathrm{C}(11)-\mathrm{C}(12)$ & $121.64(10)$ \\
\hline $\mathrm{C}(10)-\mathrm{C}(11)-\mathrm{C}(14)$ & 122.11(10) & $\mathrm{C}(12)-\mathrm{C}(11)-\mathrm{C}(14)$ & $116.24(10)$ \\
\hline $\mathrm{C}(1)-\mathrm{C}(12)-\mathrm{C}(13)$ & $119.36(10)$ & $\mathrm{C}(1)-\mathrm{C}(12)-\mathrm{C}(11)$ & $121.68(10)$ \\
\hline$C(13)-C(12)-C(11)$ & $118.95(10)$ & $\mathrm{C}(4)-\mathrm{C}(13)-\mathrm{C}(8)$ & $121.10(10)$ \\
\hline $\mathrm{C}(4)-\mathrm{C}(13)-\mathrm{C}(12)$ & 117.77(10) & $\mathrm{C}(8)-\mathrm{C}(13)-\mathrm{C}(12)$ & $121.11(10)$ \\
\hline $\mathrm{C}(19)-\mathrm{C}(14)-\mathrm{C}(15)$ & $116.18(11)$ & $\mathrm{C}(19)-\mathrm{C}(14)-\mathrm{C}(11)$ & $122.26(11)$ \\
\hline $\mathrm{C}(15)-\mathrm{C}(14)-\mathrm{C}(11)$ & $120.98(11)$ & $\mathrm{F}(5)-\mathrm{C}(15)-\mathrm{C}(16)$ & $116.97(11)$ \\
\hline $\mathrm{F}(5)-\mathrm{C}(15)-\mathrm{C}(14)$ & $120.73(11)$ & $C(16)-C(15)-C(14)$ & $122.27(12)$ \\
\hline $\mathrm{F}(4)-\mathrm{C}(16)-\mathrm{C}(17)$ & $120.12(12)$ & $\mathrm{F}(4)-\mathrm{C}(16)-\mathrm{C}(15)$ & $120.36(13)$ \\
\hline$C(17)-C(16)-C(15)$ & $119.50(12)$ & $\mathrm{F}(3)-\mathrm{C}(17)-\mathrm{C}(16)$ & $120.08(13)$ \\
\hline $\mathrm{F}(3)-\mathrm{C}(17)-\mathrm{C}(18)$ & $119.73(13)$ & $\mathrm{C}(16)-\mathrm{C}(17)-\mathrm{C}(18)$ & $120.16(11)$ \\
\hline $\mathrm{F}(2)-\mathrm{C}(18)-\mathrm{C}(17)$ & $120.02(12)$ & $\mathrm{F}(2)-\mathrm{C}(18)-\mathrm{C}(19)$ & $120.90(13)$ \\
\hline$C(17)-C(18)-C(19)$ & $119.08(12)$ & $\mathrm{F}(1)-\mathrm{C}(19)-\mathrm{C}(18)$ & $117.32(11)$ \\
\hline $\mathrm{F}(1)-\mathrm{C}(19)-\mathrm{C}(14)$ & $119.88(10)$ & $\mathrm{C}(18)-\mathrm{C}(19)-\mathrm{C}(14)$ & $122.77(12)$ \\
\hline $\mathrm{O}(1)-\mathrm{C}(20)-\mathrm{C}(21)$ & 105.82(9) & $\mathrm{O}(1)-\mathrm{C}(20)-\mathrm{H}(20 \mathrm{~A})$ & 110.6 \\
\hline $\mathrm{C}(21)-\mathrm{C}(20)-\mathrm{H}(20 \mathrm{~A})$ & 110.6 & $\mathrm{O}(1)-\mathrm{C}(20)-\mathrm{H}(20 \mathrm{~B})$ & 110.6 \\
\hline $\mathrm{C}(21)-\mathrm{C}(20)-\mathrm{H}(20 \mathrm{~B})$ & 110.6 & $\mathrm{H}(20 \mathrm{~A})-\mathrm{C}(20)-\mathrm{H}(20 \mathrm{~B})$ & 108.7 \\
\hline$C(20)-C(21)-C(22)$ & 113.71(10) & $\mathrm{C}(20)-\mathrm{C}(21)-\mathrm{H}(21 \mathrm{~A})$ & 108.8 \\
\hline $\mathrm{C}(22)-\mathrm{C}(21)-\mathrm{H}(21 \mathrm{~A})$ & 108.8 & $\mathrm{C}(20)-\mathrm{C}(21)-\mathrm{H}(21 \mathrm{~B})$ & 108.8 \\
\hline $\mathrm{C}(22)-\mathrm{C}(21)-\mathrm{H}(21 \mathrm{~B})$ & 108.8 & $\mathrm{H}(21 \mathrm{~A})-\mathrm{C}(21)-\mathrm{H}(21 \mathrm{~B})$ & 107.7 \\
\hline$C(23)-C(22)-C(21)$ & $113.90(10)$ & $\mathrm{C}(23)-\mathrm{C}(22)-\mathrm{H}(22 \mathrm{~A})$ & 108.8 \\
\hline $\mathrm{C}(21)-\mathrm{C}(22)-\mathrm{H}(22 \mathrm{~A})$ & 108.8 & $\mathrm{C}(23)-\mathrm{C}(22)-\mathrm{H}(22 \mathrm{~B})$ & 108.8 \\
\hline $\mathrm{C}(21)-\mathrm{C}(22)-\mathrm{H}(22 \mathrm{~B})$ & 108.8 & $\mathrm{H}(22 \mathrm{~A})-\mathrm{C}(22)-\mathrm{H}(22 \mathrm{~B})$ & 107.7 \\
\hline $\mathrm{C}(24)-\mathrm{C}(23)-\mathrm{C}(22)$ & $113.98(11)$ & $\mathrm{C}(24)-\mathrm{C}(23)-\mathrm{H}(23 \mathrm{~A})$ & 108.8 \\
\hline $\mathrm{C}(22)-\mathrm{C}(23)-\mathrm{H}(23 \mathrm{~A})$ & 108.8 & $\mathrm{C}(24)-\mathrm{C}(23)-\mathrm{H}(23 \mathrm{~B})$ & 108.8 \\
\hline $\mathrm{C}(22)-\mathrm{C}(23)-\mathrm{H}(23 \mathrm{~B})$ & 108.8 & $\mathrm{H}(23 \mathrm{~A})-\mathrm{C}(23)-\mathrm{H}(23 \mathrm{~B})$ & 107.7 \\
\hline$C(25)-C(24)-C(23)$ & $113.88(13)$ & $\mathrm{C}(25)-\mathrm{C}(24)-\mathrm{H}(24 \mathrm{~A})$ & 108.8 \\
\hline $\mathrm{C}(23)-\mathrm{C}(24)-\mathrm{H}(24 \mathrm{~A})$ & 108.8 & $\mathrm{C}(25)-\mathrm{C}(24)-\mathrm{H}(24 \mathrm{~B})$ & 108.8 \\
\hline $\mathrm{C}(23)-\mathrm{C}(24)-\mathrm{H}(24 \mathrm{~B})$ & 108.8 & $\mathrm{H}(24 \mathrm{~A})-\mathrm{C}(24)-\mathrm{H}(24 \mathrm{~B})$ & 107.7 \\
\hline $\mathrm{C}(24)-\mathrm{C}(25)-\mathrm{H}(25 \mathrm{~A})$ & 109.5 & $\mathrm{C}(24)-\mathrm{C}(25)-\mathrm{H}(25 \mathrm{~B})$ & 109.5 \\
\hline $\mathrm{H}(25 \mathrm{~A})-\mathrm{C}(25)-\mathrm{H}(25 \mathrm{~B})$ & 109.5 & $\mathrm{C}(24)-\mathrm{C}(25)-\mathrm{H}(25 \mathrm{C})$ & 109.5 \\
\hline
\end{tabular}




$\begin{array}{llll}\mathrm{H}(25 \mathrm{~A})-\mathrm{C}(25)-\mathrm{H}(25 \mathrm{C}) & 109.5 & \mathrm{H}(25 \mathrm{~B})-\mathrm{C}(25)-\mathrm{H}(25 \mathrm{C}) & 109.5 \\ \mathrm{C}(9) \# 1-\mathrm{C}(26)-\mathrm{C}(10) & 124.13(10) & \mathrm{C}(9) \# 1-\mathrm{C}(26)-\mathrm{H}(26) & 117.9 \\ \mathrm{C}(10)-\mathrm{C}(26)-\mathrm{H}(26) & 117.9 & & \end{array}$

Symmetry transformations used to generate equivalent atoms: $\quad \# 1-x,-y+1,-z$

Table S37. Anisotropic displacement parameters $\left(\AA^{2} \mathrm{x} 10^{3}\right)$ for HZ-F. The anisotropic displacement factor exponent takes the form: $-2 \square^{2}\left[\mathrm{~h}^{2} \mathrm{a} * 2 \mathrm{U}^{11}+\ldots+2 \mathrm{hk} \mathrm{a}^{*} \mathrm{~b} * \mathrm{U}^{12}\right]$

\begin{tabular}{|c|c|c|c|c|c|c|}
\hline & $\mathrm{U}^{11}$ & $\mathrm{U}^{22}$ & $\mathrm{U}^{33}$ & $\mathrm{U}^{23}$ & $\mathrm{U}^{13}$ & $\mathrm{U}^{12}$ \\
\hline $\mathrm{O}(1)$ & $18(1)$ & 11(1) & 11(1) & $-3(1)$ & $-4(1)$ & $-4(1)$ \\
\hline $\mathrm{F}(1)$ & $13(1)$ & $19(1)$ & $20(1)$ & $-4(1)$ & $-1(1)$ & $-7(1)$ \\
\hline $\mathrm{F}(2)$ & $39(1)$ & $24(1)$ & $32(1)$ & $-2(1)$ & $-18(1)$ & $-16(1)$ \\
\hline $\mathrm{F}(3)$ & $60(1)$ & $23(1)$ & 13(1) & $-5(1)$ & $-6(1)$ & $-15(1)$ \\
\hline $\mathrm{F}(4)$ & $32(1)$ & $26(1)$ & 21(1) & $-3(1)$ & $9(1)$ & $-7(1)$ \\
\hline$F(5)$ & $16(1)$ & $15(1)$ & 19(1) & $-2(1)$ & $-7(1)$ & $-3(1)$ \\
\hline $\mathrm{C}(1)$ & $15(1)$ & $14(1)$ & $13(1)$ & $-1(1)$ & $-3(1)$ & $-6(1)$ \\
\hline$C(2)$ & $16(1)$ & $15(1)$ & $14(1)$ & $2(1)$ & $-6(1)$ & $-6(1)$ \\
\hline$C(3)$ & $15(1)$ & 11(1) & $17(1)$ & $0(1)$ & $-4(1)$ & $-4(1)$ \\
\hline$C(4)$ & $13(1)$ & $11(1)$ & $13(1)$ & $-1(1)$ & $-1(1)$ & $-5(1)$ \\
\hline$C(5)$ & $17(1)$ & $10(1)$ & $17(1)$ & $-2(1)$ & $-2(1)$ & $-4(1)$ \\
\hline$C(6)$ & $18(1)$ & 11(1) & $14(1)$ & $-3(1)$ & $-2(1)$ & $-5(1)$ \\
\hline$C(7)$ & $13(1)$ & $12(1)$ & 11(1) & $-1(1)$ & $-2(1)$ & $-5(1)$ \\
\hline $\mathrm{C}(8)$ & $12(1)$ & $10(1)$ & 11(1) & $-1(1)$ & $-1(1)$ & $-4(1)$ \\
\hline $\mathrm{C}(9)$ & 11(1) & $10(1)$ & 11(1) & $-2(1)$ & $-1(1)$ & $-4(1)$ \\
\hline$C(10)$ & $12(1)$ & 11(1) & 11(1) & $-2(1)$ & $-1(1)$ & $-4(1)$ \\
\hline $\mathrm{C}(11)$ & $13(1)$ & 11(1) & 11(1) & $-2(1)$ & $-1(1)$ & $-5(1)$ \\
\hline \multirow[t]{2}{*}{$C(12)$} & $11(1)$ & $12(1)$ & $12(1)$ & $-1(1)$ & $-1(1)$ & $-5(1)$ \\
\hline & $\mathrm{U}^{11}$ & $\mathrm{U}^{22}$ & $\mathrm{U}^{33}$ & $\mathrm{U}^{23}$ & $\mathrm{U}^{13}$ & $\mathrm{U}^{12}$ \\
\hline
\end{tabular}

$\begin{array}{lllllll}\mathrm{C}(13) & 11(1) & 11(1) & 11(1) & -1(1) & -1(1) & -5(1) \\ \mathrm{C}(14) & 19(1) & 10(1) & 11(1) & -1(1) & -3(1) & -4(1) \\ \mathrm{C}(15) & 19(1) & 13(1) & 14(1) & 0(1) & -3(1) & -4(1) \\ \mathrm{C}(16) & 27(1) & 14(1) & 14(1) & -1(1) & 3(1) & -4(1)\end{array}$




$\begin{array}{lllllll}\mathrm{C}(17) & 40(1) & 13(1) & 11(1) & -3(1) & -6(1) & -7(1) \\ \mathrm{C}(18) & 31(1) & 14(1) & 19(1) & 0(1) & -12(1) & -9(1) \\ \mathrm{C}(19) & 20(1) & 13(1) & 16(1) & -2(1) & -4(1) & -5(1) \\ \mathrm{C}(20) & 16(1) & 12(1) & 12(1) & -4(1) & -1(1) & -5(1) \\ \mathrm{C}(21) & 16(1) & 13(1) & 14(1) & -2(1) & -3(1) & -5(1) \\ \mathrm{C}(22) & 18(1) & 19(1) & 13(1) & -3(1) & -3(1) & -8(1) \\ \mathrm{C}(23) & 19(1) & 31(1) & 14(1) & -7(1) & -1(1) & -7(1) \\ \mathrm{C}(24) & 29(1) & 35(1) & 17(1) & -11(1) & 2(1) & -9(1) \\ \mathrm{C}(25) & 26(1) & 50(1) & 15(1) & -5(1) & 0(1) & -19(1) \\ \mathrm{C}(26) & 14(1) & 11(1) & 11(1) & -2(1) & -2(1) & -4(1)\end{array}$

Table S38. Torsion angles $\left[^{\circ}\right]$ for HZ-F.

$\begin{array}{ll} & \mathrm{C}(12)-\mathrm{C}(1)-\mathrm{C}(2)-\mathrm{C}(3) \\ \mathrm{C}(1)-\mathrm{C}(2)-\mathrm{C}(3)-\mathrm{C}(4) & \mathrm{C}(2)-\mathrm{C}(3)-\mathrm{C}(4)-\mathrm{C}(5) \\ \mathrm{C}(2)-\mathrm{C}(3)-\mathrm{C}(4)-\mathrm{C}(13) & \mathrm{C}(3)-\mathrm{C}(4)-\mathrm{C}(5)-\mathrm{C}(6) \\ \mathrm{C}(13)-\mathrm{C}(4)-\mathrm{C}(5)-\mathrm{C}(6) & \mathrm{C}(4)-\mathrm{C}(5)-\mathrm{C}(6)-\mathrm{C}(7) \\ \mathrm{C}(20)-\mathrm{O}(1)-\mathrm{C}(7)-\mathrm{C}(6) & \mathrm{C}(20)-\mathrm{O}(1)-\mathrm{C}(7)-\mathrm{C}(8) \\ \mathrm{C}(5)-\mathrm{C}(6)-\mathrm{C}(7)-\mathrm{O}(1) & \mathrm{C}(5)-\mathrm{C}(6)-\mathrm{C}(7)-\mathrm{C}(8) \\ \mathrm{O}(1)-\mathrm{C}(7)-\mathrm{C}(8)-\mathrm{C}(13) & \mathrm{C}(6)-\mathrm{C}(7)-\mathrm{C}(8)-\mathrm{C}(13) \\ \mathrm{O}(1)-\mathrm{C}(7)-\mathrm{C}(8)-\mathrm{C}(9) & \mathrm{C}(6)-\mathrm{C}(7)-\mathrm{C}(8)-\mathrm{C}(9) \\ \mathrm{C}(7)-\mathrm{C}(8)-\mathrm{C}(9)-\mathrm{C}(26) \# 1 & \mathrm{C}(13)-\mathrm{C}(8)-\mathrm{C}(9)-\mathrm{C}(26) \# 1 \\ \mathrm{C}(7)-\mathrm{C}(8)-\mathrm{C}(9)-\mathrm{C}(10) & \mathrm{C}(13)-\mathrm{C}(8)-\mathrm{C}(9)-\mathrm{C}(10) \\ \mathrm{C}(26) \# 1-\mathrm{C}(9)-\mathrm{C}(10)-\mathrm{C}(11) & \mathrm{C}(8)-\mathrm{C}(9)-\mathrm{C}(10)-\mathrm{C}(11) \\ \mathrm{C}(26) \# 1-\mathrm{C}(9)-\mathrm{C}(10)-\mathrm{C}(26) & \mathrm{C}(8)-\mathrm{C}(9)-\mathrm{C}(10)-\mathrm{C}(26) \\ \mathrm{C}(26)-\mathrm{C}(10)-\mathrm{C}(11)-\mathrm{C}(12) & \mathrm{C}(9)-\mathrm{C}(10)-\mathrm{C}(11)-\mathrm{C}(12) \\ \mathrm{C}(26)-\mathrm{C}(10)-\mathrm{C}(11)-\mathrm{C}(14) & \mathrm{C}(9)-\mathrm{C}(10)-\mathrm{C}(11)-\mathrm{C}(14) \\ \mathrm{C}(2)-\mathrm{C}(1)-\mathrm{C}(12)-\mathrm{C}(13) & \mathrm{C}(2)-\mathrm{C}(1)-\mathrm{C}(12)-\mathrm{C}(11) \\ \mathrm{C}(10)-\mathrm{C}(11)-\mathrm{C}(12)-\mathrm{C}(1) & \mathrm{C}(14)-\mathrm{C}(11)-\mathrm{C}(12)-\mathrm{C}(1) \\ \mathrm{C}(10)-\mathrm{C}(11)-\mathrm{C}(12)-\mathrm{C}(13) & \mathrm{C}(14)-\mathrm{C}(11)-\mathrm{C}(12)-\mathrm{C}(13) \\ \mathrm{C}(5)-\mathrm{C}(4)-\mathrm{C}(13)-\mathrm{C}(8) & \mathrm{C}(3)-\mathrm{C}(4)-\mathrm{C}(13)-\mathrm{C}(8) \\ \mathrm{C}(5)-\mathrm{C}(4)-\mathrm{C}(13)-\mathrm{C}(12) & \mathrm{C}(3)-\mathrm{C}(4)-\mathrm{C}(13)-\mathrm{C}(12) \\ \mathrm{C}(7)-\mathrm{C}(8)-\mathrm{C}(13)-\mathrm{C}(4) & \mathrm{C}(9)-\mathrm{C}(8)-\mathrm{C}(13)-\mathrm{C}(4) \\ \mathrm{C}(7)-\mathrm{C}(8)-\mathrm{C}(13)-\mathrm{C}(12) & \mathrm{C}(9)-\mathrm{C}(8)-\mathrm{C}(13)-\mathrm{C}(12) \\ \mathrm{C}(1)-\mathrm{C}(12)-\mathrm{C}(13)-\mathrm{C}(4) & \mathrm{C}(11)-\mathrm{C}(12)-\mathrm{C}(13)-\mathrm{C}(4) \\ \mathrm{C}(1)-\mathrm{C}(12)-\mathrm{C}(13)-\mathrm{C}(8) & \mathrm{C}(11)-\mathrm{C}(12)-\mathrm{C}(13)-\mathrm{C}(8) \\ \mathrm{C}(10)-\mathrm{C}(11)-\mathrm{C}(14)-\mathrm{C}(19) & \mathrm{C}(12)-\mathrm{C}(11)-\mathrm{C}(14)-\mathrm{C}(19) \\ & \\ & \end{array}$




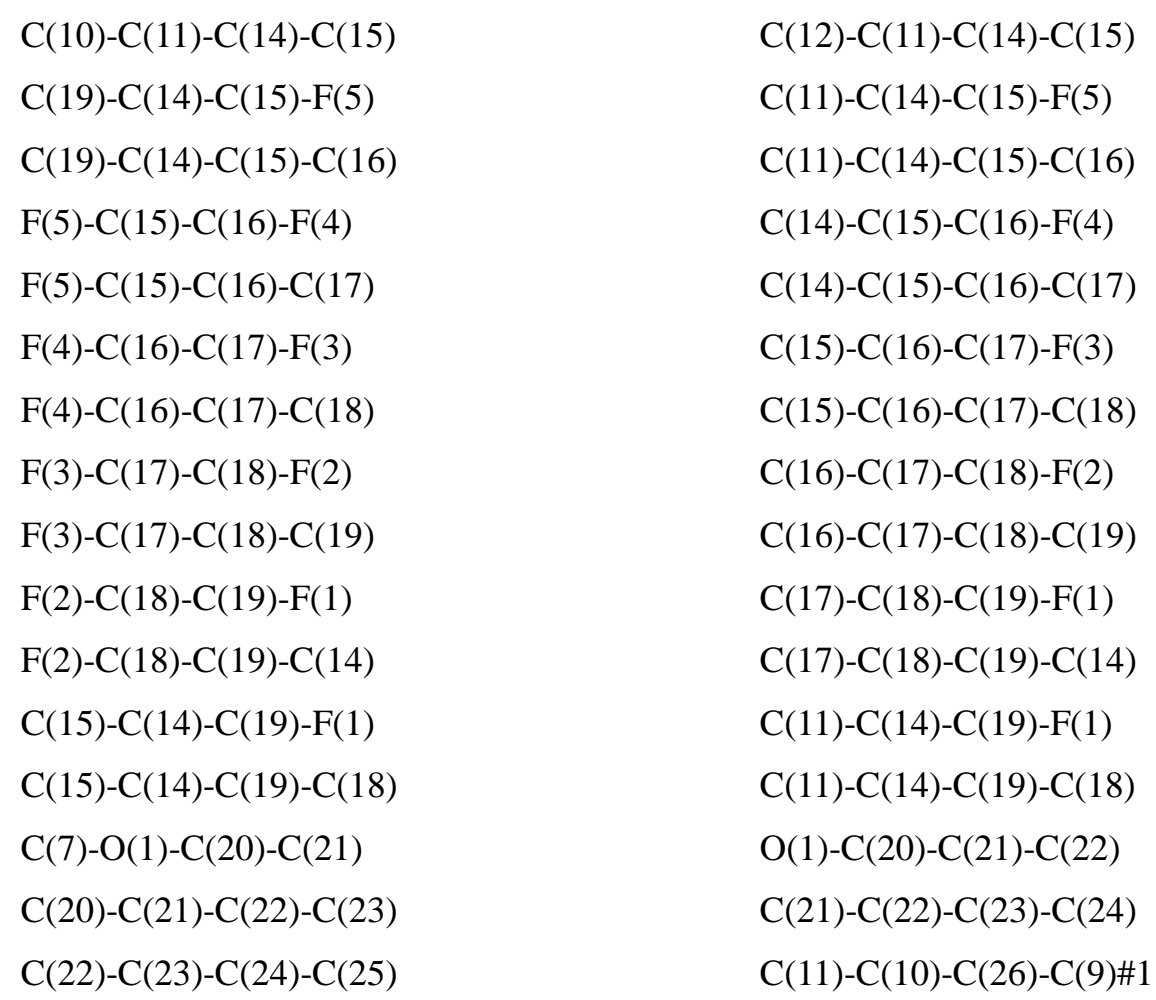

Symmetry transformations used to generate equivalent atoms: $\# 1-x,-y+1,-z$

\section{Data for PF-M}

Table S39. Crystal data and structure refinement for PF-M.

$\begin{array}{lll}\text { Empirical formula } & \mathrm{C}_{48} \mathrm{H}_{46} \mathrm{O} & \\ \text { Formula weight } & 638.90 & \\ \text { Temperature } & 100(2) \mathrm{K} & \\ \text { Wavelength } & 1.54178 \AA \\ \text { Crystal system } & \text { Monoclinic } & \\ \text { Space group } & \mathrm{P} 2(1) / \mathrm{n} & \alpha=90^{\circ} \\ \text { Unit cell dimensions } & \mathrm{a}=14.9865(5) \AA & \beta=101.364(2)^{\circ} \\ & \mathrm{b}=7.9786(3) \AA & \gamma=90^{\circ} \\ \text { Volume } & \mathrm{c}=34.6321(12) \AA & \\ \mathrm{Z} & 4059.8(2) \AA 3 & \\ \text { Density (calculated) } & 4 & \\ \text { Absorption coefficient } & 1.196 \mathrm{Mg} / \mathrm{m}^{3} & \end{array}$


$\mathrm{F}(000)$

Crystal size

Theta range for data collection

Index ranges

Reflections collected

Independent reflections

Completeness to theta $=67.679^{\circ}$

Refinement method

Data / restraints / parameters

Goodness-of-fit on $\mathrm{F}^{2}$

Final R indices [I $>2 \operatorname{sigma}(\mathrm{I})]$

$\mathrm{R}$ indices (all data)

Extinction coefficient

Largest diff. peak and hole
1568

$0.494 \times 0.156 \times 0.064 \mathrm{~mm}^{3}$

2.603 to $79.720^{\circ}$

$-19<=\mathrm{h}<=18,-9<=\mathrm{k}<=8,-43<=\mathrm{l}<=42$

35902

8417 [R(int) $=0.0463]$

$99.5 \%$

Full-matrix least-squares on $\mathrm{F}^{2}$

8417 / 0 / 513

1.027

$\mathrm{R} 1=0.0497, \mathrm{wR} 2=0.1210$

$\mathrm{R} 1=0.0689, \mathrm{wR} 2=0.1335$

$\mathrm{n} / \mathrm{a}$

0.288 and -0.264 e. $\AA^{-3}$

Table S40. Atomic coordinates ( $\mathrm{x} 10^{4}$ ) and equivalent isotropic displacement parameters $\left(\AA^{2} \mathrm{x}\right.$ $10^{3}$ )for PF-M. U(eq) is defined as one third of the trace of the orthogonalized Uij tensor.

\begin{tabular}{lcccc}
\hline & $\mathrm{x}$ & $\mathrm{y}$ & $\mathrm{z}$ & $\mathrm{U}(\mathrm{eq})$ \\
\hline & & & \\
$\mathrm{O}(1)$ & & & & \\
$\mathrm{C}(1)$ & $5826(1)$ & $6638(2)$ & $79(1)$ & $21(1)$ \\
$\mathrm{C}(2)$ & $5043(1)$ & $7483(2)$ & $89(1)$ & $18(1)$ \\
$\mathrm{C}(3)$ & $4421(1)$ & $7856(2)$ & $-262(1)$ & $21(1)$ \\
$\mathrm{C}(4)$ & $3626(1)$ & $8674(2)$ & $-252(1)$ & $21(1)$ \\
$\mathrm{C}(5)$ & $3414(1)$ & $9183(2)$ & $108(1)$ & $19(1)$ \\
$\mathrm{C}(6)$ & $2582(1)$ & $9986(2)$ & $123(1)$ & $22(1)$ \\
$\mathrm{C}(7)$ & $2378(1)$ & $10497(2)$ & $472(1)$ & $23(1)$ \\
$\mathrm{C}(8)$ & $3019(1)$ & $10265(2)$ & $823(1)$ & $20(1)$ \\
$\mathrm{C}(9)$ & $3849(1)$ & $9484(2)$ & $825(1)$ & $18(1)$ \\
$\mathrm{C}(10)$ & $4516(1)$ & $9299(2)$ & $1187(1)$ & $17(1)$ \\
$\mathrm{C}(11)$ & $5304(1)$ & $8405(2)$ & $1194(1)$ & $17(1)$ \\
$\mathrm{C}(12)$ & $5940(1)$ & $8195(2)$ & $1567(1)$ & $18(1)$ \\
$\mathrm{C}(13)$ & $6672(1)$ & $7187(2)$ & $1594(1)$ & $18(1)$ \\
$\mathrm{C}(14)$ & $7420(1)$ & $6750(2)$ & $1922(1)$ & $19(1)$ \\
$\mathrm{C}(15)$ & $7641(1)$ & $7279(2)$ & $2310(1)$ & $22(1)$ \\
& $8431(1)$ & $6646(2)$ & $2548(1)$ & $23(1)$ \\
& & & & $\mathrm{S} 136$
\end{tabular}




\begin{tabular}{|c|c|c|c|c|}
\hline$C(16)$ & 8991(1) & $5535(2)$ & 2399(1) & $23(1)$ \\
\hline$C(17)$ & $8780(1)$ & $5008(2)$ & 2008(1) & 21(1) \\
\hline $\mathrm{C}(18)$ & 7988(1) & $5617(2)$ & $1769(1)$ & $20(1)$ \\
\hline $\mathrm{C}(19)$ & $7619(1)$ & $5346(2)$ & $1348(1)$ & 19(1) \\
\hline$C(20)$ & $6835(1)$ & 6294(2) & $1249(1)$ & $19(1)$ \\
\hline $\mathrm{C}(21)$ & $6239(1)$ & $6533(2)$ & $879(1)$ & 19(1) \\
\hline $\mathrm{C}(22)$ & $5500(1)$ & $7597(2)$ & $839(1)$ & $17(1)$ \\
\hline$C(23)$ & $4878(1)$ & 7961(2) & $461(1)$ & $16(1)$ \\
\hline $\mathrm{C}(24)$ & $4058(1)$ & $8863(2)$ & $465(1)$ & $17(1)$ \\
\hline$C(25)$ & $6055(1)$ & $6245(2)$ & $-296(1)$ & $21(1)$ \\
\hline$C(26)$ & 6983(1) & $5436(2)$ & $-212(1)$ & $22(1)$ \\
\hline $\mathrm{C}(27)$ & $7289(1)$ & $4936(2)$ & $-591(1)$ & $24(1)$ \\
\hline$C(28)$ & $8266(1)$ & $4311(2)$ & $-516(1)$ & $28(1)$ \\
\hline \multirow[t]{2}{*}{ C(29) } & $8587(1)$ & 3694(3) & $-884(1)$ & $33(1)$ \\
\hline & $\mathrm{x}$ & $\mathrm{y}$ & $\mathrm{z}$ & $\mathrm{U}(\mathrm{eq})$ \\
\hline $\mathrm{C}(30)$ & $8589(2)$ & $5044(3)$ & $-1193(1)$ & $39(1)$ \\
\hline$C(31)$ & $4280(1)$ & $10049(2)$ & 1551(1) & $18(1)$ \\
\hline$C(32)$ & $4437(1)$ & $11750(2)$ & $1640(1)$ & $20(1)$ \\
\hline $\mathrm{C}(33)$ & 4114(1) & $12437(2)$ & 1957(1) & $23(1)$ \\
\hline$C(34)$ & $3645(1)$ & $11487(2)$ & 2186(1) & $24(1)$ \\
\hline$C(35)$ & $3507(1)$ & $9800(2)$ & 2095(1) & $23(1)$ \\
\hline $\mathrm{C}(36)$ & $3817(1)$ & $9057(2)$ & 1784(1) & $20(1)$ \\
\hline $\mathrm{C}(37)$ & $8093(1)$ & $4348(2)$ & 1089(1) & $20(1)$ \\
\hline$C(38)$ & $8525(1)$ & $5169(2)$ & $816(1)$ & $21(1)$ \\
\hline C(39) & $9033(1)$ & $4240(2)$ & $597(1)$ & $24(1)$ \\
\hline $\mathrm{C}(40)$ & $9135(1)$ & $2518(2)$ & $642(1)$ & $24(1)$ \\
\hline$C(41)$ & $8694(1)$ & $1725(2)$ & $908(1)$ & $24(1)$ \\
\hline $\mathrm{C}(42)$ & 8171(1) & 2601(2) & $1130(1)$ & $21(1)$ \\
\hline$C(43)$ & 4931(1) & $12853(2)$ & $1396(1)$ & $26(1)$ \\
\hline$C(44)$ & $3296(1)$ & 12231(3) & $2528(1)$ & $38(1)$ \\
\hline $\mathrm{C}(45)$ & $3650(1)$ & $7221(2)$ & $1697(1)$ & $28(1)$ \\
\hline$C(46)$ & $8461(1)$ & $7045(2)$ & $766(1)$ & $27(1)$ \\
\hline $\mathrm{C}(47)$ & $9715(1)$ & $1542(2)$ & $409(1)$ & $33(1)$ \\
\hline $\mathrm{C}(48)$ & $7673(1)$ & $1658(2)$ & 1401(1) & $27(1)$ \\
\hline
\end{tabular}


Toluene

\begin{tabular}{lrrrr}
\hline & $\mathrm{x}$ & $\mathrm{y}$ & $\mathrm{z}$ & $\mathrm{U}(\mathrm{eq})$ \\
\hline $\mathrm{C}(1 \mathrm{~T})$ & & & & \\
$\mathrm{C}(2 \mathrm{~T})$ & $-79(1)$ & $550(2)$ & $2109(1)$ & $31(1)$ \\
$\mathrm{C}(3 \mathrm{~T})$ & $-251(1)$ & $-812(2)$ & $1855(1)$ & $34(1)$ \\
$\mathrm{C}(4 \mathrm{~T})$ & $278(2)$ & $-1098(3)$ & $1575(1)$ & $41(1)$ \\
$\mathrm{C}(5 \mathrm{~T})$ & $986(2)$ & $-27(3)$ & $1541(1)$ & $44(1)$ \\
$\mathrm{C}(6 \mathrm{~T})$ & $1154(2)$ & $1329(3)$ & $1788(1)$ & $43(1)$ \\
$\mathrm{C}(7 \mathrm{~T})$ & $633(1)$ & $1623(3)$ & $2069(1)$ & $35(1)$ \\
& $-642(2)$ & $838(3)$ & $2413(1)$ & $40(1)$
\end{tabular}

Table S41. Bond lengths $[\AA]$ and angles $\left[{ }^{\circ}\right]$ for PF-M.

$\begin{array}{llll}\mathrm{O}(1)-\mathrm{C}(1) & 1.3597(19) & \mathrm{O}(1)-\mathrm{C}(25) & 1.4415(19) \\ \mathrm{C}(1)-\mathrm{C}(23) & 1.409(2) & \mathrm{C}(1)-\mathrm{C}(2) & 1.412(2) \\ \mathrm{C}(2)-\mathrm{C}(3) & 1.366(2) & \mathrm{C}(2)-\mathrm{H}(2) & 0.9300 \\ \mathrm{C}(3)-\mathrm{C}(4) & 1.403(2) & \mathrm{C}(3)-\mathrm{H}(3) & 0.9300 \\ \mathrm{C}(4)-\mathrm{C}(5) & 1.412(2) & \mathrm{C}(4)-\mathrm{C}(24) & 1.433(2) \\ \mathrm{C}(5)-\mathrm{C}(6) & 1.367(2) & \mathrm{C}(5)-\mathrm{H}(5) & 0.9300 \\ \mathrm{C}(6)-\mathrm{C}(7) & 1.405(2) & \mathrm{C}(6)-\mathrm{H}(6) & 0.9300 \\ \mathrm{C}(7)-\mathrm{C}(8) & 1.390(2) & \mathrm{C}(7)-\mathrm{H}(7) & 0.9300 \\ \mathrm{C}(8)-\mathrm{C}(24) & 1.432(2) & \mathrm{C}(8)-\mathrm{C}(9) & 1.451(2) \\ \mathrm{C}(9)-\mathrm{C}(10) & 1.375(2) & \mathrm{C}(9)-\mathrm{C}(31) & 1.499(2) \\ \mathrm{C}(10)-\mathrm{C}(11) & 1.455(2) & \mathrm{C}(10)-\mathrm{C}(22) & 1.470(2) \\ \mathrm{C}(11)-\mathrm{C}(12) & 1.349(2) & \mathrm{C}(11)-\mathrm{H}(11) & 0.9300 \\ \mathrm{C}(12)-\mathrm{C}(20) & 1.453(2) & \mathrm{C}(12)-\mathrm{C}(13) & 1.471(2) \\ \mathrm{C}(13)-\mathrm{C}(14) & 1.387(2) & \mathrm{C}(13)-\mathrm{C}(18) & 1.415(2) \\ \mathrm{C}(14)-\mathrm{C}(15) & 1.397(2) & \mathrm{C}(14)-\mathrm{H}(14) & 0.9300 \\ \mathrm{C}(15)-\mathrm{C}(16) & 1.388(2) & \mathrm{C}(15)-\mathrm{H}(15) & 0.9300 \\ \mathrm{C}(16)-\mathrm{C}(17) & 1.394(2) & \mathrm{C}(16)-\mathrm{H}(16) & 0.9300 \\ \mathrm{C}(17)-\mathrm{C}(18) & 1.395(2) & \mathrm{C}(17)-\mathrm{H}(17) & 0.9300 \\ \mathrm{C}(18)-\mathrm{C}(19) & 1.470(2) & \mathrm{C}(19)-\mathrm{C}(20) & 1.383(2) \\ \mathrm{C}(19)-\mathrm{C}(37) & 1.480(2) & \mathrm{C}(20)-\mathrm{C}(21) & 1.423(2) \\ \mathrm{C}(21)-\mathrm{C}(22) & 1.380(2) & \mathrm{C}(21)-\mathrm{H}(21) & 0.9300 \\ & & & \mathrm{~S} 138\end{array}$




\begin{tabular}{|c|c|c|c|}
\hline$C(22)-C(23)$ & $1.478(2)$ & $C(23)-C(24)$ & $1.426(2)$ \\
\hline$C(25)-C(26)$ & $1.509(2)$ & $\mathrm{C}(25)-\mathrm{H}(25 \mathrm{~A})$ & 0.9700 \\
\hline $\mathrm{C}(25)-\mathrm{H}(25 \mathrm{~B})$ & 0.9700 & $C(26)-C(27)$ & $1.526(2)$ \\
\hline $\mathrm{C}(26)-\mathrm{H}(26 \mathrm{~A})$ & 0.9700 & $\mathrm{C}(26)-\mathrm{H}(26 \mathrm{~B})$ & 0.9700 \\
\hline $\mathrm{C}(27)-\mathrm{C}(28)$ & $1.520(2)$ & $\mathrm{C}(27)-\mathrm{H}(27 \mathrm{~A})$ & 0.9700 \\
\hline $\mathrm{C}(27)-\mathrm{H}(27 \mathrm{~B})$ & 0.9700 & $C(28)-C(29)$ & $1.529(2)$ \\
\hline $\mathrm{C}(28)-\mathrm{H}(28 \mathrm{~A})$ & 0.9700 & $\mathrm{C}(28)-\mathrm{H}(28 \mathrm{~B})$ & 0.9700 \\
\hline$C(29)-C(30)$ & $1.520(3)$ & $\mathrm{C}(29)-\mathrm{H}(29 \mathrm{~A})$ & 0.9700 \\
\hline C(29)-H(29B) & 0.9700 & $\mathrm{C}(30)-\mathrm{H}(30 \mathrm{~A})$ & 0.9600 \\
\hline $\mathrm{C}(30)-\mathrm{H}(30 \mathrm{~B})$ & 0.9600 & $\mathrm{C}(30)-\mathrm{H}(30 \mathrm{C})$ & 0.9600 \\
\hline $\mathrm{C}(31)-\mathrm{C}(32)$ & $1.401(2)$ & $C(31)-C(36)$ & $1.407(2)$ \\
\hline $\mathrm{C}(32)-\mathrm{C}(33)$ & $1.397(2)$ & $C(32)-C(43)$ & $1.511(2)$ \\
\hline C(33)-C(34) & $1.386(2)$ & $\mathrm{C}(33)-\mathrm{H}(33)$ & 0.9300 \\
\hline$C(34)-C(35)$ & $1.388(2)$ & $\mathrm{C}(34)-\mathrm{C}(44)$ & $1.507(2)$ \\
\hline$C(35)-C(36)$ & $1.387(2)$ & $\mathrm{C}(35)-\mathrm{H}(35)$ & 0.9300 \\
\hline$C(36)-C(45)$ & $1.507(2)$ & $C(37)-C(42)$ & $1.404(2)$ \\
\hline $\mathrm{C}(37)-\mathrm{C}(38)$ & $1.408(2)$ & $\mathrm{C}(38)-\mathrm{C}(39)$ & $1.391(2)$ \\
\hline$C(38)-C(46)$ & $1.508(2)$ & $\mathrm{C}(39)-\mathrm{C}(40)$ & $1.388(2)$ \\
\hline C(39)-H(39) & 0.9300 & $\mathrm{C}(40)-\mathrm{C}(41)$ & $1.387(3)$ \\
\hline C(40)-C(47) & $1.513(2)$ & $\mathrm{C}(41)-\mathrm{C}(42)$ & $1.391(2)$ \\
\hline $\mathrm{C}(41)-\mathrm{H}(41)$ & 0.9300 & $\mathrm{C}(42)-\mathrm{C}(48)$ & $1.510(2)$ \\
\hline $\mathrm{C}(43)-\mathrm{H}(43 \mathrm{~A})$ & 0.9600 & $\mathrm{C}(43)-\mathrm{H}(43 \mathrm{~B})$ & 0.9600 \\
\hline $\mathrm{C}(43)-\mathrm{H}(43 \mathrm{C})$ & 0.9600 & $\mathrm{C}(44)-\mathrm{H}(44 \mathrm{~A})$ & 0.9600 \\
\hline C(44)-H(44B) & 0.9600 & $\mathrm{C}(44)-\mathrm{H}(44 \mathrm{C})$ & 0.9600 \\
\hline $\mathrm{C}(45)-\mathrm{H}(45 \mathrm{~A})$ & 0.9600 & $\mathrm{C}(45)-\mathrm{H}(45 \mathrm{~B})$ & 0.9600 \\
\hline $\mathrm{C}(45)-\mathrm{H}(45 \mathrm{C})$ & 0.9600 & $\mathrm{C}(46)-\mathrm{H}(46 \mathrm{~A})$ & 0.9600 \\
\hline $\mathrm{C}(46)-\mathrm{H}(46 \mathrm{~B})$ & 0.9600 & $\mathrm{C}(46)-\mathrm{H}(46 \mathrm{C})$ & 0.9600 \\
\hline $\mathrm{C}(47)-\mathrm{H}(47 \mathrm{~A})$ & 0.9600 & $\mathrm{C}(47)-\mathrm{H}(47 \mathrm{~B})$ & 0.9600 \\
\hline $\mathrm{C}(47)-\mathrm{H}(47 \mathrm{C})$ & 0.9600 & $\mathrm{C}(48)-\mathrm{H}(48 \mathrm{~A})$ & 0.9600 \\
\hline $\mathrm{C}(48)-\mathrm{H}(48 \mathrm{~B})$ & 0.9600 & $\mathrm{C}(48)-\mathrm{H}(48 \mathrm{C})$ & 0.9600 \\
\hline
\end{tabular}

Toluene

$$
\begin{aligned}
& \mathrm{C}(1 \mathrm{~T})-\mathrm{C}(2 \mathrm{~T}) \\
& \mathrm{C}(1 \mathrm{~T})-\mathrm{C}(7 \mathrm{~T}) \\
& \mathrm{C}(2 \mathrm{~T})-\mathrm{H}(2 \mathrm{~T}) \\
& \mathrm{C}(3 \mathrm{~T})-\mathrm{H}(3 \mathrm{~T}) \\
& \mathrm{C}(4 \mathrm{~T})-\mathrm{H}(4 \mathrm{~T}) \\
& \mathrm{C}(5 \mathrm{~T})-\mathrm{H}(5 \mathrm{~T})
\end{aligned}
$$$$
1.390(3)
$$$$
\mathrm{C}(1 \mathrm{~T})-\mathrm{C}(6 \mathrm{~T})
$$$$
1.395(3)
$$$$
1.492(3)
$$$$
\mathrm{C}(2 \mathrm{~T})-\mathrm{C}(3 \mathrm{~T})
$$$$
1.386(3)
$$$$
0.9300
$$$$
\mathrm{C}(3 \mathrm{~T})-\mathrm{C}(4 \mathrm{~T})
$$$$
1.385(3)
$$$$
0.9300
$$$$
\mathrm{C}(4 \mathrm{~T})-\mathrm{C}(5 \mathrm{~T})
$$$$
\text { 1.373(3) }
$$$$
0.9300
$$$$
\mathrm{C}(5 \mathrm{~T})-\mathrm{C}(6 \mathrm{~T})
$$$$
1.382(3)
$$$$
0.9300
$$$$
\mathrm{C}(6 \mathrm{~T})-\mathrm{H}(6 \mathrm{~T})
$$$$
0.9300
$$ 


\begin{tabular}{|c|c|c|c|}
\hline $\mathrm{C}(7 \mathrm{~T})-\mathrm{H}(7 \mathrm{~T} 1)$ & 0.9600 & $\mathrm{C}(7 \mathrm{~T})-\mathrm{H}(7 \mathrm{~T} 2)$ & 0.9600 \\
\hline $\mathrm{C}(7 \mathrm{~T})-\mathrm{H}(7 \mathrm{~T} 3)$ & 0.9600 & & \\
\hline $\mathrm{C}(1)-\mathrm{O}(1)-\mathrm{C}(25)$ & $119.41(12)$ & $\mathrm{O}(1)-\mathrm{C}(1)-\mathrm{C}(23)$ & $117.78(13)$ \\
\hline $\mathrm{O}(1)-\mathrm{C}(1)-\mathrm{C}(2)$ & $120.57(14)$ & $\mathrm{C}(23)-\mathrm{C}(1)-\mathrm{C}(2)$ & $121.65(15)$ \\
\hline $\mathrm{C}(3)-\mathrm{C}(2)-\mathrm{C}(1)$ & $120.59(15)$ & $\mathrm{C}(3)-\mathrm{C}(2)-\mathrm{H}(2)$ & 119.7 \\
\hline $\mathrm{C}(1)-\mathrm{C}(2)-\mathrm{H}(2)$ & 119.7 & $\mathrm{C}(2)-\mathrm{C}(3)-\mathrm{C}(4)$ & $120.88(15)$ \\
\hline $\mathrm{C}(2)-\mathrm{C}(3)-\mathrm{H}(3)$ & 119.6 & $\mathrm{C}(4)-\mathrm{C}(3)-\mathrm{H}(3)$ & 119.6 \\
\hline $\mathrm{C}(3)-\mathrm{C}(4)-\mathrm{C}(5)$ & $121.32(15)$ & $\mathrm{C}(3)-\mathrm{C}(4)-\mathrm{C}(24)$ & $118.81(15)$ \\
\hline $\mathrm{C}(5)-\mathrm{C}(4)-\mathrm{C}(24)$ & $119.87(15)$ & $C(6)-C(5)-C(4)$ & $121.47(15)$ \\
\hline $\mathrm{C}(6)-\mathrm{C}(5)-\mathrm{H}(5)$ & 119.3 & $\mathrm{C}(4)-\mathrm{C}(5)-\mathrm{H}(5)$ & 119.3 \\
\hline$C(5)-C(6)-C(7)$ & $119.37(16)$ & $\mathrm{C}(5)-\mathrm{C}(6)-\mathrm{H}(6)$ & 120.3 \\
\hline $\mathrm{C}(7)-\mathrm{C}(6)-\mathrm{H}(6)$ & 120.3 & $C(8)-C(7)-C(6)$ & $121.53(16)$ \\
\hline $\mathrm{C}(8)-\mathrm{C}(7)-\mathrm{H}(7)$ & 119.2 & $\mathrm{C}(6)-\mathrm{C}(7)-\mathrm{H}(7)$ & 119.2 \\
\hline $\mathrm{C}(7)-\mathrm{C}(8)-\mathrm{C}(24)$ & $119.97(14)$ & $\mathrm{C}(7)-\mathrm{C}(8)-\mathrm{C}(9)$ & $120.85(15)$ \\
\hline $\mathrm{C}(24)-\mathrm{C}(8)-\mathrm{C}(9)$ & $119.17(14)$ & $\mathrm{C}(10)-\mathrm{C}(9)-\mathrm{C}(8)$ & $120.66(14)$ \\
\hline $\mathrm{C}(10)-\mathrm{C}(9)-\mathrm{C}(31)$ & $122.48(14)$ & $\mathrm{C}(8)-\mathrm{C}(9)-\mathrm{C}(31)$ & $116.80(14)$ \\
\hline $\mathrm{C}(9)-\mathrm{C}(10)-\mathrm{C}(11)$ & $119.02(14)$ & $C(9)-C(10)-C(22)$ & $121.72(14)$ \\
\hline $\mathrm{C}(11)-\mathrm{C}(10)-\mathrm{C}(22)$ & $119.21(14)$ & $\mathrm{C}(12)-\mathrm{C}(11)-\mathrm{C}(10)$ & $120.82(14)$ \\
\hline $\mathrm{C}(12)-\mathrm{C}(11)-\mathrm{H}(11)$ & 119.6 & $\mathrm{C}(10)-\mathrm{C}(11)-\mathrm{H}(11)$ & 119.6 \\
\hline $\mathrm{C}(11)-\mathrm{C}(12)-\mathrm{C}(20)$ & $120.09(14)$ & $\mathrm{C}(11)-\mathrm{C}(12)-\mathrm{C}(13)$ & $132.83(15)$ \\
\hline$C(20)-C(12)-C(13)$ & $107.08(14)$ & $\mathrm{C}(14)-\mathrm{C}(13)-\mathrm{C}(18)$ & $120.77(15)$ \\
\hline $\mathrm{C}(14)-\mathrm{C}(13)-\mathrm{C}(12)$ & $132.64(15)$ & $\mathrm{C}(18)-\mathrm{C}(13)-\mathrm{C}(12)$ & $106.54(14)$ \\
\hline$C(13)-C(14)-C(15)$ & $118.38(16)$ & $\mathrm{C}(13)-\mathrm{C}(14)-\mathrm{H}(14)$ & 120.8 \\
\hline $\mathrm{C}(15)-\mathrm{C}(14)-\mathrm{H}(14)$ & 120.8 & $\mathrm{C}(16)-\mathrm{C}(15)-\mathrm{C}(14)$ & $121.06(15)$ \\
\hline $\mathrm{C}(16)-\mathrm{C}(15)-\mathrm{H}(15)$ & 119.5 & $\mathrm{C}(14)-\mathrm{C}(15)-\mathrm{H}(15)$ & 119.5 \\
\hline$C(15)-C(16)-C(17)$ & $121.01(15)$ & $\mathrm{C}(15)-\mathrm{C}(16)-\mathrm{H}(16)$ & 119.5 \\
\hline $\mathrm{C}(17)-\mathrm{C}(16)-\mathrm{H}(16)$ & 119.5 & $\mathrm{C}(16)-\mathrm{C}(17)-\mathrm{C}(18)$ & $118.49(16)$ \\
\hline $\mathrm{C}(16)-\mathrm{C}(17)-\mathrm{H}(17)$ & 120.8 & $\mathrm{C}(18)-\mathrm{C}(17)-\mathrm{H}(17)$ & 120.8 \\
\hline $\mathrm{C}(17)-\mathrm{C}(18)-\mathrm{C}(13)$ & $120.29(15)$ & $\mathrm{C}(17)-\mathrm{C}(18)-\mathrm{C}(19)$ & $130.42(16)$ \\
\hline $\mathrm{C}(13)-\mathrm{C}(18)-\mathrm{C}(19)$ & $109.23(14)$ & $\mathrm{C}(20)-\mathrm{C}(19)-\mathrm{C}(18)$ & $107.49(14)$ \\
\hline $\mathrm{C}(20)-\mathrm{C}(19)-\mathrm{C}(37)$ & $129.08(14)$ & $\mathrm{C}(18)-\mathrm{C}(19)-\mathrm{C}(37)$ & $123.18(14)$ \\
\hline $\mathrm{C}(19)-\mathrm{C}(20)-\mathrm{C}(21)$ & $130.45(15)$ & $\mathrm{C}(19)-\mathrm{C}(20)-\mathrm{C}(12)$ & $109.66(14)$ \\
\hline $\mathrm{C}(21)-\mathrm{C}(20)-\mathrm{C}(12)$ & $119.85(14)$ & $\mathrm{C}(22)-\mathrm{C}(21)-\mathrm{C}(20)$ & $121.45(14)$ \\
\hline $\mathrm{C}(22)-\mathrm{C}(21)-\mathrm{H}(21)$ & 119.3 & $\mathrm{C}(20)-\mathrm{C}(21)-\mathrm{H}(21)$ & 119.3 \\
\hline $\mathrm{C}(21)-\mathrm{C}(22)-\mathrm{C}(10)$ & $118.32(14)$ & $\mathrm{C}(21)-\mathrm{C}(22)-\mathrm{C}(23)$ & $124.25(14)$ \\
\hline$C(10)-C(22)-C(23)$ & $117.42(14)$ & $\mathrm{C}(1)-\mathrm{C}(23)-\mathrm{C}(24)$ & $116.88(14)$ \\
\hline $\mathrm{C}(1)-\mathrm{C}(23)-\mathrm{C}(22)$ & $124.13(14)$ & $\mathrm{C}(24)-\mathrm{C}(23)-\mathrm{C}(22)$ & $118.99(14)$ \\
\hline
\end{tabular}




\begin{tabular}{|c|c|c|c|}
\hline $\mathrm{C}(23)-\mathrm{C}(24)-\mathrm{C}(8)$ & $121.27(14)$ & $\mathrm{C}(23)-\mathrm{C}(24)-\mathrm{C}(4)$ & $121.10(15)$ \\
\hline $\mathrm{C}(8)-\mathrm{C}(24)-\mathrm{C}(4)$ & $117.63(14)$ & $\mathrm{O}(1)-\mathrm{C}(25)-\mathrm{C}(26)$ & $107.09(13)$ \\
\hline $\mathrm{O}(1)-\mathrm{C}(25)-\mathrm{H}(25 \mathrm{~A})$ & 110.3 & $\mathrm{C}(26)-\mathrm{C}(25)-\mathrm{H}(25 \mathrm{~A})$ & 110.3 \\
\hline $\mathrm{O}(1)-\mathrm{C}(25)-\mathrm{H}(25 \mathrm{~B})$ & 110.3 & $\mathrm{C}(26)-\mathrm{C}(25)-\mathrm{H}(25 \mathrm{~B})$ & 110.3 \\
\hline $\mathrm{H}(25 \mathrm{~A})-\mathrm{C}(25)-\mathrm{H}(25 \mathrm{~B})$ & 108.6 & $C(25)-C(26)-C(27)$ & $111.61(14)$ \\
\hline $\mathrm{C}(25)-\mathrm{C}(26)-\mathrm{H}(26 \mathrm{~A})$ & 109.3 & $\mathrm{C}(27)-\mathrm{C}(26)-\mathrm{H}(26 \mathrm{~A})$ & 109.3 \\
\hline $\mathrm{C}(25)-\mathrm{C}(26)-\mathrm{H}(26 \mathrm{~B})$ & 109.3 & $\mathrm{C}(27)-\mathrm{C}(26)-\mathrm{H}(26 \mathrm{~B})$ & 109.3 \\
\hline $\mathrm{H}(26 \mathrm{~A})-\mathrm{C}(26)-\mathrm{H}(26 \mathrm{~B})$ & 108.0 & $\mathrm{C}(28)-\mathrm{C}(27)-\mathrm{C}(26)$ & $112.40(14)$ \\
\hline $\mathrm{C}(28)-\mathrm{C}(27)-\mathrm{H}(27 \mathrm{~A})$ & 109.1 & $\mathrm{C}(26)-\mathrm{C}(27)-\mathrm{H}(27 \mathrm{~A})$ & 109.1 \\
\hline $\mathrm{C}(28)-\mathrm{C}(27)-\mathrm{H}(27 \mathrm{~B})$ & 109.1 & $\mathrm{C}(26)-\mathrm{C}(27)-\mathrm{H}(27 \mathrm{~B})$ & 109.1 \\
\hline $\mathrm{H}(27 \mathrm{~A})-\mathrm{C}(27)-\mathrm{H}(27 \mathrm{~B})$ & 107.9 & $\mathrm{C}(27)-\mathrm{C}(28)-\mathrm{C}(29)$ & $114.47(15)$ \\
\hline $\mathrm{C}(27)-\mathrm{C}(28)-\mathrm{H}(28 \mathrm{~A})$ & 108.6 & $\mathrm{C}(29)-\mathrm{C}(28)-\mathrm{H}(28 \mathrm{~A})$ & 108.6 \\
\hline $\mathrm{C}(27)-\mathrm{C}(28)-\mathrm{H}(28 \mathrm{~B})$ & 108.6 & $\mathrm{C}(29)-\mathrm{C}(28)-\mathrm{H}(28 \mathrm{~B})$ & 108.6 \\
\hline $\mathrm{H}(28 \mathrm{~A})-\mathrm{C}(28)-\mathrm{H}(28 \mathrm{~B})$ & 107.6 & $\mathrm{C}(30)-\mathrm{C}(29)-\mathrm{C}(28)$ & $113.81(16)$ \\
\hline $\mathrm{C}(30)-\mathrm{C}(29)-\mathrm{H}(29 \mathrm{~A})$ & 108.8 & $\mathrm{C}(28)-\mathrm{C}(29)-\mathrm{H}(29 \mathrm{~A})$ & 108.8 \\
\hline $\mathrm{C}(30)-\mathrm{C}(29)-\mathrm{H}(29 \mathrm{~B})$ & 108.8 & $\mathrm{C}(28)-\mathrm{C}(29)-\mathrm{H}(29 \mathrm{~B})$ & 108.8 \\
\hline $\mathrm{H}(29 \mathrm{~A})-\mathrm{C}(29)-\mathrm{H}(29 \mathrm{~B})$ & 107.7 & $\mathrm{C}(29)-\mathrm{C}(30)-\mathrm{H}(30 \mathrm{~A})$ & 109.5 \\
\hline $\mathrm{C}(29)-\mathrm{C}(30)-\mathrm{H}(30 \mathrm{~B})$ & 109.5 & $\mathrm{H}(30 \mathrm{~A})-\mathrm{C}(30)-\mathrm{H}(30 \mathrm{~B})$ & 109.5 \\
\hline $\mathrm{C}(29)-\mathrm{C}(30)-\mathrm{H}(30 \mathrm{C})$ & 109.5 & $\mathrm{H}(30 \mathrm{~A})-\mathrm{C}(30)-\mathrm{H}(30 \mathrm{C})$ & 109.5 \\
\hline $\mathrm{H}(30 \mathrm{~B})-\mathrm{C}(30)-\mathrm{H}(30 \mathrm{C})$ & 109.5 & $\mathrm{C}(32)-\mathrm{C}(31)-\mathrm{C}(36)$ & $119.94(15)$ \\
\hline $\mathrm{C}(32)-\mathrm{C}(31)-\mathrm{C}(9)$ & $120.92(15)$ & $\mathrm{C}(36)-\mathrm{C}(31)-\mathrm{C}(9)$ & $118.87(14)$ \\
\hline $\mathrm{C}(33)-\mathrm{C}(32)-\mathrm{C}(31)$ & $118.94(15)$ & $\mathrm{C}(33)-\mathrm{C}(32)-\mathrm{C}(43)$ & $119.63(15)$ \\
\hline $\mathrm{C}(31)-\mathrm{C}(32)-\mathrm{C}(43)$ & $121.42(15)$ & $\mathrm{C}(34)-\mathrm{C}(33)-\mathrm{C}(32)$ & $121.92(16)$ \\
\hline $\mathrm{C}(34)-\mathrm{C}(33)-\mathrm{H}(33)$ & 119.0 & $\mathrm{C}(32)-\mathrm{C}(33)-\mathrm{H}(33)$ & 119.0 \\
\hline $\mathrm{C}(33)-\mathrm{C}(34)-\mathrm{C}(35)$ & $118.09(16)$ & $\mathrm{C}(33)-\mathrm{C}(34)-\mathrm{C}(44)$ & $121.86(17)$ \\
\hline $\mathrm{C}(35)-\mathrm{C}(34)-\mathrm{C}(44)$ & $120.04(17)$ & $\mathrm{C}(36)-\mathrm{C}(35)-\mathrm{C}(34)$ & $122.13(16)$ \\
\hline $\mathrm{C}(36)-\mathrm{C}(35)-\mathrm{H}(35)$ & 118.9 & $\mathrm{C}(34)-\mathrm{C}(35)-\mathrm{H}(35)$ & 118.9 \\
\hline $\mathrm{C}(35)-\mathrm{C}(36)-\mathrm{C}(31)$ & $118.96(15)$ & $C(35)-C(36)-C(45)$ & $120.16(16)$ \\
\hline $\mathrm{C}(31)-\mathrm{C}(36)-\mathrm{C}(45)$ & $120.87(15)$ & $\mathrm{C}(42)-\mathrm{C}(37)-\mathrm{C}(38)$ & $119.36(16)$ \\
\hline $\mathrm{C}(42)-\mathrm{C}(37)-\mathrm{C}(19)$ & $120.83(15)$ & $\mathrm{C}(38)-\mathrm{C}(37)-\mathrm{C}(19)$ & $119.66(15)$ \\
\hline $\mathrm{C}(39)-\mathrm{C}(38)-\mathrm{C}(37)$ & $119.48(16)$ & $\mathrm{C}(39)-\mathrm{C}(38)-\mathrm{C}(46)$ & $119.68(16)$ \\
\hline $\mathrm{C}(37)-\mathrm{C}(38)-\mathrm{C}(46)$ & $120.83(15)$ & $\mathrm{C}(40)-\mathrm{C}(39)-\mathrm{C}(38)$ & $121.71(17)$ \\
\hline $\mathrm{C}(40)-\mathrm{C}(39)-\mathrm{H}(39)$ & 119.1 & $\mathrm{C}(38)-\mathrm{C}(39)-\mathrm{H}(39)$ & 119.1 \\
\hline $\mathrm{C}(41)-\mathrm{C}(40)-\mathrm{C}(39)$ & 118.07(16) & $\mathrm{C}(41)-\mathrm{C}(40)-\mathrm{C}(47)$ & $121.19(16)$ \\
\hline $\mathrm{C}(39)-\mathrm{C}(40)-\mathrm{C}(47)$ & $120.74(17)$ & $\mathrm{C}(40)-\mathrm{C}(41)-\mathrm{C}(42)$ & $122.16(16)$ \\
\hline $\mathrm{C}(40)-\mathrm{C}(41)-\mathrm{H}(41)$ & 118.9 & $\mathrm{C}(42)-\mathrm{C}(41)-\mathrm{H}(41)$ & 118.9 \\
\hline $\mathrm{C}(41)-\mathrm{C}(42)-\mathrm{C}(37)$ & $119.18(16)$ & $\mathrm{C}(41)-\mathrm{C}(42)-\mathrm{C}(48)$ & $119.67(15)$ \\
\hline $\mathrm{C}(37)-\mathrm{C}(42)-\mathrm{C}(48)$ & $121.12(15)$ & $\mathrm{C}(32)-\mathrm{C}(43)-\mathrm{H}(43 \mathrm{~A})$ & 109.5 \\
\hline
\end{tabular}




\begin{tabular}{|c|c|c|c|}
\hline $\mathrm{C}(32)-\mathrm{C}(43)-\mathrm{H}(43 \mathrm{~B})$ & 109.5 & $\mathrm{H}(43 \mathrm{~A})-\mathrm{C}(43)-\mathrm{H}(43 \mathrm{~B})$ & 109.5 \\
\hline $\mathrm{C}(32)-\mathrm{C}(43)-\mathrm{H}(43 \mathrm{C})$ & 109.5 & $\mathrm{H}(43 \mathrm{~A})-\mathrm{C}(43)-\mathrm{H}(43 \mathrm{C})$ & 109.5 \\
\hline $\mathrm{H}(43 \mathrm{~B})-\mathrm{C}(43)-\mathrm{H}(43 \mathrm{C})$ & 109.5 & $\mathrm{C}(34)-\mathrm{C}(44)-\mathrm{H}(44 \mathrm{~A})$ & 109.5 \\
\hline $\mathrm{C}(34)-\mathrm{C}(44)-\mathrm{H}(44 \mathrm{~B})$ & 109.5 & $\mathrm{H}(44 \mathrm{~A})-\mathrm{C}(44)-\mathrm{H}(44 \mathrm{~B})$ & 109.5 \\
\hline $\mathrm{C}(34)-\mathrm{C}(44)-\mathrm{H}(44 \mathrm{C})$ & 109.5 & $\mathrm{H}(44 \mathrm{~A})-\mathrm{C}(44)-\mathrm{H}(44 \mathrm{C})$ & 109.5 \\
\hline $\mathrm{H}(44 \mathrm{~B})-\mathrm{C}(44)-\mathrm{H}(44 \mathrm{C})$ & 109.5 & $\mathrm{C}(36)-\mathrm{C}(45)-\mathrm{H}(45 \mathrm{~A})$ & 109.5 \\
\hline $\mathrm{C}(36)-\mathrm{C}(45)-\mathrm{H}(45 \mathrm{~B})$ & 109.5 & $\mathrm{H}(45 \mathrm{~A})-\mathrm{C}(45)-\mathrm{H}(45 \mathrm{~B})$ & 109.5 \\
\hline $\mathrm{C}(36)-\mathrm{C}(45)-\mathrm{H}(45 \mathrm{C})$ & 109.5 & $\mathrm{H}(45 \mathrm{~A})-\mathrm{C}(45)-\mathrm{H}(45 \mathrm{C})$ & 109.5 \\
\hline $\mathrm{H}(45 \mathrm{~B})-\mathrm{C}(45)-\mathrm{H}(45 \mathrm{C})$ & 109.5 & $\mathrm{C}(38)-\mathrm{C}(46)-\mathrm{H}(46 \mathrm{~A})$ & 109.5 \\
\hline $\mathrm{C}(38)-\mathrm{C}(46)-\mathrm{H}(46 \mathrm{~B})$ & 109.5 & $\mathrm{H}(46 \mathrm{~A})-\mathrm{C}(46)-\mathrm{H}(46 \mathrm{~B})$ & 109.5 \\
\hline $\mathrm{C}(38)-\mathrm{C}(46)-\mathrm{H}(46 \mathrm{C})$ & 109.5 & $\mathrm{H}(46 \mathrm{~A})-\mathrm{C}(46)-\mathrm{H}(46 \mathrm{C})$ & 109.5 \\
\hline $\mathrm{H}(46 \mathrm{~B})-\mathrm{C}(46)-\mathrm{H}(46 \mathrm{C})$ & 109.5 & $\mathrm{C}(40)-\mathrm{C}(47)-\mathrm{H}(47 \mathrm{~A})$ & 109.5 \\
\hline $\mathrm{C}(40)-\mathrm{C}(47)-\mathrm{H}(47 \mathrm{~B})$ & 109.5 & $\mathrm{H}(47 \mathrm{~A})-\mathrm{C}(47)-\mathrm{H}(47 \mathrm{~B})$ & 109.5 \\
\hline $\mathrm{C}(40)-\mathrm{C}(47)-\mathrm{H}(47 \mathrm{C})$ & 109.5 & $\mathrm{H}(47 \mathrm{~A})-\mathrm{C}(47)-\mathrm{H}(47 \mathrm{C})$ & 109.5 \\
\hline $\mathrm{H}(47 \mathrm{~B})-\mathrm{C}(47)-\mathrm{H}(47 \mathrm{C})$ & 109.5 & $\mathrm{C}(42)-\mathrm{C}(48)-\mathrm{H}(48 \mathrm{~A})$ & 109.5 \\
\hline $\mathrm{C}(42)-\mathrm{C}(48)-\mathrm{H}(48 \mathrm{~B})$ & 109.5 & $\mathrm{H}(48 \mathrm{~A})-\mathrm{C}(48)-\mathrm{H}(48 \mathrm{~B})$ & 109.5 \\
\hline $\mathrm{C}(42)-\mathrm{C}(48)-\mathrm{H}(48 \mathrm{C})$ & 109.5 & $\mathrm{H}(48 \mathrm{~A})-\mathrm{C}(48)-\mathrm{H}(48 \mathrm{C})$ & 109.5 \\
\hline $\mathrm{H}(48 \mathrm{~B})-\mathrm{C}(48)-\mathrm{H}(48 \mathrm{C})$ & 109.5 & & \\
\hline
\end{tabular}

\section{Toluene}

$\begin{array}{llll}\mathrm{C}(2 \mathrm{~T})-\mathrm{C}(1 \mathrm{~T})-\mathrm{C}(6 \mathrm{~T}) & 117.91(19) & \mathrm{C}(2 \mathrm{~T})-\mathrm{C}(1 \mathrm{~T})-\mathrm{C}(7 \mathrm{~T}) & 120.45(19) \\ \mathrm{C}(6 \mathrm{~T})-\mathrm{C}(1 \mathrm{~T})-\mathrm{C}(7 \mathrm{~T}) & 121.63(18) & \mathrm{C}(3 \mathrm{~T})-\mathrm{C}(2 \mathrm{~T})-\mathrm{C}(1 \mathrm{~T}) & 120.7(2) \\ \mathrm{C}(3 \mathrm{~T})-\mathrm{C}(2 \mathrm{~T})-\mathrm{H}(2 \mathrm{~T}) & 119.6 & \mathrm{C}(1 \mathrm{~T})-\mathrm{C}(2 \mathrm{~T})-\mathrm{H}(2 \mathrm{~T}) & 119.6 \\ \mathrm{C}(4 \mathrm{~T})-\mathrm{C}(3 \mathrm{~T})-\mathrm{C}(2 \mathrm{~T}) & 120.7(2) & \mathrm{C}(4 \mathrm{~T})-\mathrm{C}(3 \mathrm{~T})-\mathrm{H}(3 \mathrm{~T}) & 119.6 \\ \mathrm{C}(2 \mathrm{~T})-\mathrm{C}(3 \mathrm{~T})-\mathrm{H}(3 \mathrm{~T}) & 119.6 & \mathrm{C}(5 \mathrm{~T})-\mathrm{C}(4 \mathrm{~T})-\mathrm{C}(3 \mathrm{~T}) & 118.8(2) \\ \mathrm{C}(5 \mathrm{~T})-\mathrm{C}(4 \mathrm{~T})-\mathrm{H}(4 \mathrm{~T}) & 120.6 & \mathrm{C}(3 \mathrm{~T})-\mathrm{C}(4 \mathrm{~T})-\mathrm{H}(4 \mathrm{~T}) & 120.6 \\ \mathrm{C}(4 \mathrm{~T})-\mathrm{C}(5 \mathrm{~T})-\mathrm{C}(6 \mathrm{~T}) & 120.9(2) & \mathrm{C}(4 \mathrm{~T})-\mathrm{C}(5 \mathrm{~T})-\mathrm{H}(5 \mathrm{~T}) & 119.6 \\ \mathrm{C}(6 \mathrm{~T})-\mathrm{C}(5 \mathrm{~T})-\mathrm{H}(5 \mathrm{~T}) & 119.6 & \mathrm{C}(5 \mathrm{~T})-\mathrm{C}(6 \mathrm{~T})-\mathrm{C}(1 \mathrm{~T}) & 120.92(19) \\ \mathrm{C}(5 \mathrm{~T})-\mathrm{C}(6 \mathrm{~T})-\mathrm{H}(6 \mathrm{~T}) & 119.5 & \mathrm{C}(1 \mathrm{~T})-\mathrm{C}(6 \mathrm{~T})-\mathrm{H}(6 \mathrm{~T}) & 119.5 \\ \mathrm{C}(1 \mathrm{~T})-\mathrm{C}(7 \mathrm{~T})-\mathrm{H}(7 \mathrm{~T} 1) & 109.5 & \mathrm{C}(1 \mathrm{~T})-\mathrm{C}(7 \mathrm{~T})-\mathrm{H}(7 \mathrm{~T} 2) & 109.5 \\ \mathrm{H}(7 \mathrm{~T} 1)-\mathrm{C}(7 \mathrm{~T})-\mathrm{H}(7 \mathrm{~T} 2) & 109.5 & \mathrm{C}(1 \mathrm{~T})-\mathrm{C}(7 \mathrm{~T})-\mathrm{H}(7 \mathrm{~T} 3) & 109.5 \\ \mathrm{H}(7 \mathrm{~T} 1)-\mathrm{C}(7 \mathrm{~T})-\mathrm{H}(7 \mathrm{~T} 3) & 109.5 & \mathrm{H}(7 \mathrm{~T} 2)-\mathrm{C}(7 \mathrm{~T})-\mathrm{H}(7 \mathrm{~T} 3) & 109.5\end{array}$


Table S42. Anisotropic displacement parameters $\left(\AA^{2} \times 10^{3}\right)$ for PF-M. The anisotropic displacement factor exponent takes the form: $-2 \square^{2}\left[h^{2} a^{* 2} U^{11}+\ldots+2 h k a^{*} b^{*} U^{12}\right]$

\begin{tabular}{|c|c|c|c|c|c|c|}
\hline & $\mathrm{U}^{11}$ & $\mathrm{U}^{22}$ & $\mathrm{U}^{33}$ & $\mathrm{U}^{23}$ & $\mathrm{U}^{13}$ & $\mathrm{U}^{12}$ \\
\hline $\mathrm{O}(1)$ & $26(1)$ & $24(1)$ & $15(1)$ & $0(1)$ & $4(1)$ & $6(1)$ \\
\hline $\mathrm{C}(1)$ & $20(1)$ & $14(1)$ & $20(1)$ & $1(1)$ & $3(1)$ & $0(1)$ \\
\hline$C(2)$ & $26(1)$ & 21(1) & $16(1)$ & $0(1)$ & $3(1)$ & $-2(1)$ \\
\hline$C(3)$ & $23(1)$ & 21(1) & $17(1)$ & $4(1)$ & $-3(1)$ & $-2(1)$ \\
\hline $\mathrm{C}(4)$ & 19(1) & $15(1)$ & 21(1) & $3(1)$ & $0(1)$ & $-2(1)$ \\
\hline$C(5)$ & 19(1) & 21(1) & $23(1)$ & $4(1)$ & $-4(1)$ & $-1(1)$ \\
\hline$C(6)$ & $17(1)$ & 21(1) & $30(1)$ & $4(1)$ & $2(1)$ & $3(1)$ \\
\hline$C(7)$ & $20(1)$ & $18(1)$ & $22(1)$ & $1(1)$ & $5(1)$ & $0(1)$ \\
\hline$C(8)$ & $18(1)$ & $14(1)$ & 20(1) & $2(1)$ & $2(1)$ & $-2(1)$ \\
\hline $\mathrm{C}(9)$ & $20(1)$ & $12(1)$ & $18(1)$ & $0(1)$ & $3(1)$ & $-1(1)$ \\
\hline$C(10)$ & 19(1) & $14(1)$ & $17(1)$ & $1(1)$ & $3(1)$ & 1(1) \\
\hline $\mathrm{C}(11)$ & 21(1) & $18(1)$ & $16(1)$ & $-2(1)$ & $3(1)$ & $1(1)$ \\
\hline$C(12)$ & 21(1) & $16(1)$ & $17(1)$ & $1(1)$ & $2(1)$ & $-1(1)$ \\
\hline$C(13)$ & $20(1)$ & $17(1)$ & $21(1)$ & $2(1)$ & $2(1)$ & $2(1)$ \\
\hline $\mathrm{C}(14)$ & $22(1)$ & $22(1)$ & $21(1)$ & $-1(1)$ & $4(1)$ & 1(1) \\
\hline$C(15)$ & $25(1)$ & $23(1)$ & $18(1)$ & $0(1)$ & $0(1)$ & $-1(1)$ \\
\hline$C(16)$ & $22(1)$ & $22(1)$ & $24(1)$ & $5(1)$ & $-2(1)$ & $0(1)$ \\
\hline$C(17)$ & 21(1) & $17(1)$ & $25(1)$ & $2(1)$ & $3(1)$ & $2(1)$ \\
\hline$C(18)$ & $22(1)$ & $15(1)$ & 21(1) & $1(1)$ & $2(1)$ & $0(1)$ \\
\hline C(19) & 21(1) & $15(1)$ & $20(1)$ & $1(1)$ & 1(1) & $2(1)$ \\
\hline$C(20)$ & 21(1) & $15(1)$ & $20(1)$ & $1(1)$ & $2(1)$ & $2(1)$ \\
\hline $\mathrm{C}(21)$ & $22(1)$ & $17(1)$ & $16(1)$ & $-2(1)$ & $3(1)$ & $2(1)$ \\
\hline $\mathrm{C}(22)$ & 19(1) & $15(1)$ & $16(1)$ & $0(1)$ & $2(1)$ & $-2(1)$ \\
\hline$C(23)$ & $20(1)$ & $12(1)$ & $17(1)$ & $0(1)$ & $2(1)$ & $-1(1)$ \\
\hline $\mathrm{C}(24)$ & $18(1)$ & $14(1)$ & 19(1) & $1(1)$ & 1(1) & $-3(1)$ \\
\hline $\mathrm{C}(25)$ & $27(1)$ & $19(1)$ & $16(1)$ & $-1(1)$ & $6(1)$ & $-1(1)$ \\
\hline$C(26)$ & $30(1)$ & $17(1)$ & $21(1)$ & 1(1) & $7(1)$ & $2(1)$ \\
\hline $\mathrm{C}(27)$ & $30(1)$ & $20(1)$ & $23(1)$ & $-2(1)$ & $8(1)$ & $-2(1)$ \\
\hline $\mathrm{C}(28)$ & $33(1)$ & $25(1)$ & $27(1)$ & 1(1) & $8(1)$ & $4(1)$ \\
\hline C(29) & $31(1)$ & $35(1)$ & $34(1)$ & $-5(1)$ & 11(1) & $4(1)$ \\
\hline & $\mathrm{U}^{11}$ & $\mathrm{U}^{22}$ & $\mathrm{U}^{33}$ & $\mathrm{U}^{23}$ & $\mathrm{U}^{13}$ & $\mathrm{U}^{12}$ \\
\hline
\end{tabular}




\begin{tabular}{|c|c|c|c|c|c|c|}
\hline $\mathrm{C}(30)$ & $38(1)$ & $46(1)$ & $35(1)$ & $-2(1)$ & $15(1)$ & $-2(1)$ \\
\hline $\mathrm{C}(31)$ & $15(1)$ & 19(1) & $18(1)$ & $0(1)$ & 1(1) & $4(1)$ \\
\hline$C(32)$ & $20(1)$ & $17(1)$ & 21(1) & $0(1)$ & $0(1)$ & $2(1)$ \\
\hline$C(33)$ & $23(1)$ & $18(1)$ & $25(1)$ & $-5(1)$ & $2(1)$ & $2(1)$ \\
\hline$C(34)$ & $20(1)$ & $30(1)$ & 21(1) & $-7(1)$ & $3(1)$ & $2(1)$ \\
\hline$C(35)$ & $20(1)$ & $29(1)$ & 21(1) & $1(1)$ & $5(1)$ & $-3(1)$ \\
\hline$C(36)$ & $18(1)$ & $20(1)$ & 21(1) & $-1(1)$ & $2(1)$ & 1(1) \\
\hline C(37) & $18(1)$ & $19(1)$ & $19(1)$ & $-2(1)$ & $-2(1)$ & $3(1)$ \\
\hline $\mathrm{C}(38)$ & 21(1) & 19(1) & $22(1)$ & $-2(1)$ & $-1(1)$ & $3(1)$ \\
\hline C(39) & 19(1) & $26(1)$ & $25(1)$ & $-2(1)$ & $3(1)$ & $-1(1)$ \\
\hline $\mathrm{C}(40)$ & $17(1)$ & $24(1)$ & $29(1)$ & $-7(1)$ & $1(1)$ & $2(1)$ \\
\hline $\mathrm{C}(41)$ & $22(1)$ & $14(1)$ & $33(1)$ & $-4(1)$ & 1(1) & $2(1)$ \\
\hline$C(42)$ & $19(1)$ & $19(1)$ & $24(1)$ & $-1(1)$ & $-1(1)$ & 1(1) \\
\hline $\mathrm{C}(43)$ & $35(1)$ & $20(1)$ & $26(1)$ & 1(1) & $7(1)$ & $-3(1)$ \\
\hline$C(44)$ & $35(1)$ & $46(1)$ & $36(1)$ & $-17(1)$ & $15(1)$ & $-5(1)$ \\
\hline$C(45)$ & $35(1)$ & 21(1) & $30(1)$ & $-1(1)$ & $10(1)$ & $-5(1)$ \\
\hline$C(46)$ & $32(1)$ & $20(1)$ & $28(1)$ & 1(1) & $6(1)$ & 1(1) \\
\hline$C(47)$ & $24(1)$ & $31(1)$ & $46(1)$ & $-12(1)$ & $10(1)$ & 2(1) \\
\hline$C(48)$ & $29(1)$ & $20(1)$ & $32(1)$ & $2(1)$ & $5(1)$ & 1(1) \\
\hline \multicolumn{7}{|c|}{ Toluene } \\
\hline & $\mathrm{U}^{11}$ & $\mathrm{U}^{22}$ & $\mathrm{U}^{33}$ & $\mathrm{U}^{23}$ & $\mathrm{U}^{13}$ & $\mathrm{U}^{12}$ \\
\hline $\mathrm{C}(1 \mathrm{~T})$ & $32(1)$ & $26(1)$ & $32(1)$ & $4(1)$ & $0(1)$ & $8(1)$ \\
\hline $\mathrm{C}(2 \mathrm{~T})$ & $32(1)$ & $26(1)$ & $41(1)$ & $3(1)$ & $-2(1)$ & $2(1)$ \\
\hline $\mathrm{C}(3 \mathrm{~T})$ & $41(1)$ & $41(1)$ & $36(1)$ & $-5(1)$ & $-2(1)$ & $8(1)$ \\
\hline $\mathrm{C}(4 \mathrm{~T})$ & $39(1)$ & $56(1)$ & $36(1)$ & $5(1)$ & 7(1) & $12(1)$ \\
\hline $\mathrm{C}(5 \mathrm{~T})$ & $36(1)$ & $43(1)$ & $48(1)$ & $12(1)$ & $5(1)$ & $-1(1)$ \\
\hline $\mathrm{C}(6 \mathrm{~T})$ & $36(1)$ & $27(1)$ & $39(1)$ & $0(1)$ & $-2(1)$ & 1(1) \\
\hline $\mathrm{C}(7 \mathrm{~T})$ & $43(1)$ & $36(1)$ & $41(1)$ & $5(1)$ & $7(1)$ & $7(1)$ \\
\hline
\end{tabular}

Table S43. Hydrogen coordinates ( x 10 $0^{4}$ ) and isotropic displacement parameters $\left(\AA^{2} \times 10^{3}\right)$ for PF-M. 


\begin{tabular}{|c|c|c|c|c|}
\hline $\mathrm{H}(2)$ & 4554 & 7541 & -503 & 26 \\
\hline $\mathrm{H}(3)$ & 3219 & 8897 & -486 & 26 \\
\hline $\mathrm{H}(5)$ & 2164 & 10172 & -110 & 26 \\
\hline $\mathrm{H}(6)$ & 1819 & 10995 & 477 & 27 \\
\hline $\mathrm{H}(7)$ & 2886 & 10642 & 1059 & 24 \\
\hline $\mathrm{H}(11)$ & 5841 & 8764 & 1789 & 22 \\
\hline $\mathrm{H}(14)$ & 7272 & 8037 & 2410 & 26 \\
\hline $\mathrm{H}(15)$ & 8585 & 6975 & 2811 & 27 \\
\hline $\mathrm{H}(16)$ & 9516 & 5135 & 2563 & 28 \\
\hline $\mathrm{H}(17)$ & 9158 & 4268 & 1909 & 26 \\
\hline $\mathrm{H}(21)$ & 6349 & 5960 & 659 & 22 \\
\hline $\mathrm{H}(25 \mathrm{~A})$ & 6066 & 7258 & -450 & 25 \\
\hline $\mathrm{H}(25 \mathrm{~B})$ & 5609 & 5484 & -443 & 25 \\
\hline $\mathrm{H}(26 \mathrm{~A})$ & 6966 & 4448 & -51 & 27 \\
\hline $\mathrm{H}(26 \mathrm{~B})$ & 7421 & 6211 & -65 & 27 \\
\hline $\mathrm{H}(27 \mathrm{~A})$ & 6890 & 4061 & -722 & 29 \\
\hline $\mathrm{H}(27 \mathrm{~B})$ & 7232 & 5895 & -766 & 29 \\
\hline $\mathrm{H}(28 \mathrm{~A})$ & 8327 & 3403 & -327 & 33 \\
\hline $\mathrm{H}(28 \mathrm{~B})$ & 8663 & 5212 & -399 & 33 \\
\hline $\mathrm{H}(29 \mathrm{~A})$ & 9198 & 3249 & -806 & 39 \\
\hline $\mathrm{H}(29 \mathrm{~B})$ & 8194 & 2785 & -1000 & 39 \\
\hline $\mathrm{H}(30 \mathrm{~A})$ & 7978 & 5436 & -1286 & 58 \\
\hline $\mathrm{H}(30 \mathrm{~B})$ & 8823 & 4587 & -1410 & 58 \\
\hline $\mathrm{H}(30 \mathrm{C})$ & 8966 & 5960 & -1080 & 58 \\
\hline $\mathrm{H}(33)$ & 4218 & 13566 & 2016 & 27 \\
\hline $\mathrm{H}(35)$ & 3196 & 9146 & 2248 & 28 \\
\hline $\mathrm{H}(39)$ & 9311 & 4786 & 415 & 28 \\
\hline $\mathrm{H}(41)$ & 8749 & 569 & 938 & 28 \\
\hline $\mathrm{H}(43 \mathrm{~A})$ & 4503 & 13308 & 1178 & 40 \\
\hline
\end{tabular}

H(43B)

H(43C)
5378

5227
12200

13751
1299

1556
40

40 


$\begin{array}{lrrrr}\mathrm{H}(44 \mathrm{~A}) & 2681 & 11869 & 2519 & 57 \\ \mathrm{H}(44 \mathrm{~B}) & 3313 & 13431 & 2513 & 57 \\ \mathrm{H}(44 \mathrm{C}) & 3673 & 11866 & 2771 & 57 \\ \mathrm{H}(45 \mathrm{~A}) & 3304 & 7089 & 1434 & 42 \\ \mathrm{H}(45 \mathrm{~B}) & 3317 & 6752 & 1881 & 42 \\ \mathrm{H}(45 \mathrm{C}) & 4222 & 6650 & 1720 & 42 \\ \mathrm{H}(46 \mathrm{~A}) & 7858 & 7341 & 631 & 40 \\ \mathrm{H}(46 \mathrm{~B}) & 8895 & 7414 & 614 & 40 \\ \mathrm{H}(46 \mathrm{C}) & 8587 & 7574 & 1020 & 40 \\ \mathrm{H}(47 \mathrm{~A}) & 9337 & 1105 & 175 & 50 \\ \mathrm{H}(47 \mathrm{~B}) & 10008 & 632 & 566 & 50 \\ \mathrm{H}(47 \mathrm{C}) & 10168 & 2269 & 339 & 50 \\ \mathrm{H}(48 \mathrm{~A}) & 7824 & 2127 & 1661 & 41 \\ \mathrm{H}(48 \mathrm{~B}) & 7848 & 499 & 1410 & 41 \\ \mathrm{H}(48 \mathrm{C}) & 7028 & 1748 & 1305 & 41\end{array}$

Toluene

$\begin{array}{lrrrr}\mathrm{H}(2 \mathrm{~T}) & -725 & -1540 & 1873 & 41 \\ \mathrm{H}(3 \mathrm{~T}) & 155 & -2018 & 1408 & 49 \\ \mathrm{H}(4 \mathrm{~T}) & 1341 & -224 & 1354 & 53 \\ \mathrm{H}(5 \mathrm{~T}) & 1625 & 2061 & 1767 & 51 \\ \mathrm{H}(6 \mathrm{~T}) & 758 & 2548 & 2234 & 42 \\ \mathrm{H}(7 \mathrm{~T} 1) & -1265 & 1017 & 2286 & 61 \\ \mathrm{H}(7 \mathrm{~T} 2) & -421 & 1807 & 2566 & 61 \\ \mathrm{H}(7 \mathrm{~T} 3) & -604 & -124 & 2582 & 61\end{array}$

Table S44. Torsion angles $\left[^{\circ}\right]$ for PF-M.

$\begin{array}{ll}\mathrm{C}(25)-\mathrm{O}(1)-\mathrm{C}(1)-\mathrm{C}(23) & \mathrm{C}(25)-\mathrm{O}(1)-\mathrm{C}(1)-\mathrm{C}(2) \\ \mathrm{O}(1)-\mathrm{C}(1)-\mathrm{C}(2)-\mathrm{C}(3) & \mathrm{C}(23)-\mathrm{C}(1)-\mathrm{C}(2)-\mathrm{C}(3) \\ \mathrm{C}(1)-\mathrm{C}(2)-\mathrm{C}(3)-\mathrm{C}(4) & \mathrm{C}(2)-\mathrm{C}(3)-\mathrm{C}(4)-\mathrm{C}(5) \\ \mathrm{C}(2)-\mathrm{C}(3)-\mathrm{C}(4)-\mathrm{C}(24) & \mathrm{C}(3)-\mathrm{C}(4)-\mathrm{C}(5)-\mathrm{C}(6)\end{array}$




\begin{tabular}{|c|c|}
\hline $\mathrm{C}(24)-\mathrm{C}(4)-\mathrm{C}(5)-\mathrm{C}(6)$ & $\mathrm{C}(4)-\mathrm{C}(5)-\mathrm{C}(6)-\mathrm{C}(7)$ \\
\hline $\mathrm{C}(5)-\mathrm{C}(6)-\mathrm{C}(7)-\mathrm{C}(8)$ & $\mathrm{C}(6)-\mathrm{C}(7)-\mathrm{C}(8)-\mathrm{C}(24)$ \\
\hline $\mathrm{C}(6)-\mathrm{C}(7)-\mathrm{C}(8)-\mathrm{C}(9)$ & $\mathrm{C}(7)-\mathrm{C}(8)-\mathrm{C}(9)-\mathrm{C}(10)$ \\
\hline $\mathrm{C}(24)-\mathrm{C}(8)-\mathrm{C}(9)-\mathrm{C}(10)$ & $\mathrm{C}(7)-\mathrm{C}(8)-\mathrm{C}(9)-\mathrm{C}(31)$ \\
\hline $\mathrm{C}(24)-\mathrm{C}(8)-\mathrm{C}(9)-\mathrm{C}(31)$ & $\mathrm{C}(8)-\mathrm{C}(9)-\mathrm{C}(10)-\mathrm{C}(11)$ \\
\hline $\mathrm{C}(31)-\mathrm{C}(9)-\mathrm{C}(10)-\mathrm{C}(11)$ & $\mathrm{C}(8)-\mathrm{C}(9)-\mathrm{C}(10)-\mathrm{C}(22)$ \\
\hline $\mathrm{C}(31)-\mathrm{C}(9)-\mathrm{C}(10)-\mathrm{C}(22)$ & $\mathrm{C}(9)-\mathrm{C}(10)-\mathrm{C}(11)-\mathrm{C}(12)$ \\
\hline $\mathrm{C}(22)-\mathrm{C}(10)-\mathrm{C}(11)-\mathrm{C}(12)$ & $\mathrm{C}(10)-\mathrm{C}(11)-\mathrm{C}(12)-\mathrm{C}(20)$ \\
\hline $\mathrm{C}(10)-\mathrm{C}(11)-\mathrm{C}(12)-\mathrm{C}(13)$ & $\mathrm{C}(11)-\mathrm{C}(12)-\mathrm{C}(13)-\mathrm{C}(14)$ \\
\hline $\mathrm{C}(20)-\mathrm{C}(12)-\mathrm{C}(13)-\mathrm{C}(14)$ & $\mathrm{C}(11)-\mathrm{C}(12)-\mathrm{C}(13)-\mathrm{C}(18)$ \\
\hline$C(20)-C(12)-C(13)-C(18)$ & $C(18)-C(13)-C(14)-C(15)$ \\
\hline $\mathrm{C}(12)-\mathrm{C}(13)-\mathrm{C}(14)-\mathrm{C}(15)$ & $\mathrm{C}(13)-\mathrm{C}(14)-\mathrm{C}(15)-\mathrm{C}(16)$ \\
\hline $\mathrm{C}(14)-\mathrm{C}(15)-\mathrm{C}(16)-\mathrm{C}(17)$ & $\mathrm{C}(15)-\mathrm{C}(16)-\mathrm{C}(17)-\mathrm{C}(18)$ \\
\hline $\mathrm{C}(16)-\mathrm{C}(17)-\mathrm{C}(18)-\mathrm{C}(13)$ & $\mathrm{C}(16)-\mathrm{C}(17)-\mathrm{C}(18)-\mathrm{C}(19)$ \\
\hline $\mathrm{C}(14)-\mathrm{C}(13)-\mathrm{C}(18)-\mathrm{C}(17)$ & $\mathrm{C}(12)-\mathrm{C}(13)-\mathrm{C}(18)-\mathrm{C}(17)$ \\
\hline $\mathrm{C}(14)-\mathrm{C}(13)-\mathrm{C}(18)-\mathrm{C}(19)$ & $\mathrm{C}(12)-\mathrm{C}(13)-\mathrm{C}(18)-\mathrm{C}(19)$ \\
\hline $\mathrm{C}(17)-\mathrm{C}(18)-\mathrm{C}(19)-\mathrm{C}(20)$ & $C(13)-C(18)-C(19)-C(20)$ \\
\hline $\mathrm{C}(17)-\mathrm{C}(18)-\mathrm{C}(19)-\mathrm{C}(37)$ & $\mathrm{C}(13)-\mathrm{C}(18)-\mathrm{C}(19)-\mathrm{C}(37)$ \\
\hline $\mathrm{C}(18)-\mathrm{C}(19)-\mathrm{C}(20)-\mathrm{C}(21)$ & $\mathrm{C}(37)-\mathrm{C}(19)-\mathrm{C}(20)-\mathrm{C}(21)$ \\
\hline $\mathrm{C}(18)-\mathrm{C}(19)-\mathrm{C}(20)-\mathrm{C}(12)$ & $\mathrm{C}(37)-\mathrm{C}(19)-\mathrm{C}(20)-\mathrm{C}(12)$ \\
\hline $\mathrm{C}(11)-\mathrm{C}(12)-\mathrm{C}(20)-\mathrm{C}(19)$ & $\mathrm{C}(13)-\mathrm{C}(12)-\mathrm{C}(20)-\mathrm{C}(19)$ \\
\hline $\mathrm{C}(11)-\mathrm{C}(12)-\mathrm{C}(20)-\mathrm{C}(21)$ & $\mathrm{C}(13)-\mathrm{C}(12)-\mathrm{C}(20)-\mathrm{C}(21)$ \\
\hline $\mathrm{C}(19)-\mathrm{C}(20)-\mathrm{C}(21)-\mathrm{C}(22)$ & $\mathrm{C}(12)-\mathrm{C}(20)-\mathrm{C}(21)-\mathrm{C}(22)$ \\
\hline $\mathrm{C}(20)-\mathrm{C}(21)-\mathrm{C}(22)-\mathrm{C}(10)$ & $C(20)-C(21)-C(22)-C(23)$ \\
\hline $\mathrm{C}(9)-\mathrm{C}(10)-\mathrm{C}(22)-\mathrm{C}(21)$ & $C(11)-C(10)-C(22)-C(21)$ \\
\hline $\mathrm{C}(9)-\mathrm{C}(10)-\mathrm{C}(22)-\mathrm{C}(23)$ & $C(11)-C(10)-C(22)-C(23)$ \\
\hline $\mathrm{O}(1)-\mathrm{C}(1)-\mathrm{C}(23)-\mathrm{C}(24)$ & $\mathrm{C}(2)-\mathrm{C}(1)-\mathrm{C}(23)-\mathrm{C}(24)$ \\
\hline $\mathrm{O}(1)-\mathrm{C}(1)-\mathrm{C}(23)-\mathrm{C}(22)$ & $\mathrm{C}(2)-\mathrm{C}(1)-\mathrm{C}(23)-\mathrm{C}(22)$ \\
\hline $\mathrm{C}(21)-\mathrm{C}(22)-\mathrm{C}(23)-\mathrm{C}(1)$ & $\mathrm{C}(10)-\mathrm{C}(22)-\mathrm{C}(23)-\mathrm{C}(1)$ \\
\hline $\mathrm{C}(21)-\mathrm{C}(22)-\mathrm{C}(23)-\mathrm{C}(24)$ & $\mathrm{C}(10)-\mathrm{C}(22)-\mathrm{C}(23)-\mathrm{C}(24)$ \\
\hline $\mathrm{C}(1)-\mathrm{C}(23)-\mathrm{C}(24)-\mathrm{C}(8)$ & $\mathrm{C}(22)-\mathrm{C}(23)-\mathrm{C}(24)-\mathrm{C}(8)$ \\
\hline $\mathrm{C}(1)-\mathrm{C}(23)-\mathrm{C}(24)-\mathrm{C}(4)$ & $\mathrm{C}(22)-\mathrm{C}(23)-\mathrm{C}(24)-\mathrm{C}(4)$ \\
\hline $\mathrm{C}(7)-\mathrm{C}(8)-\mathrm{C}(24)-\mathrm{C}(23)$ & $\mathrm{C}(9)-\mathrm{C}(8)-\mathrm{C}(24)-\mathrm{C}(23)$ \\
\hline $\mathrm{C}(7)-\mathrm{C}(8)-\mathrm{C}(24)-\mathrm{C}(4)$ & $\mathrm{C}(9)-\mathrm{C}(8)-\mathrm{C}(24)-\mathrm{C}(4)$ \\
\hline $\mathrm{C}(3)-\mathrm{C}(4)-\mathrm{C}(24)-\mathrm{C}(23)$ & $C(5)-C(4)-C(24)-C(23)$ \\
\hline $\mathrm{C}(3)-\mathrm{C}(4)-\mathrm{C}(24)-\mathrm{C}(8)$ & $\mathrm{C}(5)-\mathrm{C}(4)-\mathrm{C}(24)-\mathrm{C}(8)$ \\
\hline $\mathrm{C}(1)-\mathrm{O}(1)-\mathrm{C}(25)-\mathrm{C}(26)$ & $\mathrm{O}(1)-\mathrm{C}(25)-\mathrm{C}(26)-\mathrm{C}(27)$ \\
\hline $\mathrm{C}(25)-\mathrm{C}(26)-\mathrm{C}(27)-\mathrm{C}(28)$ & $\mathrm{C}(26)-\mathrm{C}(27)-\mathrm{C}(28)-\mathrm{C}(29)$ \\
\hline
\end{tabular}




$\begin{array}{ll}\mathrm{C}(27)-\mathrm{C}(28)-\mathrm{C}(29)-\mathrm{C}(30) & \mathrm{C}(10)-\mathrm{C}(9)-\mathrm{C}(31)-\mathrm{C}(32) \\ \mathrm{C}(8)-\mathrm{C}(9)-\mathrm{C}(31)-\mathrm{C}(32) & \mathrm{C}(10)-\mathrm{C}(9)-\mathrm{C}(31)-\mathrm{C}(36) \\ \mathrm{C}(8)-\mathrm{C}(9)-\mathrm{C}(31)-\mathrm{C}(36) & \mathrm{C}(36)-\mathrm{C}(31)-\mathrm{C}(32)-\mathrm{C}(33) \\ \mathrm{C}(9)-\mathrm{C}(31)-\mathrm{C}(32)-\mathrm{C}(33) & \mathrm{C}(36)-\mathrm{C}(31)-\mathrm{C}(32)-\mathrm{C}(43) \\ \mathrm{C}(9)-\mathrm{C}(31)-\mathrm{C}(32)-\mathrm{C}(43) & \mathrm{C}(31)-\mathrm{C}(32)-\mathrm{C}(33)-\mathrm{C}(34) \\ \mathrm{C}(43)-\mathrm{C}(32)-\mathrm{C}(33)-\mathrm{C}(34) & \mathrm{C}(32)-\mathrm{C}(33)-\mathrm{C}(34)-\mathrm{C}(35) \\ \mathrm{C}(32)-\mathrm{C}(33)-\mathrm{C}(34)-\mathrm{C}(44) & \mathrm{C}(33)-\mathrm{C}(34)-\mathrm{C}(35)-\mathrm{C}(36) \\ \mathrm{C}(44)-\mathrm{C}(34)-\mathrm{C}(35)-\mathrm{C}(36) & \mathrm{C}(34)-\mathrm{C}(35)-\mathrm{C}(36)-\mathrm{C}(31) \\ \mathrm{C}(34)-\mathrm{C}(35)-\mathrm{C}(36)-\mathrm{C}(45) & \mathrm{C}(32)-\mathrm{C}(31)-\mathrm{C}(36)-\mathrm{C}(35) \\ \mathrm{C}(9)-\mathrm{C}(31)-\mathrm{C}(36)-\mathrm{C}(35) & \mathrm{C}(32)-\mathrm{C}(31)-\mathrm{C}(36)-\mathrm{C}(45) \\ \mathrm{C}(9)-\mathrm{C}(31)-\mathrm{C}(36)-\mathrm{C}(45) & \mathrm{C}(20)-\mathrm{C}(19)-\mathrm{C}(37)-\mathrm{C}(42) \\ \mathrm{C}(18)-\mathrm{C}(19)-\mathrm{C}(37)-\mathrm{C}(42) & \mathrm{C}(20)-\mathrm{C}(19)-\mathrm{C}(37)-\mathrm{C}(38) \\ \mathrm{C}(18)-\mathrm{C}(19)-\mathrm{C}(37)-\mathrm{C}(38) & \mathrm{C}(42)-\mathrm{C}(37)-\mathrm{C}(38)-\mathrm{C}(39) \\ \mathrm{C}(19)-\mathrm{C}(37)-\mathrm{C}(38)-\mathrm{C}(39) & \mathrm{C}(42)-\mathrm{C}(37)-\mathrm{C}(38)-\mathrm{C}(46) \\ \mathrm{C}(19)-\mathrm{C}(37)-\mathrm{C}(38)-\mathrm{C}(46) & \mathrm{C}(37)-\mathrm{C}(38)-\mathrm{C}(39)-\mathrm{C}(40) \\ \mathrm{C}(46)-\mathrm{C}(38)-\mathrm{C}(39)-\mathrm{C}(40) & \mathrm{C}(38)-\mathrm{C}(39)-\mathrm{C}(40)-\mathrm{C}(41) \\ \mathrm{C}(38)-\mathrm{C}(39)-\mathrm{C}(40)-\mathrm{C}(47) & \mathrm{C}(39)-\mathrm{C}(40)-\mathrm{C}(41)-\mathrm{C}(42) \\ \mathrm{C}(47)-\mathrm{C}(40)-\mathrm{C}(41)-\mathrm{C}(42) & \mathrm{C}(40)-\mathrm{C}(41)-\mathrm{C}(42)-\mathrm{C}(37) \\ \mathrm{C}(40)-\mathrm{C}(41)-\mathrm{C}(42)-\mathrm{C}(48) & \mathrm{C}(38)-\mathrm{C}(37)-\mathrm{C}(42)-\mathrm{C}(41) \\ \mathrm{C}(19)-\mathrm{C}(37)-\mathrm{C}(42)-\mathrm{C}(41) & \mathrm{C}(38)-\mathrm{C}(37)-\mathrm{C}(42)-\mathrm{C}(48) \\ \mathrm{C}(19)-\mathrm{C}(37)-\mathrm{C}(42)-\mathrm{C}(48) & \\ & \end{array}$




\section{0. ${ }^{1} \mathrm{H},{ }^{13} \mathrm{C}$ NMR spectra, mass spectra for new compounds}
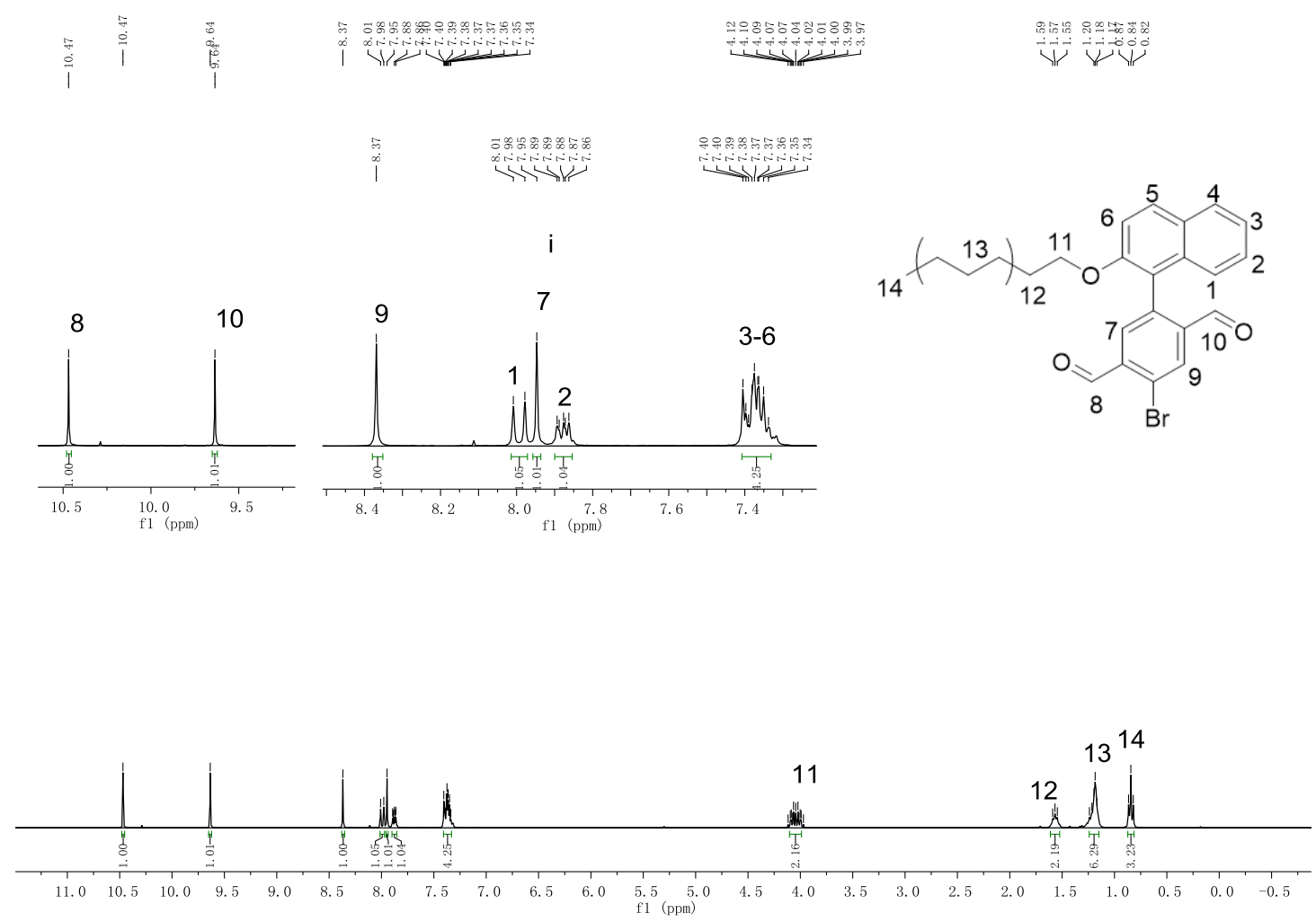

Figure S15. ${ }^{1} \mathrm{H}$ NMR spectrum (300 MHz) of compound $\mathbf{1}$ in $\mathrm{CD}_{2} \mathrm{Cl}_{2}$ at $298 \mathrm{~K}$

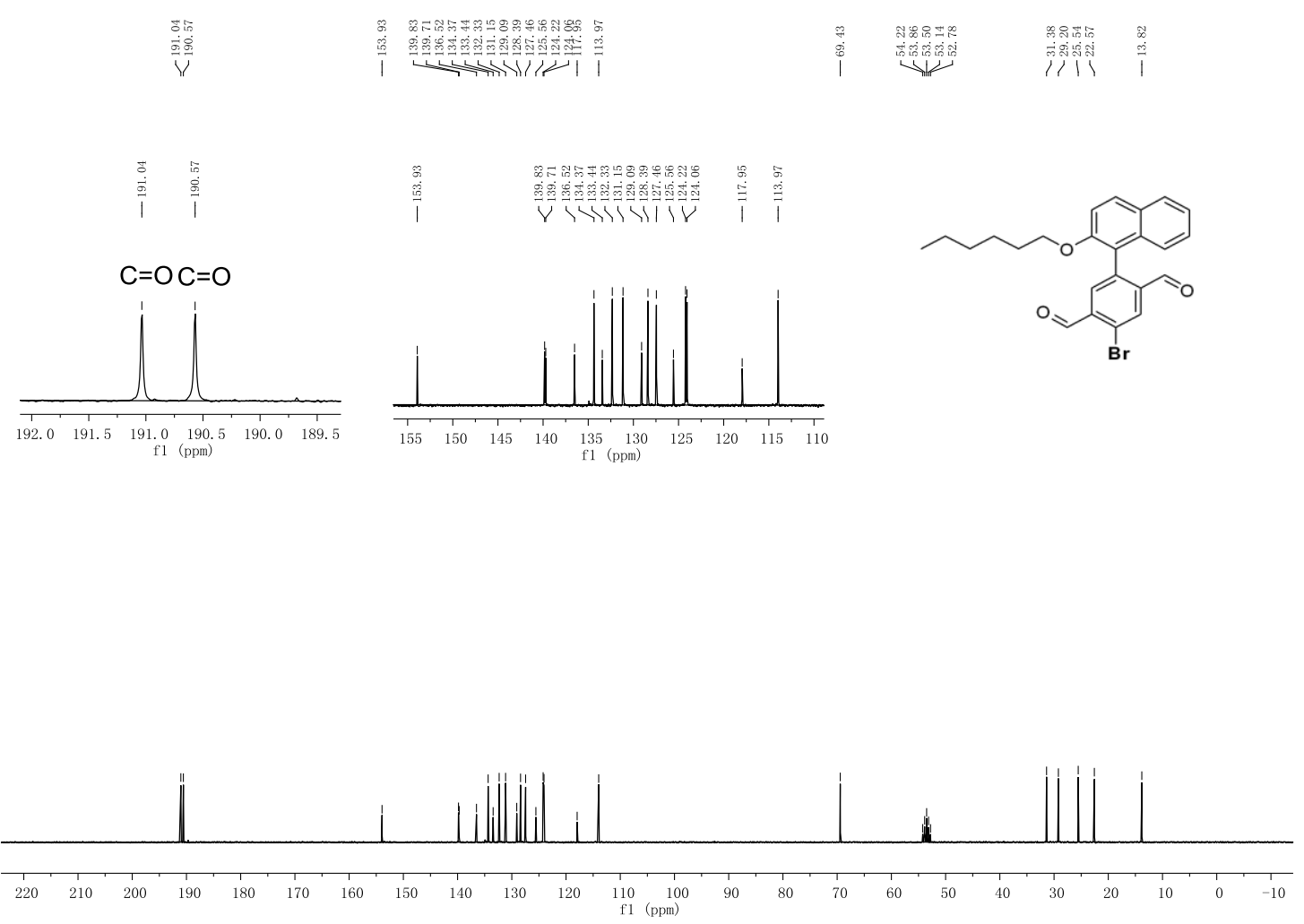

Figure S16. ${ }^{13} \mathrm{C}$ NMR spectrum (75 MHz) of compound $\mathbf{1}$ in $\mathrm{CD}_{2} \mathrm{Cl}_{2}$ at $298 \mathrm{~K}$ 


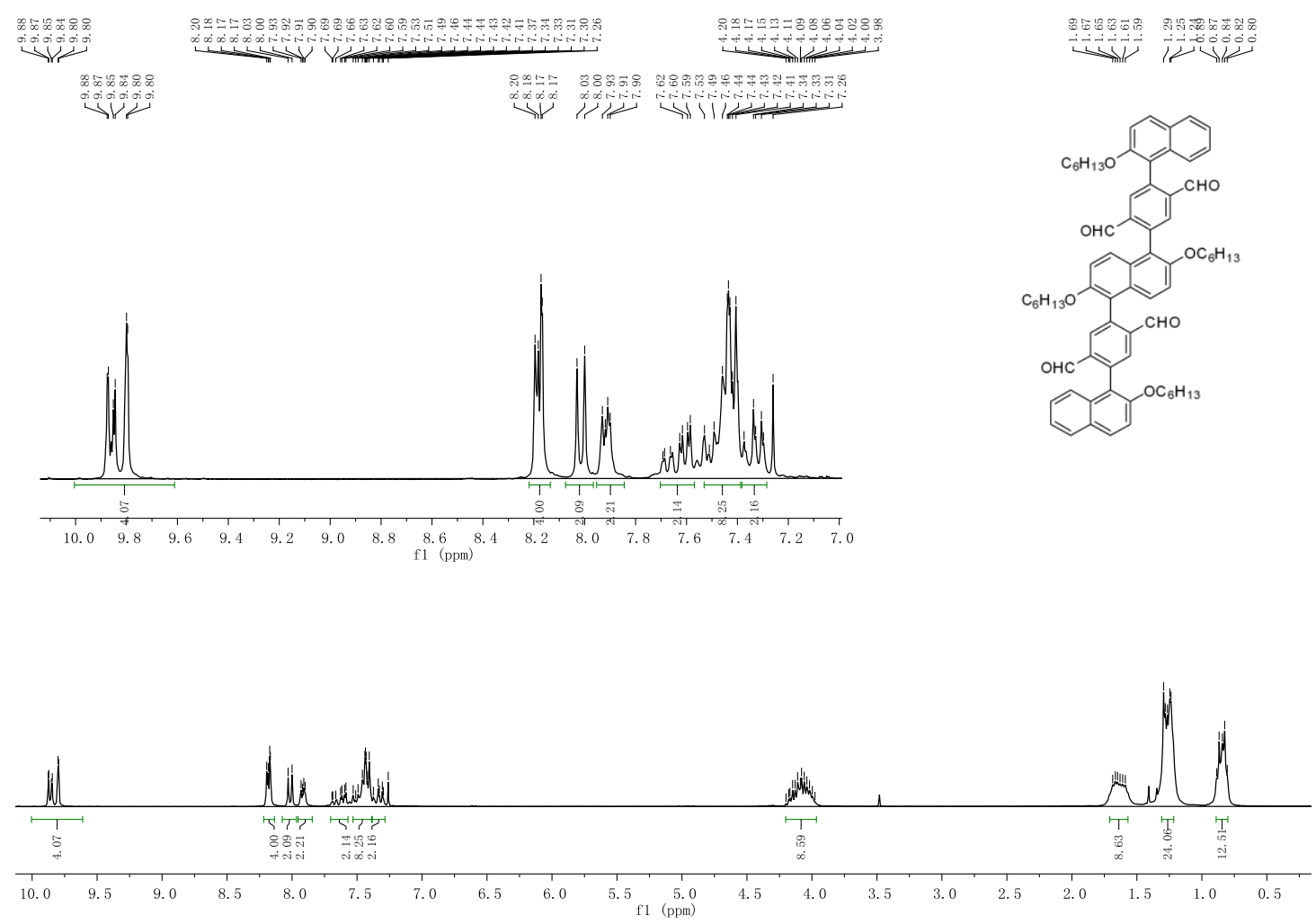

Figure S17. ${ }^{1} \mathrm{H}$ NMR spectrum (300 MHz) of compound 3 in $\mathrm{CDCl}_{3}$ at $298 \mathrm{~K}$
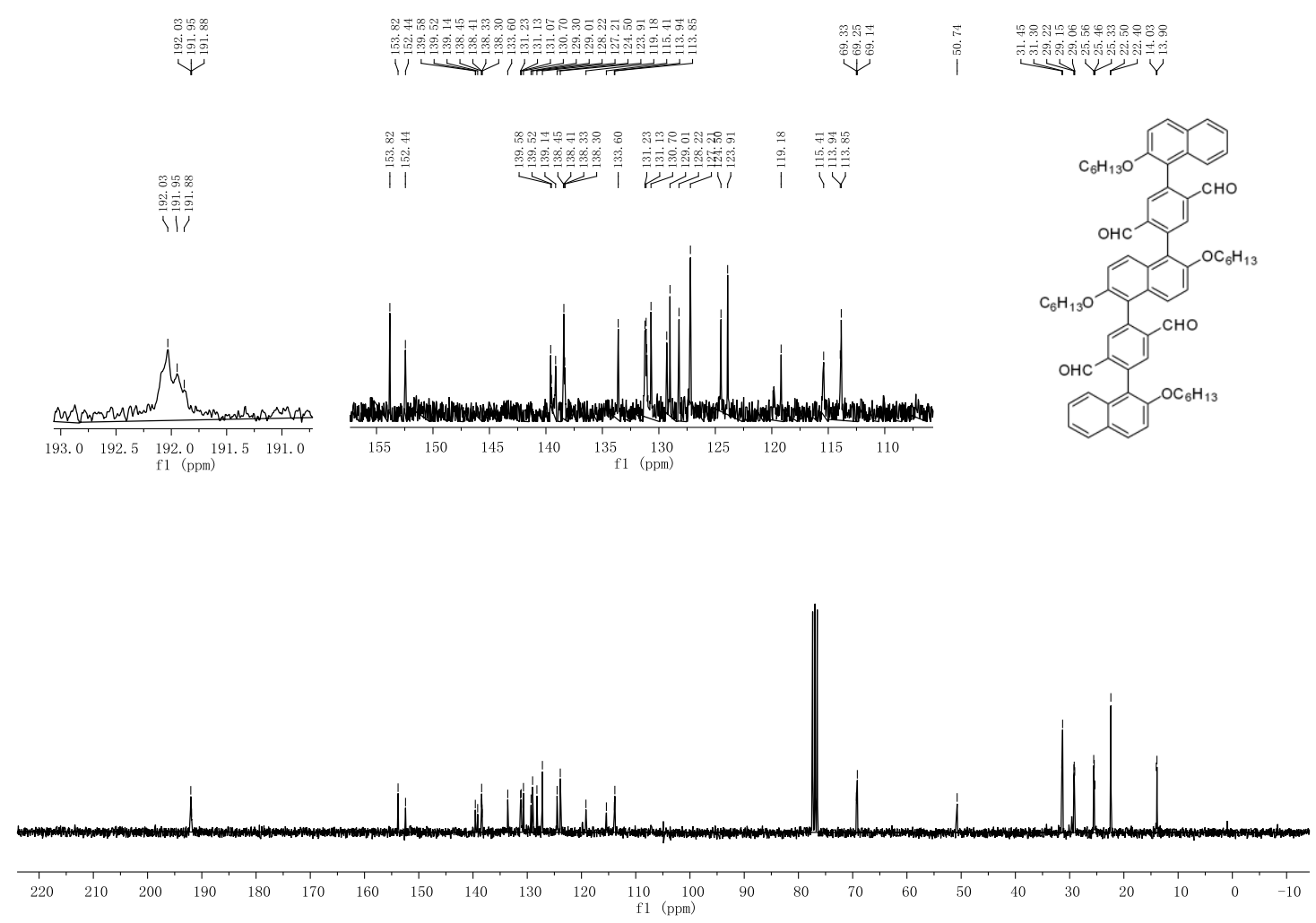

Figure S18. ${ }^{13} \mathrm{C}$ NMR spectrum (75 MHz) of compound 3 in $\mathrm{CDCl}_{3}$ at $298 \mathrm{~K}$ 

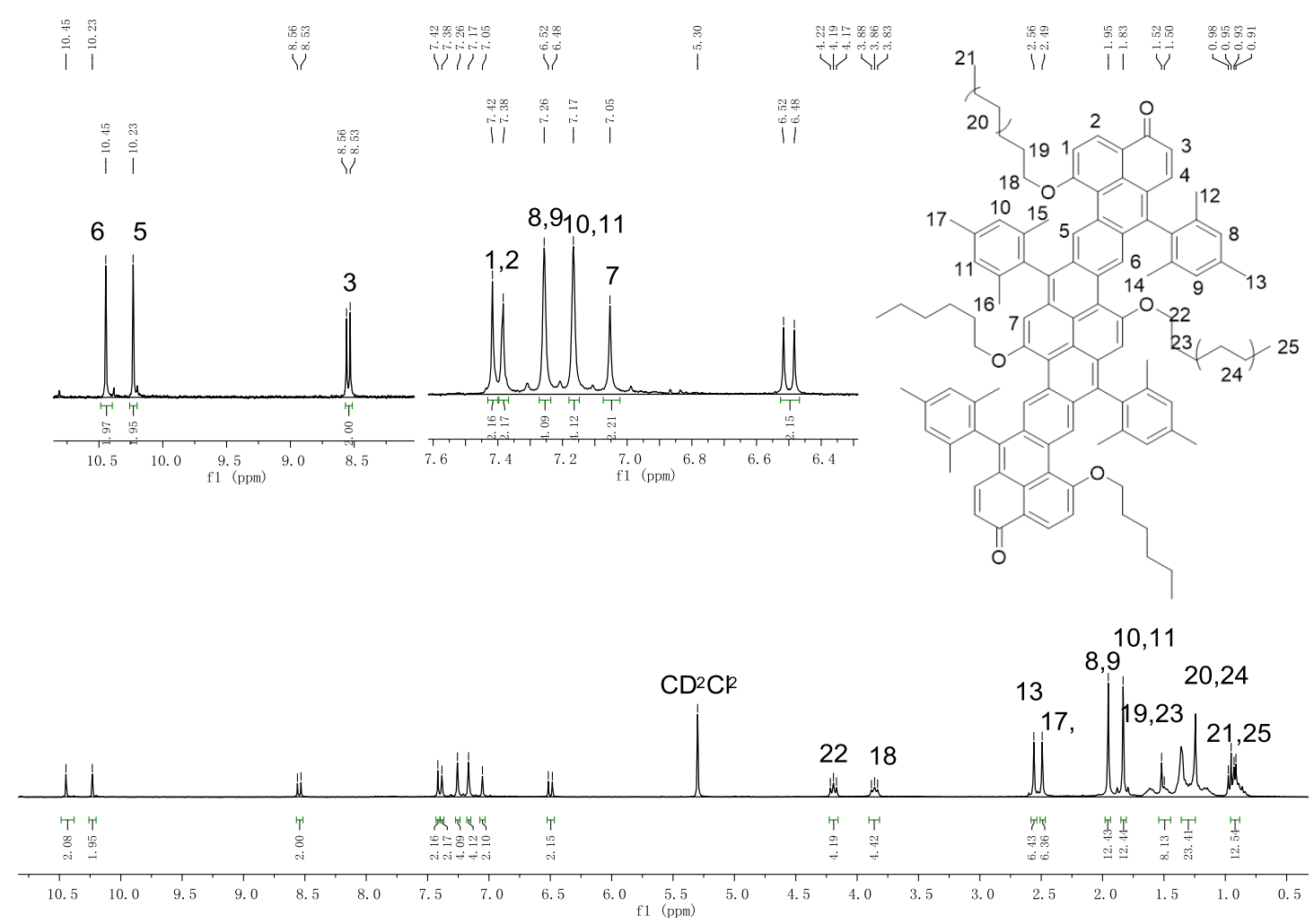

Figure S19. ${ }^{1} \mathrm{H}$ NMR spectrum (500 MHz) of HZD-M-ONE in $\mathrm{CD}_{2} \mathrm{Cl}_{2}$ at $298 \mathrm{~K}$

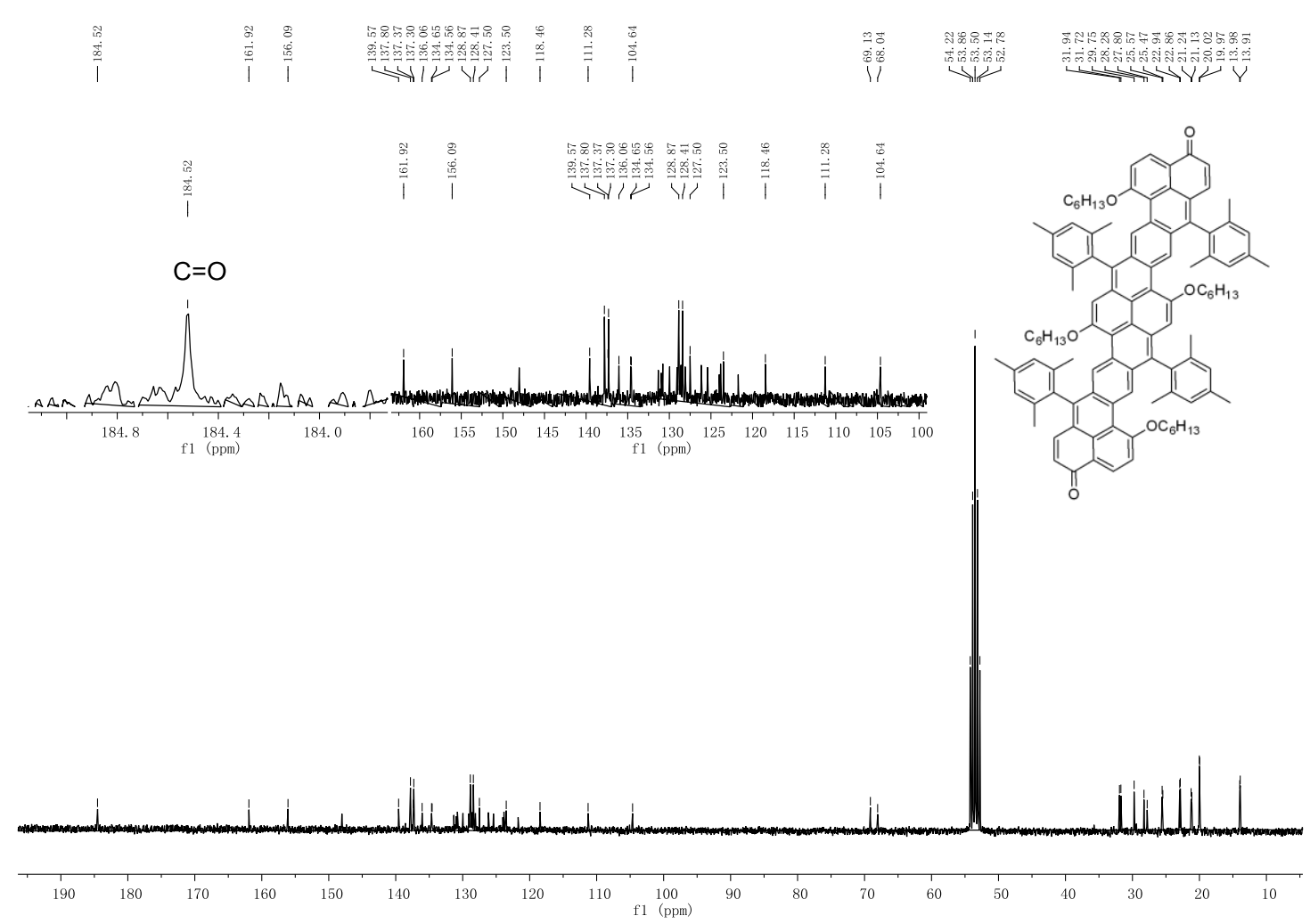

Figure S20. ${ }^{13} \mathrm{C}$ NMR spectrum $(125 \mathrm{MHz})$ of HZD-M-ONE in $\mathrm{CD}_{2} \mathrm{Cl}_{2}$ at $298 \mathrm{~K}$ 


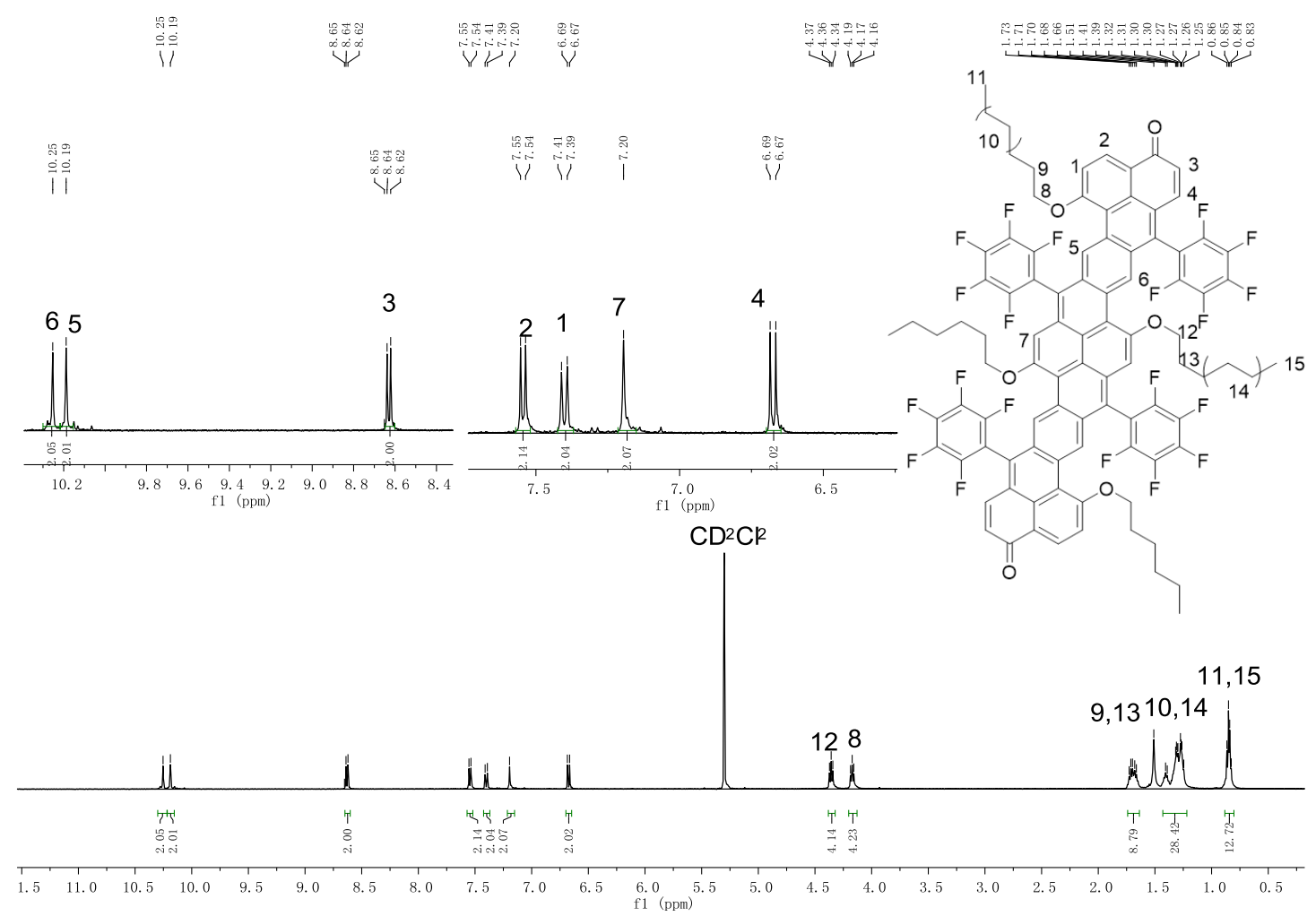

Figure S21. ${ }^{1} \mathrm{H}$ NMR spectrum (500 MHz) of HZD-F-ONE in $\mathrm{CD}_{2} \mathrm{Cl}_{2}$ at $298 \mathrm{~K}$

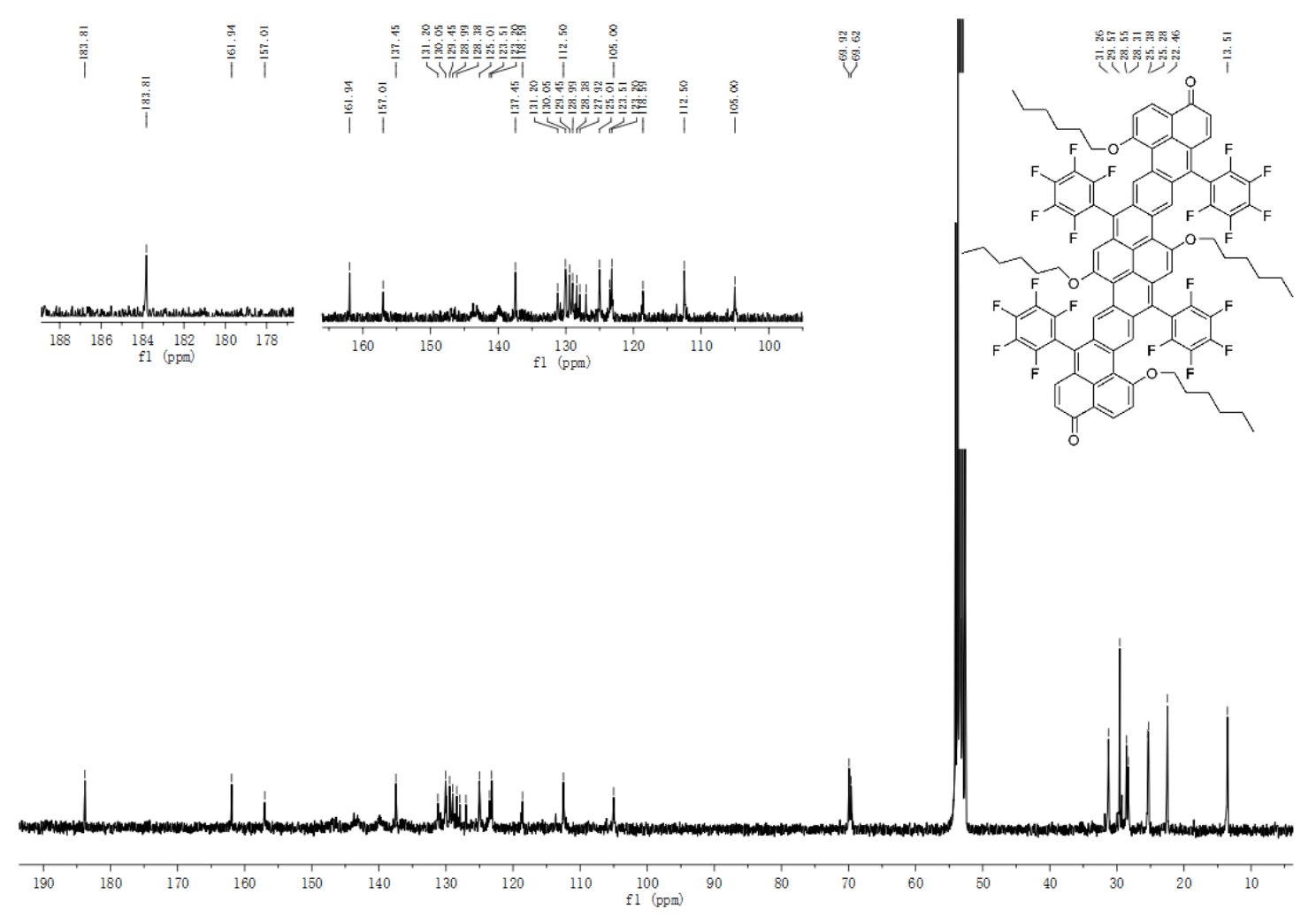

Figure S22. ${ }^{13} \mathrm{C}$ NMR spectrum (125 MHz) of HZD-F-ONE in $\mathrm{CD}_{2} \mathrm{Cl}_{2}$ at $298 \mathrm{~K}$ 

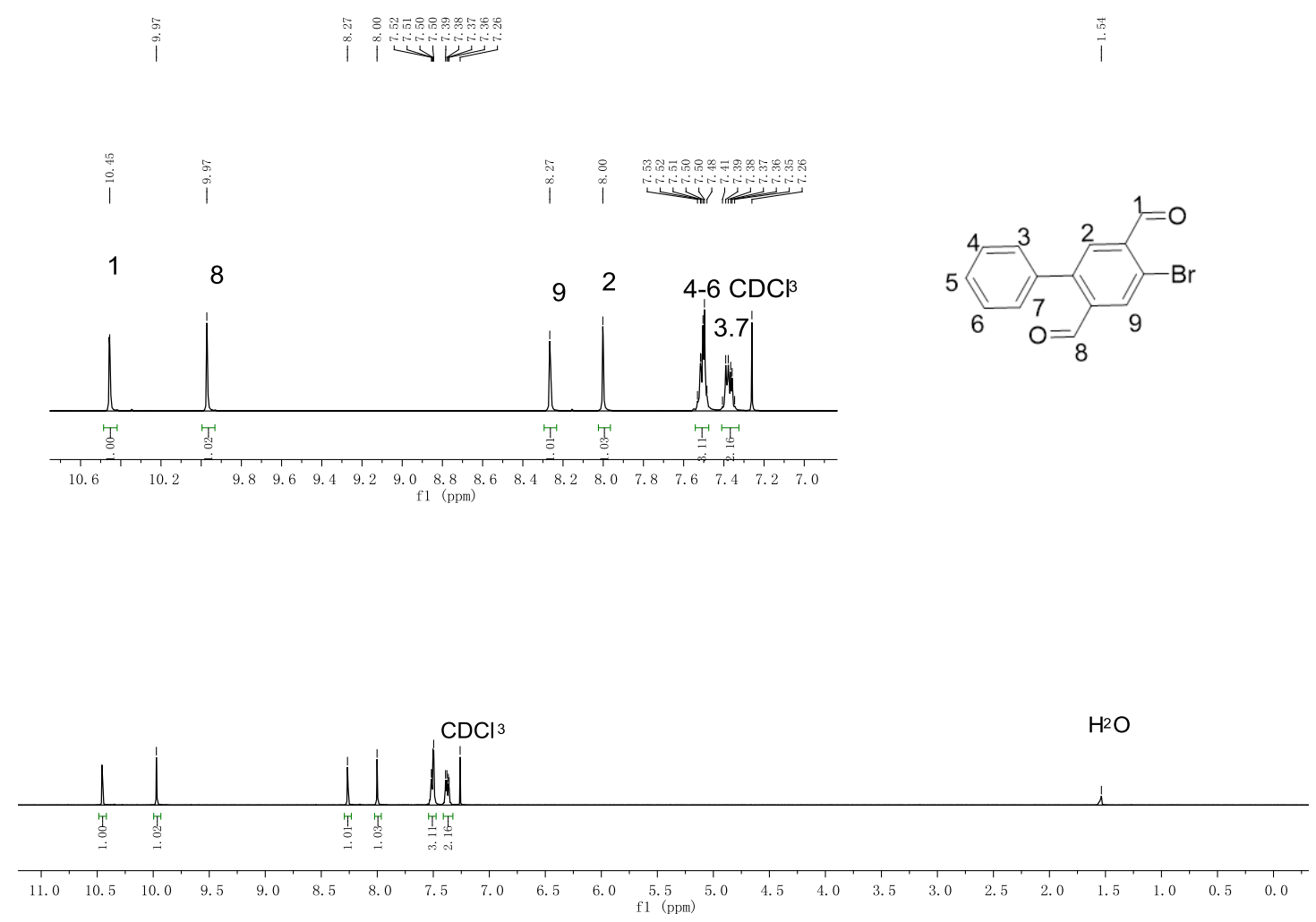

Figure S23. ${ }^{1} \mathrm{H}$ NMR spectrum $\left(300 \mathrm{MHz}\right.$ ) of compound 5 in $\mathrm{CDCl}_{3}$ at $298 \mathrm{~K}$
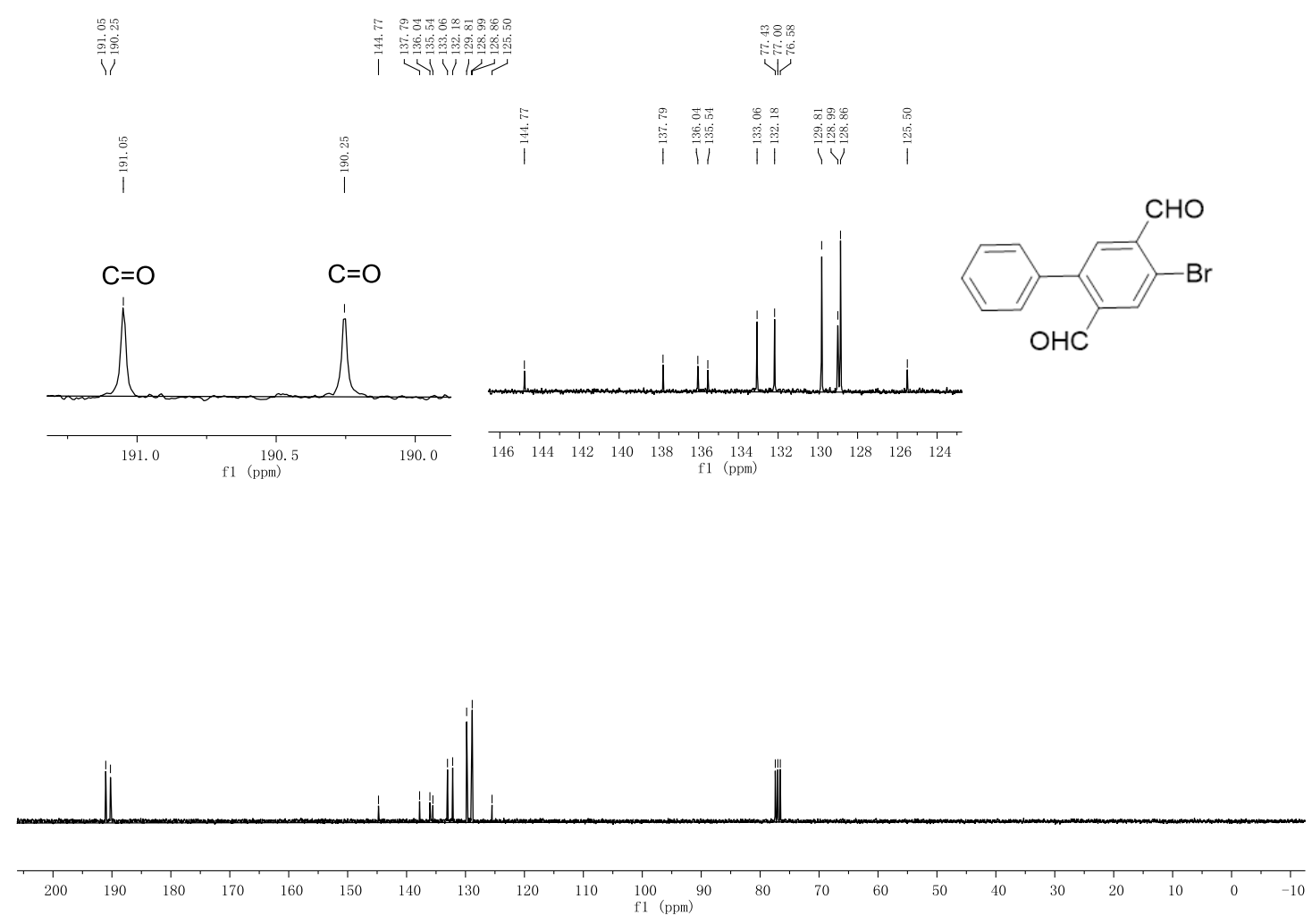

Figure S24. ${ }^{13} \mathrm{C}$ NMR spectrum (75 MHz) of compound 5 in $\mathrm{CDCl}_{3}$ at $298 \mathrm{~K}$ 

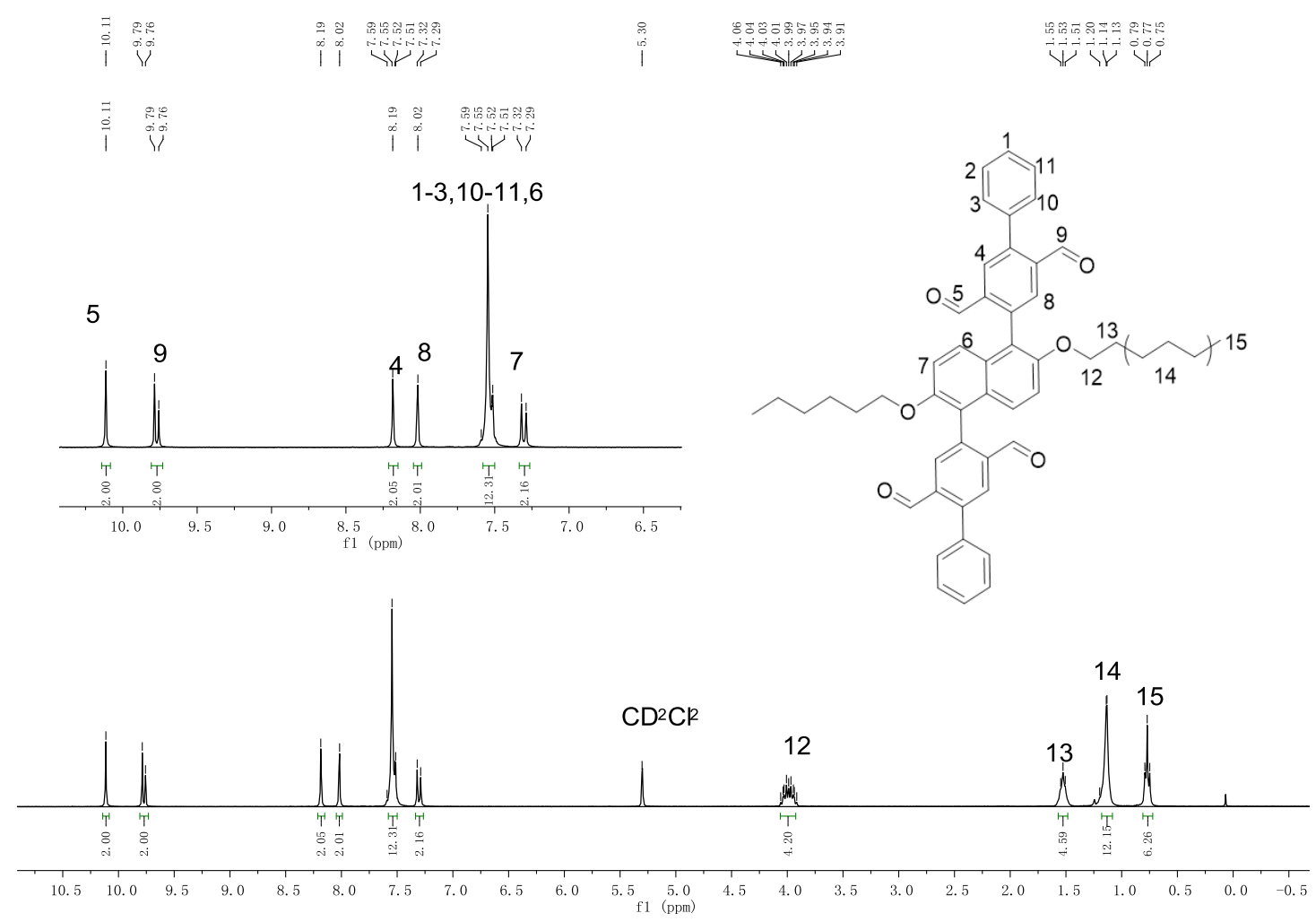

Figure S25. ${ }^{1} \mathrm{H}$ NMR spectrum (300 MHz) of compound 6 in $\mathrm{CD}_{2} \mathrm{Cl}_{2}$ at $298 \mathrm{~K}$
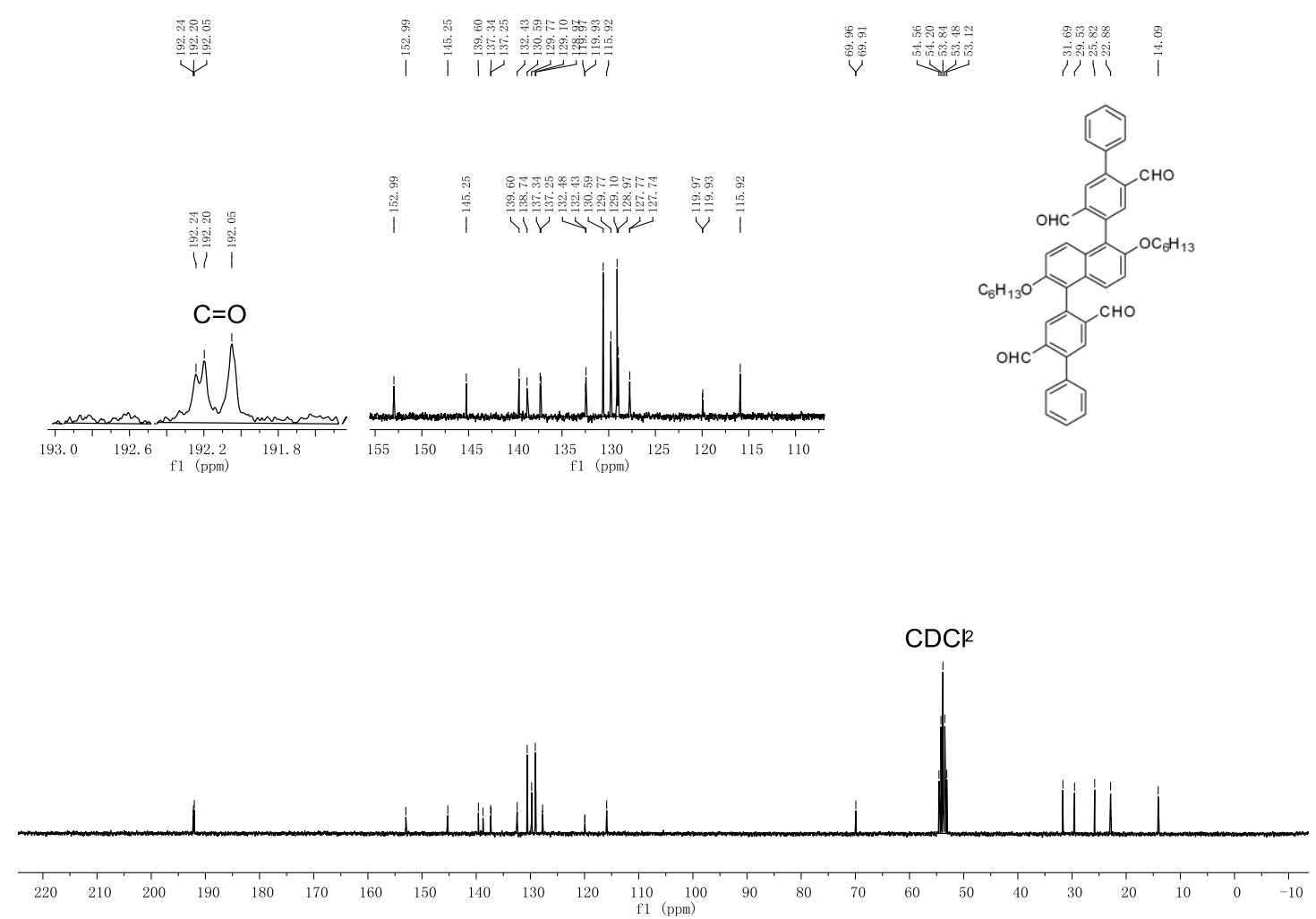

Figure S26. ${ }^{13} \mathrm{C}$ NMR spectrum (75 MHz) of compound 6 in $\mathrm{CD}_{2} \mathrm{Cl}_{2}$ at $298 \mathrm{~K}$ 

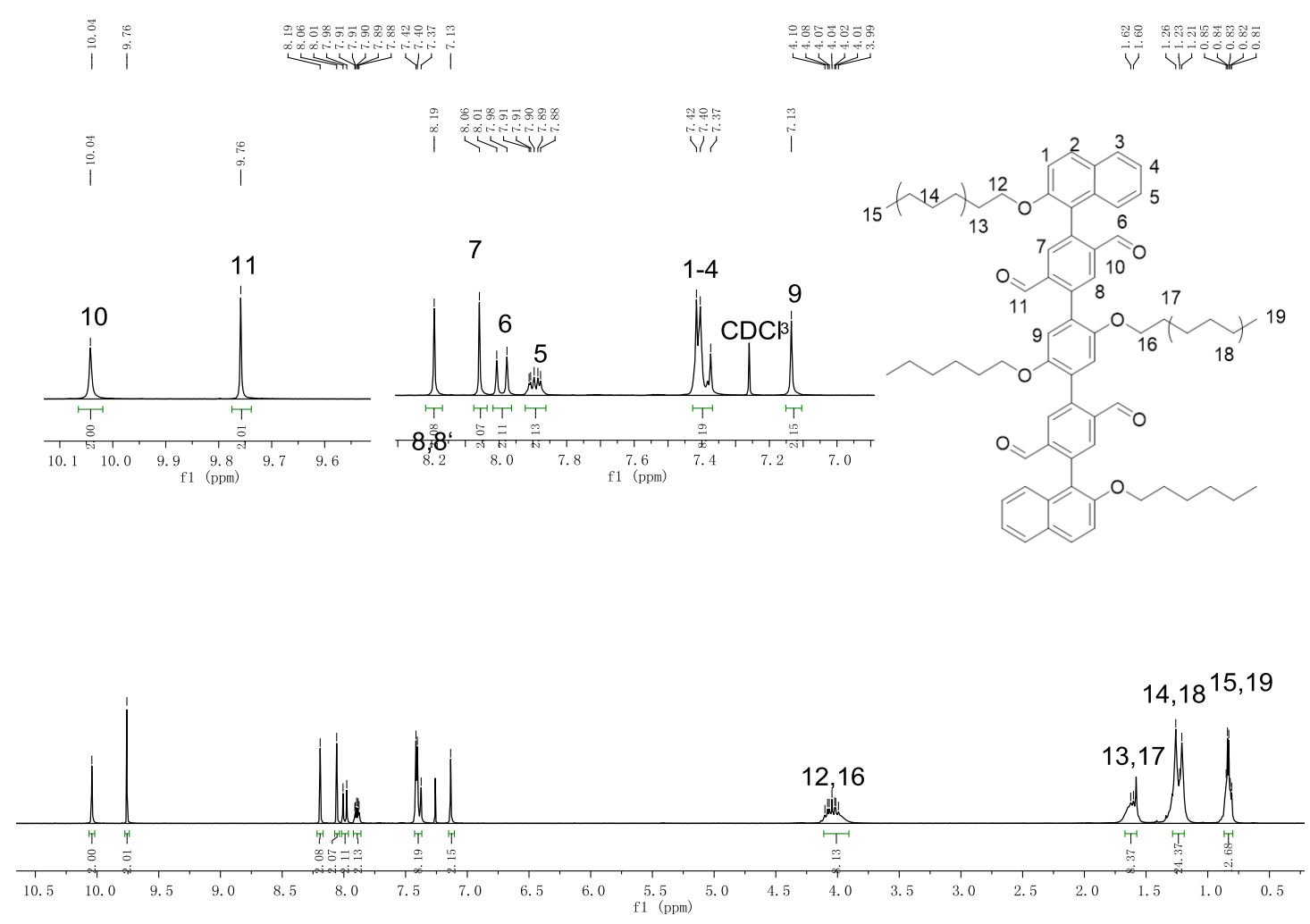

Figure S27. ${ }^{1} \mathrm{H}$ NMR spectrum (300 MHz) of compound 9 in $\mathrm{CDCl}_{3}$ at $298 \mathrm{~K}$

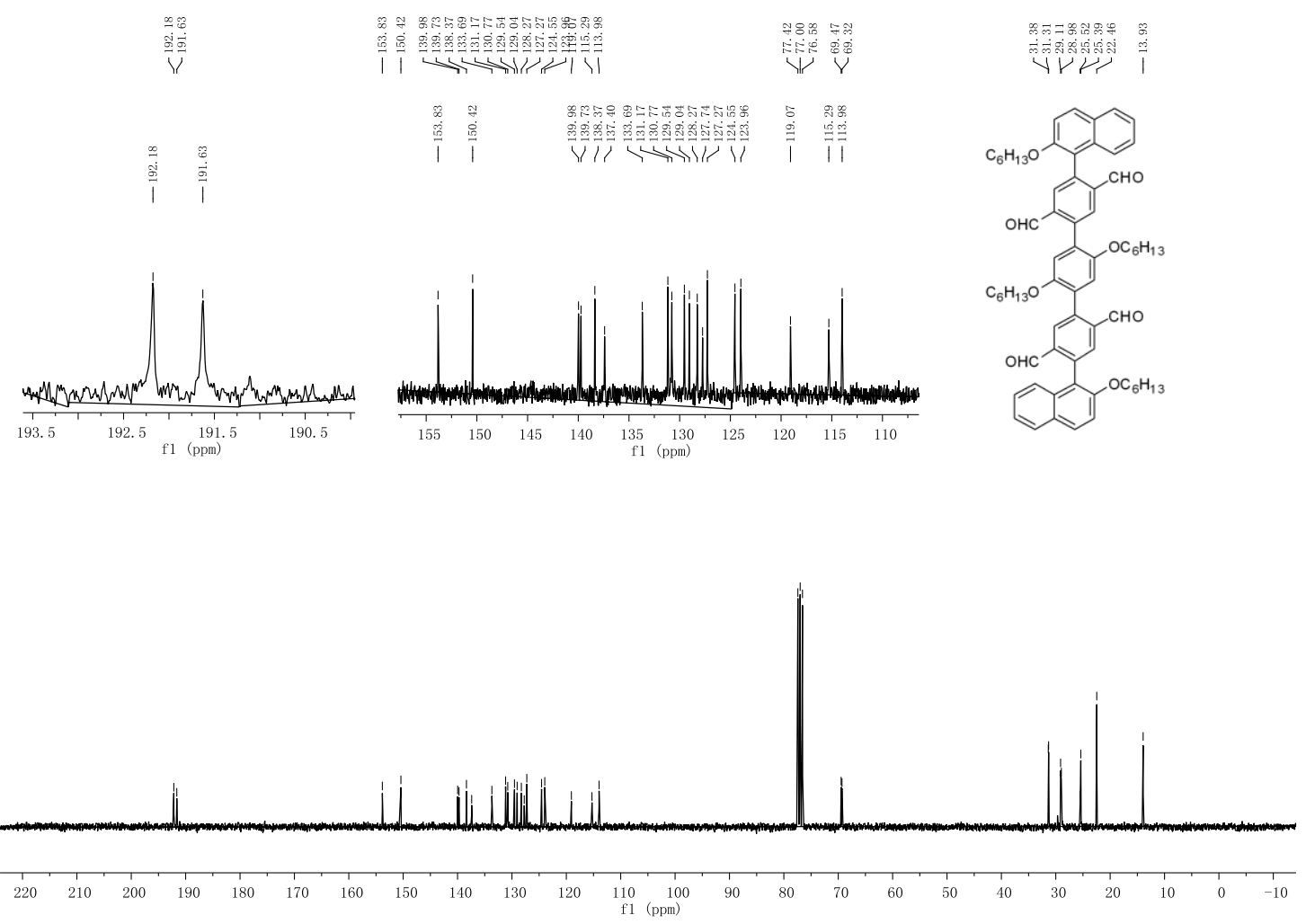

Figure S28. ${ }^{13} \mathrm{C}$ NMR spectrum $(75 \mathrm{MHz})$ of compound 9 in $\mathrm{CDCl}_{3}$ at $298 \mathrm{~K}$ 

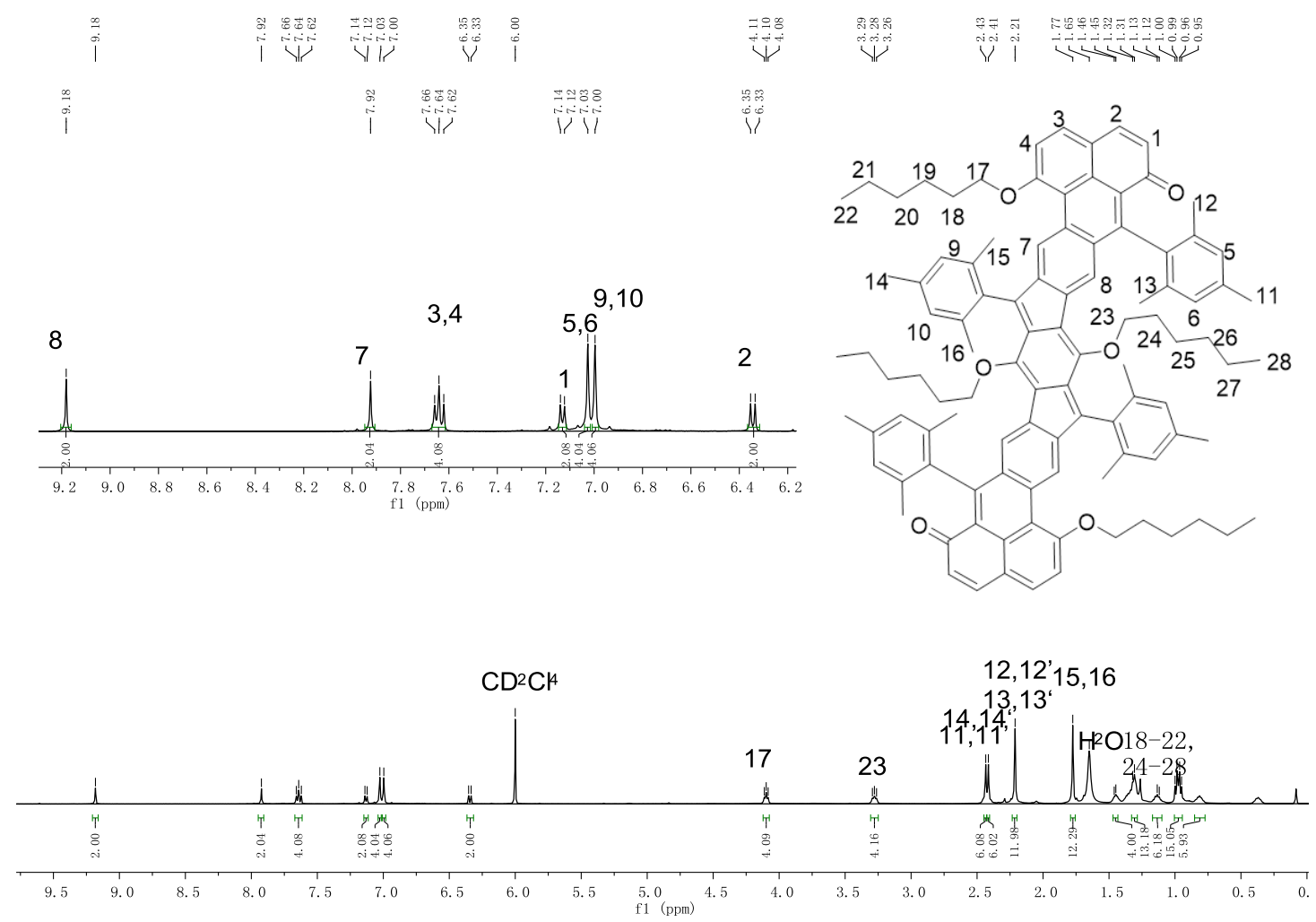

Figure S29. ${ }^{1} \mathrm{H}$ NMR spectrum $(500 \mathrm{MHz})$ of compound PIF-M-ONE in $\mathrm{CD}_{2} \mathrm{Cl}_{4}$ at $333 \mathrm{~K}$

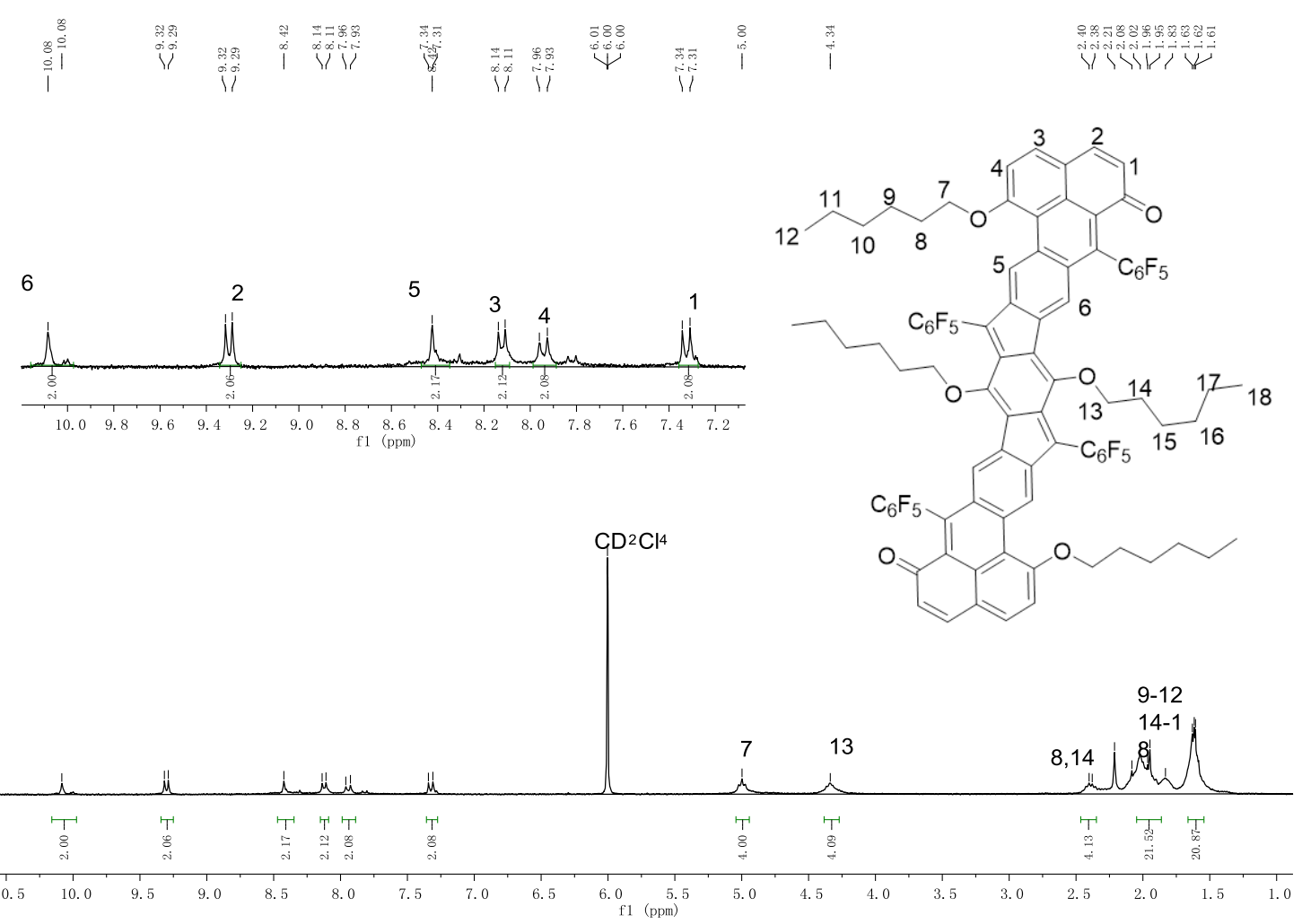

Figure S30. ${ }^{1} \mathrm{H}$ NMR spectrum (500 MHz) of compound PIF-F-ONE in $\mathrm{CD}_{2} \mathrm{Cl}_{4}$ at $333 \mathrm{~K}$ 

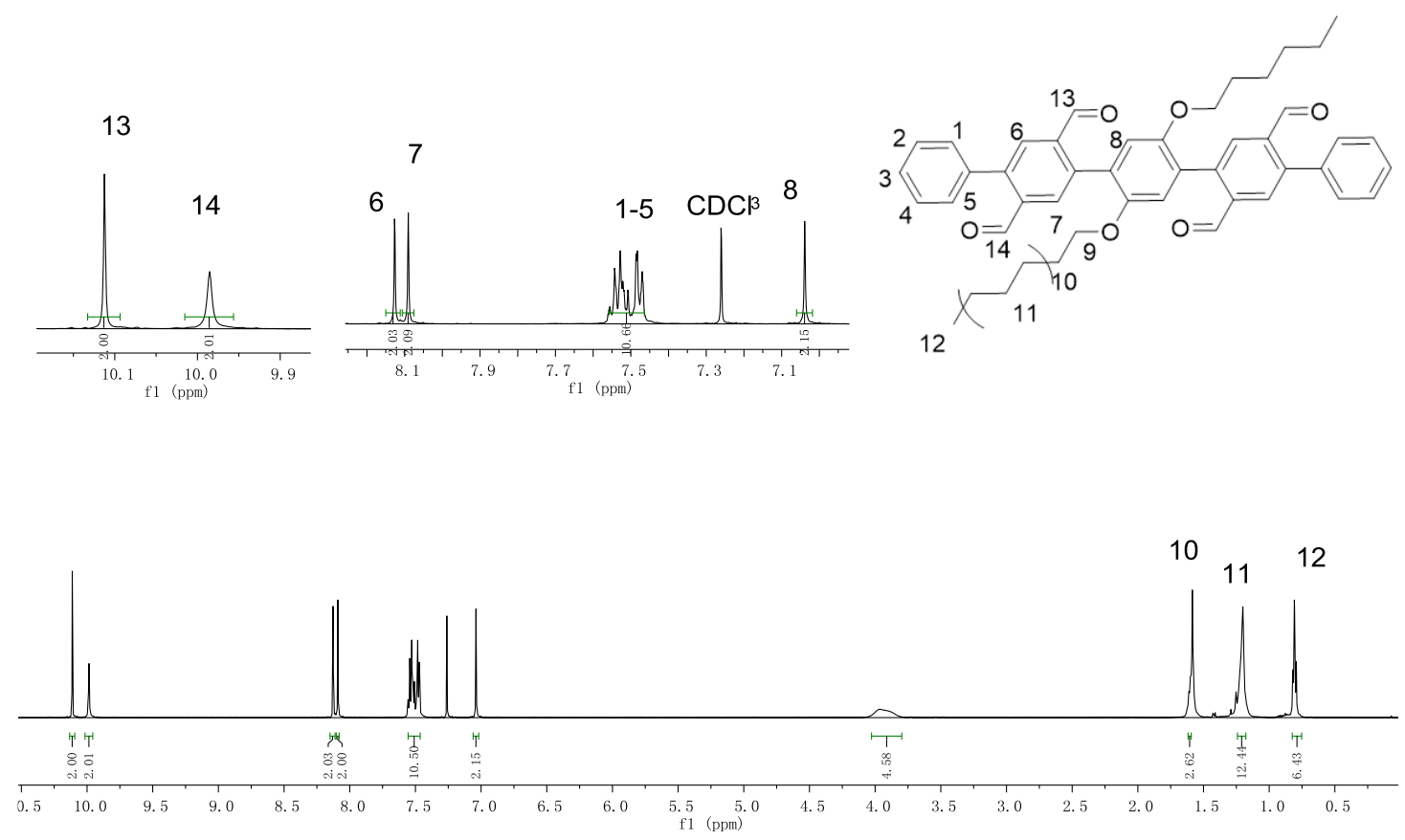

Figure S31. ${ }^{1} \mathrm{H}$ NMR spectrum (500 MHz) of compound $\mathbf{1 1}$ in $\mathrm{CDCl}_{3}$ at $298 \mathrm{~K}$
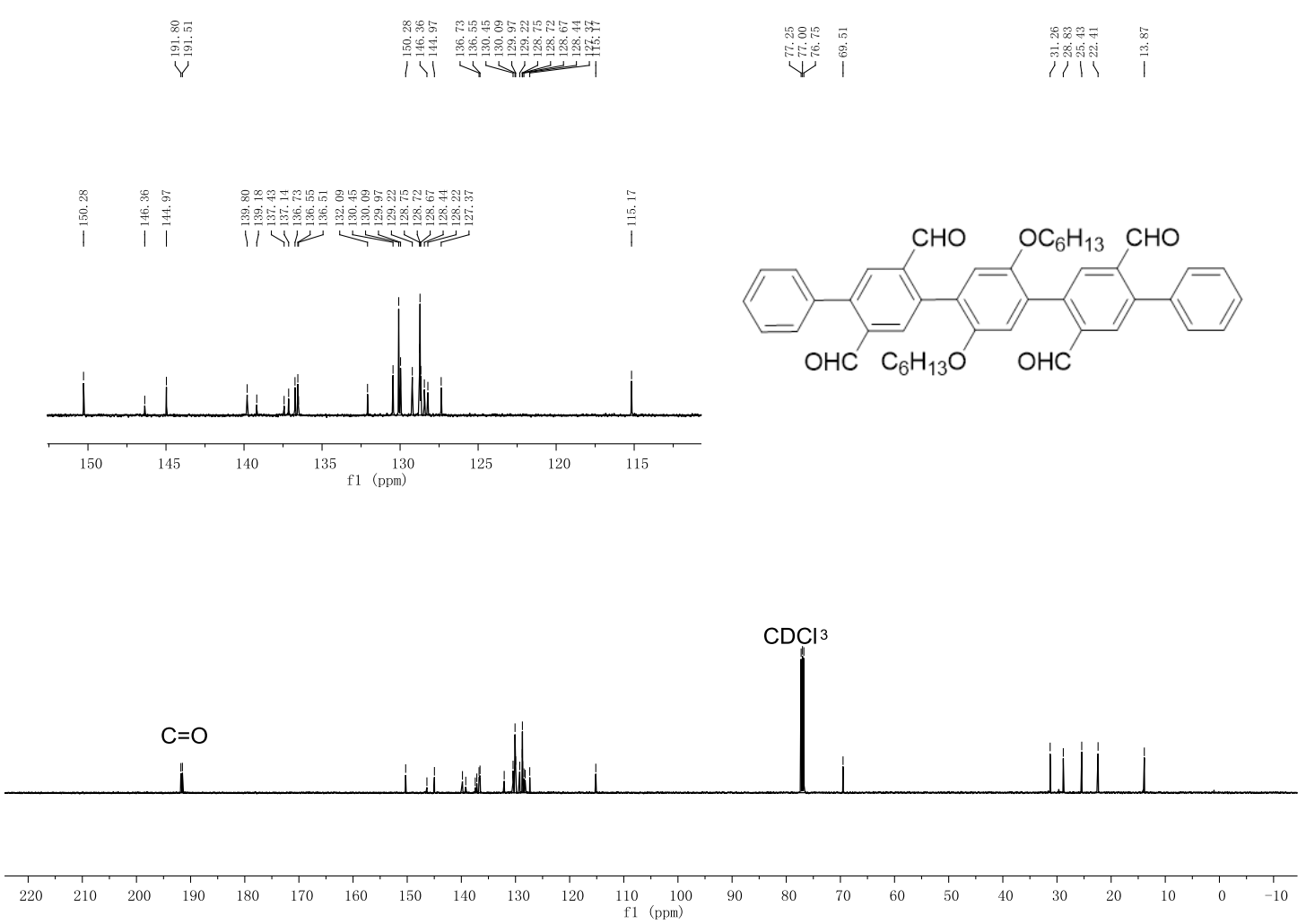

Figure S32. ${ }^{13} \mathrm{C}$ NMR spectrum $(125 \mathrm{MHz})$ of compound 11 in $\mathrm{CDCl}_{3}$ at $298 \mathrm{~K}$ 

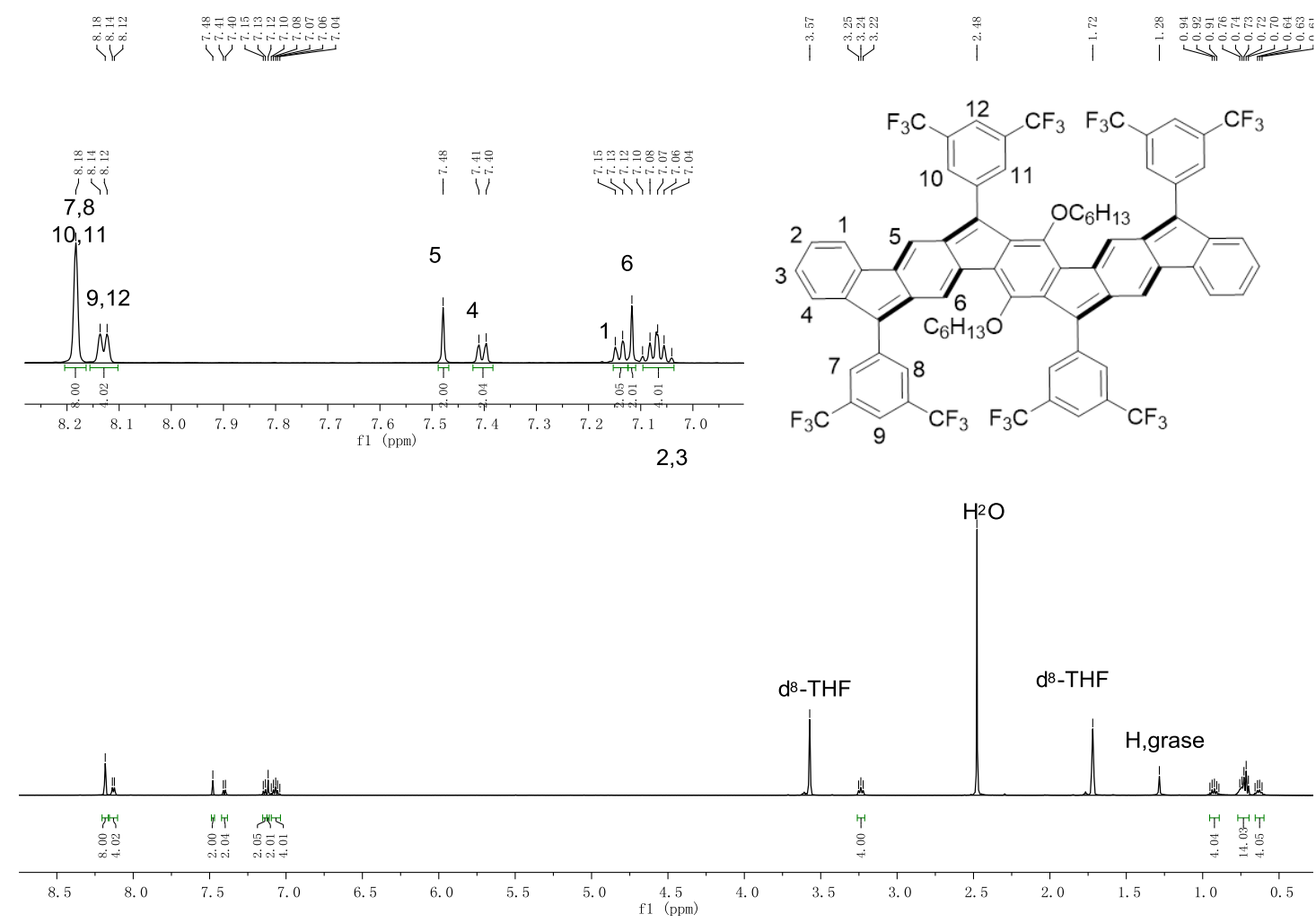

Figure S33. ${ }^{1} \mathrm{H}$ NMR spectrum (500 MHz) of compound IFD-CF 3 in $\mathrm{d}_{8}-\mathrm{THF}$ at $298 \mathrm{~K}$

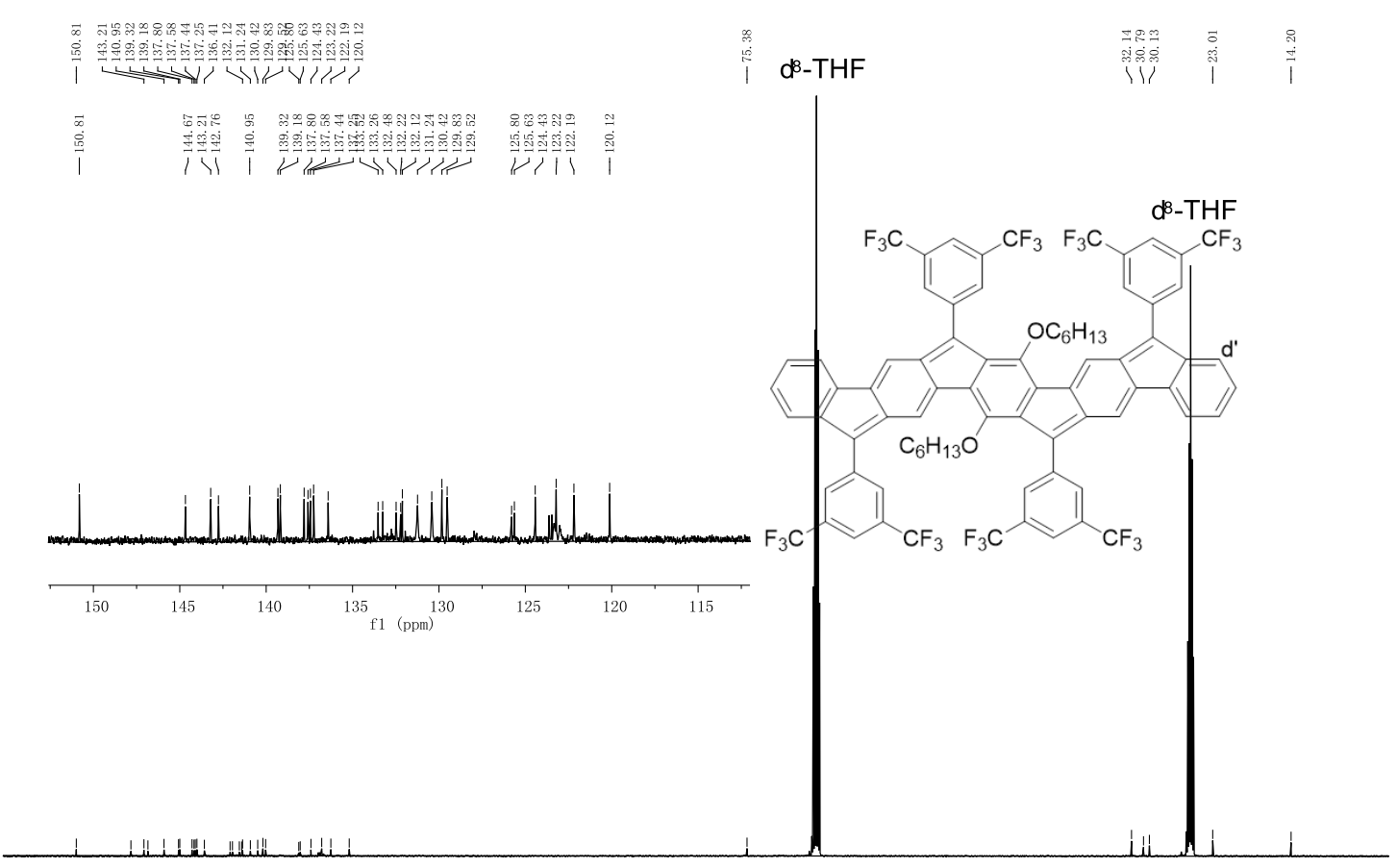

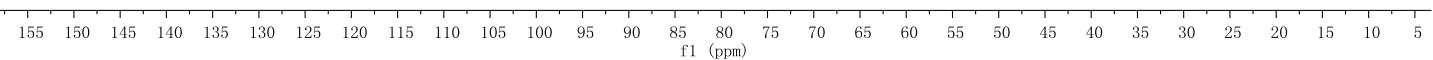

Figure S34. ${ }^{13} \mathrm{C}$ NMR spectrum $(125 \mathrm{MHz})$ of compound $\mathbf{I F D}^{-\mathbf{C F}_{3}}$ in $\mathrm{d}_{8}-\mathrm{THF}$ at $298 \mathrm{~K}$ 

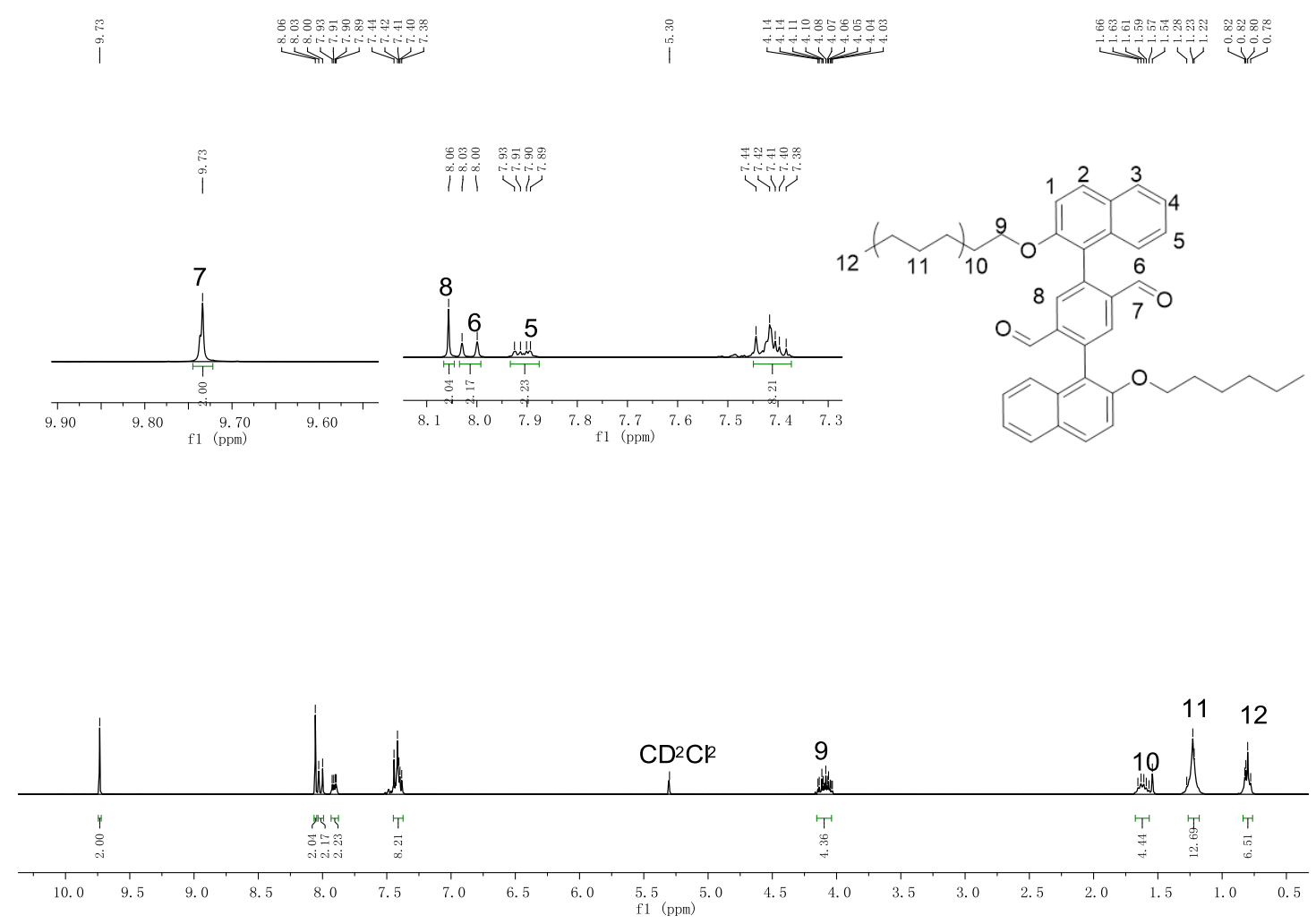

Figure S35. ${ }^{1} \mathrm{H}$ NMR spectrum (300 MHz) of compound 13 in $\mathrm{CD}_{2} \mathrm{Cl}_{2}$ at $298 \mathrm{~K}$
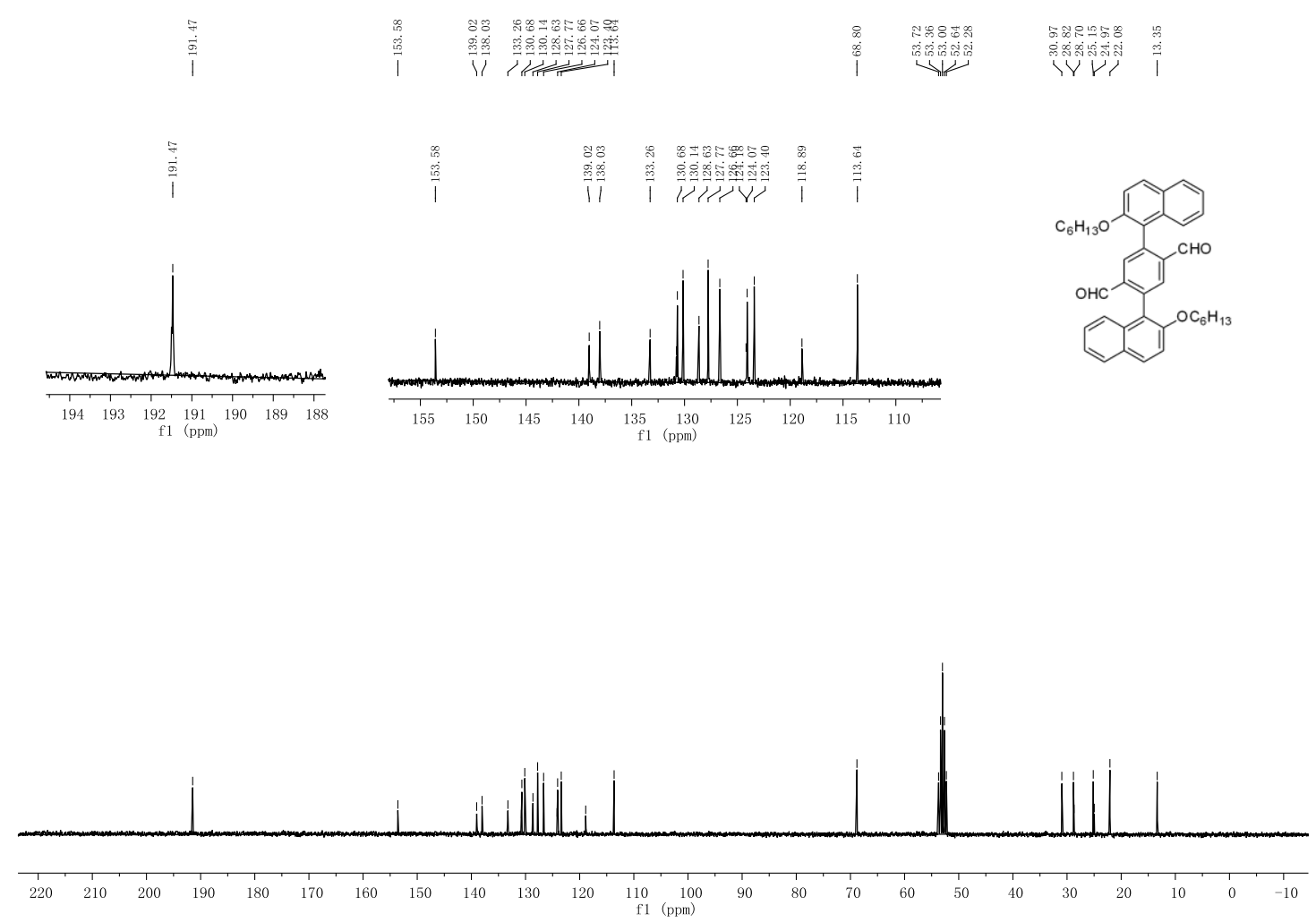

Figure S36. ${ }^{13} \mathrm{C}$ NMR spectrum $(75 \mathrm{MHz})$ of compound 13 in $\mathrm{CD}_{2} \mathrm{Cl}_{2}$ at $298 \mathrm{~K}$ 


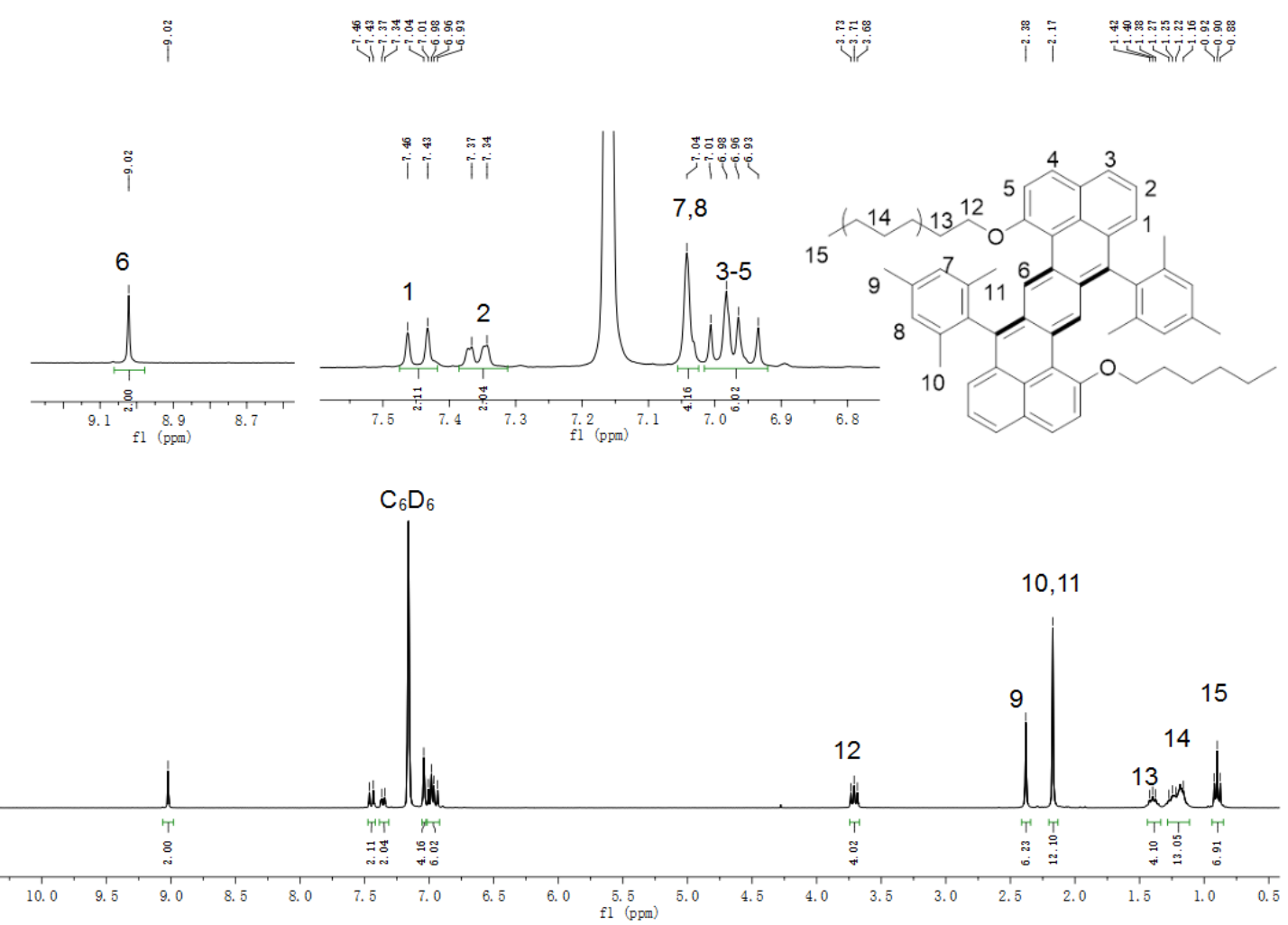

Figure S37. ${ }^{1} \mathrm{H}$ NMR spectrum (300 MHz) of HZ-M in $\mathrm{C}_{6} \mathrm{D}_{6}$ at $298 \mathrm{~K}$

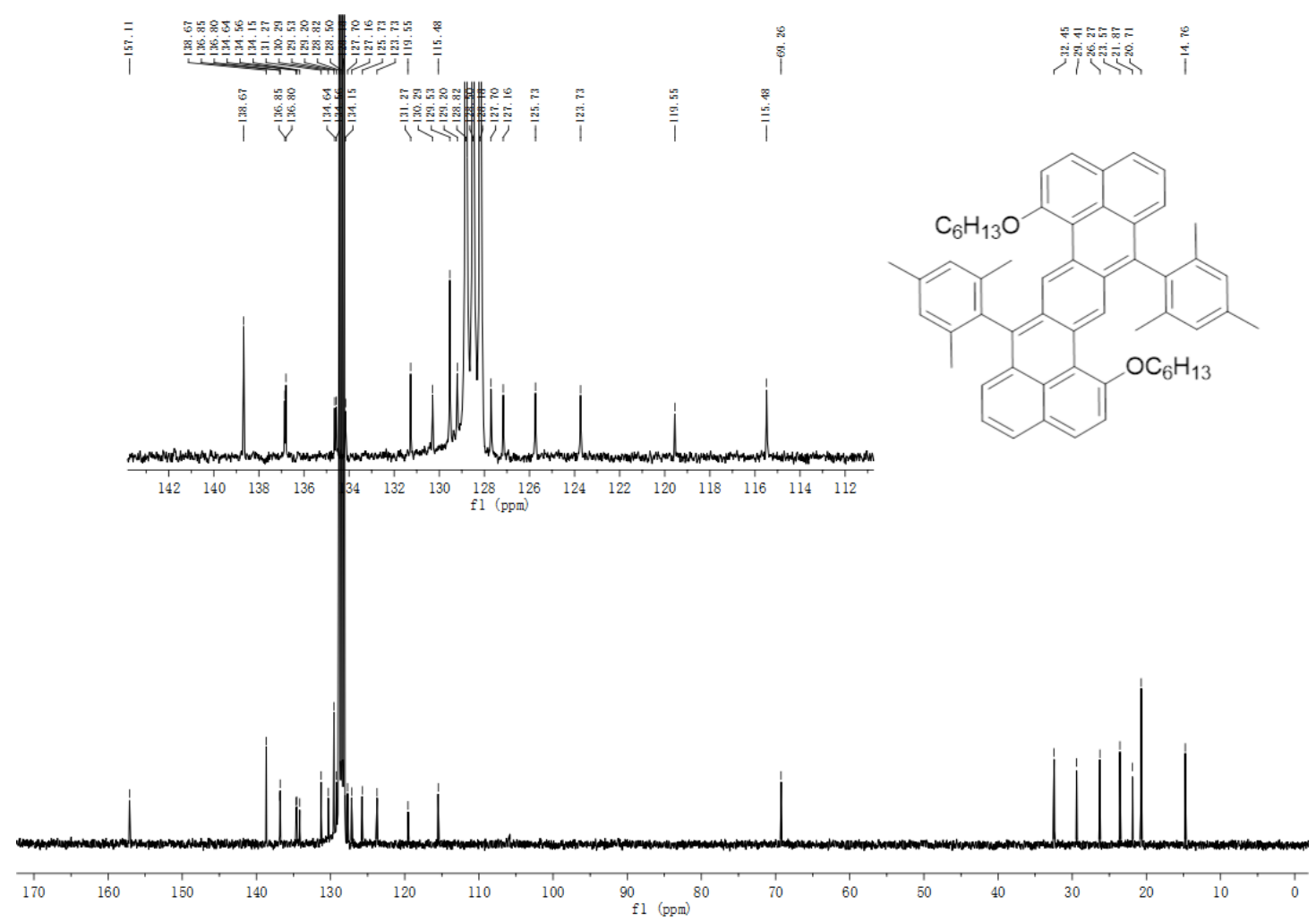

Figure S38. ${ }^{13} \mathrm{C}$ NMR spectrum $(75 \mathrm{MHz})$ of $\mathbf{H Z}-\mathbf{M}$ in $\mathrm{C}_{6} \mathrm{D}_{6}$ at $298 \mathrm{~K}$ 

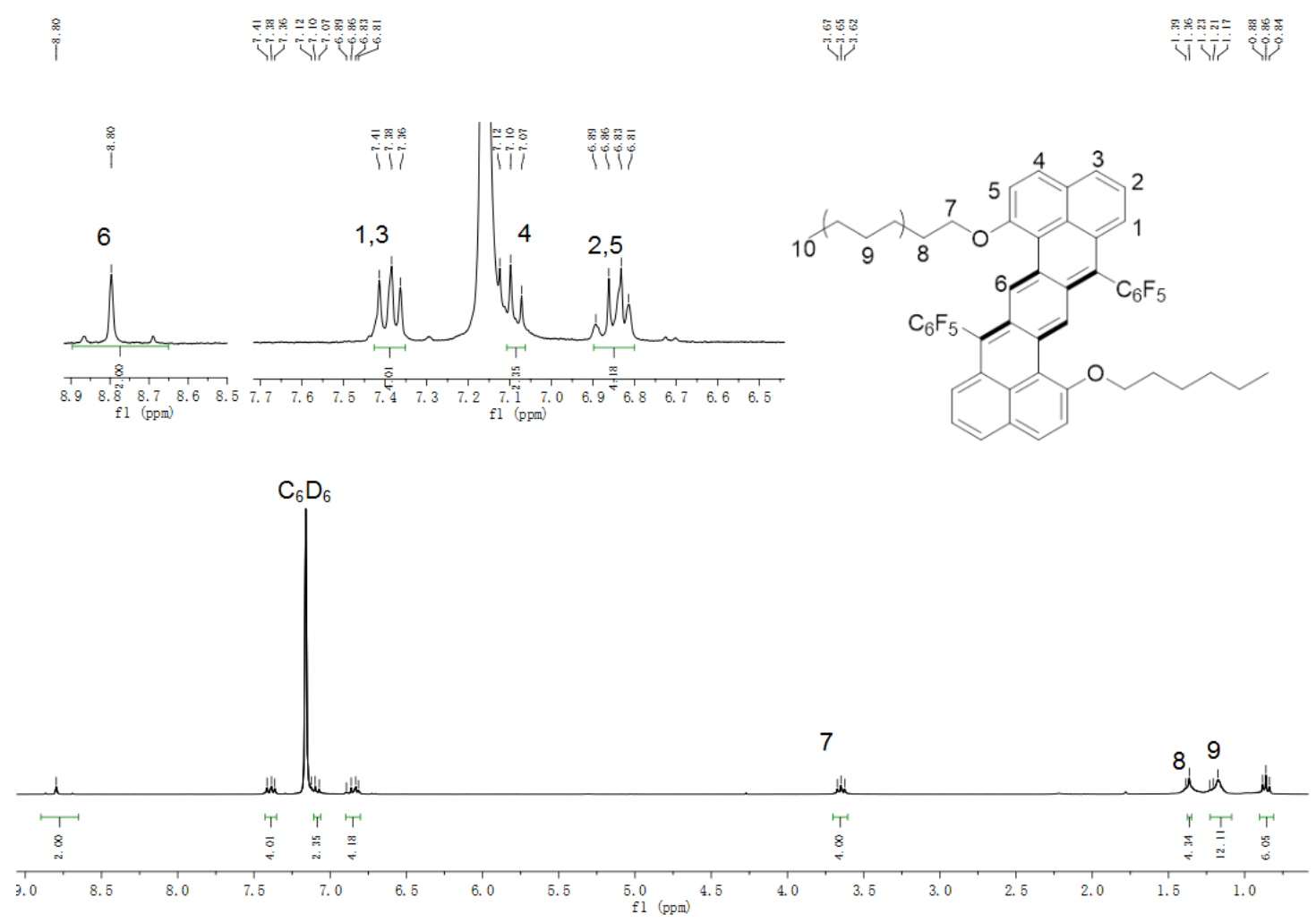

Figure S39. ${ }^{1} \mathrm{H}$ NMR spectrum (300 MHz) of HZ-F in $\mathrm{C}_{6} \mathrm{D}_{6}$ at $298 \mathrm{~K}$
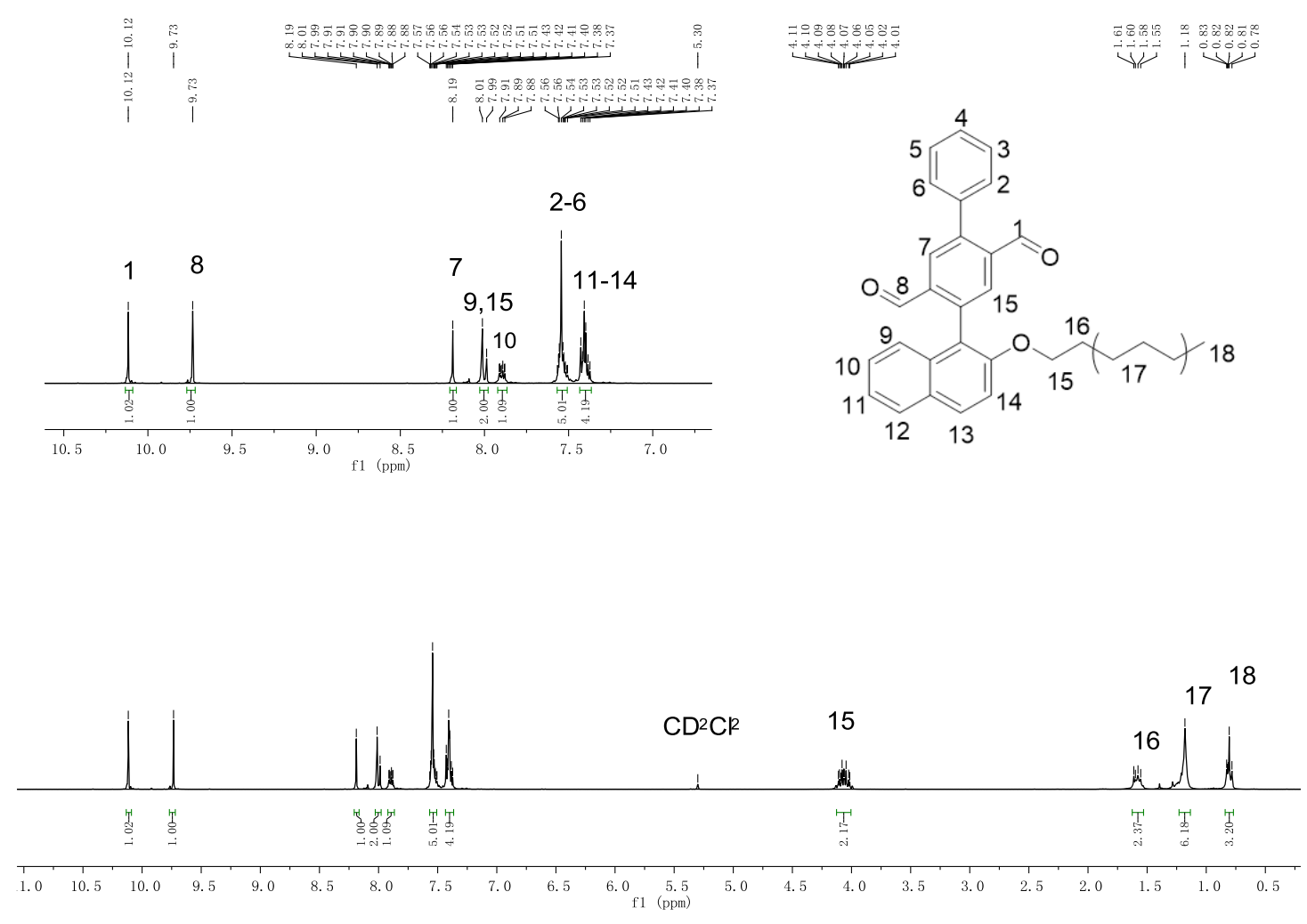

Figure S40. ${ }^{1} \mathrm{H}$ NMR spectrum (300 MHz) of compound 14 in $\mathrm{CD}_{2} \mathrm{Cl}_{2}$ at $298 \mathrm{~K}$ 

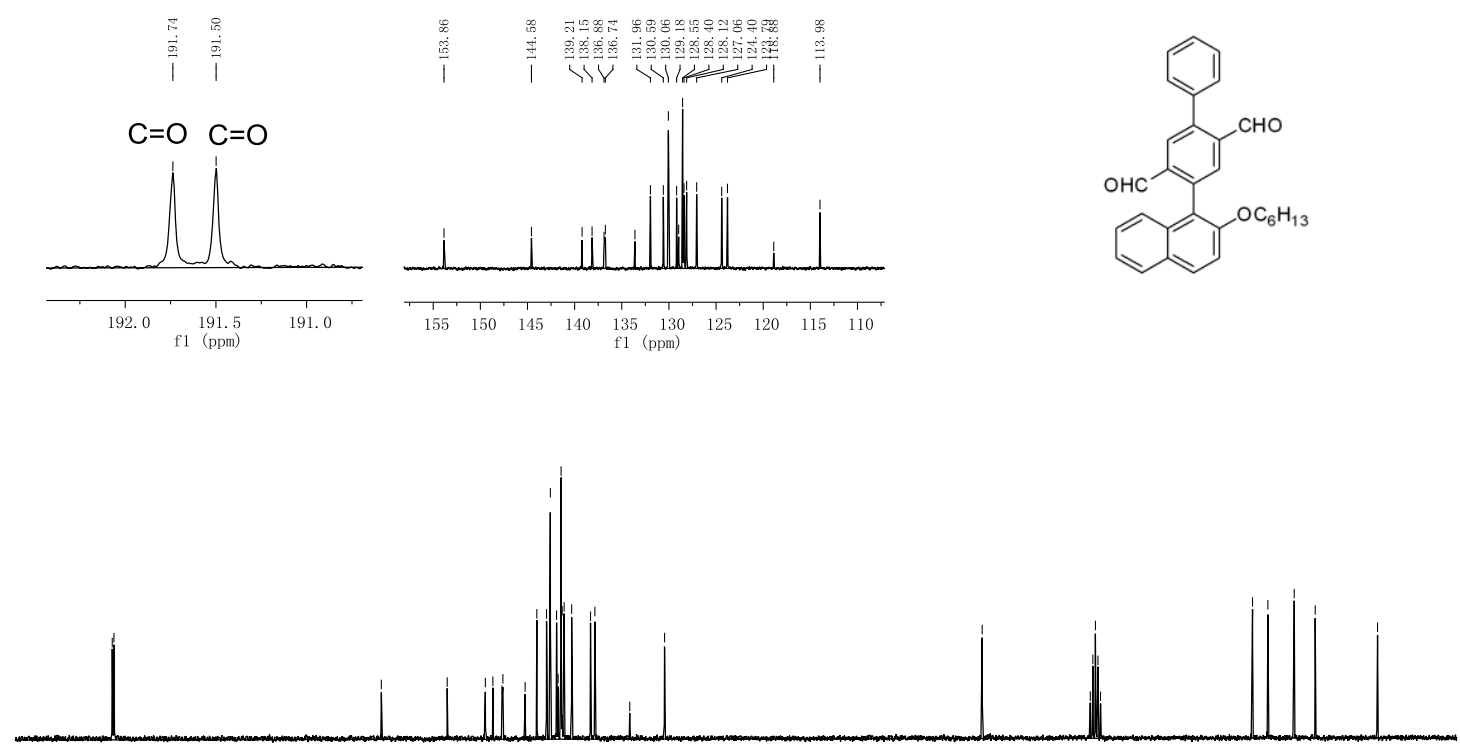

Figure S41. ${ }^{13} \mathrm{C}$ NMR spectrum $(75 \mathrm{MHz})$ of compound 14 in $\mathrm{CD}_{2} \mathrm{Cl}_{2}$ at $298 \mathrm{~K}$

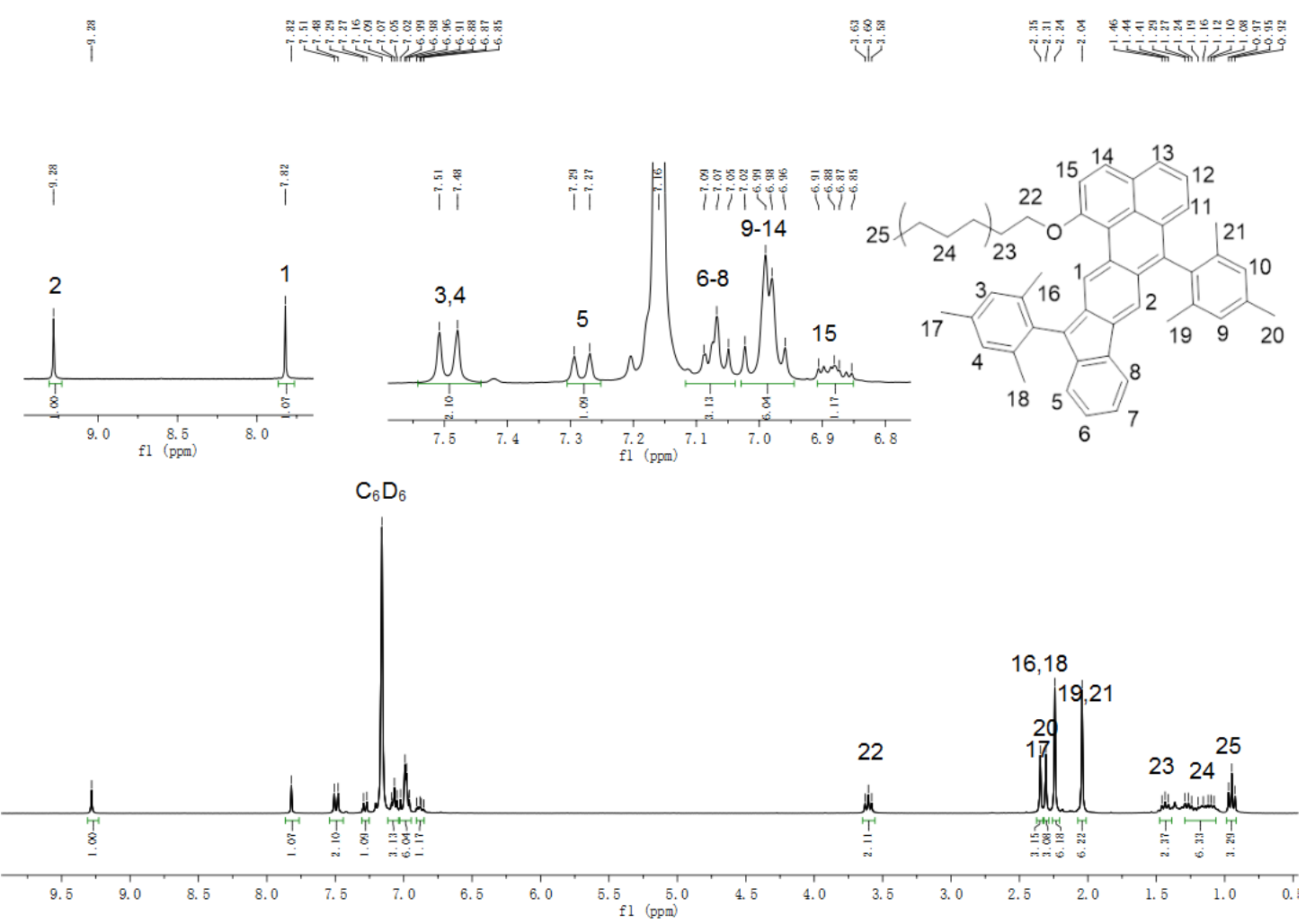

Figure S42. ${ }^{1} \mathrm{H}$ NMR spectrum (300 MHz) of compound PF-M in $\mathrm{C}_{6} \mathrm{D}_{6}$ at $298 \mathrm{~K}$ 

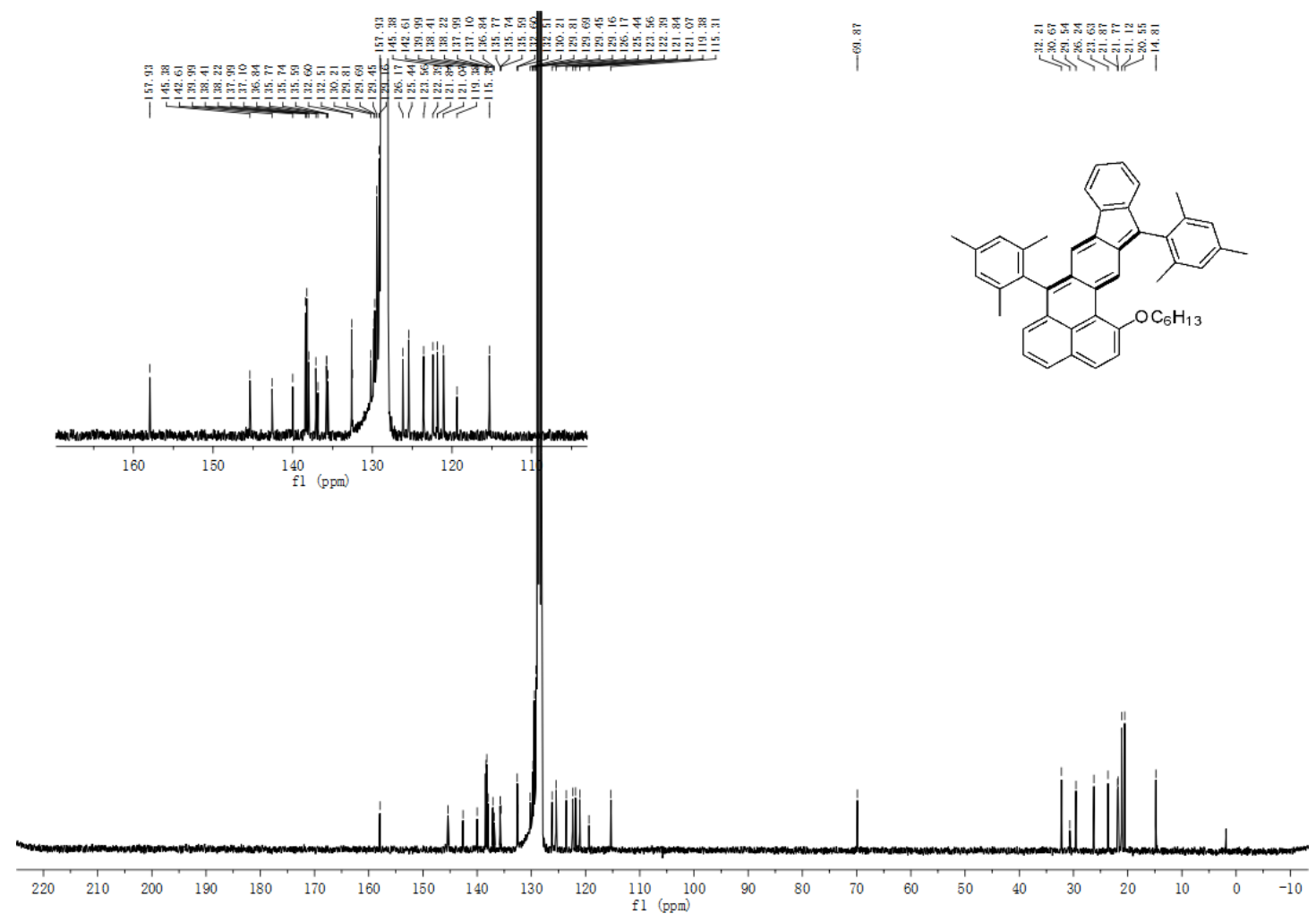

Figure S43. ${ }^{13} \mathrm{C}$ NMR spectrum $(75 \mathrm{MHz})$ of compound PF-M in $\mathrm{C}_{6} \mathrm{D}_{6}$ at $298 \mathrm{~K}$
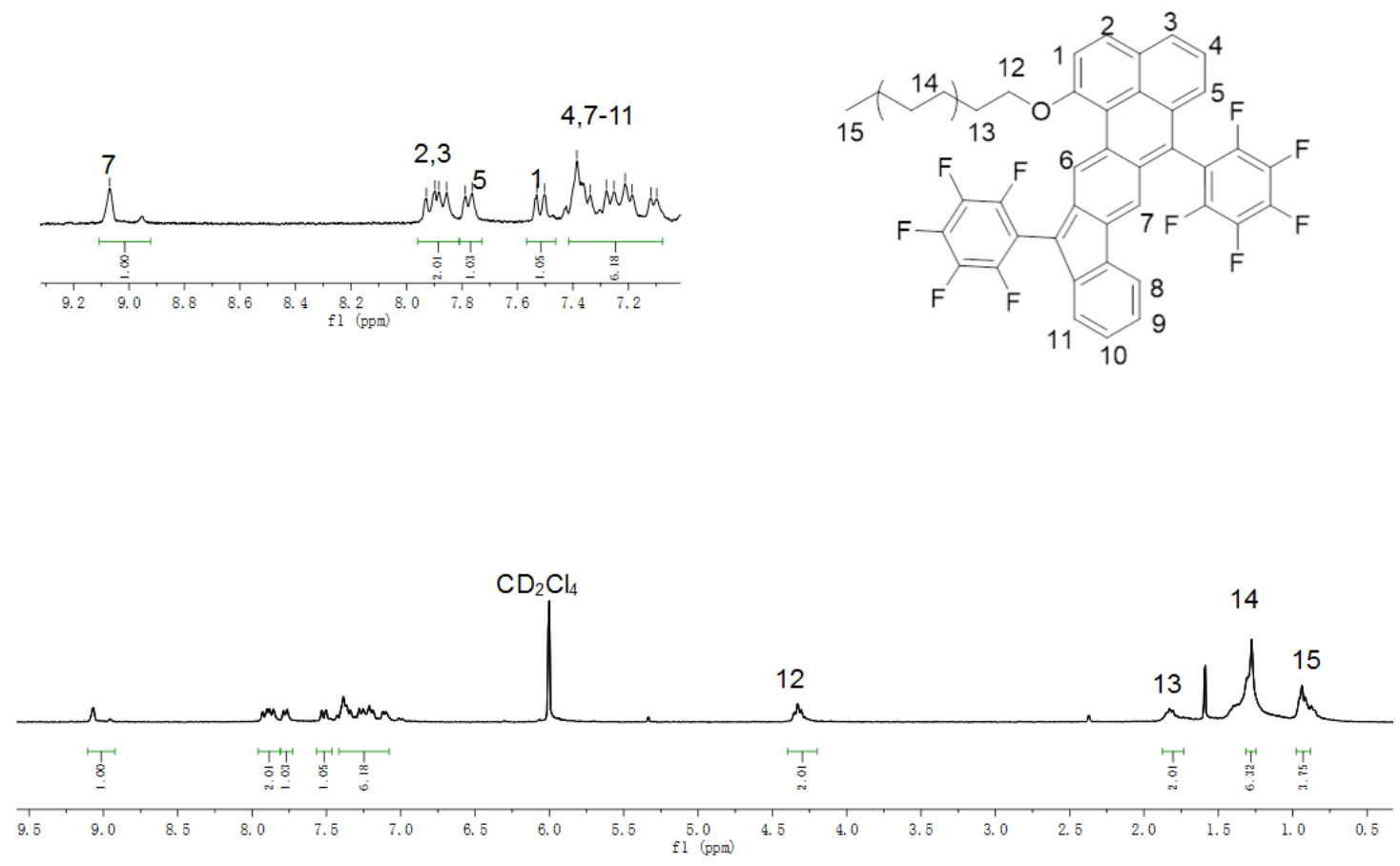

Figure S44. ${ }^{1} \mathrm{H}$ NMR spectrum (300 MHz) of compound PF-F in $\mathrm{CD}_{2} \mathrm{Cl}_{4}$ at $298 \mathrm{~K}$ 


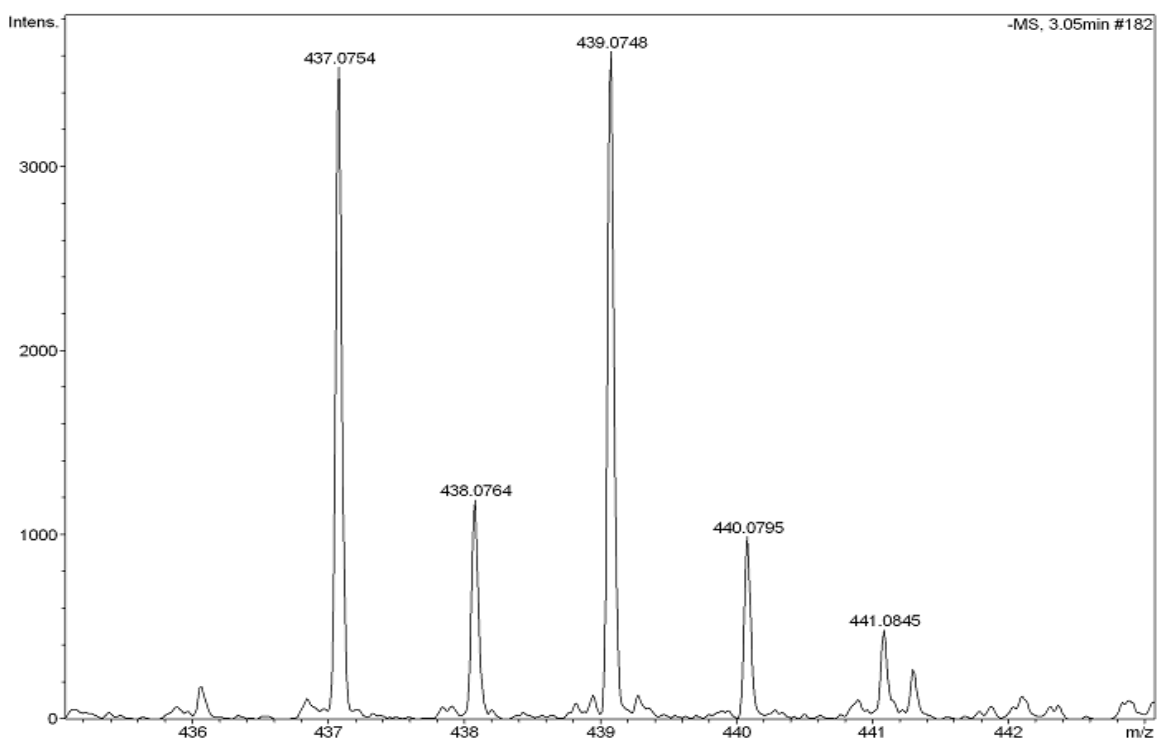

\begin{tabular}{c|c|c|c|c|c}
\hline Fomula & Mass & Error (ppm) & DblEq & N rule & Electron configuration \\
\hline $\mathrm{C}_{24} \mathrm{H}_{22} \mathrm{BrO}_{3}$ & 437.0754 & 0.8 & 13.5 & ok & even \\
\hline
\end{tabular}

Figure S45. HR mass spectrum (ESI) of compound 1

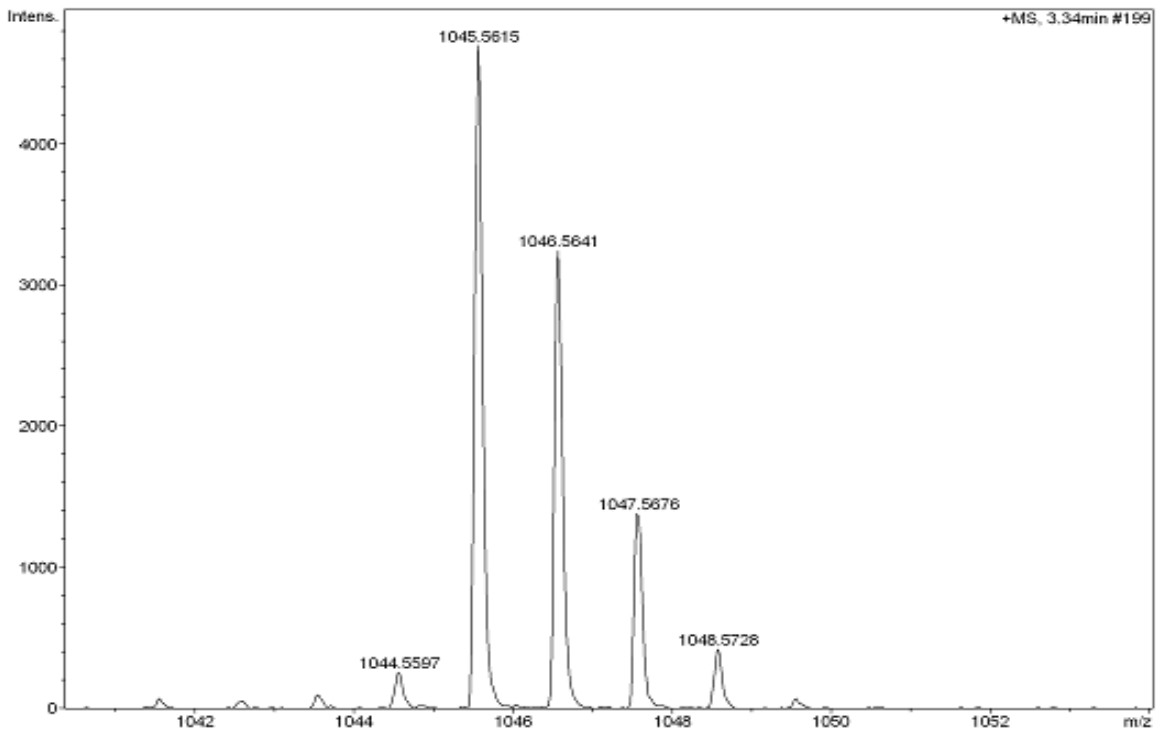

\begin{tabular}{c|c|c|c|c|c}
\hline Fomula & Mass & Error $(\mathrm{ppm})$ & DblEq & $\mathrm{N}$ rule & Electron configuration \\
\hline $\mathrm{C}_{70} \mathrm{H}_{77} \mathrm{O}_{8}$ & 1045.5615 & -0.2 & 32.5 & ok & even \\
\hline
\end{tabular}

Figure S46. HR mass spectrum (ESI) of compound $\mathbf{3}$ 


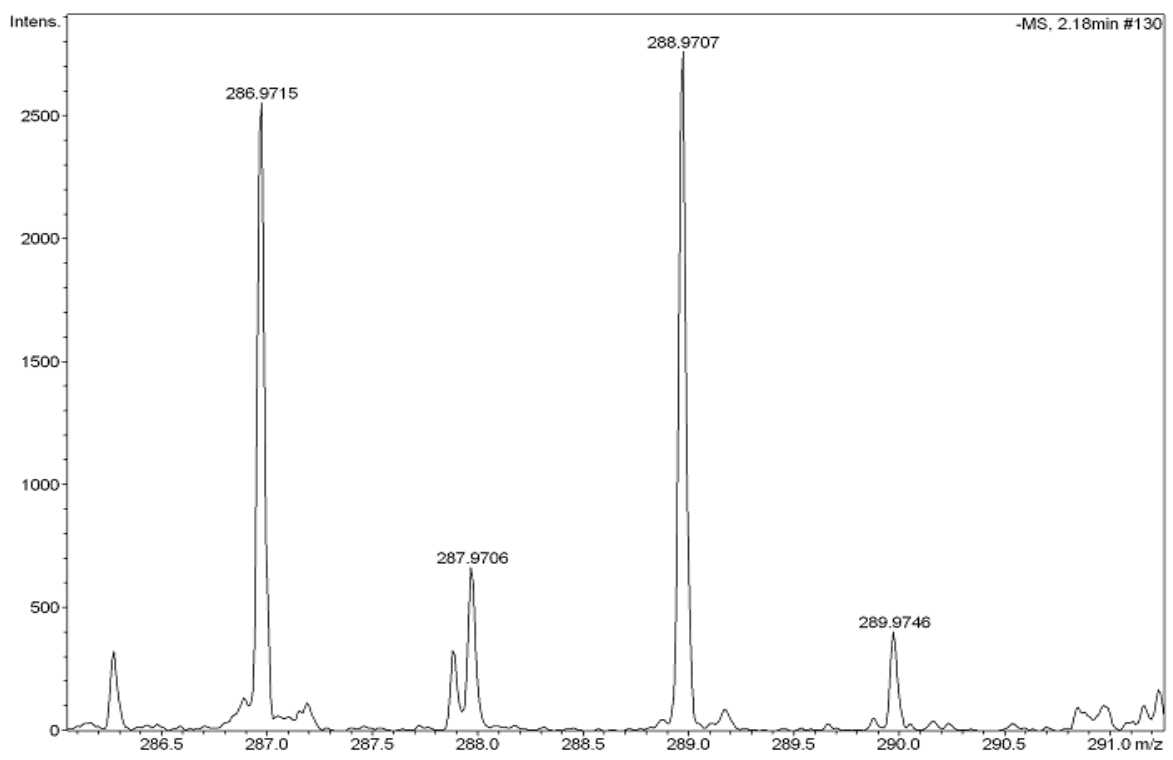

\begin{tabular}{c|c|c|c|c|c}
\hline Fomula & Mass & Error (ppm) & DblEq & N rule & Electron configuration \\
\hline $\mathrm{C}_{14} \mathrm{H}_{8} \mathrm{BrO}_{2}$ & 286.9715 & -0.7 & 10.5 & ok & even \\
\hline
\end{tabular}

Figure S47. HR mass spectrum (ESI) of compound 5

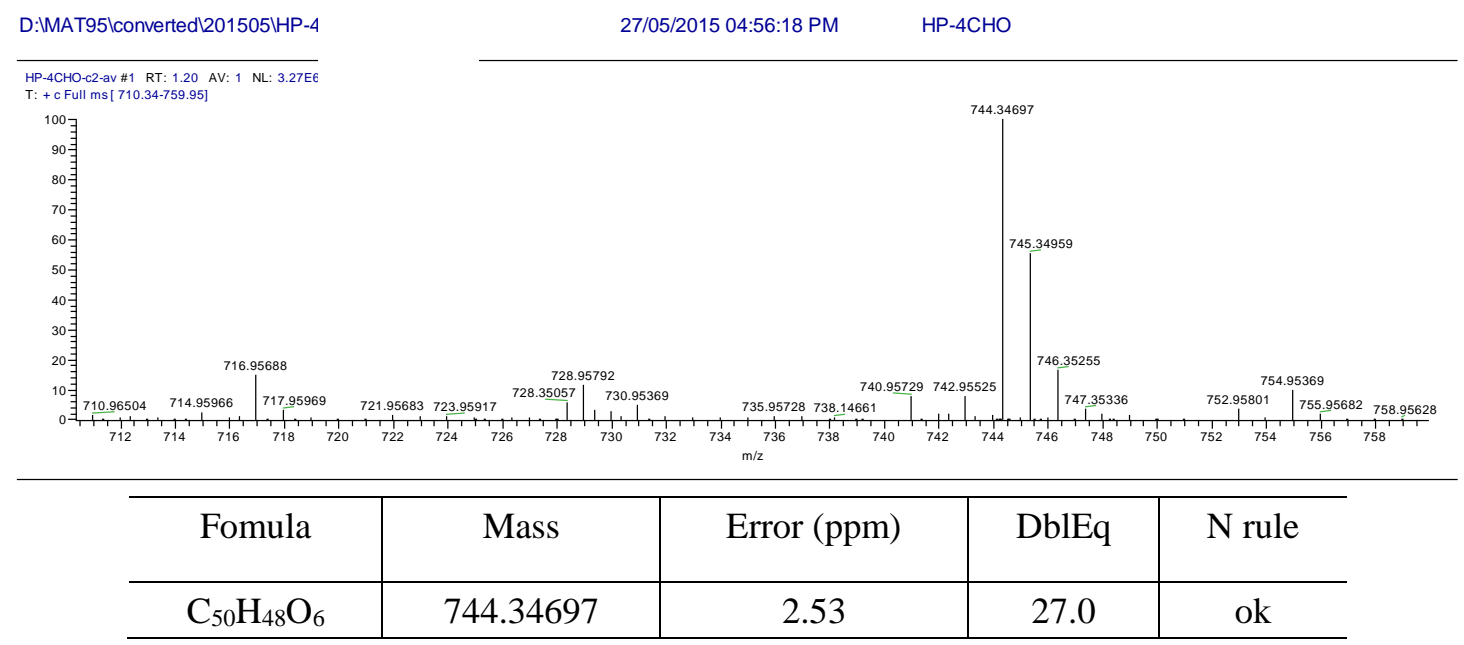

Figure S48. HR mass spectrum (EI) of compound 6 


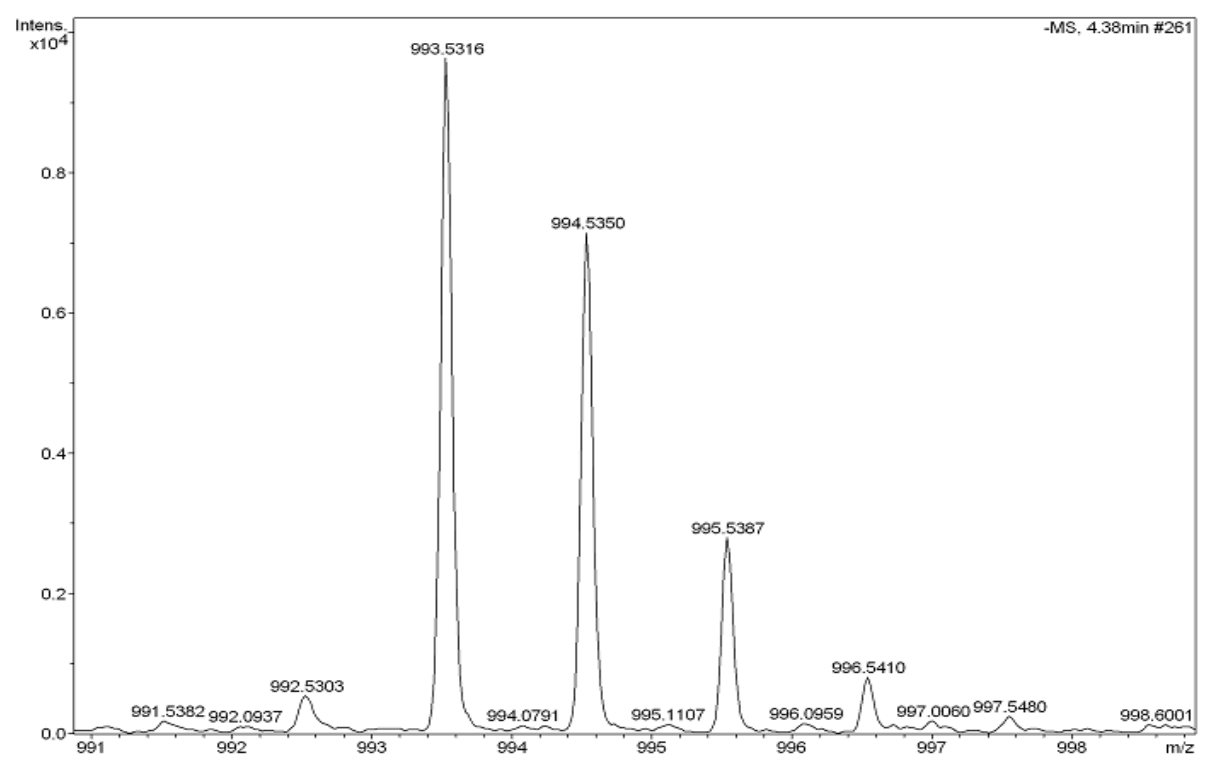

\begin{tabular}{c|c|c|c|c|c}
\hline Fomula & Mass & Error $(\mathrm{ppm})$ & DblEq & N rule & Electron configuration \\
\hline $\mathrm{C}_{66} \mathrm{H}_{73} \mathrm{O}_{8}$ & 993.5316 & -0.5 & 30.5 & ok & even \\
\hline
\end{tabular}

Figure S49. HR mass spectrum (ESI) of compound 9

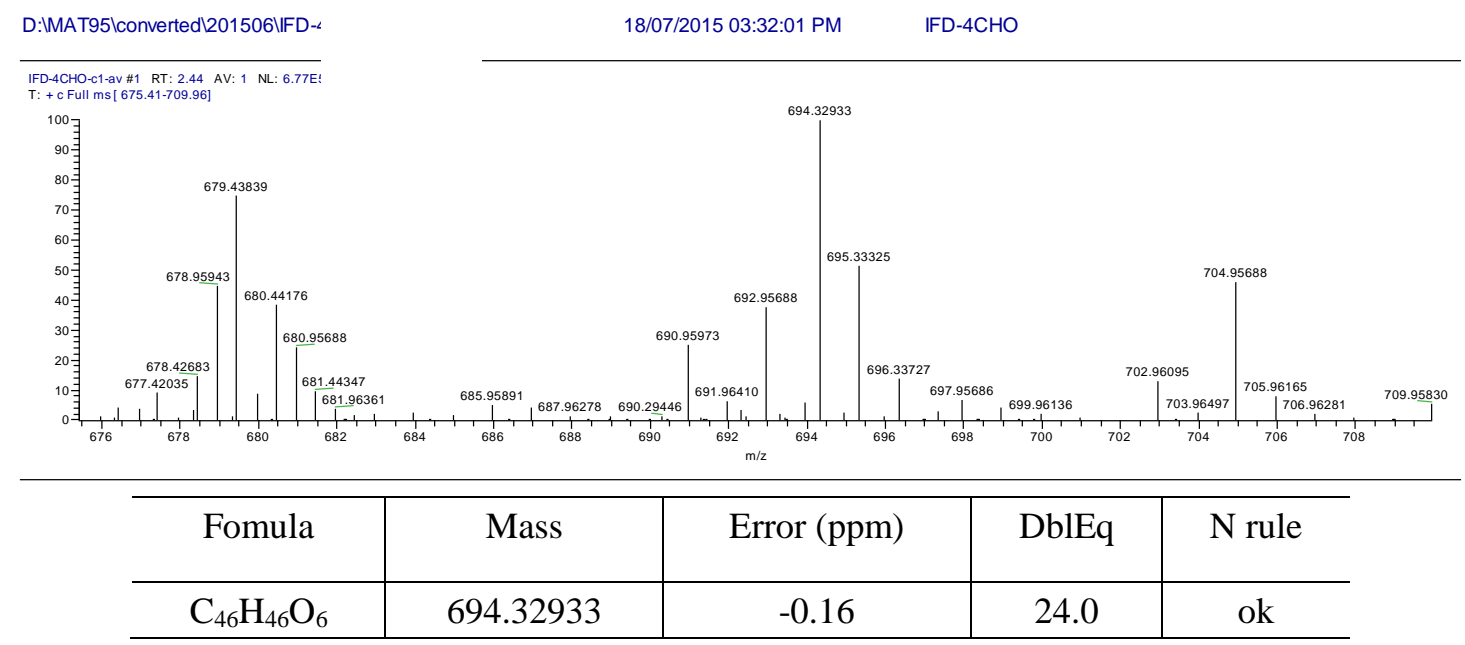

Figure S50. HR mass spectrum (EI) of compound $\mathbf{1 1}$ 
D:MAT95।converted1201505\HZ-2

27/05/2015 05:08:16 PM

$\mathrm{HZ}-2 \mathrm{CHO}$

\begin{tabular}{l}
\hline HZ-2CHO-c-av1 \#1 RT: 2.19 AV: 1 NL: 2.08E7 \\
$\mathrm{T}:+$ C Full ms [550.32-599.97]
\end{tabular}

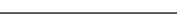

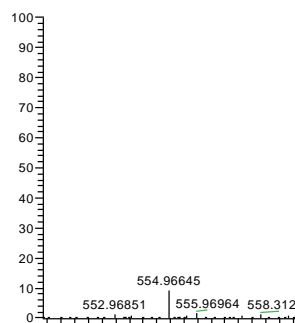

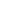

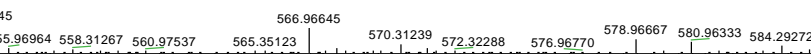

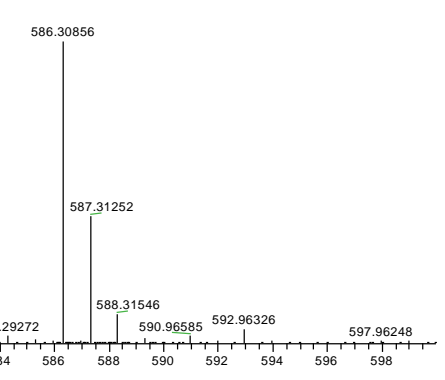

\begin{tabular}{c|c|c|c|c}
\hline Fomula & Mass & Error (ppm) & DblEq & N rule \\
\hline $\mathrm{C}_{40} \mathrm{H}_{42} \mathrm{O}_{4}$ & 586.30856 & 0.43 & 20.0 & ok \\
\hline
\end{tabular}

Figure S51. HR mass spectrum (EI) of compound 13

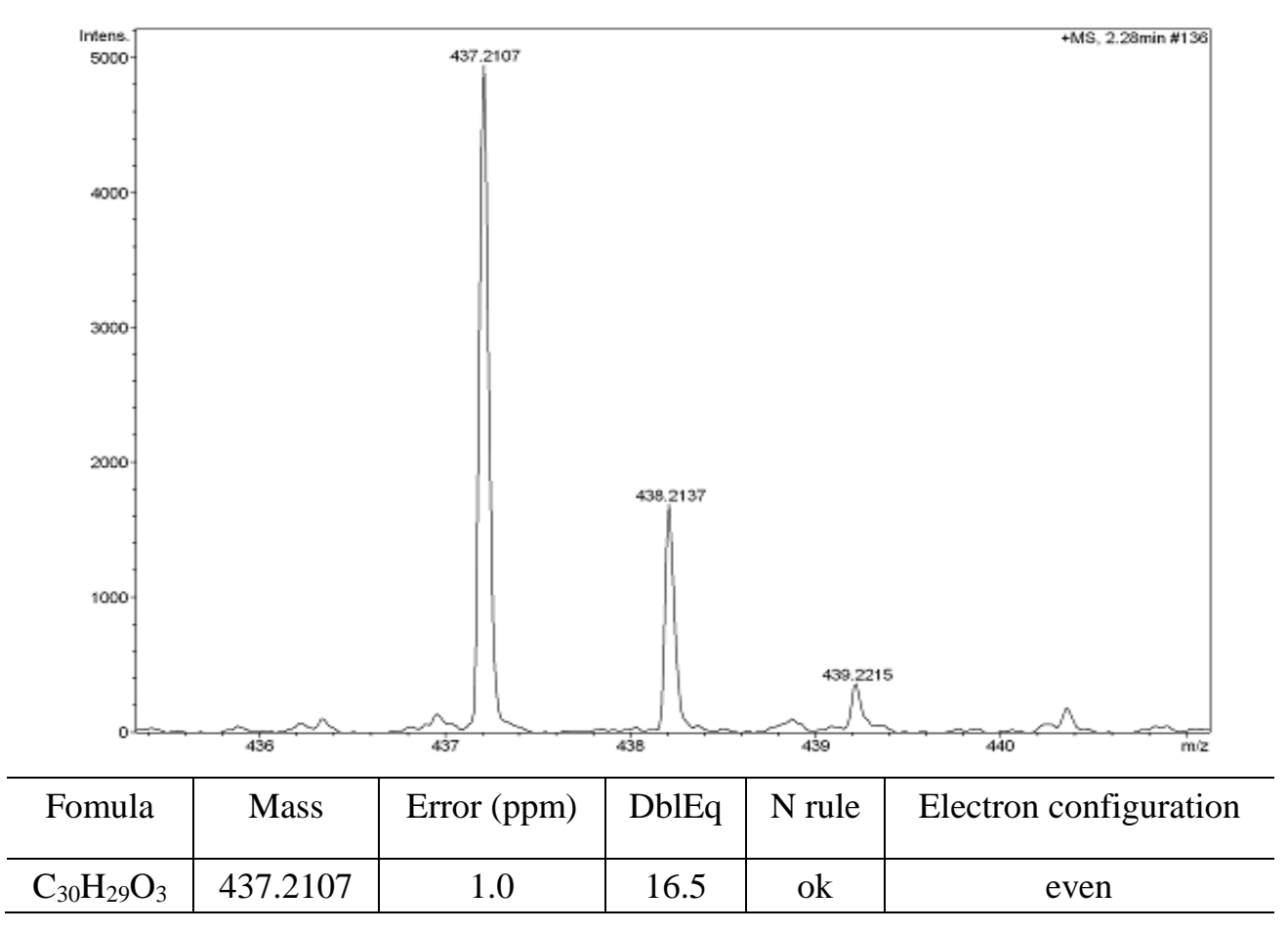

Figure S52. HR mass spectrum (ESI) of compound 14 


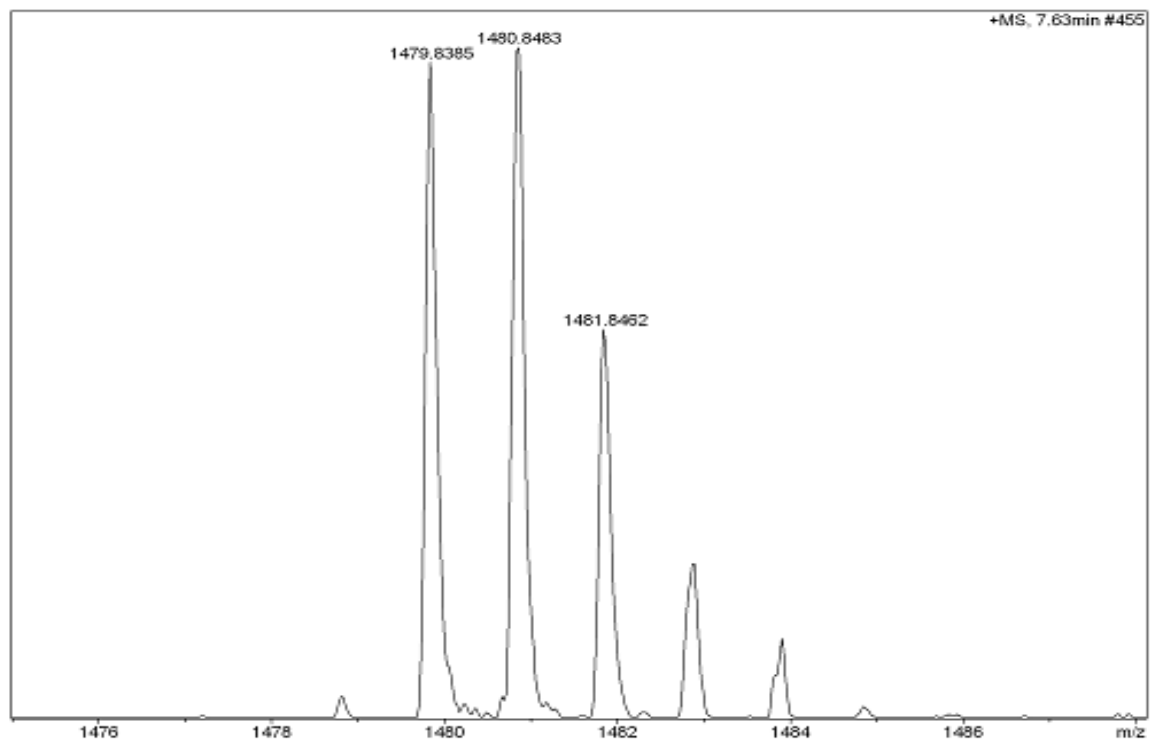

\begin{tabular}{c|c|c|c|c|c}
\hline Fomula & Mass & Error (ppm) & DblEq & N rule & Electron configuration \\
\hline $\mathrm{C}_{106} \mathrm{H}_{111} \mathrm{O}_{6}$ & 1479.8385 & -0.7 & 51.5 & ok & even \\
\hline
\end{tabular}

Figure S53. HR mass spectrum (APCI) of compound HZD-M-ONE

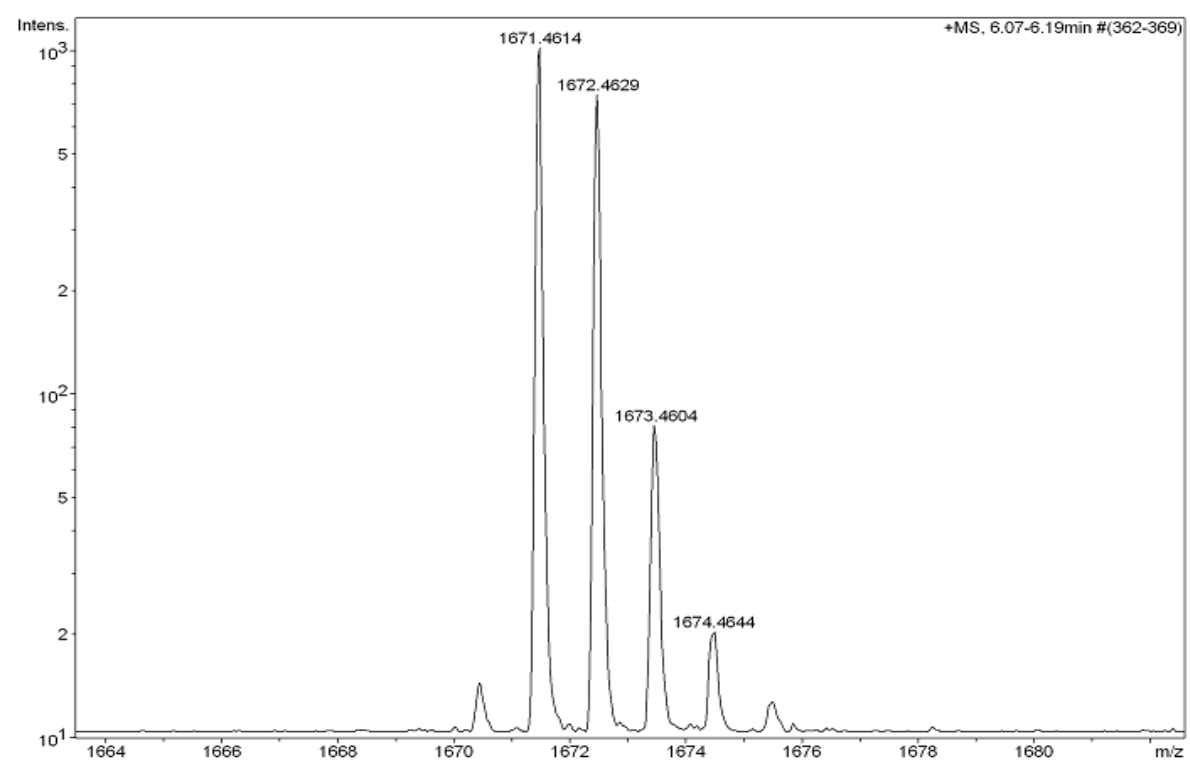

\begin{tabular}{c|c|c|c|c|c}
\hline Fomula & Mass & Error (ppm) & DblEq & $\mathrm{N}$ rule & Electron configuration \\
\hline $\mathrm{C}_{94} \mathrm{H}_{67} \mathrm{~F}_{20} \mathrm{O}_{6}$ & 1671.4641 & -0.1 & 51.5 & ok & even \\
\hline
\end{tabular}

Figure S54. HR mass spectrum (APCI) of compound HZD-F-ONE 


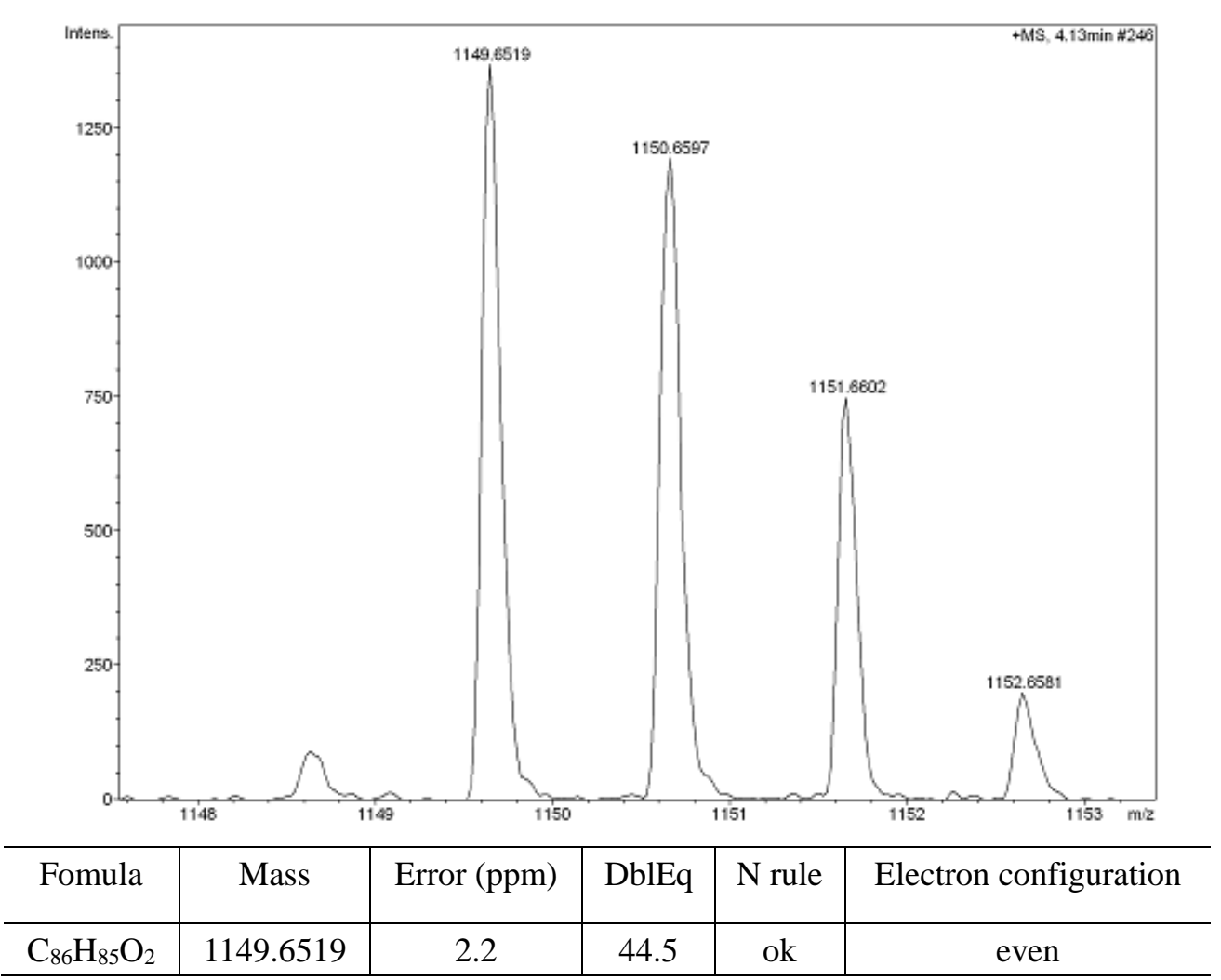

Figure S55. HR mass spectrum (APCI) of compound FP-M

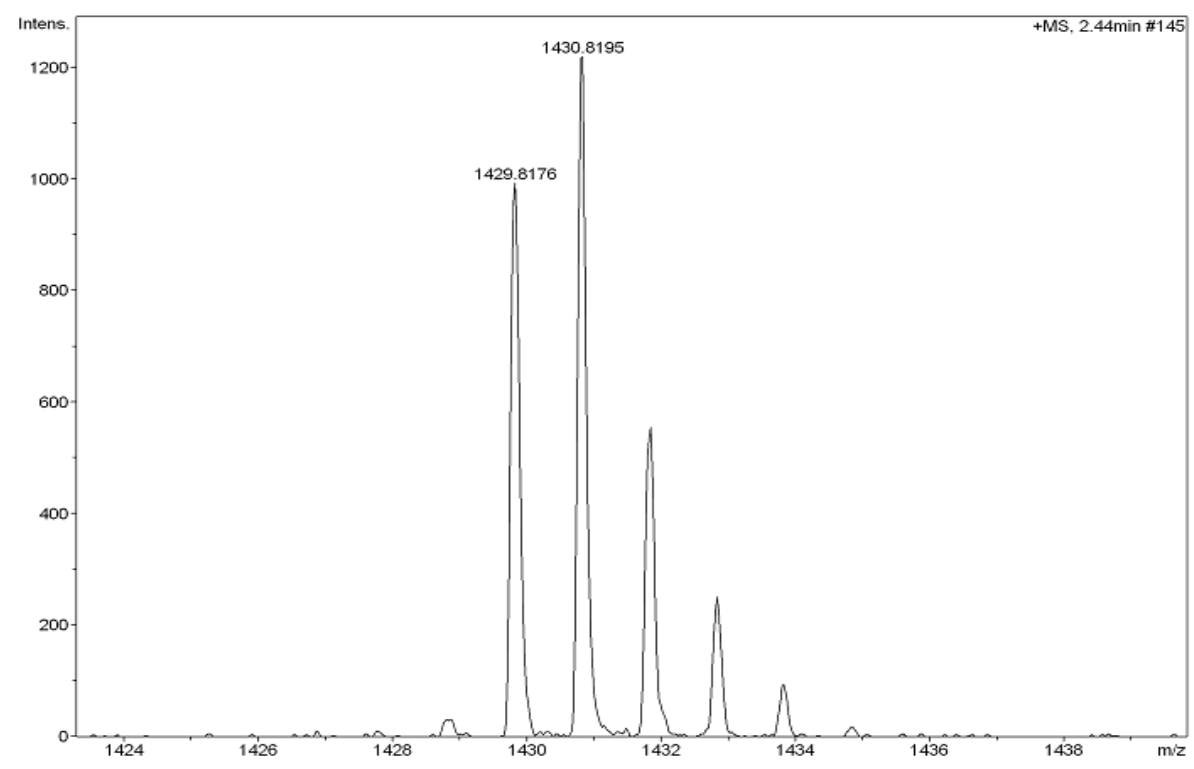

\begin{tabular}{c|c|c|c|c|c}
\hline Fomula & Mass & Error (ppm) & DblEq & N rule & Electron configuration \\
\hline $\mathrm{C}_{102} \mathrm{H}_{109} \mathrm{O}_{6}$ & 1429.8176 & 3.0 & 48.5 & ok & even \\
\hline
\end{tabular}

Figure S56. HR mass spectrum (APCI) of compound PIF-M-ONE 


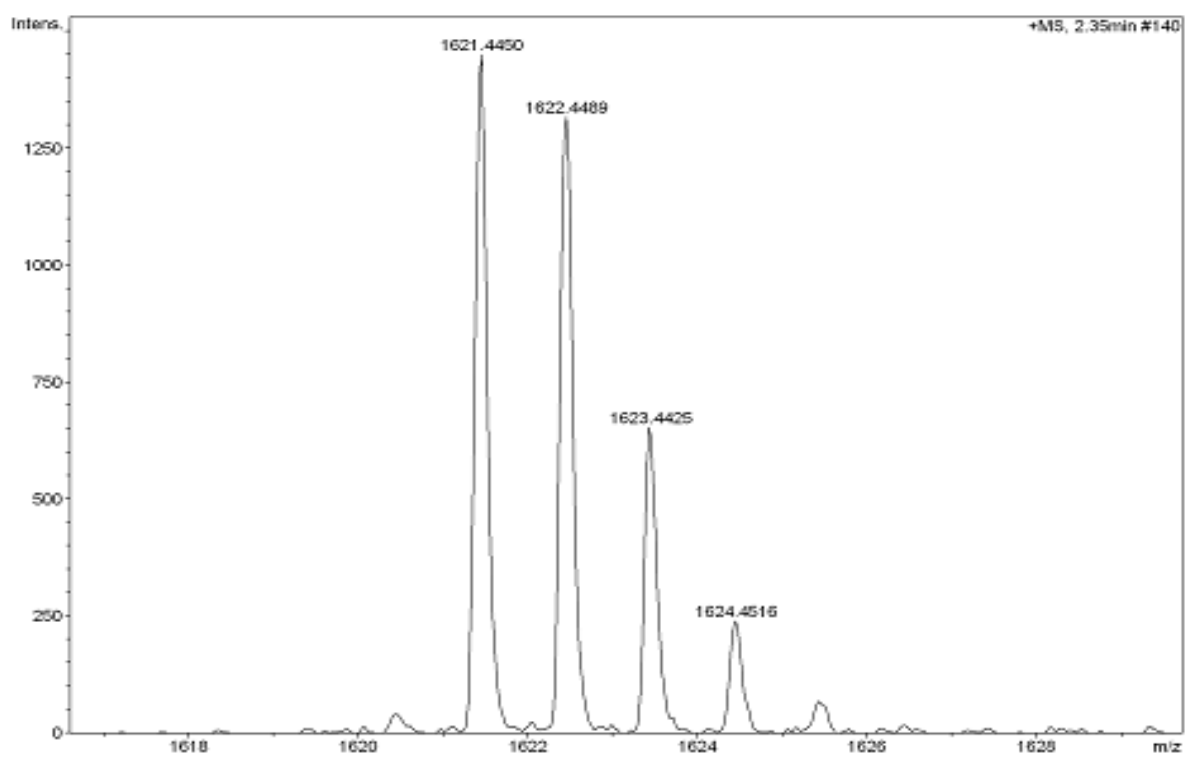

\begin{tabular}{c|c|c|c|c|c}
\hline Fomula & Mass & Error $(\mathrm{ppm})$ & DblEq & N rule & Electron configuration \\
\hline $\mathrm{C}_{90} \mathrm{H}_{65} \mathrm{~F}_{20} \mathrm{O}_{6}$ & 1621.4450 & 0.4 & 48.5 & ok & even \\
\hline
\end{tabular}

Figure S57. HR mass spectrum (APCI) of compound PIF-F-ONE

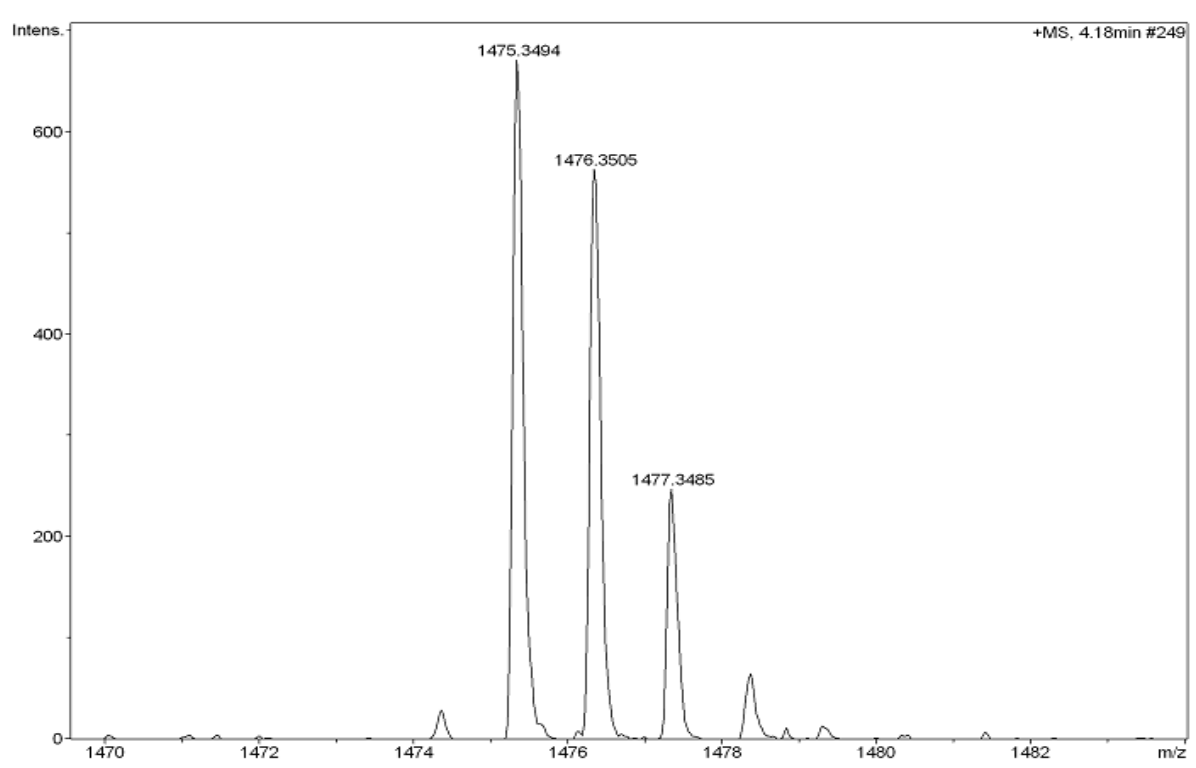

\begin{tabular}{c|c|c|c|c|c}
\hline Fomula & Mass & Error (ppm) & DblEq & N rule & Electron configuration \\
\hline $\mathrm{C}_{78} \mathrm{H}_{51} \mathrm{~F}_{24} \mathrm{O}_{2}$ & 1475.3494 & 0.4 & 41.5 & ok & even \\
\hline
\end{tabular}

Figure S58. HR mass spectrum (APCI) of compound IFD-CF $\mathbf{F}_{\mathbf{3}}$ 


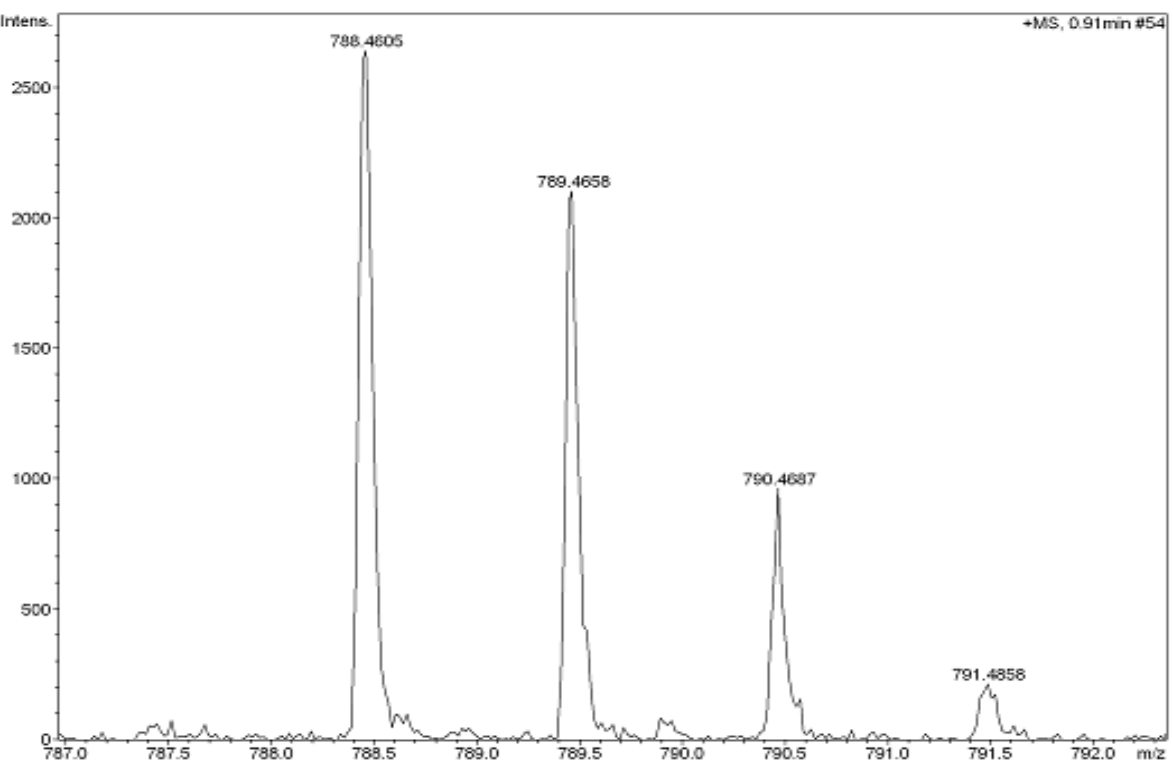

\begin{tabular}{c|c|c|c|c|c}
\hline Fomula & Mass & Error (ppm) & DblEq & N rule & Electron configuration \\
\hline $\mathrm{C}_{58} \mathrm{H}_{60} \mathrm{O}_{2}$ & 788.4605 & -2.1 & 29.0 & ok & odd \\
\hline
\end{tabular}

Figure S59. HR mass spectrum (ESI) of compound HZ-M

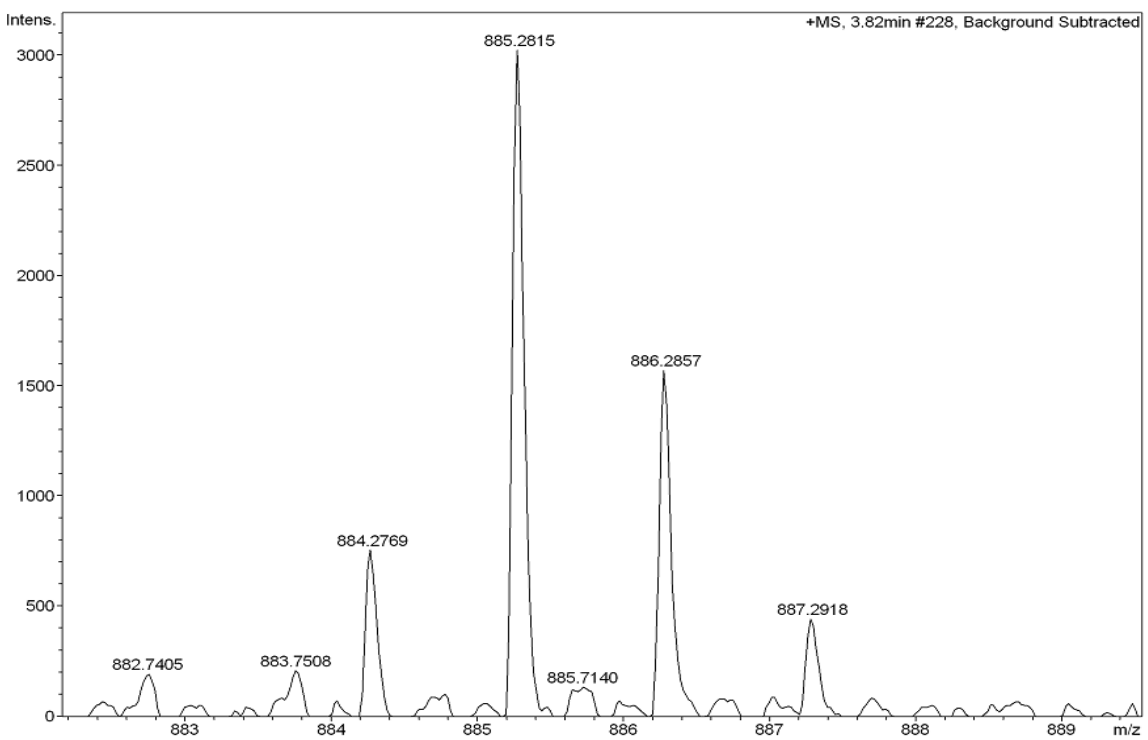

\begin{tabular}{c|c|c|c|c|c}
\hline Fomula & Mass & Error (ppm) & DblEq & N rule & Electron configuration \\
\hline $\mathrm{C}_{52} \mathrm{H}_{39} \mathrm{~F}_{10} \mathrm{O}_{2}$ & 885.2815 & -2.8 & 28.5 & ok & even \\
\hline
\end{tabular}

Figure S60. HR mass spectrum (ESI) of compound HZ-F 


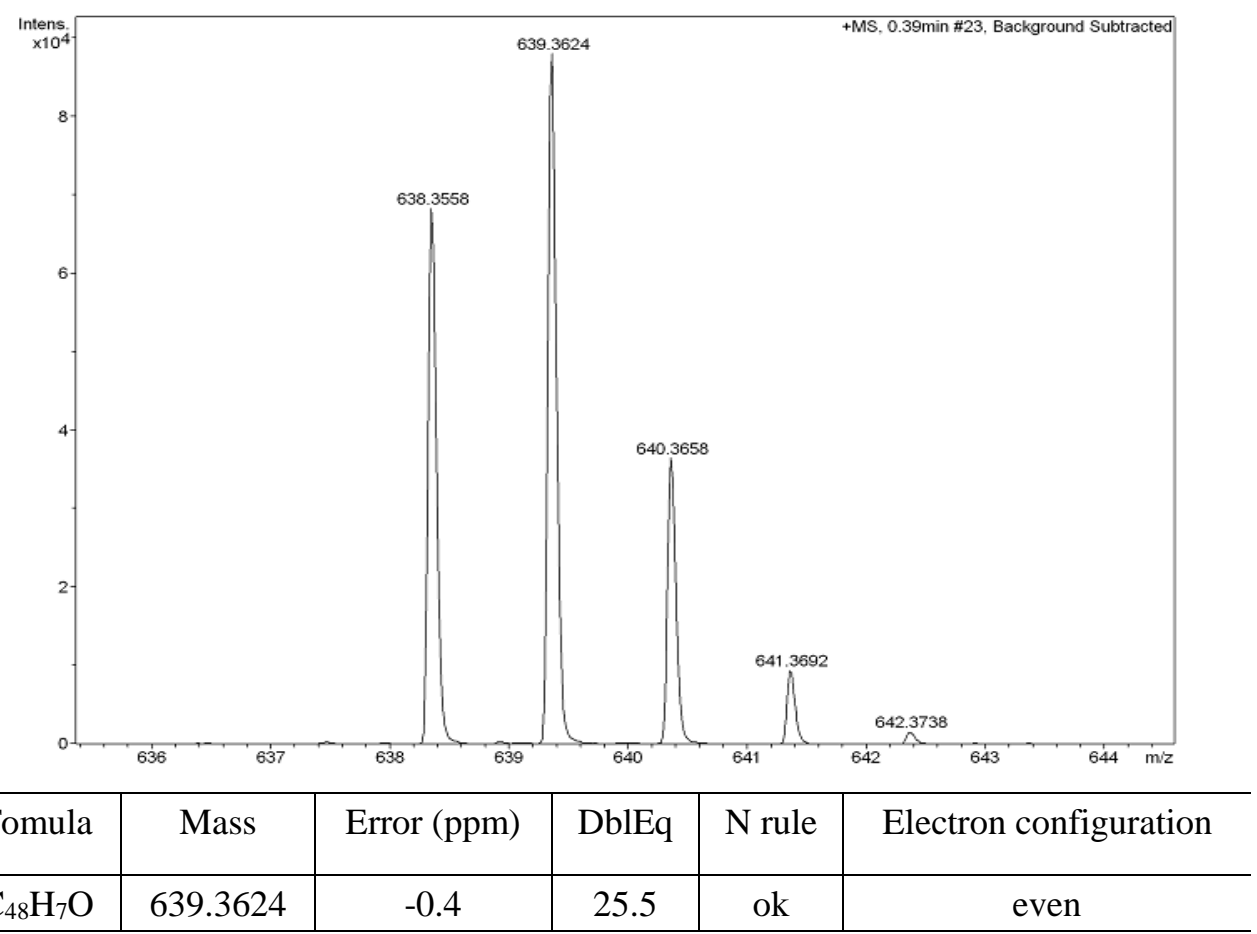

Figure S61. HR mass spectrum (ESI) of compound PF-M

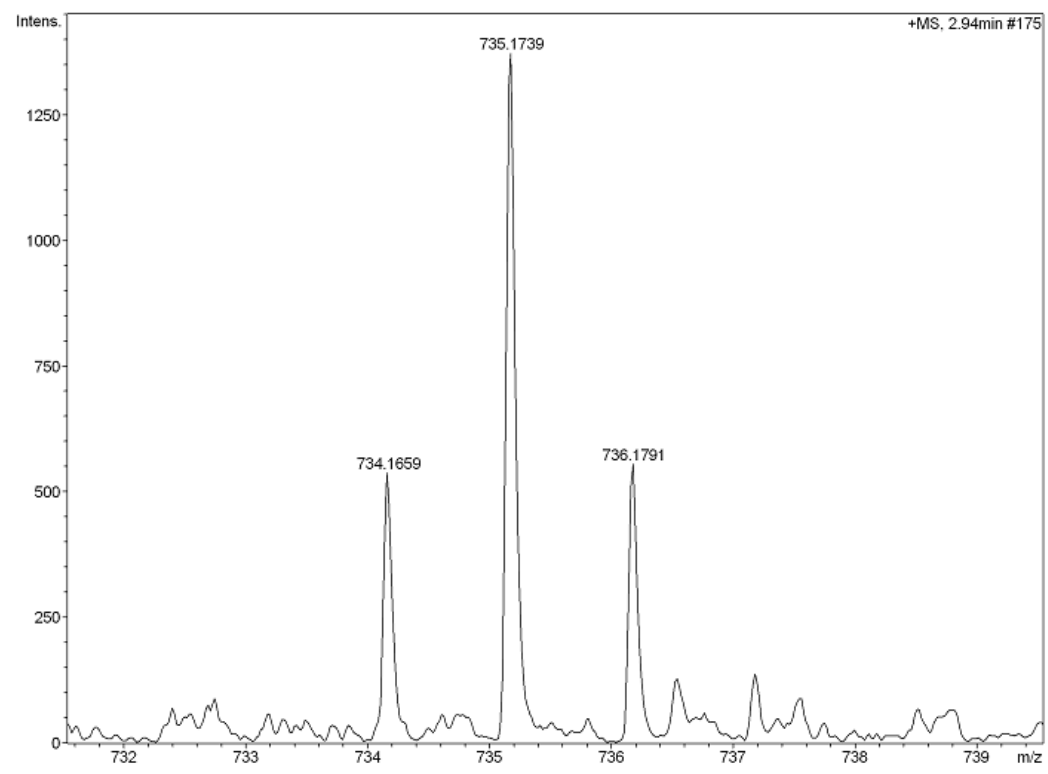

\begin{tabular}{c|c|c|c|c|c}
\hline Fomula & Mass & Error (ppm) & DblEq & N rule & Electron configuration \\
\hline $\mathrm{C}_{42} \mathrm{H}_{25} \mathrm{~F}_{10} \mathrm{O}$ & 735.1739 & 0.2 & 25.5 & ok & even \\
\hline
\end{tabular}

Figure S62. HR mass spectrum (ESI) of compound PF-F 
11. Appendix: energy, NICS data and Cartesian coordinates for all atoms of optimized geometries

Compound HZD

Natural Orbital Coefficient

HOMO: 1.14647 LUMO: 0.85353

singlet biradical character $\mathrm{y}_{0}=0.713$

\section{Singlet Biradical Open Shell:}

Sum of electronic and zero-point Energies=

$-1765.886495$

Sum of electronic and thermal Energies=

$-1765.856759$

Sum of electronic and thermal Enthalpies=

$-1765.855815$

Sum of electronic and thermal Free Energies=

$-1765.946438$

$\mathrm{S} * * 2=1.5736$

$\begin{array}{cccc}\mathrm{H} & -11.04668600 & 2.76478700 & 0.00070800 \\ \mathrm{C} & -10.27013300 & 2.00622000 & 0.00057200 \\ \mathrm{H} & -11.65425000 & 0.36779000 & 0.00044900 \\ \mathrm{C} & -10.61150700 & 0.67083700 & 0.00042700 \\ \mathrm{C} & -7.90017400 & 1.43710400 & 0.00036800 \\ \mathrm{C} & -9.60989000 & -0.33685200 & 0.00024700 \\ \mathrm{C} & -8.92913800 & 2.39170500 & 0.00054600 \\ \mathrm{C} & -8.23895800 & 0.04092300 & 0.00021100 \\ \mathrm{C} & -9.94514300 & -1.70653900 & 0.00010700 \\ \mathrm{H} & -8.66514300 & 3.44512900 & 0.00066500 \\ \mathrm{C} & -8.95568000 & -2.66334800 & -0.00005500 \\ \mathrm{H} & -10.99271300 & -1.99323500 & 0.00013300 \\ \mathrm{H} & -9.21694400 & -3.71699400 & -0.00015400 \\ \mathrm{C} & -7.60877700 & -2.29201500 & -0.00009100 \\ \mathrm{H} & -6.85959900 & -3.07473600 & -0.00021000 \\ \mathrm{C} & -7.22277000 & -0.95339600 & 0.00002900 \\ \mathrm{C} & -5.81340900 & -0.53954800 & -0.00002600 \\ \mathrm{C} & -5.50303000 & 0.86939900 & 0.00014800 \\ \mathrm{C} & -4.76285200 & -1.42689100 & -0.00024400 \\ \mathrm{H} & -4.94511400 & -2.49601400 & -0.00039700 \\ \mathrm{C} & -3.40708300 & -1.01469500 & -0.00029100 \\ \mathrm{C} & -3.09258000 & 0.38560100 & -0.00010300 \\ \mathrm{C} & -6.54379400 & 1.81333200 & 0.00034200 \\ \mathrm{H} & -6.29304900 & 2.87057900 & 0.00046600 \\ \mathrm{C} & -1.70913500 & 0.79291000 & -0.00015000 \\ \mathrm{C} & 1.03448500 & 1.57806600 & -0.00011600 \\ \mathrm{C} & -0.69005100 & -0.18843400 & -0.00033600 \\ \mathrm{C} & -1.32306800 & 2.16583800 & -0.00002300 \\ \mathrm{C} & -0.01577600 & 2.54043500 & -0.00001700\end{array}$




\begin{tabular}{|c|c|c|c|}
\hline $\mathrm{C}$ & 0.69005100 & 0.18843400 & -0.00029300 \\
\hline $\mathrm{H}$ & -2.08444100 & 2.93602300 & 0.00004100 \\
\hline $\mathrm{H}$ & 0.25018000 & 3.59357200 & 0.00005800 \\
\hline $\mathrm{C}$ & -2.37242800 & -1.95721000 & -0.00053200 \\
\hline $\mathrm{H}$ & -2.62258800 & -3.01495900 & -0.00071000 \\
\hline $\mathrm{C}$ & -1.03448600 & -1.57806600 & -0.00056200 \\
\hline $\mathrm{C}$ & 0.01577600 & -2.54043500 & -0.00087900 \\
\hline $\mathrm{H}$ & -0.25018000 & -3.59357300 & -0.00120800 \\
\hline $\mathrm{C}$ & -4.16191900 & 1.28320400 & 0.00011200 \\
\hline $\mathrm{H}$ & -3.97959300 & 2.35166400 & 0.00026400 \\
\hline $\mathrm{C}$ & 1.70913500 & -0.79291000 & -0.00043400 \\
\hline $\mathrm{C}$ & 1.32306800 & -2.16583900 & -0.00084100 \\
\hline $\mathrm{H}$ & 2.08444100 & -2.93602300 & -0.00124200 \\
\hline $\mathrm{C}$ & 3.09258000 & -0.38560100 & -0.00021200 \\
\hline $\mathrm{C}$ & 3.40708300 & 1.01469500 & -0.00013800 \\
\hline $\mathrm{C}$ & 4.16191900 & -1.28320400 & -0.00001600 \\
\hline $\mathrm{H}$ & 3.97959300 & -2.35166400 & 0.00023600 \\
\hline $\mathrm{C}$ & 5.50303000 & -0.86940000 & 0.00004800 \\
\hline $\mathrm{C}$ & 5.81340800 & 0.53954800 & -0.00011200 \\
\hline $\mathrm{C}$ & 2.37242800 & 1.95721000 & -0.00008800 \\
\hline $\mathrm{H}$ & 2.62258800 & 3.01495900 & -0.00000900 \\
\hline $\mathrm{C}$ & 7.22277000 & 0.95339600 & -0.00021400 \\
\hline $\mathrm{C}$ & 9.94514300 & 1.70653900 & -0.00014400 \\
\hline $\mathrm{C}$ & 8.23895800 & -0.04092300 & 0.00022400 \\
\hline $\mathrm{C}$ & 7.60877700 & 2.29201500 & -0.00075500 \\
\hline $\mathrm{C}$ & 8.95567900 & 2.66334800 & -0.00069600 \\
\hline $\mathrm{C}$ & 9.60989000 & 0.33685300 & 0.00029000 \\
\hline $\mathrm{H}$ & 6.85959900 & 3.07473600 & -0.00129000 \\
\hline $\mathrm{H}$ & 9.21694400 & 3.71699400 & -0.00110600 \\
\hline $\mathrm{C}$ & 6.54379400 & -1.81333200 & 0.00037200 \\
\hline $\mathrm{H}$ & 6.29304900 & -2.87057900 & 0.00053400 \\
\hline $\mathrm{C}$ & 7.90017400 & -1.43710400 & 0.00053800 \\
\hline $\mathrm{C}$ & 8.92913800 & -2.39170500 & 0.00094400 \\
\hline $\mathrm{H}$ & 8.66514300 & -3.44512900 & 0.00115400 \\
\hline $\mathrm{C}$ & 4.76285200 & 1.42689000 & -0.00008400 \\
\hline $\mathrm{H}$ & 4.94511400 & 2.49601400 & 0.00005300 \\
\hline $\mathrm{C}$ & 10.61150700 & -0.67083600 & 0.00075600 \\
\hline $\mathrm{C}$ & 10.27013400 & -2.00621900 & 0.00107200 \\
\hline $\mathrm{H}$ & 11.04668600 & -2.76478600 & 0.00139500 \\
\hline $\mathrm{H}$ & 10.99271300 & 1.99323600 & -0.00008200 \\
\hline $\mathrm{H}$ & 11.65425000 & -0.36778900 & 0.00085900 \\
\hline
\end{tabular}




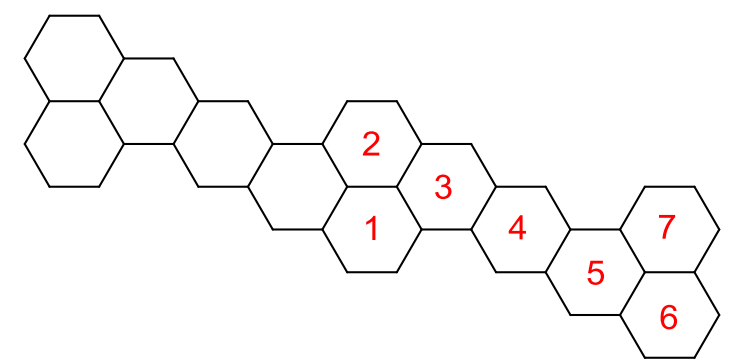

Bq distance $(\AA)$

\begin{tabular}{|c|c|c|c|c|c|c|c|}
\hline 0.000 & 10.347 & 10.347 & 2.364 & 2.491 & 24.160 & 5.546 & -2.635 \\
\hline 0.100 & 9.854 & 9.855 & 1.833 & 1.952 & 23.696 & 5.060 & -4.640 \\
\hline 0.200 & 8.436 & 8.437 & 0.313 & 0.412 & 22.347 & 3.665 & -6.886 \\
\hline 0.300 & 6.253 & 6.254 & -1.996 & -1.923 & 20.238 & 1.533 & -9.602 \\
\hline 0.400 & 3.536 & 3.538 & -4.812 & -4.757 & 17.551 & -1.093 & -12.474 \\
\hline 0.500 & 0.547 & 0.549 & -7.826 & -7.769 & 14.497 & -3.939 & -15.213 \\
\hline 0.600 & -2.467 & -2.464 & -10.755 & -10.669 & 11.290 & -6.749 & -17.600 \\
\hline 0.700 & -5.299 & -5.296 & -13.379 & -13.233 & 8.117 & -9.320 & -19.496 \\
\hline 0.800 & -7.805 & -7.802 & -15.559 & -15.321 & 5.127 & -11.511 & -20.843 \\
\hline 0.900 & -9.901 & -9.898 & -17.227 & -16.874 & 2.420 & -13.252 & -21.648 \\
\hline 1.000 & -11.560 & -11.556 & -18.384 & -17.898 & 0.055 & -14.527 & -21.962 \\
\hline 1.100 & -12.792 & -12.788 & -19.069 & -18.441 & -1.950 & -15.361 & -21.863 \\
\hline 1.200 & -13.638 & -13.634 & -19.348 & -18.577 & -3.603 & -15.808 & -21.436 \\
\hline 1.300 & -14.151 & -14.148 & -19.298 & -18.389 & -4.929 & -15.932 & -20.764 \\
\hline 1.400 & -14.391 & -14.387 & -18.993 & -17.957 & -5.965 & -15.798 & -19.923 \\
\hline 1.500 & -14.412 & -14.409 & -18.502 & -17.353 & -6.750 & -15.470 & -18.974 \\
\hline 1.600 & -14.267 & -14.263 & -17.883 & -16.637 & -7.325 & -15.002 & -17.968 \\
\hline 1.700 & -13.998 & -13.995 & -17.183 & -15.856 & -7.725 & -14.440 & -16.944 \\
\hline 1.800 & -13.642 & -13.639 & -16.439 & -15.049 & -7.983 & -13.819 & -15.931 \\
\hline 1.900 & -13.228 & -13.224 & -15.679 & -14.242 & -8.127 & -13.167 & -14.948 \\
\hline 2.000 & -12.776 & -12.773 & -14.923 & -13.455 & -8.180 & -12.507 & -14.008 \\
\hline 2.100 & -12.305 & -12.302 & -14.185 & -12.699 & -8.162 & -11.854 & -13.120 \\
\hline 2.200 & -11.827 & -11.825 & -13.475 & -11.983 & -8.088 & -11.217 & -12.286 \\
\hline 2.300 & -11.351 & -11.349 & -12.796 & -11.310 & -7.972 & -10.604 & -11.509 \\
\hline 2.400 & -10.884 & -10.881 & -12.153 & -10.682 & -7.824 & -10.019 & -10.788 \\
\hline 2.500 & -10.430 & -10.427 & -11.547 & -10.099 & -7.653 & -9.466 & -10.120 \\
\hline 2.600 & -9.991 & -9.989 & -10.977 & -9.559 & -7.465 & -8.944 & -9.503 \\
\hline 2.700 & -9.570 & -9.568 & -10.442 & -9.059 & -7.265 & -8.454 & -8.934 \\
\hline 2.800 & -9.167 & -9.165 & -9.940 & -8.597 & -7.059 & -7.994 & -8.408 \\
\hline 2.900 & -8.783 & -8.782 & -9.471 & -8.170 & -6.849 & -7.564 & -7.924 \\
\hline 3.000 & -8.418 & -8.416 & -9.031 & -7.776 & -6.637 & -7.163 & -7.476 \\
\hline 3.100 & -8.071 & -8.069 & -8.618 & -7.411 & -6.427 & -6.788 & -7.063 \\
\hline 3.200 & -7.741 & -7.740 & -8.232 & -7.073 & -6.219 & -6.438 & -6.681 \\
\hline 3.300 & -7.429 & -7.427 & -7.869 & -6.759 & -6.014 & -6.111 & -6.327 \\
\hline
\end{tabular}




$\begin{array}{llllllll}3.400 & -7.132 & -7.131 & -7.529 & -6.466 & -5.814 & -5.806 & -6.000 \\ 3.500 & -6.851 & -6.849 & -7.209 & -6.195 & -5.619 & -5.520 & -5.696 \\ 3.600 & -6.584 & -6.582 & -6.908 & -5.941 & -5.429 & -5.253 & -5.414 \\ 3.700 & -6.330 & -6.329 & -6.624 & -5.703 & -5.245 & -5.004 & -5.151 \\ 3.800 & -6.090 & -6.088 & -6.357 & -5.481 & -5.067 & -4.770 & -4.906 \\ 3.900 & -5.861 & -5.860 & -6.105 & -5.272 & -4.895 & -4.551 & -4.678 \\ 4.000 & -5.644 & -5.643 & -5.867 & -5.076 & -4.729 & -4.346 & -4.465 \\ 4.100 & -5.438 & -5.437 & -5.642 & -4.891 & -4.568 & -4.153 & -4.266 \\ 4.200 & -5.242 & -5.241 & -5.429 & -4.717 & -4.414 & -3.972 & -4.079 \\ 4.300 & -5.055 & -5.054 & -5.227 & -4.552 & -4.266 & -3.801 & -3.904 \\ 4.400 & -4.877 & -4.876 & -5.036 & -4.396 & -4.123 & -3.641 & -3.739 \\ 4.500 & -4.708 & -4.707 & -4.854 & -4.248 & -3.986 & -3.490 & -3.585 \\ 4.600 & -4.547 & -4.546 & -4.682 & -4.108 & -3.854 & -3.348 & -3.440 \\ 4.700 & -4.393 & -4.392 & -4.518 & -3.974 & -3.727 & -3.214 & -3.303 \\ 4.800 & -4.246 & -4.245 & -4.363 & -3.847 & -3.606 & -3.087 & -3.174 \\ 4.900 & -4.106 & -4.105 & -4.214 & -3.726 & -3.489 & -2.967 & -3.052 \\ 5.000 & -3.972 & -3.971 & -4.073 & -3.610 & -3.376 & -2.854 & -2.943\end{array}$

Mulliken atomic spin densities:

$\begin{array}{rrr}1 & \mathrm{H} & -0.007805 \\ 2 & \mathrm{C} & 0.206765 \\ 3 & \mathrm{H} & 0.015417 \\ 4 & \mathrm{C} & -0.338641 \\ 5 & \mathrm{C} & 0.252295 \\ 6 & \mathrm{C} & 0.155679 \\ 7 & \mathrm{C} & -0.358698 \\ 8 & \mathrm{C} & -0.153107 \\ 9 & \mathrm{C} & -0.197397 \\ 10 & \mathrm{H} & 0.015992 \\ 11 & \mathrm{C} & 0.138175 \\ 12 & \mathrm{H} & 0.008763 \\ 13 & \mathrm{H} & -0.005470 \\ 14 & \mathrm{C} & -0.213676 \\ 15 & \mathrm{H} & 0.009459 \\ 16 & \mathrm{C} & 0.170692 \\ 17 & \mathrm{C} & -0.223637 \\ 18 & \mathrm{C} & 0.260664 \\ 19 & \mathrm{C} & 0.245114 \\ 20 & \mathrm{H} & -0.009970 \\ 21 & \mathrm{C} & -0.221009 \\ 22 & \mathrm{C} & 0.228619 \\ 23 & \mathrm{C} & -0.529993 \\ 24 & \mathrm{H} & 0.022467\end{array}$




\begin{tabular}{|c|c|c|}
\hline & $\mathrm{C}$ & \\
\hline & $\mathrm{C}$ & 0.218568 \\
\hline & $\mathrm{C}$ & 0.178005 \\
\hline & $\mathrm{C}$ & 0.202982 \\
\hline & $\mathrm{C}$ & -0.209803 \\
\hline & $\mathrm{C}$ & -0.178005 \\
\hline & $\mathrm{H}$ & -0.008247 \\
\hline & $\mathrm{H}$ & 0.00878 \\
\hline & $\mathrm{C}$ & 0.306480 \\
\hline & $\mathrm{H}$ & -0.012648 \\
\hline & $\mathrm{C}$ & -0.218568 \\
\hline & $\mathrm{C}$ & \\
\hline & $\mathrm{H}$ & -0.008788 \\
\hline & $\mathrm{C}$ & -0.352329 \\
\hline & $\mathrm{H}$ & 0.014533 \\
\hline & $\mathrm{C}$ & 050 \\
\hline & $\mathrm{C}$ & 2982 \\
\hline & $\mathrm{H}$ & 0.008247 \\
\hline & $\mathrm{C}$ & -0.228619 \\
\hline & $\mathrm{C}$ & 1009 \\
\hline & $\mathrm{C}$ & 0.352329 \\
\hline & $\mathrm{H}$ & -0.01 \\
\hline & $\mathrm{C}$ & -0.260664 \\
\hline & $\mathrm{C}$ & 0.223637 \\
\hline & $\mathrm{C}$ & -0.306479 \\
\hline & $\mathrm{H}$ & 0.012648 \\
\hline & $\mathrm{C}$ & -0.170692 \\
\hline & $\mathrm{C}$ & 0.197397 \\
\hline & $\mathrm{C}$ & 0.153107 \\
\hline & $\mathrm{C}$ & 0.213676 \\
\hline & $\mathrm{C}$ & -0.138175 \\
\hline & $\mathrm{C}$ & -0.155679 \\
\hline & $\mathrm{H}$ & -0.009459 \\
\hline & $\mathrm{H}$ & 0.005470 \\
\hline & $\mathrm{C}$ & 0.529994 \\
\hline 60 & $\mathrm{H}$ & -0.022467 \\
\hline & $\mathrm{C}$ & -0.252295 \\
\hline & $\mathrm{C}$ & 0.358698 \\
\hline 63 & $\mathrm{H}$ & -0.015992 \\
\hline 64 & $\mathrm{C}$ & -0.245114 \\
\hline & $\mathrm{H}$ & 0.009970 \\
\hline 00 & $\mathrm{C}$ & 0.338641 \\
\hline 67 & $\mathrm{C}$ & -0.206765 \\
\hline & $\mathrm{H}$ & 0.00780 \\
\hline
\end{tabular}




$$
\begin{array}{lll}
69 & \mathrm{H} & -0.008763 \\
70 & \mathrm{H} & -0.015417
\end{array}
$$

\section{Singlet Closed Shell:}

Sum of electronic and zero-point Energies= Sum of electronic and thermal Energies= Sum of electronic and thermal Enthalpies= Sum of electronic and thermal Free Energies=

$\mathrm{H}$

C

$\mathrm{H}$

$\mathrm{C}$

$\mathrm{C}$

$\mathrm{C}$

$\mathrm{C}$

$\mathrm{C}$

$\mathrm{C}$

$\mathrm{H}$

$\mathrm{C}$

$\mathrm{H}$

$\mathrm{H}$

$\mathrm{C}$

$\mathrm{H}$

C

C

C

C

$\mathrm{H}$

C

C

C

$\mathrm{H}$

C

C

C

C

C

C

$\mathrm{H}$

$\mathrm{H}$

C

$\mathrm{H}$

C

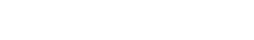

C

C

$\longrightarrow$

(

C

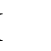

.

(

C

(1)

C

(

C

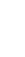

C

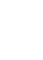

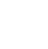

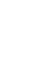

$\begin{array}{rrr}-11.04921400 & -2.78408900 & 0.00032700 \\ -10.28302100 & -2.01515400 & 0.00026600 \\ -11.68147100 & -0.39629600 & 0.00024500 \\ -10.63597900 & -0.69033900 & 0.00022100 \\ -7.92581200 & -1.43169200 & 0.00015700 \\ -9.64397200 & 0.32412100 & 0.00014100 \\ -8.92881300 & -2.38653400 & 0.00023500 \\ -8.27202500 & -0.04425900 & 0.00010800 \\ -9.98860100 & 1.69459900 & 0.00009500 \\ -8.65734800 & -3.43829300 & 0.00027200 \\ -9.00812900 & 2.65320500 & 0.00002000 \\ -11.03820200 & 1.97391100 & 0.00012100 \\ -9.27327200 & 3.70584900 & -0.00001400 \\ -7.65348700 & 2.28775600 & -0.00001300 \\ -6.91013700 & 3.07656000 & -0.00007200 \\ -7.26004000 & 0.96010500 & 0.00002700 \\ -5.84152300 & 0.55127100 & -0.00000800 \\ -5.53496800 & -0.87734600 & 0.00004900 \\ -4.80427000 & 1.42500300 & -0.00009200 \\ -4.98169700 & 2.49538000 & -0.00014100 \\ -3.42434600 & 1.01631500 & -0.00012100 \\ -3.11595600 & -0.40989300 & -0.00006400 \\ -6.53795300 & -1.79872400 & 0.00012700 \\ -6.29124000 & -2.85736100 & 0.00018200 \\ -1.70417700 & -0.81645000 & -0.00009700 \\ 1.03939500 & -1.56461900 & -0.00015300 \\ -0.68691100 & 0.18290100 & -0.00016500 \\ -1.31189100 & -2.15140400 & -0.00006900 \\ 0.02715100 & -2.51775900 & -0.00009800 \\ 0.68689700 & -0.18299200 & -0.00018400 \\ -2.05815200 & -2.93726900 & -0.00002600 \\ 0.29446400 & -3.57059800 & -0.00007800 \\ -2.41976900 & 1.93655300 & -0.00020000 \\ -2.66345000 & 2.99592100 & -0.00024900 \\ -1.03940800 & 1.56454100 & -0.00022200\end{array}$

0.00032700

0.00026600
$-1765.857088$

$-1765.827359$

$-1765.826415$

$-1765.917732$

C 


\begin{tabular}{|c|c|c|c|}
\hline $\mathrm{C}$ & -0.02716600 & 2.51767100 & -0.00032100 \\
\hline $\mathrm{H}$ & -0.29447900 & 3.57051000 & -0.00038900 \\
\hline $\mathrm{C}$ & -4.15671000 & -1.28539800 & 0.00002000 \\
\hline $\mathrm{H}$ & -3.97939800 & -2.35560900 & 0.00007000 \\
\hline $\mathrm{C}$ & 1.70417800 & 0.81637700 & -0.00020700 \\
\hline $\mathrm{C}$ & 1.31188600 & 2.15131900 & -0.00034800 \\
\hline $\mathrm{H}$ & 2.05813500 & 2.93719800 & -0.00042500 \\
\hline $\mathrm{C}$ & 3.11595600 & 0.40983000 & -0.00014700 \\
\hline $\mathrm{C}$ & 3.42436200 & -1.01637400 & -0.00015600 \\
\hline $\mathrm{C}$ & 4.15669800 & 1.28535800 & -0.00005900 \\
\hline $\mathrm{H}$ & 3.97938200 & 2.35556800 & -0.00001600 \\
\hline $\mathrm{C}$ & 5.53495600 & 0.87733100 & 0.00000200 \\
\hline $\mathrm{C}$ & 5.84153700 & -0.55128200 & -0.00005600 \\
\hline $\mathrm{C}$ & 2.41976300 & -1.93663200 & -0.00019400 \\
\hline $\mathrm{H}$ & 2.66343400 & -2.99600500 & -0.00020700 \\
\hline $\mathrm{C}$ & 7.26006300 & -0.96007700 & -0.00003000 \\
\hline $\mathrm{C}$ & 9.98864000 & -1.69450700 & 0.00006900 \\
\hline $\mathrm{C}$ & 8.27202600 & 0.04430700 & 0.00011300 \\
\hline $\mathrm{C}$ & 7.65354300 & -2.28771600 & -0.00013700 \\
\hline $\mathrm{C}$ & 9.00818100 & -2.65313900 & -0.00008300 \\
\hline $\mathrm{C}$ & 9.64398000 & -0.32404100 & 0.00016500 \\
\hline $\mathrm{H}$ & 6.91020200 & -3.07653200 & -0.00027000 \\
\hline $\mathrm{H}$ & 9.27334300 & -3.70577800 & -0.00017000 \\
\hline $\mathrm{C}$ & 6.53791800 & 1.79874300 & 0.00013100 \\
\hline $\mathrm{H}$ & 6.29118000 & 2.85737200 & 0.00019100 \\
\hline $\mathrm{C}$ & 7.92578200 & 1.43174100 & 0.00020200 \\
\hline $\mathrm{C}$ & 8.92876700 & 2.38659500 & 0.00037100 \\
\hline $\mathrm{H}$ & 8.65729700 & 3.43835300 & 0.00046700 \\
\hline $\mathrm{C}$ & 4.80430200 & -1.42503000 & -0.00011700 \\
\hline $\mathrm{H}$ & 4.98176200 & -2.49540100 & -0.00013900 \\
\hline $\mathrm{C}$ & 10.63596000 & 0.69045200 & 0.00027200 \\
\hline $\mathrm{C}$ & 10.28298100 & 2.01523200 & 0.00044500 \\
\hline $\mathrm{H}$ & 11.04916200 & 2.78418400 & 0.00060000 \\
\hline $\mathrm{H}$ & 11.03824500 & -1.97380100 & 0.00010300 \\
\hline $\mathrm{H}$ & 11.68146100 & 0.39643500 & 0.00024700 \\
\hline
\end{tabular}

\section{Triplet:}

Sum of electronic and zero-point Energies= Sum of electronic and thermal Energies= Sum of electronic and thermal Enthalpies= Sum of electronic and thermal Free Energies= $\mathrm{S}^{* * 2}=2.268$

$\begin{array}{cccc}\mathrm{H} & -11.04150800 & -2.76299500 & 0.00005200 \\ \mathrm{C} & -10.26352300 & -2.00587600 & 0.00002700\end{array}$

$-1765.880821$

$-1765.851124$

$-1765.850180$

$-1765.941727$

0.00002700 


\begin{tabular}{|c|c|c|c|}
\hline $\mathrm{H}$ & -11.64592000 & -0.36476000 & 0.00004900 \\
\hline $\mathrm{C}$ & -10.60356300 & -0.66894600 & 0.00002500 \\
\hline $\mathrm{C}$ & -7.89094300 & -1.43848900 & -0.00004300 \\
\hline $\mathrm{C}$ & -9.60043500 & 0.33840500 & -0.00000600 \\
\hline $\mathrm{C}$ & -8.92482800 & -2.39330800 & -0.00000700 \\
\hline $\mathrm{C}$ & -8.22963300 & -0.03967700 & -0.00003500 \\
\hline $\mathrm{C}$ & -9.93533600 & 1.70738700 & -0.00000800 \\
\hline $\mathrm{H}$ & -8.66180200 & -3.44691800 & -0.00001000 \\
\hline $\mathrm{C}$ & -8.94553300 & 2.66443200 & -0.00003400 \\
\hline $\mathrm{H}$ & -10.98283900 & 1.99433900 & 0.00001200 \\
\hline $\mathrm{H}$ & -9.20671500 & 3.71809500 & -0.00003300 \\
\hline $\mathrm{C}$ & -7.59939200 & 2.29260400 & -0.00005500 \\
\hline $\mathrm{H}$ & -6.84970200 & 3.07477500 & -0.00006700 \\
\hline $\mathrm{C}$ & -7.21438600 & 0.95348600 & -0.00005600 \\
\hline $\mathrm{C}$ & -5.80346300 & 0.53992500 & -0.00005400 \\
\hline $\mathrm{C}$ & -5.49155600 & -0.86779500 & -0.00006000 \\
\hline $\mathrm{C}$ & -4.75483000 & 1.42802400 & -0.00003600 \\
\hline $\mathrm{H}$ & -4.93775900 & 2.49700200 & -0.00003300 \\
\hline $\mathrm{C}$ & -3.40015000 & 1.01561100 & -0.00001500 \\
\hline $\mathrm{C}$ & -3.08440500 & -0.37919100 & -0.00001300 \\
\hline $\mathrm{C}$ & -6.54158500 & -1.81625800 & -0.00008900 \\
\hline $\mathrm{H}$ & -6.28995200 & -2.87319600 & 0.00086700 \\
\hline $\mathrm{C}$ & -1.70769500 & -0.78523500 & 0.00001000 \\
\hline $\mathrm{C}$ & 1.03500400 & -1.58160000 & 0.00005100 \\
\hline $\mathrm{C}$ & -0.69261700 & 0.18950100 & 0.00003300 \\
\hline $\mathrm{C}$ & -1.32298900 & -2.17071800 & 0.00000800 \\
\hline $\mathrm{C}$ & -0.02562800 & -2.54705600 & 0.00002500 \\
\hline $\mathrm{C}$ & 0.69261600 & -0.18951500 & 0.00005900 \\
\hline $\mathrm{H}$ & -2.08897800 & -2.93599800 & -0.00001100 \\
\hline $\mathrm{H}$ & 0.24049000 & -3.60015900 & 0.00002000 \\
\hline $\mathrm{C}$ & -2.36213300 & 1.96159400 & 0.00000300 \\
\hline $\mathrm{H}$ & -2.61386100 & 3.01903600 & -0.00000400 \\
\hline $\mathrm{C}$ & -1.03501800 & 1.58159900 & 0.00002500 \\
\hline $\mathrm{C}$ & 0.02563000 & 2.54704800 & 0.00002900 \\
\hline $\mathrm{H}$ & -0.24048800 & 3.60015200 & 0.00001200 \\
\hline $\mathrm{C}$ & -4.16025800 & -1.28217700 & -0.00003500 \\
\hline $\mathrm{H}$ & -3.97646700 & -2.35015400 & -0.00003600 \\
\hline $\mathrm{C}$ & 1.70770300 & 0.78522400 & 0.00010500 \\
\hline $\mathrm{C}$ & 1.32298400 & 2.17071300 & 0.00004700 \\
\hline $\mathrm{H}$ & 2.08897100 & 2.93600000 & 0.00004900 \\
\hline $\mathrm{C}$ & 3.08439500 & 0.37918200 & 0.00007200 \\
\hline $\mathrm{C}$ & 3.40013400 & -1.01562500 & 0.00005600 \\
\hline $\mathrm{C}$ & 4.16026200 & 1.28216900 & 0.00005600 \\
\hline $\mathrm{H}$ & 3.97647900 & 2.35015100 & 0.00007000 \\
\hline
\end{tabular}




$\begin{array}{lrrr}\text { C } & 5.49155300 & 0.86779400 & 0.00002600 \\ \mathrm{C} & 5.80346200 & -0.53994300 & 0.00000800 \\ \mathrm{C} & 2.36213300 & -1.96159900 & 0.00006900 \\ \mathrm{H} & 2.61384100 & -3.01904600 & 0.00014300 \\ \mathrm{C} & 7.21439200 & -0.95349100 & -0.00002600 \\ \mathrm{C} & 9.93535500 & -1.70737600 & -0.00008100 \\ \mathrm{C} & 8.22963800 & 0.03968100 & -0.00003400 \\ \mathrm{C} & 7.59941400 & -2.29259500 & -0.00005000 \\ \mathrm{C} & 8.94556100 & -2.66442300 & -0.00007500 \\ \mathrm{C} & 9.60043700 & -0.33839100 & -0.00006400 \\ \mathrm{H} & 6.84973200 & -3.07477500 & -0.00004900 \\ \mathrm{H} & 9.20673800 & -3.71808600 & -0.00009200 \\ \mathrm{C} & 6.54156900 & 1.81625500 & 0.00002000 \\ \mathrm{H} & 6.28994600 & 2.87319400 & 0.00003900 \\ \mathrm{C} & 7.89093800 & 1.43849300 & -0.00000600 \\ \mathrm{C} & 8.92480300 & 2.39331100 & 0.00000400 \\ \mathrm{H} & 8.66177600 & 3.44692200 & 0.00003900 \\ \mathrm{C} & 4.75484000 & -1.42803100 & 0.00002600 \\ \mathrm{H} & 4.93776600 & -2.49700900 & 0.00001600 \\ \mathrm{C} & 10.60356100 & 0.66896900 & -0.00008500 \\ \mathrm{C} & 10.26351100 & 2.00589000 & -0.00002500 \\ \mathrm{H} & 11.04148300 & 2.76302500 & -0.00000500 \\ \mathrm{H} & 10.98286200 & -1.99431300 & -0.00010300 \\ \mathrm{H} & 11.64592100 & 0.36479000 & 0.00033200\end{array}$


Compound IFD

Natural Orbital Coefficient

HOMO: 1.75667 LUMO: 0.24333

singlet biradical character $\mathrm{y}_{0}=0.038$

\section{Singlet Biradical Open Shell:}

Sum of electronic and zero-point Energies=

Sum of electronic and thermal Energies=

Sum of electronic and thermal Enthalpies=

Sum of electronic and thermal Free Energies=

$\mathrm{S} * * 2=0.6846$

$\begin{array}{lrrr}\mathrm{C} & 3.22825500 & -1.07543800 & 0.00001600 \\ \mathrm{C} & 2.81602500 & 0.32278600 & -0.00013000 \\ \mathrm{C} & 4.61482100 & -1.42128000 & 0.00012800 \\ \mathrm{C} & 3.72802700 & 1.32870700 & -0.00017500 \\ \mathrm{H} & 4.91326700 & -2.46632800 & 0.00016600 \\ \mathrm{H} & 3.42901700 & 2.37359000 & -0.00019200 \\ \mathrm{C} & 1.34852300 & 0.32897900 & -0.00005400 \\ \mathrm{C} & 0.92598300 & -1.03086900 & -0.00005900 \\ \mathrm{C} & 2.09869600 & -1.86873500 & -0.00012200 \\ \mathrm{H} & 2.08469900 & -2.95284000 & -0.00010900 \\ \mathrm{C} & 6.99618400 & -0.42259400 & 0.00007000 \\ \mathrm{C} & 9.70232900 & 0.19813500 & 0.00006300 \\ \mathrm{C} & 7.42122900 & 0.92839100 & 0.00001200 \\ \mathrm{C} & 7.92235200 & -1.44997900 & 0.00012400 \\ \mathrm{C} & 9.28178300 & -1.13007500 & 0.00012100 \\ \mathrm{C} & 8.77558400 & 1.23899800 & 0.00000500 \\ \mathrm{H} & 7.60563800 & -2.48910000 & 0.00017600 \\ \mathrm{H} & 9.10858000 & 2.27282800 & -0.00003500 \\ \mathrm{C} & 6.24134700 & 1.77682100 & -0.00001700 \\ \mathrm{C} & 5.11730700 & 0.98391700 & -0.00006900 \\ \mathrm{C} & 5.52847700 & -0.41613400 & 0.00007700 \\ \mathrm{H} & 6.25707300 & 2.86073700 & -0.00006200 \\ \mathrm{C} & -1.34852300 & -0.32897700 & -0.00007000 \\ \mathrm{C} & -0.92598300 & 1.03087000 & -0.00006700 \\ \mathrm{C} & -0.43280200 & -1.36374100 & -0.00003300 \\ \mathrm{C} & 0.43280200 & 1.36374200 & -0.00002600 \\ \mathrm{H} & -0.75096300 & -2.40278200 & -0.00005600 \\ \mathrm{H} & 0.75096100 & 2.40278400 & -0.00004800 \\ \mathrm{C} & -2.09869600 & 1.86873700 & -0.00014300 \\ \mathrm{C} & -3.22825500 & 1.07543900 & 0.00000500 \\ \mathrm{C} & -2.81602500 & -0.32278500 & -0.00013500 \\ \mathrm{H} & -2.08470000 & 2.95284200 & -0.00016100 \\ \mathrm{C} & -5.11730800 & -0.98391800 & -0.00007300\end{array}$

$-1305.276793$

$-1305.254319$

$-1305.253375$

$-1305.328543$

0.00001600

$-0.00013000$

$-0.00017500$

00016600

$-0.00005400$

$-0.00005900$

-

0.00007000

0.00006300

0.00001200

0.00012100

0.00000500

$6.24134700 \quad 1.77682100 \quad-0.00001700$

$\begin{array}{lll}5.11730700 & 0.98391700 & -0.00006900\end{array}$

$\begin{array}{llll}5.52847700 & -0.41613400 & 0.00007700\end{array}$

$\begin{array}{lll}6.25707300 & 2.86073700 & -0.00006200\end{array}$

$\begin{array}{lll}-1.34852300 & -0.32897700-0.00007000\end{array}$

$\begin{array}{lll}-.92598300 & 1.03087000 & -0.00006700\end{array}$

$\begin{array}{lll}-0.43280200 & -1.36374100 & -0.00003300\end{array}$

$0.43280200-1.36374200-0.00002600$

$\begin{array}{lll}-0.75096300 & -2.40278200 & -0.00005600\end{array}$

$\begin{array}{lll}0.75096100 & 2.40278400 & -0.00004800\end{array}$

$\begin{array}{lll}-2.09869600 & 1.86873700 & -0.00014300\end{array}$

$\begin{array}{lll}-3.22825500 & 1.07543900 & 0.00000500\end{array}$

$\begin{array}{lll}-2.08470000 & 2.95284200 & -0.00016100\end{array}$

$\begin{array}{lll}-5.11730800 & -0.98391800 & -0.00007300\end{array}$ 


$\begin{array}{lrrc}\mathrm{C} & -5.52847700 & 0.41613300 & 0.00008000 \\ \mathrm{C} & -3.72802800 & -1.32870800 & -0.00017900 \\ \mathrm{C} & -4.61482200 & 1.42127900 & 0.00013100 \\ \mathrm{H} & -3.42901700 & -2.37359100 & -0.00020800 \\ \mathrm{H} & -4.91326900 & 2.46632800 & 0.00017300 \\ \mathrm{C} & -6.99618300 & 0.42259300 & 0.00008400 \\ \mathrm{C} & -7.42122800 & -0.92839200 & 0.00002200 \\ \mathrm{C} & -6.24134700 & -1.77682200 & -0.00001400 \\ \mathrm{H} & -6.25707500 & -2.86073800 & -0.00005000 \\ \mathrm{H} & 10.76405300 & 0.42444000 & 0.00006600 \\ \mathrm{H} & 10.01942200 & -1.92649800 & 0.00016800 \\ \mathrm{C} & -7.92235100 & 1.44997800 & 0.00014900 \\ \mathrm{C} & -9.28178200 & 1.13007500 & 0.00013800 \\ \mathrm{C} & -9.70232800 & -0.19813500 & 0.00007300 \\ \mathrm{C} & -8.77558400 & -1.23899800 & 0.00002600 \\ \mathrm{H} & -9.10858000 & -2.27282800 & -0.00002300 \\ \mathrm{H} & -10.76405200 & -0.42444000 & 0.00005700 \\ \mathrm{H} & -10.01942100 & 1.92649800 & 0.00018300 \\ \mathrm{H} & -7.60563800 & 2.48909900 & 0.00019600\end{array}$

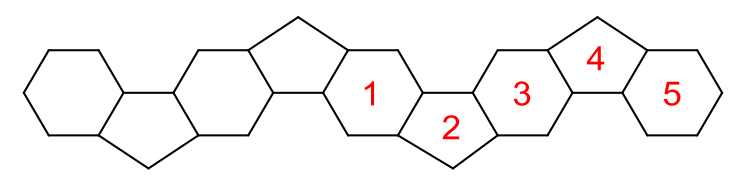

\begin{tabular}{cccccc} 
& \multicolumn{5}{c}{ NICS values (sigma zz) } \\
Bq distance $(\AA)$ & ring 1 & ring 2 & ring 3 & ring 4 & ring 5 \\
0.000 & 13.093 & 44.823 & 29.361 & 43.525 & -1.040 \\
0.100 & 12.635 & 44.385 & 28.988 & 43.095 & -1.515 \\
0.200 & 11.310 & 43.070 & 27.890 & 41.804 & -2.877 \\
0.300 & 9.262 & 40.894 & 26.129 & 39.665 & -4.948 \\
0.400 & 6.698 & 37.918 & 23.811 & 36.735 & -7.471 \\
0.500 & 3.860 & 34.268 & 21.074 & 33.136 & -10.164 \\
0.600 & 0.982 & 30.140 & 18.083 & 29.058 & -12.762 \\
0.700 & -1.734 & 25.773 & 15.003 & 24.735 & -15.056 \\
0.800 & -4.141 & 21.408 & 11.986 & 20.406 & -16.910 \\
0.900 & -6.151 & 17.254 & 9.154 & 16.278 & -18.259 \\
1.000 & -7.731 & 13.460 & 6.593 & 12.501 & -19.099 \\
1.100 & -8.891 & 10.112 & 4.349 & 9.165 & -19.473 \\
1.200 & -9.670 & 7.242 & 2.437 & 6.301 & -19.446 \\
1.300 & -10.122 & 4.838 & 0.848 & 3.902 & -19.096 \\
1.400 & -10.307 & 2.863 & -0.444 & 1.931 & -18.503 \\
1.500 & -10.284 & 1.265 & -1.471 & 0.339 & -17.738 \\
1.600 & -10.104 & -0.007 & -2.270 & -0.925 & -16.860
\end{tabular}




$\begin{array}{llllll}1.700 & -9.814 & -1.007 & -2.876 & -1.915 & -15.920 \\ 1.800 & -9.448 & -1.781 & -3.322 & -2.675 & -14.956 \\ 1.900 & -9.036 & -2.369 & -3.638 & -3.247 & -13.996 \\ 2.000 & -8.599 & -2.807 & -3.848 & -3.665 & -13.062 \\ 2.100 & -8.154 & -3.122 & -3.975 & -3.959 & -12.166 \\ 2.200 & -7.713 & -3.341 & -4.037 & -4.154 & -11.318 \\ 2.300 & -7.284 & -3.483 & -4.049 & -4.270 & -10.522 \\ 2.400 & -6.871 & -3.564 & -4.022 & -4.324 & -9.780 \\ 2.500 & -6.480 & -3.599 & -3.968 & -4.330 & -9.092 \\ 2.600 & -6.111 & -3.597 & -3.894 & -4.299 & -8.456 \\ 2.700 & -5.765 & -3.568 & -3.806 & -4.241 & -7.871 \\ 2.800 & -5.442 & -3.518 & -3.708 & -4.162 & -7.332 \\ 2.900 & -5.141 & -3.454 & -3.605 & -4.068 & -6.838 \\ 3.000 & -4.862 & -3.379 & -3.499 & -3.964 & -6.384 \\ 3.100 & -4.603 & -3.298 & -3.392 & -3.853 & -5.967 \\ 3.200 & -4.362 & -3.211 & -3.286 & -3.737 & -5.584 \\ 3.300 & -4.139 & -3.122 & -3.181 & -3.620 & -5.233 \\ 3.400 & -3.932 & -3.032 & -3.079 & -3.502 & -4.910 \\ 3.500 & -3.740 & -2.941 & -2.980 & -3.385 & -4.613 \\ 3.600 & -3.561 & -2.852 & -2.883 & -3.270 & -4.339 \\ 3.700 & -3.395 & -2.764 & -2.790 & -3.157 & -4.087 \\ 3.800 & -3.240 & -2.677 & -2.700 & -3.046 & -3.854 \\ 3.900 & -3.096 & -2.593 & -2.614 & -2.939 & -3.639 \\ 4.000 & -2.961 & -2.511 & -2.530 & -2.835 & -3.440 \\ 4.100 & -2.835 & -2.432 & -2.450 & -2.734 & -3.255 \\ 4.200 & -2.717 & -2.355 & -2.373 & -2.637 & -3.084 \\ 4.300 & -2.607 & -2.281 & -2.299 & -2.543 & -2.925 \\ 4.400 & -2.503 & -2.210 & -2.228 & -2.453 & -2.778 \\ 4.500 & -2.405 & -2.141 & -2.159 & -2.367 & -2.640 \\ 4.600 & -2.314 & -2.075 & -2.093 & -2.283 & -2.512 \\ 4.700 & -2.227 & -2.011 & -2.030 & -2.204 & -2.392 \\ 4.800 & -2.146 & -1.950 & -1.969 & -2.127 & -2.281 \\ 4.900 & -2.069 & -1.891 & -1.910 & -2.053 & -2.176 \\ 5.000 & -1.996 & -1.834 & -1.854 & -1.983 & -2.078\end{array}$

Mulliken atomic spin densities:

$\begin{array}{llr}1 & \mathrm{C} & -0.236001 \\ 2 & \mathrm{C} & 0.157372 \\ 3 & \mathrm{C} & 0.181104 \\ 4 & \mathrm{C} & -0.178030 \\ 5 & \mathrm{H} & -0.007106 \\ 6 & \mathrm{H} & 0.007103 \\ 7 & \mathrm{C} & 0.094135\end{array}$




$\begin{array}{rlc}8 & \mathrm{C} & -0.162142 \\ 9 & \mathrm{C} & 0.369295 \\ 10 & \mathrm{H} & -0.015201 \\ 11 & \mathrm{C} & -0.048782 \\ 12 & \mathrm{C} & 0.033338 \\ 13 & \mathrm{C} & 0.081801 \\ 14 & \mathrm{C} & 0.022509 \\ 15 & \mathrm{C} & -0.079019 \\ 16 & \mathrm{C} & -0.086394 \\ 17 & \mathrm{H} & -0.000765 \\ 18 & \mathrm{H} & 0.003859 \\ 19 & \mathrm{C} & -0.383222 \\ 20 & \mathrm{C} & 0.212635 \\ 21 & \mathrm{C} & -0.177314 \\ 22 & \mathrm{H} & 0.016042 \\ 23 & \mathrm{C} & -0.094135 \\ 24 & \mathrm{C} & 0.162141 \\ 25 & \mathrm{C} & 0.109839 \\ 26 & \mathrm{C} & -0.109839 \\ 27 & \mathrm{H} & -0.004333 \\ 28 & \mathrm{H} & 0.004333 \\ 29 & \mathrm{C} & -0.369297 \\ 30 & \mathrm{C} & 0.236001 \\ 31 & \mathrm{C} & -0.157374 \\ 32 & \mathrm{H} & 0.015201 \\ 33 & \mathrm{C} & -0.212636 \\ 34 & \mathrm{C} & 0.177314 \\ 35 & \mathrm{C} & 0.178032 \\ 36 & \mathrm{C} & -0.181105 \\ 37 & \mathrm{H} & -0.007103 \\ 38 & \mathrm{H} & 0.007106 \\ 39 & \mathrm{C} & 0.048783 \\ 40 & \mathrm{C} & -0.081802 \\ 41 & \mathrm{C} & 0.383225 \\ 42 & \mathrm{H} & -0.016042 \\ 43 & \mathrm{H} & -0.001067 \\ 44 & \mathrm{H} & 0.003888 \\ 45 & \mathrm{C} & -0.022509 \\ & \mathrm{C} & 0.079019 \\ 4 & -0.033339 \\ 4038385\end{array}$


$\begin{array}{lll}52 & \mathrm{H} & 0.000765\end{array}$

\section{Singlet Closed Shell:}

Sum of electronic and zero-point Energies= Sum of electronic and thermal Energies= Sum of electronic and thermal Enthalpies= Sum of electronic and thermal Free Energies=

$\begin{array}{cccc}\mathrm{C} & 3.22739600 & -1.08301800 & -0.00003600 \\ \mathrm{C} & 2.81207700 & 0.32701700 & 0.00000700 \\ \mathrm{C} & 4.62666100 & -1.42598200 & -0.00005400 \\ \mathrm{C} & 3.71717100 & 1.32790000 & 0.00003500 \\ \mathrm{H} & 4.92454500 & -2.47123300 & -0.00006700 \\ \mathrm{H} & 3.41922100 & 2.37312900 & 0.00003200 \\ \mathrm{C} & 1.34522400 & 0.32895400 & -0.00004100 \\ \mathrm{C} & 0.92815700 & -1.02738600 & -0.00003700 \\ \mathrm{C} & 2.11475000 & -1.86858900 & -0.00002500 \\ \mathrm{H} & 2.10003600 & -2.95277700 & -0.00012900 \\ \mathrm{C} & 6.99969400 & -0.42522000 & -0.00001200 \\ \mathrm{C} & 9.70065900 & 0.20946500 & 0.00003800 \\ \mathrm{C} & 7.41649900 & 0.92662800 & 0.00003100 \\ \mathrm{C} & 7.93138000 & -1.44818300 & -0.00003000 \\ \mathrm{C} & 9.28852300 & -1.12106300 & -0.00000500 \\ \mathrm{C} & 8.76758600 & 1.24532700 & 0.00005600 \\ \mathrm{H} & 7.61982200 & -2.48887600 & -0.00006300 \\ \mathrm{H} & 9.09474100 & 2.28101500 & 0.00009000 \\ \mathrm{C} & 6.22746000 & 1.77207000 & 0.00004100 \\ \mathrm{C} & 5.11738700 & 0.98521200 & 0.00000600 \\ \mathrm{C} & 5.53208800 & -0.42566100 & -0.00002900 \\ \mathrm{H} & 6.24177000 & 2.85617500 & 0.00007300 \\ \mathrm{C} & -1.34522900 & -0.32892100 & -0.00002000 \\ \mathrm{C} & -0.92817200 & 1.02742400 & 0.00000300 \\ \mathrm{C} & -0.42445100 & -1.36433700 & -0.00006700 \\ \mathrm{C} & 0.42444400 & 1.36438200 & -0.00004000 \\ \mathrm{H} & -0.74097500 & -2.40385300 & -0.00006200 \\ \mathrm{H} & 0.74097800 & 2.40389600 & -0.00004100 \\ \mathrm{C} & -2.11476300 & 1.86861100 & 0.00004300 \\ \mathrm{C} & -3.22740500 & 1.08303600 & 0.00001800 \\ \mathrm{C} & -2.81208300 & -0.32699100 & 0.00002600 \\ \mathrm{H} & -2.10004900 & 2.95279800 & 0.00006500 \\ \mathrm{C} & -5.11736300 & -0.98519800 & 0.00005000 \\ \mathrm{C} & -5.53208900 & 0.42567000 & -0.00001200 \\ \mathrm{C} & -31714400 & -1.32785900 & 0.00007900 \\ & & & -0.00003900\end{array}$




$\begin{array}{lrrr}\mathrm{H} & -3.41917700 & -2.37308300 & 0.00011500 \\ \mathrm{H} & -4.92455600 & 2.47125000 & -0.00007500 \\ \mathrm{C} & -6.99970200 & 0.42520500 & -0.00003200 \\ \mathrm{C} & -7.41648500 & -0.92665300 & 0.00004200 \\ \mathrm{C} & -6.22742600 & -1.77207600 & 0.00006700 \\ \mathrm{H} & -6.24171300 & -2.85618100 & 0.00006300 \\ \mathrm{H} & 10.76101000 & 0.44227200 & 0.00005700 \\ \mathrm{H} & 10.03084500 & -1.91309600 & -0.00001800 \\ \mathrm{C} & -7.93140500 & 1.44815300 & -0.00008300 \\ \mathrm{C} & -9.28854500 & 1.12101000 & -0.00005500 \\ \mathrm{C} & -9.70066200 & -0.20953000 & 0.00001700 \\ \mathrm{C} & -8.76756900 & -1.24537900 & 0.00003500 \\ \mathrm{H} & -9.09470400 & -2.28107300 & 0.00015600 \\ \mathrm{H} & -10.76100900 & -0.44235500 & 0.00017000 \\ \mathrm{H} & -10.03087700 & 1.91303400 & -0.00008700 \\ \mathrm{H} & -7.61986400 & 2.48885100 & -0.00013600\end{array}$

\section{Triplet:}

Sum of electronic and zero-point Energies=

$-1305.262277$

Sum of electronic and thermal Energies=

$-1305.239632$

Sum of electronic and thermal Enthalpies=

$-1305.238687$

Sum of electronic and thermal Free Energies=

$-1305.315198$ $\mathrm{S} * * 2=2.2307$

$\begin{array}{ccrc}\mathrm{C} & 3.22395200 & -1.03000100 & 0.00023100 \\ \mathrm{C} & 2.83072500 & 0.34660600 & 0.00016500 \\ \mathrm{C} & 4.57846400 & -1.39630500 & 0.00026300 \\ \mathrm{C} & 3.76293600 & 1.35539600 & 0.00015400 \\ \mathrm{H} & 4.86841700 & -2.44351500 & 0.00031200 \\ \mathrm{H} & 3.46972100 & 2.40171800 & 0.00009400 \\ \mathrm{C} & 1.36261500 & 0.36865500 & 0.00011200 \\ \mathrm{C} & 0.92573000 & -1.03039200 & 0.00016600 \\ \mathrm{C} & 2.03904200 & -1.84034200 & 0.00025200 \\ \mathrm{H} & 2.03205300 & -2.92457700 & 0.00031900 \\ \mathrm{C} & 6.98562600 & -0.42510200 & -0.00002300 \\ \mathrm{C} & 9.70794500 & 0.13566500 & -0.00032800 \\ \mathrm{C} & 7.44166500 & 0.92008600 & 0.00002900 \\ \mathrm{C} & 7.88813800 & -1.47177800 & -0.00022300 \\ \mathrm{C} & 9.25586300 & -1.18304700 & -0.00037500 \\ \mathrm{C} & 8.80682300 & 1.19724400 & -0.00012700 \\ \mathrm{H} & 7.54869200 & -2.50372200 & -0.00026300 \\ \mathrm{H} & 9.16372300 & 2.22304500 & -0.00009000 \\ \mathrm{C} & 6.29169200 & 1.78722800 & 0.00025000 \\ \mathrm{C} & 5.12879800 & 0.99495700 & 0.00028900 \\ \mathrm{C} & 5.51825200 & -0.38934500 & 0.00016700\end{array}$




$\begin{array}{lrcc}\mathrm{H} & 6.32162300 & 2.87044600 & 0.00016700 \\ \mathrm{C} & -1.36582200 & -0.33976100 & 0.00002700 \\ \mathrm{C} & -0.93070300 & 1.06275700 & -0.00006300 \\ \mathrm{C} & -0.46796700 & -1.35586600 & 0.00009300 \\ \mathrm{C} & 0.47215800 & 1.38477200 & -0.00008200 \\ \mathrm{H} & -0.78036800 & -2.39674100 & 0.00020100 \\ \mathrm{H} & 0.78629700 & 2.42509900 & -0.00014300 \\ \mathrm{C} & -2.03171200 & 1.86716500 & -0.00019100 \\ \mathrm{C} & -3.23187800 & 1.03847600 & -0.00011400 \\ \mathrm{C} & -2.83011200 & -0.32613300 & 0.00005400 \\ \mathrm{H} & -2.03198700 & 2.95113300 & -0.00018000 \\ \mathrm{C} & -5.12028900 & -0.99485300 & -0.00011900 \\ \mathrm{C} & -5.51717900 & 0.37732500 & -0.00011900 \\ \mathrm{C} & -3.76227100 & -1.34728900 & -0.00002400 \\ \mathrm{C} & -4.57880600 & 1.39654400 & -0.00005300 \\ \mathrm{H} & -3.46041200 & -2.39107400 & -0.00006400 \\ \mathrm{H} & -4.87823000 & 2.44099200 & -0.00003400 \\ \mathrm{C} & -6.98540800 & 0.40792300 & -0.00016600 \\ \mathrm{C} & -7.43981600 & -0.94065200 & -0.00015300 \\ \mathrm{C} & -6.29566100 & -1.80227000 & -0.00021100 \\ \mathrm{H} & -6.31929100 & -2.88542700 & -0.00015700 \\ \mathrm{H} & 10.77477200 & 0.33634400 & -0.00044900 \\ \mathrm{H} & 9.97460200 & -1.99654300 & -0.00053200 \\ \mathrm{C} & -7.88909200 & 1.45221200 & -0.00005600 \\ \mathrm{C} & -9.25765100 & 1.16186100 & 0.00009300 \\ \mathrm{C} & -9.70894200 & -0.15767500 & 0.00012500 \\ \mathrm{C} & -8.80748000 & -1.21800700 & -0.00006700 \\ \mathrm{H} & -9.16337200 & -2.24414200 & 0.00010200 \\ \mathrm{H} & -10.77564100 & -0.35895100 & 0.00072800 \\ \mathrm{H} & -9.97696900 & 1.97482700 & 0.00020500 \\ \mathrm{H} & -7.55144200 & 2.48474400 & -0.00006600\end{array}$


Compound PIF

Natural Orbital Coefficient

HOMO: 1.36832 LUMO: 0.63168

singlet biradical character $\mathrm{y}_{0}=0.351$

\section{Singlet Biradical Open Shell:}

Sum of electronic and zero-point Energies= Sum of electronic and thermal Energies= Sum of electronic and thermal Enthalpies= Sum of electronic and thermal Free Energies= $\mathrm{S} * * 2=1.5338$

$\mathrm{H}$

C

$\mathrm{H}$

C

$\mathrm{C}$

C

$\mathrm{C}$

$\mathrm{C}$

$\mathrm{C}$

$\mathrm{H}$

C

$\mathrm{H}$

$\mathrm{H}$

$\mathrm{C}$

$\mathrm{H}$

$\mathrm{C}$

C

C

C

C

$\mathrm{C}$

C

$\mathrm{H}$

C

C

C

C

$\mathrm{H}$

$\mathrm{H}$

$\mathrm{H}$

C

C

C

$\begin{array}{rrr}10.62208800 & -2.92557900 & 0.00069900 \\ 9.88058000 & -2.13280400 & 0.00054100 \\ 11.33657400 & -0.55908200 & 0.00072200 \\ 10.28092300 & -0.81397300 & 0.00055700 \\ 7.54377000 & -1.45414300 & 0.00012700 \\ 9.32836700 & 0.23784100 & 0.00034700 \\ 8.52281800 & -2.45593600 & 0.00032000 \\ 7.94094500 & -0.07912600 & 0.00014800 \\ 9.72496100 & 1.59139700 & 0.00031500 \\ 8.20974300 & -3.49590200 & 0.00029300 \\ 8.77669300 & 2.58995200 & 0.00004600 \\ 10.78401300 & 1.83182200 & 0.00049000 \\ 9.08378000 & 3.63123100 & -0.00002000 \\ 7.41525600 & 2.27983800 & -0.00015400 \\ 6.70352700 & 3.09647400 & -0.00042400 \\ 6.96535700 & 0.95802600 & -0.00005800 \\ 6.16385800 & -1.76568300 & -0.00010600 \\ 5.17961400 & -0.78632100 & -0.00022500 \\ 5.54429200 & 0.60902700 & -0.00015000 \\ 4.53211700 & 1.56550600 & -0.00013200 \\ 2.82946300 & -0.21257800 & -0.00041300 \\ 3.79580300 & -1.16385900 & -0.00037800 \\ 3.55276600 & -2.22334500 & -0.00044500 \\ 1.36266800 & -0.28162500 & -0.00057200 \\ 0.88697300 & 1.07517000 & -0.00015900 \\ 3.18740800 & 1.18561900 & -0.00025400 \\ 2.00024000 & 1.94805700 & -0.00036600 \\ 1.94741400 & 3.03068500 & -0.00030700 \\ 5.86717900 & -2.81126500 & -0.00015700 \\ 4.77179500 & 2.62283700 & 0.00002300 \\ 0.49771700 & -1.34695900 & -0.00075200 \\ -0.88697300 & -1.07517000 & -0.00045600 \\ -1.36266800 & 0.28162500 & -0.00006300\end{array}$

$-1612.330833$

$-1612.303385$

$-1612.302441$

$-1612.388403$

0.00069900

.0054100

0.00055700

0.00012700

0.00032000

0.00014800

0.00029300

0.00004600

$-0.00002000$

$-0.00015400$

2400

$-0.00005800$

$-0.00010600$

.0022500

$-0.00013200$

00041300

$-0.00037800$

.00044500

$-0.00057200$

$-0.00025400$

$-0.00036600$

$-0.00030700$

.00015700

$-0.00075200$

$-0.00006300$ 


$\begin{array}{lrrr}\mathrm{C} & -0.49771700 & 1.34695900 & 0.00006800 \\ \mathrm{H} & 0.85803600 & -2.37215200 & -0.00087500 \\ \mathrm{H} & -0.85803800 & 2.37215100 & 0.00022400 \\ \mathrm{C} & -2.00024000 & -1.94805600 & -0.00029400 \\ \mathrm{C} & -2.82946300 & 0.21257800 & -0.00002300 \\ \mathrm{C} & -3.18740800 & -1.18561900 & -0.00017400 \\ \mathrm{H} & -1.94741500 & -3.03068500 & -0.00039600 \\ \mathrm{C} & -4.53211700 & -1.56550600 & -0.00012100 \\ \mathrm{C} & -5.54429200 & -0.60902800 & 0.00009000 \\ \mathrm{H} & -3.55276500 & 2.22334400 & 0.00017800 \\ \mathrm{C} & -3.79580300 & 1.16385900 & 0.00010900 \\ \mathrm{C} & -5.17961400 & 0.78632100 & 0.00007500 \\ \mathrm{C} & -6.16385700 & 1.76568300 & 0.00016200 \\ \mathrm{C} & -7.54377000 & 1.45414300 & 0.00017200 \\ \mathrm{C} & -8.52281700 & 2.45593600 & 0.00018700 \\ \mathrm{C} & -7.94094500 & 0.07912600 & 0.00013300 \\ \mathrm{C} & -6.96535700 & -0.95802600 & 0.00014600 \\ \mathrm{C} & -9.32836700 & -0.23784000 & 0.00016400 \\ \mathrm{C} & -10.28092300 & 0.81397300 & 0.00018200 \\ \mathrm{C} & -9.88058000 & 2.13280400 & 0.00018200 \\ \mathrm{C} & -7.41525600 & -2.27983800 & 0.00019300 \\ \mathrm{C} & -8.77669300 & -2.58995100 & 0.00019800 \\ \mathrm{C} & -9.72496100 & -1.59139700 & 0.00017800 \\ \mathrm{H} & -4.77179500 & -2.62283700 & -0.00027100 \\ \mathrm{H} & -6.70352700 & -3.09647400 & 0.00027800 \\ \mathrm{H} & -9.08378000 & -3.63123100 & 0.00024500 \\ \mathrm{H} & -10.78401300 & -1.83182200 & 0.00018000 \\ \mathrm{H} & -11.33657300 & 0.55908300 & 0.00020100 \\ \mathrm{H} & -10.62208800 & 2.92557900 & 0.00019000 \\ \mathrm{H} & -8.20974200 & 3.49590200 & 0.00020700 \\ & -5.86718000 & 2.81126500 & 0.00021200\end{array}$

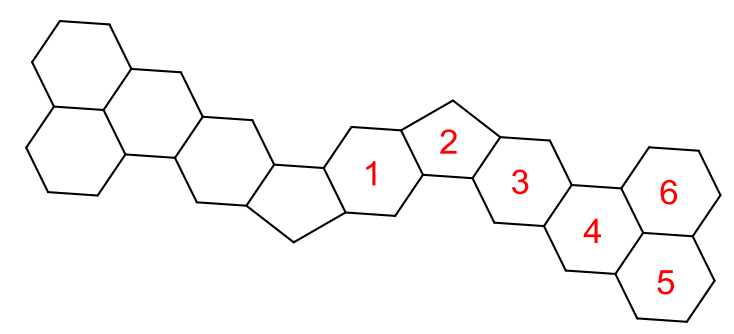

$\begin{array}{ccccccc}\text { Bq distance }(\AA) & \text { ring 1 } & \text { ring 2 } & \text { ring 3 } & \text { ring 4 } & \text { ring 5 } & \text { ring 6 } \\ 0.000 & 13.097 & 36.478 & 12.120 & 21.731 & 3.503 & -1.905 \\ 0.100 & 12.650 & 35.956 & 11.643 & 21.273 & 3.018 & -2.419 \\ 0.200 & 11.358 & 34.423 & 10.270 & 19.941 & 1.624 & -3.886\end{array}$ 


\begin{tabular}{|c|c|c|c|c|c|c|}
\hline 0.300 & 9.356 & 31.974 & 8.155 & 17.859 & -0.504 & -6.110 \\
\hline 0.400 & 6.841 & 28.767 & 5.524 & 15.208 & -3.119 & -8.809 \\
\hline 0.500 & 4.044 & 25.017 & 2.632 & 12.201 & -5.944 & -11.673 \\
\hline 0.600 & 1.193 & 20.967 & -0.276 & 9.050 & -8.722 & -14.423 \\
\hline 0.700 & -1.517 & 16.867 & -2.999 & 5.945 & -11.246 & -16.838 \\
\hline 0.800 & -3.425 & 12.932 & -5.392 & 3.035 & -13.378 & -18.781 \\
\hline 0.900 & -5.987 & 9.326 & -7.373 & 0.422 & -15.048 & -20.190 \\
\hline 1.000 & -7.620 & 6.150 & -8.917 & -1.840 & -16.241 & -21.068 \\
\hline 1.100 & -8.843 & 3.447 & -10.040 & -3.731 & -16.988 & -21.463 \\
\hline 1.200 & -9.691 & 1.214 & -10.783 & -5.263 & -17.343 & -21.446 \\
\hline 1.300 & -10.213 & -0.585 & -11.204 & -6.465 & -17.373 & -21.100 \\
\hline 1.400 & -10.467 & -2.000 & -11.363 & -7.376 & -17.147 & -20.505 \\
\hline 1.500 & -10.507 & -3.088 & -11.320 & -8.038 & -16.728 & -19.734 \\
\hline 1.600 & -10.385 & -3.904 & -11.126 & -8.493 & -16.173 & -18.849 \\
\hline 1.700 & -10.144 & -4.499 & -10.826 & -8.779 & -15.528 & -17.900 \\
\hline 1.800 & -9.821 & -4.916 & -10.453 & -8.929 & -14.829 & -16.926 \\
\hline 1.900 & -9.444 & -5.192 & -10.037 & -8.971 & -14.105 & -15.954 \\
\hline 2.000 & -9.036 & -5.357 & -9.599 & -8.929 & -13.377 & -15.006 \\
\hline 2.100 & -8.613 & -5.436 & -9.154 & -8.824 & -12.660 & -14.094 \\
\hline 2.200 & -8.189 & -5.447 & -8.713 & -8.670 & -11.965 & -13.228 \\
\hline 2.300 & -7.771 & -5.409 & -8.284 & -8.480 & -11.299 & -12.412 \\
\hline 2.400 & -7.367 & -5.333 & -7.872 & -8.265 & -10.665 & -11.648 \\
\hline 2.500 & -6.980 & -5.229 & -7.481 & -8.032 & -10.065 & -10.935 \\
\hline 2.600 & -6.613 & -5.107 & -7.110 & -7.789 & -9.501 & -10.273 \\
\hline 2.700 & -6.267 & -4.971 & -6.762 & -7.539 & -8.972 & -9.658 \\
\hline 2.800 & -5.942 & -4.828 & -6.436 & -7.287 & -8.477 & -9.089 \\
\hline 2.900 & -5.638 & -4.680 & -6.131 & -7.035 & -8.014 & -8.562 \\
\hline 3.000 & -5.354 & -4.531 & -5.846 & -6.787 & -7.581 & -8.074 \\
\hline 3.100 & -5.090 & -4.383 & -5.580 & -6.542 & -7.177 & -7.622 \\
\hline 3.200 & -4.844 & -4.236 & -5.332 & -6.303 & -6.800 & -7.203 \\
\hline 3.300 & -4.615 & -4.093 & -5.100 & -6.071 & -6.448 & -6.815 \\
\hline 3.400 & -4.402 & -3.954 & -4.883 & -5.845 & -6.120 & -6.454 \\
\hline 3.500 & -4.203 & -3.820 & -4.681 & -5.627 & -5.813 & -6.119 \\
\hline 3.600 & -3.846 & -3.690 & -4.491 & -5.417 & -5.525 & -5.807 \\
\hline 3.700 & -3.685 & -3.565 & -4.312 & -5.214 & -5.256 & -5.517 \\
\hline 3.800 & -3.534 & -3.445 & -4.145 & -5.018 & -5.005 & -5.246 \\
\hline 3.900 & -3.394 & -3.330 & -3.987 & -4.830 & -4.769 & -4.994 \\
\hline 4.000 & -3.262 & -3.220 & -3.839 & -4.650 & -4.547 & -4.757 \\
\hline 4.100 & -3.138 & -3.114 & -3.699 & -4.477 & -4.340 & -4.536 \\
\hline 4.200 & -3.022 & -3.014 & -3.566 & -4.311 & -4.144 & -4.329 \\
\hline 4.300 & -2.912 & -2.917 & -3.441 & -4.152 & -3.961 & -4.134 \\
\hline 4.400 & -2.809 & -2.825 & -3.322 & -4.000 & -3.788 & -3.952 \\
\hline 4.500 & -2.712 & -2.737 & -3.210 & -3.854 & -3.625 & -3.780 \\
\hline
\end{tabular}




$\begin{array}{lllllll}4.600 & -2.621 & -2.652 & -3.102 & -3.714 & -3.471 & -3.618 \\ 4.700 & -2.534 & -2.571 & -3.001 & -3.580 & -3.326 & -3.466 \\ 4.800 & -2.452 & -2.494 & -2.903 & -3.452 & -3.189 & -3.323 \\ 4.900 & -2.374 & -2.420 & -2.811 & -3.329 & -3.060 & -3.187 \\ 5.000 & -2.256 & -2.350 & -2.723 & -3.212 & -2.938 & -3.059\end{array}$

Mulliken atomic spin densities:

$\begin{array}{rlc}1 & \mathrm{H} & -0.007233 \\ 2 & \mathrm{C} & 0.190586 \\ 3 & \mathrm{H} & 0.013862 \\ 4 & \mathrm{C} & -0.306311 \\ 5 & \mathrm{C} & 0.224915 \\ 6 & \mathrm{C} & 0.152035 \\ 7 & \mathrm{C} & -0.325696 \\ 8 & \mathrm{C} & -0.128802 \\ 9 & \mathrm{C} & -0.222204 \\ 10 & \mathrm{H} & 0.014508 \\ 11 & \mathrm{C} & 0.148078 \\ 12 & \mathrm{H} & 0.009914 \\ 13 & \mathrm{H} & -0.005778 \\ 14 & \mathrm{C} & -0.240350 \\ 15 & \mathrm{H} & 0.010663 \\ 16 & \mathrm{C} & 0.181239 \\ 17 & \mathrm{C} & -0.428598 \\ 18 & \mathrm{C} & 0.245961 \\ 19 & \mathrm{C} & -0.270735 \\ 20 & \mathrm{C} & 0.293812 \\ 21 & \mathrm{C} & 0.145242 \\ 22 & \mathrm{C} & -0.152585 \\ 23 & \mathrm{H} & 0.006046 \\ 24 & \mathrm{C} & 0.175836 \\ 25 & \mathrm{C} & -0.261041 \\ 26 & \mathrm{C} & -0.279334 \\ 27 & \mathrm{C} & 0.489847 \\ 28 & \mathrm{H} & -0.020113 \\ 29 & \mathrm{H} & 0.018205 \\ 30 & \mathrm{H} & -0.011818 \\ 31 & \mathrm{C} & -0.185653 \\ 32 & \mathrm{C} & 0.261041 \\ 33 & \mathrm{C} & -0.175836 \\ 34 & \mathrm{C} & 0.185653 \\ 35 & \mathrm{H} & 0.007333 \\ 36 & \mathrm{H} & -0.007333\end{array}$




$\begin{array}{llr}37 & \mathrm{C} & -0.489846 \\ 38 & \mathrm{C} & -0.145242 \\ 39 & \mathrm{C} & 0.279334 \\ 40 & \mathrm{H} & 0.020113 \\ 41 & \mathrm{C} & -0.293812 \\ 42 & \mathrm{C} & 0.270735 \\ 43 & \mathrm{H} & -0.006046 \\ 44 & \mathrm{C} & 0.152586 \\ 45 & \mathrm{C} & -0.245961 \\ 46 & \mathrm{C} & 0.428598 \\ 47 & \mathrm{C} & -0.224915 \\ 48 & \mathrm{C} & 0.325695 \\ 49 & \mathrm{C} & 0.128802 \\ 50 & \mathrm{C} & -0.181239 \\ 51 & \mathrm{C} & -0.152034 \\ 52 & \mathrm{C} & 0.306310 \\ 53 & \mathrm{C} & -0.190586 \\ 54 & \mathrm{C} & 0.240350 \\ 55 & \mathrm{C} & -0.148077 \\ 56 & \mathrm{C} & 0.222203 \\ 57 & \mathrm{H} & 0.011818 \\ 58 & \mathrm{H} & -0.010663 \\ 59 & \mathrm{H} & 0.005778 \\ 60 & \mathrm{H} & -0.009914 \\ 61 & \mathrm{H} & -0.013862 \\ 62 & \mathrm{H} & 0.007233 \\ 63 & \mathrm{H} & -0.014508 \\ 64 & \mathrm{H} & -0.018205\end{array}$

\section{Singlet Closed Shell:}

Sum of electronic and zero-point Energies=

$-1612.318096$

Sum of electronic and thermal Energies=

Sum of electronic and thermal Enthalpies=

$-1612.290777$

Sum of electronic and thermal Free Energies=

$-1612.289833$

$-1612.376032$

$\begin{array}{crrc}\mathrm{H} & -10.58924400 & 2.95758700 & 0.00019900 \\ \mathrm{C} & -9.86178900 & 2.15213400 & 0.00017200 \\ \mathrm{H} & -11.33606500 & 0.60399900 & 0.00030500 \\ \mathrm{C} & -10.27720400 & 0.84601300 & 0.00023100 \\ \mathrm{C} & -7.53990500 & 1.44933200 & 0.00004100 \\ \mathrm{C} & -9.33825100 & -0.21788600 & 0.00019700 \\ \mathrm{C} & -8.48968200 & 2.45456400 & 0.00007700 \\ \mathrm{C} & -7.94991800 & 0.08177200 & 0.00010000 \\ \mathrm{C} & -9.75290600 & -1.56855000 & 0.00025600\end{array}$




\begin{tabular}{|c|c|c|c|}
\hline $\mathrm{H}$ & -8.16458300 & 3.49106700 & 0.00003100 \\
\hline $\mathrm{C}$ & -8.81961800 & -2.57286600 & 0.00021900 \\
\hline $\mathrm{H}$ & -10.81502700 & -1.79513100 & 0.00033000 \\
\hline $\mathrm{H}$ & -9.13504600 & -3.61155100 & 0.00026400 \\
\hline $\mathrm{C}$ & -7.44835800 & -2.27465800 & 0.00012300 \\
\hline $\mathrm{H}$ & -6.74675800 & -3.10058200 & 0.00009600 \\
\hline $\mathrm{C}$ & -6.98514400 & -0.96925600 & 0.00006400 \\
\hline $\mathrm{C}$ & -6.13250300 & 1.74738000 & -0.00005100 \\
\hline $\mathrm{C}$ & -5.17546800 & 0.78489300 & -0.00008700 \\
\hline $\mathrm{C}$ & -5.54688400 & -0.63554900 & -0.00004100 \\
\hline $\mathrm{C}$ & -4.56078600 & -1.57567800 & -0.00013700 \\
\hline $\mathrm{C}$ & -2.81720900 & 0.21168300 & -0.00017600 \\
\hline $\mathrm{C}$ & -3.77369500 & 1.15676100 & -0.00015900 \\
\hline $\mathrm{H}$ & -3.53200000 & 2.21677200 & -0.00018200 \\
\hline $\mathrm{C}$ & -1.35433200 & 0.27444800 & -0.00021900 \\
\hline $\mathrm{C}$ & -0.88924300 & -1.06941200 & -0.00016900 \\
\hline $\mathrm{C}$ & -3.18071100 & -1.20679200 & -0.00014200 \\
\hline $\mathrm{C}$ & -2.03798400 & -1.95518100 & -0.00011500 \\
\hline $\mathrm{H}$ & -1.98247900 & -3.03773100 & -0.00008500 \\
\hline $\mathrm{H}$ & -5.83634500 & 2.79354400 & -0.00008600 \\
\hline $\mathrm{H}$ & -4.79996900 & -2.63367500 & 0.00022700 \\
\hline $\mathrm{C}$ & -0.47809400 & 1.34697400 & -0.00020100 \\
\hline $\mathrm{C}$ & 0.88920100 & 1.06936300 & -0.00013000 \\
\hline $\mathrm{C}$ & 1.35430700 & -0.27450600 & -0.00028800 \\
\hline $\mathrm{C}$ & 0.47801000 & -1.34703800 & -0.00027700 \\
\hline $\mathrm{H}$ & -0.83921500 & 2.37201200 & -0.00018500 \\
\hline $\mathrm{H}$ & 0.83909000 & -2.37208900 & -0.00021000 \\
\hline $\mathrm{C}$ & 2.03795300 & 1.95513700 & -0.00014000 \\
\hline $\mathrm{C}$ & 2.81719500 & -0.21173100 & -0.00022200 \\
\hline $\mathrm{C}$ & 3.18070700 & 1.20672800 & -0.00014600 \\
\hline $\mathrm{H}$ & 1.98245200 & 3.03768800 & -0.00052000 \\
\hline $\mathrm{C}$ & 4.56077500 & 1.57561900 & 0.00002800 \\
\hline $\mathrm{C}$ & 5.54689400 & 0.63550300 & 0.00003600 \\
\hline $\mathrm{H}$ & 3.53207800 & -2.21684200 & -0.00040800 \\
\hline $\mathrm{C}$ & 3.77376200 & -1.15685100 & -0.00026900 \\
\hline $\mathrm{C}$ & 5.17552100 & -0.78495000 & -0.00024200 \\
\hline $\mathrm{C}$ & 6.13255700 & -1.74740000 & -0.00009000 \\
\hline $\mathrm{C}$ & 7.53995100 & -1.44931200 & 0.00005300 \\
\hline $\mathrm{C}$ & 8.48976000 & -2.45451100 & 0.00013300 \\
\hline $\mathrm{C}$ & 7.94993500 & -0.08173700 & 0.00010000 \\
\hline $\mathrm{C}$ & 6.98513100 & 0.96925900 & 0.00008600 \\
\hline $\mathrm{C}$ & 9.33824800 & 0.21796000 & 0.00018100 \\
\hline $\mathrm{C}$ & 10.27722100 & -0.84590200 & 0.00024400 \\
\hline $\mathrm{C}$ & 9.86184900 & -2.15203300 & 0.00023600 \\
\hline
\end{tabular}




$\begin{array}{lrrr}\mathrm{C} & 7.44829500 & 2.27467600 & 0.00012900 \\ \mathrm{C} & 8.81954900 & 2.57292700 & 0.00019100 \\ \mathrm{C} & 9.75286800 & 1.56863600 & 0.00022100 \\ \mathrm{H} & 4.79994800 & 2.63362000 & 0.00007400 \\ \mathrm{H} & 6.74667300 & 3.10058300 & 0.00013000 \\ \mathrm{H} & 9.13493600 & 3.61162500 & 0.00022500 \\ \mathrm{H} & 10.81498300 & 1.79524300 & 0.00027700 \\ \mathrm{H} & 11.33607300 & -0.60385100 & 0.00020600 \\ \mathrm{H} & 10.58932700 & -2.95746500 & 0.00040300 \\ \mathrm{H} & 8.16470500 & -3.49102900 & 0.00010300 \\ \mathrm{H} & 5.83641000 & -2.79356700 & -0.00009500\end{array}$

Triplet:

Sum of electronic and zero-point Energies=

Sum of electronic and thermal Energies=

Sum of electronic and thermal Enthalpies=

Sum of electronic and thermal Free Energies=

$\mathrm{S} * * 2=2.3534$

$\begin{array}{lrrc}\mathrm{H} & -10.65781100 & -2.88460200 & -0.00031100 \\ \mathrm{C} & -9.90468700 & -2.10274600 & -0.00024200 \\ \mathrm{H} & -11.34120300 & -0.50740700 & -0.00009800 \\ \mathrm{C} & -10.28900700 & -0.77576700 & -0.00012300 \\ \mathrm{C} & -7.55606600 & -1.45252400 & -0.00018300 \\ \mathrm{C} & -9.32234500 & 0.26315300 & -0.00002800 \\ \mathrm{C} & -8.55563000 & -2.44469700 & -0.00027000 \\ \mathrm{C} & -7.93808900 & -0.07132900 & -0.00006000 \\ \mathrm{C} & -9.70080600 & 1.62083700 & 0.00009700 \\ \mathrm{H} & -8.25589000 & -3.48848100 & -0.00035900 \\ \mathrm{C} & -8.73954600 & 2.61002600 & 0.00018600 \\ \mathrm{H} & -10.75668300 & 1.87490200 & 0.00012100 \\ \mathrm{H} & -9.03537900 & 3.65455500 & 0.00028100 \\ \mathrm{C} & -7.38451400 & 2.28436200 & 0.00015200 \\ \mathrm{H} & -6.66248900 & 3.09158400 & 0.00022000 \\ \mathrm{C} & -6.95115400 & 0.95276500 & 0.00003000 \\ \mathrm{C} & -6.19011300 & -1.77963500 & -0.00019400 \\ \mathrm{C} & -5.18619700 & -0.80135600 & -0.00011100 \\ \mathrm{C} & -5.53860500 & 0.58770900 & -0.00002600 \\ \mathrm{C} & -4.51032200 & 1.54515800 & 0.00016400 \\ \mathrm{C} & -2.83735600 & -0.23709100 & 0.00004700 \\ \mathrm{C} & -3.81298500 & -1.18767700 & -0.00008400 \\ \mathrm{H} & -3.57362500 & -2.24785500 & -0.00015400 \\ \mathrm{C} & -1.37163500 & -0.31304900 & 0.00013300 \\ \mathrm{C} & -0.88847000 & 1.07497500 & 0.00017200 \\ \mathrm{C} & -3.18543500 & 1.15230200 & 0.00016100\end{array}$

$-1612.323140$

$-1612.295599$

$-1612.294654$

$-1612.381747$

$-0.00031100$

24200

-

$-0.00012300$

(2)

0.00002800

$-0.00006000$

0.00009700

0.00018600

0.00012100

0.00015200

$-7.38451400 \quad 2.28436200$

0.00022000

.00003000

$-0.00019400$

$-0.00002600$

0.00016400

.

$-0.00008400$

0.00013300

0.00016100 


\begin{tabular}{|c|c|c|c|}
\hline $\mathrm{C}$ & -1.96529200 & 1.92312900 & 0.00024700 \\
\hline $\mathrm{H}$ & -1.91859200 & 3.00624200 & 0.00033400 \\
\hline $\mathrm{H}$ & -5.90122200 & -2.82722300 & -0.00024700 \\
\hline $\mathrm{H}$ & -4.74411600 & 2.60351600 & 0.00020200 \\
\hline $\mathrm{C}$ & -0.52108200 & -1.36110500 & 0.00014300 \\
\hline $\mathrm{C}$ & 0.89347200 & -1.09193500 & 0.00015400 \\
\hline $\mathrm{C}$ & 1.37586800 & 0.29755100 & 0.00008400 \\
\hline $\mathrm{C}$ & 0.51935800 & 1.34642500 & 0.00010900 \\
\hline $\mathrm{H}$ & -0.87490000 & -2.38853100 & 0.00021300 \\
\hline $\mathrm{H}$ & 0.87224200 & 2.37417900 & 0.00032400 \\
\hline $\mathrm{C}$ & 1.96203100 & -1.93779600 & 0.00020000 \\
\hline $\mathrm{C}$ & 2.83795100 & 0.22703000 & 0.00000200 \\
\hline $\mathrm{C}$ & 3.19108000 & -1.15796700 & 0.00007700 \\
\hline $\mathrm{H}$ & 1.91838700 & -3.02087400 & 0.00035700 \\
\hline $\mathrm{C}$ & 4.51030200 & -1.54742200 & -0.00004100 \\
\hline $\mathrm{C}$ & 5.53992000 & -0.58236000 & -0.00010200 \\
\hline $\mathrm{H}$ & 3.57011300 & 2.24208300 & -0.00048300 \\
\hline $\mathrm{C}$ & 3.81381800 & 1.18287900 & -0.00015300 \\
\hline $\mathrm{C}$ & 5.18381300 & 0.80106100 & -0.00016000 \\
\hline $\mathrm{C}$ & 6.19216400 & 1.78572600 & -0.00025600 \\
\hline $\mathrm{C}$ & 7.55243000 & 1.45991700 & -0.00019500 \\
\hline $\mathrm{C}$ & 8.55656800 & 2.45409500 & -0.00027000 \\
\hline $\mathrm{C}$ & 7.93630000 & 0.07793800 & -0.00005100 \\
\hline $\mathrm{C}$ & 6.95063900 & -0.94583500 & 0.00000000 \\
\hline $\mathrm{C}$ & 9.32118900 & -0.25609300 & 0.00001600 \\
\hline $\mathrm{C}$ & 10.28811300 & 0.78267300 & -0.00005900 \\
\hline $\mathrm{C}$ & 9.90297600 & 2.11154200 & -0.00018100 \\
\hline $\mathrm{C}$ & 7.38445900 & -2.27968900 & 0.00015500 \\
\hline $\mathrm{C}$ & 8.73823400 & -2.60412600 & 0.00023700 \\
\hline $\mathrm{C}$ & 9.69952500 & -1.61317400 & 0.00016300 \\
\hline $\mathrm{H}$ & 4.74853700 & -2.60465500 & 0.00016200 \\
\hline $\mathrm{H}$ & 6.66253500 & -3.08680900 & 0.00020900 \\
\hline $\mathrm{H}$ & 9.03540400 & -3.64828700 & 0.00035400 \\
\hline $\mathrm{H}$ & 10.75546200 & -1.86707500 & 0.00022400 \\
\hline $\mathrm{H}$ & 11.34030600 & 0.51452100 & 0.00001500 \\
\hline $\mathrm{H}$ & 10.65687100 & 2.89274100 & -0.00024500 \\
\hline $\mathrm{H}$ & 8.25661500 & 3.49777600 & -0.00038900 \\
\hline $\mathrm{H}$ & 5.90019600 & 2.83240600 & -0.00036900 \\
\hline
\end{tabular}


Compound FP

Natural Orbital Coefficient

HOMO: 1.18888 LUMO: 0.81112

Singlet biradical character $\mathrm{y}_{0}=0.635$

Singlet Biradical Open Shell:

Sum of electronic and zero-point Energies= Sum of electronic and thermal Energies= Sum of electronic and thermal Enthalpies= Sum of electronic and thermal Free Energies= $\mathrm{S} * * 2=1.5338$

$\begin{array}{lrrr}\mathrm{C} & 0.409123000 & 8.253580000 & 0.000000000 \\ \mathrm{C} & 1.256032000 & 7.160484000 & 0.000000000 \\ \mathrm{C} & 2.666280000 & 7.336807000 & 0.000000000 \\ \mathrm{C} & 3.208734000 & 8.623043000 & 0.000000000 \\ \mathrm{C} & 2.347667000 & 9.714712000 & 0.000000000 \\ \mathrm{C} & 0.963311000 & 9.535231000 & 0.000000000 \\ \mathrm{H} & -0.669489000 & 8.124761000 & 0.000000000 \\ \mathrm{H} & 4.284799000 & 8.769492000 & 0.000000000 \\ \mathrm{H} & 2.756232000 & 10.720532000 & 0.000000000 \\ \mathrm{H} & 0.309932000 & 10.402012000 & 0.000000000 \\ \mathrm{C} & 3.293236000 & 6.043191000 & 0.000000000 \\ \mathrm{C} & 0.998520000 & 5.716328000 & 0.000000000 \\ \mathrm{C} & 2.287031000 & 5.055325000 & 0.000000000 \\ \mathrm{C} & -0.141542000 & 4.985407000 & 0.000000000 \\ \mathrm{C} & 2.362242000 & 3.667250000 & 0.000000000 \\ \mathrm{C} & -0.080933000 & 3.552263000 & 0.000000000 \\ \mathrm{C} & 1.191003000 & 2.882094000 & 0.000000000 \\ \mathrm{C} & 1.216129000 & 1.443490000 & 0.000000000 \\ \mathrm{C} & -1.256032000 & 2.804566000 & 0.000000000 \\ \mathrm{C} & -0.000287000 & 0.715160000 & 0.000000000 \\ \mathrm{C} & -1.246209000 & 1.411113000 & 0.000000000 \\ \mathrm{C} & 2.437557000 & 0.705141000 & 0.000000000 \\ \mathrm{C} & 0.000287000 & -0.715160000 & 0.000000000 \\ \mathrm{C} & -2.452037000 & 0.654414000 & 0.000000000 \\ \mathrm{C} & 2.452037000 & -0.654414000 & 0.000000000 \\ \mathrm{C} & 1.246209000 & -1.411113000 & 0.000000000 \\ \mathrm{C} & -2.437557000 & -0.705141000 & 0.000000000 \\ \mathrm{C} & -1.216129000 & -1.443490000 & 0.000000000 \\ \mathrm{C} & -1.191003000 & -2.882094000 & 0.000000000 \\ \mathrm{C} & 1.256032000 & -2.804566000 & 0.000000000 \\ \mathrm{C} & 0.080933000 & -3.552263000 & 0.000000000\end{array}$

0.000000000

0.000000000

0.000000000

0.000000000

0.000000000

0.000000000

0.000000000

0.000000000

年

$-1458.834338$

$-1458.809580$

$-1458.808636$

$-1458.887817$

(1)

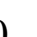

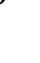

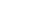




$\begin{array}{lrrr}\mathrm{C} & 0.141542000 & -4.985407000 & 0.000000000 \\ \mathrm{C} & -2.362242000 & -3.667250000 & 0.000000000 \\ \mathrm{C} & -2.287031000 & -5.055325000 & 0.000000000 \\ \mathrm{C} & -0.998520000 & -5.716328000 & 0.000000000 \\ \mathrm{C} & -3.293236000 & -6.043191000 & 0.000000000 \\ \mathrm{C} & -1.256032000 & -7.160484000 & 0.000000000 \\ \mathrm{C} & -2.666280000 & -7.336807000 & 0.000000000 \\ \mathrm{C} & -3.208734000 & -8.623043000 & 0.000000000 \\ \mathrm{C} & -0.409123000 & -8.253580000 & 0.000000000 \\ \mathrm{C} & -2.347667000 & -9.714712000 & 0.000000000 \\ \mathrm{C} & -0.963311000 & -9.535231000 & 0.000000000 \\ \mathrm{H} & 4.361524000 & 5.862892000 & 0.000000000 \\ \mathrm{H} & -1.120589000 & 5.457614000 & 0.000000000 \\ \mathrm{H} & 3.339574000 & 3.199673000 & 0.000000000 \\ \mathrm{H} & -2.210699000 & 3.324409000 & 0.000000000 \\ \mathrm{H} & 3.382802000 & 1.233002000 & 0.000000000 \\ \mathrm{H} & 3.397353000 & -1.189453000 & 0.000000000 \\ \mathrm{H} & -3.397353000 & 1.189453000 & 0.000000000 \\ \mathrm{H} & -3.382802000 & -1.233002000 & 0.000000000 \\ \mathrm{H} & 2.210699000 & -3.324409000 & 0.000000000 \\ \mathrm{H} & -3.339574000 & -3.199673000 & 0.000000000 \\ \mathrm{H} & 1.120589000 & -5.457614000 & 0.000000000 \\ \mathrm{H} & -4.361524000 & -5.862892000 & 0.000000000 \\ \mathrm{H} & 0.669489000 & -8.124761000 & 0.000000000 \\ \mathrm{H} & -0.309932000 & -10.402012000 & 0.000000000 \\ \mathrm{H} & -2.756232000 & -10.720532000 & 0.000000000 \\ \mathrm{H} & -4.284799000 & -8.769492000 & 0.000000000\end{array}$

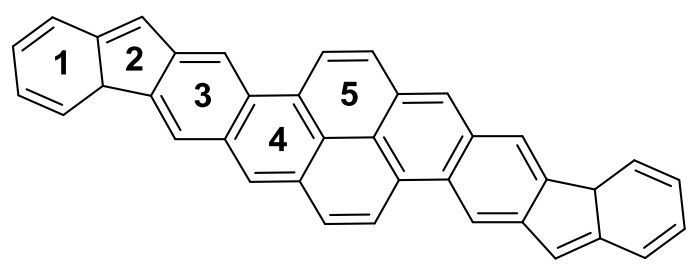

FP

NICS values (sigma zz)

$\begin{array}{rrlrrr}\text { Bq distance }(\AA) & \text { ring 1 } & \text { ring 2 } & \text { ring 3 } & \text { ring 4 } & \text { ring 5 } \\ 0.0000 & -1.7028 & 36.1304 & 8.5865 & 0.3362 & 9.0015 \\ 0.1000 & -2.1798 & 35.6233 & 8.1109 & -0.1871 & 8.5139 \\ 0.2000 & -3.5459 & 34.1306 & 6.7411 & -1.6861 & 7.1082 \\ 0.3000 & -5.6202 & 31.7398 & 4.6367 & -3.9635 & 4.9435 \\ 0.4000 & -8.1446 & 28.5981 & 2.0281 & -6.7396 & 2.2494\end{array}$




\begin{tabular}{|c|c|c|c|c|c|}
\hline 0.5000 & -10.8332 & 24.9060 & -0.8255 & -9.7076 & -0.7152 \\
\hline 0.6000 & -13.4212 & 20.8978 & -3.6778 & -12.5860 & -3.7032 \\
\hline 7000 & -15.6993 & 16.8132 & -6.3262 & -15.1554 & -6.5104 \\
\hline 8000 & -17.5311 & 12.8659 & -8.6291 & -17.2742 & -8.9912 \\
\hline 9000 & -18.8535 & 9.2213 & -10.5085 & -18.8776 & -11.0621 \\
\hline 0000 & -19.6646 & 5.9857 & -11.9417 & -19.9633 & -12.6938 \\
\hline 1000 & -20.0068 & 3.2083 & -12.9476 & -20.5729 & \\
\hline 1.2000 & -19.9484 & 0.8926 & -13.5720 & -20.7733 & -14.7150 \\
\hline 1.3000 & -19.5683 & -0.9900 & -13.8743 & -20.6418 & -15.1975 \\
\hline & -18.9 & 355 & -13 & & -15 . \\
\hline 5000 & -18.1514 & 474 & -13.7605 & -19.6824 & 3926 \\
\hline 6000 & -17.2476 & -4.5292 & -13.4568 & -18. & -15 . \\
\hline 7000 & -16.2 & 798 & $-13 .($ & -18.2064 & 094 \\
\hline 00 & -15.2 & 25 & -12.5 & 88 & 180 \\
\hline 9000 & -14.3 & 541 & -12.06 & & 677 \\
\hline 0000 & -13.3659 & 451 & -11.5 & -15 & -13.5809 \\
\hline & -12. & -6. & $-11 .($ & 71 & -13 \\
\hline 2000 & -11.5 & -6.2627 & -10.4 & -14 & 5622 \\
\hline 3000 & -10.7846 & -6.2272 & -9.9715 & -13.4364 & -12.0523 \\
\hline 4000 & -10.0323 & -6.1 & -9.4851 & -12 . & -11 . \\
\hline 5000 & -9. & -6. & $-9 .($ & -12 & -11 \\
\hline 6000 & & & & & -10.5952 \\
\hline 7000 & -8.1005 & 527 & -8.1734 & -10.9047 & -10.1439 \\
\hline 2.8000 & -7.5 & -5 . & -7.7 & -10 & -9.7120 \\
\hline & & & & & \\
\hline 3.0000 & -6.6002 & -5. & -7.0 & 34 & -8.9076 \\
\hline 3.1000 & -6.1 & -5.0826 & -6.7672 & 527 & -8.5344 \\
\hline 3.2000 & $-5.7 c$ & -4.9128 & -6.4698 & & \\
\hline 3000 & -5.4426 & & -6.1 & & \\
\hline 3.4000 & -5.1182 & -4.5823 & -5.9303 & -7.7900 & -7.5235 \\
\hline 3.5000 & -4.8201 & -4.4233 & -5.6854 & -7.4490 & -7.2201 \\
\hline 3.6000 & -4.5458 & & -5.4554 & & -6.9320 \\
\hline 7000 & -4.2931 & -4.1200 & -5.2 & -6.8267 & -6.6585 \\
\hline 3.8000 & -4.0600 & -3.9762 & -5.0355 & -6.5425 & -6.3987 \\
\hline 3.9000 & -3.8448 & -3.8378 & & & -6.1518 \\
\hline 4.0000 & -3.6458 & -3.7047 & -4.6623 & -6.0214 & -5.9172 \\
\hline 4.1000 & -3.4615 & -3.5769 & -4.4911 & -5.7823 & -5.6941 \\
\hline 4.2000 & -3.2907 & -3.4543 & -4.3290 & -5.5561 & -5.4818 \\
\hline 4.3000 & -3.1321 & -3.3367 & -4.1754 & -5.3420 & -5.2798 \\
\hline 4.4000 & -2.9847 & -3.2241 & -4.0297 & -5.1391 & -5.0874 \\
\hline 4.5000 & -2.8474 & -3.1161 & -3.8913 & -4.9466 & -4.9042 \\
\hline 4.6000 & -2.7195 & -3.0127 & -3.7596 & -4.7640 & -4.7295 \\
\hline 4.7000 & -2.6001 & -2.9137 & -3.6344 & -4.5905 & -4.5629 \\
\hline
\end{tabular}




$\begin{array}{llllll}4.8000 & -2.4885 & -2.8188 & -3.5150 & -4.4255 & -4.4039 \\ 4.9000 & -2.3841 & -2.7279 & -3.4011 & -4.2686 & -4.2522 \\ 5.0000 & -2.2863 & -2.6409 & -3.2924 & -4.1191 & -4.1074\end{array}$

Mulliken atomic spin densities:

\begin{tabular}{rlr}
1 & C & -0.089252 \\
2 & C & 0.145916 \\
3 & C & -0.174577 \\
4 & C & 0.181901 \\
5 & C & -0.100772 \\
6 & C & 0.179133 \\
7 & H & 0.003415 \\
8 & H & -0.008072 \\
9 & H & 0.003792 \\
10 & H & -0.008564 \\
11 & C & 0.624686 \\
12 & C & 0.122812 \\
13 & C & -0.262984 \\
14 & C & -0.114474 \\
15 & C & 0.375211 \\
16 & C & 0.196351 \\
17 & C & -0.217419 \\
18 & C & 0.230775 \\
19 & C & -0.248303 \\
20 & C & -0.145403 \\
21 & C & 0.201118 \\
22 & C & -0.189692 \\
23 & C & 0.145403 \\
24 & C & -0.189161 \\
25 & C & 0.189161 \\
26 & C & -0.201118 \\
27 & C & 0.189692 \\
28 & C & -0.230775 \\
29 & C & 0.217419 \\
30 & C & 0.248303 \\
31 & C & -0.196351 \\
32 & C & 0.114474 \\
33 & C & -0.375211 \\
34 & C & 0.262984 \\
35 & C & -0.122812 \\
36 & C & -0.624686 \\
37 & C & -0.145916 \\
38 & C & 0.174577 \\
& \\
\hline
\end{tabular}




$\begin{array}{llc}39 & \mathrm{C} & -0.181901 \\ 40 & \mathrm{C} & 0.089252 \\ 41 & \mathrm{C} & 0.100772 \\ 42 & \mathrm{C} & -0.179133 \\ 43 & \mathrm{H} & -0.026047 \\ 44 & \mathrm{H} & 0.004480 \\ 45 & \mathrm{H} & -0.015442 \\ 46 & \mathrm{H} & 0.010171 \\ 47 & \mathrm{H} & 0.007750 \\ 48 & \mathrm{H} & -0.007877 \\ 49 & \mathrm{H} & 0.007877 \\ 50 & \mathrm{H} & -0.007750 \\ 51 & \mathrm{H} & -0.010171 \\ 52 & \mathrm{H} & 0.015442 \\ 53 & \mathrm{H} & -0.004480 \\ 54 & \mathrm{H} & 0.026047 \\ 55 & \mathrm{H} & -0.003415 \\ 56 & \mathrm{H} & 0.008564 \\ 57 & \mathrm{H} & -0.003792 \\ 58 & \mathrm{H} & 0.008072\end{array}$

\section{Singlet Closed Shell:}

Sum of electronic and zero-point Energies= Sum of electronic and thermal Energies= Sum of electronic and thermal Enthalpies= Sum of electronic and thermal Free Energies=

$\begin{array}{lrrr}\mathrm{C} & 0.355127000 & 8.293525000 & 0.000000000 \\ \mathrm{C} & 1.203282000 & 7.199144000 & 0.000000000 \\ \mathrm{C} & 2.609247000 & 7.371462000 & 0.000000000 \\ \mathrm{C} & 3.153296000 & 8.651975000 & 0.000000000 \\ \mathrm{C} & 2.293494000 & 9.746887000 & 0.000000000 \\ \mathrm{C} & 0.909568000 & 9.572962000 & 0.000000000 \\ \mathrm{H} & -0.723644000 & 8.164487000 & 0.000000000 \\ \mathrm{H} & 4.229603000 & 8.797505000 & 0.000000000 \\ \mathrm{H} & 2.705728000 & 10.751396000 & 0.000000000 \\ \mathrm{H} & 0.258460000 & 10.441429000 & 0.000000000 \\ \mathrm{C} & 3.237390000 & 6.059960000 & 0.000000000 \\ \mathrm{C} & 0.952362000 & 5.756734000 & 0.000000000 \\ \mathrm{C} & 2.263002000 & 5.102328000 & 0.000000000 \\ \mathrm{C} & -0.172793000 & 5.020702000 & 0.000000000 \\ \mathrm{C} & 2.333269000 & 3.677124000 & 0.000000000 \\ \mathrm{C} & -0.103733000 & 3.573317000 & 0.000000000\end{array}$

$-1458.812838$ $-1458.788104$ $-1458.787160$ $-1458.866837$ 


\begin{tabular}{|c|c|c|c|}
\hline $\mathrm{C}$ & 1.203282000 & 2.909492000 & 0.000000000 \\
\hline $\mathrm{C}$ & 1.228302000 & 1.439168000 & 0.000000000 \\
\hline $\mathrm{C}$ & -1.245414000 & 2.835877000 & 0.000000000 \\
\hline $\mathrm{C}$ & 0.000473000 & 0.710305000 & 0.000000000 \\
\hline $\mathrm{C}$ & -1.242406000 & 1.404280000 & 0.000000000 \\
\hline $\mathrm{C}$ & 2.415807000 & 0.714498000 & 0.000000000 \\
\hline $\mathrm{C}$ & -0.000473000 & -0.710305000 & 0.000000000 \\
\hline $\mathrm{C}$ & -2.423490000 & 0.674840000 & 0.000000000 \\
\hline $\mathrm{C}$ & 2.423490000 & -0.674840000 & 0.000000000 \\
\hline $\mathrm{C}$ & 1.242406000 & -1.404280000 & 0.000000000 \\
\hline $\mathrm{C}$ & -2.415807000 & -0.714498000 & 0.000000000 \\
\hline $\mathrm{C}$ & -1.228302000 & -1.439168000 & 0.000000000 \\
\hline $\mathrm{C}$ & -1.203282000 & -2.909492000 & 0.000000000 \\
\hline $\mathrm{C}$ & 1.245414000 & -2.835877000 & 0.000000000 \\
\hline $\mathrm{C}$ & 0.103733000 & -3.573317000 & 0.000000000 \\
\hline $\mathrm{C}$ & 0.172793000 & -5.020702000 & 0.000000000 \\
\hline $\mathrm{C}$ & -2.333269000 & -3.677124000 & 0.000000000 \\
\hline $\mathrm{C}$ & -2.263002000 & -5.102328000 & 0.000000000 \\
\hline $\mathrm{C}$ & -0.952362000 & -5.756734000 & 0.000000000 \\
\hline $\mathrm{C}$ & -3.237390000 & -6.059960000 & 0.000000000 \\
\hline $\mathrm{C}$ & -1.203282000 & -7.199144000 & 0.000000000 \\
\hline $\mathrm{C}$ & -2.609247000 & -7.371462000 & 0.000000000 \\
\hline $\mathrm{C}$ & -3.153296000 & -8.651975000 & 0.000000000 \\
\hline $\mathrm{C}$ & -0.355127000 & -8.293525000 & 0.000000000 \\
\hline $\mathrm{C}$ & -2.293494000 & -9.746887000 & 0.000000000 \\
\hline $\mathrm{C}$ & -0.909568000 & -9.572962000 & 0.000000000 \\
\hline $\mathrm{H}$ & 4.307121000 & 5.886157000 & 0.000000000 \\
\hline $\mathrm{H}$ & -1.158203000 & 5.479990000 & 0.000000000 \\
\hline $\mathrm{H}$ & 3.317155000 & 3.221053000 & 0.000000000 \\
\hline $\mathrm{H}$ & -2.207507000 & 3.342335000 & 0.000000000 \\
\hline $\mathrm{H}$ & 3.368125000 & 1.230818000 & 0.000000000 \\
\hline $\mathrm{H}$ & 3.371927000 & -1.204392000 & 0.000000000 \\
\hline $\mathrm{H}$ & -3.371927000 & 1.204392000 & 0.000000000 \\
\hline $\mathrm{H}$ & -3.368125000 & -1.230818000 & 0.000000000 \\
\hline $\mathrm{H}$ & 2.207507000 & -3.342335000 & 0.000000000 \\
\hline $\mathrm{H}$ & -3.317155000 & -3.221053000 & 0.000000000 \\
\hline $\mathrm{H}$ & 1.158203000 & -5.479990000 & 0.000000000 \\
\hline $\mathrm{H}$ & -4.307121000 & -5.886157000 & 0.000000000 \\
\hline $\mathrm{H}$ & 0.723644000 & -8.164487000 & 0.000000000 \\
\hline $\mathrm{H}$ & -0.258460000 & -10.441429000 & 0.000000000 \\
\hline $\mathrm{H}$ & -2.705728000 & -10.751396000 & 0.000000000 \\
\hline $\mathrm{H}$ & -4.229603000 & -8.797505000 & 0.000000000 \\
\hline
\end{tabular}

Triplet: 
Sum of electronic and zero-point Energies= Sum of electronic and thermal Energies= Sum of electronic and thermal Enthalpies= Sum of electronic and thermal Free Energies= $\mathrm{S}^{* * 2}=2.3534$

$\begin{array}{lrrr}\mathrm{C} & 0.428673000 & 8.242788000 & 0.000000000 \\ \mathrm{C} & 1.273507000 & 7.147567000 & 0.000000000 \\ \mathrm{C} & 2.686633000 & 7.322368000 & 0.000000000 \\ \mathrm{C} & 3.230086000 & 8.611683000 & 0.000000000 \\ \mathrm{C} & 2.370828000 & 9.702282000 & 0.000000000 \\ \mathrm{C} & 0.984498000 & 9.523281000 & 0.000000000 \\ \mathrm{H} & -0.650085000 & 8.115657000 & 0.000000000 \\ \mathrm{H} & 4.306345000 & 8.756475000 & 0.000000000 \\ \mathrm{H} & 2.779223000 & 10.708135000 & 0.000000000 \\ \mathrm{H} & 0.332281000 & 10.390936000 & 0.000000000 \\ \mathrm{C} & 3.311787000 & 6.036425000 & 0.000000000 \\ \mathrm{C} & 1.011146000 & 5.705466000 & 0.000000000 \\ \mathrm{C} & 2.295470000 & 5.039997000 & 0.000000000 \\ \mathrm{C} & -0.132444000 & 4.977951000 & 0.000000000 \\ \mathrm{C} & 2.370828000 & 3.662473000 & 0.000000000 \\ \mathrm{C} & -0.076009000 & 3.546559000 & 0.000000000 \\ \mathrm{C} & 1.187862000 & 2.873959000 & 0.000000000 \\ \mathrm{C} & 1.211655000 & 1.443334000 & 0.000000000 \\ \mathrm{C} & -1.257676000 & 2.797751000 & 0.000000000 \\ \mathrm{C} & 0.000204000 & 0.717115000 & 0.000000000 \\ \mathrm{C} & -1.246560000 & 1.413720000 & 0.000000000 \\ \mathrm{C} & 2.443555000 & 0.701090000 & 0.000000000 \\ \mathrm{C} & -0.000204000 & -0.717115000 & 0.000000000 \\ \mathrm{C} & -2.459365000 & 0.650016000 & 0.000000000 \\ \mathrm{C} & 2.459365000 & -0.650016000 & 0.000000000 \\ \mathrm{C} & 1.246560000 & -1.413720000 & 0.000000000 \\ \mathrm{C} & -2.443555000 & -0.701090000 & 0.000000000 \\ \mathrm{C} & -1.211655000 & -1.443334000 & 0.000000000 \\ \mathrm{C} & -1.187862000 & -2.873959000 & 0.000000000 \\ \mathrm{C} & 1.257676000 & -2.797751000 & 0.000000000 \\ \mathrm{C} & 0.076009000 & -3.546559000 & 0.000000000 \\ \mathrm{C} & 0.132444000 & -4.977951000 & 0.000000000 \\ \mathrm{C} & -2.370828000 & -3.662473000 & 0.000000000 \\ \mathrm{C} & -2.295470000 & -5.039997000 & 0.000000000 \\ \mathrm{C} & -1.011146000 & -5.705466000 & 0.000000000 \\ \mathrm{C} & -3.311787000 & -6.036425000 & 0.000000000 \\ \mathrm{C} & & -7.147567000 & 0.000000000\end{array}$

$-1458.829773$

$-1458.805031$

$-1458.804087$

$-1458.884223$

0.000000000

0.000000000

0.000000000

0.000000000

0.000000000

0

0.000000000

0.000000000

(0.0000000000000000

0.000000000

0.000000000

0.000000000

0.000000000

0.000000000

0.000000000

0.000000000

0.000000000

0.000000000

0.000000000

0.000000000

0.000000000

0.000000000

0.000000000

.

0.000000000

0.000000000

0.000000000

0.000000000

0.000000000

0.000000000 


$\begin{array}{lrrr}\mathrm{C} & -2.686633000 & -7.322368000 & 0.000000000 \\ \mathrm{C} & -3.230086000 & -8.611683000 & 0.000000000 \\ \mathrm{C} & -0.428673000 & -8.242788000 & 0.000000000 \\ \mathrm{C} & -2.370828000 & -9.702282000 & 0.000000000 \\ \mathrm{C} & -0.984498000 & -9.523281000 & 0.000000000 \\ \mathrm{H} & 4.379307000 & 5.852638000 & 0.000000000 \\ \mathrm{H} & -1.109634000 & 5.453958000 & 0.000000000 \\ \mathrm{H} & 3.345662000 & 3.190326000 & 0.000000000 \\ \mathrm{H} & -2.210558000 & 3.320895000 & 0.000000000 \\ \mathrm{H} & 3.386482000 & 1.232717000 & 0.000000000 \\ \mathrm{H} & 3.403368000 & -1.187409000 & 0.000000000 \\ \mathrm{H} & -3.403368000 & 1.187409000 & 0.000000000 \\ \mathrm{H} & -3.386482000 & -1.232717000 & 0.000000000 \\ \mathrm{H} & 2.210558000 & -3.320895000 & 0.000000000 \\ \mathrm{H} & -3.345662000 & -3.190326000 & 0.000000000 \\ \mathrm{H} & 1.109634000 & -5.453958000 & 0.000000000 \\ \mathrm{H} & -4.379307000 & -5.852638000 & 0.000000000 \\ \mathrm{H} & 0.650085000 & -8.115657000 & 0.000000000 \\ \mathrm{H} & -0.332281000 & -10.390936000 & 0.000000000 \\ \mathrm{H} & -2.779223000 & -10.708135000 & 0.000000000 \\ \mathrm{H} & -4.306345000 & -8.756475000 & 0.000000000\end{array}$


Compound PF

Natural Orbital Coefficient

HOMO: 1.72234 LUMO: 0.27766

Singlet biradical character $\mathrm{y}_{0}=0.051$

Singlet Biradical Open Shell:

Sum of electronic and zero-point Energies=

Sum of electronic and thermal Energies=

Sum of electronic and thermal Enthalpies=

Sum of electronic and thermal Free Energies= $\mathrm{S} * * 2=0.581$

$\begin{array}{lrrr}\mathrm{C} & 0.762665000 & -0.451900000 & 0.000000000 \\ \mathrm{C} & 0.000000000 & 0.785036000 & 0.000000000 \\ \mathrm{C} & -1.377626000 & 0.717026000 & 0.000000000 \\ \mathrm{C} & -2.048129000 & -0.528003000 & 0.000000000 \\ \mathrm{C} & -1.276352000 & -1.760723000 & 0.000000000 \\ \mathrm{C} & 0.073734000 & -1.717976000 & 0.000000000 \\ \mathrm{H} & -1.977261000 & 1.620443000 & 0.000000000 \\ \mathrm{H} & 0.673621000 & -2.624546000 & 0.000000000 \\ \mathrm{C} & -3.395120000 & -0.846229000 & 0.000000000 \\ \mathrm{C} & -3.533833000 & -2.289082000 & 0.000000000 \\ \mathrm{C} & -4.661213000 & -3.105549000 & 0.000000000 \\ \mathrm{C} & -4.489911000 & -4.487363000 & 0.000000000 \\ \mathrm{C} & -3.214712000 & -5.051361000 & 0.000000000 \\ \mathrm{C} & -2.078304000 & -4.240890000 & 0.000000000 \\ \mathrm{C} & -2.239363000 & -2.867044000 & 0.000000000 \\ \mathrm{H} & -5.658052000 & -2.674405000 & 0.000000000 \\ \mathrm{H} & -5.361109000 & -5.135336000 & 0.000000000 \\ \mathrm{H} & -3.104576000 & -6.131218000 & 0.000000000 \\ \mathrm{H} & -1.087983000 & -4.687603000 & 0.000000000 \\ \mathrm{C} & 0.738697000 & 2.054488000 & 0.000000000 \\ \mathrm{C} & 2.137665000 & -0.419972000 & 0.000000000 \\ \mathrm{C} & 2.163764000 & 2.036236000 & 0.000000000 \\ \mathrm{C} & 2.877497000 & 0.797551000 & 0.000000000 \\ \mathrm{C} & 0.102625000 & 3.292492000 & 0.000000000 \\ \mathrm{C} & 2.898510000 & 3.253577000 & 0.000000000 \\ \mathrm{C} & 4.270693000 & 0.803253000 & 0.000000000 \\ \mathrm{H} & -0.979405000 & 3.347850000 & 0.000000000 \\ \mathrm{C} & 0.825312000 & 4.490565000 & 0.000000000 \\ \mathrm{C} & 2.199945000 & 4.480095000 & 0.000000000 \\ \mathrm{C} & 4.316835000 & 3.208033000 & 0.000000000 \\ \mathrm{C} & 4.985623000 & 2.006563000 & 0.000000000 \\ \mathrm{H} & 0.287771000 & 5.433681000 & 0.000000000 \\ & & & \\ \mathrm{H} & & & \\ \mathrm{H} & & & \end{array}$

$-922.162645$

$-922.146979$

$-922.146035$

$-922.205571$

0.000000000

0.000000000

(2)

0.000000000

0.000000000

0.000000000

0.000000000

0.000000000

0.000000000

0.000000000

0.000000000

0.000000000

0.000000000

0.000000000

0.000000000

0.000000000

0.000000000

0.000000000

0.000000000

0.000000000

0.000000000

0.000000000

0.000000000

0.000000000

0.000000000

0.000000000

0.000000000

0.000000000 


$\begin{array}{lrrr}\mathrm{H} & 2.762787000 & 5.408849000 & 0.000000000 \\ \mathrm{H} & 4.866640000 & 4.144648000 & 0.000000000 \\ \mathrm{H} & 6.070858000 & 1.987046000 & 0.000000000 \\ \mathrm{H} & 4.801750000 & -0.144208000 & 0.000000000 \\ \mathrm{H} & 2.689248000 & -1.356626000 & 0.000000000 \\ \mathrm{H} & -4.218309000 & -0.141513000 & 0.000000000\end{array}$

\begin{tabular}{|c|c|c|c|c|c|c|}
\hline & & NICS va & es (siing & $\mathrm{Zz})$ & & \\
\hline Bq distance $(\AA)$ & ring 1 & ring 2 & ring 3 & ring 4 & ring 5 & ring 6 \\
\hline 0.0000 & -5.1296 & 32.4701 & 16.5170 & 25.9071 & -3.7070 & 0.5585 \\
\hline 0.1000 & -5.6151 & 31.9322 & 16.0591 & 25.4658 & -4.2225 & 0.0680 \\
\hline 0.2000 & -7.0025 & 30.3579 & 14.7344 & 24.1785 & -5.6956 & -1.3381 \\
\hline 0.3000 & -9.1006 & 27.8630 & 12.6800 & 22.1507 & -7.9237 & -3.4788 \\
\hline 0.4000 & -11.6371 & 24.6315 & 10.0970 & 19.5409 & -10.6192 & -6.0955 \\
\hline 0.5000 & -14.3132 & 20.8972 & 7.2167 & 16.5393 & -13.4687 & -8.9027 \\
\hline 0.6000 & -16.8548 & 16.9168 & 4.2659 & 13.3435 & -16.1864 & -11.6356 \\
\hline 0.7000 & -19.0491 & 12.9370 & 1.4390 & 10.1357 & -18.5522 & -14.0848 \\
\hline 0.8000 & -20.7613 & 9.1660 & -1.1194 & 7.0662 & -20.4281 & -16.1120 \\
\hline 0.9000 & -21.9340 & 5.7545 & -3.3195 & 4.2448 & -21.7556 & -17.6506 \\
\hline 1.0000 & -22.5731 & 2.7902 & -5.1227 & 1.7394 & -22.5 & -18.6933 \\
\hline 1.1000 & -22.7293 & 0.3042 & -6.5304 & -0.4191 & -22.8358 & -19.2762 \\
\hline 1.2000 & -22.4785 & -1.7158 & -7.5722 & -2.2294 & -22.7158 & -19.4604 \\
\hline 1.3000 & -21.9061 & -3.3099 & -8.2937 & -3.7101 & -22.2664 & -19.3188 \\
\hline 1.4000 & -21.0958 & -4.5325 & -8.7469 & -4.8922 & -21.5710 & -18.9245 \\
\hline 1.5000 & -20.1229 & -5.4418 & -8.9838 & -5.8122 & -20.7045 & -18.3448 \\
\hline 1.6000 & -19.0508 & -6.0936 & -9.0520 & -6.5077 & -19.7298 & -17.6380 \\
\hline 1.7000 & -17.9302 & -6.5378 & -8.9928 & -7.0147 & -18.6975 & -16.8515 \\
\hline 1.8000 & -16.8001 & -6.8169 & -8.8407 & -7.3657 & -17.6464 & -16.0231 \\
\hline 1.9000 & -15.6892 & -6.9664 & -8.6234 & -7.5892 & -16.6051 & -15.1812 \\
\hline 2.0000 & -14.6172 & -7.0154 & -8.3628 & -7.7097 & -15.5937 & -14.3466 \\
\hline 2.1000 & -13.5972 & -6.9874 & -8.0758 & -7.7482 & -14.6256 & -13.5339 \\
\hline 2.2000 & -12.6370 & -6.9013 & -7.7750 & -7.7217 & -13.7087 & -12.7530 \\
\hline 2.3000 & -11.7402 & -6.7723 & -7.4697 & -7.6447 & -12.8472 & -12.0099 \\
\hline 2.4000 & -10.9077 & -6.6123 & -7.1667 & -7.5289 & -12.0426 & -11.3077 \\
\hline 2.5000 & -10.1383 & -6.4310 & -6.8705 & -7.3838 & -11.2941 & -10.6477 \\
\hline 2.6000 & -9.4296 & -6.2357 & -6.5842 & -7.2173 & -10.5998 & -10.0297 \\
\hline 2.7000 & -8.7782 & -6.0322 & -6.3098 & -7.0354 & -9.9571 & -9.4526 \\
\hline 2.8000 & -8.1804 & -5.8249 & -6.0482 & -6.8431 & -9.3627 & -8.9147 \\
\hline
\end{tabular}




$\begin{array}{lllllll}2.9000 & -7.6323 & -5.6172 & -5.7998 & -6.6445 & -8.8133 & -8.4138 \\ 3.0000 & -7.1298 & -5.4114 & -5.5646 & -6.4424 & -8.3054 & -7.9476 \\ 3.1000 & -6.6691 & -5.2095 & -5.3423 & -6.2393 & -7.8357 & -7.5139 \\ 3.2000 & -6.2467 & -5.0126 & -5.1323 & -6.0371 & -7.4011 & -7.1102 \\ 3.3000 & -5.8591 & -4.8216 & -4.9340 & -5.8372 & -6.9986 & -6.7344 \\ 3.4000 & -5.5030 & -4.6372 & -4.7467 & -5.6406 & -6.6254 & -6.3843 \\ 3.5000 & -5.1757 & -4.4595 & -4.5697 & -5.4483 & -6.2790 & -6.0579 \\ 3.6000 & -4.8745 & -4.2888 & -4.4022 & -5.2607 & -5.9571 & -5.7534 \\ 3.7000 & -4.5969 & -4.1251 & -4.2437 & -5.0782 & -5.6576 & -5.4691 \\ 3.8000 & -4.3409 & -3.9683 & -4.0934 & -4.9012 & -5.3785 & -5.2033 \\ 3.9000 & -4.1044 & -3.8183 & -3.9507 & -4.7298 & -5.1183 & -4.9547 \\ 4.0000 & -3.8856 & -3.6750 & -3.8151 & -4.5642 & -4.8752 & -4.7219 \\ 4.1000 & -3.6830 & -3.5380 & -3.6861 & -4.4043 & -4.6478 & -4.5036 \\ 4.2000 & -3.4951 & -3.4072 & -3.5633 & -4.2501 & -4.4349 & -4.2988 \\ 4.3000 & -3.3206 & -3.2824 & -3.4460 & -4.1015 & -4.2354 & -4.1065 \\ 4.4000 & -3.1584 & -3.1632 & -3.3341 & -3.9585 & -4.0481 & -3.9256 \\ 4.5000 & -3.0074 & -3.0494 & -3.2271 & -3.8208 & -3.8721 & -3.7555 \\ 4.6000 & -2.8666 & -2.9408 & -3.1247 & -3.6885 & -3.7065 & -3.5952 \\ 4.7000 & -2.7352 & -2.8372 & -3.0266 & -3.5613 & -3.5507 & -3.4441 \\ 4.8000 & -2.6124 & -2.7382 & -2.9326 & -3.4390 & -3.4037 & -3.3016 \\ 4.9000 & -2.4975 & -2.6436 & -2.8424 & -3.3216 & -3.2650 & -3.1670 \\ 5.0000 & -2.3899 & -2.5533 & -2.7558 & -3.2088 & -3.1341 & -3.0398 \\ 5.1000 & -2.2889 & -2.4670 & -2.6725 & -3.1004 & -3.0102 & -2.9194 \\ 5.2000 & -2.1941 & -2.3845 & -2.5924 & -2.9963 & -2.8931 & -2.8055 \\ 5.3000 & -2.1051 & -2.3056 & -2.5154 & -2.8964 & -2.7821 & -2.6976 \\ 5.4000 & -2.0212 & -2.2302 & -2.4413 & -2.8004 & -2.6769 & -2.5953 \\ 5.5000 & -1.9422 & -2.1580 & -2.3698 & -2.7083 & -2.5771 & -2.4982 \\ 5.6000 & -1.8677 & -2.0889 & -2.3010 & -2.6198 & -2.4824 & -2.4060 \\ 5.7000 & -1.7974 & -2.0227 & -2.2346 & -2.5347 & -2.3924 & -2.3184 \\ 5.8000 & -1.7310 & -1.9593 & -2.1706 & -2.4530 & -2.3068 & -2.2351 \\ 5.9000 & -1.6682 & -1.8985 & -2.1088 & -2.3746 & -2.2254 & -2.1559\end{array}$

Mulliken atomic spin densities:

$\begin{array}{rrc}1 & \mathrm{C} & 0.243026 \\ 2 & \mathrm{C} & -0.257301 \\ 3 & \mathrm{C} & 0.305066 \\ 4 & \mathrm{C} & -0.253861 \\ 5 & \mathrm{C} & 0.158206 \\ 6 & \mathrm{C} & -0.157844 \\ 7 & \mathrm{H} & -0.012403 \\ 8 & \mathrm{H} & 0.006208 \\ 9 & \mathrm{C} & 0.463767 \\ 10 & \mathrm{C} & -0.111859\end{array}$




$\begin{array}{llc}11 & \mathrm{C} & 0.114752 \\ 12 & \mathrm{C} & -0.054477 \\ 13 & \mathrm{C} & 0.108520 \\ 14 & \mathrm{C} & -0.043698 \\ 15 & \mathrm{C} & 0.078772 \\ 16 & \mathrm{H} & -0.005122 \\ 17 & \mathrm{H} & 0.001938 \\ 18 & \mathrm{H} & -0.005277 \\ 19 & \mathrm{H} & 0.001614 \\ 20 & \mathrm{C} & 0.162754 \\ 21 & \mathrm{C} & -0.366366 \\ 22 & \mathrm{C} & -0.112962 \\ 23 & \mathrm{C} & 0.197594 \\ 24 & \mathrm{C} & -0.201650 \\ 25 & \mathrm{C} & 0.125268 \\ 26 & \mathrm{C} & -0.265136 \\ 27 & \mathrm{H} & 0.008920 \\ 28 & \mathrm{C} & 0.125279 \\ 29 & \mathrm{C} & -0.183702 \\ 30 & \mathrm{C} & -0.243170 \\ 31 & \mathrm{C} & 0.157091 \\ 32 & \mathrm{H} & -0.004908 \\ 33 & \mathrm{H} & 0.008184 \\ 34 & \mathrm{H} & 0.010969 \\ 35 & \mathrm{H} & -0.006001 \\ 36 & \mathrm{H} & 0.011759 \\ 37 & \mathrm{H} & 0.015450 \\ 38 & \mathrm{H} & -0.019402\end{array}$

\section{Singlet Closed Shell:}

Sum of electronic and zero-point Energies=

$-922.159087$

Sum of electronic and thermal Energies=

$-922.143485$

Sum of electronic and thermal Enthalpies=

$-922.142541$

Sum of electronic and thermal Free Energies=

$-922.202136$

$\begin{array}{lrrc}\mathrm{C} & 0.226157000 & -0.860074000 & 0.000000000 \\ \mathrm{C} & 0.547619000 & 0.573511000 & 0.000000000 \\ \mathrm{C} & -0.469478000 & 1.477990000 & 0.000000000 \\ \mathrm{C} & -1.838505000 & 1.060921000 & 0.000000000 \\ \mathrm{C} & -2.150968000 & -0.371214000 & 0.000000000 \\ \mathrm{C} & -1.162377000 & -1.281188000 & 0.000000000 \\ \mathrm{H} & -0.267814000 & 2.543699000 & 0.000000000 \\ \mathrm{H} & -1.366304000 & -2.349109000 & 0.000000000 \\ \mathrm{C} & -3.003255000 & 1.769347000 & 0.000000000\end{array}$




$\begin{array}{lrrc}\mathrm{C} & -4.122905000 & 0.837583000 & 0.000000000 \\ \mathrm{C} & -5.497055000 & 1.050503000 & 0.000000000 \\ \mathrm{C} & -6.347814000 & -0.052140000 & 0.000000000 \\ \mathrm{C} & -5.840063000 & -1.350837000 & 0.000000000 \\ \mathrm{C} & -4.463575000 & -1.573677000 & 0.000000000 \\ \mathrm{C} & -3.611254000 & -0.482510000 & 0.000000000 \\ \mathrm{H} & -5.902634000 & 2.058063000 & 0.000000000 \\ \mathrm{H} & -7.422789000 & 0.101256000 & 0.000000000 \\ \mathrm{H} & -6.522165000 & -2.195205000 & 0.000000000 \\ \mathrm{H} & -4.073093000 & -2.587601000 & 0.000000000 \\ \mathrm{C} & 1.974334000 & 0.956909000 & 0.000000000 \\ \mathrm{C} & 1.215676000 & -1.787664000 & 0.000000000 \\ \mathrm{C} & 2.974832000 & -0.060017000 & 0.000000000 \\ \mathrm{C} & 2.612577000 & -1.440821000 & 0.000000000 \\ \mathrm{C} & 2.391757000 & 2.277265000 & 0.000000000 \\ \mathrm{C} & 4.351731000 & 0.287448000 & 0.000000000 \\ \mathrm{C} & 3.595996000 & -2.412398000 & 0.000000000 \\ \mathrm{H} & 1.661943000 & 3.078375000 & 0.000000000 \\ \mathrm{C} & 3.752031000 & 2.623111000 & 0.000000000 \\ \mathrm{C} & 4.719337000 & 1.651699000 & 0.000000000 \\ \mathrm{C} & 5.327018000 & -0.743327000 & 0.000000000 \\ \mathrm{C} & 4.957190000 & -2.062903000 & 0.000000000 \\ \mathrm{H} & 4.031137000 & 3.672088000 & 0.000000000 \\ \mathrm{H} & 5.772956000 & 1.914978000 & 0.000000000 \\ \mathrm{H} & 6.376848000 & -0.464737000 & 0.000000000 \\ \mathrm{H} & 5.711939000 & -2.842781000 & 0.000000000 \\ \mathrm{H} & 3.306966000 & -3.459521000 & 0.000000000 \\ \mathrm{H} & 0.956576000 & -2.843628000 & 0.000000000 \\ & -3.097599000 & 2.849016000 & 0.000000000\end{array}$

Triplet:

Sum of electronic and zero-point Energies=

$-922.147250$

Sum of electronic and thermal Energies=

$-922.131511$

$-922.130567$

Sum of electronic and thermal Enthalpies=

$-922.191090$ $\mathrm{S} * * 2=2.1433$
$\mathrm{C}$
0.783297000
$-0.424349000$
0.000000000
C $\quad 0.000000000$
0.761091000
0.000000000
C $\quad-1.414190000$
0.642132000
0.000000000
C $\quad-2.006444000$
$-0.596979000$
0.000000000
C $\quad-1.210553000$
$-1.787796000$
0.000000000
C $\quad 0.152518000$
$-1.697720000$
0.000000000
$\mathrm{H} \quad-2.038939000$
1.527379000
0.000000000
$\mathrm{H}$
0.778073000
$-2.586549000$
0.000000000 


$\begin{array}{lrrr}\mathrm{C} & -3.400915000 & -0.987010000 & 0.000000000 \\ \mathrm{C} & -3.462908000 & -2.401004000 & 0.000000000 \\ \mathrm{C} & -4.559126000 & -3.280373000 & 0.000000000 \\ \mathrm{C} & -4.324475000 & -4.644960000 & 0.000000000 \\ \mathrm{C} & -3.016577000 & -5.149176000 & 0.000000000 \\ \mathrm{C} & -1.916285000 & -4.291507000 & 0.000000000 \\ \mathrm{C} & -2.132099000 & -2.924184000 & 0.000000000 \\ \mathrm{H} & -5.573585000 & -2.892910000 & 0.000000000 \\ \mathrm{H} & -5.161765000 & -5.335809000 & 0.000000000 \\ \mathrm{H} & -2.858564000 & -6.223090000 & 0.000000000 \\ \mathrm{H} & -0.908511000 & -4.696634000 & 0.000000000 \\ \mathrm{C} & 0.669241000 & 2.051450000 & 0.000000000 \\ \mathrm{C} & 2.199322000 & -0.332011000 & 0.000000000 \\ \mathrm{C} & 2.087044000 & 2.101850000 & 0.000000000 \\ \mathrm{C} & 2.859378000 & 0.891135000 & 0.000000000 \\ \mathrm{C} & -0.037592000 & 3.267554000 & 0.000000000 \\ \mathrm{C} & 2.763461000 & 3.356740000 & 0.000000000 \\ \mathrm{C} & 4.277816000 & 0.978449000 & 0.000000000 \\ \mathrm{H} & -1.120286000 & 3.267931000 & 0.000000000 \\ \mathrm{C} & 0.623843000 & 4.489397000 & 0.000000000 \\ \mathrm{C} & 2.005812000 & 4.542893000 & 0.000000000 \\ \mathrm{C} & 4.182319000 & 3.383784000 & 0.000000000 \\ \mathrm{C} & 4.917147000 & 2.206968000 & 0.000000000 \\ \mathrm{H} & 0.046085000 & 5.408495000 & 0.000000000 \\ \mathrm{H} & 2.521186000 & 5.498840000 & 0.000000000 \\ \mathrm{H} & 4.685147000 & 4.346056000 & 0.000000000 \\ \mathrm{H} & 6.002014000 & 2.249370000 & 0.000000000 \\ \mathrm{H} & 4.854283000 & 0.058285000 & 0.000000000 \\ \mathrm{H} & 2.780625000 & -1.249997000 & 0.000000000 \\ \mathrm{H} & -4.245958000 & -0.309612000 & 0.000000000\end{array}$


Compound HZ

Natural Orbital Coefficient

HOMO: 1.53579 LUMO: 0.46421

Singlet biradical character $\mathrm{y}_{0}=0.167$

\section{Singlet Biradical Open Shell:}

Sum of electronic and zero-point Energies=

Sum of electronic and thermal Energies=

Sum of electronic and thermal Enthalpies=

Sum of electronic and thermal Free Energies= $\mathrm{S}^{* * 2}=1.5736$

$\begin{array}{lrrr}\mathrm{C} & -1.344192000 & -0.509244000 & 0.000002000 \\ \mathrm{C} & -0.265037000 & -1.362226000 & 0.000010000 \\ \mathrm{C} & 1.080448000 & -0.909148000 & 0.000008000 \\ \mathrm{C} & 1.344192000 & 0.509244000 & 0.000005000 \\ \mathrm{C} & 0.265037000 & 1.362226000 & -0.000009000 \\ \mathrm{C} & -1.080448000 & 0.909148000 & -0.000009000 \\ \mathrm{H} & -0.413524000 & -2.436626000 & 0.000017000 \\ \mathrm{H} & 0.413524000 & 2.436626000 & -0.000025000 \\ \mathrm{C} & -2.739107000 & -0.966546000 & 0.000002000 \\ \mathrm{C} & -3.785740000 & -0.002068000 & 0.000000000 \\ \mathrm{C} & -3.085393000 & -2.315373000 & 0.000003000 \\ \mathrm{C} & -3.488998000 & 1.400526000 & -0.000009000 \\ \mathrm{C} & -5.144850000 & -0.420845000 & 0.000004000 \\ \mathrm{C} & -4.421386000 & -2.726851000 & 0.000006000 \\ \mathrm{H} & -2.313176000 & -3.075478000 & -0.000001000 \\ \mathrm{C} & -4.540203000 & 2.323509000 & -0.000010000 \\ \mathrm{C} & -6.174610000 & 0.556739000 & 0.000003000 \\ \mathrm{C} & -5.438882000 & -1.800754000 & 0.000008000 \\ \mathrm{H} & -4.650716000 & -3.787909000 & 0.000007000 \\ \mathrm{C} & -5.871828000 & 1.899869000 & -0.000004000 \\ \mathrm{H} & -4.307026000 & 3.384259000 & -0.000017000 \\ \mathrm{H} & -7.208266000 & 0.223825000 & 0.000007000 \\ \mathrm{H} & -6.477415000 & -2.118592000 & 0.000012000 \\ \mathrm{H} & -6.669211000 & 2.636513000 & -0.000005000 \\ \mathrm{C} & 2.739107000 & 0.966546000 & 0.000014000 \\ \mathrm{C} & 3.785740000 & 0.002068000 & -0.000002000 \\ \mathrm{C} & 3.085393000 & 2.315373000 & 0.000040000 \\ \mathrm{C} & 3.488998000 & -1.400526000 & -0.000010000 \\ \mathrm{C} & 5.144850000 & 0.420845000 & -0.000005000 \\ \mathrm{C} & 4.421386000 & 2.726851000 & 0.000038000 \\ \mathrm{H} & 2.313176000 & 3.075478000 & 0.000067000\end{array}$

$-1075.688769$

$-1075.670604$

$-1075.669659$

$-1075.734715$

0.000002000

.000008000

$-0.000009000$

$-0.000025000$

0.000002000

0.000003000

0.000004000

0.000006000

$-0.000001000$

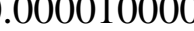

0.000003000

0.000007000

$-0.000004000$

0.000017000

0.000007000

$-0.000005000$

0.000014000

0.000040000

$-0.000010000$

0.000038000

0.000067000 


$\begin{array}{lrrr}\mathrm{C} & 4.540203000 & -2.323509000 & -0.000026000 \\ \mathrm{C} & 6.174611000 & -0.556739000 & -0.000023000 \\ \mathrm{C} & 5.438882000 & 1.800754000 & 0.000013000 \\ \mathrm{H} & 4.650716000 & 3.787909000 & 0.000056000 \\ \mathrm{C} & 5.871828000 & -1.899869000 & -0.000034000 \\ \mathrm{H} & 4.307026000 & -3.384259000 & -0.000032000 \\ \mathrm{H} & 7.208266000 & -0.223825000 & -0.000027000 \\ \mathrm{H} & 6.477415000 & 2.118593000 & 0.000009000 \\ \mathrm{H} & 6.669211000 & -2.636512000 & -0.000047000 \\ \mathrm{C} & -2.135305000 & 1.815523000 & -0.000015000 \\ \mathrm{C} & 2.135305000 & -1.815523000 & 0.000001000 \\ \mathrm{H} & -1.917592000 & 2.880231000 & -0.000024000 \\ \mathrm{H} & 1.917592000 & -2.880231000 & 0.000000000\end{array}$

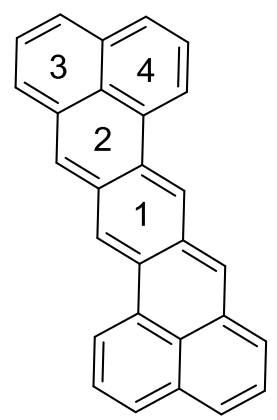

HZ

\begin{tabular}{rrrrr} 
& \multicolumn{4}{l}{ NICS values (sigma zz) } \\
Bq distance $(\AA)$ & ring 1 & \multicolumn{1}{l}{ ring 2 } & ring 3 & ring 4 \\
0.0000 & 10.8506 & 24.8921 & 3.3048 & -3.1309 \\
0.1000 & 10.3248 & 24.4272 & 2.8131 & -3.6508 \\
0.2000 & 8.8151 & 23.0760 & 1.4021 & -5.1363 \\
0.3000 & 6.5102 & 20.9613 & -0.7497 & -7.3821 \\
0.4000 & 3.6784 & 18.2646 & -3.3878 & -10.0977 \\
0.5000 & 0.6158 & 15.1977 & -6.2303 & -12.9665 \\
0.6000 & -2.4038 & 11.9729 & -9.0157 & -15.7006 \\
0.7000 & -5.1638 & 8.7790 & -11.5358 & -18.0791 \\
0.8000 & -7.5211 & 5.7645 & -13.6522 & -19.9642 \\
0.9000 & -9.4053 & 3.0321 & -15.2960 & -21.2984 \\
1.0000 & -10.8063 & 0.6396 & -16.4570 & -22.0889 \\
1.1000 & -11.7570 & -1.3924 & -17.1672 & -22.3882 \\
1.2000 & -12.3157 & -3.0714 & -17.4840 & -22.2730 \\
1.3000 & -12.5517 & -4.4232 & -17.4763 & -21.8294 \\
1.4000 & -12.5351 & -5.4836 & -17.2143 & -21.1410 \\
1.5000 & -12.3301 & -6.2923 & -16.7631 & -20.2828
\end{tabular}




$\begin{array}{lllll}1.6000 & -11.9921 & -6.8888 & -16.1789 & -19.3177 \\ 1.7000 & -11.5664 & -7.3095 & -15.5082 & -18.2962 \\ 1.8000 & -11.0885 & -7.5868 & -14.7880 & -17.2571 \\ 1.9000 & -10.5854 & -7.7485 & -14.0464 & -16.2286 \\ 2.0000 & -10.0767 & -7.8182 & -13.3044 & -15.2308 \\ 2.1000 & -9.5761 & -7.8154 & -12.5767 & -14.2766 \\ 2.2000 & -9.0929 & -7.7562 & -11.8735 & -13.3740 \\ 2.3000 & -8.6326 & -7.6536 & -11.2012 & -12.5270 \\ 2.4000 & -8.1984 & -7.5183 & -10.5635 & -11.7368 \\ 2.5000 & -7.7916 & -7.3587 & -9.9622 & -11.0025 \\ 2.6000 & -7.4122 & -7.1816 & -9.3975 & -10.3223 \\ 2.7000 & -7.0593 & -6.9925 & -8.8689 & -9.6933 \\ 2.8000 & -6.7317 & -6.7957 & -8.3750 & -9.1123 \\ 2.9000 & -6.4276 & -6.5944 & -7.9141 & -8.5758 \\ 3.0000 & -6.1454 & -6.3915 & -7.4845 & -8.0803 \\ 3.1000 & -5.8833 & -6.1888 & -7.0840 & -7.6227 \\ 3.2000 & -5.6395 & -5.9879 & -6.7107 & -7.1996 \\ 3.3000 & -5.4124 & -5.7901 & -6.3627 & -6.8081 \\ 3.4000 & -5.2004 & -5.5963 & -6.0381 & -6.4455 \\ 3.5000 & -5.0021 & -5.4071 & -5.7352 & -6.1092 \\ 3.6000 & -4.8163 & -5.2229 & -5.4522 & -5.7970 \\ 3.7000 & -4.6417 & -5.0441 & -5.1878 & -5.5068 \\ 3.8000 & -4.4774 & -4.8710 & -4.9403 & -5.2366 \\ 3.9000 & -4.3224 & -4.7035 & -4.7087 & -4.9848 \\ 4.0000 & -4.1758 & -4.5419 & -4.4916 & -4.7498 \\ 4.1000 & -4.0370 & -4.3859 & -4.2879 & -4.5301 \\ 4.2000 & -3.9052 & -4.2357 & -4.0967 & -4.3246 \\ 4.3000 & -3.7799 & -4.0910 & -3.9170 & -4.1321 \\ 4.4000 & -3.6606 & -3.9519 & -3.7480 & -3.9515 \\ 4.5000 & -3.5467 & -3.8180 & -3.5889 & -3.7819 \\ 4.6000 & -3.4379 & -3.6893 & -3.4389 & -3.6225 \\ 4.7000 & -3.3337 & -3.5657 & -3.2975 & -3.4724 \\ 4.8000 & -3.2340 & -3.4469 & -3.1641 & -3.3310 \\ 4.9000 & -3.1383 & -3.3328 & -3.0380 & -3.1976 \\ 5.0000 & -3.0465 & -3.2232 & -2.9188 & -3.0717\end{array}$

Mulliken atomic spin densities:

$\begin{array}{llc}1 & \mathrm{C} & -0.257098 \\ 2 & \mathrm{C} & 0.298622 \\ 3 & \mathrm{C} & -0.276717 \\ 4 & \mathrm{C} & 0.257098 \\ 5 & \mathrm{C} & -0.298622 \\ 6 & \mathrm{C} & 0.276717\end{array}$




$\begin{array}{rlc}7 & \mathrm{H} & -0.012142 \\ 8 & \mathrm{H} & 0.012142 \\ 9 & \mathrm{C} & 0.174466 \\ 10 & \mathrm{C} & -0.138859 \\ 11 & \mathrm{C} & -0.215566 \\ 12 & \mathrm{C} & 0.235153 \\ 13 & \mathrm{C} & 0.145507 \\ 14 & \mathrm{C} & 0.136596 \\ 15 & \mathrm{H} & 0.009542 \\ 16 & \mathrm{C} & -0.322451 \\ 17 & \mathrm{C} & -0.299775 \\ 18 & \mathrm{C} & -0.197610 \\ 19 & \mathrm{H} & -0.005383 \\ 20 & \mathrm{C} & 0.188123 \\ 21 & \mathrm{H} & 0.014361 \\ 22 & \mathrm{H} & 0.013616 \\ 23 & \mathrm{H} & 0.008793 \\ 24 & \mathrm{H} & -0.007154 \\ 25 & \mathrm{C} & -0.174466 \\ 26 & \mathrm{C} & 0.138859 \\ 27 & \mathrm{C} & 0.215566 \\ 28 & \mathrm{C} & -0.235153 \\ 29 & \mathrm{C} & -0.145506 \\ 30 & \mathrm{C} & -0.136596 \\ 31 & \mathrm{H} & -0.009542 \\ 32 & \mathrm{C} & 0.322451 \\ 33 & \mathrm{C} & 0.299774 \\ 34 & \mathrm{C} & 0.197610 \\ 35 & \mathrm{H} & 0.005383 \\ 36 & \mathrm{C} & -0.188123 \\ 37 & \mathrm{H} & -0.014361 \\ 38 & \mathrm{H} & -0.013616 \\ 39 & \mathrm{H} & -0.008793 \\ 40 & \mathrm{H} & 0.007154 \\ 41 & \mathrm{C} & -0.470998 \\ 42 & \mathrm{C} & 0.470998 \\ 43 & \mathrm{H} & 0.019895 \\ 44 & \mathrm{H} & -0.019895\end{array}$

\section{Singlet Closed Shell:}

Sum of electronic and zero-point Energies= Sum of electronic and thermal Energies= Sum of electronic and thermal Enthalpies=
$-1075.680828$

$-1075.662714$

$-1075.661770$ 
Sum of electronic and thermal Free Energies=

\begin{tabular}{|c|c|c|c|}
\hline $\mathrm{C}$ & -1.347594000 & -0.522614000 & -0.000001000 \\
\hline $\mathrm{C}$ & -0.285628000 & -1.364062000 & -0.000004000 \\
\hline $\mathrm{C}$ & 1.084852000 & -0.915534000 & -0.000003000 \\
\hline $\mathrm{C}$ & 1.347594000 & 0.522614000 & 0.000001000 \\
\hline $\mathrm{C}$ & 0.285628000 & 1.364062000 & 0.000004000 \\
\hline $\mathrm{C}$ & -1.084852000 & 0.915534000 & 0.000003000 \\
\hline $\mathrm{H}$ & -0.431124000 & -2.439298000 & -0.000008000 \\
\hline $\mathrm{H}$ & 0.431124000 & 2.439298000 & 0.000008000 \\
\hline $\mathrm{C}$ & -2.754068000 & -0.973467000 & -0.000002000 \\
\hline $\mathrm{C}$ & -3.795527000 & 0.000937000 & 0.000001000 \\
\hline $\mathrm{C}$ & -3.108162000 & -2.311597000 & -0.000006000 \\
\hline $\mathrm{C}$ & -3.490747000 & 1.397577000 & 0.000006000 \\
\hline $\mathrm{C}$ & -5.155868000 & -0.407956000 & 0.000001000 \\
\hline $\mathrm{C}$ & -4.451710000 & -2.717084000 & -0.000007000 \\
\hline $\mathrm{H}$ & -2.341748000 & -3.078068000 & -0.000009000 \\
\hline $\mathrm{C}$ & -4.519904000 & 2.322015000 & 0.000009000 \\
\hline $\mathrm{C}$ & -6.177417000 & 0.576996000 & 0.000004000 \\
\hline $\mathrm{C}$ & -5.459965000 & -1.788067000 & -0.000004000 \\
\hline $\mathrm{H}$ & -4.685457000 & -3.777148000 & -0.000011000 \\
\hline $\mathrm{C}$ & -5.863584000 & 1.910996000 & 0.000008000 \\
\hline $\mathrm{H}$ & -4.279554000 & 3.381356000 & 0.000013000 \\
\hline $\mathrm{H}$ & -7.213800000 & 0.252214000 & 0.000003000 \\
\hline $\mathrm{H}$ & -6.500916000 & -2.098057000 & -0.000004000 \\
\hline $\mathrm{H}$ & -6.651797000 & 2.657369000 & 0.000011000 \\
\hline $\mathrm{C}$ & 2.754068000 & 0.973467000 & 0.000002000 \\
\hline $\mathrm{C}$ & 3.795527000 & -0.000937000 & -0.000001000 \\
\hline $\mathrm{C}$ & 3.108162000 & 2.311597000 & 0.000006000 \\
\hline $\mathrm{C}$ & 3.490747000 & -1.397577000 & -0.000006000 \\
\hline $\mathrm{C}$ & 5.155868000 & 0.407956000 & -0.000001000 \\
\hline $\mathrm{C}$ & 4.451710000 & 2.717084000 & 0.000007000 \\
\hline $\mathrm{H}$ & 2.341748000 & 3.078068000 & 0.000009000 \\
\hline $\mathrm{C}$ & 4.519904000 & -2.322015000 & -0.000009000 \\
\hline $\mathrm{C}$ & 6.177417000 & -0.576996000 & -0.000004000 \\
\hline $\mathrm{C}$ & 5.459965000 & 1.788067000 & 0.000004000 \\
\hline $\mathrm{H}$ & 4.685457000 & 3.777148000 & 0.000011000 \\
\hline $\mathrm{C}$ & 5.863584000 & -1.910996000 & -0.000008000 \\
\hline $\mathrm{H}$ & 4.279554000 & -3.381356000 & -0.000013000 \\
\hline $\mathrm{H}$ & 7.213800000 & -0.252214000 & -0.000003000 \\
\hline $\mathrm{H}$ & 6.500916000 & 2.098057000 & 0.000005000 \\
\hline $\mathrm{H}$ & 6.651797000 & -2.657369000 & -0.000011000 \\
\hline $\mathrm{C}$ & -2.111378000 & 1.805493000 & 0.000007000 \\
\hline $\mathrm{C}$ & 2.111378000 & -1.805493000 & -0.000007000 \\
\hline
\end{tabular}

$-1075.727103$

$-0.000001000$

0.000001000

(2)

0.000008000$$
-0.000002000
$$

0.000001000

.000006000

0.000001000

$-0.000007000$

$-0.000009000$

0.000004000

$-0.000004000$

000

0.000013000

0.00003000

0.000011000

0.000002000

0.000001000

006000

$-0.000001000$

0007000

$-0.000009000$

(2.00004000

0.000011000

$-0.000008000$ 


$\begin{array}{lrrr}\mathrm{H} & -1.896556000 & 2.871121000 & 0.000010000 \\ \mathrm{H} & 1.896556000 & -2.871121000 & -0.000010000\end{array}$

\section{Triplet:}

SCF Done: $\quad$ E(UCAM-B3LYP $)=-1076.02418006$

$$
\mathrm{S} * * 2=2.1661
$$

a.u. after 1 cycles

Sum of electronic and zero-point Energies=

Sum of electronic and thermal Energies=

Sum of electronic and thermal Enthalpies=

Sum of electronic and thermal Free Energies= $\mathrm{S} * * 2=2.268$

$\begin{array}{lrrr}\text { C } & -1.338296000 & -0.497883000 & -0.000001000 \\ \mathrm{C} & -0.246824000 & -1.359761000 & -0.000004000 \\ \mathrm{C} & 1.074840000 & -0.901815000 & -0.000003000 \\ \mathrm{C} & 1.338296000 & 0.497883000 & 0.000001000 \\ \mathrm{C} & 0.246824000 & 1.359761000 & 0.000004000 \\ \mathrm{C} & -1.074840000 & 0.901815000 & 0.000003000 \\ \mathrm{H} & -0.397620000 & -2.433517000 & -0.000008000 \\ \mathrm{H} & 0.397620000 & 2.433517000 & 0.000008000 \\ \mathrm{C} & -2.727000000 & -0.961486000 & -0.000002000 \\ \mathrm{C} & -3.774843000 & -0.007625000 & 0.000001000 \\ \mathrm{C} & -3.063898000 & -2.319664000 & -0.000006000 \\ \mathrm{C} & -3.484504000 & 1.404306000 & 0.000006000 \\ \mathrm{C} & -5.133061000 & -0.434656000 & 0.000001000 \\ \mathrm{C} & -4.392306000 & -2.736733000 & -0.000007000 \\ \mathrm{H} & -2.285762000 & -3.073192000 & -0.000009000 \\ \mathrm{C} & -4.562377000 & 2.327018000 & 0.000009000 \\ \mathrm{C} & -6.171252000 & 0.536730000 & 0.000004000 \\ \mathrm{C} & -5.417948000 & -1.812721000 & -0.000004000 \\ \mathrm{H} & -4.617971000 & -3.798600000 & -0.000010000 \\ \mathrm{C} & -5.879105000 & 1.890448000 & 0.000008000 \\ \mathrm{H} & -4.336105000 & 3.389002000 & 0.000012000 \\ \mathrm{H} & -7.202168000 & 0.196128000 & 0.000003000 \\ \mathrm{H} & -6.454271000 & -2.137622000 & -0.000004000 \\ \mathrm{H} & -6.686282000 & 2.616472000 & 0.000011000 \\ \mathrm{C} & 2.727000000 & 0.961486000 & 0.000002000 \\ \mathrm{C} & 3.774843000 & 0.007625000 & -0.000001000 \\ \mathrm{C} & 3.063898000 & 2.319664000 & 0.000006000 \\ \mathrm{C} & 3.484504000 & -1.404306000 & -0.000006000 \\ \mathrm{C} & 5.133061000 & 0.434656000 & -0.000001000 \\ \mathrm{C} & 4.392306000 & 2.736733000 & 0.000007000 \\ \mathrm{H} & 2.285762000 & 3.073192000 & 0.000009000 \\ \mathrm{C} & 4.562377000 & -2.327018000 & -0.000009000\end{array}$

$-\mathrm{V} / \mathrm{T}=2.009$

$-1075.674976$

$-1075.656776$

$-1075.655832$

$-1075.721844$

$-0.000001000$

$-0.000004000$

0.000001000

0.000004000

0.000003000

.000008000

0.000002000

0.000001000

$-0.000006000$

0.000006000

0.000001000

$-0.000007000$

0.000009000

0.000004000

$-0.000004000$

$-0.000010000$

0.000008000

0.000012000

0.000003000

0.000004000

0.000002000

$-0.000001000$

0.000006000

$-0.000006000$

$-0.000001000$

0.00000700

$-0.000009000$ 


$\begin{array}{lrrr}\mathrm{C} & 6.171252000 & -0.536730000 & -0.000004000 \\ \mathrm{C} & 5.417948000 & 1.812721000 & 0.000004000 \\ \mathrm{H} & 4.617971000 & 3.798600000 & 0.000010000 \\ \mathrm{C} & 5.879105000 & -1.890448000 & -0.000008000 \\ \mathrm{H} & 4.336105000 & -3.389002000 & -0.000013000 \\ \mathrm{H} & 7.202168000 & -0.196128000 & -0.000003000 \\ \mathrm{H} & 6.454271000 & 2.137622000 & 0.000005000 \\ \mathrm{H} & 6.686282000 & -2.616472000 & -0.000011000 \\ \mathrm{C} & -2.161861000 & 1.825862000 & 0.000007000 \\ \mathrm{C} & 2.161861000 & -1.825862000 & -0.000007000 \\ \mathrm{H} & -1.940572000 & 2.889544000 & 0.000010000 \\ \mathrm{H} & 1.940572000 & -2.889544000 & -0.000010000\end{array}$




\section{Compound IF}

\section{Singlet Closed Shell:}

Sum of electronic and zero-point Energies= Sum of electronic and thermal Energies= Sum of electronic and thermal Enthalpies= Sum of electronic and thermal Free Energies=

$\begin{array}{lrrr}\mathrm{C} & 0.964919000 & 1.016583000 & 0.000618000 \\ \mathrm{C} & 1.352581000 & -0.402874000 & 0.000058000 \\ \mathrm{C} & 0.429543000 & -1.385718000 & -0.000537000 \\ \mathrm{C} & -0.964919000 & -1.016583000 & -0.000618000 \\ \mathrm{C} & -1.352581000 & 0.402874000 & -0.000058000 \\ \mathrm{C} & -0.429543000 & 1.385718000 & 0.000537000 \\ \mathrm{C} & 2.089201000 & 1.781320000 & 0.001153000 \\ \mathrm{C} & 3.262653000 & 0.912972000 & 0.000975000 \\ \mathrm{C} & 2.820296000 & -0.430375000 & 0.000303000 \\ \mathrm{C} & -2.089201000 & -1.781320000 & -0.001153000 \\ \mathrm{C} & -3.262653000 & -0.912972000 & -0.000975000 \\ \mathrm{C} & -2.820296000 & 0.430375000 & -0.000303000 \\ \mathrm{C} & 4.619380000 & 1.205791000 & 0.001342000 \\ \mathrm{C} & 5.532434000 & 0.152052000 & 0.001036000 \\ \mathrm{C} & 5.095036000 & -1.170274000 & 0.000375000 \\ \mathrm{C} & 3.731835000 & -1.471199000 & 0.000003000 \\ \mathrm{C} & -4.619380000 & -1.205791000 & -0.001342000 \\ \mathrm{C} & -5.532434000 & -0.152052000 & -0.001036000 \\ \mathrm{C} & -5.095036000 & 1.170274000 & -0.000375000 \\ \mathrm{C} & -3.731835000 & 1.471199000 & -0.000003000 \\ \mathrm{H} & 0.707646000 & -2.436367000 & -0.000952000 \\ \mathrm{H} & -0.707646000 & 2.436367000 & 0.000952000 \\ \mathrm{H} & 2.124523000 & 2.864943000 & 0.001638000 \\ \mathrm{H} & -2.124523000 & -2.864943000 & -0.001638000 \\ \mathrm{H} & 4.966336000 & 2.234992000 & 0.001858000 \\ \mathrm{H} & 6.597048000 & 0.364544000 & 0.001317000 \\ \mathrm{H} & 5.822055000 & -1.976369000 & 0.000146000 \\ \mathrm{H} & 3.400193000 & -2.505651000 & -0.000512000 \\ \mathrm{H} & -4.966336000 & -2.234992000 & -0.001858000 \\ \mathrm{H} & -6.597048000 & -0.364544000 & -0.001317000 \\ \mathrm{H} & -5.822055000 & 1.976369000 & -0.000146000 \\ \mathrm{H} & -3.400193000 & 2.505651000 & 0.000512000\end{array}$

$-768.636492$

$-768.623381$

$-768.622437$

$-768.676299$

0.000618000 


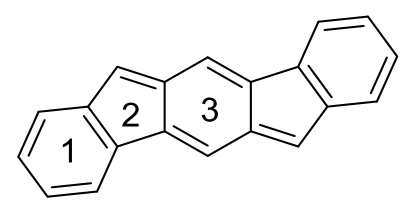

IF

\begin{tabular}{|c|c|c|c|}
\hline \multirow[b]{2}{*}{ Bq distance $(\AA)$} & \multirow[b]{2}{*}{ ring 1} & \multicolumn{2}{|c|}{ NICS values (sigma zz) } \\
\hline & & ring 2 & ring 3 \\
\hline 0.0000 & -1.5435 & 44.7697 & 34.5711 \\
\hline 0.1000 & -2.0222 & 44.3507 & 34.2269 \\
\hline 0.2000 & -3.3929 & 43.0894 & 33.2059 \\
\hline 0.3000 & -5.4742 & 40.9870 & 31.5458 \\
\hline 0.4000 & -8.0064 & 38.0850 & 29.3172 \\
\hline 0.5000 & -10.7026 & 34.4937 & 26.6267 \\
\hline 0.6000 & -13.2964 & 30.3973 & 23.6124 \\
\hline 0.7000 & -15.5777 & 26.0296 & 20.4287 \\
\hline 0.8000 & -17.4097 & 21.6345 & 17.2281 \\
\hline 0.9000 & -18.7295 & 17.4258 & 14.1428 \\
\hline 1.0000 & -19.5358 & 13.5616 & 11.2735 \\
\hline 1.1000 & -19.8716 & 10.1363 & 8.6853 \\
\hline 1.2000 & -19.8058 & 7.1869 & 6.4102 \\
\hline 1.3000 & -19.4182 & 4.7069 & 4.4540 \\
\hline 1.4000 & -18.7882 & 2.6623 & 2.8033 \\
\hline 1.5000 & -17.9877 & 1.0045 & 1.4336 \\
\hline 1.6000 & -17.0783 & -0.3196 & 0.3144 \\
\hline 1.7000 & -16.1094 & -1.3617 & -0.5866 \\
\hline 1.8000 & -15.1196 & -2.1688 & -1.3006 \\
\hline 1.9000 & -14.1373 & -2.7825 & -1.8571 \\
\hline 2.0000 & -13.1828 & -3.2382 & -2.2823 \\
\hline 2.1000 & -12.2697 & -3.5660 & -2.5994 \\
\hline 2.2000 & -11.4063 & -3.7911 & -2.8288 \\
\hline 2.3000 & -10.5972 & -3.9343 & -2.9875 \\
\hline 2.4000 & -9.8440 & -4.0132 & -3.0901 \\
\hline 2.5000 & -9.1464 & -4.0418 & -3.1486 \\
\hline 2.6000 & -8.5026 & -4.0317 & -3.1728 \\
\hline 2.7000 & -7.9100 & -3.9922 & -3.1707 \\
\hline 2.8000 & -7.3655 & -3.9307 & -3.1488 \\
\hline 2.9000 & -6.8657 & -3.8531 & -3.1119 \\
\hline 3.0000 & -6.4072 & -3.7640 & -3.0641 \\
\hline 3.1000 & -5.9866 & -3.6670 & -3.0084 \\
\hline 3.2000 & -5.6008 & -3.5649 & -2.9472 \\
\hline 3.3000 & -5.2466 & -3.4599 & -2.8822 \\
\hline
\end{tabular}




$\begin{array}{llll}3.4000 & -4.9212 & -3.3536 & -2.8149 \\ 3.5000 & -4.6220 & -3.2472 & -2.7463 \\ 3.6000 & -4.3466 & -3.1417 & -2.6772 \\ 3.7000 & -4.0929 & -3.0377 & -2.6081 \\ 3.8000 & -3.8588 & -2.9359 & -2.5395 \\ 3.9000 & -3.6426 & -2.8366 & -2.4718 \\ 4.0000 & -3.4426 & -2.7400 & -2.4051 \\ 4.1000 & -3.2575 & -2.6463 & -2.3397 \\ 4.2000 & -3.0858 & -2.5556 & -2.2756 \\ 4.3000 & -2.9265 & -2.4680 & -2.2130 \\ 4.4000 & -2.7784 & -2.3835 & -2.1518 \\ 4.5000 & -2.6405 & -2.3021 & -2.0922 \\ 4.6000 & -2.5120 & -2.2237 & -2.0342 \\ 4.7000 & -2.3922 & -2.1482 & -1.9776 \\ 4.8000 & -2.2803 & -2.0756 & -1.9227 \\ 4.9000 & -2.1756 & -2.0059 & -1.8692 \\ 5.0000 & -2.0775 & -1.9388 & -1.8172\end{array}$

\section{Triplet:}

Sum of electronic and zero-point Energies= Sum of electronic and thermal Energies= Sum of electronic and thermal Enthalpies= Sum of electronic and thermal Free Energies= $\mathrm{S} * * 2=2.1433$

$\begin{array}{lrrr}\mathrm{C} & 0.950633000 & 1.008303000 & 0.000612000 \\ \mathrm{C} & 1.341035000 & -0.356618000 & 0.000077000 \\ \mathrm{C} & 0.394780000 & -1.373345000 & -0.000538000 \\ \mathrm{C} & -0.950633000 & -1.008303000 & -0.000612000 \\ \mathrm{C} & -1.341035000 & 0.356618000 & -0.000077000 \\ \mathrm{C} & -0.394780000 & 1.373345000 & 0.000538000 \\ \mathrm{C} & 2.151508000 & 1.822182000 & 0.001182000 \\ \mathrm{C} & 3.275016000 & 0.956750000 & 0.000996000 \\ \mathrm{C} & 2.808302000 & -0.393250000 & 0.000318000 \\ \mathrm{C} & -2.151508000 & -1.822182000 & -0.001182000 \\ \mathrm{C} & -3.275016000 & -0.956750000 & -0.000996000 \\ \mathrm{C} & -2.808302000 & 0.393250000 & -0.000318000 \\ \mathrm{C} & 4.651623000 & 1.221449000 & 0.001355000 \\ \mathrm{C} & 5.541336000 & 0.155937000 & 0.001040000 \\ \mathrm{C} & 5.076373000 & -1.162409000 & 0.000375000 \\ \mathrm{C} & 3.705893000 & -1.442915000 & 0.000011000 \\ \mathrm{C} & -4.651623000 & -1.221449000 & -0.001355000 \\ \mathrm{C} & -5.541336000 & -0.155937000 & -0.001040000 \\ \mathrm{C} & -5.076373000 & 1.162409000 & -0.000375000\end{array}$

$-768.614432$

$-768.601121$

$-768.600176$

$-768.655309$

0.000612000

0.000077000

$-0.000077000$

0.001182000

0.000996000$$
-0.001182000
$$

$-0.000996000$

0318000

$-0.000375000$ 


$\begin{array}{lrcc}\mathrm{C} & -3.705893000 & 1.442915000 & -0.000011000 \\ \mathrm{H} & 0.688999000 & -2.419188000 & -0.000948000 \\ \mathrm{H} & -0.688999000 & 2.419188000 & 0.000948000 \\ \mathrm{H} & 2.177963000 & 2.905025000 & 0.001665000 \\ \mathrm{H} & -2.177963000 & -2.905025000 & -0.001665000 \\ \mathrm{H} & 5.014883000 & 2.244929000 & 0.001871000 \\ \mathrm{H} & 6.609991000 & 0.346069000 & 0.001311000 \\ \mathrm{H} & 5.789247000 & -1.981055000 & 0.000138000 \\ \mathrm{H} & 3.361619000 & -2.473170000 & -0.000505000 \\ \mathrm{H} & -5.014883000 & -2.244929000 & -0.001871000 \\ \mathrm{H} & -6.609991000 & -0.346069000 & -0.001311000 \\ \mathrm{H} & -5.789247000 & 1.981055000 & -0.000138000 \\ \mathrm{H} & -3.361619000 & 2.473170000 & 0.000505000\end{array}$

